\title{
DIGITALCOMMONS
}

$5-1-2012$

\section{Vol. 11, No. 1 (Full Issue)}

JMASM Editors

Follow this and additional works at: http://digitalcommons.wayne.edu/jmasm

\section{Recommended Citation}

Editors, JMASM (2012) "Vol. 11, No. 1 (Full Issue)," Journal of Modern Applied Statistical Methods: Vol. 11 : Iss. 1 , Article 29. DOI: $10.22237 /$ jmasm/1335846480

Available at: http://digitalcommons.wayne.edu/jmasm/vol11/iss1/29 


\section{Journal Of Modern Applied Statistical Methods}

Shlomo S. Sawilowsky

Editor

College of Education

Wayne State University

Harvey Keselman

Associate Editor

Department of Psychology

University of Manitoba

Bruno D. Zumbo

Associate Editor

Measurement, Evaluation, \& Research Methodology

University of British Columbia

Vance W. Berger

Assistant Editor

Biometry Research Group

National Cancer Institute

John L. Cuzzocrea

Assistant Editor

Educational Research

University of Akron

Todd C. Headrick Assistant Editor

Educational Psychology and Special Education

Southern Illinois University-Carbondale

Alan Klockars
Assistant Editor

Educational Psychology

University of Washington 


\section{Journal Of Modern Applied Statistical Methods}

Regular Articles

$2-23$

$24-41$

$42-53$

$54-68$

$69-77$

$78-94$

$95-105$

$106-122$

$123-143$

$144-151$
Tim Moses

Dandan Wang, Tiffany A. Whittaker, S. Natasha Beretvas

Marcelo Angelo Cirillo, Lúcia Pereira Barroso

Abdul R. Othman, Teh Sin Yin, H. J. Keselman, Rand R. Wilcox, James Algina

Sami Khedhiri, Ghassen EI Montasser

Ayman Alzaatreh, Felix Famoye, Carl Lee

Maher Qumsiyeh, Gerald Shaughnessy

Cheng C. Chen, Sarjinder Singh

Justice I. Odiase

Faisal Ababneh, Omar M. Eidous
Underlying Distributions in Loglinear Models of Discrete Data

The Impact of Violating Factor Scaling Method Assumptions on Latent Mean Difference Testing in Structured Means Models

Robust Regression Estimates in the Prediction of Latent Variables in Structural Equation Models

Robust Modifications of the Levene and O’Brien Tests for Spread

An Extension of the Seasonal KPSS Test

Gamma-Pareto Distribution and Its Applications

Comparison of Re-Sampling Methods to Generalized Linear Models and Transformations in Factorial and Fractional Factorial Designs

Estimation of Multinomial Proportions Using Higher Order Moments of Scrambling Variables in Randomized Response Sampling

Empirical Sampling from Permutation Space with Unique Patterns

A Weighted Exponential Detection Function Model for Line Transect Data 


\begin{tabular}{|c|c|}
\hline $152-157$ & Ghadban Khalaf \\
\hline $158-166$ & Vincent A. R. Camara \\
\hline $167-178$ & $\begin{array}{l}\text { W. Holmes Finch, } \\
\text { Brian F. French }\end{array}$ \\
\hline $179-189$ & $\begin{array}{l}\text { Parviz Nasiri, } \\
\text { Saman Hosseini }\end{array}$ \\
\hline $190-202$ & Gyan Prakash \\
\hline $203-210$ & Ameen Jameel Alawneh \\
\hline $211-217$ & $\begin{array}{l}\text { Jennifer L. Brown, } \\
\text { Gerald Halpin, } \\
\text { Glennelle Halpin }\end{array}$ \\
\hline $218-232$ & $\begin{array}{l}\text { Folefac Atem, } \\
\text { Julius S. Ngwa, } \\
\text { Abidemi Adeniji }\end{array}$ \\
\hline
\end{tabular}

$233-241$

$242-254$

$255-260$

$261-267$
W. Holmes Finch,

Parviz Nasiri, Saman Hosseini

Jennifer L. Brown, Gerald Halpin,

Folefac Atem, Abidemi Adeniji

Tze-San Lee

Xing Liu, Hari Koirala

Makarand Ratnaparkhi, Uttara Naik-Nimbalkar

Lin Zhang, Linjuan Tang
Improved Estimator in the Presence of Multicollinearity

New Approximate Bayesian Confidence Intervals for the Coefficient of Variation of a Gaussian Distribution

Parameter Estimation with Mixture Item Response Theory Models: A Monte Carlo Comparison of Maximum Likelihood and Bayesian Methods

Statistical Inferences for Lomax Distribution Based on Record Values (Bayesian and Classical)

Inverted Exponential Distribution Under a Bayesian Viewpoint

Steady State Probabilities of a Three Preemptive Single Server Queue

Weight: Does it Really Matter?

Regression Models for Mixed Over-Dispersed Poisson in Continuous Clustered Data: Modeling BMI and Number of Cigarettes Smoked Per Day

A Poisson Regression Model for Female Radium Dial Workers

Ordinal Regression Analysis: Using Generalized Ordinal Logistic Regression Models to Estimate Educational Data

The Length-Biased Lognormal Distribution and Its Application in the Analysis of Data from Oil Field Exploration Studies

A Study on Underwriting Cycle of Property Insurance Industry of China 
$268-273$

Abdel-Razzaq Mugdadi

James F. Reed III

$279-283$

Rajesh Tailor, Sunil Chouhan

JMASM Algorithms and Code

$284-295$

Hyewon Chung,

Jiseon Kim,

Ryoungsun Park
Four Period Crossover Designs

The Weighted Hellinger Distance for Kernal Distribution Estimator of Function of Observations

Ratio Type Estimator of Ratio of Two Population Means in Stratified Random Sampling

JMASM 32: SAS Template for Single-Subject Experimental Designs

$J M A S M$ is an independent print and electronic journal (http://www.jmasm.com/), publishing (1) new statistical tests or procedures, or the comparison of existing statistical tests or procedures, using computer-intensive Monte Carlo, bootstrap, jackknife, or resampling methods, (2) the study of nonparametric, robust, permutation, exact, and approximate randomization methods, and (3) applications of computer programming, preferably in Fortran (all other programming environments are welcome), related to statistical algorithms, pseudorandom number generators, simulation techniques, and self-contained executable code to carry out new or interesting statistical methods.

Editorial Assistant: Julie M. Smith, Ph. D. 


\title{
Regular Articles \\ Underlying Distributions in Loglinear Models of Discrete Data
}

\author{
Tim Moses \\ Educational Testing Service, \\ Princeton, NJ
}

The implications of loglinear models based on underlying uniform and binomial distribution are assessed with respect to modeling eight distributions. Regarding statistical selection of the loglinear models' parameterizations, results indicate that better fitting models are obtained when the distribution being modeled is dissimilar to the underlying distribution used. For loglinear models with predetermined numbers of parameters, results suggest that better fitting models can be obtained when the distribution being modeled is similar to the underlying distribution.

Key words: Loglinear models, uniform distribution, binomial distribution.

\section{Introduction}

Loglinear models are used to estimate the distributions of discrete data that occur in applied research involving political questionnaires, biomedical data and psychometric testing (Agresti, 2002; Bishop, Fienberg \& Holland, 1975; Holland \& Thayer, 2000; Kolen \& Brennan, 2004). The choice of most interest when selecting a plausible loglinear model for a particular discrete distribution is usually the number of moments of the observed distribution to preserve in the modeled distribution. A less familiar choice pertains to the distribution that underlies the loglinear model, which is obtained when most or all of the loglinear model's parameters are set to zero. This study considers the implications of using different underlying distributions specifically uniform and binomial distributions for loglinear models of discrete distributions.

Tim Moses is a Senior Psychometrician at Educational Testing Service where he works on several testing programs. He completed his Ph.D. in Educational Psychology at the University of Washington. Please send correspondence regarding this manuscript to Tim Moses, Educational Testing Service, Rosedale Road MS 03-P, Princeton, NJ 08541. Email him at: tmoses@ets.org.
Loglinear Models of Discrete Distributions

Loglinear models of discrete distributions relate the log of a model's expected probabilities, $\rho$, to a linear function of a categorical variable's values, for example the scores of a psychometric test,

$$
\log _{e}(\boldsymbol{\rho})=\alpha+\boldsymbol{\mu}+\mathbf{X} \boldsymbol{\beta}
$$

where $\boldsymbol{\rho}$ is an $I$-by- 1 column vector of the probabilities, $\alpha$ is a normalizing constant which ensures that the sum of the entries of $\boldsymbol{\rho}$ is 1 , $\sum_{i} \rho_{i}=1, \boldsymbol{\mu}$ is an $I$-by- 1 column vector of known constants, $\mathbf{X}$ is an $I$-by- $K$ design matrix containing $K$ functions of categorical variable $X$, and $\boldsymbol{\beta}$ is a $K$-by-1 column vector of free parameters. The $k=1$ to $K$ columns of $\mathbf{X}$ give the first through $K^{\text {th }}$ degrees of the $X$ values that can be expressed as power functions, $x_{i}^{k}$, or as used in this study, the more numerically stable and less collinear orthogonal polynomials. When maximum likelihood estimation is used for model 1 then the estimation results in the first derivative of the log-likelihood being set to zero, or,

$$
\sum_{i} \widehat{\rho}_{i} x_{i}^{k}=\sum_{i} \frac{n_{i}}{N} x_{i}^{k},
$$


where $n_{i}$ is the observed frequency of the $i^{\text {th }}$ category value of $X$ and $N$ is the total sample size. Equation 2 implies that the first $K$ moments of $X$ 's observed distribution will be preserved in the loglinear model's distribution (Agresti, 2002; Holland \& Thayer, 2000).

\section{Loglinear Models’ Underlying Distributions}

Specific values of $\boldsymbol{\mu}$ can result in

loglinear models such as model 1 reflecting different underlying distributions. When $\boldsymbol{\mu}$ is a vector of zeros or of any constant and $\boldsymbol{\beta}$ is also zero the loglinear model resolves into a uniform distribution, $\log _{e}(\boldsymbol{\rho})=\alpha$. The loglinear model that produces a uniform distribution reflects the notion that the $i$-level probabilities are all equal and independent of $X$.

Another choice for the loglinear model's underlying distribution is available when $\boldsymbol{\mu}$ is defined as $I$ constants that vary by the categories of $X, \mu_{i}$. Holland and Thayer (2000, pp. 139140) showed that when model 1 has a $\boldsymbol{\mu}$ with entries

$$
\mu_{i}=\log _{e}\left(\begin{array}{l}
x_{I} \\
x_{i}
\end{array}\right)
$$

where $\left(\begin{array}{l}x_{I} \\ x_{i}\end{array}\right)$ denotes the binomial coefficient,

" $x_{I}$ choose $x_{i}$," then a binomial distribution can be produced by defining $\boldsymbol{\beta}$ as $\beta_{1}$ and defining $\mathbf{X}$ as a single column of values for fitting the first degree of $X,\left(x_{1}^{1}, x_{2}^{1}, \ldots, x_{I}^{1}\right)^{t}$. With these definitions model 1 can be expressed as,

$$
\rho_{i}=\left(\begin{array}{c}
x_{I} \\
x_{i}
\end{array}\right) \pi^{x_{i}}(1-\pi)^{x_{I}-x_{i}}
$$

where $\pi$ is a function of the mean of $X$,

$$
\pi=\frac{1}{x_{I}} \sum_{i} \rho_{i} x_{i}=\frac{1}{x_{I}} \bar{x}
$$

and a function of $\beta_{1}$,

$$
\pi=\frac{\exp \left(\beta_{1}\right)}{1+\exp \left(\beta_{1}\right)}
$$

Thus, equation 3 implies that for fixed value, $x_{I}$, and a parameter based on the mean, $\pi$, the probability of obtaining a particular value of $X$ is a variate from a binomial distribution based on $x_{I}$ trials and success probability $\pi$.

Assessing the Role of the Loglinear Model's Underlying Distribution in Models of Population Distributions

The role of the underlying distribution used in loglinear models has not been extensively studied. The focus of loglinear modeling applications to psychometric test score distributions tends to be on fitting several of the observed distributions' moments - that is, more than three (Holland \& Thayer, 2000; Kolen \& Brennan, 2004); thus, the relatively simple uniform and binomial distributions underlying the fitted distributions have not received much attention. It is possible, however, that the uniform and binomial distributions underlying the models that fit observed distributions have subtle influences on the overall fit of the loglinear model.

To illustrate the influence of uniform and binomial distributions, consider how loglinear models based on each distribution fit eight different population distributions. Table 1 shows the eight population distributions of $X$ variables with 10 categories. Six of the population distributions were obtained from Steele and Chasling's (2006) study: the decreasing, step, triangular, platykurtic and leptokurtic distributions. Two other population distributions are based on both considered underlying distributions (the uniform distribution and the binomial distribution with $\pi$ $=0.5$ ). An additional under-dispersed binomial distribution was created to be similar to the binomial distribution, but with a relatively small variance.

For each of the eight distributions, loglinear models similar to model 1 were fit based on the uniform distribution $\left(K=0, \mu_{i}=0\right)$, and on fitting $K=1,2,3$ and 4 moments with the loglinear model based on the uniform 


\section{UNDERLYING DISTRIBUTIONS IN LOGLINEAR MODELS OF DISCRETE DATA}

distribution (equation 1 where $\boldsymbol{\mu}$ is a vector of zeros). Other loglinear models comparable to model 1 were fit based on the binomial distribution, $K=1, \mu_{i}=\log _{e}\left(\begin{array}{l}x_{I} \\ x_{i}\end{array}\right)$, and on fitting $K=2,3$ and 4 moments with the loglinear model based on the binomial distribution (equation 1 where $\boldsymbol{\mu}$ has entries

$$
\left.\mu_{i}=\log _{e}\left(\begin{array}{l}
x_{I} \\
x_{i}
\end{array}\right)\right)
$$

Table 1's population distributions and the fits of the loglinear models to the population distributions for a hypothetical sample size of $N$ $=100$ are illustrated in Figures 1-16. These figures show the fits of the considered models in terms of individual score values and each model's summarized likelihood ratio Chi-square statistic:

$$
G^{2}=2 \sum_{i} n_{i} \log _{e}\left(\frac{n_{i}}{N \hat{\rho}_{i}}\right)
$$

\begin{tabular}{|c|c|c|c|c|c|c|c|c|}
\hline $\begin{array}{c}X ' s \\
\text { Categories } \\
\& \\
\text { Moments }\end{array}$ & Uniform & Decreasing & Step & Triangular & Platykurtic & Leptokurtic & Binomial & $\begin{array}{c}\text { Under- } \\
\text { Dispersed } \\
\text { Binomial }\end{array}$ \\
\hline 1 & 0.10 & 0.32 & 0.05 & 0.17 & 0.04 & 0.05 & 0.01 & 0.00 \\
\hline 2 & 0.10 & 0.13 & 0.05 & 0.13 & 0.11 & 0.05 & 0.04 & 0.03 \\
\hline 3 & 0.10 & 0.10 & 0.05 & 0.10 & 0.11 & 0.05 & 0.12 & 0.13 \\
\hline 4 & 0.10 & 0.08 & 0.05 & 0.07 & 0.12 & 0.05 & 0.21 & 0.22 \\
\hline 5 & 0.10 & 0.07 & 0.05 & 0.03 & 0.12 & 0.30 & 0.25 & 0.26 \\
\hline 6 & 0.10 & 0.07 & 0.15 & 0.03 & 0.12 & 0.30 & 0.21 & 0.21 \\
\hline 7 & 0.10 & 0.06 & 0.15 & 0.07 & 0.12 & 0.05 & 0.12 & 0.12 \\
\hline 8 & 0.10 & 0.06 & 0.15 & 0.10 & 0.11 & 0.05 & 0.04 & 0.04 \\
\hline 9 & 0.10 & 0.05 & 0.15 & 0.13 & 0.11 & 0.05 & 0.01 & 0.00 \\
\hline 10 & 0.10 & 0.05 & 0.15 & 0.17 & 0.04 & 0.05 & 0.00 & 0.00 \\
\hline Mean & 5.50 & 3.86 & 6.75 & 5.50 & 5.50 & 5.50 & 5.00 & 5.00 \\
\hline Std. Dev. & 2.87 & 2.91 & 2.59 & 3.41 & 2.51 & 2.06 & 1.57 & 1.41 \\
\hline Skew & 0.00 & 0.69 & -0.68 & 0.00 & 0.00 & 0.00 & 0.02 & 0.04 \\
\hline Kurtosis & 1.78 & 2.17 & 2.56 & 1.38 & 1.91 & 3.35 & 2.75 & 2.40 \\
\hline
\end{tabular}

Table 1: Population Distributions 


\section{TIM MOSES}

Figures 1-16 suggest that model fit is a function of the number of moments fit in the model and also show how closely the underlying distribution reflects the distribution being modeled. Loglinear models that fit $K=3$ and 4 moments tend to have better fits (lower $G^{2}$ values) compared to models that fit $K=0,1$ and 2 moments, however, the loglinear model's underlying distribution appears to moderate the influence of $K$.

For population distributions more similar to the uniform distribution (i.e., the uniform, decreasing, step, triangular and platykurtic population distributions), models based on an underlying uniform distribution can closely fit the population distributions with fewer moments than those required by models based on an underlying binomial distribution (Figures 1, 3, 5, 7 \& 9 vs. Figures 2, 4, 6, 8 \& 10). For population distributions similar to the binomial distribution (i.e., the leptokurtic, binomial and under-dispersed binomial population distributions), models based on an underlying binomial distribution can closely fit the population distributions with fewer moments than those required by models based on an underlying uniform distribution (Figures 11, 13 $\& 15$ vs. Figures $12,14 \& 16$ ).

\section{Methodology}

To better understand the implications of results shown in Figures 1-16, a series of simulations was conducted. For the simulations of interest, 1,000 datasets of sample sizes 30,100 and 1,000 were drawn from each of Table 1's population distributions. For each of the randomly drawn datasets, loglinear models were fit based on an underlying uniform distribution with $K=0-4$, and also based on an underlying binomial distribution with $K=1-4$. For models reflecting one of the two underlying distributions, the $K$ values were selected based on nested Chi-square tests for differences in models' $G^{2}$ statistics (Haberman, 1974) and also on minimizing models' AIC statistics (Akaike, 1981).

To consider the influence of the underlying distribution for situations similar to what might be encountered in psychometric testing practice, where the moments to be fit in a test score distribution might be predetermined rather than statistically selected, modeling results were also produced by always fitting $K=$ 4 moments based on the both the uniform and the binomial distributions. The results of interest for each combination of sample size, underlying distribution and moment selection method were the percentages of datasets where specific $K$ values were selected, the mean $K$ values across all 1,000 datasets and the average model fit (i.e., mean $G^{2}$ values) across all 1,000 datasets.

\section{Results}

Simulation results are summarized in Tables 2-9. Each table presents the simulation results for one of Table 1's eight population distributions; rows show the simulation results for a specific combination of sample size $(30,100$ or 1,000$)$, underlying distribution (the uniform or binomial distribution) and selection method for $K\left(G^{2}\right.$, $A I C$, or $K=4)$. Each row's results show the percentage of moments $(K)$ selected in the 1,000 simulated datasets, the mean of the selected $K$ 's and the mean model fit (mean $G^{2}$ ). Because the percentages in Tables 2-9 are presented in rounded form, they do not always sum to exactly $100 \%$ within each row.

Some results shown in Tables 2-9 have been shown elsewhere (Moses \& Holland, 2010). $K$ selections based on the $A I C$ result in larger $K$ values than selections based on the $G^{2}$. Selections based on sample sizes of 1,000 result in larger $K$ values than selections based on smaller sample sizes. Models with larger $K$ values fit the sample distributions more closely; that is, they result in smaller $G^{2}$.

Tables 2-9 show that the influences of the $G^{2}$ and $A I C$ selection strategies and the sample sizes are moderated by how closely the loglinear model's underlying distribution reflects the population distribution. For population distributions that closely reflect the uniform distribution (i.e., the uniform and decreasing population distributions, Tables 2-3), using the uniform distribution results in $A I C$ and $G^{2}$ model selections with smaller mean $K$ values and larger mean $G^{2}$ values than using the binomial distribution. These results were also partially obtained for the step population distribution (Table 4, N=30 and 100), the triangular population distribution (Table 5, N $=30$ ), and the leptokurtic population distribution (Table 7, $N=30$ and 100). 
Figure 1: Uniform Population Distribution Modeling Results

Based on an Underlying Uniform Distribution

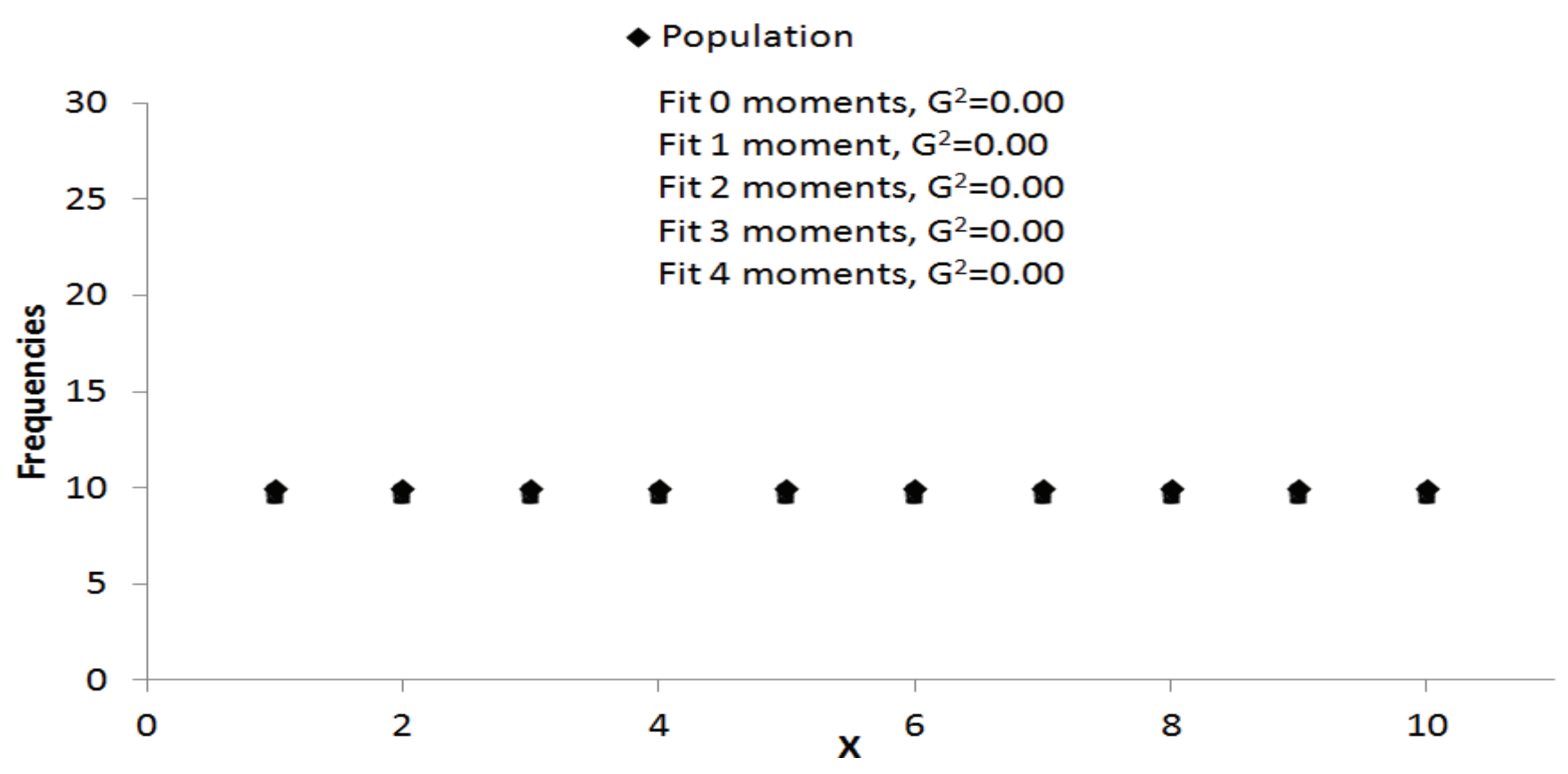

Figure 2: Uniform Population Distribution Modeling Results Based on an Underlying Binomial Distribution

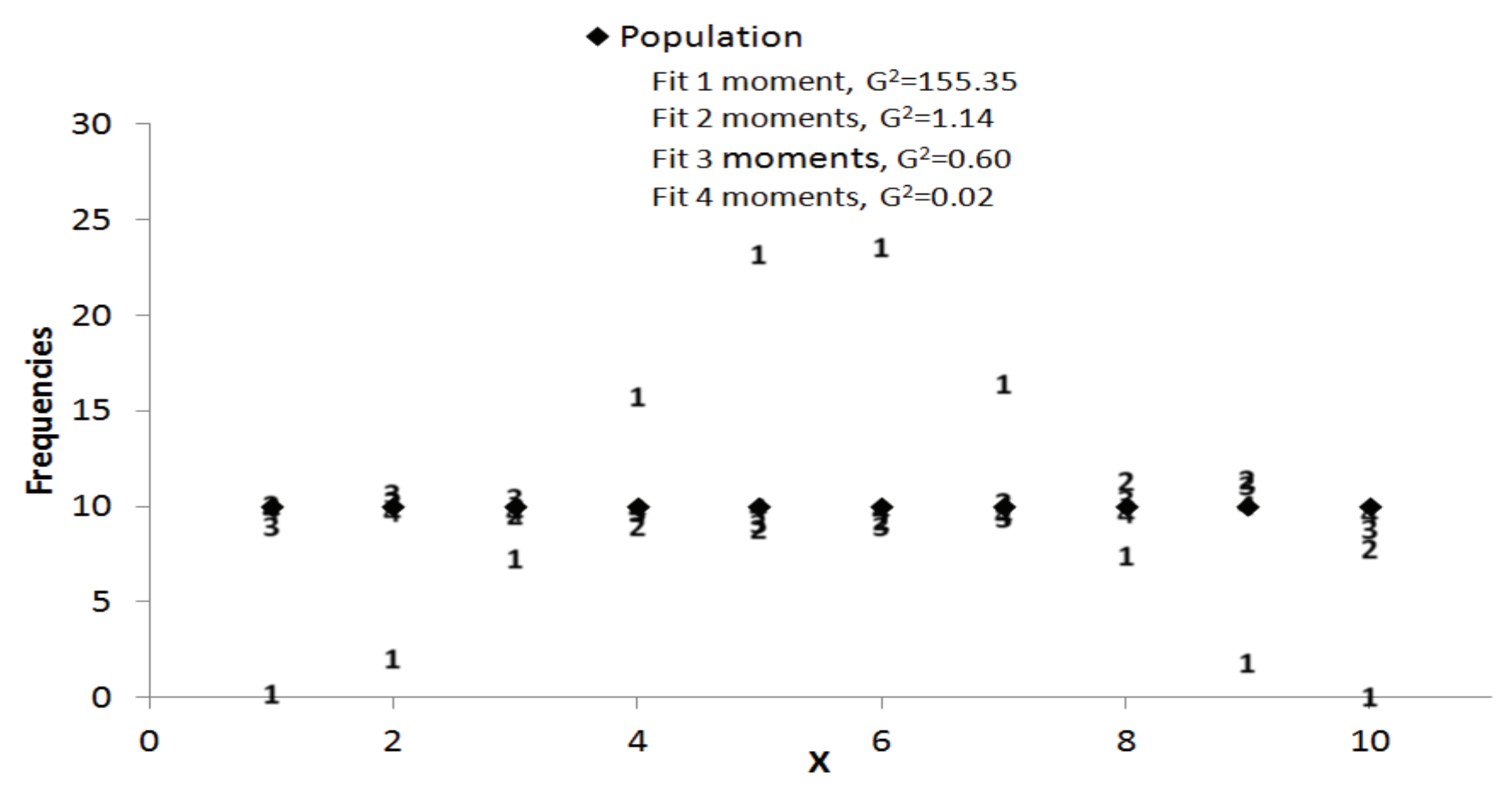




\section{TIM MOSES}

Figure 3: Decreasing Population Distribution Modeling Results Based on an Underlying Uniform Distribution

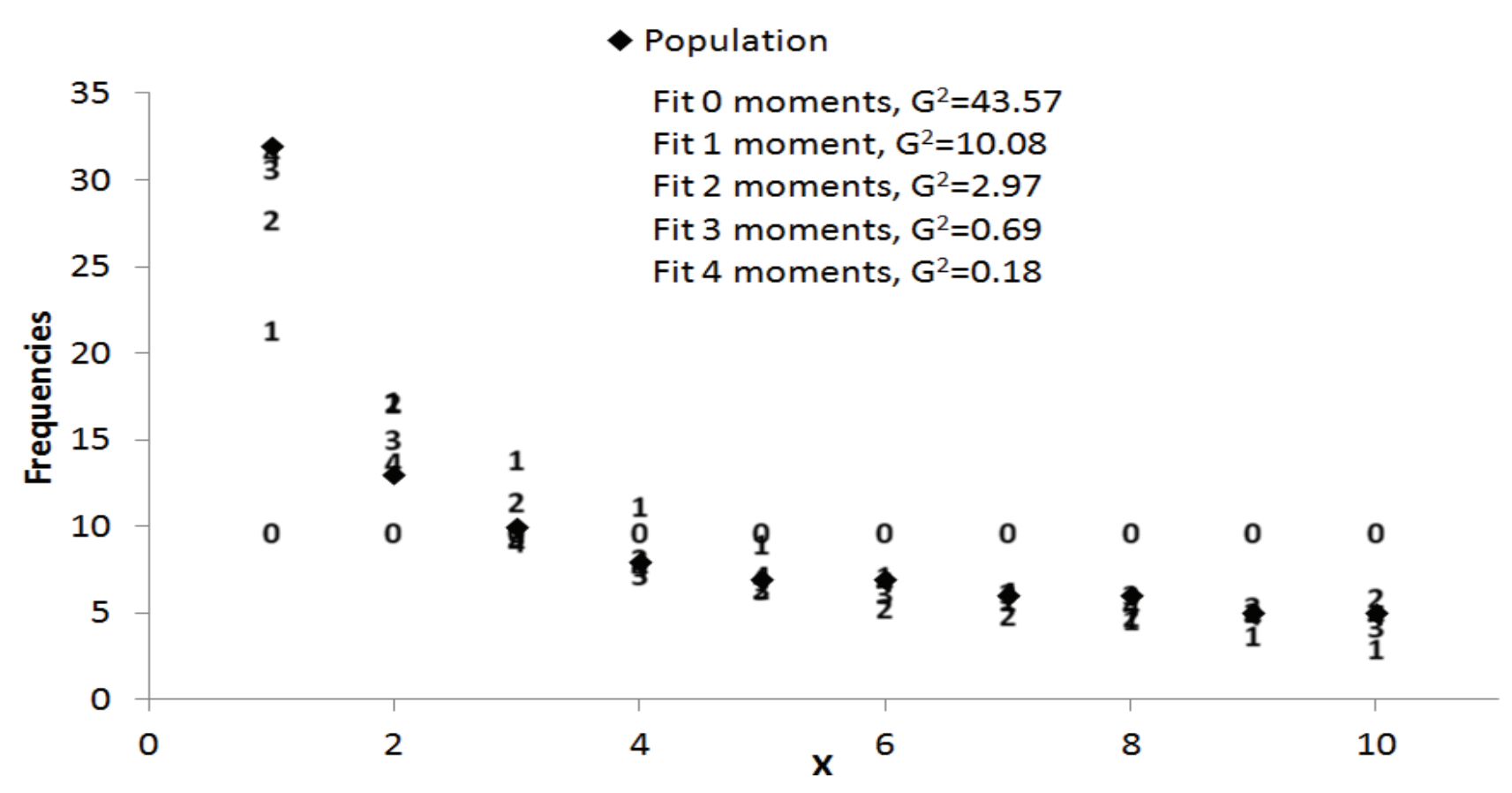

Figure 4: Decreasing Population Distribution Modeling Results

Based on an Underlying Binomial Distribution

- Population

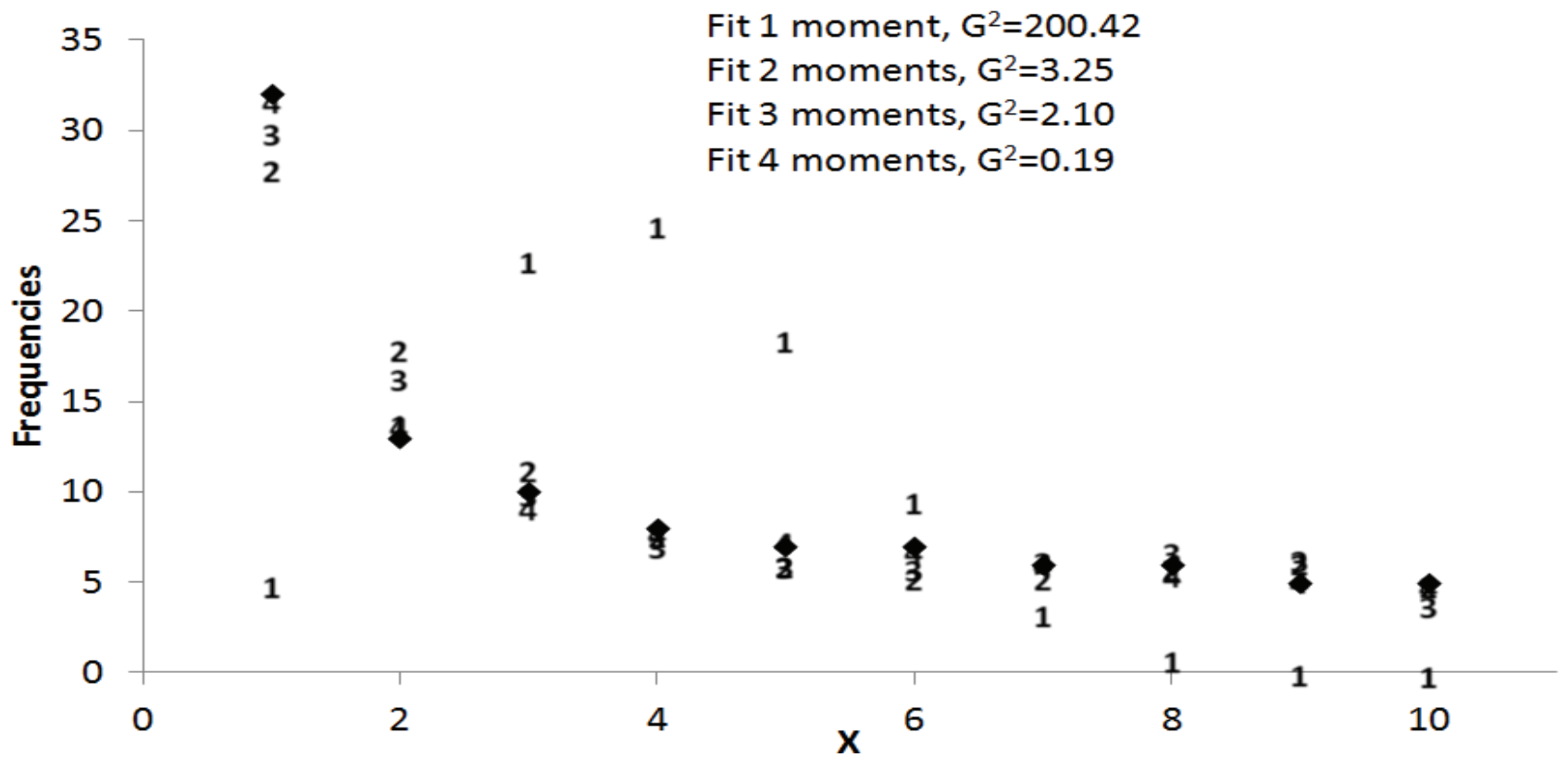


Figure 5: Step Population Distribution Modeling Results

Based on an Underlying Uniform Distribution

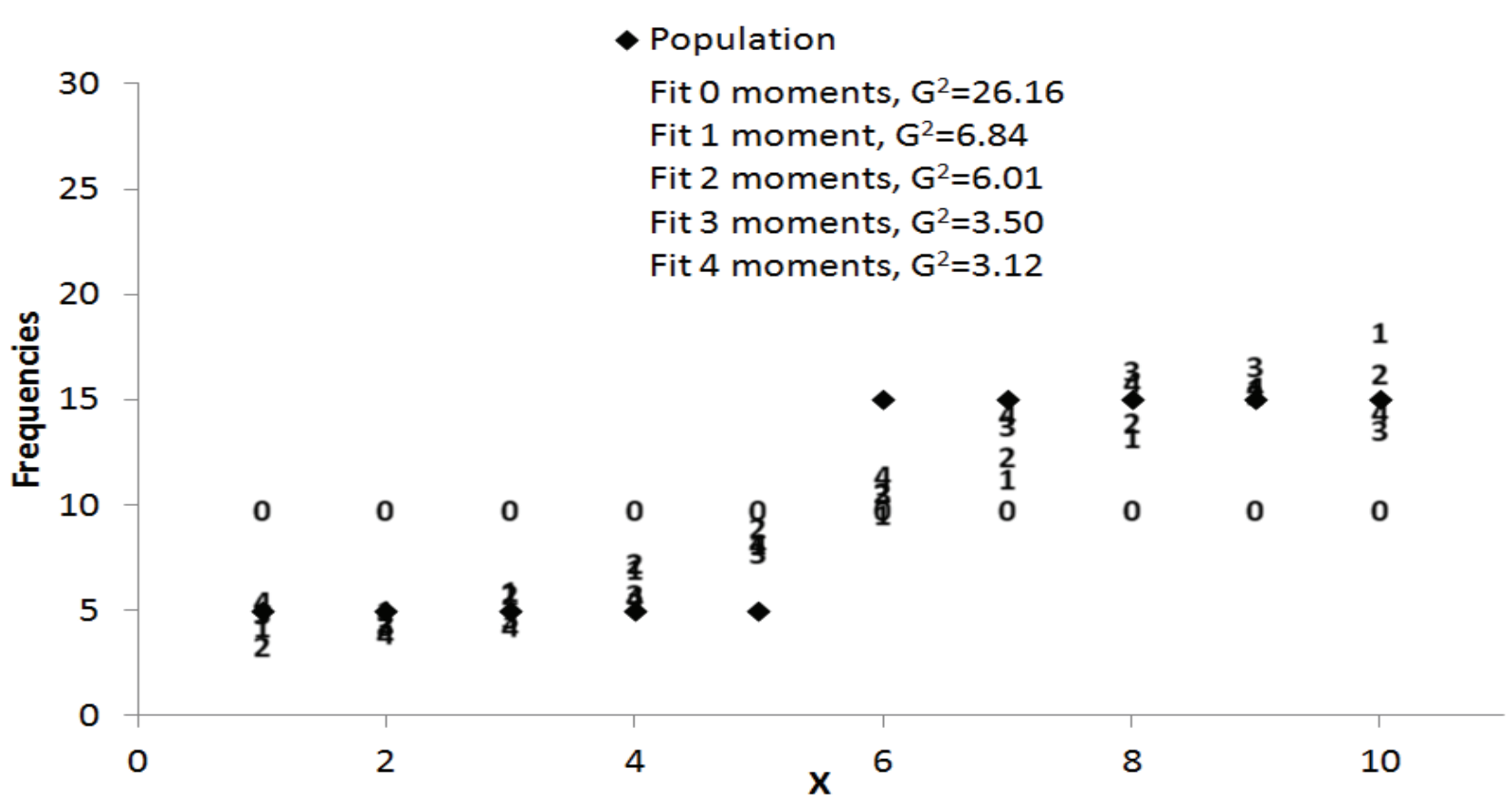

Figure 6: Step Population Distribution Modeling Results Based on an Underlying Binomial Distribution

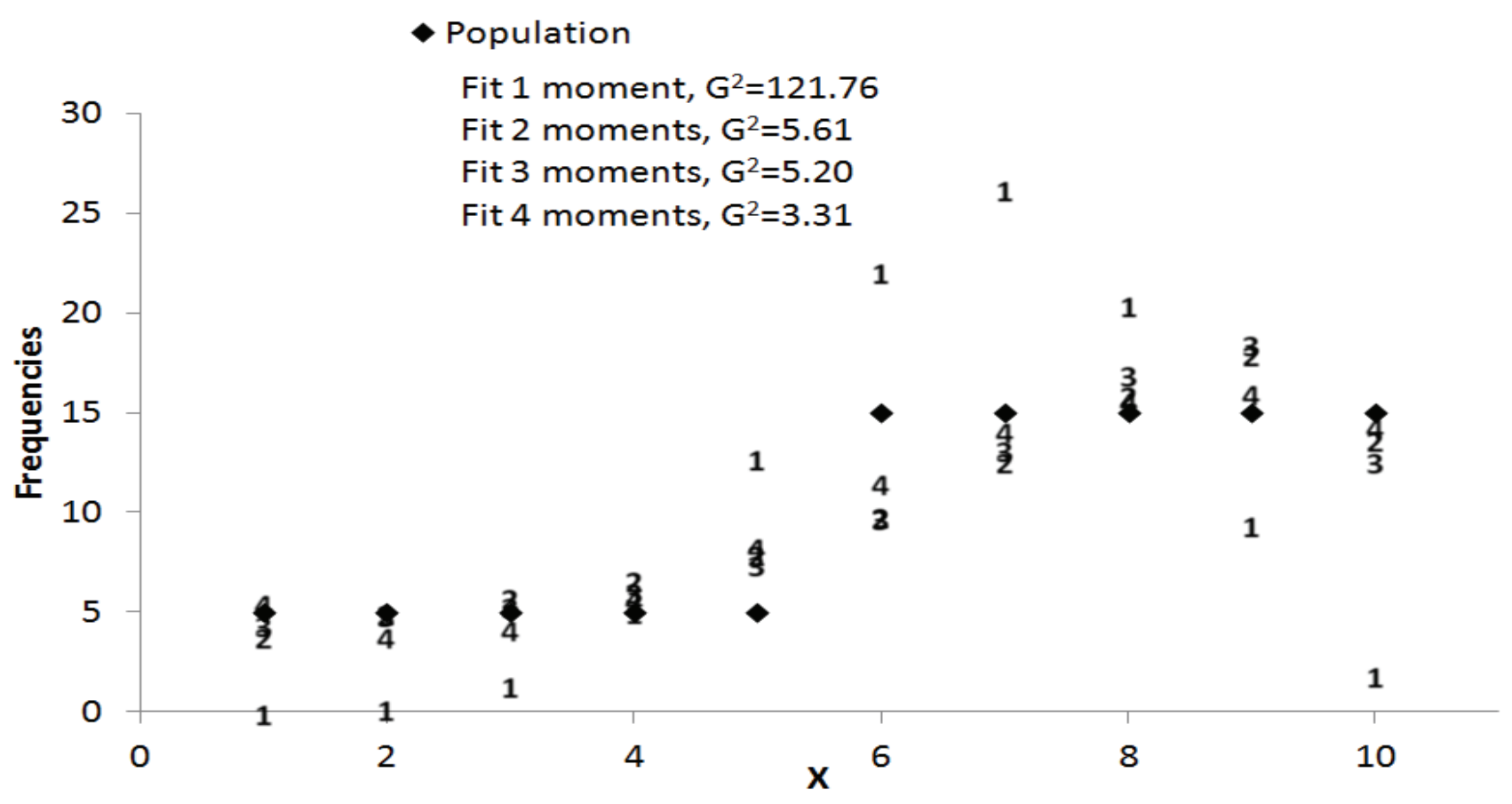




\section{TIM MOSES}

Figure 7: Triangular Population Distribution Modeling Results

Based on an Underlying Uniform Distribution

- Population

Fit 0 moments, $\mathrm{G}^{2}=25.29$

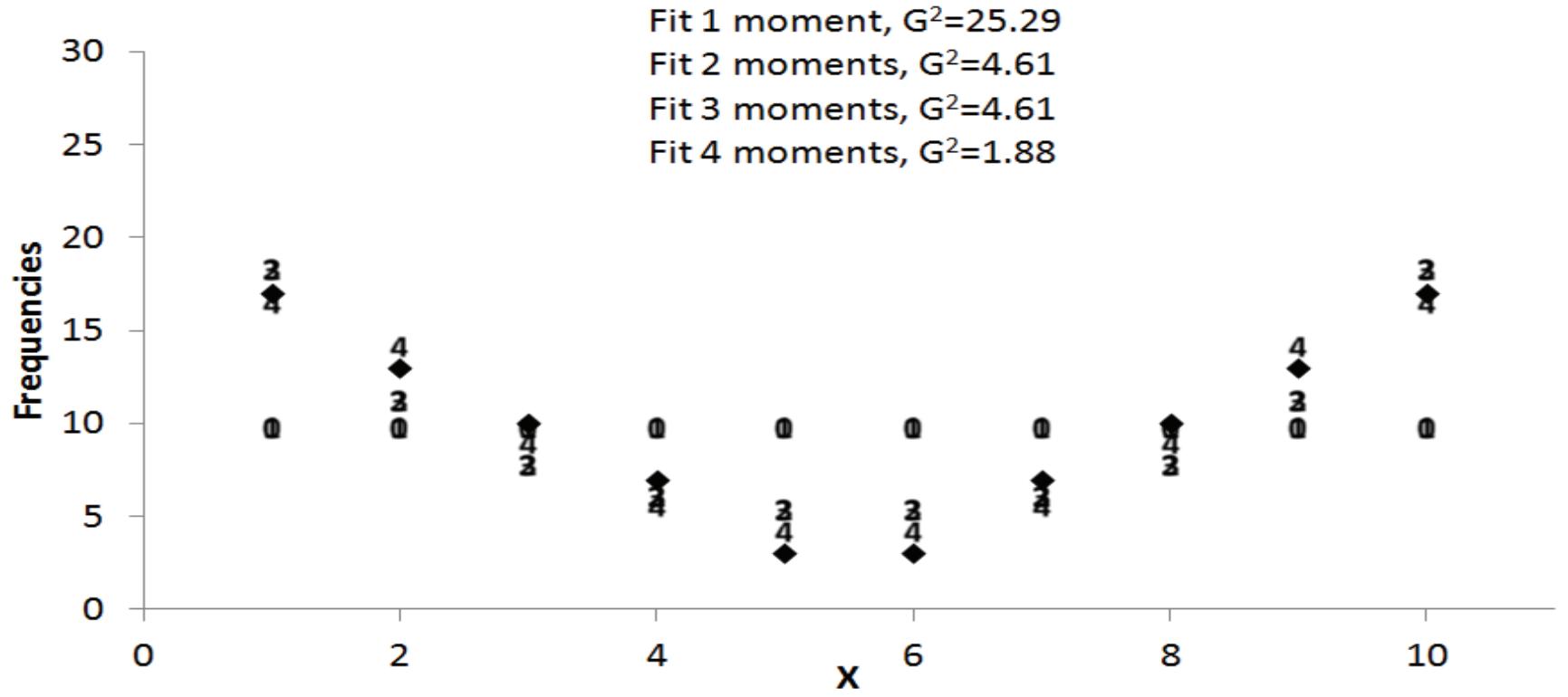

Figure 8: Triangular Population Distribution Modeling Results Based on an Underlying Binomial Distribution

- Population

Fit 1 moment, $\mathrm{G}^{2}=324.83$

Fit 2 moments, $\mathrm{G}^{2}=3.44$

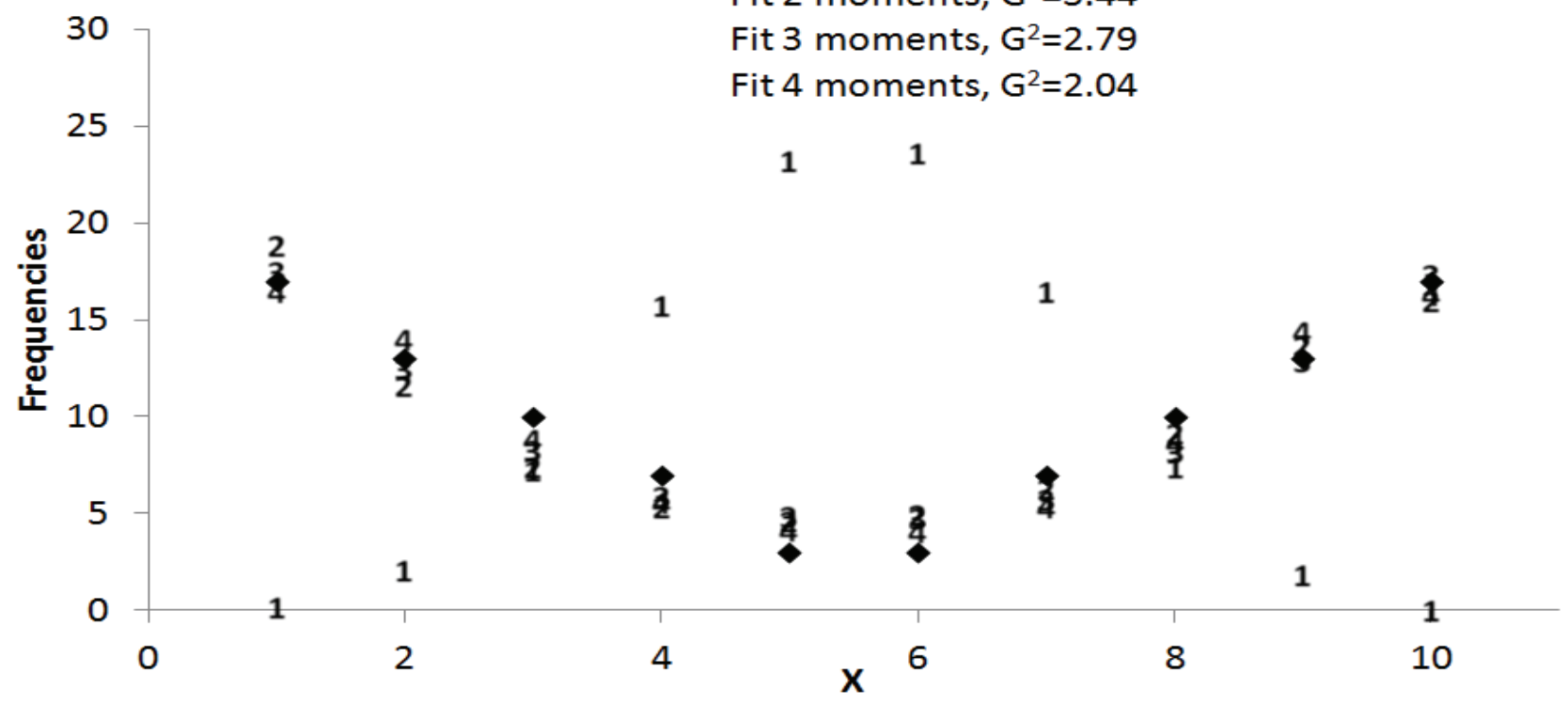


Figure 9: Platykurtic Population Distribution Modeling Results

Based on an Underlying Uniform Distribution

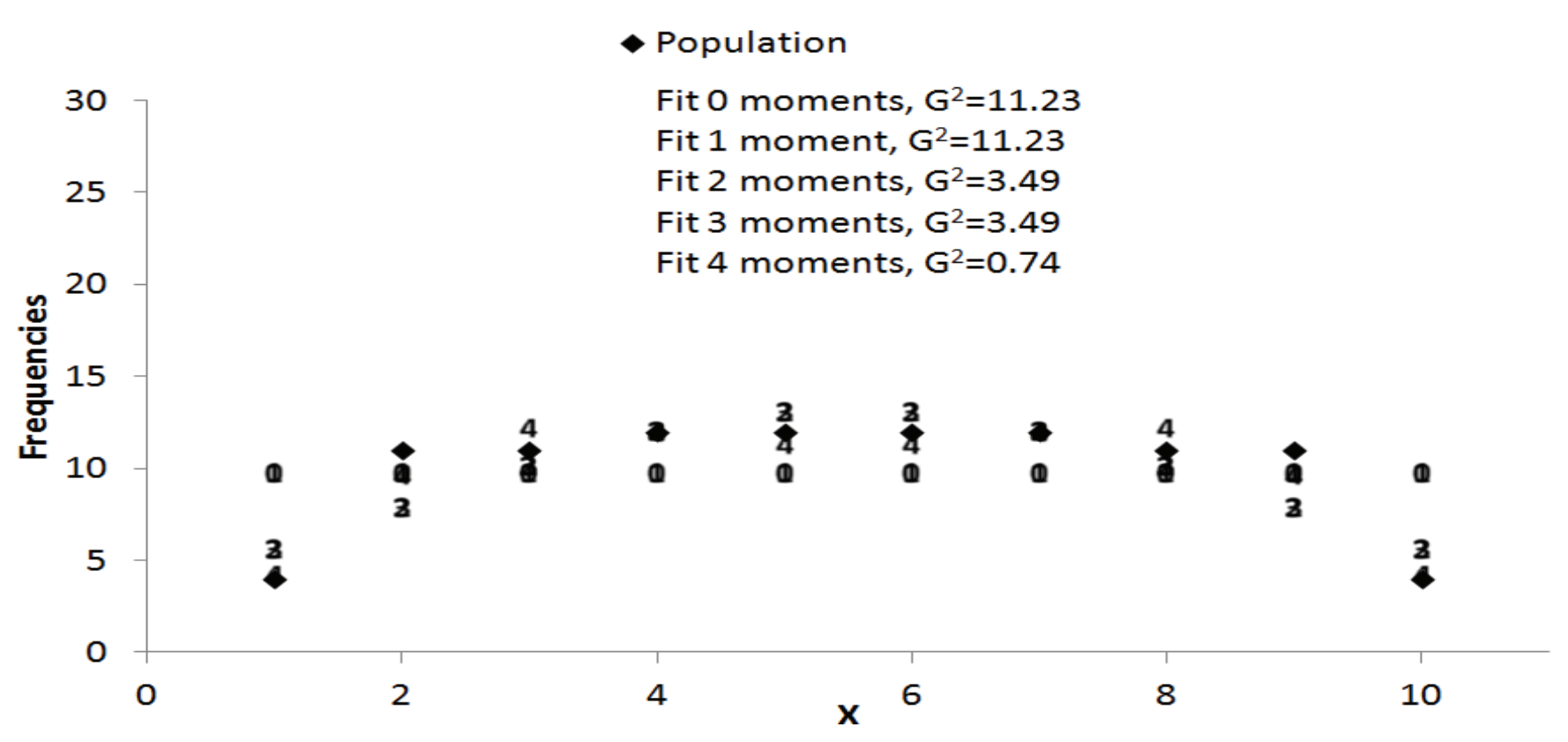

Figure 10: Platykurtic Population Distribution Modeling Results

Based on an Underlying Binomial Distribution

- Population

Fit 1 moment, $\mathrm{G}^{2}=80.76$

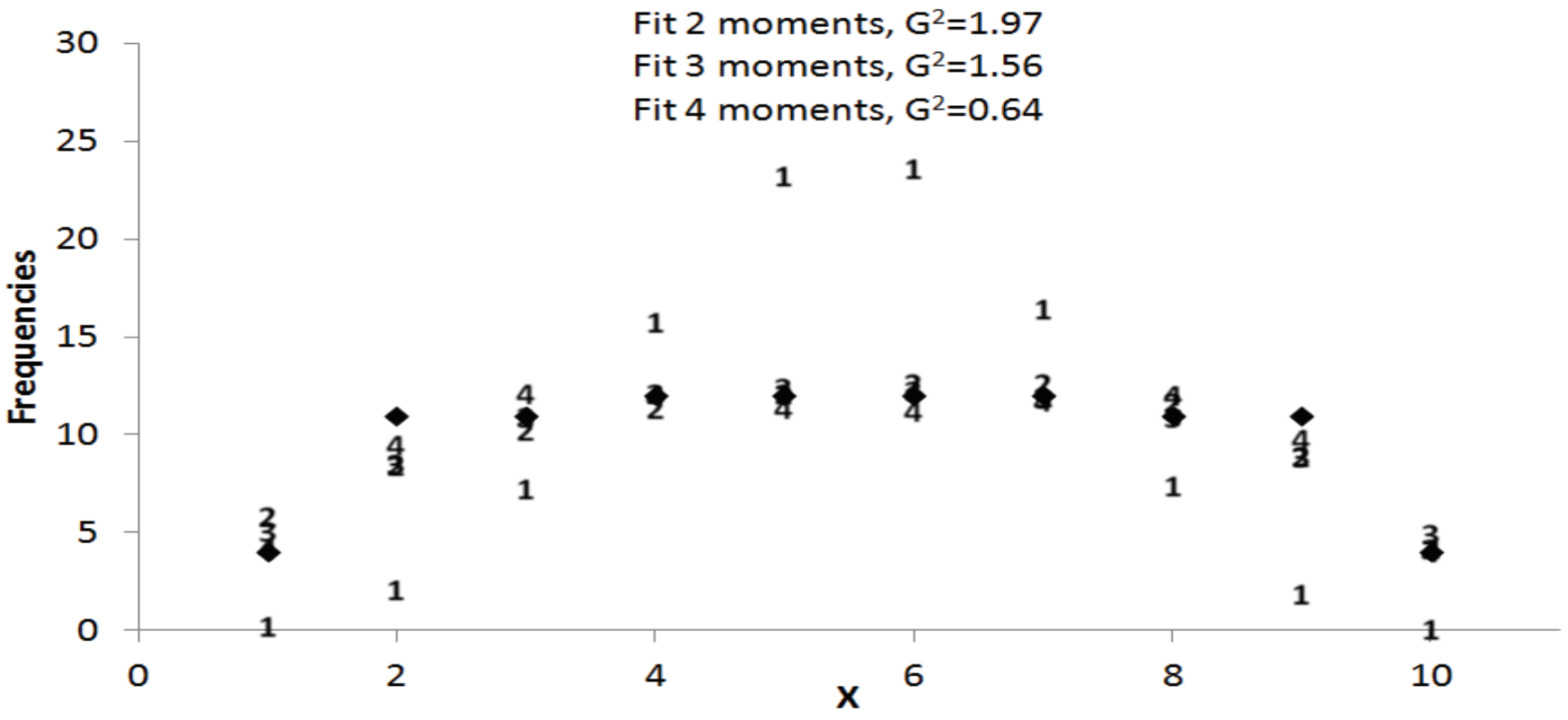




\section{TIM MOSES}

Figure 11: Leptokurtic Population Distribution Modeling Results Based on an Underlying Uniform Distribution

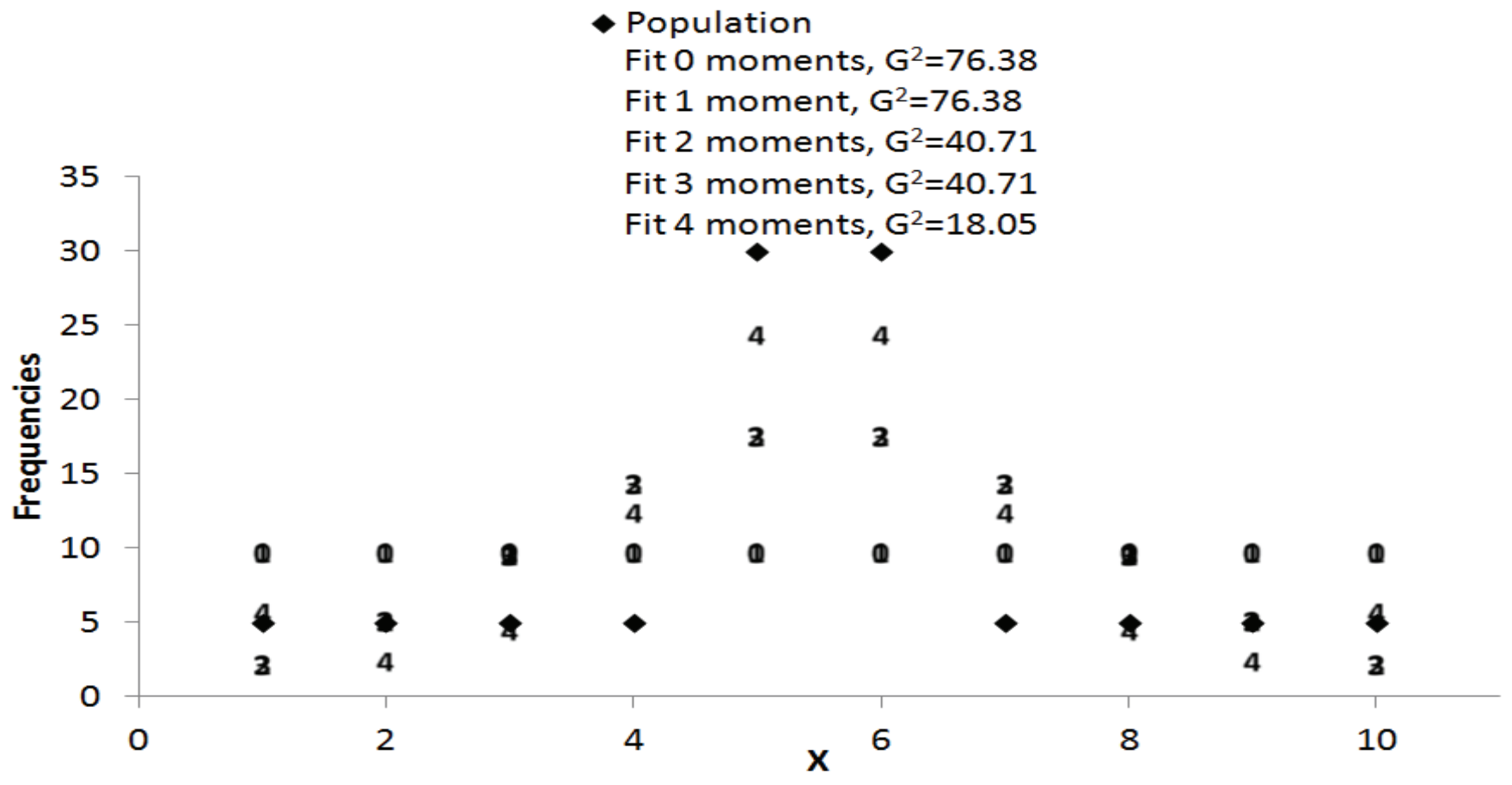

Figure 12: Leptokurtic Population Distribution Modeling Results

Based on an Underlying Binomial Distribution

- Population

Fit 1 moment, $\mathrm{G}^{2}=68.08$

Fit 2 moments, $\mathrm{G}^{2}=46.53$

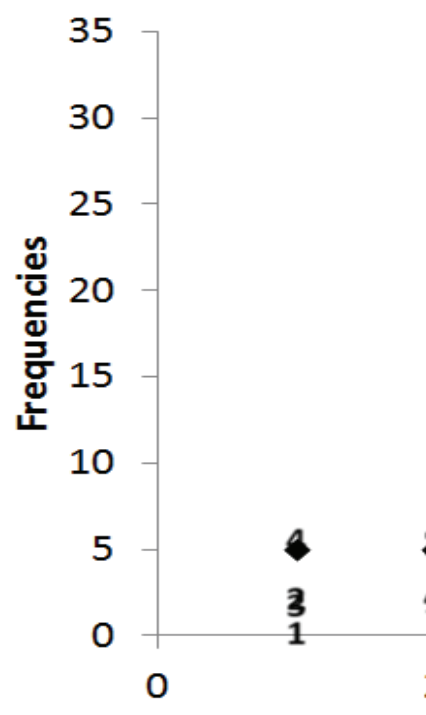

Fit 3 moments, $\mathrm{G}^{2}=46.30$

Fit 4 moments, $\mathrm{G}^{2}=17.70$
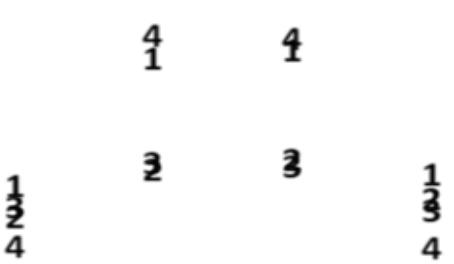
Figure 13: Binomial Population Distribution Modeling Results

Based on an Underlying Uniform Distribution

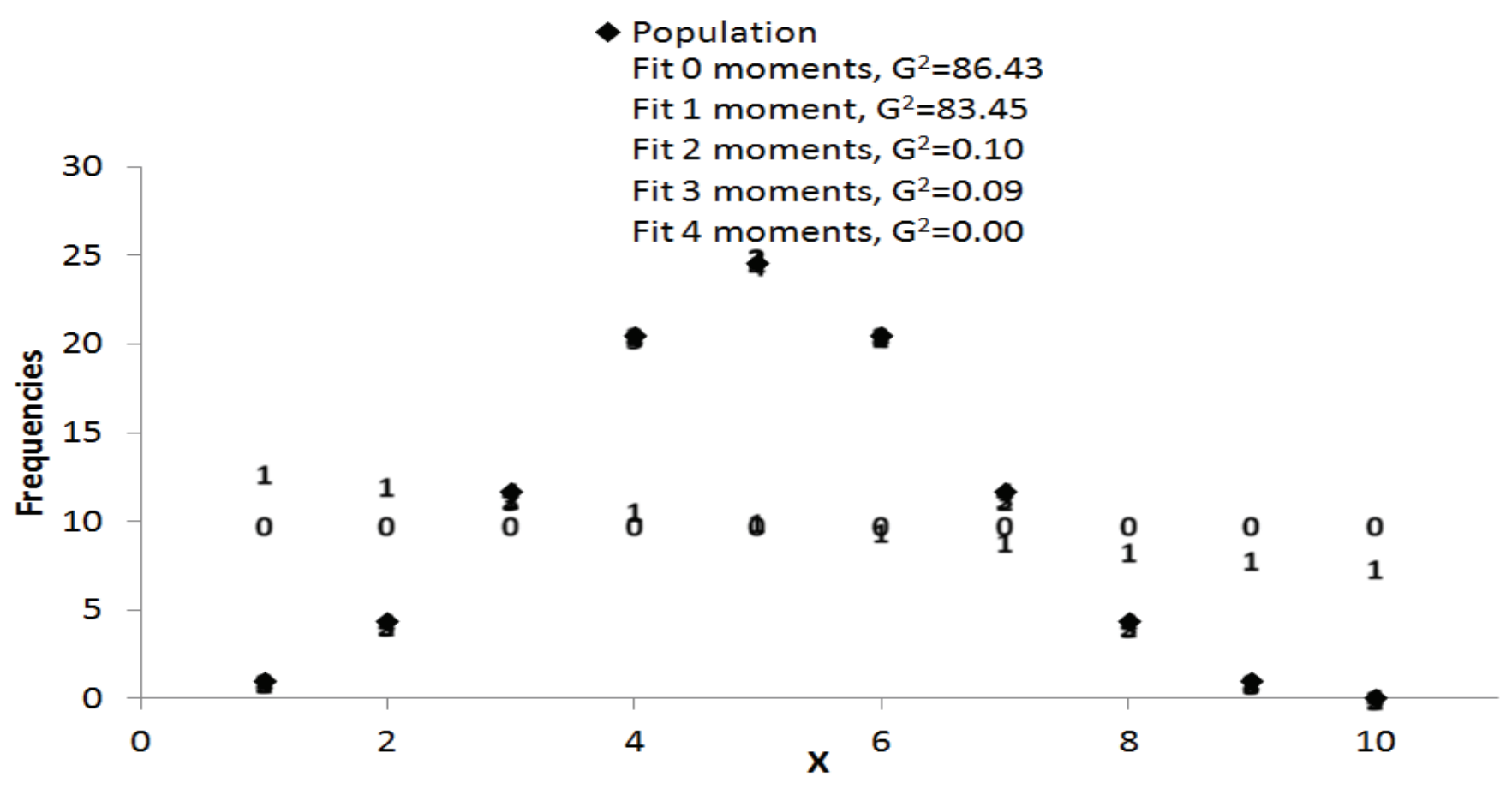

Figure 14: Binomial Population Distribution Modeling Results Based on an Underlying Binomial Distribution

Population

Fit 1 moment, $\mathrm{G}^{2}=0.00$

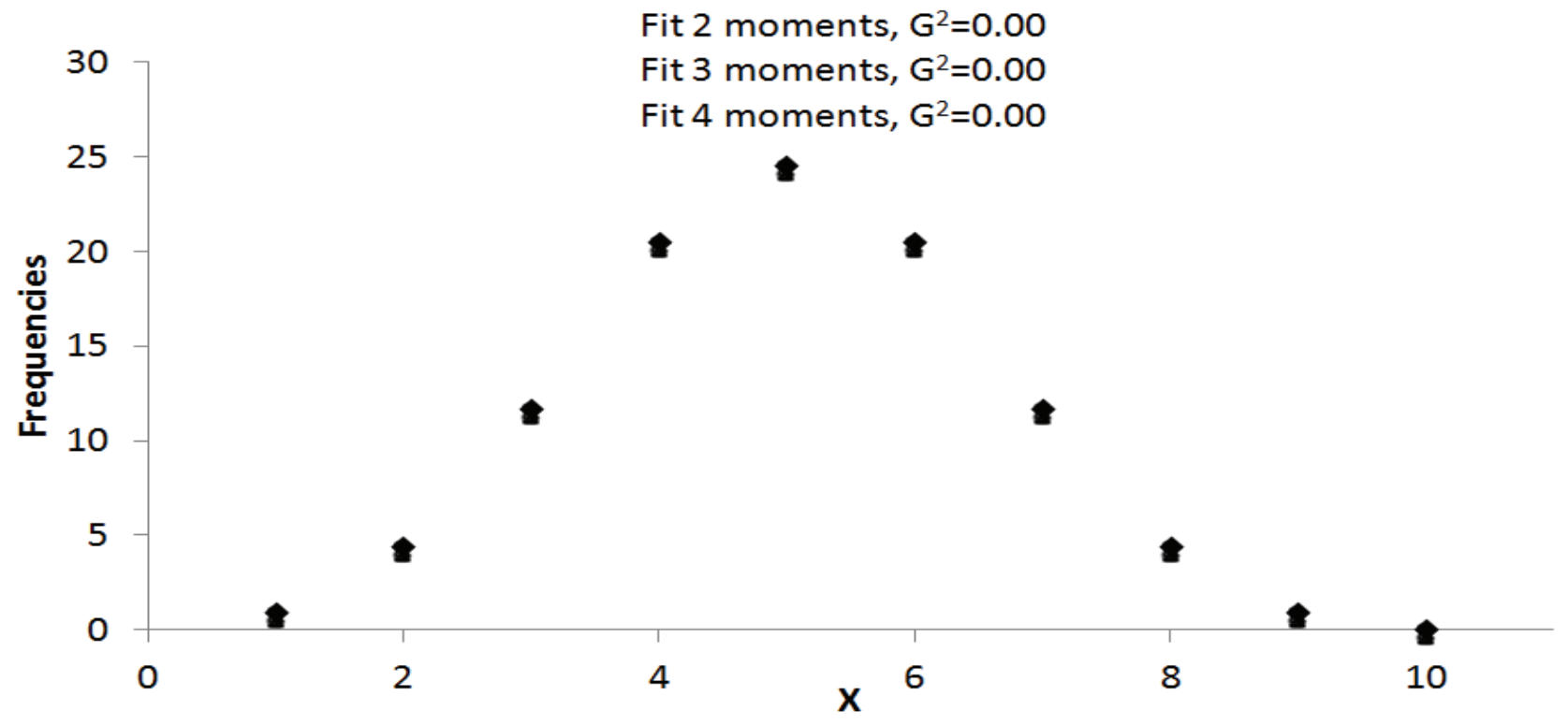




\section{TIM MOSES}

Figure 15: Under-Dispersed Binomial Population Distribution Modeling Results Based on an Underlying Uniform Distribution

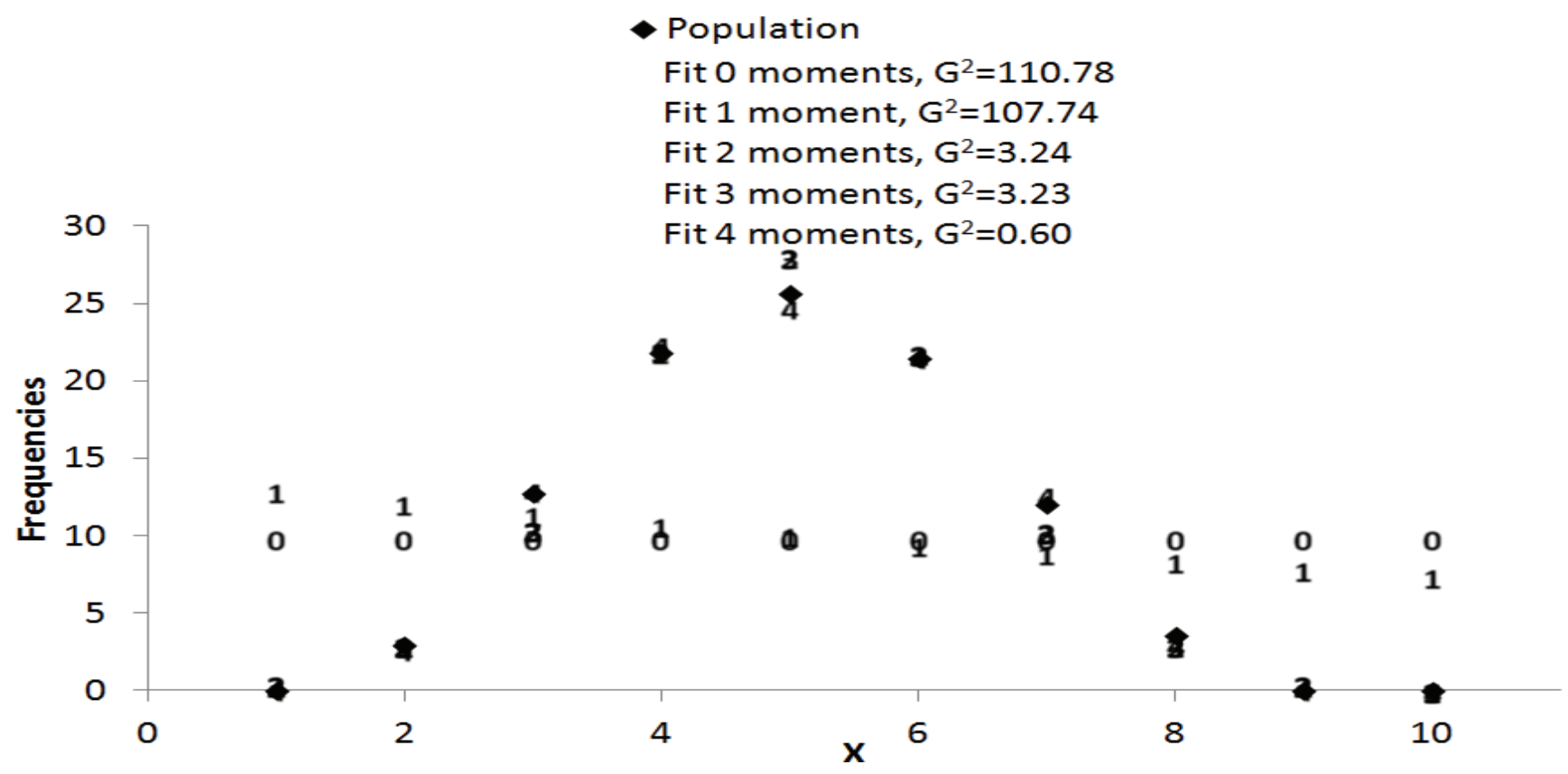

Figure 16: Under-Dispersed Binomial Population Distribution Modeling Results

Based on an Underlying Binomial Distribution

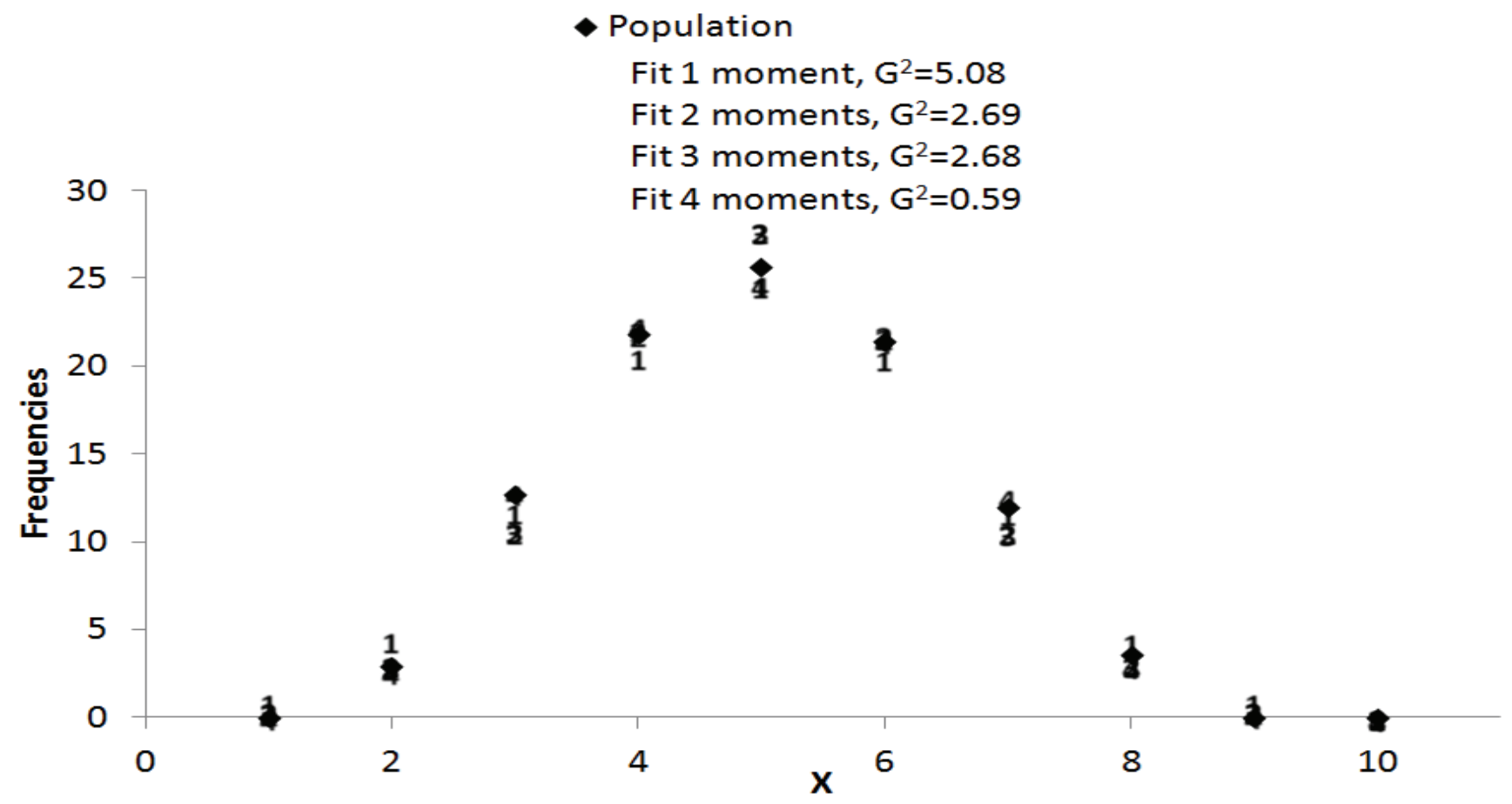


Table 2: Simulation Results for the Uniform Population Distribution

\begin{tabular}{|c|c|c|c|c|c|c|c|c|}
\hline \multirow{2}{*}{$N$} & \multirow{2}{*}{$\begin{array}{l}\text { Underlying } \\
\text { Distribution }\end{array}$} & \multicolumn{5}{|c|}{$\begin{array}{l}\text { Percentage of } K \text { Moments Selected } \\
\text { (out of } 1,000 \text { replications) }\end{array}$} & \multirow{2}{*}{$\begin{array}{c}\text { Mean } \\
\text { Moments }(K)\end{array}$} & \multirow{2}{*}{$\underset{G^{2}}{\operatorname{Mean}}$} \\
\hline & & 0 & 1 & 2 & 3 & 4 & & \\
\hline \multicolumn{9}{|c|}{$G^{2}$ Selections of $K$} \\
\hline \multirow{2}{*}{30} & Uniform & $94 \%$ & $1 \%$ & $1 \%$ & $2 \%$ & $2 \%$ & 0.16 & 9.37 \\
\hline & Binomial & & $0 \%$ & $94 \%$ & $3 \%$ & $3 \%$ & 2.08 & 7.75 \\
\hline \multirow{2}{*}{100} & Uniform & $96 \%$ & $1 \%$ & $1 \%$ & $1 \%$ & $1 \%$ & 0.11 & 8.97 \\
\hline & Binomial & & $0 \%$ & $91 \%$ & $4 \%$ & $5 \%$ & 2.15 & 7.56 \\
\hline \multirow{2}{*}{1,000} & Uniform & $96 \%$ & $2 \%$ & $0 \%$ & $1 \%$ & $1 \%$ & 0.08 & 8.38 \\
\hline & Binomial & & $0 \%$ & $26 \%$ & $25 \%$ & $50 \%$ & 3.24 & 7.11 \\
\hline \multicolumn{9}{|c|}{$A I C$ Selections of $K$} \\
\hline \multirow{2}{*}{30} & Uniform & $74 \%$ & $12 \%$ & $6 \%$ & $4 \%$ & $4 \%$ & 0.52 & 8.35 \\
\hline & Binomial & & $0 \%$ & $72 \%$ & $16 \%$ & $12 \%$ & 2.41 & 6.80 \\
\hline \multirow{2}{*}{100} & Uniform & $73 \%$ & $13 \%$ & $8 \%$ & $4 \%$ & $3 \%$ & 0.51 & 7.84 \\
\hline & Binomial & & $0 \%$ & $62 \%$ & $21 \%$ & $18 \%$ & 2.56 & 6.28 \\
\hline \multirow{2}{*}{1,000} & Uniform & $74 \%$ & $13 \%$ & $5 \%$ & $5 \%$ & $3 \%$ & 0.49 & 7.28 \\
\hline & Binomial & & $0 \%$ & $5 \%$ & $13 \%$ & $82 \%$ & 3.77 & 5.22 \\
\hline \multicolumn{9}{|c|}{ Always Fit $K=4$} \\
\hline \multirow{2}{*}{30} & Uniform & $0 \%$ & $0 \%$ & $0 \%$ & $0 \%$ & $100 \%$ & 4.00 & 5.78 \\
\hline & Binomial & $0 \%$ & $0 \%$ & $0 \%$ & $0 \%$ & $100 \%$ & 4.00 & 5.78 \\
\hline \multirow{2}{*}{100} & Uniform & $0 \%$ & $0 \%$ & $0 \%$ & $0 \%$ & $100 \%$ & 4.00 & 5.22 \\
\hline & Binomial & $0 \%$ & $0 \%$ & $0 \%$ & $0 \%$ & $100 \%$ & 4.00 & 5.24 \\
\hline \multirow{2}{*}{1,000} & Uniform & $0 \%$ & $0 \%$ & $0 \%$ & $0 \%$ & $100 \%$ & 4.00 & 4.80 \\
\hline & Binomial & $0 \%$ & $0 \%$ & $0 \%$ & $0 \%$ & $100 \%$ & 4.00 & 4.96 \\
\hline
\end{tabular}




\section{TIM MOSES}

Table 3: Simulation Results for the Decreasing Population Distribution

\begin{tabular}{|c|c|c|c|c|c|c|c|c|}
\hline \multirow{2}{*}{$N$} & \multirow{2}{*}{$\begin{array}{l}\text { Underlying } \\
\text { Distribution }\end{array}$} & \multicolumn{5}{|c|}{$\begin{array}{l}\text { Percentage of } K \text { Moments Selected } \\
\text { (out of } 1,000 \text { replications) }\end{array}$} & \multirow{2}{*}{$\begin{array}{c}\text { Mean } \\
\text { Moments }(K)\end{array}$} & \multirow{2}{*}{$\begin{array}{c}\text { Mean } \\
G^{2}\end{array}$} \\
\hline & & 0 & 1 & 2 & 3 & 4 & & \\
\hline \multicolumn{9}{|c|}{$G^{2}$ Selections of $K$} \\
\hline \multirow{2}{*}{30} & Uniform & $19 \%$ & $62 \%$ & $13 \%$ & $5 \%$ & $1 \%$ & 1.08 & 10.62 \\
\hline & Binomial & & $0 \%$ & $92 \%$ & $5 \%$ & $4 \%$ & 2.12 & 8.21 \\
\hline \multirow{2}{*}{100} & Uniform & $0 \%$ & $35 \%$ & $46 \%$ & $16 \%$ & $3 \%$ & 1.86 & 9.55 \\
\hline & Binomial & & $0 \%$ & $78 \%$ & $9 \%$ & $13 \%$ & 2.35 & 8.17 \\
\hline \multirow{2}{*}{1,000} & Uniform & $0 \%$ & $0 \%$ & $1 \%$ & $69 \%$ & $30 \%$ & 3.29 & 8.61 \\
\hline & Binomial & & $0 \%$ & $1 \%$ & $3 \%$ & $96 \%$ & 3.96 & 6.84 \\
\hline \multicolumn{9}{|c|}{$A I C$ Selections of $K$} \\
\hline \multirow{2}{*}{30} & Uniform & $3 \%$ & $36 \%$ & $33 \%$ & $21 \%$ & $8 \%$ & 1.96 & 7.58 \\
\hline & Binomial & & $0 \%$ & $65 \%$ & $22 \%$ & $13 \%$ & 2.49 & 7.09 \\
\hline \multirow{2}{*}{100} & Uniform & $0 \%$ & $6 \%$ & $35 \%$ & $40 \%$ & $19 \%$ & 2.72 & 6.35 \\
\hline & Binomial & & $0 \%$ & $44 \%$ & $21 \%$ & $35 \%$ & 2.91 & 6.38 \\
\hline \multirow{2}{*}{1,000} & Uniform & $0 \%$ & $0 \%$ & $0 \%$ & $27 \%$ & $73 \%$ & 3.73 & 6.84 \\
\hline & Binomial & & $0 \%$ & $0 \%$ & $0 \%$ & $100 \%$ & 4.00 & 6.67 \\
\hline \multicolumn{9}{|c|}{ Always Fit $K=4$} \\
\hline \multirow{2}{*}{30} & Uniform & $0 \%$ & $0 \%$ & $0 \%$ & $0 \%$ & $100 \%$ & 4.00 & 6.04 \\
\hline & Binomial & $0 \%$ & $0 \%$ & $0 \%$ & $0 \%$ & $100 \%$ & 4.00 & 6.04 \\
\hline \multirow{2}{*}{100} & Uniform & $0 \%$ & $0 \%$ & $0 \%$ & $0 \%$ & $100 \%$ & 4.00 & 5.43 \\
\hline & Binomial & $0 \%$ & $0 \%$ & $0 \%$ & $0 \%$ & $100 \%$ & 4.00 & 5.43 \\
\hline \multirow{2}{*}{1,000} & Uniform & $0 \%$ & $0 \%$ & $0 \%$ & $0 \%$ & $100 \%$ & 4.00 & 6.59 \\
\hline & Binomial & $0 \%$ & $0 \%$ & $0 \%$ & $0 \%$ & $100 \%$ & 4.00 & 6.67 \\
\hline
\end{tabular}


UNDERLYING DISTRIBUTIONS IN LOGLINEAR MODELS OF DISCRETE DATA

Table 4: Simulation Results for the Step Population Distribution

\begin{tabular}{|c|c|c|c|c|c|c|c|c|}
\hline \multirow{2}{*}{$N$} & \multirow{2}{*}{$\begin{array}{l}\text { Underlying } \\
\text { Distribution }\end{array}$} & \multicolumn{5}{|c|}{$\begin{array}{l}\text { Percentage of } K \text { Moments Selected } \\
\quad \text { (out of } 1,000 \text { replications) }\end{array}$} & \multirow{2}{*}{$\begin{array}{c}\text { Mean } \\
\text { Moments }(K)\end{array}$} & \multirow{2}{*}{$\begin{array}{c}\text { Mean } \\
G^{2}\end{array}$} \\
\hline & & 0 & 1 & 2 & 3 & 4 & & \\
\hline \multicolumn{9}{|c|}{$G^{2}$ Selections of $K$} \\
\hline \multirow{2}{*}{30} & Uniform & $45 \%$ & $44 \%$ & $4 \%$ & $6 \%$ & $3 \%$ & 0.78 & 11.57 \\
\hline & Binomial & & $1 \%$ & $91 \%$ & $3 \%$ & $5 \%$ & 2.13 & 8.95 \\
\hline \multirow{2}{*}{100} & Uniform & $2 \%$ & $72 \%$ & $7 \%$ & $17 \%$ & $3 \%$ & 1.48 & 12.34 \\
\hline & Binomial & & $0 \%$ & $81 \%$ & $4 \%$ & $15 \%$ & 2.33 & 10.90 \\
\hline \multirow{2}{*}{1,000} & Uniform & $0 \%$ & $0 \%$ & $0 \%$ & $69 \%$ & $30 \%$ & 3.30 & 37.72 \\
\hline & Binomial & & $0 \%$ & $2 \%$ & $1 \%$ & $97 \%$ & 3.95 & 38.01 \\
\hline \multicolumn{9}{|c|}{$A I C$ Selections of $K$} \\
\hline \multirow{2}{*}{30} & Uniform & $10 \%$ & $50 \%$ & $16 \%$ & $17 \%$ & $8 \%$ & 1.63 & 8.65 \\
\hline & Binomial & & $0 \%$ & $68 \%$ & $16 \%$ & $16 \%$ & 2.48 & 7.91 \\
\hline \multirow{2}{*}{100} & Uniform & $0 \%$ & $35 \%$ & $15 \%$ & $34 \%$ & $16 \%$ & 2.32 & 9.68 \\
\hline & Binomial & & $0 \%$ & $54 \%$ & $12 \%$ & $34 \%$ & 2.81 & 9.50 \\
\hline \multirow{2}{*}{1,000} & Uniform & $0 \%$ & $0 \%$ & $0 \%$ & $30 \%$ & $70 \%$ & 3.70 & 36.16 \\
\hline & Binomial & & $0 \%$ & $0 \%$ & $0 \%$ & $100 \%$ & 4.00 & 37.84 \\
\hline \multicolumn{9}{|c|}{ Always Fit $K=4$} \\
\hline \multirow{2}{*}{30} & Uniform & $0 \%$ & $0 \%$ & $0 \%$ & $0 \%$ & $100 \%$ & 4.00 & 6.83 \\
\hline & Binomial & $0 \%$ & $0 \%$ & $0 \%$ & $0 \%$ & $100 \%$ & 4.00 & 6.89 \\
\hline \multirow{2}{*}{100} & Uniform & $0 \%$ & $0 \%$ & $0 \%$ & $0 \%$ & $100 \%$ & 4.00 & 8.32 \\
\hline & Binomial & $0 \%$ & $0 \%$ & $0 \%$ & $0 \%$ & $100 \%$ & 4.00 & 8.52 \\
\hline \multirow{2}{*}{1,000} & Uniform & $0 \%$ & $0 \%$ & $0 \%$ & $0 \%$ & $100 \%$ & 4.00 & 35.89 \\
\hline & Binomial & $0 \%$ & $0 \%$ & $0 \%$ & $0 \%$ & $100 \%$ & 4.00 & 37.84 \\
\hline
\end{tabular}




\section{TIM MOSES}

Table 5: Simulation Results for the Triangular Population Distribution

\begin{tabular}{|c|c|c|c|c|c|c|c|c|}
\hline \multirow{2}{*}{$N$} & \multirow{2}{*}{$\begin{array}{l}\text { Underlying } \\
\text { Distribution }\end{array}$} & \multicolumn{5}{|c|}{$\begin{array}{l}\text { Percentage of } K \text { Moments Selected } \\
\text { (out of } 1,000 \text { replications) }\end{array}$} & \multirow{2}{*}{$\begin{array}{c}\text { Mean } \\
\text { Moments }(K)\end{array}$} & \multirow{2}{*}{$\begin{array}{c}\text { Mean } \\
G^{2}\end{array}$} \\
\hline & & 0 & 1 & 2 & 3 & 4 & & \\
\hline \multicolumn{9}{|c|}{$G^{2}$ Selections of $K$} \\
\hline \multirow{2}{*}{30} & Uniform & $46 \%$ & $2 \%$ & $44 \%$ & $2 \%$ & $7 \%$ & 1.21 & 10.83 \\
\hline & Binomial & & $0 \%$ & $93 \%$ & $4 \%$ & $3 \%$ & 2.10 & 8.56 \\
\hline \multirow{2}{*}{100} & Uniform & $1 \%$ & $0 \%$ & $75 \%$ & $1 \%$ & $22 \%$ & 2.43 & 9.69 \\
\hline & Binomial & & $0 \%$ & $88 \%$ & $6 \%$ & $6 \%$ & 2.19 & 9.66 \\
\hline \multirow{2}{*}{1,000} & Uniform & $0 \%$ & $0 \%$ & $0 \%$ & $0 \%$ & $100 \%$ & 4.00 & 23.55 \\
\hline & Binomial & & $0 \%$ & $14 \%$ & $19 \%$ & $66 \%$ & 3.52 & 26.68 \\
\hline \multicolumn{9}{|c|}{$A I C$ Selections of $K$} \\
\hline \multirow{2}{*}{30} & Uniform & $17 \%$ & $2 \%$ & $50 \%$ & $11 \%$ & $19 \%$ & 2.13 & 8.13 \\
\hline & Binomial & & $0 \%$ & $71 \%$ & $16 \%$ & $12 \%$ & 2.41 & 7.62 \\
\hline \multirow{2}{*}{100} & Uniform & $0 \%$ & $0 \%$ & $46 \%$ & $7 \%$ & $46 \%$ & 3.00 & 7.98 \\
\hline & Binomial & & $0 \%$ & $56 \%$ & $21 \%$ & $23 \%$ & 2.66 & 8.20 \\
\hline \multirow{2}{*}{1,000} & Uniform & $0 \%$ & $0 \%$ & $0 \%$ & $0 \%$ & $100 \%$ & 4.00 & 23.55 \\
\hline & Binomial & & $0 \%$ & $2 \%$ & $6 \%$ & $92 \%$ & 3.91 & 25.23 \\
\hline \multicolumn{9}{|c|}{ Always Fit $K=4$} \\
\hline \multirow{2}{*}{30} & Uniform & $0 \%$ & $0 \%$ & $0 \%$ & $0 \%$ & $100 \%$ & 4.00 & 6.50 \\
\hline & Binomial & $0 \%$ & $0 \%$ & $0 \%$ & $0 \%$ & $100 \%$ & 4.00 & 6.55 \\
\hline \multirow{2}{*}{100} & Uniform & $0 \%$ & $0 \%$ & $0 \%$ & $0 \%$ & $100 \%$ & 4.00 & 7.08 \\
\hline & Binomial & $0 \%$ & $0 \%$ & $0 \%$ & $0 \%$ & $100 \%$ & 4.00 & 7.24 \\
\hline \multirow{2}{*}{1,000} & Uniform & $0 \%$ & $0 \%$ & $0 \%$ & $0 \%$ & $100 \%$ & 4.00 & 23.55 \\
\hline & Binomial & $0 \%$ & $0 \%$ & $0 \%$ & $0 \%$ & $100 \%$ & 4.00 & 25.12 \\
\hline
\end{tabular}


UNDERLYING DISTRIBUTIONS IN LOGLINEAR MODELS OF DISCRETE DATA

Table 6: Simulation Results for the Platykurtic Population Distribution

\begin{tabular}{|c|c|c|c|c|c|c|c|c|}
\hline \multirow{2}{*}{$N$} & \multirow{2}{*}{$\begin{array}{l}\text { Underlying } \\
\text { Distribution }\end{array}$} & \multicolumn{5}{|c|}{$\begin{array}{l}\text { Percentage of } K \text { Moments Selected } \\
\text { (out of } 1,000 \text { replications) }\end{array}$} & \multirow{2}{*}{$\begin{array}{c}\text { Mean } \\
\text { Moments }(K)\end{array}$} & \multirow{2}{*}{$\begin{array}{c}\text { Mean } \\
G^{2}\end{array}$} \\
\hline & & 0 & 1 & 2 & 3 & 4 & & \\
\hline \multicolumn{9}{|c|}{$G^{2}$ Selections of $K$} \\
\hline \multirow{2}{*}{30} & Uniform & $74 \%$ & $0 \%$ & $17 \%$ & $0 \%$ & $9 \%$ & 0.71 & 10.38 \\
\hline & Binomial & & $2 \%$ & $91 \%$ & $1 \%$ & $6 \%$ & 2.11 & 8.00 \\
\hline \multirow{2}{*}{100} & Uniform & $26 \%$ & $0 \%$ & $50 \%$ & $1 \%$ & $24 \%$ & 1.96 & 9.44 \\
\hline & Binomial & & $0 \%$ & $86 \%$ & $4 \%$ & $10 \%$ & 2.24 & 7.94 \\
\hline \multirow{2}{*}{1,000} & Uniform & $0 \%$ & $0 \%$ & $0 \%$ & $0 \%$ & $100 \%$ & 3.99 & 12.24 \\
\hline & Binomial & & $0 \%$ & $16 \%$ & $8 \%$ & $76 \%$ & 3.60 & 12.41 \\
\hline \multicolumn{9}{|c|}{$A I C$ Selections of $K$} \\
\hline \multirow{2}{*}{30} & Uniform & $44 \%$ & $3 \%$ & $29 \%$ & $5 \%$ & $19 \%$ & 1.53 & 8.22 \\
\hline & Binomial & & $0 \%$ & $70 \%$ & $13 \%$ & $16 \%$ & 2.46 & 6.93 \\
\hline \multirow{2}{*}{100} & Uniform & $7 \%$ & $0 \%$ & $41 \%$ & $5 \%$ & $47 \%$ & 2.85 & 6.95 \\
\hline & Binomial & & $0 \%$ & $60 \%$ & $15 \%$ & $26 \%$ & 2.66 & 6.72 \\
\hline \multirow{2}{*}{1,000} & Uniform & $0 \%$ & $0 \%$ & $0 \%$ & $0 \%$ & $100 \%$ & 4.00 & 12.22 \\
\hline & Binomial & & $0 \%$ & $2 \%$ & $3 \%$ & $96 \%$ & 3.94 & 11.25 \\
\hline \multicolumn{9}{|c|}{ Always Fit $K=4$} \\
\hline \multirow{2}{*}{30} & Uniform & $0 \%$ & $0 \%$ & $0 \%$ & $0 \%$ & $100 \%$ & 4.00 & 5.88 \\
\hline & Binomial & $0 \%$ & $0 \%$ & $0 \%$ & $0 \%$ & $100 \%$ & 4.00 & 5.84 \\
\hline \multirow{2}{*}{100} & Uniform & $0 \%$ & $0 \%$ & $0 \%$ & $0 \%$ & $100 \%$ & 4.00 & 5.78 \\
\hline & Binomial & $0 \%$ & $0 \%$ & $0 \%$ & $0 \%$ & $100 \%$ & 4.00 & 5.67 \\
\hline \multirow{2}{*}{1,000} & Uniform & $0 \%$ & $0 \%$ & $0 \%$ & $0 \%$ & $100 \%$ & 4.00 & 12.22 \\
\hline & Binomial & $0 \%$ & $0 \%$ & $0 \%$ & $0 \%$ & $100 \%$ & 4.00 & 11.18 \\
\hline
\end{tabular}




\section{TIM MOSES}

Table 7: Simulation Results for the Leptokurtic Population Distribution

\begin{tabular}{|c|c|c|c|c|c|c|c|c|}
\hline \multirow{2}{*}{$N$} & \multirow{2}{*}{$\begin{array}{l}\text { Underlying } \\
\text { Distribution }\end{array}$} & \multicolumn{5}{|c|}{$\begin{array}{l}\text { Percentage of } K \text { Moments Selected } \\
\text { (out of } 1,000 \text { replications) }\end{array}$} & \multirow{2}{*}{$\begin{array}{c}\text { Mean } \\
\text { Moments }(K)\end{array}$} & \multirow{2}{*}{$\begin{array}{c}\text { Mean } \\
G^{2}\end{array}$} \\
\hline & & 0 & 1 & 2 & 3 & 4 & & \\
\hline \multicolumn{9}{|c|}{$G^{2}$ Selections of $K$} \\
\hline \multirow{2}{*}{30} & Uniform & $11 \%$ & $0 \%$ & $42 \%$ & $7 \%$ & $41 \%$ & 2.68 & 13.94 \\
\hline & Binomial & & $21 \%$ & $16 \%$ & $9 \%$ & $54 \%$ & 2.97 & 13.21 \\
\hline \multirow{2}{*}{100} & Uniform & $0 \%$ & $0 \%$ & $3 \%$ & $0 \%$ & $97 \%$ & 3.94 & 23.57 \\
\hline & Binomial & & $0 \%$ & $1 \%$ & $0 \%$ & $99 \%$ & 3.98 & 23.12 \\
\hline \multirow{2}{*}{1,000} & Uniform & $0 \%$ & $0 \%$ & $0 \%$ & $0 \%$ & $100 \%$ & 4.00 & 185.28 \\
\hline & Binomial & & $0 \%$ & $0 \%$ & $0 \%$ & $100 \%$ & 4.00 & 181.76 \\
\hline \multicolumn{9}{|c|}{$A I C$ Selections of $K$} \\
\hline \multirow{2}{*}{30} & Uniform & $3 \%$ & $0 \%$ & $21 \%$ & $10 \%$ & $66 \%$ & 3.35 & 11.86 \\
\hline & Binomial & & $8 \%$ & $11 \%$ & $8 \%$ & $73 \%$ & 3.47 & 11.65 \\
\hline \multirow{2}{*}{100} & Uniform & $0 \%$ & $0 \%$ & $1 \%$ & $0 \%$ & $99 \%$ & 3.99 & 23.41 \\
\hline & Binomial & & $0 \%$ & $0 \%$ & $0 \%$ & $100 \%$ & 4.00 & 23.05 \\
\hline \multirow{2}{*}{1,000} & Uniform & $0 \%$ & $0 \%$ & $0 \%$ & $0 \%$ & $100 \%$ & 4.00 & 185.28 \\
\hline & Binomial & & $0 \%$ & $0 \%$ & $0 \%$ & $100 \%$ & 4.00 & 181.76 \\
\hline \multicolumn{9}{|c|}{ Always Fit $K=4$} \\
\hline \multirow{2}{*}{30} & Uniform & $0 \%$ & $0 \%$ & $0 \%$ & $0 \%$ & $100 \%$ & 4.00 & 11.17 \\
\hline & Binomial & $0 \%$ & $0 \%$ & $0 \%$ & $0 \%$ & $100 \%$ & 4.00 & 11.07 \\
\hline \multirow{2}{*}{100} & Uniform & $0 \%$ & $0 \%$ & $0 \%$ & $0 \%$ & $100 \%$ & 4.00 & 23.40 \\
\hline & Binomial & $0 \%$ & $0 \%$ & $0 \%$ & $0 \%$ & $100 \%$ & 4.00 & 23.05 \\
\hline \multirow{2}{*}{1,000} & Uniform & $0 \%$ & $0 \%$ & $0 \%$ & $0 \%$ & $100 \%$ & 4.00 & 185.28 \\
\hline & Binomial & $0 \%$ & $0 \%$ & $0 \%$ & $0 \%$ & $100 \%$ & 4.00 & 181.76 \\
\hline
\end{tabular}


UNDERLYING DISTRIBUTIONS IN LOGLINEAR MODELS OF DISCRETE DATA

Table 8: Simulation Results for the Binomial Population Distribution

\begin{tabular}{|c|c|c|c|c|c|c|c|c|}
\hline \multirow{2}{*}{$N$} & \multirow{2}{*}{$\begin{array}{l}\text { Underlying } \\
\text { Distribution }\end{array}$} & \multicolumn{5}{|c|}{$\begin{array}{l}\text { Percentage of } K \text { Moments Selected } \\
\text { (out of } 1,000 \text { replications) }\end{array}$} & \multirow{2}{*}{$\begin{array}{c}\text { Mean } \\
\text { Moments }(K)\end{array}$} & \multirow{2}{*}{$\underset{G^{2}}{\operatorname{Mean}}$} \\
\hline & & 0 & 1 & 2 & 3 & 4 & & \\
\hline \multicolumn{9}{|c|}{$G^{2}$ Selections of $K$} \\
\hline \multirow{2}{*}{30} & Uniform & $0 \%$ & $0 \%$ & $96 \%$ & $1 \%$ & $4 \%$ & 2.08 & 6.09 \\
\hline & Binomial & & $94 \%$ & $1 \%$ & $1 \%$ & $4 \%$ & 1.15 & 6.86 \\
\hline \multirow{2}{*}{100} & Uniform & $0 \%$ & $0 \%$ & $95 \%$ & $1 \%$ & $4 \%$ & 2.09 & 6.89 \\
\hline & Binomial & & $94 \%$ & $2 \%$ & $1 \%$ & $3 \%$ & 1.13 & 7.62 \\
\hline \multirow{2}{*}{1,000} & Uniform & $0 \%$ & $0 \%$ & $92 \%$ & $1 \%$ & $7 \%$ & 2.14 & 7.46 \\
\hline & Binomial & & $96 \%$ & $1 \%$ & $1 \%$ & $2 \%$ & 1.10 & 7.69 \\
\hline \multicolumn{9}{|c|}{$A I C$ Selections of $K$} \\
\hline \multirow{2}{*}{30} & Uniform & $0 \%$ & $0 \%$ & $74 \%$ & $9 \%$ & $17 \%$ & 2.43 & 5.11 \\
\hline & Binomial & & $72 \%$ & $12 \%$ & $6 \%$ & $10 \%$ & 1.53 & 5.82 \\
\hline \multirow{2}{*}{100} & Uniform & $0 \%$ & $0 \%$ & $77 \%$ & $10 \%$ & $13 \%$ & 2.36 & 6.12 \\
\hline & Binomial & & $75 \%$ & $12 \%$ & $6 \%$ & $7 \%$ & 1.46 & 6.70 \\
\hline \multirow{2}{*}{1,000} & Uniform & $0 \%$ & $0 \%$ & $68 \%$ & $11 \%$ & $21 \%$ & 2.53 & 6.31 \\
\hline & Binomial & & $76 \%$ & $12 \%$ & $7 \%$ & $5 \%$ & 1.42 & 6.78 \\
\hline \multicolumn{9}{|c|}{ Always Fit $K=4$} \\
\hline \multirow{2}{*}{30} & Uniform & $0 \%$ & $0 \%$ & $0 \%$ & $0 \%$ & $100 \%$ & 4.00 & 4.01 \\
\hline & Binomial & $0 \%$ & $0 \%$ & $0 \%$ & $0 \%$ & $100 \%$ & 4.00 & 3.99 \\
\hline \multirow{2}{*}{100} & Uniform & $0 \%$ & $0 \%$ & $0 \%$ & $0 \%$ & $100 \%$ & 4.00 & 4.99 \\
\hline & Binomial & $0 \%$ & $0 \%$ & $0 \%$ & $0 \%$ & $100 \%$ & 4.00 & 4.96 \\
\hline \multirow{2}{*}{1,000} & Uniform & $0 \%$ & $0 \%$ & $0 \%$ & $0 \%$ & $100 \%$ & 4.00 & 5.13 \\
\hline & Binomial & $0 \%$ & $0 \%$ & $0 \%$ & $0 \%$ & $100 \%$ & 4.00 & 5.08 \\
\hline
\end{tabular}




\section{TIM MOSES}

Table 9: Simulation Results for the Under-Dispersed Binomial Population Distribution

\begin{tabular}{|c|c|c|c|c|c|c|c|c|}
\hline \multirow{2}{*}{$N$} & \multirow{2}{*}{$\begin{array}{l}\text { Underlying } \\
\text { Distribution }\end{array}$} & \multicolumn{5}{|c|}{$\begin{array}{l}\text { Percentage of } K \text { Moments Selected } \\
\text { (out of } 1,000 \text { replications) }\end{array}$} & \multirow{2}{*}{$\begin{array}{c}\text { Mean } \\
\text { Moments }(K)\end{array}$} & \multirow{2}{*}{$\begin{array}{c}\text { Mean } \\
G^{2}\end{array}$} \\
\hline & & 0 & 1 & 2 & 3 & 4 & & \\
\hline \multicolumn{9}{|c|}{$G^{2}$ Selections of $K$} \\
\hline \multirow{2}{*}{30} & Uniform & $0 \%$ & $0 \%$ & $91 \%$ & $0 \%$ & $9 \%$ & 2.19 & 4.70 \\
\hline & Binomial & & $85 \%$ & $5 \%$ & $0 \%$ & $10 \%$ & 1.36 & 5.70 \\
\hline \multirow{2}{*}{100} & Uniform & $0 \%$ & $0 \%$ & $79 \%$ & $0 \%$ & $21 \%$ & 2.42 & 5.80 \\
\hline & Binomial & & $64 \%$ & $18 \%$ & $0 \%$ & $18 \%$ & 1.73 & 7.13 \\
\hline \multirow{2}{*}{1,000} & Uniform & $0 \%$ & $0 \%$ & $0 \%$ & $0 \%$ & $100 \%$ & 4.00 & 8.49 \\
\hline & Binomial & & $0 \%$ & $0 \%$ & $0 \%$ & $100 \%$ & 4.00 & 8.37 \\
\hline \multicolumn{9}{|c|}{$A I C$ Selections of $K$} \\
\hline \multirow{2}{*}{30} & Uniform & $0 \%$ & $0 \%$ & $71 \%$ & $1 \%$ & $27 \%$ & 2.56 & 3.72 \\
\hline & Binomial & & $57 \%$ & $24 \%$ & $1 \%$ & $18 \%$ & 1.81 & 4.40 \\
\hline \multirow{2}{*}{100} & Uniform & $0 \%$ & $0 \%$ & $49 \%$ & $1 \%$ & $50 \%$ & 3.01 & 4.26 \\
\hline & Binomial & & $23 \%$ & $40 \%$ & $1 \%$ & $36 \%$ & 2.49 & 4.85 \\
\hline \multirow{2}{*}{1,000} & Uniform & $0 \%$ & $0 \%$ & $0 \%$ & $0 \%$ & $100 \%$ & 4.00 & 8.49 \\
\hline & Binomial & & $0 \%$ & $0 \%$ & $0 \%$ & $100 \%$ & 4.00 & 8.35 \\
\hline \multicolumn{9}{|c|}{ Always Fit $K=4$} \\
\hline \multirow{2}{*}{30} & Uniform & $0 \%$ & $0 \%$ & $0 \%$ & $0 \%$ & $100 \%$ & 4.00 & 2.56 \\
\hline & Binomial & $0 \%$ & $0 \%$ & $0 \%$ & $0 \%$ & $100 \%$ & 4.00 & 2.55 \\
\hline \multirow{2}{*}{100} & Uniform & $0 \%$ & $0 \%$ & $0 \%$ & $0 \%$ & $100 \%$ & 4.00 & 3.20 \\
\hline & Binomial & $0 \%$ & $0 \%$ & $0 \%$ & $0 \%$ & $100 \%$ & 4.00 & 3.18 \\
\hline \multirow{2}{*}{1,000} & Uniform & $0 \%$ & $0 \%$ & $0 \%$ & $0 \%$ & $100 \%$ & 4.00 & 8.49 \\
\hline & Binomial & $0 \%$ & $0 \%$ & $0 \%$ & $0 \%$ & $100 \%$ & 4.00 & 8.35 \\
\hline
\end{tabular}




\section{UNDERLYING DISTRIBUTIONS IN LOGLINEAR MODELS OF DISCRETE DATA}

For population distributions that closely reflect the binomial distribution (i.e., the binomial and under-dispersed binomial population distributions, Tables 8-9), using the binomial distribution results in $A I C$ and $G^{2}$ model selections with smaller mean $K$ values and larger mean $G^{2}$ values than using the uniform distribution. Model selection results for the platykurtic population distribution were mixed (Table 6).

When loglinear models were fit with each underlying distribution using a predetermined $K=4$, rather than a statistically selected $K$ value, the results depended on how closely the underlying distribution reflected the population distribution. For the uniform, decreasing, step and triangular population distributions (Tables 2-5), the use of a predetermined $K$ value of 4 resulted in slightly smaller mean $G^{2}$ values with the uniform distribution than the binomial distribution. For the platykurtic, leptokurtic, binomial and underdispersed binomial population distributions (Tables 6-9), the use of a predetermined $K$ value of 4 resulted in slightly smaller mean $G^{2}$ values with the binomial distribution than the uniform distribution.

\section{Conclusion}

The results of this study show that the loglinear model's underlying distribution has a small but real - effect in modeling discrete distributions. This effect depends on the population distribution and on how the number of moments is determined. For models fitting a predetermined number of moments, this study shows that the use of a binomial underlying distribution can result in better fits for distributions that are similar to the binomial distribution, whereas the use of a uniform underlying distribution can result in better fits for distributions similar to the uniform distribution.

For the statistical selection of loglinear models in sample distributions, results suggest that using a distribution less similar to the distribution being modeled (e.g., using the binomial as the underlying distribution for uniformly distributed populations and samples) results in more moments being chosen and slightly better model fit. The implications for modeling distributions more likely to resemble binomial distributions than uniform distributions (e.g., psychometric tests) are that better fitting models can be statistically selected when using an underlying uniform distribution, and better fitting models for a predetermined number of moments can be obtained using an underlying binomial distribution.

Results obtained herein are useful replications and extensions of other studies that have assessed statistical power for detecting departures from uniform distributions (Choulakian, Lockhart \& Stephens, 1994; Pettitt \& Stephens, 1977; Steele \& Chaseling, 2006). This study also showed that the likelihood ratio $\left(G^{2}\right)$ selection strategy had relatively moderate power levels and the $A I C$ selection strategies had relatively high power levels compared to the strategies considered in Steele and Chaeseling's study (i.e., the Kolmogorov-Smirnov, nominal Kolmogorov-Smirnov, Cramer-von Mises, Anderson-Darling, Pearson Chi-square and Watson's tests). Similar to the prior studies, this study found that power is higher when the underlying distribution is less similar to the distribution being modeled.

This study extends prior power studies by considering Type I error, where this study shows that Type I error rates were closer to $5 \%$ for the $G^{2}$ selection strategy than the AIC selection strategy. The more controlled Type I error rates of the $G^{2}$ selection strategy were observed both for the uniform distribution (underlying and population, Table 2) and also for the binomial distribution (underlying and population, Table 8). This study's findings that the $G^{2}$ and $A I C$ selection strategies' Type I error and power tendencies for assessing binomial distributions are similar to those for assessing uniform distributions also extend prior studies that have primarily focused on detecting departures from uniform distributions. Results suggest that future studies considering loglinear models' underlying distributions would be useful for comparing other distributions and statistical selection strategies. 


\section{TIM MOSES}

\section{References}

Agresti, A. (2002). Categorical data analysis $\left(2^{\text {nd }} E d\right.$.). New York, NY: Wiley.

Akaike, H. (1981). Likelihood of a model and information criteria. Journal of Econometrics, 16, 3-14.

Bishop, Y. M. M., Fienberg, S. E., \& Holland, P. W. (1975). Discrete multivariate analysis. Cambridge, MA: MIT Press.

Choulakian, V., Lockhart, R. A., \& Stephens, M. A. (1994). Cramer-von Mises statistics for discrete distributions. The Canadian Journal of Statistics, 22, 125-137.

Haberman, S. J. (1974). Log-linear models for frequency tables with ordered classifications. Biometrics, 30, 589-600.

Holland, P. W., \& Thayer, D. T. (2000). Univariate and bivariate loglinear models for discrete test score distributions. Journal of Educational and Behavioral Statistics, 25, 133183.
Kolen, M. J., \& Brennan, R. J. (2004). Test equating, scaling and linking $\left(2^{\text {nd }} E d\right.$. $)$. New York, NY: Springer-Verlag.

Moses, T., \& Holland, P. W. (2010). A comparison of statistical selection strategies for univariate and bivariate log-linear models. British Journal of Mathematical and Statistical Psychology, 63, 557-574.

Pettitt, A. N., \& Stephens, M. A. (1977). The Komogorov-Smirnov goodness-of-fit statistic with discrete and grouped data. Technometrics, 19, 205-210.

Steele, M., \& Chaseling, J. (2006). Powers of discrete goodness-of-fit test statistics for a uniform null against a selection of alternative distributions. Communications in Statistics-Simulation and Computation, 35, 1067-1075. 


\title{
The Impact of Violating Factor Scaling Method Assumptions On Latent Mean Difference Testing in Structured Means Models
}

\author{
Dandan Wang Tiffany A. Whittaker S. Natasha Beretvas \\ The University of Texas at Austin, \\ Austin, TX
}

Type I error rates and power of the likelihood ratio test and bias of the standardized effect size measure associated with the latent mean difference in structured means modeling are examined when violating the assumptions underlying the two available factor scaling methods under various conditions. Implications and recommendations are discussed.

Key words: Structured means modeling, latent mean difference testing, non-invariant factor loadings, factor scaling methods, reference indicator.

Introduction

Many social science studies focus on comparing outcomes for groups categorized by observed variables such as gender, race or treatment group membership. Structural equation modeling (SEM) and, more specifically, structured means modeling (SMM; Sörbom, 1974) may be used to compare, for example, male and female high school students' latent variable means on math anxiety. The SMM approach is a multiple-group confirmatory factor analysis (MG-CFA) model in which the mean structure is incorporated into the model for testing the difference in latent variable means across groups.

Dandan Wang received her Ph.D. in Quantitative Methods in the Department of Educational Psychology at the University of Texas at Austin. She currently works at International Baccalaureate. Email her at: dwvv4@utexas.edu. Tiffany Whittaker is an Assistant Professor of Quantitative Methods in the Department of Educational Psychology. Email her at: t.whittaker@austin.utexas.edu. S. Natasha Beretvas is a Professor of Quantitative Methods in the Department of Educational Psychology. Her interests are in multilevel and meta-analytic modeling techniques. Email her at: tasha.beretvas@mail.utexas.edu.
As with traditional CFA techniques, each latent variable must be assigned a scale of measurement in SMM. This may be accomplished by either constraining one loading per factor to a value of one across groups or constraining each factor's variance to a value of one across groups (Kline, 2011). Both factor scaling methods require meeting certain assumptions. For example, the reference indicator (RI) strategy involves an assumption that the RI has invariant factor loadings across groups. The factor-variance scaling method, by contrast, is based on an assumption that the factor variances are equal across groups. To date, no published study has examined the effect on latent mean comparisons of constraining unequal factor loadings or unequal factor variances to a value of one across groups. The focus of this study is to investigate the impact of violating the assumptions underlying two factor scaling methods on the latent mean difference test and the standardized effect size measure associated with the latent mean difference in SMM.

Structured Means Model and Testing Latent Mean Differences

A single-factor, $p$-indicator structured means model can be expressed in matrix notation using the following measurement equation:

$$
x_{g}=v_{g}+\bigwedge_{g} \xi_{g}+\delta_{g}
$$


where $g(g=1,2, \ldots, G)$ represents group membership, $\boldsymbol{x}$ is a vector containing $p \times 1$ observed variable scores, $\boldsymbol{v}$ is a $p \times 1$ vector containing indicator variable intercepts, $\boldsymbol{\Lambda}$ is a $p \times 1$ vector of factor loadings that relates the observed indicator variables to the latent variable, $\boldsymbol{\xi}$ is a latent variable score and $\boldsymbol{\delta}$ is a $p \times 1$ vector of normally distributed random measurement errors associated with the observed indicator variables.

In a structured means model (SMM), certain constraints are imposed in order to validly compare latent means across groups as well as to ensure model identification. The factor loadings and observed indicator variables' intercepts are constrained to be equal across groups in SMM. This allows latent mean differences detected between groups to be attributed to actual differences in the constructs as opposed to differences in the measurement of the constructs across the groups (Rock, Werts \& Flaugher, 1978; Sörbom, 1974). In addition, it is not possible to estimate the intercepts of all observed indicator variables separately across groups as this would result in the underidentification of the means portion of the model (Sörbom, 1974).

Under the assumption of factor loading and intercept invariance, and assuming that the mean of the measurement errors within each group is equal to zero, the expected values of observed variables in each group can be expressed in matrix notation as:

$$
E\left[x_{g}\right]=\mu_{g}=v+\Lambda \kappa_{g},
$$

where $\boldsymbol{\kappa}_{\boldsymbol{g}}$ is the latent variable mean for group $g, \boldsymbol{v}$ is a $p \times 1$ vector containing the observed variables' intercepts which are invariant across groups, and $\Lambda$ represents a $p \times 1$ vector containing invariant factor loadings (Yoon \& Millsap, 2007). In addition, assuming that the measurement errors are uncorrelated and that the measurement errors are uncorrelated with the latent variable in each group, $g$, the covariances among observed variables in each group can be expressed in matrix notation as:

$$
\begin{aligned}
E\left[\left(x_{g}-\mu_{g}\right)\left(x_{g}-\mu_{g}\right)^{\prime}\right] & =\Sigma_{g} \\
& =\Lambda \Phi_{g} \Lambda^{\prime}+\Theta_{g}
\end{aligned}
$$

where $\boldsymbol{\Phi}$ is the latent variable variance, $\boldsymbol{\Theta}$ is a $p \times p$ diagonal matrix containing $p$ measurement error variances associated with the observed indicator variables and $\boldsymbol{\Lambda}$ represents a $p \times 1$ vector containing invariant factor loadings (Sörbom, 1974; Yoon \& Millsap, 2007).

If factor loading and/or intercept invariance is not supported by means of model fit assessment and/or model comparisons, Byrne, Shavelson and Muthén (1989) suggested that some of the constraints may be relaxed and that partial invariance suffices when using the SMM approach to compare latent means across groups. It is important to note that there exists a debate concerning the issue of partial measurement invariance and the meaningful interpretation of latent mean differences in SMM. Some contend that strict invariance is required for valid interpretations of latent mean differences (Meredith, 1993), whereas others maintain that strong invariance is sufficient (Hancock, 1997; Thompson \& Green, 2006). (In addition, some researchers have argued - and demonstrated that meaningful interpretations of latent mean differences may be rendered under partial factor loading and partial intercept invariance; this debate is beyond the scope of this work but the interested reader is referred to: Byrne, Shavelson \& Muthén, 1989; Muthén \& Christoffersson, 1981; Steenkamp \& Baumgartner, 1998.)

The means portion of the model is estimated through use of a constant which is modeled to have direct effects on the latent and observed indicator variables. The constant's direct effect on a latent variable represents the latent variable mean and its effects on observed indicator variables represent observed variables' intercepts (Kline, 2011). An added constraint must also be imposed for identification of the means portion of the model. Namely, the latent mean in one group, treated as the reference group, must be constrained to zero whereas the latent means in the remaining $G-1$ comparison groups are estimated. Therefore, the test of the latent mean of the $G-1$ comparison groups 


\section{IMPACT OF VIOLATING FACTOR SCALING METHOD ASSUMPTIONS}

corresponds to a test of the latent mean difference between each of the $G-1$ comparison group's and the reference group's latent mean (Hancock, 1997). For simplicity, a two-group comparison of the latent means in a single-factor model will be assumed for the ensuing discussion of latent mean comparisons in SMM.

Because the expected value of the latent variable is given by:

$$
E\left(\xi_{g}\right)=\kappa_{g},
$$

the latent variable mean in the reference group for a two-group comparison of a single-factor model is:

$$
E(\xi)=\kappa_{1}=0
$$

and the latent variable mean in the comparison group (group two) is:

$$
E(\xi)=\kappa_{2}-\kappa_{1}=\kappa_{2} .
$$

Accordingly, the null hypothesis tested in SMM is that the two groups' means are equal:

$$
H_{0}: \kappa_{1}=\kappa_{2} \text {. }
$$

The $z$ test statistic is commonly used to evaluate the statistical significance of the latent mean difference estimate in SMM. If the $z$ test statistic associated with the estimated latent mean difference is statistically significant, then it is inferred that there is a significant difference between the two groups' latent variable means. Unfortunately, the $z$ test statistic is not invariant to the choice of factor scaling method. Consequently, the likelihood ratio test, $\mathrm{LRT}_{\kappa}$, has been suggested to evaluate the statistical significance of the latent mean difference estimate in SMM because it is invariant to factor scaling procedures (Gonzalez \& Griffin, 2001; Hancock, Lawrence \& Nevitt, 2000).

When calculating the $\mathrm{LRT}_{\kappa}$, two models must be estimated: The parameter of interest (the latent variable mean difference) is freely estimated in group two in one model but is constrained to be equal to the reference group's latent variable mean value of zero in the second model. The $\mathrm{LRT}_{\kappa}$ is calculated as the difference between the two nested models' Chisquare $\left(\chi^{2}\right)$ statistics, and the $\mathrm{LRT}_{\kappa}$ is asymptotically distributed as a non-central $\chi^{2}$ statistic:

$$
\mathrm{LRT}_{\kappa}=\Delta \chi^{2}=\chi_{\text {restricted }}^{2}-\chi_{\text {baseline model }}^{2},
$$

where $\chi_{\text {restricted }}^{2}$ is the $\chi^{2}$ statistic associated with the model in which the latent variable mean difference is constrained to a value of zero and $\chi_{\text {baseline model }}^{2}$ is the $\chi^{2}$ statistic associated with the model in which the latent mean is freely estimated in group two. The $\mathrm{LRT}_{\kappa}$ has corresponding degrees of freedom equal to the difference in the degrees of freedom associated with each model and is calculated to evaluate whether there is a statistically significant drop in model fit when constraining a particular parameter (the latent variable mean difference) to zero. A significant $\mathrm{LRT}_{\kappa}$ indicates that the parameter of interest differs significantly from zero.

Although the $\mathrm{LRT}_{\kappa}$ may be used to evaluate whether there is a statistically significant difference between two groups' latent means, it does not provide any information about the practical significance of the latent mean difference. Hancock (2001) suggested using a standardized effect size measure, $\hat{\delta}_{\kappa}$, to describe the practical difference between two groups' latent means. When using the SMM approach, the standardized latent mean difference effect size, $\hat{\delta}_{\kappa}$, is estimated as follows:

$$
\begin{aligned}
& \hat{\delta}_{\kappa}=\left(\hat{k}_{1}-\hat{k}_{2}\right) / \hat{\phi}^{1 / 2}=\hat{k}_{2} / \hat{\phi}^{1 / 2}, \\
& \hat{\delta}_{k}=\left|\hat{k}_{1}-\hat{k}_{2}\right| / \hat{\phi}^{1 / 2}=\hat{k}_{2} / \hat{\phi}^{1 / 2}
\end{aligned}
$$


where $\hat{k}_{1}$ and $\hat{k}_{2}$ represent groups one and two latent mean estimates, respectively. For model identification purposes, the latent mean of the reference group (group one) is typically constrained to a value of zero, but the latent mean of the comparison group (group two) is estimated (Hancock, 1997), resulting in the rightmost expression of Equation 9. The pooled factor variance estimate, $\hat{\phi}$, is determined as follows:

$$
\hat{\phi}=\left(n_{1} \hat{\phi}_{1}+n_{2} \hat{\phi}_{2}\right) /\left(n_{1}+n_{2}\right)
$$

where $\hat{\phi}_{1}$ and $\hat{\phi}_{2}$ are the estimated factor variances for groups one and two, respectively, and $n_{1}$ and $n_{2}$ represent the sample sizes for groups one and two, respectively. It is important to note that the calculation and use of the pooled factor variance involves an assumption of homogeneity of the two groups' factor variances. The interpretation of $\hat{\delta}_{\kappa}$ is similar to that in conventional univariate analyses. For example, $\hat{\delta}_{\kappa}=0.5$ can be interpreted as indicating that the two groups' latent mean estimates differ by half a standard deviation (Hancock, 2001).

Factor Scaling Method Implications and Related Research

Both factor scaling methods may be used to scale the latent variable in a structured means model and both involve strict assumptions. For example, the factor-variancebased scaling method is grounded on the assumption that the factor variance is invariant across groups. If the factor variances are not equal across groups, the scale of the factor loadings will be changed, possibly making truly invariant factor loadings falsely appear noninvariant across groups. This could also make the metric invariance test less accurate (Cheung \& Rensvold, 1999; Kline, 2011; Yoon \& Millsap, 2007).

The reference indicator (RI) strategy is based on the assumption that the RI's loading is invariant across groups. If this assumption is violated, all other factor loadings in a structured means model will be rescaled. Constraining the RI's non-invariant factor loadings to a value of one across groups will result in different metrics for the two groups' factor loadings and can lead to incorrect inferences about the changed loadings' invariance; therefore, it is important to select an item that has invariant factor loadings across groups to serve as the RI in a structured means model (Johnson, Meade \& DuVernet, 2009).

Although assumptions associated with the two factor scaling methods are important, researchers have not examined the issue to a great extent. For example, Johnson, Meade and DuVernet (2009) conducted a literature review of studies published between 2005 and 2007 that involved measurement invariance (MI) tests; only 17 out of 153 studies referenced Cheung and Rensvold's (1999) study in which a new technique to select invariant item sets to serve as the RI was recommended. Most research simply assumed that the selected RI variable had invariant factor loadings across groups; consequently, it is essential that the impact of violating the assumptions associated with the two factor scaling methods be inspected in order to better inform applied users of SMM.

Previous studies germane to this study include those in which the effect of partial metric invariance on latent mean difference testing was assessed. For example, Kaplan and George (1995) conducted a population study to assess the power to detect latent mean differences between two groups in the SMM approach while manipulating the magnitude of the latent mean difference, group sample size ratio, frequency of non-invariant factor loadings, factor loading size, factor loading pattern and the number of observed indicators per factor. Because factor loadings were varied in the study, the determinant of the covariance matrix $[\operatorname{det}(\Sigma)]$ was also varied. The determinant corresponds to the generalized variance, which indicates the amount of variance shared among a set of variables. Two combinations of ratios of larger sample size $\left(n_{\text {Larger }}\right)$ to generalized variance $[\operatorname{det}(\Sigma)]$ conditions were examined, including a positive, $n_{\text {Larger }}: \operatorname{det}(\boldsymbol{\Sigma})$, condition in which the group with the larger sample size was paired with the larger generalized variance, and 


\section{IMPACT OF VIOLATING FACTOR SCALING METHOD ASSUMPTIONS}

a negative, $n_{\text {Larger }}: \operatorname{det}(\Sigma)$, condition in which the group with the larger sample size was paired with the smaller generalized variance.

The findings demonstrated that when the magnitude of the latent mean difference increased, the power of the latent mean difference test increased and the sample size ratio between the two groups tended to influence the power of the latent mean difference test. When the group sample sizes were equal, the power of the latent mean difference test was less affected by non-invariant factor loadings. However, when unequal sample sizes were present, the power associated with the latent mean difference test was low even though factor loading invariance held. A large drop in power was observed as the group sample size ratio increased which was observed in both positive and negative conditions. Nonetheless, higher power always occurred in the positive $n_{\text {Larger }}: \operatorname{det}(\boldsymbol{\Sigma})$ condition as compared to the negative $n_{\text {Larger }}: \operatorname{det}(\boldsymbol{\Sigma})$ condition. Finally, the power of the latent mean difference test increased when the model consisted of more indicator variables per factor.

Hancock, Lawrence and Nevitt (2000) conducted both a Monte Carlo simulation study and a population study investigating how partial metric invariance affected the Type I error rates and the power, respectively, of the latent mean difference test between two groups using SMM, multiple-indicator multiple-cause (MIMIC) modeling, and MANOVA approaches. Manipulated conditions included latent mean difference magnitude, total sample size, group sample size ratio, frequency of non-invariant factor loadings, factor loading size and factor loading pattern. Factor loading pattern manipulations resulted in four scenarios: (1) metric invariance with equal factor loadings across and within two groups; (2) metric invariance with equal factor loadings across two groups but unequal within groups; (3) metric non-invariance with approximately equivalent generalized variances for the two groups; and (4) metric non-invariance with different generalized variances for the two groups.

Type I error rates of the latent mean difference tests in all three approaches were well controlled under metric invariance, approximately equivalent group generalized variance, and equal group sample size conditions. When both the sample size and the generalized variance were unequal between the two groups, however, Type I error rates of the latent mean difference test in the three approaches varied. The SMM approach was the only one in which Type I error rates were well controlled under all manipulated conditions. The Type I error rates when using the MIMIC approach were too low under the negative $n_{\text {Larger }}: \operatorname{det}(\boldsymbol{\Sigma})$ condition (larger sample size paired with smaller generalized variance) and were too high under the positive $n_{\text {Larger }}: \operatorname{det}(\boldsymbol{\Sigma})$ condition (larger sample size paired with larger generalized variance). The opposite pattern of Type I error rates were observed when using the MANOVA approach.

The power of the latent mean difference test in the three approaches increased when the sample size, magnitude of the factor loadings, and magnitude of the latent mean difference increased. When the sample size ratio between the two groups became larger, the power of the latent mean difference test in the three approaches decreased. Overall, the power of the latent mean difference test when using the MIMIC technique tended to be approximately equal to, or marginally higher than, the power when using the SMM technique, but the power associated with the MANOVA approach was the lowest. When different generalized variances were paired with unequal sample sizes, results indicated that the SMM approach had greater power in the negative $n_{\text {Larger }}: \operatorname{det}(\boldsymbol{\Sigma})$ condition whereas the MIMIC approach had greater power in the positive $n_{\text {Larger }}: \operatorname{det}(\Sigma)$ condition.

Hancock et al. (2000) reported that both SMM and MIMIC approaches were acceptable under equal group sample sizes. The SMM approach, however, was recommended under unequal group sample sizes. The choice of the SMM approach was based on its flexibility in accommodating non-invariant factor loadings. Additionally, the SMM approach had satisfactory power without sacrificing the Type I error rate. In contrast, the MIMIC approach's slightly higher power was marred by the potential cost of Type I error inflation (Hancock, et al., 2000). 
Previous studies investigating the effects of partial measurement invariance on latent mean difference detection under various conditions have found that group sample size ratio, factor loading pattern, loading difference magnitude, and latent mean difference magnitude can affect both or either the Type I error rate and power of latent mean difference tests. However, previous simulation studies have not devoted much attention to the assumption underlying the RI strategy and - to the authors' knowledge - no published study has investigated the effect of violating the assumption underlying the factor-variance scaling method. The purpose of this Monte Carlo simulation study is to investigate the performance of the likelihood ratio test $\left(\mathrm{LRT}_{\kappa}\right)$ and the standardized latent mean difference effect size measure $\left(\hat{\delta}_{\kappa}\right)$ when violating the assumptions fundamental to the two factor scaling methods and using the SMM approach to test latent mean differences.

\section{Methodology}

The impact of violating the assumptions associated with the two factor scaling methods on the performance of the $\mathrm{LRT}_{\kappa}$ and $\hat{\delta}_{\kappa}$ were examined under varied conditions, including group sample size ratio, factor loading pattern, loading difference magnitude, latent mean difference magnitude and group factor variance ratio. For each generated sample of data, two factor scaling methods (constraining one loading per factor to a value of one for both groups and assigning a value of one to each factor's variance for both groups) were implemented. The performance of the $\mathrm{LRT}_{\kappa}$ was evaluated via an assessment of its Type I error rates and power under specified conditions. The performance of the $\hat{\delta}_{\kappa}$ in terms of the parameter bias and relative parameter bias under certain conditions was also evaluated.

For simplicity, two groups' latent variable means were compared using the SMM approach. The model used for data generation and estimation was a simple, single-factor model with six observed indicator variables. The choice of the six observed indicator variables was based on designs of previous simulation studies (e.g.,
Kaplan \& George, 1995) and reflects what has been found in applied research (Hinkin, 1995). The values of all invariant factor loadings were set to 0.4 to represent factor loadings commonly observed in applied studies (Enders \& Finney, 2003) and because a relatively large loading difference value across groups was included and resulted in markedly large non-invariant factor loadings.

All observed variable intercepts were set to zero across groups in the generating models. Residual variances associated with the observed variables were calculated as one minus the squared condition-specific standardized factor loading. Error covariances were not modeled in the generating or estimating models. Total sample size was 500 and was not varied. This sample size was used because it is in the range of sample sizes utilized in previous simulation research in which adequate power was obtained (e.g., Hancock, et al., 2000) and permits the examination of reasonably disparate group sample sizes.

Manipulated Conditions: Group Sample Size Ratio

Three group sample size ratio conditions $\left(n_{1}: n_{2}\right)$ were used when generating the data. The equal sample size condition (1:1) served as a baseline condition in which the sample size in each group was equal to 250 . Two unequal sample size ratio conditions (1:4 and 4:1) were also used to generate the data: data in the $1: 4$ condition were generated such that the sample size was 100 and 400 in group one and in group two, respectively, and data in the $4: 1$ condition were generated in which the two groups' sample sizes were reversed.

Manipulated Conditions: Factor Loading Pattern Five factor loading patterns were manipulated in this study. In the equal factor loading pattern condition, all factor loadings were generated to be invariant across groups to serve as a baseline condition. In the $1^{\text {st }}$ loading unequal pattern condition, the RI's (here, the first observed indicator variable's) factor loading was set to be higher in group two than in group one by the condition-specific factor loading difference. In the $2^{\text {nd }}$ loading unequal pattern condition, the factor loading of a non-RI 


\section{IMPACT OF VIOLATING FACTOR SCALING METHOD ASSUMPTIONS}

variable (here, the second observed indicator variable) was set to be higher in group two than in group one by the condition-specific factor loading difference. In the all lower pattern condition and the mixed pattern condition, both the RI and the second observed indicator variable had non-invariant factor loadings across groups in the generating models. In the all lower pattern condition, both of the non-invariant factor loadings had lower true values in group one. In the mixed pattern condition, the true factor loading value for the RI was higher in group one and the true factor loading value for the second observed indicator variable was higher in group two.

Manipulated Conditions: Loading Difference Magnitude

Two factor loading difference values $\left(\left|\lambda_{1}-\lambda_{2}\right|=0.1\right.$ and $\left.\left|\lambda_{1}-\lambda_{2}\right|=0.4\right)$ were investigated in the current simulation study to represent small and large differences. These two values are in the range of factor loading difference values investigated in previous simulation research (Hancock, et al., 2000; Kaplan \& George, 1995). These factor loading differences were added to the invariant factor loading value of 0.4 , resulting in factor loading non-invariance across groups (with loading values of 0.5 and 0.8 , respectively).

Manipulated Conditions: Latent Mean Difference Magnitude

This study considered two latent mean difference values $\left(\kappa_{2}-\kappa_{1}=0\right.$ and $\kappa_{2}-\kappa_{1}=$ $0.5)$. The condition of equal latent means $\left(\kappa_{2}-\kappa_{1}=0\right)$ across groups was included because this permits an assessment of the Type I error rates associated with the $\mathrm{LRT}_{\kappa}$ and the performance of the $\hat{\delta}_{\kappa}$ in terms of parameter bias. Scenarios with unequal latent means across groups were also investigated in order to assess the power of the $\mathrm{LRT}_{\kappa}$ and the performance of the $\hat{\delta}_{\kappa}$ in terms of relative parameter bias. A moderately large latent mean difference value $\left(\kappa_{2}-\kappa_{1}=0.5\right)$ was included because previous simulation studies found sufficient power with this latent mean difference value (Hancock, et al., 2000; Kaplan \& George, 1995).
Manipulated Conditions: Group Factor Variance Ratio

In the simulation study, three factor variance ratio conditions $\left(\Phi_{1}: \Phi_{2}\right)$ were considered. In the first factor variance ratio condition, the factor variances for the two groups were set to be equal (1:1). In the second and third factor variance ratio conditions, the factor variances for the two groups were set to be unequal with a ratio of 0.8:1.2 and 1.2:0.8. These two unequal factor variance conditions represent a realistic yet moderate difference (Kim, Cramond \& Bandalos, 2006) between the two groups' factor variances which provides a starting point for this line of research.

Data Generation

Raw data for the two groups were generated in SAS (Version 9.2; SAS Institute Inc., 2008) according to the specified population parameters for a single-factor, six-indicator CFA model using the Kaiser and Dickman (1962) matrix decomposition procedure (Fan \& Fan, 2005). Thus, each generated sample of data consisted of $n_{1} \times 6$ and $n_{2} \times 6$ matrices for group one and group two, respectively, where $n_{1}$ and $n_{2}$ represent the condition-specific sample size for each of the two groups. One thousand $(1,000)$ raw data sets were generated for each of the 162 combinations of manipulated conditions. After raw data for the two groups were generated, SAS 9.2 was programmed to call DOS to run Mplus (Version 6.1; Muthén \& Muthén, 2010), as described by Gagné and Furlow (2009), to estimate the models. Maximum likelihood (ML) estimation was used to estimate all model parameters.

When estimating the model parameters, cross-group constraints were imposed on all factor loadings and observed variable intercepts whereas error variances were freely estimated in both groups. When using the RI strategy to scale the factor, the RI's loading was constrained to a value of one in both groups. Two different structured means models were estimated. The traditional structured means model was estimated in which the latent mean of group one was constrained to be equal to zero but the latent mean of group two was estimated freely (the $\mathrm{SMM}_{\mathrm{K}^{*}}$ model) and another model in which the 
latent means for both groups were constrained to zero (the $\mathrm{SMM}_{\mathrm{\kappa} 0}$ model) was estimated. Also, two factor scaling methods were used to set the scale of the latent variable for each generated data set. When using the RI strategy, the first factor loading was constrained to a value of one across groups, all other factor loadings were constrained to be equal across groups, and factor variances for the two groups were freely estimated. When the factor-variance-based scaling method was implemented, the factor variance was instead constrained to a value of one across groups and all factor loadings were estimated yet constrained to be equal across groups. Thus, for each generated data set, four models (two factor scaling methods $\times$ two latent mean constraints) were estimated. It is important to note that the models using the factor-variance scaling method had one degree of freedom more than the models using the RI strategy.

\section{Data Analysis}

The $\chi^{2}$ statistic associated with each estimated model from each replication was saved to calculate the $\mathrm{LRT}_{\kappa}$ (see Equation 8) between the two estimated models $\left(\mathrm{SMM}_{\mathrm{K}^{*}}\right.$ and $\mathrm{SMM}_{\mathrm{\kappa} 0}$ ) when using each of the two factor scaling methods. The performance of the $\mathrm{LRT}_{\kappa}$ was evaluated by summarizing its Type I error rates and power. Type I error rates of the $\mathrm{LRT}_{\kappa}$, defined as the proportion of incorrect rejections of the null hypothesis $\left(H_{0}: \kappa_{1}=\kappa_{2}\right)$ out of the 1,000 replications in equal latent mean conditions $\left(\kappa_{2}-\kappa_{1}=0\right)$, were evaluated using Bradley's (1978) liberal criterion of $\alpha \pm 1 / 2 \alpha$ (where $\alpha=0.05$ ) such that rates less than 2.5\% were considered overly conservative and rates greater than $7.5 \%$ were considered overly liberal.

The power of the $\mathrm{LRT}_{\kappa}$ is defined as the proportion of correct rejections of the null hypothesis $\left(H_{0}: \kappa_{1}=\kappa_{2}\right)$ out of the 1,000 replications in unequal latent mean conditions $\left(\kappa_{2}-\kappa_{1}=0.5\right)$. A minimum power criterion of 0.8 is traditionally recommended as a reasonable level of power (Cohen, 1988), whereas others have recommended a minimum power criterion of 0.95 as a more appropriate level of power (Cashen \& Geiger 2004; Rossi, 1990). In this study, a minimum power criterion of 0.9 was selected to gauge the adequacy of the power associated with the $\mathrm{LRT}_{\kappa}$ as a compromise between the traditional and more stringent power recommendations.

The latent mean estimate in group two and the factor variance estimates in both groups were saved from the $\mathrm{SMM}_{\mathrm{K}^{*}}$ model, which were used to estimate the standardized latent mean difference effect size, $\hat{\delta}_{\kappa}$ (see Equations 9 and 10). The performance of the $\hat{\delta}_{\kappa}$ was examined through an assessment of its parameter bias and relative parameter bias under specific latent mean difference magnitude conditions. In conditions in which the latent mean difference was equal to zero $\left(\kappa_{2}-\kappa_{1}=0\right)$, the parameter bias of $\hat{\delta}_{\kappa}$ was calculated as follows:

$$
B\left(\hat{\delta}_{\kappa}\right)=\overline{\hat{\delta}}_{\kappa}-0
$$

where $\overline{\hat{\delta}}_{\kappa}$ is the mean of the $\delta_{\kappa}$ estimates across the 1,000 replications in each relevant condition (Hoogland \& Boomsma, 1998). The relative parameter bias of the $\hat{\delta}_{\kappa}$ was calculated with conditions in which the latent mean difference was equal to $0.5\left(\kappa_{2}-\kappa_{1}=0.5\right)$ as:

$$
\operatorname{RPB}\left(\hat{\delta}_{\kappa}\right)=\frac{\overline{\hat{\delta}}_{\kappa}-0.5}{0.5}
$$

(Hoogland \& Boomsma, 1998). According to Hoogland and Boomsma (1998), conditions in which the $\left|B\left(\hat{\delta}_{\kappa}\right)\right|$ or the $\left|R P B\left(\hat{\delta}_{\kappa}\right)\right|$ is less than 0.05 indicates acceptable levels of bias in the $\hat{\delta}_{\kappa}$.

\section{Results}

The results describing the performance of the $\mathrm{LRT}_{\kappa}$ are presented first, including Type I error 


\section{IMPACT OF VIOLATING FACTOR SCALING METHOD ASSUMPTIONS}

Table 1: Explanations of Abbreviations Used in the Tables of Results

\begin{tabular}{|c|l|}
\hline Abbreviation & \multicolumn{1}{|c|}{ Explanation } \\
\hline RI & Reference indicator strategy implemented \\
\hline FV & Factor-variance-based scaling method implemented \\
\hline Equal Loading & All factor loadings were equal/invariant across groups \\
\hline $1^{\text {st }}$ Loading Unequal & $\begin{array}{c}\text { The first factor loading (RI) was higher in group two than in group one } \\
\text { with the condition-specific loading difference }\end{array}$ \\
\hline 2nd Loading Unequal & $\begin{array}{c}\text { The second (non-RI) factor loading was higher in group two than in } \\
\text { group one with the condition-specific loading difference }\end{array}$ \\
\hline All Lower & $\begin{array}{c}\text { Both the first (RI) and second (non-RI) factor loading were higher in } \\
\text { group two than in group one with the condition-specific loading } \\
\text { difference }\end{array}$ \\
\hline Mixed & $\begin{array}{c}\text { The first factor loading (RI) was higher in group one than in group two } \\
\text { and the second (non-RI) factor loading was higher in group two } \\
\text { than in group one with the condition-specific loading difference }\end{array}$ \\
\hline
\end{tabular}

rates and power. The results describing the performance of the $\hat{\delta}_{\kappa}$, including parameter and relative parameter bias, are subsequently presented. Table 1 provides the explanations of abbreviations used in all the Tables illustrating the performance of the $\mathrm{LRT}_{\kappa}$ and the $\hat{\delta}_{\kappa}$.

\section{Performance of the $\mathrm{LRT}_{\kappa}$ : Type I Error Rates}

Table 2 presents the observed Type I error rates associated with the $\mathrm{LRT}_{\kappa}$ under equal latent mean conditions $\left(\kappa_{2}-\kappa_{1}=0\right)$. Values above the dashed line in Table 2 are the Type I error rates in the equal/invariant factor loading conditions and, thus, for scenarios in which the covariance structures are appropriately modeled. Values below the dashed line in Table 2 are the Type I error rates in the unequal/non-invariant factor loading conditions and, thus, for scenarios in which the covariance structures are not modeled appropriately. In each design cell, Type I error rates when implementing the RI strategy and when implementing the factor-variance (FV) scaling method are both reported. Employing Bradley's (1978) criterion, Table 2 shows overly conservative Type I error rates (i.e., less than or equal to $2.5 \%$ ) denoted with boldface and italics; overly liberal rates (i.e., greater than or equal to $7.5 \%$ ) are underlined.

In the equal/invariant factor loading conditions, all observed Type I error rates when using the RI strategy were within the criterion of $0.05 \pm 0.025$. Type I error rates did not appear to vary substantially or systematically as a function of group sample size ratio or group factor variance ratio. Upon implementing the factorvariance scaling method, one Type I error rate was found to be overly liberal (0.079) in the condition with the group sample size ratio of $1: 4$ and the group factor variance ratio of 1.2:0.8.

In the unequal/non-invariant factor loading conditions, the Type I error rates when the RI strategy was implemented were within the 
acceptable range of $0.05 \pm 0.025$. When the factor-variance scaling method was used, however, twelve observed Type I error rates were beyond the criterion of $0.05 \pm 0.025$. These unacceptable Type I error rates all occurred in the unequal sample size conditions such that liberal rates tended to occur in the 4:1 group sample size ratio scenarios and conservative rates tended to occur in the 1:4 group sample size ratio scenarios. Further, the majority $(83 \%)$ of these unacceptable Type I error rates were found in the large loading difference $\left(\left|\lambda_{1}-\lambda_{2}\right|=\right.$ $0.4)$ magnitude conditions (see Table 2 ).

Power of the $\mathrm{LRT}_{\mathrm{k}}$

Table 3 presents the observed power rates associated with the $\mathrm{LRT}_{\kappa}$ under conditions in which the latent mean difference was unequal across groups $\left(\kappa_{2}-\kappa_{1}=0.5\right)$. A criterion of 0.90 was used to evaluate the power of the $\mathrm{LRT}_{\kappa}$; hence, power rates below 0.90 were deemed too low (see Table 3 ). In the equal factor loading conditions, three power rates fell below the 0.90 cutoff when the RI strategy was implemented. These occurred in the 1:1 group factor variance ratio with the 4:1 group sample size ratio condition, in the 1.2:0.8 group factor variance ratio with the 1:4 group sample size ratio condition, and in the $0.8: 1.2$ group factor variance ratio with the $4: 1$ group sample size ratio condition. Although these values were lower than the cutoff criterion, they were not substantially lower than a value of 0.90 , ranging from 0.866 to 0.888 . Power tended to be higher in the equal group sample size conditions, but it did not vary substantially or systematically as a function of the group factor variance ratio under the RI strategy.

When the factor-variance scaling method was implemented under equal factor loadings, five out of nine power rates were lower than 0.90. Nonetheless, these values did not deviate substantially from 0.90 (range was from 0.891 to 0.894 ) and all were found in the unequal sample size conditions. Power rates were higher in the equal sample size conditions than in the unequal sample size conditions. Additionally, power rates when using the factorvariance scaling method did not differ markedly as a function of the group factor variance ratio (see Table 3).

In the unequal factor loading conditions, five power rates were lower than 0.90 when using the RI strategy which all occurred in conditions in which the loading difference was small $\left(\left|\lambda_{1}-\lambda_{2}\right|=0.1\right)$ and the group sample sizes were unequal (1:4 or 4:1). Again, these power rates were not substantially lower than the cutoff criterion, ranging from 0.890 to 0.898 (see Table 3 ). Power tended to be marginally higher when the loading difference was large $\left(\left|\lambda_{1}-\lambda_{2}\right|=0.4\right)$ than when small $\left(\left|\lambda_{1}-\lambda_{2}\right|=0.1\right)$. Across the three group sample size ratios, power rates were slightly higher when sample sizes were equal across groups than when they were unequal. Further, power rates under the RI strategy did not vary substantially as a function of the group factor variance ratios or the factor loading patterns.

When the factor-variance scaling method was implemented under unequal factor loadings, two observed power rates were lower than the cutoff criterion, although they did not differ substantially from the 0.90 criterion (0.890 and 0.892). These two low power rates were found in conditions in which the loading difference was small $\left(\left|\lambda_{1}-\lambda_{2}\right|=0.1\right)$ with the 0.8:1.2 group factor variance ratio and 1:4 group sample size ratio (see Table 3). Power when using the factor-variance scaling method was consistent with the power found when using the RI strategy. Specifically, power rates were marginally higher when the loading difference was large than when it was small and when sample sizes were equal across groups than when unequal. In addition, power rates did not vary considerably as a function of group factor variance ratio or the factor loading pattern when implementing the factor-variance scaling method.

Performance of the $\hat{\delta}_{\mathrm{\kappa}}$ : Parameter Bias of the $\hat{\delta}_{\kappa}$

Parameter bias of the standardized latent mean difference effect size measure $\left(\hat{\delta}_{\mathrm{\kappa}}\right)$ was calculated in conditions in which the true latent mean difference was equal to zero 
IMPACT OF VIOLATING FACTOR SCALING METHOD ASSUMPTIONS

Table 2: Type I Error Rates Associated with the Likelihood Ratio Test as a Function of Manipulated Conditions $\left(\kappa_{2}-\kappa_{1}=0\right)$

\begin{tabular}{|c|c|c|c|c|c|c|c|c|}
\hline \multirow{3}{*}{$\begin{array}{l}\text { Loading } \\
\text { Difference }\end{array}$} & \multirow{3}{*}{$\begin{array}{l}\text { Loading } \\
\text { Pattern }\end{array}$} & \multirow{3}{*}{$\begin{array}{c}\text { Group } \\
\text { Sample } \\
\text { Size Ratio }\end{array}$} & \multicolumn{6}{|c|}{ Group Factor Variance Ratio } \\
\hline & & & \multicolumn{2}{|c|}{$1: 1$} & \multicolumn{2}{|c|}{$1.2: 0.8$} & \multicolumn{2}{|c|}{$0.8: 1.2$} \\
\hline & & & RI & FV & RI & FV & RI & FV \\
\hline \multirow{3}{*}{0} & \multirow{3}{*}{$\begin{array}{l}\text { Equal } \\
\text { Loading }\end{array}$} & $250: 250$ & 0.056 & 0.057 & 0.049 & 0.051 & 0.058 & 0.058 \\
\hline & & $100: 400$ & 0.062 & 0.052 & 0.060 & $\underline{0.079}$ & 0.059 & 0.044 \\
\hline & & 400:100 & 0.068 & 0.067 & 0.051 & 0.037 & 0.055 & 0.070 \\
\hline \multirow{12}{*}{0.1} & \multirow{3}{*}{$\begin{array}{c}1^{\text {st }} \text { Loading } \\
\text { Unequal }\end{array}$} & $250: 250$ & 0.047 & 0.045 & 0.050 & 0.049 & 0.057 & 0.058 \\
\hline & & $100: 400$ & 0.047 & 0.043 & 0.046 & 0.052 & 0.068 & 0.046 \\
\hline & & 400:100 & 0.043 & 0.044 & 0.062 & 0.054 & 0.056 & 0.068 \\
\hline & \multirow{3}{*}{$\begin{array}{c}2^{\text {nd }} \text { Loading } \\
\text { Unequal }\end{array}$} & $250: 250$ & 0.046 & 0.046 & 0.046 & 0.046 & 0.050 & 0.051 \\
\hline & & $100: 400$ & 0.052 & 0.051 & 0.047 & 0.058 & 0.059 & 0.047 \\
\hline & & 400:100 & 0.038 & 0.045 & 0.052 & 0.040 & 0.064 & $\underline{0.083}$ \\
\hline & \multirow{3}{*}{ All Lower } & $250: 250$ & 0.068 & 0.067 & 0.057 & 0.060 & 0.071 & 0.071 \\
\hline & & $100: 400$ & 0.056 & 0.049 & 0.054 & 0.064 & 0.045 & 0.029 \\
\hline & & 400:100 & 0.058 & 0.070 & 0.049 & 0.038 & 0.051 & 0.068 \\
\hline & \multirow{3}{*}{ Mixed } & $250: 250$ & 0.048 & 0.048 & 0.054 & 0.055 & 0.047 & 0.046 \\
\hline & & $100: 400$ & 0.050 & 0.049 & 0.049 & 0.059 & 0.052 & 0.037 \\
\hline & & 400:100 & 0.053 & 0.054 & 0.056 & 0.040 & 0.059 & $\underline{0.081}$ \\
\hline \multirow{12}{*}{0.4} & \multirow{3}{*}{$\begin{array}{c}1^{\mathrm{st}} \text { Loading } \\
\text { Unequal }\end{array}$} & $250: 250$ & 0.044 & 0.043 & 0.058 & 0.059 & 0.044 & 0.042 \\
\hline & & $100: 400$ & 0.048 & 0.030 & 0.053 & 0.044 & 0.060 & 0.019 \\
\hline & & 400:100 & 0.058 & 0.070 & 0.054 & 0.054 & 0.061 & $\underline{0.096}$ \\
\hline & \multirow{3}{*}{$\begin{array}{c}2^{\text {nd }} \text { Loading } \\
\text { Unequal }\end{array}$} & $250: 250$ & 0.053 & 0.050 & 0.054 & 0.054 & 0.055 & 0.053 \\
\hline & & 100:400 & 0.055 & 0.027 & 0.053 & 0.050 & 0.049 & 0.025 \\
\hline & & 400:100 & 0.050 & 0.064 & 0.045 & 0.043 & 0.051 & $\underline{0.085}$ \\
\hline & \multirow{3}{*}{ All Lower } & $250: 250$ & 0.055 & 0.050 & 0.048 & 0.044 & 0.050 & 0.040 \\
\hline & & 100:400 & 0.044 & 0.016 & 0.066 & 0.046 & 0.061 & 0.016 \\
\hline & & 400:100 & 0.045 & $\underline{0.082}$ & 0.047 & 0.059 & 0.051 & $\underline{0.113}$ \\
\hline & \multirow{3}{*}{ Mixed } & $250: 250$ & 0.055 & 0.056 & 0.052 & 0.052 & 0.042 & 0.041 \\
\hline & & 100:400 & 0.050 & 0.041 & 0.045 & 0.058 & 0.040 & 0.021 \\
\hline & & $400: 100$ & 0.038 & 0.029 & 0.045 & 0.024 & 0.046 & 0.052 \\
\hline
\end{tabular}

Note: Type I error rates less than 0.025 are bold and italicized. Type I error rates greater than 0.075 are underlined. Abbreviations used in this table are described in Table 1. 
Table 3: Power Associated with the Likelihood Ratio Test as a Function of Manipulated Conditions $\left(\kappa_{2}-\kappa_{1}=0.5\right)$

\begin{tabular}{|c|c|c|c|c|c|c|c|c|}
\hline \multirow{3}{*}{$\begin{array}{l}\text { Loading } \\
\text { Difference }\end{array}$} & \multirow{3}{*}{$\begin{array}{l}\text { Loading } \\
\text { Pattern }\end{array}$} & \multirow{3}{*}{$\begin{array}{c}\text { Group } \\
\text { Sample } \\
\text { Size Ratio }\end{array}$} & \multicolumn{6}{|c|}{ Group Factor Variance Ratio } \\
\hline & & & \multicolumn{2}{|c|}{$1: 1$} & \multicolumn{2}{|c|}{$1.2: 0.8$} & \multicolumn{2}{|c|}{$0.8: 1.2$} \\
\hline & & & RI & FV & RI & FV & RI & $\mathrm{FV}$ \\
\hline \multirow{3}{*}{0} & \multirow{3}{*}{$\begin{array}{c}\text { Equal } \\
\text { Loading }\end{array}$} & $250: 250$ & 0.979 & 0.979 & 0.983 & 0.983 & 0.982 & 0.982 \\
\hline & & $100: 400$ & 0.906 & 0.906 & $\underline{0.866}$ & $\underline{0.892}$ & 0.911 & $\underline{0.893}$ \\
\hline & & 400:100 & $\underline{0.888}$ & $\underline{0.891}$ & 0.920 & $\underline{0.894}$ & $\underline{0.873}$ & $\underline{0.893}$ \\
\hline \multirow{12}{*}{0.1} & \multirow{3}{*}{$\begin{array}{c}1^{\text {st }} \text { Loading } \\
\text { Unequal }\end{array}$} & $250: 250$ & 0.987 & 0.988 & 0.983 & 0.982 & 0.980 & 0.981 \\
\hline & & $100: 400$ & 0.924 & 0.926 & 0.927 & 0.937 & 0.941 & 0.921 \\
\hline & & 400:100 & $\underline{0.898}$ & 0.905 & 0.929 & 0.916 & $\underline{0.897}$ & 0.927 \\
\hline & \multirow{3}{*}{$\begin{array}{c}2^{\text {nd }} \text { Loading } \\
\text { Unequal }\end{array}$} & $250: 250$ & 0.984 & 0.984 & 0.988 & 0.988 & 0.982 & 0.982 \\
\hline & & $100: 400$ & 0.925 & 0.917 & $\underline{0.890}$ & 0.911 & 0.920 & $\underline{0.890}$ \\
\hline & & $400: 100$ & 0.904 & 0.912 & 0.947 & 0.936 & 0.912 & 0.937 \\
\hline & \multirow{3}{*}{ All Lower } & $250: 250$ & 0.991 & 0.991 & 0.993 & 0.993 & 0.994 & 0.994 \\
\hline & & $100: 400$ & 0.935 & 0.928 & 0.939 & 0.942 & 0.953 & 0.926 \\
\hline & & $400: 100$ & 0.919 & 0.931 & 0.938 & 0.927 & 0.916 & 0.929 \\
\hline & \multirow{3}{*}{ Mixed } & $250: 250$ & 0.983 & 0.984 & 0.985 & 0.986 & 0.986 & 0.986 \\
\hline & & $100: 400$ & 0.906 & 0.901 & $\underline{0.890}$ & 0.903 & 0.908 & $\underline{0.892}$ \\
\hline & & 400:100 & 0.911 & 0.908 & 0.942 & 0.924 & $\underline{0.892}$ & 0.910 \\
\hline \multirow{12}{*}{0.4} & \multirow{3}{*}{$\begin{array}{c}1^{\text {st }} \text { Loading } \\
\text { Unequal }\end{array}$} & $250: 250$ & 0.998 & 0.998 & 1.000 & 1.000 & 0.999 & 0.999 \\
\hline & & $100: 400$ & 0.971 & 0.967 & 0.975 & 0.978 & 0.989 & 0.971 \\
\hline & & 400:100 & 0.964 & 0.973 & 0.973 & 0.979 & 0.940 & 0.962 \\
\hline & \multirow{3}{*}{$\begin{array}{c}2^{\text {nd }} \text { Loading } \\
\text { Unequal }\end{array}$} & $250: 250$ & 0.997 & 0.997 & 0.999 & 0.999 & 0.998 & 0.998 \\
\hline & & $100: 400$ & 0.984 & 0.967 & 0.976 & 0.979 & 0.984 & 0.966 \\
\hline & & 400:100 & 0.965 & 0.974 & 0.974 & 0.974 & 0.944 & 0.962 \\
\hline & \multirow{3}{*}{ All Lower } & $250: 250$ & 1.000 & 1.000 & 0.999 & 0.999 & 1.000 & 1.000 \\
\hline & & $100: 400$ & 0.993 & 0.980 & 0.993 & 0.989 & 0.995 & 0.981 \\
\hline & & 400:100 & 0.982 & 0.995 & 0.993 & 0.995 & 0.971 & 0.990 \\
\hline & \multirow{3}{*}{ Mixed } & $250: 250$ & 0.995 & 0.996 & 0.995 & 0.997 & 0.998 & 0.998 \\
\hline & & $100: 400$ & 0.961 & 0.957 & 0.953 & 0.964 & 0.972 & 0.959 \\
\hline & & 400:100 & 0.925 & 0.920 & 0.931 & 0.906 & 0.924 & 0.932 \\
\hline
\end{tabular}

Note: Power rates below 0.90 are underlined. Abbreviations used in this table are described in Table 1. 


\section{IMPACT OF VIOLATING FACTOR SCALING METHOD ASSUMPTIONS}

$\left(\kappa_{2}-\kappa_{1}=0\right)$. A cutoff value of 0.05 was used to evaluate the acceptability of the parameter bias, meaning that absolute parameter bias values less than 0.05 indicated acceptable bias (Hoogland \& Boomsma, 1998). No substantial parameter bias was found across conditions examined with bias values ranging from -0.010 to 0.013 . Although both negative and positive parameter bias was observed, no clear trend was noticed.

Performance of the $\hat{\delta}_{\kappa}$ : Relative Parameter Bias of the $\hat{\delta}_{\kappa}$

In conditions where the true latent mean difference was equal to $0.5\left(\kappa_{2}-\kappa_{1}=0.5\right)$, relative parameter bias of the $\hat{\delta}_{\kappa}$ was calculated. Table 4 presents the relative parameter bias of the $\hat{\delta}_{\kappa}$ for each combination of manipulated conditions. Following Hoogland \& Boomsma (1998), a minimum cutoff of 0.05 was used to represent a substantial degree of bias (see Table 4).

In the equal factor loading conditions, relative parameter bias was acceptable, regardless of the factor scaling method used. Relative parameter bias when using the RI strategy and the factor-variance scaling method showed consistent trends. Negative relative parameter bias values occurred in conditions in which the group factor variance ratio was $1: 1$ or $0.8: 1.2$ and positive relative parameter bias values emerged in conditions in which the group factor variance ratio was 1.2:0.8 (see Table 4). Although the relative parameter bias values were in opposite directions, their absolute values did not differ substantially as a function of group factor variance ratio or group sample size ratio.

Unacceptable relative parameter bias was found when implementing the RI strategy in 33 conditions under the unequal factor loading scenarios. Unacceptable and substantial relative parameter bias was found more often in conditions in which the loading difference was large $\left(\left|\lambda_{1}-\lambda_{2}\right|=0.4\right)$ than when it was small $\left(\mid \lambda_{1}\right.$ $\left.-\lambda_{2} \mid=0.1\right)$. Relative parameter bias varied as a function of factor loading pattern as well. For example, relative parameter bias was more substantial in the all lower factor loading pattern conditions than in the remaining three factor loading scenarios (i.e., $1^{\text {st }}$ loading unequal, $2^{\text {nd }}$ loading unequal and mixed pattern conditions) whereas relative parameter bias was the least substantial in the mixed pattern scenarios (see Table 4). Relative parameter bias values were the smallest in the 1:4 group sample size ratio scenarios whereas they were more substantial in the 4:1 group sample size ratio scenarios. In addition, no clear trend was exhibited across the three group factor variance ratios when using the RI scaling method. When the factor-variance scaling strategy was implemented, relative parameter bias trends closely resembled those found when using the RI scaling strategy as previously described (see Table 4).

\section{Conclusion}

The primary question addressed in this study was whether violating the assumptions underlying the RI strategy and/or the factorvariance scaling method (i.e., using a RI with non-invariant factor loadings or constraining unequal factor variances to a value of one across groups) would affect the testing and description of the latent mean difference across groups. When implementing the RI strategy, the Type I error rates associated with the $\mathrm{LRT}_{\kappa}$ were not adversely affected by factor loading difference magnitude, factor loading pattern, group sample size ratio, or group factor variance ratio. This result indicates that violating the assumption of equivalent reference indicator loadings underlying the RI strategy did not affect Type I error rates associated with the $\mathrm{LRT}_{\kappa}$ for conditions and models examined here. This is consistent with the findings from the study conducted by Hancock, et al. (2000).

Previous research on SMM has not thoroughly investigated the factor-variance scaling method. The study found that when implementing the factor-variance scaling method, group factor variance ratio, group sample size ratio and loading difference magnitude did affect the Type I error rates associated with the $\mathrm{LRT}_{\kappa}$. More specifically, when factor loadings were noninvariant/unequal, all Type I error rates that 


\section{WANG, WHITTAKER \& BERETVAS}

Table 4: Relative Parameter Bias of the Standardized Latent Mean Difference Effect Size Measure as a Function of Manipulated Conditions $\left(\kappa_{2}-\kappa_{1}=0.5\right)$

\begin{tabular}{|c|c|c|c|c|c|c|c|c|}
\hline \multirow{3}{*}{$\begin{array}{c}\text { Loading } \\
\text { Difference }\end{array}$} & \multirow{3}{*}{$\begin{array}{l}\text { Loading } \\
\text { Pattern }\end{array}$} & \multirow{3}{*}{$\begin{array}{c}\text { Group } \\
\text { Sample } \\
\text { Size Ratio }\end{array}$} & \multicolumn{6}{|c|}{ Group Factor Variance Ratio } \\
\hline & & & \multicolumn{2}{|c|}{$1: 1$} & \multicolumn{2}{|c|}{$1.2: 0.8$} & \multicolumn{2}{|c|}{$0.8: 1.2$} \\
\hline & & & RI & FV & RI & FV & RI & FV \\
\hline \multirow{3}{*}{0} & \multirow{3}{*}{$\begin{array}{c}\text { Equal } \\
\text { Loading }\end{array}$} & $250: 250$ & -0.008 & -0.008 & 0.012 & 0.012 & -0.004 & -0.003 \\
\hline & & $100: 400$ & -0.011 & -0.011 & 0.004 & 0.005 & -0.013 & -0.012 \\
\hline & & 400:100 & -0.006 & -0.006 & 0.004 & 0.004 & -0.004 & -0.008 \\
\hline \multirow{12}{*}{0.1} & \multirow{3}{*}{$\begin{array}{c}1^{\text {st }} \text { Loading } \\
\text { Unequal }\end{array}$} & $250: 250$ & 0.017 & 0.016 & 0.022 & 0.024 & 0.033 & 0.033 \\
\hline & & $100: 400$ & 0.019 & 0.019 & $\underline{0.050}$ & $\underline{0.051}$ & 0.013 & 0.014 \\
\hline & & 400:100 & $\underline{0.050}$ & $\underline{0.050}$ & 0.049 & $\underline{0.051}$ & 0.037 & 0.037 \\
\hline & \multirow{3}{*}{$\begin{array}{c}2^{\text {nd }} \text { Loading } \\
\text { Unequal }\end{array}$} & $250: 250$ & 0.013 & 0.013 & 0.034 & 0.035 & 0.008 & 0.009 \\
\hline & & $100: 400$ & 0.010 & 0.010 & -0.004 & -0.003 & -0.014 & -0.013 \\
\hline & & 400:100 & 0.041 & 0.041 & $\underline{0.050}$ & $\underline{0.051}$ & $\underline{0.057}$ & $\underline{0.057}$ \\
\hline & \multirow{3}{*}{ All Lower } & $250: 250$ & 0.046 & 0.045 & $\underline{0.059}$ & $\underline{0.061}$ & 0.037 & 0.036 \\
\hline & & $100: 400$ & 0.015 & 0.014 & 0.042 & 0.043 & 0.010 & 0.010 \\
\hline & & 400:100 & $\underline{0.066}$ & $\underline{0.066}$ & $\underline{0.074}$ & $\underline{0.076}$ & $\underline{0.070}$ & $\underline{0.068}$ \\
\hline & \multirow{3}{*}{ Mixed } & $250: 250$ & 0.0004 & 0.0002 & -0.013 & -0.011 & 0.015 & 0.015 \\
\hline & & $100: 400$ & -0.002 & -0.002 & -0.017 & -0.017 & -0.015 & -0.014 \\
\hline & & 400:100 & 0.009 & 0.009 & 0.016 & 0.017 & 0.014 & 0.011 \\
\hline \multirow{12}{*}{0.4} & \multirow{3}{*}{$\begin{array}{c}1^{\text {st }} \text { Loading } \\
\text { Unequal }\end{array}$} & $250: 250$ & $\underline{0.111}$ & $\underline{0.103}$ & $\underline{0.155}$ & $\underline{0.154}$ & $\underline{0.097}$ & $\underline{0.088}$ \\
\hline & & $100: 400$ & 0.039 & 0.034 & $\underline{0.052}$ & $\underline{0.051}$ & 0.043 & 0.037 \\
\hline & & 400:100 & $\underline{0.215}$ & $\underline{0.204}$ & $\underline{0.229}$ & $\underline{0.229}$ & $\underline{0.175}$ & $\underline{0.155}$ \\
\hline & \multirow{3}{*}{$\begin{array}{c}2^{\text {nd }} \text { Loading } \\
\text { Unequal }\end{array}$} & $250: 250$ & $\underline{0.129}$ & $\underline{0.121}$ & $\underline{0.147}$ & $\underline{0.145}$ & $\underline{0.092}$ & $\underline{0.083}$ \\
\hline & & $100: 400$ & 0.025 & 0.021 & $\underline{0.077}$ & $\underline{0.076}$ & 0.038 & 0.032 \\
\hline & & $400: 100$ & $\underline{0.198}$ & $\underline{0.187}$ & $\underline{0.213}$ & $\underline{0.214}$ & $\underline{0.180}$ & $\underline{0.162}$ \\
\hline & \multirow{3}{*}{ All Lower } & $250: 250$ & $\underline{0.184}$ & $\underline{0.153}$ & $\underline{0.230}$ & $\underline{0.212}$ & $\underline{0.130}$ & $\underline{0.091}$ \\
\hline & & $100: 400$ & $\underline{0.060}$ & 0.046 & $\underline{0.068}$ & $\underline{0.059}$ & 0.033 & 0.019 \\
\hline & & 400:100 & $\underline{0.344}$ & $\underline{0.313}$ & $\underline{0.390}$ & $\underline{0.374}$ & $\underline{0.299}$ & $\underline{0.255}$ \\
\hline & \multirow{3}{*}{ Mixed } & $250: 250$ & -0.005 & -0.006 & -0.011 & 0.008 & 0.030 & 0.022 \\
\hline & & $100: 400$ & 0.008 & 0.006 & $\underline{0.050}$ & $\underline{0.058}$ & -0.007 & -0.012 \\
\hline & & $400: 100$ & $\underline{-0.064}$ & $\underline{-0.058}$ & $\underline{-0.087}$ & $\underline{-0.072}$ & -0.015 & -0.026 \\
\hline
\end{tabular}

Note: Relative parameter bias values equal to or greater than 0.05 are underlined which represent unacceptable bias. Abbreviations used in this table are described in Table 1. 


\section{IMPACT OF VIOLATING FACTOR SCALING METHOD ASSUMPTIONS}

were deemed unacceptable occurred when group sample sizes were unequal. Additionally, the majority of unacceptable Type I error rates were found in conditions in which the group factor variance ratio was lower in group one than in group two and the loading difference was large. However, when the sample sizes were equal across groups, violating the equal factorvariance assumption did not have any substantial impact on Type I error rates associated with the $\mathrm{LRT}_{\kappa}$.

Power associated with the LRT $_{\kappa}$ was affected by group sample size ratio and loading difference magnitude. For example, when factor loadings were either equal or unequal across groups, power rates below the cutoff criterion of 0.90 were found only in the unequal sample size conditions; this finding is consistent with Kaplan and George (1995). Both group sample size ratio and loading difference magnitude influenced the power of the $\mathrm{LRT}_{\kappa}$ when factor loadings were unequal. That is, power was low only in unequal sample size scenarios and low factor loading difference scenarios. These low power rates, nonetheless, were not considerably lower than 0.90 and would in fact be deemed as acceptable if the traditional, less stringent 0.80 power criterion had been used as the benchmark. High power was particularly observed in the large latent mean difference conditions, as would be expected.

Previous studies have not investigated the performance of the standardized effect size measure, $\hat{\delta}_{\kappa}$, under varying conditions, particularly when the assumptions underlying the RI strategy and the factor-variance scaling method are violated. The findings in this study demonstrate that the $\hat{\delta}_{\kappa}$ is not biased in conditions in which there was no latent mean difference between the two groups. Thus, violating the assumptions associated with the RI strategy and the factor-variance scaling method did not have any substantial or systematic impact on the parameter bias of the $\hat{\delta}_{\kappa}$. Further, the parameter bias of the $\hat{\delta}_{\kappa}$ was not affected by loading difference magnitude, group sample size ratio, group factor variance ratio, or factor loading pattern.

When there was a latent mean difference between the two groups, the $\hat{\delta}_{\kappa}$ was not biased in the baseline conditions in which factor loadings were equal/invariant. However, substantial relative parameter bias of the $\hat{\delta}_{\kappa}$ was found in the partial metric invariance conditions in which factor loadings were unequal. In addition, the relative parameter bias of the $\hat{\delta}_{\kappa}$ in these partial invariance conditions varied as a function of loading difference magnitude, factor loading pattern, and group sample size ratio, regardless of the factor scaling method used. Overall, the relative parameter bias was more unacceptable when the factor loading difference magnitude was large, when the non-invariant factor loadings were higher in group two, and when sample size in group one was larger than sample size in group two.

Study Limitations

The assumptions underlying the RI strategy and the factor-variance scaling method have not been widely investigated in previous simulation studies. Thus, as a starting point for this line of research, this study included a relatively simple model and investigated latent mean difference comparisons under relatively ideal conditions. Due to the preliminary nature of the research, there are several limitations inherent in this study. For example, only a moderately large latent mean difference was included when investigating the power of the $\mathrm{LRT}_{\kappa}$.

As a result, power associated with the LRT $_{\kappa}$ was high in these conditions and did not differ systematically as a function of the factor loading pattern or group factor variance ratio. It was found that violating the assumptions underlying the two factor-scaling methods did not influence the power of the $\mathrm{LRT}_{\kappa}$. However, it is not clear whether the same findings would be obtained with smaller latent mean differences (e.g., 0.1 and 0.3). In future simulation studies, researchers could include smaller latent mean differences and examine how violating the 
assumptions underlying the two factor-scaling methods may affect the power of the $\mathrm{LRT}_{\kappa}$.

Neither model size nor model complexity was varied in this study. For simplicity, a two-group, one-factor CFA model with six indicator variables was the true generating model. Future researchers could consider more complex models (for example, more observed indicators and/or additional latent variables) to investigate whether varying the model size and/or model complexity would affect the testing and description of the latent mean difference across groups. Future research that includes models with more observed indicators could likewise investigate more severe loading non-invariance conditions. Further, mean comparisons between more than two groups are not uncommon and, hence, the impact of including more than two groups on latent mean comparisons could be examined in future investigations. In addition, multivariate normal data were generated. Future studies could also explore the implications of violating the assumption of normality when using the LRT $_{\kappa}$ and the $\hat{\delta}_{\kappa}$ to test and describe groups' latent mean differences.

The results of this study suggest that researchers do not necessarily need to be concerned about violating the assumption underlying the RI strategy given that it does not adversely affect the performance of the $\mathrm{LRT}_{\kappa}$. The results also suggest that researchers do not necessarily need to be concerned about violating the assumptions underlying either of the two factor scaling methods when using the $\hat{\delta}_{\kappa}$ in order to describe the latent mean difference across groups.

The findings concerning the RI factor scaling method are notable because the assumption underlying the RI strategy may be frequently violated given the difficulty of identifying an item with truly invariant factor loadings (Hancock, Stapleton \& ArnoldBerkovits, 2009). Nonetheless, more research is necessary in order to assuredly know that violating the RI assumption does not impact latent mean difference testing and that violating either of the factor scaling method assumptions does not impact latent mean difference descriptions. By contrast, the results clearly indicate that researchers should be aware of the assumption underlying the factor-variance scaling method. In particular, when the sample sizes for the two groups being compared are unequal, constraining unequal factor variances to a value of one across groups is likely to produce overly conservative or liberal Type I error rates associated with the latent mean difference test $\left(\mathrm{LRT}_{\kappa}\right)$. Additionally, researchers should cautiously interpret the $\hat{\delta}_{\kappa}$ when factor loadings are non-invariant across groups in combination with unequal group sample sizes, regardless of factor scaling method employed.

\section{References}

Bradley, J. V. (1978). Robustness? British Journal of Mathematical and Statistical Psychology, 31, 144-152.

Byrne, B. M., Shavelson, R. J., \& Muthén, B. (1989). Testing for the equivalence of factor covariance and mean structures: The issue of partial measurement invariance. Psychological Bulletin, 105, 456-466.

Cashen, L. H., \& Geiger, S. W. (2004). Statistical power and the testing of null hypotheses: A review of contemporary management research and recommendations for future studies. Organizational Research Methods, 7, 151-167.

Cheung, G. W., \& Rensvold, R. B. (1999). Testing factorial invariance across groups: A conceptualization and proposed new method. Journal of Management, 25, 1-27.

Cheung, G. W., \& Rensvold, R. B. (2002). Evaluating goodness-of-fit indexes for testing measurement invariance. Structural Equation Modeling, 9, 233-255.

Cohen, J. (1988). Statistical power analysis for the behavioral sciences ( $2^{\text {nd }} E d$.). Hillsdale, NJ: Lawrence Erlbaum Associates.

Cole, D. A., Maxwell, S. E., Arvey, R., \& Salas, E. (1993). Multivariate group comparisons of variable systems: MANOVA and structural equation modeling. Psychological Bulletin, 114, 174-184. 


\section{IMPACT OF VIOLATING FACTOR SCALING METHOD ASSUMPTIONS}

Enders, C. K., \& Finney, S. J. (2003, April). SEM fit index criteria re-examined: An investigation of $M L$ and robust fit indices in complex models. Paper presented at the annual meeting of the American Educational Research Association, Chicago, IL.

Fan, X., \& Fan, X. (2005). Using SAS for Monte Carlo simulation research in SEM. Structural Equation Modeling, 12, 299-333.

Gagné, P., \& Furlow, C. F. (2009). Automating multiple software packages in simulation research for structural equation modeling and hierarchical linear modeling. Structural Equation Modeling, 16, 179-185.

Gonzalez, R., \& Griffin, D. (2001). Testing parameters in structural equation modeling: Every "one" matters. Psychological Methods, 6, 258-269.

Hancock, G. R. (1997). Structural equation modeling methods of hypothesis testing of latent variable means. Measurement and Evaluation in Counseling and Development, 30, 91-105.

Hancock, G. R. (2001). Effect size, power, and sample size determination for structured means modeling and MIMIC approaches to between-groups hypothesis testing of means on a single latent construct. Psychometrika, 66, 373-388.

Hancock, G. R., Lawrence, F. R., \& Nevitt, J. (2000). Type I error and power of latent mean methods and MANOVA in factorially invariant and noninvariant latent variable systems. Structural Equation Modeling, 7, 534-556.

Hancock, G. R., Stapleton, L. M., \& Arnold-Berkovits, I. (2009). The tenuousness of invariance tests within multisample covariance and mean structure models. In Structural equation modeling: Concepts and applications in educational research, T. Teo \& M. S. Khine (Eds.), 137-174. Rotterdam, Netherlands: Sense Publishers.

Hinkin, T. R. (1995). A review of scale development practices in the study of organizations. Journal of Management, 21, 967988.

Hoogland, J. J., \& Boomsma, A. (1998). Robustness studies in covariance structure modeling. Sociological Methods and Research, 26, 329-367.
Johnson, E. C., Meade, A. W., \& DuVernet, A. M. (2009). The role of referent indicators in tests of measurement invariance. Structural Equation Modeling, 16, 642-657.

Kaiser, H. F., \& Dickman, K. (1962). Sample and population score matrices and sample correlation matrices from an arbitrary population correlation matrix. Psychomerika, 27, 179-182.

Kaplan, D., \& George, R. (1995). A study of power associated with testing factor mean differences under violations of factorial invariance. Structural Equation Modeling, 2, 101-118.

Kim, K. H., Cramond, B., \& Bandalos, D. L. (2006). The latent structure and measurement invariance of scores on the Torrance tests of creative thinking-Figural. Educational and Psychological Measurement, 66, 459-477.

Kline, R. (2011). Principles and Practice of Structural Equation Modeling ( ${ }^{\text {rd }}$ $E d$.). New York, NY: Guilford Publications.

Lawrence, F. R., \& Hancock, G. R. (1998). Finite sample behavior of the likelihood ratio, Wald, and Lagrange Multiplier tests: Bias and variability in univariate noncentrality parameter estimation. Paper presented at the annual meeting of the American Educational Research Association, April, San Diego, CA.

Meredith, W. (1993). Measurement invariance, factor analysis and factorial invariance. Psychometrika, 58, 525-543.

Muthén, B., \& Christoffersson, A. (1981). Simultaneous factor analysis of dichotomous variables in several groups. Psychometrika, 46, 407-419.

Muthén, L. K., \& Muthén, B. O. (2010). Mplus (Version 6.1) [computer software]. Los Angeles, CA: Muthén \& Muthén.

Rensvold, R. B. \& Cheung, G. W. (2001). Testing for metric invariance using structural equation models: Solving the standardization problem. In Research in management: Vol. 1. Equivalence in measurement, C. A. Schriesheim \& L. L. Neider (Eds.), 21-50. Greenwich, CT: Information Age. 


\section{WANG, WHITTAKER \& BERETVAS}

Rock, D. A., Werts, C. E., \& Flaugher, R. L. (1978). The use of analysis of covariance structures for comparing the psychometric properties of multiple variables across populations. Multivariate Behavioral Research, 13, 403-418.

Rossi, J. S. (1990). Statistical power of psychological research: What have we gained in 20 years? Journal of Consulting and Clinical Psychology, 58, 646-656.

SAS. (2008). SAS (Version 9.2) [computer software]. Cary, NC: SAS Institute Inc.

Sörbom, D. (1974). A general method for studying differences in factor means and factor structure between groups. British Journal of Mathematical and Statistical Psychology, 27, 229-239.

Steenkamp, J-B., \& Baumgartner, H. (1998). Assessing measurement invariance in cross-national consumer research. Journal of Consumer Research, 25, 78-90.
Thompson, M. S., \& Green, S. B. (2006). Evaluating between-group differences in latent variable means. In Structural equation modeling: A second course, G. R. Hancock \& R. O. Mueller (Eds.), 119-169. Greenwood, CT: Information Age Publishing, Inc.

Vandenberg, R. J. (2002). Toward a further understanding of and improvement in measurement invariance methods and procedures. Organizational Research Methods, $5,139-158$.

Yoon, M. \& Millsap, R. E. (2007). Detecting violations of factorial invariance using data-based specification searches: A Monte Carlo study. Structural Equation Modeling, 14(3), 435-463. 


\section{Robust Regression Estimates in the Prediction of Latent Variables in Structural Equation Models}

\author{
Marcelo Angelo Cirillo \\ Federal University of Lavras, \\ Lavras, Brazil
}

\author{
Lúcia Pereira Barroso \\ University of São Paulo, \\ São Paulo, Brazil
}

The incorporation of the robust regression methods Least Median Square (LMS) and Least Trimmed Squares (LTS) is proposed in structural equation modeling. Results show that, in situations of high deviations of symmetry, the evaluated methods would be recommended for applications including smaller sample sizes.

Key words: Accuracy, Monte Carlo simulation, normal asymmetry, precision.

\section{Introduction}

Structural equation modeling seeks to reproduce a population covariance matrix through the sample covariance associated with parameter constraints determined by a researcher. The most commonly used estimation methods are based on maximum likelihood, ordinary least squares and partial least squares. These methods, however, show a sensitivity to various factors, for example: violation of the normality assumption (Lei \& Lomax, 2005), presence of outliers (Yuan \& Bentler, 2001) and samples that show the effects of asymmetry and excess kurtosis compared to the multivariate normal distribution (Gao, et al., 2008).

It is reasonable to assume that the presence of these effects can be explained by observing outliers in the sample and it is also relevant to consider sample size because large data sets are subject to a large number of these observations. Moreover, the violation of the assumption of normality can also be caused by these observations; therefore, a practical (but not

Marcelo Cirillo is Adjunct Lecturer IV in the Exact Sciences Department. E-mail him at: macufla@dex.ufla.br. Lúcia Barroso is Associate Professor in the in the Institute of Mathematics and Statistics. E-mail her at: lbarroso@ime.usp.br. always feasible) alternative is to apply a transformation to the data. Gao, et al. (2008) state that - depending on the transformation to be used - the relationships between variables may be nonlinear. However, applying this statement to structural equation models, where the nature of the relationships between the variables are linear, the application of a transformation may complicate the interpretation of results as well as affect the quality of model fit.

Pilati and Laros (2007) state that, if the assumption of normality is not met, the researcher may choose to use other estimation methods such as the method of partial least squares (PLS) estimators. According to O'Loughlin \& Coenders (2004) the disadvantage of this method is that they provide biased estimates. Cassel, et al. (1999) note that the properties of robustness must be further studied due to the fact that the estimates are sensitive to deviations from symmetry in observable variables, multicollinearity among observable variables and incorrect specification of measurement models.

As noted, not all variable distributions are normal and - in the presence of outliers - not all are appropriate to use for robust regression estimators that would be incorporated in structural equation models (SEMs). However, robust regression estimators that have a high breaking point are constructed based on the size of the subsets of data, thus, Hawkins, et al. (1984) state that this size should be minimal, 


\section{CIRILLO \& BARROSO}

that is, the total number of observations belonging to each set should be equal to the number of model parameters. This recommendation is justified due to the fact that the proportion of subsets that will contain at least one observational discrepancy will grow rapidly as a function of the size of the subset. For this reason, by keeping the subsets as small as possible the number of estimates that are not influenced by outliers is maximized.

As a base for this theory, the method of Least Median of Squares (LMS) is cited (Gervini \& Yohai, 2002). This method involves minimizing the sum of squares of the ordinary residuals obtained by the method in such a way that the subset formed from the residuals resulting in the lowest median value are considered for the minimization processes. Other robust regression estimators, adequate for incorporation in structural equation modeling (SEM) are defined by the Least Trimmed Squares (LTS) method (Agulló, 2001). This method proposes to minimize the sum of the first $h$ residuals sorted and squared, and $h$ the size of the subset defined by the number of model parameters and sample size (Maronna, et al., 2006).

This article incorporates robust estimators LMS and LTS in structural SEM. For this purpose, the performance of the incorporation of these estimators was evaluated by Monte Carlo simulation considering different degrees of asymmetry in the distributions of the observable variables and errors of the structural and measurement models.

\section{Methodology}

Error Distribution of the Structural Equation Model using Monte Carlo Simulation

Following the definition of a structural equation model (SEM), structural error and measurement models were generated following the asymmetric standard normal distribution with probability functions defined respectively as

$$
\begin{aligned}
& f_{\zeta}=2 \phi(\zeta) \Phi(a \zeta), \zeta \in \mathfrak{R}, a \in \mathfrak{N} \\
& f_{\delta}=2 \phi(\delta) \Phi(a \delta), \delta \in \mathfrak{R}, a \in \mathfrak{R} ;
\end{aligned}
$$

and

$$
f_{\varepsilon}=2 \phi(\varepsilon) \Phi(a \varepsilon), \varepsilon \in \mathfrak{R}, a \in \mathfrak{R},
$$

where $\phi($.$) and \Phi($.$) represent the probability$ density function and standard normal cumulative distribution $\mathrm{N}(0,1)$ and $a$ is the asymmetry parameter. Thus, the notation $\zeta \sim \mathrm{N}(0,1, a), \delta \sim$ $\mathrm{N}(0,1, a)$ and $\varepsilon \sim \mathrm{N}(0,1, a)$ was used. Arbitrarily, the values were set at $a=20,0$ and -20 implying the errors presented positive asymmetry, symmetry, and negative asymmetry, respectively; these situations are illustrated in Figures 1-3.

Figure $1: \zeta, \delta, \varepsilon \sim \mathrm{N}(0,1,20)$ Error Histogram

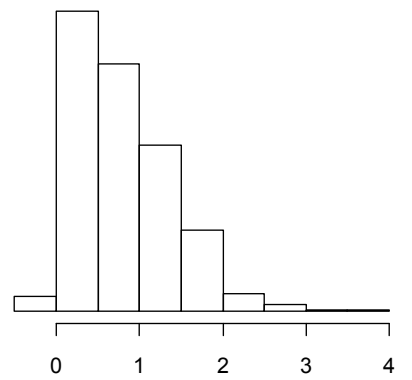

Figure 2: $\zeta, \delta, \varepsilon \sim \mathrm{N}(0,1)$ Error Histogram

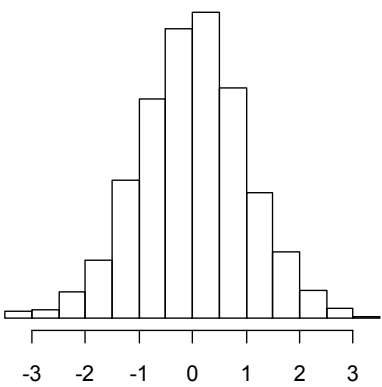

Figure 3: $\zeta, \delta, \varepsilon \sim \mathrm{N}(0,1,-20)$ Error Histogram

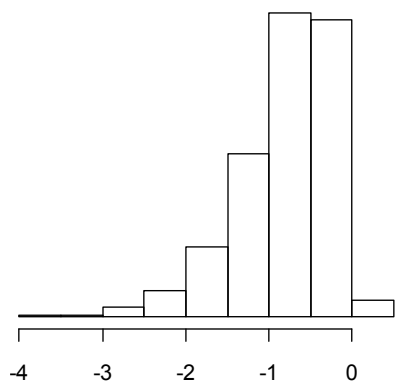


Structural Equation Models Used in the Monte Carlo Simulation

Keeping the specifications of the error distributions consistent with the proposed objective in the Monte Carlo simulation process, the structural model

$$
\eta=\gamma_{1} \xi_{1}+\gamma_{2} \xi_{2}+\gamma_{3} \xi_{3}+\zeta
$$

where $\gamma_{i}$ for $i=1,2,3$ represents the regression coefficients and $\zeta$ is the structural error with distribution is $\mathrm{N}(0,1, a)$ was considered. The observable variables were defined by $\mathrm{x}_{\mathrm{k}}(\mathrm{k}=1$, ..., 9), such that each equation of the measurement model at $\mathrm{x}$ was composed by

$$
\begin{aligned}
& \xi_{1}=\pi_{1} x_{1}+\pi_{2} x_{2}+\pi_{3} x_{3}+\delta_{1}, \\
& \xi_{2}=\pi_{4} x_{4}+\pi_{5} x_{5}+\pi_{6} x_{6}+\delta_{2}, \\
& \xi_{3}=\pi_{7} x_{7}+\pi_{8} x_{8}+\pi_{9} x_{9}+\delta_{3} .
\end{aligned}
$$

and where $\delta_{\mathrm{i}}(\mathrm{i}=1,2,3)$ was generated by a normal distribution.

In the case of the measurement model for $\eta$, the definition of the equations was given as:

$$
\begin{aligned}
& y_{1}=\lambda_{1} \eta+\varepsilon_{1}, \\
& y_{2}=\lambda_{2} \eta+\varepsilon_{2}, \\
& y_{3}=\lambda_{3} \eta+\varepsilon_{3},
\end{aligned}
$$

and

$$
y_{4}=\lambda_{4} \eta+\varepsilon_{4}
$$

Based on these equations, along with assumptions of the structural model in which the expected values of the error vectors and latent variables are equal to zero, $\zeta$ and $\xi_{\mathrm{i}},(\mathrm{i}=1,2$, 3) are uncorrelated, $\varepsilon_{j}(j=1,2,3,4)$ are uncorrelated with $\eta, \xi_{i}$ and $\delta_{i}$, being $\delta_{i}$ uncorrelated with $\xi_{i}, \eta$ and $\varepsilon_{j}$. With these specifications, the regression coefficient estimators were obtained by robust LMS and
LTS methods and the sample generation was characterized by different degrees of asymmetry for $\mathrm{x}$ observable variables distributed as a Beta $(\alpha, \beta)$ distribution, whose parameters were fixed in accordance with Table 1.

Table 1: Beta $(\alpha, \beta)$ Distribution Used in the Simulation of the Observable Variables with Different Degrees of Asymmetry

\begin{tabular}{|c|c|c|}
\hline Case & Distribution & $\begin{array}{c}\text { Degree of } \\
\text { Asymmetry }\end{array}$ \\
\hline 0 & Beta $(6,6)$ & $\begin{array}{c}\text { Perfectly } \\
\text { Symmetrical }\end{array}$ \\
\hline 1 & Beta $(9,4)$ & $\begin{array}{c}\text { Moderate } \\
\text { Asymmetry to } \\
\text { the Right }\end{array}$ \\
\hline 2 & Beta $(9,1)$ & $\begin{array}{c}\text { High Asymmetry } \\
\text { to the Right }\end{array}$ \\
\hline
\end{tabular}

The illustration of each case is shown in Figures 4-6 and the parametric values used in the simulation of the structural model are shown in Figure 7.

Figure 4: Beta $(6,6)$

\section{Case 0}

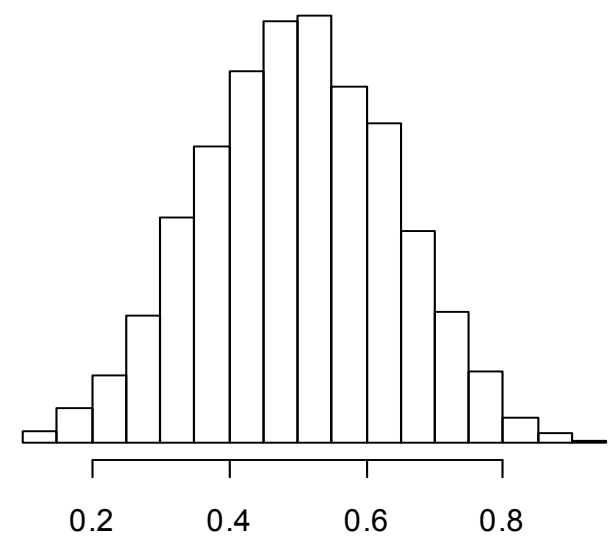




\section{CIRILLO \& BARROSO}

Figure 5: Beta $(9,4)$

\section{Case 1}

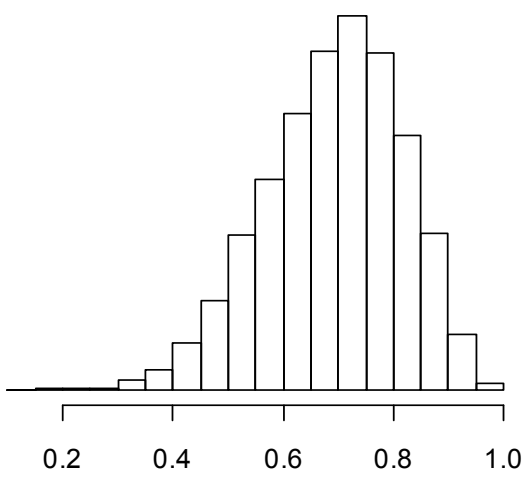

Figure 6: Beta $(9,1)$

Case 2

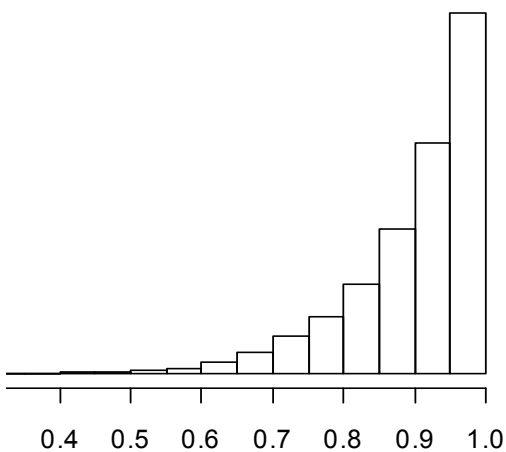

Robust Regression Estimators Incorporated into the Structural Equation Model

The LMS estimators (Rousseeuw \& Leroy, 2003) applied in SEM were incorporated considering structural model (4) and the measurement models related to the independent (models (5)-(7)) and the dependent (models (8)(11)) variables. For sample sizes fixed at $n=50$, 200 and 1,000, estimates were obtained by minimizing equations (12)-(14). Thus, with the exception of the structural model, each of the measurement model equations was specified by the index $\mathrm{s}=1, \ldots, \mathrm{Q}$, where $\mathrm{Q}$ is the total number of equations and the parameter estimates of each equation were obtained by the LMS method.

$$
\begin{gathered}
\min \left\{\operatorname{median}\left(\zeta_{1}^{2}, \ldots, \zeta_{\mathrm{n}}^{2}\right)\right\} \\
\min \left\{\operatorname{median}\left(\varepsilon_{\mathrm{s} 1}^{2}, \ldots, \varepsilon_{\mathrm{sn}}^{2}\right)\right\}, \mathrm{s}=1, \ldots,(\mathrm{Q}=4),
\end{gathered}
$$

and

$$
\min \left\{\operatorname{median}\left(\delta_{\mathrm{s} 1}^{2}, \ldots, \delta_{\mathrm{sn}}^{2}\right)\right\} \mathrm{s}=1, \ldots,(\mathrm{Q}=3)
$$

In the case of the LTS method (Rousseeuw \& Leroy, 2003) the parameter estimates were obtained from expressions:

$$
\min \left\{\sum_{\mathrm{r}=1}^{\mathrm{h}} \zeta_{\mathrm{r}}^{2}, \ldots, \zeta_{\mathrm{h}}^{2}\right\}
$$

$$
\min \left\{\sum_{\mathrm{r}=1}^{\mathrm{h}} \varepsilon_{\mathrm{sr}}^{2}, \ldots, \varepsilon_{\mathrm{sh}}^{2}\right\} ; \mathrm{s}=1, \ldots,(\mathrm{Q}=4)
$$

and

$$
\min \left\{\sum_{\mathrm{r}=1}^{\mathrm{h}}\left(\delta_{\mathrm{sr}}^{2}, \ldots, \delta_{\mathrm{sh}}^{2}\right)\right\} ; \mathrm{s}=1, \ldots,(\mathrm{Q}=3)
$$

In all situations, the values of $h$ were recommended by Rousseeuw and Hubert (1994), according to the expression:

$$
\mathrm{h}=\left[\frac{\mathrm{n}+\mathrm{p}+1}{2}\right]
$$

where $p$ is the total number of parameters relating to each equation.

Statistical Measurements to Compare the Results

Based on the procedure described, the robust estimators were incorporated into the structural equation model and a program was constructed in software $\mathrm{R}$ version 2.11.1 (R Development Core Team, 2010), in which 1,000 Monte Carlo simulations were performed for each sample size for each degree of asymmetry in the errors distributions (see Figures 1-3) and 
Figure 7: Graphical Representation of the Structural Equation Model with the Parametric Values Used in the Monte Carlo Simulation Process

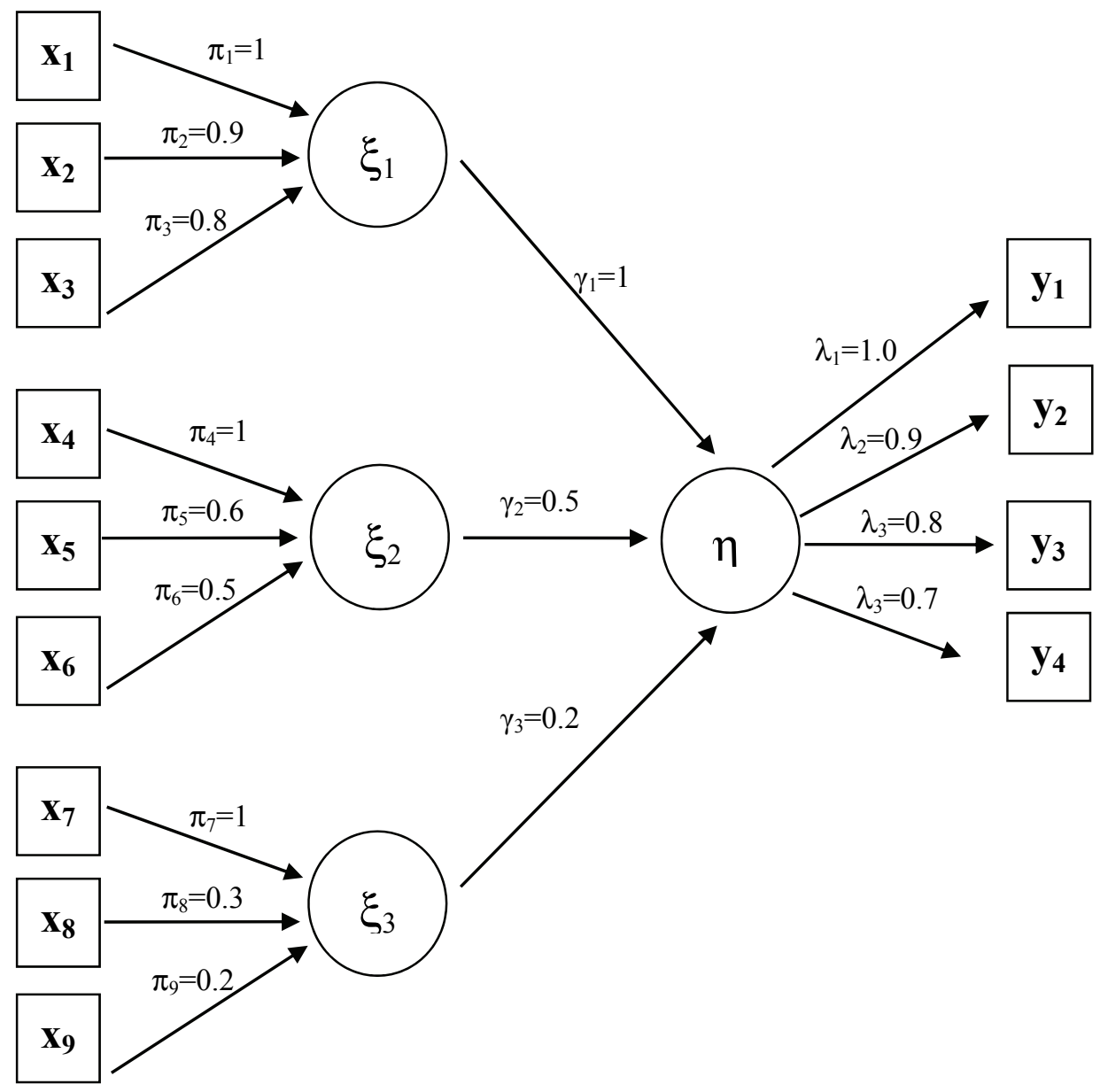

in the observable variables distributions (see Figures 4-6). To evaluate the accuracy for each parameter of structural equation (4), the relative deviations were computed as:

$$
\mathrm{B}=\frac{\sum_{\mathrm{b}=1}^{1000}\left(\frac{\gamma_{\mathrm{ib}}-\hat{\gamma}_{\mathrm{ib}}}{\gamma_{\mathrm{ib}}}\right)}{1000}, \mathrm{i}=1,2,3 .
$$

In terms of the precision of the study, the range given in (20) was used considering the difference between the highest and lowest standard deviation value $\mathrm{S} \gamma_{\mathrm{i}}$ obtained by means of the empirical distribution of estimates of each parameter $\gamma_{\mathrm{i}}(\mathrm{i}=1,2,3)$ generated in the simulation process:

$$
\begin{aligned}
& \mathrm{A}_{\mathrm{sd}}=\max \left(\mathrm{S}_{\gamma_{\mathrm{i}}}\right)-\min \left(\mathrm{S}_{\gamma_{\mathrm{i}}}\right), \\
& \mathrm{i}=1,2,3
\end{aligned}
$$

\section{Results}

Considering the observable variables generated with perfect symmetry (Case 0 ; see Table 2), it was observed that, in the situation where errors were symmetrical (Figure 2), the results were similar; the three estimators being considered accurate for all methods evaluated in terms of sample sizes. 


\section{CIRILLO \& BARROSO}

Table 2: Estimates of the Relative Bias (B) Considering Different Degrees of Asymmetry of the Distribution of Errors with the Observable Variables Generated by Beta $(6,6)$

\begin{tabular}{|c|c|c|c|c|c|c|c|c|c|}
\hline \multicolumn{10}{|c|}{ LS Method } \\
\hline \multirow{2}{*}{$\mathrm{n}$} & \multicolumn{3}{|c|}{$\zeta ; \varepsilon_{j} ; \delta_{\mathrm{i}} \sim \mathrm{N}(0,1)$} & \multicolumn{3}{|c|}{$\zeta ; \varepsilon_{\mathrm{j}} ; \delta_{\mathrm{i}} \sim \mathrm{N}(0,1,20)$} & \multicolumn{3}{|c|}{$\zeta ; \varepsilon_{\mathrm{j}} ; \delta_{\mathrm{i}} \sim \mathrm{N}(0,1,-20)$} \\
\hline & $\gamma_{1}$ & $\gamma_{2}$ & $\gamma_{3}$ & $\gamma_{1}$ & $\gamma_{2}$ & $\gamma_{3}$ & $\gamma_{1}$ & $\gamma_{2}$ & $\gamma_{3}$ \\
\hline 50 & 0.0023 & -0.0007 & -0.0426 & 0.1561 & 0.2666 & 0.6163 & -0.5592 & -0.5830 & 0.1774 \\
\hline 200 & -0.0017 & -0.0015 & 0.0076 & 0.1557 & 0.2684 & 0.6019 & -0.5597 & -0.5406 & 0.2067 \\
\hline 1000 & -0.0001 & -0.0004 & 0.0081 & 0.1551 & 0.2755 & 0.5831 & -0.5625 & -0.5335 & 0.2511 \\
\hline \multicolumn{10}{|c|}{ LMS Method } \\
\hline \multirow{2}{*}{$\mathrm{n}$} & \multicolumn{3}{|c|}{$\zeta ; \varepsilon_{\mathrm{j}} ; \delta_{\mathrm{i}} \sim \mathrm{N}(0,1)$} & \multicolumn{3}{|c|}{$\zeta ; \varepsilon_{j} ; \delta_{\mathrm{i}} \sim \mathrm{N}(0,1,20)$} & \multicolumn{3}{|c|}{$\zeta ; \varepsilon_{j} ; \delta_{\mathrm{i}} \sim \mathrm{N}(0,1,-20)$} \\
\hline & $\gamma_{1}$ & $\gamma_{2}$ & $\gamma_{3}$ & $\gamma_{1}$ & $\gamma_{2}$ & $\gamma_{3}$ & $\gamma_{1}$ & $\gamma_{2}$ & $\gamma_{3}$ \\
\hline 50 & 0.0081 & -0.0481 & -0.0202 & 0.0994 & 0.2009 & 0.4488 & -0.4828 & -0.5342 & 0.0770 \\
\hline 200 & 0.0091 & -0.0346 & 0.0044 & 0.0927 & 0.1684 & 0.3605 & -0.4619 & -0.3981 & 0.0890 \\
\hline 1000 & 0.0006 & 0.0027 & 0.0087 & 0.0852 & 0.1642 & 0.3227 & 0.0782 & 0.1142 & 0.1043 \\
\hline \multicolumn{10}{|c|}{ LTS Method } \\
\hline \multirow{2}{*}{$\mathrm{n}$} & \multicolumn{3}{|c|}{$\zeta ; \varepsilon_{\mathrm{j}} ; \delta_{\mathrm{i}} \sim \mathrm{N}(0,1)$} & \multicolumn{3}{|c|}{$\zeta ; \varepsilon_{j} ; \delta_{\mathrm{i}} \sim \mathrm{N}(0,1,20)$} & \multicolumn{3}{|c|}{$\zeta ; \varepsilon_{\mathrm{j}} ; \delta_{\mathrm{i}} \sim \mathrm{N}(0,1,-20)$} \\
\hline & $\gamma_{1}$ & $\gamma_{2}$ & $\gamma_{3}$ & $\gamma_{1}$ & $\gamma_{2}$ & $\gamma_{3}$ & $\gamma_{1}$ & $\gamma_{2}$ & $\gamma_{3}$ \\
\hline 50 & 0.0245 & -0.0596 & -0.0452 & 0.0999 & 0.1863 & 0.4770 & -0.4754 & -0.5223 & 0.0238 \\
\hline 200 & -0.0007 & -0.0156 & -0.0039 & 0.0834 & 0.1557 & 0.3758 & -0.4334 & -0.3834 & 0.0548 \\
\hline 1000 & -0.0018 & -0.0033 & 0.0164 & 0.0712 & 0.1479 & 0.3087 & -0.3976 & -0.3352 & 0.1547 \\
\hline
\end{tabular}

This result is consistent with findings by Cassel, et al. (1999) that the parameter estimates resulting from the application of the Partial Least Squares method showed similar results, that is, estimates were not affected by increasing sample size. However, in considering the errors distributed as the normal asymmetric with the parameters of asymmetry $a=20$ (see Figure 1) and $a=-20$ (see Figure 3), it was observed that the values of the biases in situations where the LMS and LTS methods were used were smaller those when considering the method of Least Squares (LS) estimation. However, it should be emphasized that the robust regression methods in the case of negative asymmetry of the errors retained results that characterize an inaccuracy and show evidence of a tendency to provide inflated or deflated estimates related to the parameters of the structural equation. This deficiency was corrected by increasing the sample size to $\mathrm{n}=1,000$ with emphasis on the LMS method which provided more accurate results compared to the parametric values imposed on the structural model.

Because results were more accurate for robust methods, in situations where the errors were generated by asymmetric normal distributions precision was evaluated using the statistic given by (20). Thus, the results illustrated in Figure 8 show that the LMS and 
Figure 8: Precision of the Robust Estimates Considering Positive and Negative Asymmetric Errors with Observable Variables Generated by Beta $(6,6)$

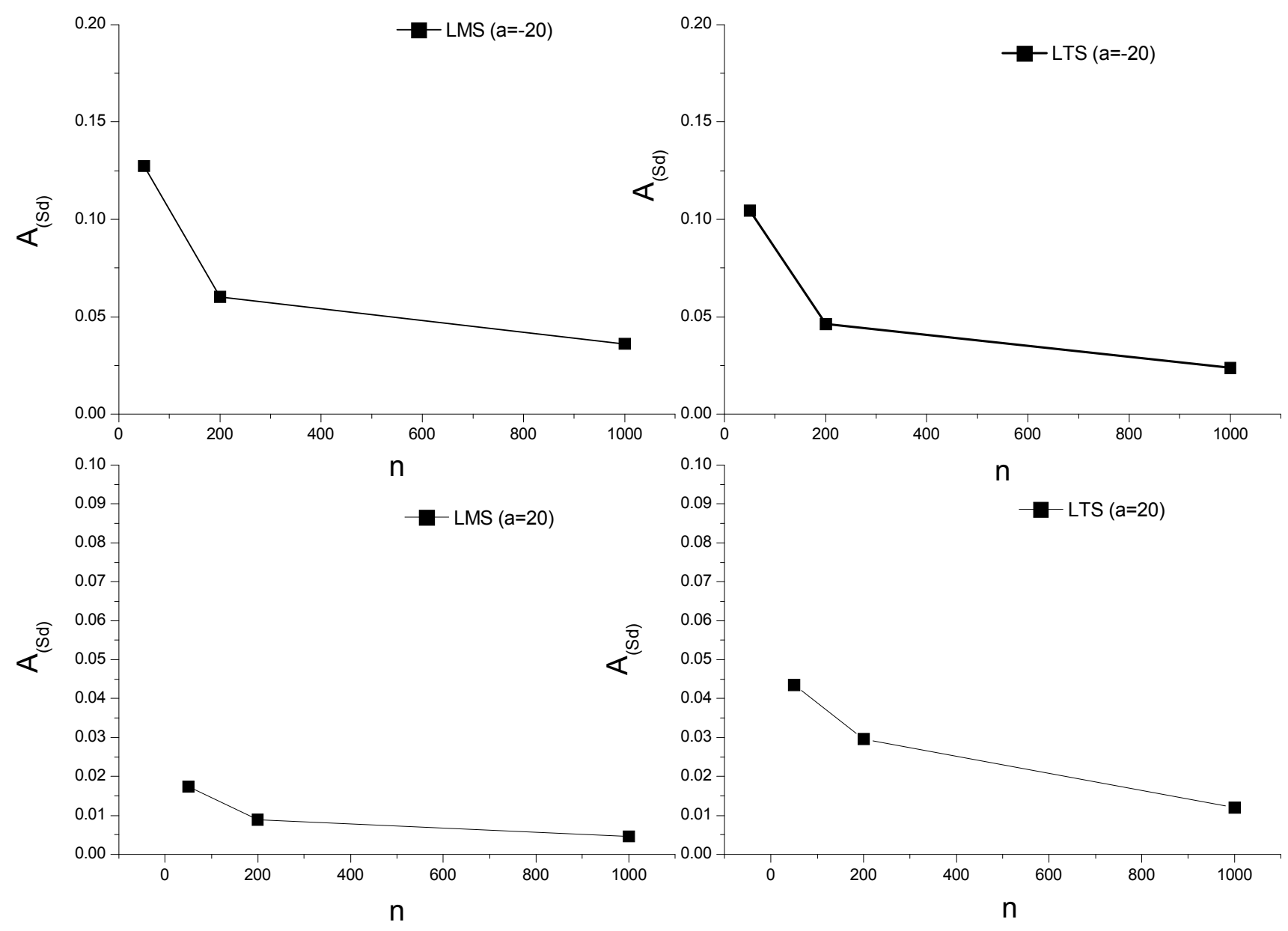

LTS methods presented similar precision when the errors have a negative asymmetry effect, and the LMS method showed better performance in the case of positive asymmetry.

Considering the distribution of observable variables with a moderate degree of asymmetry (Case 1), the results in Table 3 show that, in the situation where structural errors and measurement were simulated assuming normality without deviations of asymmetry (Figure 2), the LMS and LTS methods continued to provide good accuracy. However, when considering the errors generated by the normal asymmetric distribution, it was observed that the robust estimation methods were less accurate than those outlined in Table 2, but showed better performance than the least squares estimator. Maintaining the focus on the study of the precision afforded by the robust LMS and LTS methods (see Figure 9) the performance of the two estimators is similar.

Supplementing these findings, in a comparative study of maximum likelihood estimators (MLE) and robust estimation methods applied in the setting of a confirmatory factor analysis, Zhong \& Yuan (2011) concluded that, in the case of non-normal distributions, the MLE estimates are biased and inefficient and the use of robust methods provide an improvement on these properties; however, they stressed that the 


\section{CIRILLO \& BARROSO}

Table 3: Estimates of Relative Bias (B) Considering Different Degrees of Asymmetry of the

Distribution of Errors with the Observable Variables Generated by Beta $(9,4)$

\begin{tabular}{|c|c|c|c|c|c|c|c|c|c|}
\hline \multicolumn{10}{|c|}{ LS Method } \\
\hline \multirow{2}{*}{$\mathrm{n}$} & \multicolumn{3}{|c|}{$\zeta ; \varepsilon_{\mathrm{j} j} ; \delta_{\mathrm{i}} \sim \mathrm{N}(0,1)$} & \multicolumn{3}{|c|}{$\zeta ; \varepsilon_{\mathrm{j}} ; \delta_{\mathrm{i}} \sim \mathrm{N}(0,1,20)$} & \multicolumn{3}{|c|}{$\zeta ; \varepsilon_{j} ; \delta_{\mathrm{i}} \sim \mathrm{N}(0,1,-20)$} \\
\hline & $\gamma_{1}$ & $\gamma_{2}$ & $\gamma_{3}$ & & $\gamma_{1}$ & $\gamma_{2}$ & $\gamma_{3}$ & & $\gamma_{1}$ \\
\hline 50 & 0.0000 & 0.0024 & -0.0078 & 50 & 0.0000 & 0.0024 & -0.0078 & 50 & 0.0000 \\
\hline 200 & -0.0018 & 0.0047 & 0.0043 & 200 & -0.0018 & 0.0047 & 0.0043 & 200 & -0.0018 \\
\hline 1000 & -0.0007 & 0.0051 & -0.0064 & 1000 & -0.0007 & 0.0051 & -0.0064 & 1000 & -0.0007 \\
\hline \multicolumn{10}{|c|}{ LMS Method } \\
\hline \multirow{2}{*}{$\mathrm{n}$} & \multicolumn{3}{|c|}{$\zeta ; \varepsilon_{\mathrm{j}} ; \delta_{\mathrm{i}} \sim \mathrm{N}(0,1)$} & \multicolumn{3}{|c|}{$\zeta ; \varepsilon_{\mathrm{j}} ; \delta_{\mathrm{i}} \sim \mathrm{N}(0,1,20)$} & \multicolumn{3}{|c|}{$\zeta ; \varepsilon_{j} ; \delta_{\mathrm{i}} \sim \mathrm{N}(0,1,-20)$} \\
\hline & $\gamma_{1}$ & $\gamma_{2}$ & $\gamma_{3}$ & & $\gamma_{1}$ & $\gamma_{2}$ & $\gamma_{3}$ & & $\gamma_{1}$ \\
\hline 50 & 0.0035 & -0.0042 & -0.0505 & 50 & 0.0035 & -0.0042 & -0.0505 & 50 & 0.0035 \\
\hline 200 & -0.0054 & 0.0187 & 0.0039 & 200 & -0.0054 & 0.0187 & 0.0039 & 200 & -0.0054 \\
\hline 1000 & -0.0008 & -0.0023 & 0.0137 & 1000 & -0.0008 & -0.0023 & 0.0137 & 1000 & -0.0008 \\
\hline \multicolumn{10}{|c|}{ LTS Method } \\
\hline \multirow{2}{*}{$\mathrm{n}$} & \multicolumn{3}{|c|}{$\zeta ; \varepsilon_{\mathrm{j}} ; \delta_{\mathrm{i}} \sim \mathrm{N}(0,1)$} & \multicolumn{3}{|c|}{$\zeta ; \varepsilon_{j} ; \delta_{\mathrm{i}} \sim \mathrm{N}(0,1,20)$} & \multicolumn{3}{|c|}{$\zeta ; \varepsilon_{\mathrm{j}} ; \delta_{\mathrm{i}} \sim \mathrm{N}(0,1,-20)$} \\
\hline & $\gamma_{1}$ & $\gamma_{2}$ & $\gamma_{3}$ & & $\gamma_{1}$ & $\gamma_{2}$ & $\gamma_{3}$ & & $\gamma_{1}$ \\
\hline 50 & -0.0134 & 0.0130 & -0.0012 & 50 & -0.0134 & 0.0130 & -0.0012 & 50 & -0.0134 \\
\hline 200 & -0.0045 & 0.0082 & 0.0160 & 200 & -0.0045 & 0.0082 & 0.0160 & 200 & -0.0045 \\
\hline 1000 & -0.0037 & -0.0026 & 0.0385 & 1000 & -0.0037 & -0.0026 & 0.0385 & 1000 & -0.0037 \\
\hline
\end{tabular}

estimates of standard errors must be studied. In this sense, the study of the precision of the estimators is justified, confirming the claims made by Kaplan (1969) referring to the fact that the refinement of precision does not necessarily lead to increased reliability. In addition, in some situations, there could occur a reduction in the reliability of the estimates by a reduction of the accuracy because in practical situations the researcher may encounter systematic errors, for example, errors in the definition of the measurement or recording of the observation and random errors associated with uncontrollable variations.

When a high degree of asymmetry is imposed on the observable variables through distribution Beta $(9,1)$ (Case 2; see Table 4), maintaining the structural error and measurement errors distributed according to a normal symmetry (Figure 2), the LMS and LTS methods are accurate and similar results are obtained using the LS method. However, considering the asymmetric error (see Figures 1 and 3), based on the bias, the robust methods were more suitable for use in replacement of the LS method.

In the same situations with different degrees of asymmetry (see Table 1) assuming the measurement errors of the equations ((5)-(7)) and ((8)-(11)) are uniformly distributed, Cassel, et al. (1999) evaluated the biases of the parameter estimates of the structural equation model 
Figure 9: Precision of the Robust Estimates Considering the Positive and Negative Asymmetric Errors with Observable Variables Generated by Beta $(9,4)$

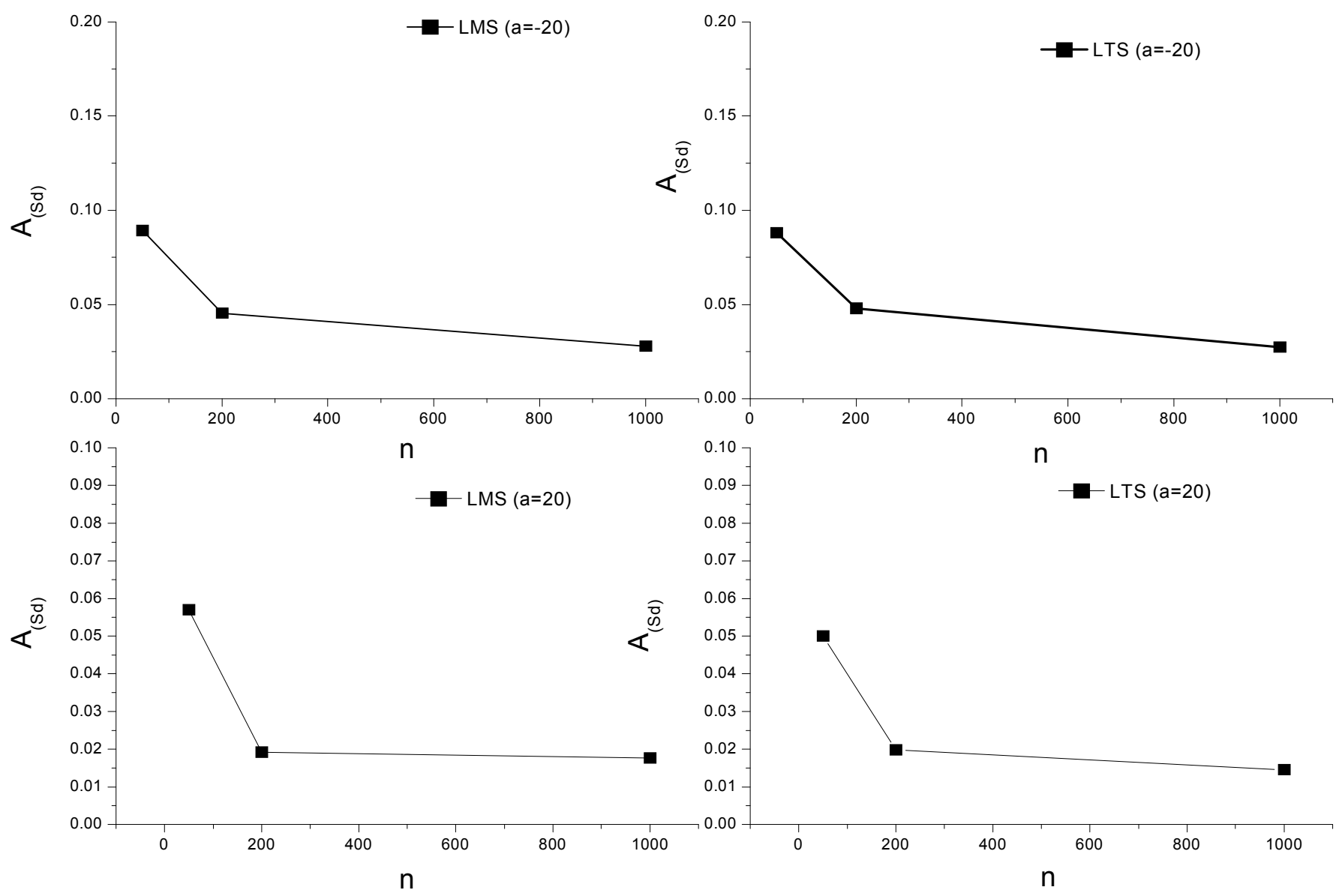

resulting from the application of Partial Least Squares method and concluded that the increase in the degree of asymmetry reflected an increase in bias and this is more emphasized in a situation of a high degree of asymmetry (Case 2; see Figure 6). Results obtained in this work are consistent with the Cassel, et al. (1999) findings and there is statistical evidence to argue that robust regression methods are consistent and worthy of being adapted to be used in structural equation modeling.

Relevant to the discussion of the estimates accuracy provided by the LMS and LTS methods it is important to note that, in the violation of the normality assumption, there are numerous works relating other methods that can be used as alternatives. Engel, et al. (2003) stated that the maximum likelihood method can be applied when the normality deviation is relatively moderate. In this situation the authors recommend $n>400$. Moreover, Muthén and Muthén (2002) stated that the maximum likelihood estimators can be consistent but not necessarily efficient.

For variables with non-normal distributions, the recommendations are divergent. The minimum sample size for the weighted least squares method to be reliable, should be at least $\mathrm{n}=1,000$ (Hoogland \& Boomsma, 1998), and depending on the model and data being analyzed; in some cases even exceeding 4,000 or 5,000 (Boomsma \& Hoogland, 2001; Hu, et al., 1992). In general, when considering small sample sizes, Bollen, et 


\section{CIRILLO \& BARROSO}

Table 4: Estimates of Relative Biases (B) Considering Different Degrees of Asymmetry of the Distribution of Errors with the Observable Variables Generated by Beta $(9,1)$

\begin{tabular}{|c|c|c|c|c|c|c|c|c|c|}
\hline \multicolumn{10}{|c|}{ LS Method } \\
\hline \multirow{2}{*}{$\mathrm{n}$} & \multicolumn{3}{|c|}{$\zeta ; \varepsilon_{\mathrm{j}} ; \delta_{\mathrm{i}} \sim \mathrm{N}(0,1)$} & \multicolumn{3}{|c|}{$\zeta ; \varepsilon_{j} ; \delta_{i} \sim \mathrm{N}(0,1,20)$} & \multicolumn{3}{|c|}{$\zeta ; \varepsilon_{j} ; \delta_{\mathrm{i}} \sim \mathrm{N}(0,1,-20)$} \\
\hline & $\gamma_{1}$ & $\gamma_{2}$ & $\gamma_{3}$ & $\gamma_{1}$ & $\gamma_{2}$ & $\gamma_{3}$ & $\gamma_{1}$ & $\gamma_{2}$ & $\gamma_{3}$ \\
\hline 50 & -0.0030 & 0.0059 & -0.0037 & -0.0030 & 0.0059 & -0.0037 & -0.0030 & 0.0059 & -0.0037 \\
\hline 200 & -0.0004 & 0.0035 & -0.0044 & -0.0004 & 0.0035 & -0.0044 & -0.0004 & 0.0035 & -0.0044 \\
\hline 1000 & 0.0001 & 0.0007 & -0.0009 & 0.0001 & 0.0007 & -0.0009 & 0.0001 & 0.0007 & -0.0009 \\
\hline \multicolumn{10}{|c|}{ LMS Method } \\
\hline \multirow{2}{*}{$\mathrm{n}$} & \multicolumn{3}{|c|}{$\zeta ; \varepsilon_{\mathrm{j}} ; \delta_{\mathrm{i}} \sim \mathrm{N}(0,1)$} & \multicolumn{3}{|c|}{$\zeta ; \varepsilon_{j} ; \delta_{\mathrm{i}} \sim \mathrm{N}(0,1,20)$} & \multicolumn{3}{|c|}{$\zeta ; \varepsilon_{j} ; \delta_{\mathrm{i}} \sim \mathrm{N}(0,1,-20)$} \\
\hline & $\gamma_{1}$ & $\gamma_{2}$ & $\gamma_{3}$ & $\gamma_{1}$ & $\gamma_{2}$ & $\gamma_{3}$ & $\gamma_{1}$ & $\gamma_{2}$ & $\gamma_{3}$ \\
\hline 50 & -0.0022 & -0.0019 & 0.0717 & -0.0022 & -0.0019 & 0.0717 & -0.0022 & -0.0019 & 0.0717 \\
\hline 200 & -0.0039 & 0.0106 & -0.0166 & -0.0039 & 0.0106 & -0.0166 & -0.0039 & 0.0106 & -0.0166 \\
\hline 1000 & 0.0028 & -0.0083 & -0.0160 & 0.0028 & -0.0083 & -0.0160 & 0.0028 & -0.0083 & -0.0160 \\
\hline \multicolumn{10}{|c|}{ LTS Method } \\
\hline \multirow{2}{*}{$\mathrm{n}$} & \multicolumn{3}{|c|}{$\zeta ; \varepsilon_{\mathrm{j}} ; \delta_{\mathrm{i}} \sim \mathrm{N}(0,1)$} & \multicolumn{3}{|c|}{$\zeta ; \varepsilon_{j} ; \delta_{\mathrm{i}} \sim \mathrm{N}(0,1,20)$} & \multicolumn{3}{|c|}{$\zeta ; \varepsilon_{j} ; \delta_{\mathrm{i}} \sim \mathrm{N}(0,1,-20)$} \\
\hline & $\gamma_{1}$ & $\gamma_{2}$ & $\gamma_{3}$ & $\gamma_{1}$ & $\gamma_{2}$ & $\gamma_{3}$ & $\gamma_{1}$ & $\gamma_{2}$ & $\gamma_{3}$ \\
\hline 50 & -0.0160 & 0.0236 & 0.0612 & -0.0160 & 0.0236 & 0.0612 & -0.0160 & 0.0236 & 0.0612 \\
\hline 200 & 0.0006 & 0.0046 & -0.0094 & 0.0006 & 0.0046 & -0.0094 & 0.0006 & 0.0046 & -0.0094 \\
\hline 1000 & -0.0002 & 0.0015 & -0.0110 & -0.0002 & 0.0015 & -0.0110 & -0.0002 & 0.0015 & -0.0110 \\
\hline
\end{tabular}

al. (2007) recommended that results should be interpreted with caution. Considering these arguments, it may be stated that the use of the robust estimation methods evaluated in this work are worthy of being considered in SEM, as the results show that the increase in the degree of asymmetry in the distribution of observed variables provided an improvement in the precision of the LMS and LTS estimates.
Conclusion

In situations where the observed variables and errors in a structural equation model show asymmetry deviations, the robust estimation methods LMS and LTS are suitable for application, especially with smaller sample sizes. 
Figure 10: Precision of the Robust Estimates Considering Positive and Negative Asymmetric Errors with

Observable Variables Generated by Beta $(9,1)$

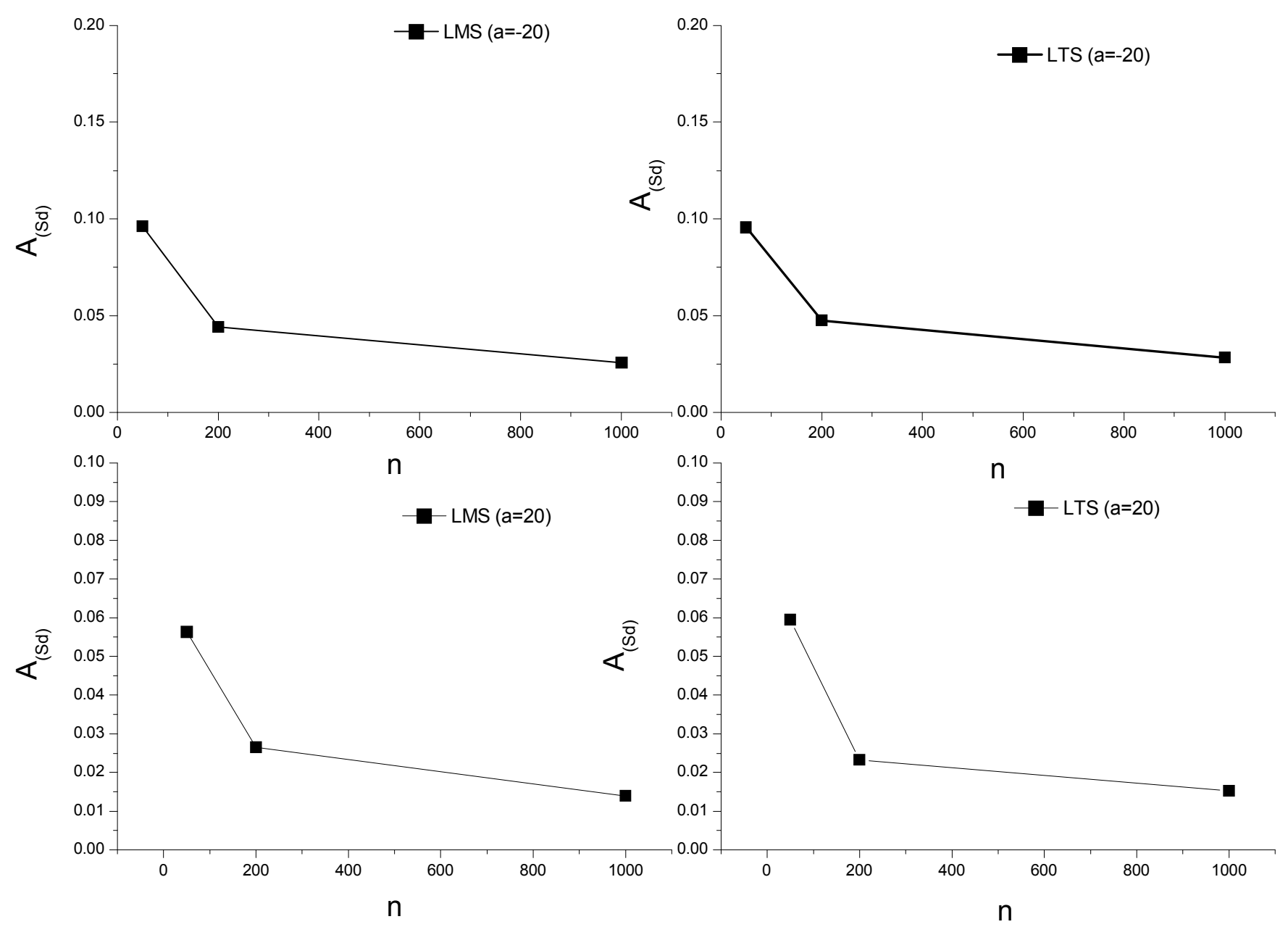

\section{References}

Agulló, J. (2001). New algorithms for computing the least trimmed squares regression estimator. Computational Statistics and Data Analysis, 36, 425-439.

Bollen, K. A., Kirby, J. B., Curran, P. J., Paxton, P. M., \& Chen, F. (2007). Latent variable models under misspecification twostage least squares (2SLS) and maximum likelihood (ML) estimators. Sociological Methods \& Research, I, 48-86.
Boomsma, A., \& Hoogland, J. J. (2001). The robustness of LISREL modeling revisited. In Structural equation models: Present and future: A Festschrift in honor of Karl Jöreskog, R. Cudeck, S. du Toit, \& D. Sörbom (Eds.), 139168. Chicago, IL: Scientific Software International.

Cassel, C., Hackl, P., \& Westlund, A. H. (1999). Robustness of partial least-squares method for estimating latent variable quality structures. Journal of Applied Statistics, 26, 435446. 


\section{CIRILLO \& BARROSO}

Engel, K. S., Moosbrugger, H., \& Muller, K. (2003). Evaluating the fit of structural equation models: tests of significance and descriptive goodness-of-fit measures. Methods of Psychological Research Online, 2, 23-74.

Gao, S., Mokhtarian, P. L., \& Johnston, R. A. (2008). Nonnormality of data in structural equation. Transportation Research Record: Journal of the Transportation Research Board, 2082, 116-124.

Gervini, D. M., \& Yohai, V. J. (2002). A class of robust and fully efficient regression estimators. Annals of Statistics, 30, 583-616.

Hawkins, D. M., Bradu, D., \& Kass, G. (1984). Location of several outliers in multiple regression data using element al sets. Technometrics, 26, 197-208.

Hoogland, J. J., \& Boomsma, A. (1998). Robustness studies in covariance structure modeling: An overview and a meta-analysis. Sociological Methods \& Research, 26, 329-367.

Hu, L., Bentler, P. M., \& Kano, Y. (1992). Can test statistics in covariance structure analysis be trusted? Psychological Bulletin, 112, 351-362.

Kaplan, A. A. (1969). The conduct of research. Sao Paulo, Brazil: Edusp.

Lei, M., \& Lomax, R. G. (2005). The effect of varying degrees of nonnormality in structural equation modeling. Structural Equation Modeling: A Multidisciplinary Journal, 12, 1-27.

Maronna, R. A., Martin, D., \& Yohai, V. J. (2006). Robust statistics theory and methods. New York, NY: Wiley.
Muthén, L. K., \& Muthén, B. O. (2002). How to use a Monte Carlo study to decide on sample size and determine power. Structural Equation Modeling, 4, 599-620.

O’Loughlin, C., \& Coenders, G. (2004). Estimation of the European customer satisfaction index: maximum likelihood versus partial least squares: Application to postal services. Total Quality Management \& Business Excellence, 15, 1231-1255.

Pilati, R., \& Laros, J. A. (2007). Modelos de equações estruturais em psicologia: conceitos e aplicações. Psicologia: Teoria $e$ Pesquisa, 23, 205-216.

R Development Core Team (2010). $R$ : A language and environment for statistical computing. Vienna, Austria: R Foundation for Statistical Computing. http://www.R-project.org.

Rousseeuw, P., \& Hubert, M. (1994). Recent developments in PROGRESS. Institute of Mathematical Statistics Lecture NotesMonograph Series, Hayward, California, 31, 201-214.

Rousseeuw, P., \& Leroy, A. (2003). Robust regression and outlier detection. New York, NY: Wiley.

Yuan, K. H., \& Bentler, P. M. (2001). Effects of "outliers" on estimators and tests in covariance structure analysis. British Journal of Mathematical and Statistical Psychology, 54, 161-175.

Zhong, X., \& Yuan, K. H. (2011). Bias and efficiency in structural equation modeling: maximum likelihood versus robust methods. Multivariate Behavioral Research, 46, 229-265. 


\section{Robust Modifications of the Levene and O'Brien Tests for Spread}

\author{
Abdul R. Othman Teh Sin Yin \\ Universiti Sains Malaysia, \\ Georgetown, Penang, Malaysia \\ Rand R. Wilcox \\ University of Southern California, \\ Los Angeles, CA
}

\author{
H. J. Keselman \\ University of Manitoba, \\ Winnipeg, Manitoba Canada \\ James Algina \\ University of Florida, \\ Gainesville, FL
}

Variants of Levene's and O'Brien's procedures not investigated by Keselman, Wilcox \& Algina (2008) were examined. Simulations indicate that a new O'Brien variant provides very good Type I error control and is simpler for applied researchers to compute than the method recommended by Keselman, et al.

Key words: Levene test of spread, O’Brien test of spread, Type I error.

\section{Introduction}

Keselman, Wilcox, Algina, et al. (2008) compared a number of tests for spread that were based on either least squares or trimmed estimates of central tendency and variability. These estimators were based on either the original data or transformations suggested by Levene (1960) and O'Brien (1981). The adaptive trimming estimators they used were defined by Reed and Stark (1996), estimators which rely on procedures that determine whether data should be trimmed symmetrically, asymmetrically, or not at all. The transformed scores were used in an analysis of variance (ANOVA) F-test, a Welch (1951) test, and a robust ANOVA test due to Lee and Fung (1985). Based on their extensive simulation study, Keselman, et al. recommended a Levene-type

Abdul R. Othman is a Professor of Statistics. Email him at: arothman60@yahoo.com. Teh Sin Yin is a Senior Lecturer of Statistics. Email her at: syin.teh@gmail.com. H. J. Keselman is a Professor of Psychology. His research interests are in applied statistics. Email him at: kesel@ms.umanitoba.ca. Rand R. Wilcox is a Professor of Psychology. Email him at: rwilcox@usc.edu. James Algina is a Professor of Educational Psychology Research and Evaluation Methodology at the University of Florida. Email him at: algina@ufl.edu. transformation based on empirically determined $20 \%$ asymmetric trimmed means, involving a particular adaptive estimator, where the transformed scores are then used with an ANOVA F test.

In their investigation, Keselman, et al. only examined a limited number of variations of the Levene (1960) and O'Brien (1979) methods - variations where, by-in-large, the transformed variables were obtained via the application of asymmetrically trimmed means involving one of the seven hinge estimators defined by Reed and Stark (1996). However, there are many other ways in which the transformed variables may be created. For example, the transformed variables may be based on symmetrically trimmed means and then these transformed variables may be symmetrically/asymmetrically transformed with one of the seven hinge estimators. Thus, the purpose of this study was to examine other variants of the Levene and O'Brien methods not examined by Keselman, et al. (2008).

\section{Background}

As Keselman, et al. (2008), and others, have noted, the traditional test for equality of variances, e.g., $F=\frac{s_{1}^{2}}{s_{2}^{2}}$, where $s_{j}^{2}$ is the usual unbiased sample variance for the $\mathrm{j}^{\text {th }}$ group, is affected adversely when the data in the groups are not normally distributed (i.e., it is sensitive to kurtosis). That is, the actual level of 


\section{OTHMAN, YIN, KESELMAN, WILCOX \& ALGINA}

significance can differ substantially from the nominal significance level. In addition, power can be low.

Levene (1960) suggested an alternative test statistic that can be used to assess equality of spread across independent treatment groups. For the one-way layout with model $X_{i j}=\mu_{j}+\varepsilon_{i j}$ $\left(i=1, \ldots, n_{j} ; j=1, \ldots, J\right)$, where $\mu_{j}$ is the population mean for the $\mathrm{j}^{\text {th }}$ group and $\varepsilon_{\mathrm{ij}}$ is random error, Levene suggested that the scores could be modified with the transformation $\mathrm{Z}_{\mathrm{ij}}=\left|\mathrm{X}_{\mathrm{ij}}-\overline{\mathrm{X}}_{. \mathrm{j}}\right|$, where $\overline{\mathrm{X}}_{\mathrm{j}}$ is the $\mathrm{j}^{\mathrm{th}}$ sample mean, and then these scores can be used in an ANOVA test. That is, the test suggested by Levene is

$$
\mathrm{W}_{0}=\frac{\sum_{\mathrm{j}=1}^{\mathrm{J}} \mathrm{n}_{\mathrm{j}}\left(\overline{\mathrm{z}}_{\mathrm{j} .}-\overline{\mathrm{z}}_{\ldots .}\right)^{2} /(\mathrm{J}-1)}{\sum_{\mathrm{j}=1}^{\mathrm{J}} \sum_{\mathrm{i}=1}^{\mathrm{n}_{\mathrm{j}}}\left(\mathrm{z}_{\mathrm{ij}}-\overline{\mathrm{z}}_{\mathrm{j} .}\right)^{2} / \sum_{\mathrm{j}=1}^{\mathrm{J}}\left(\mathrm{n}_{\mathrm{j}}-1\right)},
$$

where

$$
\bar{z}_{j}=\sum_{1=1}^{n_{j}} \frac{z_{i j}}{n_{j}} \text { and } \bar{z}_{. .}=\frac{\sum_{j=1}^{J} \sum_{i=1}^{n_{j}} z_{i j}}{\sum_{j=1}^{J} n_{j}} .
$$

Critical values for $\mathrm{W}_{0}$ are obtained from the $\mathrm{F}$ distribution based on $\mathrm{J}-1$ and $\sum_{\mathrm{j}=1}^{\mathrm{J}}\left(\mathrm{n}_{\mathrm{j}}-1\right)$ degrees of freedom. Another statistic relevant to this article is $\mathrm{W}_{\overline{\mathrm{x}}_{t}}$. This statistic replaces the group mean in obtaining the transformed $\mathrm{Z}_{\mathrm{ij}} \mathrm{S}$ with the group trimmed mean.

Other methods have also appeared in the literature in addition to Levene's (1960) procedure. Lee and Fung (1985) presented a robust ANOVA F-test based on trimmed means. Keselman, et al. (1979) and others (e.g., O'Brien, 1981) have indicated that a Welch statistic can be adopted instead of the usual ANOVA F-test to assess spread across independent groups. O'Brien (1979) also suggested that a Welch test can be used with his transformation of the data, $\mathrm{X}_{\mathrm{ij}}$, namely

$$
\mathrm{r}_{\mathrm{ij}}=\frac{\left(\mathrm{n}_{\mathrm{j}}-1.5\right) \mathrm{n}_{\mathrm{j}}\left(\mathrm{X}_{\mathrm{ij}}-\overline{\mathrm{X}}_{\mathrm{j}}\right)^{2}-.5 \mathrm{~s}_{\mathrm{j}}^{2}\left(\mathrm{n}_{\mathrm{j}}-1\right)}{\left(\mathrm{n}_{\mathrm{j}}-1\right)\left(\mathrm{n}_{\mathrm{j}}-2\right)}
$$

Adaptive Trimming Methods

Keselman, et al. (2008) provided a detailed description of adaptive trimming methods. Reed and Stark (1996) defined seven adaptive location estimators based on measures of tail-length and skewness for a set of $n$ observations based on the work of Hogg (1974, 1982). To define these estimators, measures of tail-length and skewness must first be defined. Using the notation of Hogg $(1974,1982)$ and Reed and Stark (1996) and based on the ordered values, let $L_{\alpha}=$ the mean of the smallest $[\alpha \mathrm{n}$ ] observations, where $[\alpha \mathrm{n}]$ denotes the greatest integer less than $\alpha \mathrm{n}$ and $\mathrm{U}_{\alpha}=$ the mean of the largest $\left[\alpha \mathrm{n}\right.$ ] observations. When $\alpha=.05, \mathrm{~L}_{(.05)}$ is the mean of the smallest $[0.05 n]$ observations, $\mathrm{B}$ is the mean of the next largest $0.15 \mathrm{n}$ observations, $\mathrm{C}$ is the mean of the next largest 0.30 n observations, $\mathrm{D}$ is the mean of the next largest $0.30 \mathrm{n}$ observations, $\mathrm{E}$ the mean of the next largest $0.15 \mathrm{n}$ observations, and $\mathrm{U}_{(.05)}$ the mean of the largest $0.05 n$ observations.

\section{Tail-Length Measures}

Hogg (1974) defined two measures of tail-length, $\mathrm{Q}$ and $\mathrm{Q}_{1}$, where

$$
\begin{gathered}
\mathrm{Q}=\left(\mathrm{U}_{(.05)}-\mathrm{L}_{(.05)}\right) /\left(\mathrm{U}_{(.5)}-\mathrm{L}_{(.5)}\right) \\
\text { and } \\
\mathrm{Q}_{1}=\left(\mathrm{U}_{(.2)}-\mathrm{L}_{(.2)}\right) /\left(\mathrm{U}_{(.5)}-\mathrm{L}_{(.5)}\right) .
\end{gathered}
$$

$\mathrm{Q}$ and $\mathrm{Q}_{1}$ are location free statistics, are uncorrelated with location statistics and can be used to classify symmetric distributions as lighttailed, medium-tailed or heavy-tailed (Reed \& Stark, 1996). According to Hogg (1974) and Reed and Stark (1996), values of $\mathrm{Q}<2$ imply a light-tailed distribution, $2.0 \leq \mathrm{Q} \leq 2.6 \quad \mathrm{a}$ medium-tailed distribution, $2.6 \leq \mathrm{Q} \leq 3.2$ a heavy-tailed distribution, and $Q>3.2$ a very 


\section{ROBUST MODIFICATIONS OF THE LEVENE AND O'BRIEN TESTS FOR SPREAD}

heavy-tailed distribution. The cutoffs for $\mathrm{Q}_{1}$ are: $\mathrm{Q}_{1}<1.81 \quad$ (light-tailed), $\quad 1.81 \leq \mathrm{Q}_{1} \leq 1.87$ (medium-tailed) and $\mathrm{Q}_{1}>1.87$ (heavy-tailed).

Hogg (1982) introduced yet another measure of tail-length:

$$
\mathrm{H}_{3}=\frac{\left(\mathrm{U}_{(.05)}-\mathrm{L}_{(.05)}\right)}{(\mathrm{E}-\mathrm{B})} .
$$

With this measure, values of $\mathrm{H}_{3}<1.26$ suggest that the tails of the distribution are similar to a uniform distribution; values of 1.26 through 1.76 suggest a normal distribution, and values greater than 1.76 suggest the tails are similar to those of a double exponential distribution.

Measures of Skewness

Reed and Stark (1996) defined four measures of skewness as:

$$
\begin{aligned}
& \mathrm{Q}_{2}=\left(\mathrm{U}_{(.05)}-\mathrm{T}_{(.25)}\right) /\left(\mathrm{T}_{(.25)}-\mathrm{L}_{(.05)}\right), \\
& \mathrm{H}_{1}=\left(\mathrm{U}_{(.05)}-\mathrm{D}\right) /\left(\mathrm{C}-\mathrm{L}_{(.05)}\right), \\
& \mathrm{SK}_{2}=\left(\mathrm{X}_{(1)}-\mathrm{XMD}\right) /\left(\mathrm{XMD}-\mathrm{X}_{(\mathrm{n})}\right)
\end{aligned}
$$

and

$$
\mathrm{SK}_{5}=\left(\mathrm{X}_{(1)}-\mathrm{XM}\right) /\left(\mathrm{XM}-\mathrm{X}_{(\mathrm{n})}\right) \text {, }
$$

where $\mathrm{XMD}$ is the median, $\mathrm{XM}$ is the arithmetic mean, $\mathrm{T}_{(.25)}$ is the 0.25 -trimmed mean $\left(\mathrm{T}_{\alpha}\right)$ and $X_{(1)}$ and $X_{(n)}$ are the first and last ordered observations, respectively. Reed (1998) defined the $\alpha$-trimmed mean as:

$$
\mathrm{T}_{\alpha}=\frac{1}{\mathrm{n}(1-2 \alpha)}\left[\sum_{\mathrm{i}=\mathrm{k}+1}^{\mathrm{n}-\mathrm{k}} \mathrm{X}_{\mathrm{i}}+(\mathrm{k}-\alpha \mathrm{n})\left(\mathrm{X}_{\mathrm{k}}+\mathrm{X}_{\mathrm{n}-\mathrm{k}+1}\right)\right] .
$$

In this definition a proportion, $\alpha$, has been trimmed from each tail and the accompanying Winsorized variance $\mathrm{S}^{2}$ is defined as:

$$
\begin{aligned}
& \mathrm{S}^{2}= \\
& \frac{1}{(\mathrm{n}-1)(1-2 \alpha)^{2}}\left[\sum_{\mathrm{i}=\mathrm{k}+1}^{\mathrm{n}-\mathrm{k}}\left(\begin{array}{l}
\left(\mathrm{X}_{\mathrm{i}}-\mathrm{T}_{\alpha}\right)^{2}+\mathrm{k}\left(\mathrm{X}_{\mathrm{k}}-\mathrm{T}_{\alpha}\right)^{2} \\
+\mathrm{k}\left(\mathrm{X}_{\mathrm{n}-\mathrm{k}+1}-\mathrm{T}_{\alpha}\right)^{2}
\end{array}\right)\right],
\end{aligned}
$$

where $\mathrm{k}=[\alpha \mathrm{n}]+1$.

Based on the definitions of tail-length and skewness, Reed and Stark proposed a set of adaptive linear estimators "that have the capability of asymmetric trimming" (1996, p. 13). They defined a general scheme for their approach as follows:

1. Set the value for the total amount of trimming from the sample, $\alpha$.

2. Determine the proportion to be trimmed from the lower end of the sample $\left(\alpha_{1}\right)$ by the following proportion:

$\alpha_{1}=\alpha\left[\mathrm{UW}_{\mathrm{X}} /\left(\mathrm{UW}_{\mathrm{X}}+\mathrm{LW}_{\mathrm{X}}\right)\right], \quad$ where $\mathrm{UW}_{\mathrm{X}}$ and $\mathrm{LW}_{\mathrm{X}}$ are the numerator and denominator of the defined selector statistics (i.e., tail-length and skewness).

3. The upper trimming proportion is: $\alpha_{2}=\alpha-$ $\alpha_{1}$.

Based on this general schema, Reed and Stark (1996) defined seven hinge estimators, which are trimmed means, as:

1. $\mathrm{HQ} \alpha_{1}=\alpha\left[\mathrm{UW}_{\mathrm{Q}} /\left(\mathrm{UW}_{\mathrm{Q}}+\mathrm{LW}_{\mathrm{Q}}\right)\right]$,

2. $\mathrm{HQ}_{1} \quad \alpha_{1}=\alpha\left[\mathrm{UW}_{\mathrm{Q}_{1}} /\left(\mathrm{UW}_{\mathrm{Q}_{1}}+\mathrm{LW}_{\mathrm{Q}_{1}}\right)\right]$,

3. $\mathrm{HH}_{3} \quad \alpha_{1}=\alpha\left[\mathrm{UW}_{\mathrm{H}_{3}} /\left(\mathrm{UW}_{\mathrm{H}_{3}}+\mathrm{LW}_{\mathrm{H}_{3}}\right)\right]$,

4. $\mathrm{HQ}_{2} \quad \alpha_{1}=\alpha\left[\mathrm{UW}_{\mathrm{Q}_{2}} /\left(\mathrm{UW}_{\mathrm{Q}_{2}}+\mathrm{LW}_{\mathrm{Q}_{2}}\right)\right]$,

5. $\mathrm{HH}_{1} \quad \alpha_{1}=\alpha\left[\mathrm{UW}_{\mathrm{H}_{1}} /\left(\mathrm{UW}_{\mathrm{H}_{1}}+\mathrm{LW}_{\mathrm{H}_{1}}\right)\right]$,

6. $\mathrm{HSK}_{2} \quad \alpha_{1}=\alpha\left[\mathrm{UW}_{\mathrm{SK}_{2}} /\left(\mathrm{UW}_{\mathrm{SK}_{2}}+\mathrm{LW}_{\mathrm{SK}_{2}}\right)\right]$, and

7. $\mathrm{HSK}_{5} \quad \alpha_{1}=\alpha\left[\mathrm{UW}_{\mathrm{SK}_{5}} /\left(\mathrm{UW}_{\mathrm{SK}_{5}}+\mathrm{LW}_{\mathrm{SK}_{5}}\right)\right]$. 


\section{OTHMAN, YIN, KESELMAN, WILCOX \& ALGINA}

Keselman, et al. (2008), investigating Type I error rates of procedures for testing spread, examined the Reed and Stark (1996) procedure with various values for $\alpha$ because the literature varies on the amount of recommended (symmetric) trimming. Rosenberger and Gasko (1983) recommend 25\% when sample sizes are small (although they state that 20\% generally suffices), Wilcox (2005) recommends $20 \%$ and Mudholkar, Mudholkar and Srivastava (1991) suggest $15 \%$. Ten percent has been considered by Hill and Dixon (1982), Huber (1977), Stigler (1977) and Staudte and Sheather (1990); results reported by Keselman, et al. (2002) also support $10 \%$ trimming. In addition, Keselman, et al. (2005) obtained good results with 5\% symmetric trimming.

According to Keselman, et al. (2007), Reed and Stark's (1996) tail-length and skewness measures may be modified for the multi-group problem and applied to the modified multi-group measures to the hinge estimators. In particular, they indicated that each of the measures can be modified by taking weighted averages in a manner analogous to the modifications of tail-length and symmetry measures suggested by Babu, Padmanaban and Puri (1999) of each numerator and denominator term. For example, for the multi-group problem, where $n_{j}$ represents the number of observations in each group, $\mathrm{Q}_{1}$ and $\mathrm{Q}_{2}$ can be defined as:

$$
\begin{gathered}
\mathrm{Q}_{1}=\frac{\left[\sum_{\mathrm{j}} \mathrm{n}_{\mathrm{j}}\left(\mathrm{U}_{(.2)}-\mathrm{L}_{(.2)}\right) / \sum_{\mathrm{j}} \mathrm{n}_{\mathrm{j}}\right]}{\left[\sum_{\mathrm{j}} \mathrm{n}_{\mathrm{j}}\left(\mathrm{U}_{(.5)}-\mathrm{L}_{(.5)}\right) / \sum_{\mathrm{j}} \mathrm{n}_{\mathrm{j}}\right]}, \\
\mathrm{Q}_{2}=\frac{\left[\sum_{\mathrm{j}} \mathrm{n}_{\mathrm{j}}\left(\mathrm{U}_{(.05)}-\mathrm{T}_{(.25)}\right) / \sum_{\mathrm{j}} \mathrm{n}_{\mathrm{j}}\right]}{\left[\sum_{\mathrm{j}} \mathrm{n}_{\mathrm{j}}\left(\mathrm{T}_{(.25)}-\mathrm{L}_{(.05)}\right) / \sum_{\mathrm{j}} \mathrm{n}_{\mathrm{j}}\right]} .
\end{gathered}
$$

The other measures would be similarly modified and it is these multi-group measures of taillength and skewness that are applied to the general scheme proposed by Reed and Stark
(1996), treating the transformed $\mathrm{Z}_{\mathrm{ij}} \mathrm{s}$ as the original variables

One could go a step further than merely applying the transformed $Z_{\mathrm{ij}} \mathrm{s}$ in a Welch test. It is suggested that the transformed $Z_{\mathrm{ij}} \mathrm{s}$ be treated as the original random variable in a test statistic that has been found to be generally insensitive to nonnormality, namely a Welch test based on trimmed means, that is, Yuen's (1974) test).

Thus, consider the following. The $\alpha$ trimmed means and Winsorized variances can be defined in a number of different ways (see Hogg, 1974; Reed, 1998; Keselman, et al., 2007; Wilcox, 2003). Let $Z_{(1) \mathrm{j}} \leq Z_{(2) \mathrm{j}} \leq \ldots \leq \mathrm{Z}_{\left(\mathrm{n}_{\mathrm{j}}\right) \mathrm{j}}$ represent the ordered observations associated with the $j^{\text {th }}$ group. Reed's (1998) approach is based on the work of Hogg (1974). Hogg defined the $\alpha$-trimmed mean as:

$$
\mathrm{m}(\alpha)=(1 / \mathrm{h}) \sum_{\mathrm{i}=\mathrm{g}+1}^{\mathrm{n}_{\mathrm{j}} \mathrm{g}} \mathrm{Z}_{(\mathrm{i})},
$$

where $\alpha$ is selected so that $g=\left[n_{j} \alpha\right]$ and $\mathrm{h}=\mathrm{n}_{\mathrm{j}}-2 \mathrm{~g}=\mathrm{n}_{\mathrm{j}}-2\left[\mathrm{n}_{\mathrm{j}} \alpha\right]$. The standard error of $\mathrm{m}(\alpha)$ Hogg suggests is based on the works of Tukey and McLaughlin (1963) and Huber (1970) and is estimated by:

$$
\mathrm{S}_{\mathrm{m}(\alpha)}=\sqrt{\frac{\mathrm{SS}(\alpha)}{\mathrm{h}(\mathrm{h}-1)}},
$$

where $\operatorname{SS}(\alpha)$ is the Winsorized sum of squares defined as:

$$
\begin{aligned}
& (\mathrm{g}+1)\left[\mathrm{Z}_{(\mathrm{g}+1)}-\mathrm{m}(\alpha)\right]^{2} \\
& \quad+\left[\mathrm{Z}_{(\mathrm{g}+2)}-\mathrm{m}(\alpha)\right]^{2} \\
& \quad+\ldots \\
& \quad+\left[\mathrm{Z}_{\left(\mathrm{n}_{\mathrm{j}-\mathrm{g}-1)}-\mathrm{m}(\alpha)\right]^{2}}\right. \\
& \quad+(\mathrm{g}+1)\left[\mathrm{Z}_{\left(\mathrm{n}_{\mathrm{j}} \mathrm{g}\right)}-\mathrm{m}(\alpha)\right]^{2} .
\end{aligned}
$$


When allowing for different amounts of trimming in each tail of the distribution, Hogg (1974) defines the trimmed mean as:

$$
\mathrm{m}\left(\alpha_{1}, \alpha_{2}\right)=\left(\frac{1}{\mathrm{~h}}\right) \sum_{\mathrm{i}=\mathrm{g}_{1}+1}^{\mathrm{n}_{\mathrm{j}}-\mathrm{g}_{2}} \mathrm{z}_{(\mathrm{i})},
$$

where $\quad g_{1}=\left[n_{j} \alpha_{1}\right], \quad g_{2}=\left[n_{j} \alpha_{2}\right] \quad$ and $\mathrm{h}_{\mathrm{j}}=\mathrm{n}_{\mathrm{j}}-\mathrm{g}_{1}-\mathrm{g}_{2}$. Hogg suggests that the standard deviation of $\mathrm{m}\left(\alpha_{1}, \alpha_{2}\right)$ can be estimated as:

$$
\mathrm{S}_{\mathrm{m}\left(\alpha_{1}, \alpha_{2}\right)}=\sqrt{\frac{\mathrm{SS}\left(\alpha_{1}, \alpha_{2}\right)}{\mathrm{h}(\mathrm{h}-1)}}
$$

where $\operatorname{SS}\left(\alpha_{1}, \alpha_{2}\right)$ can be calculated as

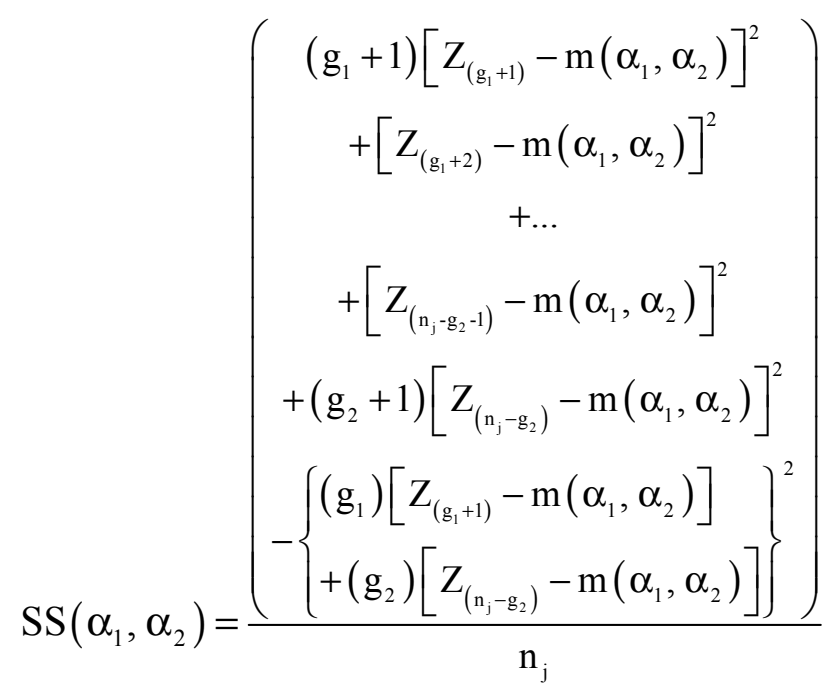

Test Statistics

$$
\text { Let } \quad \hat{\mu}_{\mathrm{j}}=\sum_{\mathrm{i}=1}^{\mathrm{n}_{\mathrm{j}}} \mathrm{Z}_{\mathrm{ij}} / \mathrm{n}_{\mathrm{j}} \quad \text { and }
$$
$\mathrm{s}_{\mathrm{j}}^{2}=\sum_{\mathrm{i}=1}^{\mathrm{n}}\left(\mathrm{Z}_{\mathrm{i}}-\overline{\mathrm{Z}}_{\mathrm{j}}\right)^{2} /\left(\mathrm{n}_{\mathrm{j}}-1\right)$ where $\hat{\mu}_{\mathrm{j}}$ is the estimate of $\mu_{\mathrm{j}}$ and $\mathrm{s}_{\mathrm{j}}^{2}$ is the unbiased estimate of the variance for population $j$. A heteroscedastic statistic (Welch, 1951) can be defined as:

$$
F_{W}=\frac{\sum_{j=1}^{J} w_{j}\left(\hat{\mu}_{j}-\hat{\mu}\right)^{2} /(J-1)}{1+\frac{2(J-2)}{\left(J^{2}-1\right)} \sum_{j=1}^{J} \frac{\left(1-w_{j} / W\right)^{2}}{n_{j}-1}},
$$

where

$$
\begin{gathered}
\hat{\mu}=\sum_{j=1}^{J} w_{j} \hat{\mu}_{j} / W, \\
W=\sum_{j=1}^{J} w_{j} a, .
\end{gathered}
$$

and

$$
\mathrm{w}_{\mathrm{j}}=\mathrm{n}_{\mathrm{j}} / \mathrm{s}_{\mathrm{j}}^{2} \text {. }
$$

The test statistic is approximately distributed as an $\mathrm{F}$ variate and is referred to the critical value $\mathrm{F}\left[(1-\alpha) ;(\mathrm{J}-1), \mathrm{v}_{\mathrm{W}}\right]$, the $(1-\alpha)$ quantile of the $F$ distribution, where error degrees of freedom are obtained from

$$
v_{w}=\frac{J^{2}-1}{3 \sum_{j=1}^{J} \frac{\left(1-w_{j} / W\right)^{2}}{n_{j}-1}} .
$$

\section{A Robust ANOVA F-Test}

Lee and Fung (1985) defined an ANOVA F-test based on trimmed means. Because the ANOVA F-test can be more powerful than the Welch F-test, this statistic was chosen for this investigation. The Lee and Fung (1985) statistic is defined as:

$$
F_{t}=\frac{\sum_{j=1}^{J} h_{j}\left(\hat{m}_{t j}-\hat{M}_{t}\right)^{2} /(J-1)}{\sum_{j=1}^{J} S_{j}\left(a_{1}, a_{2}\right) /(H-J)}
$$

where

$$
\begin{gathered}
H=\sum_{j=1}^{J} h_{j}, \\
\hat{M}_{t}=\sum_{j=1}^{J} h_{j} \hat{m}_{t j} / H,
\end{gathered}
$$

and

$$
\operatorname{SS}_{\mathrm{j}}\left(\alpha_{1}, \alpha_{2}\right)=\text { the }\left(\alpha_{1}, \alpha_{2}\right) \text {. }
$$




\section{OTHMAN, YIN, KESELMAN, WILCOX \& ALGINA}

The Winsorized sum of squared deviations for the $\mathrm{j}^{\text {th }}$ group; $\mathrm{h}_{\mathrm{j}}$ and $\hat{\mathrm{m}}_{\mathrm{t} j}$ are defined the same as previously. Note that, when $\alpha_{1}=\alpha_{2}=0, F_{t}=F$ (O’Brien, 1979).

O'Brien (1979) indicated that the $\mathrm{r}_{\mathrm{ij}} \mathrm{s}$ can be used in the Welch test. Accordingly, the trimmed mean version is given by:

$$
\mathrm{r}_{\mathrm{t}_{\mathrm{ij}}}=\frac{\left(\mathrm{h}_{\mathrm{j}}-1.5\right) \mathrm{n}_{\mathrm{j}}\left(\mathrm{X}_{\mathrm{ij}}-\hat{\mathrm{m}}_{\mathrm{tj}}\right)^{2}-.5 \mathrm{~s}_{\mathrm{mj}}^{2}\left(\mathrm{~h}_{\mathrm{j}}-1\right)}{\left(\mathrm{h}_{\mathrm{j}}-1\right)\left(\mathrm{h}_{\mathrm{j}}-2\right)} .
$$

where $\mathrm{h}_{\mathrm{j}}=\mathrm{n}_{\mathrm{j}}-\mathrm{g}_{1}-\mathrm{g}_{2}, \quad \hat{\mathrm{m}}_{\mathrm{tj}}=$ the $\left(\alpha_{1}, \alpha_{2}\right)$ trimmed mean of the $\mathrm{j}^{\text {th }}$ group and $\mathrm{s}_{\mathrm{mj}}^{2}=\mathrm{s}_{\mathrm{m}\left(\alpha_{1}, \alpha_{2}\right)}^{2}$ for group $\mathrm{j}$. The $\mathrm{r}_{\mathrm{ij}} \mathrm{s}$ and $\mathrm{r}_{\mathrm{t}_{\mathrm{ij}}} \mathrm{s}$ were also used with the Lee and Fung (1985) test.

\section{Methodology}

A total of 170 new Levene (1960) type procedures were created. These procedures were (see Table 2 for a summary of the Levene methods examined):

(A1) Let $Z_{i j}=\left|X_{i j}-\bar{X}_{. j}\right|$. The $Z_{i j} s$ were then trimmed symmetrically $\alpha \%$ and the robust F-test, $\mathrm{F}_{\mathrm{t} \alpha}$ was computed. There are 4 variants with this designation because there are four symmetric trimming percentages: $5 \%, 10 \%, 15 \%$ and $20 \%$. For example, $A_{1} 15$ signifies $Z_{i j}=\left|X_{i j}-\bar{X}_{. j}\right|$, the $Z_{i j} s$ were trimmed symmetrically $15 \%$ and $F_{t \alpha}$ was computed.

(A2) Let $Z_{i j}=\left|X_{i j}-\bar{X}_{. j}\right|$, the $Z_{i j} s$ were trimmed asymmetrically $\beta \%$ with a hinge estimator $\mathrm{H}$ and the robust F-test, $\mathrm{F}_{\mathrm{t} \beta} \quad$ computed. $\mathrm{A}_{2} 10 \mathrm{HSK}_{5}$ signifies transformation $\mathrm{Z}_{\mathrm{ij}}=\left|\mathrm{X}_{\mathrm{ij}}-\overline{\mathrm{X}}_{\mathrm{j}}\right|$ and these values were subjected to $10 \%$ asymmetric trimming with hinge estimator $\mathrm{HSK}_{5}$ before computing $\mathrm{F}_{\mathrm{t} \beta}$.
Because there are four asymmetrical trimming percentages and seven hinge estimators, there are 28 variants with this designation.

Let $Z_{\mathrm{ij}}=\left|\mathrm{X}_{\mathrm{ij}}-\overline{\mathrm{X}}_{. \mathrm{j}}\right|$. The $\mathrm{Z}_{\mathrm{ij}} \mathrm{s}$ were used with the Welch test, $F_{W}$.

Let $Z_{\mathrm{ij}}=\left|\mathrm{X}_{\mathrm{ij}}-\mathrm{M}_{\mathrm{j}}\right|$. The $\mathrm{Z}_{\mathrm{ij}} \mathrm{s}$ were used with the Welch test, $\mathrm{F}_{\mathrm{W}}$.

These variants are designated $\mathrm{E}_{1} \alpha \mathrm{H}$, where $Z_{\mathrm{ij}}=\left|\mathrm{X}_{\mathrm{ij}}-\overline{\mathrm{X}}_{\mathrm{t}_{\alpha}}\right|, \quad \overline{\mathrm{X}}_{\mathrm{t}_{\alpha}}$ are group $\alpha \%$ symmetric trimmed means. The $\mathrm{Z}_{\mathrm{ij}} \mathrm{s}$ were used with the usual $F$-test, W, hence variant $\mathrm{E}_{1} 20$ signifies transformed values $\mathrm{Z}_{\mathrm{ij}}=\left|\mathrm{X}_{\mathrm{ij}}-\overline{\mathrm{X}}_{\mathrm{t}_{20}}\right|$, where $\overline{\mathrm{X}}_{\mathrm{t}_{20}}$ are group $20 \%$ symmetrically trimmed at tail, computed with W. Because there are four symmetric trimming percentages $(5 \%, 10 \%, 15 \%$ and $20 \%)$, there are four variants with this designation.

(E2) In variants $E_{2} \alpha_{1} \alpha_{2}$ let $Z_{\mathrm{ij}}=\left|\mathrm{X}_{\mathrm{ij}}-\overline{\mathrm{X}}_{\mathrm{t}_{\mathrm{\alpha} 1}}\right|$. The transformed values were trimmed symmetrically $\alpha_{2} \%$ and used with $\mathrm{F}_{\mathrm{t}_{2}}$. Therefore, variant $E_{2} 1520$ signifies transformation $\mathrm{Z}_{\mathrm{ij}}=\left|\mathrm{X}_{\mathrm{ij}}-\overline{\mathrm{X}}_{\mathrm{t}_{15}}\right|$, where $\bar{X}_{t_{15}}$ are group $15 \%$ symmetric trimmed means and these values were subjected to $20 \%$ symmetric trimming before being used with $F_{t a_{2}}$. Because there are four different $\overline{\mathrm{X}}_{\mathrm{t}_{\mathrm{a} 1}}$ 's and four symmetric trimming percentages, there are 16 variants with this designation.

(E3) In variants $\mathrm{E}_{3} \alpha \beta \mathrm{H}$, let $\mathrm{Z}_{\mathrm{ij}}=\left|\mathrm{X}_{\mathrm{ij}}-\overline{\mathrm{X}}_{\mathrm{t}_{\alpha}}\right|$. The transformed values were asymmetrically trimmed at $\beta \%$ involving seven hinge estimators (HQ, $\mathrm{HQ}_{1}, \mathrm{HH}_{3}, \mathrm{HQ}_{2}, \mathrm{HH}_{1}, \mathrm{HSK}_{2}, \mathrm{HSK}_{5}$ ) and used with $\mathrm{F}_{\mathrm{t} \beta}$. Hence, $\mathrm{E}_{3} 1025 \mathrm{HH}_{3}$ 


\section{ROBUST MODIFICATIONS OF THE LEVENE AND O'BRIEN TESTS FOR SPREAD}

signifies transformation $\mathrm{Z}_{\mathrm{ij}}=\left|\mathrm{X}_{\mathrm{ij}}-\overline{\mathrm{X}}_{\mathrm{t}_{10}}\right|$, where $\bar{X}_{t_{10}}$ are group $10 \%$ symmetric trimmed means; these values were subjected to $25 \%$ asymmetric trimming calculated using the $\mathrm{HH}_{3}$ hinge estimator before applying $\mathrm{F}_{\mathrm{t} \beta}$. Because there are four different $\overline{\mathrm{X}}_{\mathrm{t}_{\alpha}}$ 's and four asymmetric trimming percentages with seven different hinge estimators, there are 112 variants for this designation.

(E4) Variants $E_{4} \alpha H$ use $\bar{X}_{t_{\alpha}}$ in place of $\bar{X}_{. j}$ $\left(A_{3}\right)$ or $M_{j}\left(B_{3}\right)$, in getting the $Z_{i j}$ values. The $\mathrm{Z}_{\mathrm{ij}} \mathrm{s}$ were then used with the Welch test, $\mathrm{F}_{\mathrm{W}}$. Hence $\mathrm{E}_{4} 10$ signifies transformed values $\mathrm{Z}_{\mathrm{ij}}=\left|\mathrm{X}_{\mathrm{ij}}-\overline{\mathrm{X}}_{\mathrm{t}_{10}}\right|$, where $\bar{X}_{t_{10}}$ are group $10 \%$ symmetric trimmed means and used with $\mathrm{F}_{\mathrm{W}}$. Since there are four symmetric trimming percentages, there are four variants with this designation.

A total of 165 O'Brien-type procedures were created (see Table 2 for a summary of the O'Brien methods examined):

(J) O'Brien transformation based upon group means and variances used with the usual F-test, W (that is, $\mathrm{r}_{\mathrm{ij}}$ in W).

(Q1) Variants are designated $\mathrm{Q}_{1} \alpha$. The O'Brien transformation based upon symmetric trimmed means and Winsorized variances of $X_{\mathrm{ij}}$. These trimmed means were calculated at $\alpha=$ $5 \%, \quad 10 \%, \quad 15 \%$ and $20 \%$. The transformed values, $r_{i j}$, were used with the usual F-test (that is, $r_{i j}$ in $W$ ). Because there are four symmetrical trimming percentages, there are four variants with this designation. Therefore, variants $\mathrm{Q}_{1} 10$ signifies transformation of $\mathrm{X}_{\mathrm{ij}}$ with $10 \%$ symmetric trimmed mean before used with W.

(Q2) Variants are designated $\mathrm{Q}_{2} \alpha_{1} \alpha_{2}$. The O'Brien transformation based upon group symmetric trimmed means and Winsorized variances of $X_{i j}$. These trimmed means and Winsorized variances were calculated at symmetric trimming percentages $(5 \%, 10 \%, 15 \%$ and $20 \%$ ). The resultant transformed values, $r_{\mathrm{ij}}$, were symmetrically trimmed based on the same percentages used for $\mathrm{X}_{\mathrm{ij}}$ and used with the robust ANOVA $F_{t}$ test (that is, symmetrically trimmed $r_{i j}$ in $F_{t}$ ). Because there are four symmetric trimming percentages used twice, there are 16 variants with this designation. Hence, variant $\mathrm{Q}_{2} 515$ signifies transformation of $\mathrm{X}_{\mathrm{ij}}$ with $5 \%$ symmetric trimmed mean and $15 \%$ symmetric trimmed mean for the transform value, $r_{i j}$, before used with $\mathrm{F}_{\mathrm{t}}$.

(Q3) Variants are designated $\mathrm{Q}_{3} \alpha \beta \mathrm{H}$. The O'Brien transformation based upon symmetric trimmed means and Winsorized variances of $X_{\mathrm{ij}}$. These trimmed means were calculated with the four symmetric trimming percentages $(5 \%, 10 \%, 15 \%$ and $20 \%)$. The resultant transformed values, $r_{i j}$, were then asymmetrically trimmed at $\beta=10 \%$, $15 \%, 20 \%$ and $25 \%$ involving seven hinge estimators and used with the robust ANOVA $\mathrm{F}_{\mathrm{t}}$-test (that is, asymmetrically trimmed $r_{i j}$ in $F_{t}$ ). Because there are four symmetric trimming percentages, on $\mathrm{X}_{\mathrm{ij}}$, four asymmetric trimming percentages on $r_{i j}$ with seven hinge estimators, there are 112 variants with this designation. 


\section{OTHMAN, YIN, KESELMAN, WILCOX \& ALGINA}

(Q4) Because variants are designated $\mathrm{Q}_{4} \alpha$. The O'Brien transformation based upon symmetric trimmed means Winsorized variances of $\mathrm{X}_{\mathrm{ij}}$. These trimmed means were calculated at the four symmetric trimming percentages $(5 \%, 10 \%, 15 \%$ and $20 \%$ ). The transformed values, $r_{i j}$, were then used with the Welch test, $\mathrm{F}_{\mathrm{W}}$ (that is, $r_{i j}$ in $F_{W}$ ). Because there are four symmetrical trimming percentages, there are four variants with this designation. Hence, variants $\mathrm{Q}_{4} 05$ signifies transformation of $\mathrm{X}_{\mathrm{ij}}$ with $5 \%$ symmetric trimmed mean before used with $\mathrm{F}_{\mathrm{W}}$.

Variants are designated $\mathrm{K} \beta \mathrm{H}$. The O'Brien transformation based upon asymmetric trimmed means Winsorized variances of $X_{\mathrm{ij}}$. These trimmed means were calculated at $\beta=$ $10 \%, 15 \%, 20 \%$ and $25 \%$ involving seven hinge estimators (HQ, $\mathrm{HQ}_{1}, \mathrm{HH}_{3}, \mathrm{HQ}_{2}, \mathrm{HH}_{1}, \mathrm{HSK}_{2}$, $\mathrm{HSK}_{5}$ ). The transformed values, $\mathrm{r}_{\mathrm{ij}}$, were used with the usual F-test (that is, $r_{i j}$ in W). Because there are four asymmetrical trimming percentages and seven hinge estimators, there are 28 variants with this designation. Hence, variants $\mathrm{K}_{15 \mathrm{HSK}_{2}}$ signifies transformation of $\mathrm{X}_{\mathrm{ij}}$ with $15 \%$ asymmetric trimmed mean calculated using the $\mathrm{HSK}_{2}$ hinge estimator before used with W.

\section{Study Conditions}

Four variables were employed in the $\mathrm{J}=$ 3 study: (a) total sample size; (b) degree of sample size inequality; (c) shape of the population distribution; and (d) type and amount of total trimming.

\section{Total Sample Size}

The effect of sample size on the performance of the various procedures was evaluated by varying the total sample size $(\mathrm{N})$. The total sample size was manipulated, setting the average group size to $n_{j}=20$ and 40 . The average group-sizes correspond to total sample sizes of $\mathrm{N}=60$ and $\mathrm{N}=120$.

Degree of Sample Size Inequality

Three conditions of sample size equality/inequality were investigated which are referred to as: equal $n_{j}$, moderately unequal $n_{j}$ and extremely unequal $n_{j}$ (see below for values). These conditions were evaluated because Keselman, et al. (1998) found that unbalanced designs were more common than balanced designs.

\begin{tabular}{|c|}
\hline $\mathrm{n}_{\mathrm{j}} \mathrm{s}$ \\
\hline $20,20,20$ \\
\hline $15,20,25$ \\
\hline $10,20,30$ \\
\hline $40,40,40$ \\
\hline $35,40,45$ \\
\hline $30,40,50$ \\
\hline
\end{tabular}

Shape of the Population Distribution

This study investigated distributions ranging from symmetric to skewed and platykurtic to normal-tailed to leptokurtic distributions. In total, seven distributions were employed to compare the procedures. The distributions used were: (i) the Fleishman (1978) transformation of the standard normal distribution into a skewed platykurtic distribution with skewness, $\gamma_{1}=0.5$ and kurtosis, $\gamma_{2}=-0.5$; (ii) a second Fleishman transformation of the standard normal distribution into a skewed normal-tailed distribution with $\gamma_{1}=0.75$ and $\gamma_{2}=0$; (iii) the Beta $(0.5,0.5)$ distribution representing symmetric platykurtic distributions; (iv) a $\mathrm{g}$ and $\mathrm{h}$ distribution (Hoaglin, 1985) where $\mathrm{g}=\mathrm{h}=0$, which is the standard normal distribution with $\gamma_{1}=\gamma_{2}=0$; (v) a $\mathrm{g}=0$ and $\mathrm{h}=0.225$ long-tailed distribution with $\gamma_{1}=0$ and $\gamma_{2}=154.84$, representing symmetric leptokurtic distributions; (vi) a $\mathrm{g}=0.76$ and $\mathrm{h}=-0.098$ distribution with skew and kurtosis equal to that of an exponential 
Table 1: Description of the Levene (1960) Transformations Used In the Simulations

\begin{tabular}{|c|c|c|}
\hline Designation & Description & Cases \\
\hline A1 & $\begin{array}{l}X_{i j} \rightarrow Z_{i j} \text { : use group means from } X_{i j} \text {. } \\
Z_{i j} \text { : symmetrically trimmed at tail proportions: } 0.05,0.10,0.15, \\
0.20, \text { apply robust ANOVA } F \text {-test. }\end{array}$ & 4 \\
\hline $\mathrm{A} 2$ & 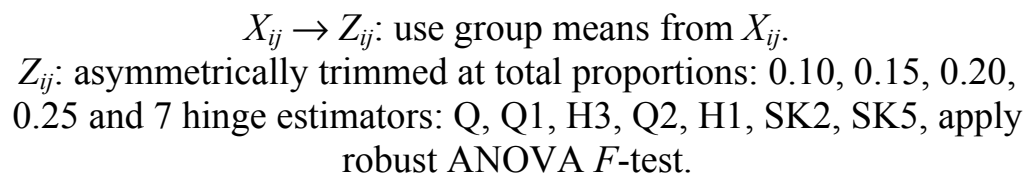 & 28 \\
\hline A3 & $\begin{array}{c}X_{i j} \rightarrow Z_{i j}: \text { use group means from } X_{i j} . \\
Z_{i j} \text { : apply Welch F-test. }\end{array}$ & 1 \\
\hline B3 & $\begin{array}{c}X_{i j} \rightarrow Z_{i j}: \text { use group medians from } X_{i j} . \\
Z_{i j:} \text { apply Welch F-test. }\end{array}$ & 1 \\
\hline E1 & $\begin{array}{c}X_{i j} \rightarrow Z_{i j}: \text { use group symmetric trimmed means from } X_{i j} . X_{i j} \\
\text { symmetrically trimmed at tail proportions: } 0.05,0.10,0.15,0.20 \text {, } \\
Z_{i j} \text { : apply usual ANOVA } F \text {-test. }\end{array}$ & 4 \\
\hline E2 & $\begin{array}{c}X_{i j} \rightarrow Z_{i j} \text { : use group symmetric trimmed means from } X_{i j} . X_{i j} \\
\text { symmetrically trimmed at tail proportions: } 0.05,0.10,0.15,0.20 \text {, } \\
Z_{i j}: \text { symmetrically trimmed at tail proportions: } 0.05,0.10,0.15 \text {, } \\
0.20 \text {, apply robust ANOVA } F \text {-test. }\end{array}$ & 16 \\
\hline E3 & $\begin{array}{c}X_{i j} \rightarrow Z_{i j} \text { : use group symmetric trimmed means from } X_{i j} . X_{i j} \\
\text { symmetrically trimmed at tail proportions: } 0.05,0.10,0.15,0.20 \text {, } \\
Z_{i j} \text { : asymmetrically trimmed at total proportions: } 0.10,0.15,0.20 \text {, } \\
0.25 \text {, keeping hinge estimator constant, apply robust ANOVA F- } \\
\text { test. }\end{array}$ & 112 \\
\hline E4 & $\begin{array}{c}X_{i j} \rightarrow Z_{i j} \text { : use group symmetric trimmed means from } X_{i j} . X_{i j} \\
\text { symmetrically trimmed at tail proportions: } 0.05,0.10,0.15,0.20, \\
Z_{i j} \text { : apply Welch F-test. }\end{array}$ & 4 \\
\hline & Total & 170 \\
\hline
\end{tabular}


OTHMAN, YIN, KESELMAN, WILCOX \& ALGINA

Table 2: Description of the O'Brien (1979) Designations Used In the Simulations

\begin{tabular}{|c|c|c|}
\hline Designation & Description & Cases \\
\hline $\mathrm{J}$ & $\begin{array}{l}X_{i j} \rightarrow R_{i j}: \text { use group means and variances from } X_{i j} . \\
\qquad R_{i j} \text { : apply usual ANOVA } F \text {-test. }\end{array}$ & 1 \\
\hline Q1 & $\begin{array}{l}X_{i j} \rightarrow R_{i j} \text { : use group symmetric trimmed means and Winsorized } \\
\text { variances from } X_{i j} . X_{i j} \text { symmetrically trimmed at tail proportions: } \\
\quad 0.05,0.10,0.15,0.20, R_{i j}: \text { apply usual } F \text {-test. }\end{array}$ & 4 \\
\hline Q2 & $\begin{array}{l}X_{i j} \rightarrow R_{i j}: \text { use group symmetric trimmed means and Winsorized } \\
\text { variances from } X_{i j} . X_{i j} \text { symmetrically trimmed at tail proportions: } \\
\quad 0.05,0.10,0.15,0.20, R_{i j}: \text { symmetrically trimmed at tail } \\
\text { proportions: } 0.05,0.10,0.15,0.20 \text {, apply robust ANOVA } F \text {-test. }\end{array}$ & 16 \\
\hline Q3 & $\begin{array}{c}X_{i j} \rightarrow R_{i j} \text { : use group symmetric trimmed means and Winsorized } \\
\text { variances from } X_{i j} . X_{i j} \text { symmetrically trimmed at tail proportions: } \\
0.05,0.10,0.15,0.20, R_{i j}: \text { asymmetrically trimmed at total } \\
\text { proportions: } 0.10,0.15,0.20,0.25 \text { and } 7 \text { hinge estimators: Q, Q1, } \\
\text { H3, Q2, H1, SK2, SK5. Apply robust ANOVA } F \text {-test. }\end{array}$ & 112 \\
\hline Q4 & $\begin{array}{c}X_{i j} \rightarrow R_{i j} \text { : use group symmetric trimmed means and Winsorized } \\
\text { variances from } X_{i j} . X_{i j} \text { symmetrically trimmed at tail proportions: } \\
\quad 0.05,0.10,0.15,0.20, R_{i j} \text { : apply Welch } F \text {-test. }\end{array}$ & 4 \\
\hline \multirow[t]{2}{*}{$\mathrm{K}$} & $\begin{array}{c}X_{i j} \rightarrow R_{i j}: \text { use group asymmetric trimmed means and Winsorized } \\
\text { variances from } X_{i j} . X_{i j} \text { asymmetrically trimmed at total proportions: } \\
\begin{aligned} 0.10,0.15,0.20,0.25 \text { and } 7 \text { hinge estimators: Q, Q1, H3, Q2, H1, } \\
\text { SK2, SK5. } \\
R_{i j} \text { : apply usual } F \text {-test. }\end{aligned}\end{array}$ & 28 \\
\hline & Total: & 165 \\
\hline
\end{tabular}




\section{ROBUST MODIFICATIONS OF THE LEVENE AND O'BRIEN TESTS FOR SPREAD}

distribution $\left(\gamma_{1}=2, \gamma_{2}=6\right)$; and (vii) a $\mathrm{g}=$ 0.225 and $\mathrm{h}=0.225$ distribution, which is also a long-tailed skewed distribution $\left(\gamma_{1}=4.90, \gamma_{2}=4673.80\right)$. The last two distributions represent skewed leptokurtic distributions, with (vii) more severe than (vi). These distribution conditions were selected in order to evaluate the operating characteristics of the procedures across a variety of distributions and because they have been examined in other studies (e.g., Algina, Keselman \& Penfield, 2007).

The Fleishman (1978) power transformation is of the form $\mathrm{Y}=\mathrm{a}+\mathrm{bZ}+\mathrm{cZ}^{2}+$ $\mathrm{dZ}^{3}$, where $Z$ are standard normal variates. Fleishman provided a table of values for the coefficients, b, c, and d that enables the standard normal distribution to be transformed into a nonnormal distribution, also having mean zero and variance one, but with different degrees of skewness and kurtosis. The extra coefficient a is obtained through the relation $\mathrm{a}=-\mathrm{c}$ as a direct result of constraining $\mathrm{E}(\mathrm{Y})=0$. Two sets of coefficients (b, c, d) were selected from Fleishman (1978) and used in the preceding equation to generate $Z_{\mathrm{ij}}$ s from the RANDGEN function (SAS, 2006) with the normal distribution option to produce distributions (i) and (ii). This RANDGEN SAS subroutine allows a user to generate 20 known distributions, both discrete and continuous. Data from the third distribution was also generated using the RANDGEN function but with the beta distribution option. Beta $(0.5,0.5)$ is a symmetric u-shaped distribution, hence the negative kurtosis.

To generate data from $\mathrm{a} g$ and $\mathrm{h}$ distribution, standard unit normal variables $\left(Z_{\mathrm{ij}}\right)$ were converted to $\mathrm{g}$ and $\mathrm{h}$ distributed random variables via

$$
Y_{i j}=\frac{\exp \left(g Z_{i j}\right)-1}{g} \exp \left(\frac{h Z_{i j}^{2}}{2}\right),
$$

where both $\mathrm{g}$ and $\mathrm{h}$ are non-zero. When $\mathrm{g}$ is zero

$$
\mathrm{Y}_{\mathrm{ij}}=\mathrm{Z}_{\mathrm{ij}} \exp \left(\frac{\mathrm{h} \mathrm{Z}_{\mathrm{ij}}^{2}}{2}\right)
$$

The $Z_{\mathrm{ij}}$ scores were generated using the generator RANDGEN with the normal distribution option.

Observations generated for distributions (iii), (v), (vi) and (vii), where the variances were not equal to one, were standardized so that they were one, to reflect the null hypothesis, $\mathrm{H}_{0}$ : $\sigma_{1}^{2}=\sigma_{2}^{2}=\sigma_{3}^{2}$; that is, in the simulations, $\sigma_{1}^{2}=\sigma_{2}^{2}=\sigma_{3}^{2}=1$.

Percentages of Total Trimming

Four values of total trimming, namely $10 \%, 15 \%, 20 \%$ and $25 \%$ were examined when data were asymmetrically trimmed, whether to obtain the values used in the transformation of the $X_{\mathrm{ij}}$ data or when trimming was carried out on the Levene transformed values $Z_{\mathrm{ij}}$ or O'Brien's transformed values, $r_{i j}$ and $r_{t_{i j}}$. Symmetric trimming values of $5 \%, 10 \%, 15 \%$ and $20 \%$ were also investigated. As noted, the literature varies on the amount of recommended (symmetric) trimming and thus these values were chosen to cover the range of values recommended. For each condition 5,000 replications were conducted and the nominal levels of significance for all tests were 0.05 and 0.10 .

Results

Tables 4 and 5 summarize the ten best results for the modified Levene (1960) tests for spread. Table 4 shows the average rates of Type I error, the absolute values of the difference between the average rates and 0.05 , and the percent of cases falling in three intervals $-(0.025,0.050),(0.045$, $0.055)$, and $(0.045,0.050)$. The last column indicates total percentage of cases falling in $(0.025,0.055)$; using simple set theory algebra, this is just percent of cases in $(0.025,0.050)$ and in $(0.045,0.055)$ minus percent of cases in $(0.045,0.055)$. Based on these findings the following are noted:

1. All ten methods examined provided very good Type I error control. Indeed, the 


\section{OTHMAN, YIN, KESELMAN, WILCOX \& ALGINA}

empirical rates ranged from 0.046 to 0.0579 ; and

2. In order to identify the best method(s) the percentages reported in the last column were relied upon. From this information, $\mathrm{B}_{3}$ was identified as the best of the Levene (1960) modifications defined and examined.

Table 5 presents Type I error rates for each characteristic of the distributions investigated, as well as the overall rate, indicates that the method that selected as best, contains average Type I errors of 0.048 .

The same information is presented in Tables 6 and 7 for the ten best modified O'Brien (1981) tests for spread. Based on these findings, the ten best O'Brien variants provided tight Type I error control ranging from 0.490 to 0.0508 . The last column of Table 6 identifies two of the modified procedures, $\mathrm{Q}_{3} 1025 \mathrm{HQ}_{1}$ and $\mathrm{K}_{2} \mathrm{HHH}_{3}$, as the best of the O'Brien (1981) modifications. Table 7 presents Type I error rates for each characteristic of the distributions investigated, as well as the overall rate, and indicates that the both methods that selected as best contain Type I errors averaging 0.050.

\section{Conclusion}

This study examined the Type I error rate (for $\alpha$ $=0.05)$ of various modifications of Levene's (1960) and O'Brien's (1981) procedures that could be used to compare variability across groups in independent groups designs, specifically variations not examined by Keselman, et al. (2008). The procedures examined used Levene (1960) or O'Brien (1981) type transformations of the original scores or transformed scores, except as opposed to using the measures of central tendency and variability suggested by Levene and O'Brien, robust measures of central tendency and/or variability were adopted.

The robust values of central tendency and variability (i.e., the trimmed means and Winsorized variances) were based on symmetric or asymmetric trimming rules, that is, rules that either set a priori the amount of total trimming or determined empirically the amount to be trimmed from the tails (if at all) based on varied recommendations for total trimming. These approaches were also applied to various test statistics: the ANOVA F-test, a robust F-test (Lee \& Fung, 1985), the Welch (1951) test, and bootstrapped versions of these statistics. The procedures were compared under seven distributions when group sizes were equal, moderately, or very unequal. The skewness and kurtosis of the distributions examined varied from the normal distribution $\left(\gamma_{1}=0, \gamma_{2}=0\right.$ respectively) to distributions that were nonnormal, $\gamma_{1}=4.9$ and $\gamma_{2}=4673.80$, respectively).

The procedures were compared on four measures: the average rate of Type I error across the 42 conditions examined, the percentage of empirical Type I errors that fell within the intervals $(0.025,0.05),(0.045,0.055)$ and $(0.045,0.05)$, and the absolute value of the difference between the mean Type I error rate and 0.05 . Finally, it should be noted that though it was intended to examine bootstrapped versions of these procedures, this was not pursued because very good Type I error control was achieved without resorting to bootstrapping. Results indicated that the results reported by Keselman, et al. (2008) could not be improved upon with respect to the Levene (1960) test. That is, though the new Levene modifications all worked very well in controlling Type I error rates, they did not result in as many cases falling into the three intervals defined for good Type I error control as reported by Keselman, et al. (2008).

Conversely, two of the O'Brien (1960) modifications did perform well, at least as well as the variants examined by Keselman, et al. (2008) and their recommended Levene variant. These were $\mathrm{Q}_{3} 1025 \mathrm{HQ}_{1}$ and $\mathrm{K}_{2} \mathrm{OHH}$ with tighter Type I error control and a decent number of cases falling into the three intervals defined for good Type I error control by Keselman, et al. (2008).

\section{Acknowledgements}

This research was supported by grants provided by the Social Sciences and Humanities Research Council of Canada and the Fundamental Research Grant Scheme of Malaysia. 


\section{ROBUST MODIFICATIONS OF THE LEVENE AND O’BRIEN TESTS FOR SPREAD}

Table 4: Type I Error Rates for the 10 Best Performing New Levene's Variants and Percentages of Type I

Error Rates within Various Intervals

\begin{tabular}{|c|c|c|c|c|c|c|c|}
\hline No. & Variant & $\begin{array}{c}\text { Average } \\
\text { p-Values }\end{array}$ & $\mid$ Mean-0.05| & $\begin{array}{c}\text { Percent } \\
\text { Within } \\
2.5,5.0\end{array}$ & $\begin{array}{c}\text { Percent } \\
\text { Within } \\
4.5,5.5\end{array}$ & $\begin{array}{c}\text { Percent } \\
\text { Within } \\
4.5,5.0\end{array}$ & Total \% \\
\hline 1 & $\mathrm{~B}_{3}$ & .0476 & .0024 & 54.76 & 30.95 & 14.29 & 71 \\
\hline 2 & $\mathrm{E}_{1} 20$ & .0531 & .0031 & 47.62 & 40.48 & 23.81 & 64 \\
\hline 3 & $\mathrm{E}_{3} 0525 \mathrm{HH}_{1}$ & .0555 & .0055 & 33.33 & 28.57 & 14.29 & 48 \\
\hline 4 & $\mathrm{E}_{3} 1025 \mathrm{HH}_{1}$ & .0564 & .0064 & 26.19 & 21.43 & 4.76 & 43 \\
\hline 5 & $\mathrm{E}_{3} 0525 \mathrm{HH}_{3}$ & .0566 & .0066 & 38.10 & 23.81 & 16.67 & 45 \\
\hline 6 & $\mathrm{E}_{3} 0525 \mathrm{HHQ}_{2}$ & .0566 & .0066 & 28.57 & 26.19 & 9.52 & 45 \\
\hline 7 & $\mathrm{E}_{3} 0525 \mathrm{HHQ}$ & .0568 & .0068 & 33.33 & 23.81 & 9.52 & 48 \\
\hline 8 & $\mathrm{E}_{3} 1025 \mathrm{HHQ}$ & .0577 & .0077 & 28.57 & 19.05 & 7.14 & 41 \\
\hline 9 & $\mathrm{E}_{1} 15$ & .0579 & .0079 & 35.71 & 47.62 & 28.57 & 55 \\
\hline 10 & $\mathrm{E}_{3} 1025 \mathrm{HHQ}$ & .0579 & .0079 & 26.19 & 40.48 & 21.43 & 45 \\
\hline
\end{tabular}

Table 5: Type I Error Rates for the 10 Best Performing Variants of Levene's Procedure

\begin{tabular}{|c|c|c|c|c|c|c|c|}
\hline No. & Variant & Overall & Skewed & Symmetric & Leptokurtic & $\begin{array}{c}\text { Normal } \\
\text { Tailed }\end{array}$ & Platykurtic \\
\hline 1 & $\mathrm{~B}_{3}$ & .048 & .053 & .042 & .055 & .048 & .038 \\
\hline 2 & $\mathrm{E}_{1} 20$ & .053 & .057 & .048 & .052 & .055 & .052 \\
\hline 3 & $\mathrm{E}_{3} 0525 \mathrm{HH}_{1}$ & .056 & .060 & .050 & .052 & .058 & .058 \\
\hline 4 & $\mathrm{E}_{3} 1025 \mathrm{HH}_{1}$ & .056 & .059 & .053 & .052 & .058 & .062 \\
\hline 5 & $\mathrm{E}_{3} 0525 \mathrm{HH}_{3}$ & .057 & .061 & .051 & .055 & .058 & .058 \\
\hline 6 & $\mathrm{E}_{3} 0525 \mathrm{HHQ}_{2}$ & .057 & .061 & .051 & .053 & .059 & .059 \\
\hline 7 & $\mathrm{E}_{3} 0525 \mathrm{HHQ}_{2}$ & .057 & .062 & .050 & .054 & .060 & .058 \\
\hline 8 & $\mathrm{E}_{3} 1025 \mathrm{HHQ}$ & \\
\hline 9 & $\mathrm{E}_{1} 15$ & .058 & .061 & .053 & .053 & .059 & .063 \\
\hline 10 & $\mathrm{E}_{3} 1025 \mathrm{HHQ}$ & .058 & .064 & .050 & .058 & .059 & .057 \\
\hline
\end{tabular}

Table 6: Type I Error Rates for the 10 Best Performing O'Brien (1979) Variants and Percentages of Type I Error Rates within Various Intervals

\begin{tabular}{|c|c|c|c|c|c|c|c|}
\hline No. & Variant & $\begin{array}{c}\text { Average } \\
\text { p-Values }\end{array}$ & $\mid$ Mean-0.05 & $\begin{array}{c}\text { Percent } \\
\text { Within } \\
2.5,5.0\end{array}$ & $\begin{array}{c}\text { Percent } \\
\text { Within } \\
4.5,5.5\end{array}$ & $\begin{array}{c}\text { Percent } \\
\text { Within } \\
4.5,5.0\end{array}$ & Total \% \\
\hline 1 & $\mathrm{Q}_{3} 2015 \mathrm{HH}_{3}$ & .0499 & .0001 & 35.71 & 11.90 & 9.52 & 38 \\
\hline 2 & $\mathrm{Q}_{3} 1515 \mathrm{HH}_{3}$ & .0498 & .0002 & 35.71 & 19.05 & 9.52 & 45 \\
\hline 3 & $\mathrm{~K}_{10 \mathrm{HQ}}$ & .0503 & .0003 & 54.76 & 23.81 & 16.67 & 62 \\
\hline 4 & $\mathrm{Q}_{3} 1025 \mathrm{HQ}_{1}$ & .0503 & .0003 & 57.14 & 26.19 & 11.90 & 71 \\
\hline 5 & $\mathrm{Q}_{2} 0515$ & .0504 & .0004 & 52.38 & 19.05 & 9.52 & 62 \\
\hline 6 & $\mathrm{~K}_{20 H}$ & .0496 & .0004 & 54.76 & 28.57 & 14.29 & 69 \\
\hline 7 & $\mathrm{~K}_{3} 0 \mathrm{HH}_{3}$ & .0506 & .0006 & 54.76 & 23.81 & 16.67 & 62 \\
\hline 8 & $\mathrm{Q}_{3} 1010 \mathrm{HQ}$ & .0507 & .0007 & 42.86 & 28.57 & 16.67 & 55 \\
\hline 9 & $\mathrm{Q}_{3} 2015 \mathrm{HQ}$ & .0508 & .0008 & 33.33 & 16.67 & 9.52 & 40 \\
\hline 10 & $\mathrm{~K} 25 \mathrm{HQ}$ & .0490 & .0010 & 54.76 & 9.52 & 9.52 & 55 \\
\hline
\end{tabular}




\section{OTHMAN, YIN, KESELMAN, WILCOX \& ALGINA}

Table 7: Type I Error Rates for the 10 Best Performing Variants of O’Brien's (1979) Procedure

\begin{tabular}{|c|c|c|c|c|c|c|c|}
\hline No. & Variant & Overall & Skewed & Symmetric & Leptokurtic & $\begin{array}{c}\text { Normal } \\
\text { Tailed }\end{array}$ & Platykurtic \\
\hline 1 & $\mathrm{Q}_{3} 2015 \mathrm{HH}_{3}$ & .050 & .051 & .049 & .035 & .056 & .066 \\
\hline 2 & $\mathrm{Q}_{3} 1515 \mathrm{HH}_{3}$ & .050 & .051 & .049 & .036 & .058 & .063 \\
\hline 3 & $\mathrm{~K} 10 H Q_{1}$ & .050 & .056 & .043 & .045 & .056 & .053 \\
\hline 4 & $\mathrm{Q}_{3} 1025 \mathrm{HQ}_{1}$ & .050 & .052 & .048 & .045 & .059 & .051 \\
\hline 5 & $\mathrm{Q}_{2} 0515$ & .050 & .054 & .046 & .043 & .059 & .053 \\
\hline 6 & $\mathrm{~K}_{2} 0 \mathrm{HH}_{3}$ & .050 & .055 & .042 & .043 & .058 & .052 \\
\hline 7 & $\mathrm{~K}_{10 H}$ & .051 & .057 & .043 & .046 & .056 & .053 \\
\hline 8 & $\mathrm{Q}_{3} 1010 \mathrm{HQ}$ & .051 & .052 & .049 & .040 & .058 & .059 \\
\hline 9 & $\mathrm{Q}_{3} 2015 \mathrm{HQ}$ & .051 & .053 & .048 & .038 & .059 & .062 \\
\hline 10 & $\mathrm{~K} 25 \mathrm{HQ}$ & .049 & .058 & .038 & .042 & .058 & .052 \\
\hline
\end{tabular}

References

Algina, J., Keselman, H. J., \& Penfield, R. D. (2007). Confidence intervals for an effect size measure in multiple linear regression. Educational and Psychological Measurement, 67, 207-218.

Babu, J. G., Padmanabhan A. R., \& Puri, M. P. (1999). Robust one-way ANOVA under possibly non-regular conditions. Biometrical Journal, 41(1978), 321-339.

Fleishman, A. I. (1978). A method for simulating non-normal distributions. Psychometrika, 43, 521-532.

Hill, M., \& Dixon, W. J. (1982). Robustness in real life: A study of clinical laboratory data. Biometrics, 38, 377-396.

Hoaglin, D. C. (1985). Summarizing shape numerically: The g- and h distributions. In D. Hoaglin, F. Mosteller, and J. Tukey (Eds), Exploring data tables, trends, and shapes, 461513. New York, NY: Wiley.

Hogg, R. V. (1974). Adaptive robust procedures: A partial review and some suggestions for future applications and theory. Journal of the American Statistical Association, 69, 909-927.

Hogg, R. V. (1982). On adaptive statistical inferences. Communications in Statistics: Theory and Methods, 11, 2531-2542.

Huber, P. J. (1970). Studentizing robust estimates. In M. L. Puri, (Ed.) Nonparametric techniques in statistical inference. London, England: Cambridge University Press.
Huber, P. J. (1977). Discussion. The Annals of Statistics, 5, 1090-1091.

Keselman, H. J., Algina, J., \& Fradette, K. (2005). Robust confidence intervals for effect size in the two-group case. Journal of Modern Applied Statistical Methods, 4, 353-371.

Keselman, H. J., Games, P. A., \& Clinch, J. J. (1979). Tests for homogeneity of variance. Communications in Statistics, Simulation and Computation, B8(2), 113-129.

Keselman, H. J., et al. (1998). Statistical practices of Educational Researchers: An analysis of their ANOVA, MANOVA and ANCOVA analyses. Review of Educational Research, 68(3), 350-386.

Keselman, H. J., Wilcox, R. R., Algina, J., Othman, A. R., \& Fradette, K. (2008). A comparative study of robust tests for spread: Asymmetric trimming strategies. British Journal of Mathematical and Statistical Psychology, 61, 235-253.

Keselman, H. J., Wilcox, R. R., Lix, L. M., Algina, J., \& Fradette, K. (2007). Adaptive robust estimation and testing. British Journal of Mathematical and Statistical Psychology, 60, 267-293.

Keselman, H. J., Wilcox, R. R., Othman, A. R., \& Fradette, K. (2002). Trimming, transforming statistics, and bootstrapping: Circumventing the biasing effects of heteroscedasticity and nonnormality. Journal of Modern Applied Statistical Methods, 1, 288-309. 


\section{ROBUST MODIFICATIONS OF THE LEVENE AND O'BRIEN TESTS FOR SPREAD}

Lee, H., \& Fung, K. Y. (1985). Behaviour of trimmed $\mathrm{F}$ and sine-wave $\mathrm{F}$ statistics in one-way ANOVA. Sankhya: The Indian Journal of Statistics, 47, Series B, 186201.

Levene, H. (1960). Robust tests for the equality of variances. In I. Olkin (Ed.), Contributions to probability and statistics. Palo Alto, CA: Stanford University Press.

Mudholkar, A., Mudholkar, G. S., \& Srivastava, D. K. (1991). A construction and appraisal of pooled trimmed-t statistics. Communications in Statistics: Theory and Methods, 20, 1345-1359.

O’Brien, R. G. (1979). A general ANOVA method for robust tests of additive models for variances, Journal of the American Statistical Association, 74, 877-880.

O’Brien, R. G. (1981). A simple test for variance effects in experimental designs. Psychological Bulletin, 89, 570-574.

Olejnik, S. F., \& Algina, J. (1987). Type

I error rates and power estimates of selected parametric and nonparametric tests of scale. Journal of Educational Statistics, 12, 45-61.

Reed III, J. F. (1998). Contributions to adaptive estimation. Journal of Applied Statistics, 25, 651-669.

Reed III, J. F., \& Stark, D. B. (1996). Hinge estimators of location: Robust to asymmetry. Computer Methods and Programs in Biomedicine, 49, 11-17.

Rosenberger, J. L., \& Gasko, M. (1983). Comparing location estimators: Trimmed means, medians and trimean. In D. Hoaglin, F. Mosteller and J. Tukey, (Eds.), Understanding robust and exploratory data analysis, 297-336. New York, NY: Wiley.
Rousseeuw, P. J., \& van Zomeren, B. C. (1990). Unmasking multivariate outliers and leverage points. Journal of the American Statistical Association, 85, 633-639.

SAS Institute Inc. (2006). SAS OnlineDoc ${ }^{\circledR}$ 9.1.3. Cary, $\mathrm{NC}$ : SAS Institute Inc.

Staudte, R. G., \& Sheather, S. J. (1990). Robust estimation and testing. New York: Wiley.

Stigler, S. M. (1977). Do robust estimators work with real data? The Annals of Statistics, 5, 1055-1098.

Tukey, J. W., \& McLaughlin, D. H. (1963). Less vulnerable confidence and significance procedures for location based on a single sample: Trimming/Winsorization. Sankhya: The Indian Journal of Statistics, 25, Series A, 331-352.

Welch, B. L. (1951). On the comparison of several mean values: An alternative approach. Biometrika, 38, 330-336.

Wilcox, R. R. (2003). Applying contemporary statistical methods. San Diego, CA: Academic Press.

Wilcox, R. R. (2005). Introduction to robust estimation and hypothesis testing $\left(2^{\text {nd }}\right.$ $E d$.). San Diego, CA: Academic Press.

Yuen, K. K. (1974). The two-sample trimmed $\mathrm{t}$ for unequal population variances. Biometrika, 61, 165-170. 


\section{An Extension of the Seasonal KPSS Test}

\author{
Sami Khedhiri \\ University of Prince Edward Island, \\ Charlottetown, PEI, Canada
}

\author{
Ghassen El Montasser \\ University of Manouba, \\ Tunis, Tunisia
}

The limit theory of the seasonal KPSS test is established under the null hypothesis using seasonal dummy variables. Taking these variables into account can result in improved finite sample performance of the test. The seasonal KPSS test can be interpreted as a test of deterministic seasonality and it may be used in addition to seasonal unit root tests to analyze the dynamic properties of time series. The seasonal indicator variables provide the test with an explicit model-based regression that in itself constitutes a support for its limit theory.

Key words: KPSS test, deterministic seasonality, Brownian motion, C32 time series models.

Introduction

The use of seasonally unadjusted data has become increasingly popular in empirical studies; there are several possible reasons for this. One key reason is the argument that seasonal adjustment distorts inference in dynamic models, for example, seasonal unit roots can be seriously affected when working with seasonally adjusted data. In this respect, Ghysels and Perron (1993) showed that seasonal adjustment filters affect finite sample distributions of unit root test statistics under the null hypothesis. Further, the seasonal component is an unobserved part of a time series, thus it must be taken into consideration because its elimination can lead to detrimental information loss. It was found in several cases that the seasonal component and other systematic components, such as trend and cycle, are in fact non-separable. From a statistical viewpoint this could be attributed to the fact that economic propagation mechanisms transmitting seasonal

Sami Khedhiri is an Associate Professor in Statistics in the Department of Mathematics and Statistics. Email him at: samikhedhiri@hotmail.com._Ghassen El Montasser is an Assistant Professor in Econometrics at the Ecole Supérieure de Commerce. Email him at: montas_1999@yahoo.fr. fluctuations from exogenous to endogenous variables are systematically related to business cycle fluctuations. Beaulieu, MacKie-Mason and Miron (1992) and Miron (1996) showed this in their studies of international economic aggregates such as output, labor input, interest rates, and prices. Canova and Ghysels (1994) also found that seasonality tends to differ across business cycle stages of recessions and expansions referring to an empirical study of $U$. $\mathrm{S}$. macroeconomic time series. Consequently, a forced seasonal adjustment may lead to inaccurate predictions, which in turn may result in wrong decisions.

The literature presents several different models of seasonality. As highlighted by Canova and Hansen (1995), the first approach is to model seasonality as a deterministic component. This approach is generally adopted by macroeconomists, as shown by Barsky and Miron (1989). The second approach is to consider seasonality as a deterministic process along with its stationary stochastic pattern as illustrated by Canova (1992). The third approach is to consider seasonality as a stochastic component by allowing for seasonal unit roots.

A famous testing framework proposed by Hylleberg, et al. (1990) used the null hypothesis of seasonal non-stationarity induced by the presence of seasonal unit root(s) to make the distinction between unit roots at different seasonal frequencies. The subsequent rejection of their null hypothesis implies a strong result 


\section{AN EXTENSION OF THE KPSS TEST WITH DETERMINISTIC SEASONALITY}

that the series exhibits a stationary seasonal pattern but their test was found to suffer from the problem of low power with moderate sample sizes. Because testing for seasonal unit roots is an important step in time series analysis these tests are often used as a pre-test for seasonal cointegration (Johansen \& Schaumberg, 1999), several authors have contributed to the development of these types of tests (Canova \& Hansen, 1995; Caner, 1998). In the testing approach the rejection of the null hypothesis would show evidence that the data are nonstationary. Another reason why this testing method is interesting to practitioners could be explained by the necessity to take into account the cost of spurious inference when testing the dummy variables model as argued in Franses, Hylleberg and Lee (1995).

Canova and Hansen (1995) and Taylor (2003) generalized the KPSS testing framework to seasonal data to test the stationarity hypothesis against (seasonal) unit roots. After specifying a general regression for their tests, the authors examined specific cases related to testing stationarity against (seasonal) unit root or some unit roots among a well-defined set. Lyhagen (2006) proposed another version of the KPSS test in the seasonal context which results in a frequency-based test and tested the hypothesis of level stationarity against a single seasonal unit root. In this study, seasonal indicator variables are included in the seasonal KPSS suggested by Lyhagen (2006). This approach may several have advantages over existing methods: It provides a model-based regression to the test, which is different from Lyhagen's method, where the limit theory based on an explicit form of the model was not established. The novelty of results from this study is the development of an asymptotic theory of the test in the presence of seasonal dummies which leads to a natural extension of the SKPSS to include deterministic seasonality.

Preliminaries on the Seasonal KPSS Test

Let $y_{t}$ be a time series observed quarterly. This frequency was chosen because it provides a simple and clear analysis, however it should be noted that results of this study are valid for all seasonal frequencies (e.g., monthly or daily data) by simply defining seasonal unit roots according to their corresponding seasonal frequencies. Because the goal of this research is to test for the presence of negative unit root, it would be suitable to use the appropriate filter in order to isolate the effects of other unit roots in the series. Therefore, the test will be applied to the transformed series:

$$
y_{t}^{(1)}=\left(1-L+L^{2}-L^{3}\right) y_{t},
$$

where $L$ is the lag operator.

Next, test the unit root of -1 in the series

$$
\begin{aligned}
& y_{t}^{(1)}=x_{t}^{\prime} \beta+r_{t}+u_{t}, \\
& t=1, \ldots, T
\end{aligned}
$$

where $T=4 N, \beta^{\prime} x_{t}=\sum_{i=1}^{4} a_{i} D_{i t}$, the shorthand notation $D_{i t}=\delta(i, t-4[(t-1) / 4])$, [.] denotes the largest integer function and $\delta(i, j)$ is the Kronecker's $\delta$ function. The term $u_{t}$ is zero mean weakly dependent process with autocovariogram $\gamma_{h}=E\left(u_{t} u_{t+h}\right)$ and a strictly positive long run variance $\omega_{u}^{2}$.

The component $r_{t}$ is drawn from the following process:

$$
r_{t}=-r_{t-1}+v_{t},
$$

where $v_{t}$ is zero mean weakly process with variance $\sigma_{v}^{2}$ and long run variance $\omega_{v}^{2}>0$. The transformation required to carry out the seasonal KPSS test for complex unit roots $\pm i$ is given by the variable, $y_{t}^{(2)}=\left(1-L^{2}\right) y_{t}$. The test of such complex unit roots is based on the regression,

$$
y_{t}^{(2)}=x_{t}^{\prime} \lambda+c_{t}+e_{t}
$$




\section{KHEDHIRI \& EL MONTASSER}

where $e_{t}$ is zero mean weakly dependent process with long run variance $\omega_{e}^{2}>0$ and $\lambda^{\prime} x_{t}=\sum_{i=1}^{4} b_{i} D_{i t}$. The component $c_{t}$ is given by

$$
c_{t}=-c_{t-2}+\varepsilon_{t},
$$

where $\varepsilon_{t}$ is another zero mean weakly dependent process with variance $\sigma_{\varepsilon}^{2}$ and strictly positive long run variance $\omega_{\varepsilon}^{2}$.

Adding the deterministic terms in (1) and (3) is very important because it enables the seasonal KPSS test to be extended to include deterministic seasonality. The testing procedure follows in two steps: First, test for the existence of unit root -1 , and second, test for the complex roots where the null hypothesis will be specified thereafter.

The seasonal KPSS test is a Lagrange Multiplier-based test, hence, the null hypothesis of a root equal to -1 is $H_{0}: \sigma_{v}^{2}=0$. Under this null hypothesis

$$
y_{t}^{(1)}=x_{t}^{\prime} \beta+u_{t} \text {, }
$$

where the series is trend stationary after seasonal mean correction. Under the alternative hypothesis $H_{1}: \sigma_{v}^{2}>0, y_{t}^{(1)}$ has a unit root corresponding to Nyquist frequency.

Let $\tilde{u}_{t}$ be the residual series obtained from least squares regression applied to equation (5), $t=1,2, \ldots, T$. Following Breitung and Franses (1998, Eq. (18), p. 209), Busetti and Harvey (2003, Eq. (8), p. 422) and Taylor (2003, Eq. (38), p. 605), replace the long-run variance $\omega_{u}^{2}$ by an estimate of ( $2 \pi$ times) the spectrum at the observed frequency in order to deal with unconditional heteroscedasticity and serial correlation. This nonparametric estimation of the long-run variance is a useful solution to the nuisance parameter problem (Taylor, 2003). Thus, the Nyquist frequency is

$$
\begin{aligned}
& \tilde{\omega}_{u}^{2}(l)= \\
& T^{-1} \sum_{t=1}^{T} \tilde{u}_{t}^{2}+2 T^{-1} \sum_{k=1}^{l} w(k, l)\left(\sum_{t=k+1}^{T} \tilde{u}_{t} \tilde{u}_{t-k}\right) \cos (\pi k),
\end{aligned}
$$

where the weight function $w(k, l)=1-\frac{k}{l+1}$ and $l$ is a lag truncation parameter such that $l \rightarrow \infty$ as $T \rightarrow \infty$ and $l=o\left(n^{1 / 2}\right)$. From equation (6), a Bartlett kernel following Newey and West (1987) can now be chosen. It should be noted that Andrews (1991) and Kwaitkowski, et al. (1992) showed that that such a truncation lag can produce good results in practice. Similarly, the null hypothesis of the test regarding complex unit roots is given by $H_{0}: \sigma_{\varepsilon}^{2}=0$; under this null hypothesis

$$
y_{t}^{(2)}=x_{t}^{\prime} \lambda+e_{t}
$$

Using the residuals $\widetilde{e}_{t}$ obtained from the least squares regression of equation (7), the Bartlett kernel estimator $\omega_{e}^{2}$ is computed as:

$$
\begin{aligned}
& \tilde{\omega}_{e}^{2}(l)= \\
& T^{-1} \sum_{t=1}^{T} \tilde{e}_{t}^{2}+2 T^{-1} \sum_{k=1}^{l} w(k, l)\left(\sum_{t=k+1}^{T} \tilde{e}_{t} \tilde{e}_{t-k}\right) \cos \left(\frac{\pi}{2} k\right),
\end{aligned}
$$

with the partial sums defined as $\widetilde{S}_{t}=\sum_{j=1}^{t} e^{i \pi j} \widetilde{u}_{j}$ and $\widetilde{P}_{t}=\sum_{j=1}^{t} e^{i \frac{\pi}{2} j} \widetilde{e}_{t}$.

It follows that the test statistics for unit root of -1 is given by:

$$
\eta^{(-1)}=\frac{1}{T^{2}} \frac{\sum_{t=1}^{T} \widetilde{S}_{t} \overline{\widetilde{S}}_{t}}{\widetilde{\omega}_{u}^{2}(l)} .
$$

This statistic may be written for the complex unit roots, as 


$$
\eta^{( \pm i)}=\frac{1}{T^{2}} \frac{\sum_{t=1}^{T} \widetilde{P}_{t} \widetilde{\widetilde{P}}_{t}}{\widetilde{\omega}_{e}^{2}(l)}
$$

where $\overline{\widetilde{S}}_{t}$ and $\widetilde{\widetilde{P}}_{t}$ are the conjugate numbers of $\widetilde{S}_{t}$ and $\widetilde{P}_{t}$, respectively.

Asymptotic Results

Next, the asymptotic distribution of $\eta^{(1)}$ and $\eta^{( \pm i)}$ is shown.

Theorem

a) Under

$$
H_{0}: \sigma_{v}^{2}=0, \eta^{(-1)} \rightarrow_{d} \int_{0}^{1} V(r)^{2} d r
$$

where $V(r)$ is a standard Brownian bridge, and $\rightarrow_{d}$ denotes weak convergence in probability and $r \in[0,1]$.

b) Under

$$
\begin{aligned}
& H_{0}: \sigma_{\varepsilon}^{2}=0, \\
& \eta^{( \pm i)} \rightarrow_{d} \frac{1}{2} \int_{0}^{1}\left[V_{R}^{c}(\tau)^{2}+V_{I}^{c}(\tau)^{2}\right] d \tau
\end{aligned}
$$

where $V_{R}^{c}(\tau)$ and $V_{I}^{c}(\tau)$ are two independent standard Brownian bridges and $\tau \in[0,1]$.

Proof

Starting with the first part of the theorem and referring to Jin and Phillips (2002) whose results showed that seasonal dummies maintain the asymptotic properties of the KPSS test unchanged. Also, given the mirror image of negative unit roots,

$$
\frac{1}{\sqrt{T}} \sum_{j=1}^{[T r]}(-1)^{j} u_{j} \rightarrow{ }_{d} B(r)
$$

where $B(r)$ is a Brownian motion. The standardized partial sum process can be written as follows:

$$
\begin{aligned}
\frac{\tilde{S}_{[T r]}}{\sqrt{T}} & =\frac{1}{\sqrt{T}} \sum_{j=1}^{[T r]} e^{i(\pi j)} \tilde{u}_{j} \\
& =\frac{1}{\sqrt{T}} \sum_{j=1}^{[T r]}(-1)^{j} \tilde{u}_{j} \\
& =\frac{1}{\sqrt{T}} \sum_{j=1}^{[T r]}(-1)^{j}\left[u_{j}-x_{j}^{\prime}(\tilde{\beta}-\beta)\right] \\
& =\frac{1}{\sqrt{T}} \sum_{j=1}^{[T r]}(-1)^{j} u_{j}- \\
& \frac{1}{\sqrt{T}} \sum_{j=1}^{[T r]}(-1)^{j} x_{j}^{\prime}\left(X^{\prime} X\right)^{-1}\left(X^{\prime} u\right),
\end{aligned}
$$

where $r \in[0,1]$. Thus the following is obtained,

$$
\begin{aligned}
\frac{\tilde{S}_{[T r]}}{\sqrt{T}}= & \frac{1}{\sqrt{T}} \sum_{j=1}^{[T r]}(-1)^{j} u_{j}- \\
& \left.\frac{\sum_{j=1}^{[T r]}(-1)^{j} x_{j}^{\prime}}{T}\right)\left(\frac{X^{\prime} X}{T}\right)^{-1}\left(\frac{X^{\prime} u}{\sqrt{T}}\right) .
\end{aligned}
$$

In addition,

$$
\begin{gathered}
\frac{1}{T} \sum_{j=1}^{[T r]}(-1)^{j} x_{j}^{\prime} \rightarrow\left(-\frac{r}{4}, \frac{r}{4},-\frac{r}{4}, \frac{r}{4}\right), \\
T^{-1} X^{\prime} X \rightarrow(1 / 4) I_{4},
\end{gathered}
$$

and 


\section{KHEDHIRI \& EL MONTASSER}

$$
\begin{aligned}
\frac{1}{\sqrt{T}} X^{\prime} u & =\frac{1}{2} \frac{1}{\sqrt{\frac{T}{4}}}\left(\begin{array}{l}
\sum_{j=1}^{\frac{T}{4}} u_{4 j-3} \\
\sum_{j=1}^{\frac{T}{4}} u_{4 j-2} \\
\frac{T}{4} u_{4 j-1} \\
\sum_{j=1}^{T} \\
\frac{T}{4} u_{4 j} \\
\sum_{j=1} u_{4 j}
\end{array}\right) \rightarrow\left(\begin{array}{l}
B_{u .1} \\
B_{u .2} \\
B_{u .3} \\
B_{u .4}
\end{array}\right) \\
\equiv & \left.\frac{1}{2}\left(\begin{array}{llll}
\omega_{0} & \omega_{1} & \omega_{2} & \omega_{3} \\
\omega_{1} & \omega_{0} & \omega_{1} & \omega_{2} \\
\omega_{2} & \omega_{1} & \omega_{0} & \omega_{1} \\
\omega_{3} & \omega_{2} & \omega_{1} & \omega_{0}
\end{array}\right)\right),
\end{aligned}
$$

where $\quad B_{u . i}(1)={ }_{d} N\left(0, \omega_{0}\right), \quad i=1, \ldots, 4$, $\omega_{0}=\gamma_{0}+2 \sum_{h=1}^{\infty} \gamma_{4 h}, \omega_{1}=\sum_{h=1}^{\infty} \gamma_{2 h-1}=\omega_{3}, \quad$ and $\omega_{2}=2 \sum_{h=1}^{\infty} \gamma_{4 h-2}$. It follows that $\left(\frac{\sum_{j=1}^{[T r]}(-1)^{j} x_{j}^{\prime}}{T}\right)\left(\frac{X^{\prime} X}{T}\right)^{-1}\left(\frac{X^{\prime} u}{\sqrt{T}}\right) \rightarrow$ ${ }_{d}\left(-\frac{r}{4}, \frac{r}{4},-\frac{r}{4}, \frac{r}{4}\right)\left(\begin{array}{cccc}4 & 0 & 0 & 0 \\ 0 & 4 & 0 & 0 \\ 0 & 0 & 4 & 0 \\ 0 & 0 & 0 & 4\end{array}\right) \frac{1}{2}\left(\begin{array}{c}B_{u .1}(1) \\ B_{u .2}(1) \\ B_{u .3}(1) \\ B_{u .4}(1)\end{array}\right)$

$\rightarrow_{d} \frac{1}{2} r\left[-B_{u .1}(1)+B_{u .2}(1)-B_{u .3}(1)+B_{u .4}(1)\right]$ $\rightarrow_{d} \frac{1}{2} r\left[-B_{1}(1)+B_{2}(1)-B_{3}(1)+B_{4}(1)\right]=r B(1)$ and

$$
\frac{1}{\sqrt{T}} \widetilde{S}_{[T r]} \rightarrow_{d} B(r)-r B(1)
$$

which results in the following:

$$
T^{-2} \sum_{t=1}^{T} \widetilde{S}_{t}^{2} \rightarrow_{d} \omega_{u}^{2} \int_{0}^{1} V(r)^{2} d r
$$

where $V(r)$ is a standard Brownian bridge process. Further, because $\widetilde{\omega}_{u}^{2}(l)$ is a consistent estimate of $\omega_{u}^{2}$, it can be shown that $\eta^{(-1)} \rightarrow_{d} \int_{0}^{1} V(r)^{2} d r$

Next it is necessary to prove the second part of the theorem. Because complex-valued roots come in conjugate pairs, it is only necessary to consider the complex root $i$ associated with frequency $\frac{\pi}{2}$. In this case the standardized partial sum process can be written as follows:

$$
\begin{aligned}
\frac{\tilde{P}_{[T \tau]}}{\sqrt{T}} & =\frac{1}{\sqrt{T}} \sum_{j=1}^{[T \tau]} e^{i\left(j \frac{\pi}{2}\right)} \tilde{e}_{j} \\
& =\frac{1}{\sqrt{T}} \sum_{j=1}^{[T \tau]} e^{i\left(j \frac{\pi}{2}\right)} \tilde{e}_{j} \\
& =\frac{1}{\sqrt{T}} \sum_{j=1}^{[T \tau]} e^{i\left(j \frac{\pi}{2}\right)}\left[e_{j}-x_{j}^{\prime}(\tilde{\lambda}-\lambda)\right]
\end{aligned}
$$

where

$$
\frac{\tilde{S}_{[T r]}}{\sqrt{T}}=
$$

$\frac{1}{\sqrt{T}} \sum_{j=1}^{[T \tau]} e^{i\left(j \frac{\pi}{2}\right)} e_{j}-\left(\frac{\sum_{j=1}^{[T \tau]} e^{i\left(j \frac{\pi}{2}\right)} x_{j}^{\prime}}{T}\right)\left(\frac{X^{\prime} X}{T}\right)^{-1}\left(\frac{X^{\prime} e}{\sqrt{T}}\right)$ 
Chan and Wei (1988) showed that

$$
\frac{1}{\sqrt{T}} \sum_{j=1}^{[T \tau]} e^{i\left(j \frac{\pi}{2}\right)} e_{j} \rightarrow_{d} B^{*}(\tau)
$$

where $B^{*}(\tau)=B_{R}^{*}(\tau)+i B_{I}^{*}(\tau)$ and $B_{R}^{*}(\tau)$ and $B_{I}^{*}(\tau)$ are two independent real Brownian motions and

$\frac{1}{T} \sum_{j=1}^{[T \tau]} e^{i\left(j \frac{\pi}{2}\right)} x_{j}^{\prime} \rightarrow\left(0,-\frac{r}{4}, 0, \frac{r}{4}\right)+i\left(\frac{r}{4}, 0,-\frac{r}{4}, 0\right)$

Therefore,

$$
\left.\begin{array}{c}
\left(\frac{\sum_{j=1}^{[T \tau]} e^{i\left(j \frac{\pi}{2}\right)} x_{j}^{\prime}}{T}\right)\left(\frac{X^{\prime} X}{T}\right)^{-1}\left(\frac{X^{\prime} e}{\sqrt{T}}\right) \rightarrow \\
{\left[\begin{array}{l}
d \\
4
\end{array} \times\left(0,-\frac{r}{4}, 0, \frac{r}{4}\right) I_{4} \times \frac{1}{2}\left(\begin{array}{l}
B_{e .1} \\
B_{e .2} \\
B_{e .3} \\
B_{e .4}
\end{array}\right)\right.} \\
+i\left[4 \times\left(\frac{r}{4}, 0,-\frac{r}{4}, 0\right) I_{4} \times \frac{1}{2}\left(\begin{array}{l}
B_{e .1} \\
B_{e .2} \\
B_{e .3} \\
B_{e .4}
\end{array}\right)\right]
\end{array}\right],
$$

where $B_{e . i}$ are defined similarly to $B_{u . i}$ in equation (12), $i=1, \ldots, 4$. It follows that

$$
\begin{aligned}
& \left(\frac{\sum_{j=1}^{[T \tau]} e^{i\left(j \frac{\pi}{2}\right)} x_{j}^{\prime}}{T}\right)\left(\frac{X^{\prime} X}{T}\right)^{-1}\left(\frac{X^{\prime} e}{\sqrt{T}}\right) \rightarrow \\
& \left(\begin{array}{l}
d \frac{1}{\sqrt{2}} \frac{\sqrt{2}}{2} \tau\left(-B^{(2)}(1)+B^{(4)}(1)\right) \\
+i \frac{1}{\sqrt{2}} \frac{\sqrt{2}}{2} \tau\left(B^{(1)}(1)-B^{(3)}(1)\right)
\end{array}\right) \\
& =\frac{1}{\sqrt{2}} \tau\left(B_{R}^{*}(1)+i B_{I}^{*}(1)\right)=\frac{1}{\sqrt{2}} \tau B^{*}(1) \text {, }
\end{aligned}
$$

where $B^{(i)}(\tau), i=1, . ., 4, B_{R}^{*}(\tau)$ and $B_{I}^{*}(\tau)$ are all real Brownian motions and the last two processes are independent.

$\frac{\widetilde{P}_{[T \tau]}}{\sqrt{T}} \rightarrow_{d} \sqrt{\frac{\omega_{e}^{2}}{2}} V^{c}(\tau)$, where $V^{c}(\tau)$ is a complex Brownian bridge that can be written as $V^{c}(\tau)=V_{R}^{c}(\tau)+i V_{I}^{c}(\tau) \quad V_{R}^{c}(\tau)$ and $V_{I}^{c}(\tau)$ are two independent real standard Brownian bridges. As a result,

$$
\frac{1}{T^{2}} \sum_{t=1}^{T} \tilde{P}_{t} \overline{\tilde{P}}_{t} \rightarrow_{d} \frac{\omega_{e}^{2}}{2} \int_{0}^{1}\left[\left(V_{R}^{c}\right)^{2}(\tau)+\left(V_{I}^{c}\right)^{2}(\tau)\right] d \tau
$$

because $\widetilde{\omega}_{e}^{2}(l)$ is a convergent estimate of $\omega_{e}^{2}$, it may be concluded that

$$
\eta^{( \pm i)} \rightarrow_{d} \frac{1}{2} \int_{0}^{1}\left[\left(V_{R}^{c}\right)^{2}(\tau)+\left(V_{I}^{c}\right)^{2}(\tau)\right] d \tau
$$

as claimed.

It should be noted that asymptotically $\eta^{(-1)}$ has the Cramer-von Mises distribution (CvM) under the null hypothesis, although the limit theory of $\eta^{( \pm i)}$ was shown as a function of a generalized $\mathrm{CvM}$ with two degrees, specifically, $\eta^{( \pm i)} \rightarrow_{d} \frac{1}{2} C v M(2)$. The critical values of the seasonal KPSS test with seasonal 


\section{KHEDHIRI \& EL MONTASSER}

dummies can be computed from Nyblom (1989) or from Canova and Hansen (1995). These critical values are shown in Table 1 .

Table 1: Critical Values of the Seasonal KPSS Test

\begin{tabular}{|c|c|c|c|}
\cline { 2 - 4 } \multicolumn{1}{c|}{} & $1 \%$ & $5 \%$ & $10 \%$ \\
\hline Root -1 & 0.743 & 0.461 & 0.347 \\
\hline Roots $\pm i$ & 0.537 & 0.374 & 0.3035 \\
\hline
\end{tabular}

\section{Monte Carlo Analysis}

To evaluate the size performance of the seasonal KPSS statistic, Monte Carlo simulation experiments were conducted using seasonal roots of a quarterly process. The data generating process (DGP) for the negative unit root is

$$
\begin{aligned}
& y_{t}=x_{t}^{\prime} \beta+r_{t}, \\
& t=1, \ldots, T,
\end{aligned}
$$

where $x_{t}^{\prime} \beta$ is defined as in (1) and the autoregressive process $r_{t}$ is given by:

$$
r_{t}=\alpha r_{t-1}+v_{t}
$$

The error terms $v_{t}$ are normally distributed with zero mean and unit variance. The DGP for complex unit roots is given by:

$$
\begin{aligned}
& y_{t}=x_{t}^{\prime} \lambda+c_{t}, \\
& t=1, \ldots, T,
\end{aligned}
$$

where $x_{t}^{\prime} \lambda$ is defined in (3) and the process $c_{t}$ is given by:

$$
c_{t}=\alpha c_{t-2}+\varepsilon_{t},
$$

and $\varepsilon_{t}$ are normally distributed with zero mean and unit variance.

Alternative values of $\alpha \in$ $\{-1,-0.8,-0.2,0,0.2,0.8\}$ were chosen and only the $5 \%$ nominal size was considered. The bandwidth values chosen in these experiments are given by: $\quad l 0=0, \quad l 4=$ integer $\left[4(T / 100)^{1 / 4}\right] \quad$ and $\quad l 12=$ integer $\left[12(T / 100)^{1 / 4}\right]$. Twenty-thousand $(20,000)$ replications were conducted and all the simulation experiments were carried out with Matlab programs.

Results in Table 2 show that the test size increases as values of $\alpha$ decrease. Also note that larger data samples do not significantly affect the test size. These simulations raised another point, which was observed by Lyhagen (2006) for similar testing but without deterministic components: they show that, as opposed to the original KPSS testing framework, $l 4$ and $l 12$ do not have better size performance than $l 0$. In fact, in the seasonal KPSS framework, the test size deterioration induces substantial power. Results of the simulation experiments performed in this study (see Table 1) suggest an overall good power performance of the seasonal KPSS test, particularly against near seasonal unit root alternatives.

\section{Conclusion}

The joint use of unit root and stationarity tests is recommended in empirical studies. Such a joint use can lead to a more in-depth analysis of the dynamic properties of the time series. This article established asymptotic theory of the seasonal KPSS test in the presence of seasonal dummies and extended SKPSS to include deterministic seasonality. Given that seasonal unit root tests have low power in moderate samples, the test represents an adequate solution as illustrated by the simulation results. Lyhagen (2006) also showed good power properties of the test when there is no deterministic term in the model, however, it would be interesting to study both power and size performance of the test when factors affecting the time series such as measurement errors and additive outliers are present. Khedhiri and El Montasser (2010) used 


\section{AN EXTENSION OF THE KPSS TEST WITH DETERMINISTIC SEASONALITY}

Monte Carlo methods to show that the seasonal KPSS test is robust to the magnitude and the number of additive outliers. Furthermore, the statistical results obtained demonstrate overall good performance on the finite-sample properties of the test.

\section{References \\ Andrews, D. W. K. (1991).} Heteroskedasticity and autocorrelation consistent covariance matrix estimation. Econometrica, 59, 817-858.

Barsky, R. B., \& Miron, J. A. (1989). The seasonal cycle and the business cycle. Journal of Political Economy, 97, 503-533.

Beaulieu, J. J., MacKie-Mason, J. K., \& Miron, J. A. (1992). Why do countries and industries with large seasonal cycles also have large business cycles? Quarterly Journal of Economics, 107, 621-651.
Breitung, J., \& Franses, P. H. (1998). On Phillips-Perron-type tests for seasonal unit roots. Econometric Theory, 14, 200-221.

Busetti, F., \& Harvey, A. (2003). Seasonality tests. Journal of Business and Economic Statistics, 21, 420-436.

Caner, M. (1998). A locally optimal seasonal unit root test, Journal of Business and Economic Statistics, 16, 349-356.

Canova, F. (1992). An alternative approach to modelling and forecasting seasonal time series. Journal of Business and Economic Statistics, 10, 97-108.

Canova, F., \& Ghysels, E. (1994). Changes in seasonal patterns: Are they cyclical? Journal of Economic Dynamics and Control, 18, 1143-1171.

Canova, F., \& Hansen, B. (1995). Are seasonal patterns constant over time? A test for seasonal stability. Journal of Business and Economic Statistics, 13, 237-252.

Table 2: Rejection Frequencies for the Seasonal KPSS Test with Seasonal Dummies for Seasonal Quarterly Unit Roots

\begin{tabular}{|c|c|c|c|c|c|c|c|}
\hline \multicolumn{4}{|c|}{$\eta^{(-1)}$} & \multicolumn{4}{|c|}{$\eta^{( \pm i)}$} \\
\hline \multirow{2}{*}{-1} & $\mathrm{~T}$ & $l 0$ & $l 4$ & $l 12$ & $l 0$ & $l 4$ & $l 12$ \\
\cline { 2 - 8 } & 80 & 0.99 & 0.8192 & 0.5314 & 0.9969 & 0.9708 & 0.7755 \\
\hline \multirow{3}{*}{-0.9} & 200 & 0.9997 & 0.9469 & 0.7268 & 1 & 0.9970 & 0.9264 \\
\cline { 2 - 8 } & 200 & 0.9729 & 0.4780 & 0.1409 & 0.9736 & 0.8436 & 0.3735 \\
\hline \multirow{3}{*}{-0.2} & 80 & 0.1359 & 0.0558 & 0.0279 & 0.1598 & 0.0851 & 0.0423 \\
\cline { 2 - 8 } & 200 & 0.1262 & 0.0575 & 0.0425 & 0.1645 & 0.0861 & 0.0515 \\
\hline \multirow{3}{*}{0} & 80 & 0.0543 & 0.0418 & 0.0240 & 0.0505 & 0.0420 & 0.0325 \\
\cline { 2 - 8 } & 200 & 0.0526 & 0.0449 & 0.0389 & 0.0508 & 0.0467 & 0.0413 \\
\hline \multirow{2}{*}{0.2} & 80 & 0.0183 & 0.0308 & 0.0205 & 0.0112 & 0.0223 & 0.0246 \\
\cline { 2 - 8 } & 200 & 0.0144 & 0.0360 & 0.0351 & 0.0086 & 0.0251 & 0.0326 \\
\hline \multirow{2}{*}{0.9} & 80 & 0.00 & 0.0066 & 0.0038 & 0.00 & 0.0055 & 0.0050 \\
\cline { 2 - 8 } & 200 & 0.00 & 0.0002 & 0.0066 & 0.00 & 0.0001 & 0.0012 \\
\hline
\end{tabular}




\section{KHEDHIRI \& EL MONTASSER}

Chan, N. H., \& Wei, C. Z. (1988). Limiting distributions of least squares estimates of unstable autoregressive processes. Annals of Statistics, 16, 367-401.

Franses, P. H., Hylleberg, S., Lee, H. S. (1995). Spurious deterministic seasonality. Economics Letters, 48, 241-248.

Ghysels, E., \& Perron, P. (1993). The effect of seasonal adjustment filters on tests for a unit root. Journal of Econometrics, 55, 57-99.

Hylleberg, S., Engle, R. F., Granger, C. W. J., \&Yoo, B. S. (1990). Seasonal integration and cointegration. Journal of Econometrics, 44, 215-238.

Jin, S., \& Phillips, P. C. B. (2002). The KPSS test with seasonal dummies. Economics Letters, 77, 239-243.

Johansen, S., \& Schaumberg, E. (1999). Likelihood Analysis of Seasonal Cointegration. Journal of Econometrics, 54, 1-49.

Khedhiri, S., \& El Montasser, G. (2010), The effects of additive outliers on the seasonal KPSS test: A Monte Carlo analysis. Journal of Statistical Computation and Simulation, 80, 643-651.
Kwiatkowski, D., Phillips, P. C. B., Schmidt, P., \& Shin, Y. (1992). Testing the null hypothesis of stationarity against the alternative of a unit root. Journal of Econometrics, 54, 159178.

Lyhagen, J. (2006). The seasonal KPSS statistic. Economics Bulletin, 13, 1-9.

Miron, J. J. (1996). The Economics of Seasonal Cycles. Cambridge, MA: MIT Press.

Newey, W. K., \& West, K. D. (1987). A simple positive semi-definite, heteroscedasticity and autocorrelation consistent covariance matrix. Econometrica, 55, 703-708.

Nyblom, J. (1989). Testing for the constancy of parameters over time. Journal of the American Statistical Association, 84, 223230.

Taylor, A. M. R. (2003). Locally optimal tests against unit roots in seasonal time series. Journal of Time Series Analysis, 24, 591612. 


\title{
Gamma-Pareto Distribution and Its Applications
}

\author{
Ayman Alzaatreh \\ Felix Famoye Carl Lee \\ Austin Peay State University, Central Michigan University, \\ Clarksville, TN \\ Mount Pleasant, MI
}

A new distribution, the gamma-Pareto, is defined and studied and various properties of the distribution are obtained. Results for moments, limiting behavior and entropies are provided. The method of maximum likelihood is proposed for estimating the parameters and the distribution is applied to fit three real data sets.

Key words: Estimation, moments, T-X family, unimodality.

Introduction

The Pareto distribution was named after Swiss economist Vilfredo Pareto (1848-1923) who discovered it while studying distributions for modeling income in Switzerland. Since that time, the Pareto distribution has been widely used in modeling heavy-tailed distributions, such as income distribution. Many applications of the Pareto distribution in economics, biology and physics can be found throughout the literature. Burroughs and Tebbens (2001) discussed applications of the Pareto distribution in modeling earthquakes, forest fire areas and oil and gas field sizes, and Schroeder, et al. (2010) presented an application of the Pareto distribution in modeling disk drive sector errors. To add flexibility to the Pareto distribution, various generalizations of the distribution have been derived, including: the generalized Pareto distribution (Pickands, 1975), the beta-Pareto distribution (Akinsete, et al., 2008), and the beta generalized Pareto distribution (Mahmoudi, 2011).

Ayman Alzaatreh is an Assistant Professor in the Department of Mathematics \& Statistics. Email him at: alzaatreha@apsu.edu. Felix Famoye is a Professor in the Department of Mathematics. Email him at: felix.famoye@cmich.edu. Carl Lee is a Professor in the Department of Mathematics. Email him at: carl.lee@cmich.edu.
Let $F(x)$ be the cumulative distribution function (CDF) of any random variable $X$ and $r(t)$ be the probability density function (PDF) of a random variable, $T$, defined on $[0, \infty)$. The CDF of the $T-X$ family of distributions defined by Alzaatreh, et al. (2012) is given by

$$
G(x)=\int_{0}^{-\log (1-F(x))} r(t) d t .
$$

Alzaatreh, et al. (2012) named this family of distributions the Transformed-Transformer family (or $T-X$ family). When $X$ is a continuous random variable, the probability density function of the $T$ - $X$ family is

$$
\begin{aligned}
g(x) & =\frac{f(x)}{1-F(x)} r(-\log (1-F(x))) \\
& =h(x) r(H(x)) .
\end{aligned}
$$

If a random variable $T$ follows the gamma distribution with parameters $\alpha$ and $\beta$, then

$$
r(t)=\left(\beta^{\alpha} \Gamma(\alpha)\right)^{-1} t^{\alpha-1} e^{-t / \beta}, \quad t \geq 0,
$$

and the definition in (1.2) leads to the gamma- $X$ family with PDF 


$$
\begin{aligned}
& g(x)= \\
& \frac{f(x)(-\log (1-F(x)))^{\alpha-1}(1-F(x))^{\frac{1}{\beta}-1}}{\Gamma(\alpha) \beta^{\alpha}} .
\end{aligned}
$$

When $\beta=1$, the gamma- $X$ family in (1.3) reduces to

$$
g(x)=\frac{1}{\Gamma(\alpha)} f(x)(-\log (1-F(x)))^{\alpha-1} .
$$

Alzaatreh, et al. (2012) noted that, when $\alpha=n$ where $n$ is a positive integer, the distribution in (1.4) can be written as

$$
g(x)=\frac{1}{(n-1) !} f(x)(-\log (1-F(x)))^{n-1},
$$

which is the density function of the upper record values, $X_{U(n)}$, arising from a sequence $\left\{X_{i}\right\}_{i=1}^{n}$ of identically independent random variables with PDF $f(x)$ and CDF $F(x)$ (Johnson, et al., 1994).

The Gamma-Pareto Distribution

If $X$ is a Pareto random variable with density function $f(x)=k \theta^{k} / x^{k+1}, x>\theta$, then (1.3) results in

$$
g(x)=\frac{k^{\alpha}}{x \Gamma(\alpha) \beta^{\alpha}}\left(\frac{\theta}{x}\right)^{k / \beta}\left(\log \left(\frac{x}{\theta}\right)\right)^{\alpha-1},
$$$$
x>\theta \text {. }
$$

Setting $\beta / k=c,(2.1)$ reduces to

$$
\begin{aligned}
& g(x)=\frac{1}{x \Gamma(\alpha) c^{\alpha}}\left(\frac{\theta}{x}\right)^{1 / c}\left(\log \left(\frac{x}{\theta}\right)\right)^{\alpha-1}, \\
& \alpha, c, \theta>0 ; x>\theta
\end{aligned}
$$

A random variable $X$ with the PDF $g(x)$ in (2.2) is said to follow the gamma-Pareto distribution. When $\alpha=1$ the gamma-Pareto distribution reduces to the Pareto distribution with parameters $1 / c$ and $\theta$, and when $\alpha=n+1$, it reduces to the upper record value distribution arising from Pareto identically independent random variables (Ahsanullah \& Houchens, 1989). From (2.2), the CDF of the gammaPareto distribution is obtained as

$$
G(x)=\gamma\left\{\alpha, c^{-1} \log (x / \theta)\right\} / \Gamma(\alpha),
$$

where $\gamma(\alpha, t)=\int_{0}^{t} u^{\alpha-1} e^{-u} d u$ is the incomplete gamma function.

Johnson, et al. (1994) discussed different types of Pareto distributions and their CDFs. These are

$$
\begin{array}{ll}
\text { Pareto } & F(x)=1-\frac{\theta^{a}}{(x+\theta)^{a}}, x>0 . \\
\text { II: } & \\
\text { Pareto } & F(x)=1-\frac{\theta e^{-b x}}{(x+\theta)^{a}}, x>0 . \\
\text { III: } & \\
\text { Pareto } & F(x)=1-\left[1+\left(\frac{x-\mu}{\sigma}\right)^{1 / \gamma}\right]^{-a}, x>\mu . \\
\text { IV: } &
\end{array}
$$

Using equation (1.3), the corresponding PDF of gamma-Pareto II, gamma-Pareto III, and gamma-Pareto IV can be written as shown in Table 1.

Some relationships among these distributions are:

- If $Y$ follows the gamma-Pareto distribution in (2.2), then the translation $X=Y-\theta$ follows the gamma-Pareto II distribution.

- When $a=1$ and $b=0$, the gamma-Pareto III distribution reduces to the gamma-Pareto II distribution. 
Table 1: Corresponding PDFs of gamma-Pareto Distributions

gamma-Pareto II: $\quad g(x)=\frac{\theta^{1 / c}}{c^{\alpha} \Gamma(\alpha)}(x+\theta)^{-1-1 / c}[\log (1+x / \theta)]^{\alpha-1}, x>0$, where $c=\frac{\beta}{a}$.

gamma-Pareto III: $\quad g(x)=\frac{1}{\beta^{\alpha} \Gamma(\alpha)}\left(b+\frac{a}{x+\theta}\right)\left[\frac{\theta e^{-b x}}{(x+\theta)^{a}}\right]^{1 / \beta}\left[-\log \left(\frac{\theta e^{-b x}}{(x+\theta)^{a}}\right)\right]^{\alpha-1}, x>0$.

gamma-Pareto IV: $\quad g(x)=\frac{1}{\gamma \sigma c^{\alpha} \Gamma(\alpha)}\left(\frac{x-\mu}{\sigma}\right)^{-1+1 / \gamma}\left(1+\left(\frac{x-\mu}{\sigma}\right)^{1 / \gamma}\right)^{-1-1 / c}\left[\log \left(1+\left(\frac{x-\mu}{\sigma}\right)^{1 / \gamma}\right]^{\alpha-1}\right.$,

$x>\mu$, where $c=\beta / a$.

- When $\gamma=1$ and $\mu=\sigma=\theta$, the gammaPareto IV distribution reduces to the gamma-Pareto distribution in (2.2) with parameters $\alpha, c$ and $\theta$.

- When $\gamma=1$ and $\mu=0$, the gamma-Pareto IV distribution reduces to the gamma-Pareto II distribution.

Properties of the gamma-Pareto distribution

The following Lemma shows the relationship between the gamma-Pareto distribution and the gamma distribution.

Lemma 1

If a random variable $Y$ follows the gamma distribution with parameters $\alpha$ and $c$, then the random variable $X=\theta e^{Y}$ follows the gamma-Pareto distribution with parameters $\alpha$, $c$ and $\theta$.
Lemma 1 Proof

The result follows by using the transformation technique.

The hazard function associated with the gamma-Pareto distribution is

$$
\begin{aligned}
h(x) & =\frac{g(x)}{1-G(x)} \\
& =\frac{\theta^{1 / c}(\log (x / \theta))^{\alpha-1}}{x^{1+1 / c} c^{\alpha}\left(\Gamma(\alpha)-\gamma\left\{\alpha, c^{-1} \log (x / \theta)\right\}\right)}, \\
x & >\theta
\end{aligned}
$$

and the limiting behaviors of the gamma-Pareto PDF and the hazard function are given in the following theorem.

Theorem 1

The limit of the gamma-Pareto density function and the gamma-Pareto hazard function 
as $x \rightarrow \infty$ is 0 and the limit as $x \rightarrow \theta^{+}$is given by

$$
\lim _{x \rightarrow \theta^{+}} g(x)=\lim _{x \rightarrow \theta^{+}} h(x)= \begin{cases}0, & \alpha>1 \\ 1 /(c \theta), & \alpha=1 \\ \infty, & \alpha<1\end{cases}
$$

Theorem 1 Proof

First it can be shown that $\lim _{x \rightarrow \infty} g(x)=0$. If $\alpha \leq 1$, then from definition (2.2) $\lim _{x \rightarrow \infty} g(x)=0$, and if $\alpha>1$, then

$$
\begin{aligned}
& \lim _{x \rightarrow \infty} g(x) \\
& =\lim _{x \rightarrow \infty} \frac{(\theta / x)^{1 / c}}{\Gamma(\alpha) c^{\alpha}} \times \lim _{x \rightarrow \infty}\left(\log \left(\frac{x}{\theta}\right)\right)^{\alpha-1} \frac{1}{x} \\
& =0 \times \lim _{x \rightarrow \infty}\left(\log \left(\frac{x}{\theta}\right) / x^{1 /(\alpha-1)}\right)^{\alpha-1} .
\end{aligned}
$$

Using L'Hôpital's rule, it can be shown that

$$
\lim _{x \rightarrow \infty}\left(\log (x / \theta) / x^{1 /(\alpha-1)}\right)^{\alpha-1}=0 .
$$

To show that $\lim _{x \rightarrow \infty} h(x)=0$, we have $\lim _{x \rightarrow \infty} h(x)=\lim _{x \rightarrow \infty} g(x) /(1-G(x)) . \quad$ Because $\lim _{x \rightarrow \infty} g(x)=0$, L'Hôpital's rule can be applied and implies that

$$
\begin{aligned}
\lim _{x \rightarrow \infty} h(x) & =\lim _{x \rightarrow \infty} g^{\prime}(x) / g(x) \\
& =\lim _{x \rightarrow \infty}\left\{\frac{\alpha-1}{x \log (x / \theta)}-\frac{c+1}{c x}\right\} \\
& =0 .
\end{aligned}
$$

The result in (3.2) follows directly from the definition of (2.2) and $h(x)=g(x) /(1-G(x))$. The following theorem shows that the gammaPareto distribution is unimodal.
Theorem 2

The gamma-Pareto distribution has a unique mode at $x=x_{0}$. When $\alpha \leq 1$ the mode is $x_{0}=\theta$ and when $\alpha>1$ the mode is $x_{0}=\theta \mathrm{e}^{c(\alpha-1) /(c+1)}$.

Theorem 2 Proof

The derivative with respect to $x$ of equation (2.2) is given by

$$
\begin{aligned}
& g^{\prime}(x)=\theta^{1 / c} x^{2-1 / c} \Gamma(\alpha) \\
& \quad \times\left(\log \left(\frac{x}{\theta}\right)\right)^{\alpha-2}\left\{c(\alpha-1)-(c+1) \log \left(\frac{x}{\theta}\right)\right\} .
\end{aligned}
$$

From (3.3) the critical points of $g(x)$ are $x=\theta$ and $x=\theta \mathrm{e}^{c(\alpha-1) /(c+1)}$. For $\alpha \leq 1$, it may be observed from (3.3) that $g^{\prime}(x)<0$, therefore $g(x)$ is strictly decreasing. Also, from Theorem 1, $\lim _{x \rightarrow \theta^{+}} g(x)=1 /(c \theta) \quad$ when $\quad \alpha=1$ and $\lim _{x \rightarrow \theta^{+}} g(x)=\infty$ when $\alpha<1$. Thus, $g(x)$ has a unique mode at $x=\theta$. Using Theorem 1, for $\alpha>1, \quad \lim _{x \rightarrow \theta^{+}} g(x)=0$ implies that $x=\theta$ cannot be a modal point, hence the mode is $x_{0}=\theta \mathrm{e}^{c(\alpha-1) /(c+1)}$.

Graphs of $g(x)$ and $h(x)$ are displayed in Figures 1-3. The plots show that the gamma-Pareto PDF has a very long right tail and also that when parameters $c$ and $\theta$ increase, the peak of the distribution decreases. In addition, the graphs of the gamma-Pareto PDF indicate that $g(x)$ is a right skewed distribution. The plots in Figure 3 illustrate that the gamma-Pareto hazard function is either monotone decreasing or upside-down bathtub.

The entropy of a random variable $X$ is a measure of variation of uncertainty (Rényi, 1961). Shannon entropy (Shannon, 1948) for a random variable $X$ with PDF $g(x)$ is defined as $E\{-\log (g(X))\}$. Shannon showed important applications of this entropy in communication theory and many applications have been used in 
GAMMA-PARETO DISTRIBUTION AND ITS APPLICATIONS

Figure 1: The gamma-Pareto PDF for Various Values of $\alpha, c$ and $\theta$

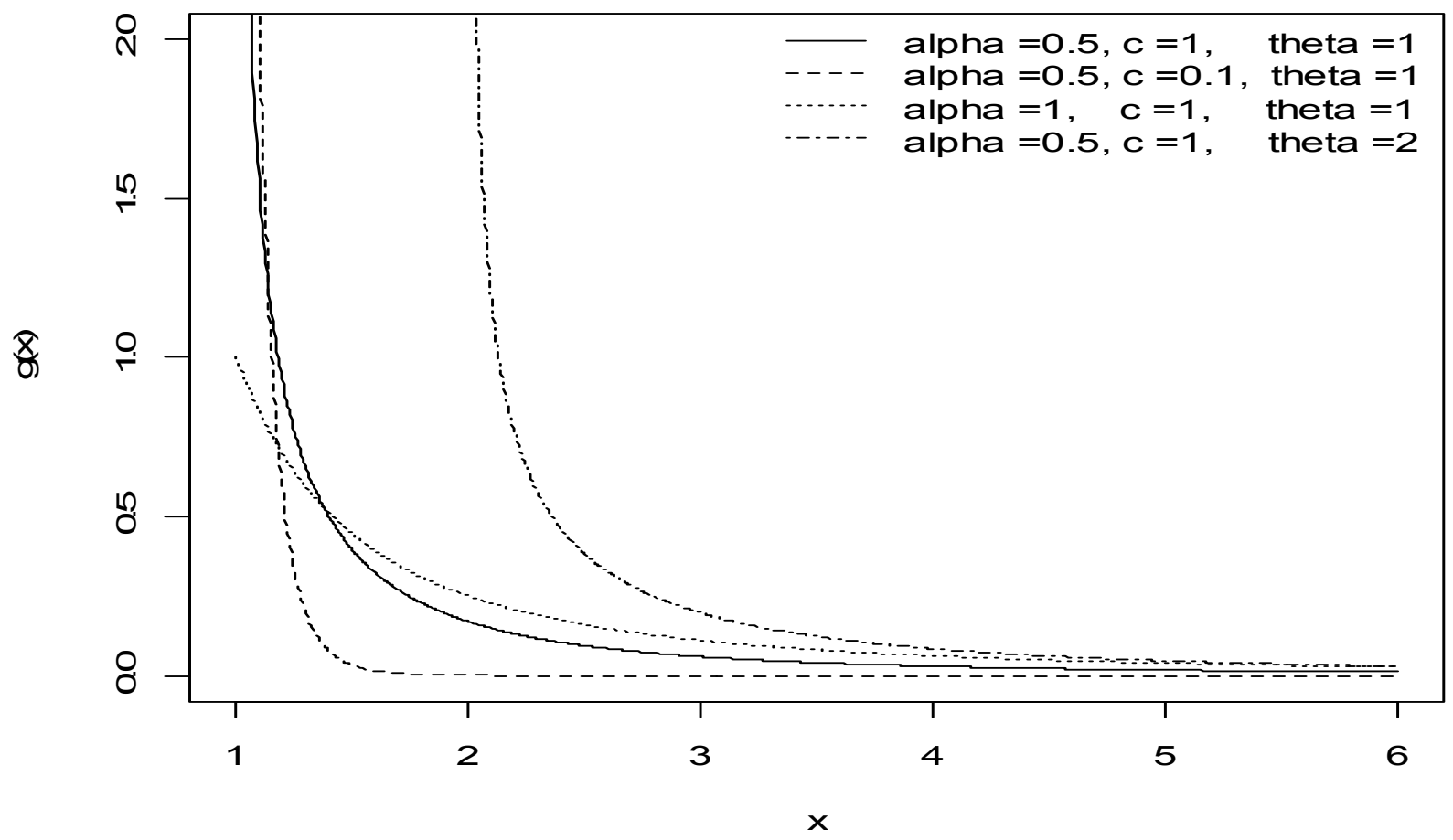

Figure 2: The gamma-Pareto PDF for Various Values of $\alpha, c$ and $\theta$

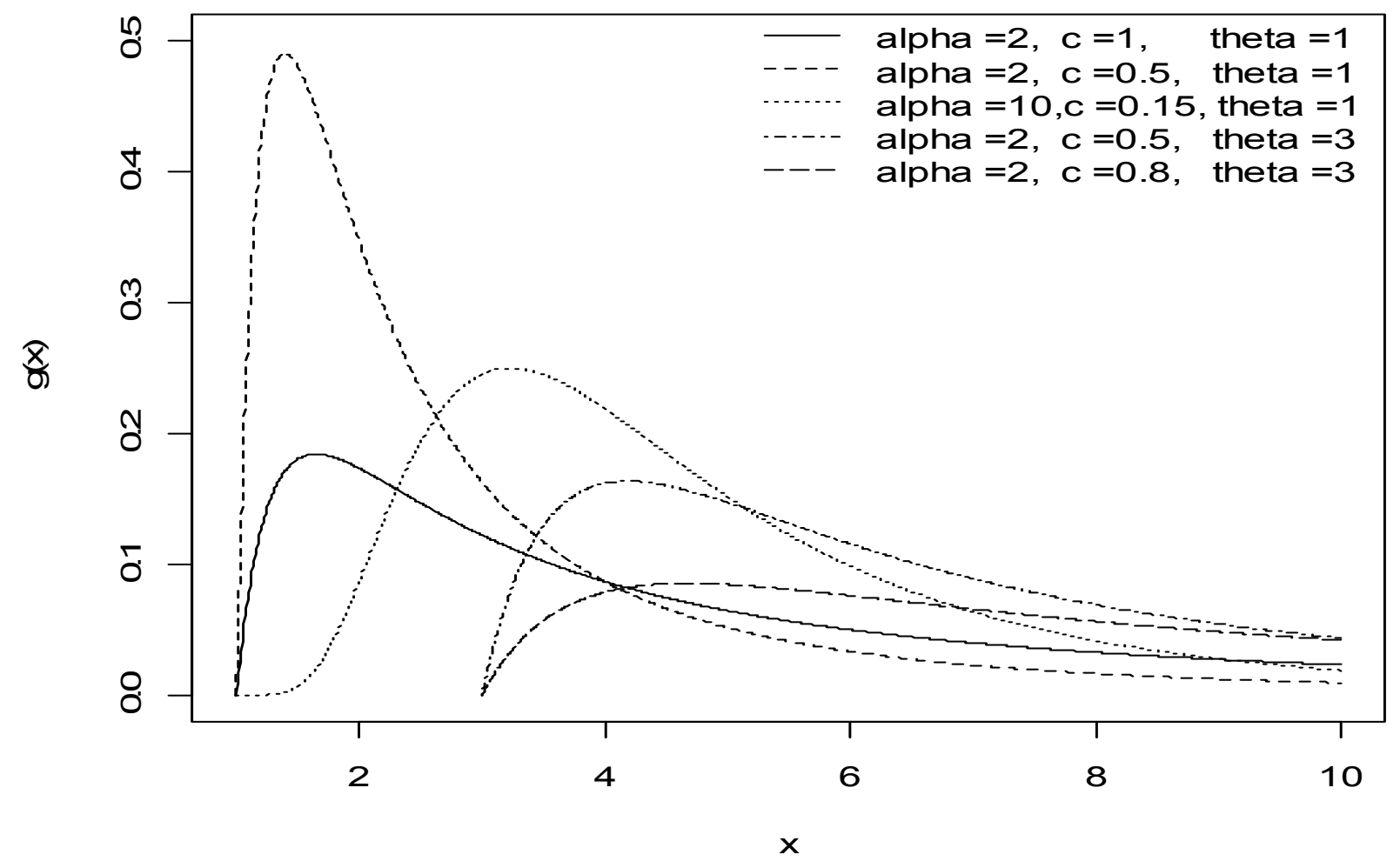


Figure 3: The gamma-Pareto Hazard Function for Various Values of $\alpha, c$ and $\theta$

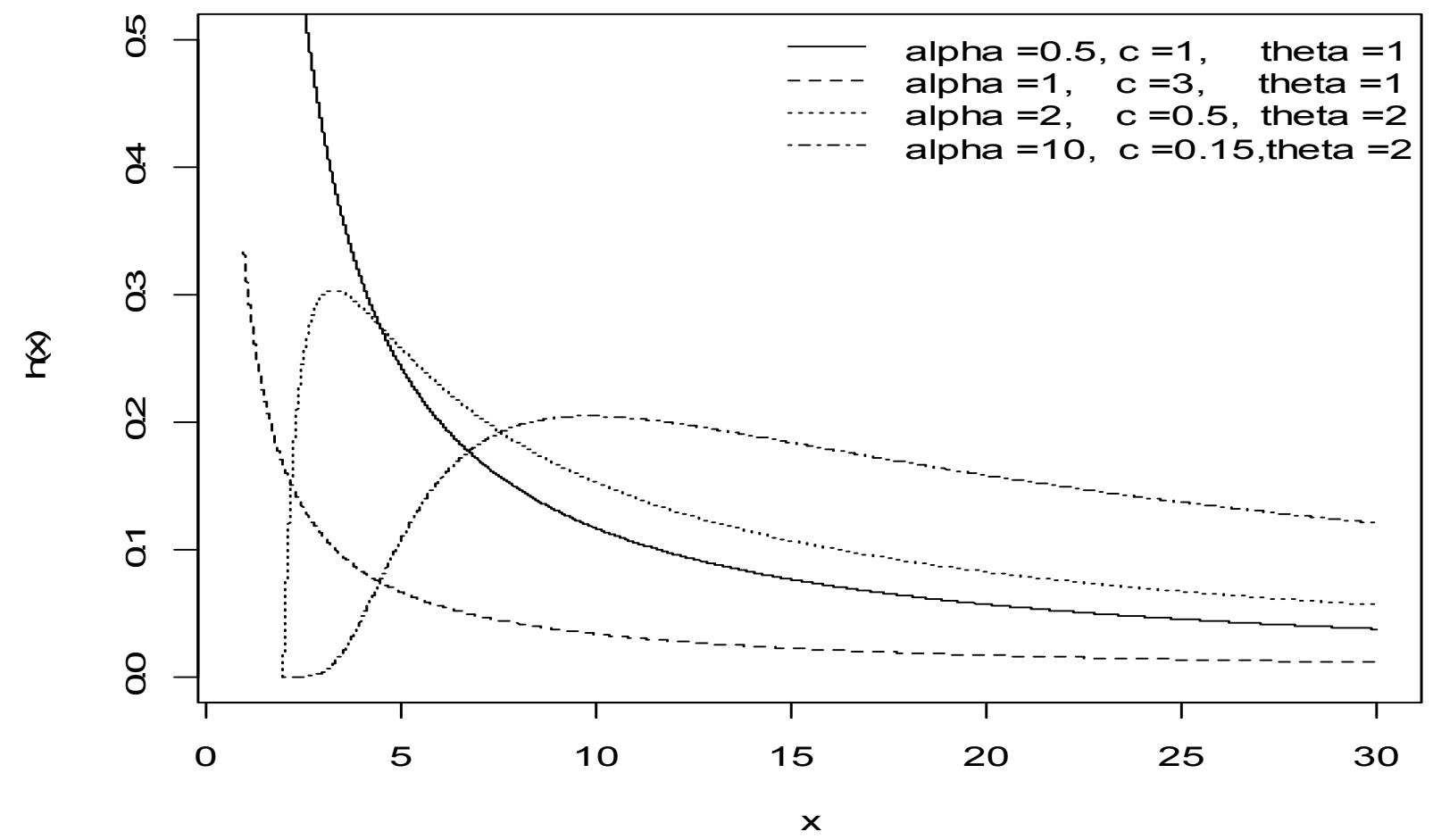

different areas such as engineering, physics, biology and economics.

Lemma 2

The Shannon entropy of the gamma- $X$ family of distributions is given by

$$
\begin{aligned}
\eta_{X}= & -E\left\{\log f\left(F^{-1}\left(1-e^{-T}\right)\right)\right\}+\alpha(1-\beta) \\
& +\log \beta+\log \Gamma(\alpha)+(1-\alpha) \psi(\alpha),
\end{aligned}
$$

where $\psi$ is the digamma function and $T$ is the gamma random variable with parameters $\alpha$ and $\beta$.

\section{Lemma 2 Proof} details.

See Alzaatreh, et al. (2012) for proof

\section{Theorem 3}

The Shannon entropy for the gammaPareto distribution is given by

$$
\begin{aligned}
\eta_{X}= & \log c+\log \theta+\log \Gamma(\alpha) \\
& +\alpha(c+1)+(1-\alpha) \psi(\alpha) .
\end{aligned}
$$

Theorem 3 Proof

$$
\begin{gathered}
\text { First it is necessary to find } \\
-E\left\{\log f\left(F^{-1}\left(1-e^{-T}\right)\right)\right\}, \\
f(x)=k \theta^{k} / x^{k+1} \text {. It can be shown that } \\
F^{-1}(x)=\theta(1-x)^{-1 / k} \text {, thus } \\
-E\left\{\log f\left(F^{-1}\left(1-e^{-T}\right)\right)\right\}= \\
\log \theta-\log k+(1+1 / k) E(T)
\end{gathered}
$$

The result follows from Lemma 2 by noting that $E(T)=\alpha \beta$ and $c=\beta / k$ (see equation 2.1).

The Rényi (1961) entropy for the random variable $X$ with PDF $g(x)$ is defined as 


$$
I_{R}(s)=\frac{1}{1-s} \log \left\{\int g^{s}(x) d x\right\}, s>0, s \neq 1 .
$$

By using the gamma-Pareto PDF in (2.2), we have

$$
\begin{aligned}
\int_{\theta}^{\infty} g^{s}(x) d x & =c^{-s \alpha} \Gamma^{-s}(\alpha) \\
\times \int_{\theta}^{\infty} x^{-s}\left(\frac{\theta}{x}\right)^{s / c} & \left.\log \left(\frac{x}{\theta}\right)\right]^{s(\alpha-1)} d x .
\end{aligned}
$$

Substituting $u=\log (x / \theta)$, (3.6) can be rewritten as

$$
\int_{\theta}^{\infty} g^{s}(x) d x=\frac{\theta^{1-s} \Gamma(s(\alpha-1)+1)}{c^{s \alpha} \Gamma^{s}(\alpha)[s(1+1 / c)-1]^{s(\alpha-1)+1}} .
$$

Using equation (3.7), the Rényi entropy in (3.5) can be written as

$$
I_{R}(s)=\log \theta-\frac{1}{1-s}\left\{\begin{array}{l}
s \alpha \log c+s \log \Gamma(\alpha) \\
-\log \Gamma(\xi)+\xi \log \left(s\left(1+c^{-1}\right)\right.
\end{array}\right\}
$$

where $\xi=s(\alpha-1)+1$. Shannon entropy is a special case of Rényi entropy obtained by taking the limit of Rényi entropy as $s \rightarrow 1$. The result in (3.4) follows by using the L'Hôpital's rule for evaluating the limit of equation (3.8) as $s \rightarrow 1$.

Moments and Mean Deviations

The non-central moments for the gamma-Pareto distribution in (2.2) can be written as

$$
\begin{aligned}
E\left(X^{r}\right) & =c^{-\alpha} \Gamma^{-1}(\alpha) \\
& \times \int_{\theta}^{\infty} x^{r-1}\left(\frac{\theta}{x}\right)^{1 / c}\left[\log \left(\frac{x}{\theta}\right)\right]^{\alpha-1} d x .
\end{aligned}
$$

Substituting $u=\log (x / \theta)$ reduces (4.1) to

$$
E\left(X^{r}\right)=\theta^{r}(1-r c)^{-\alpha}, \quad c<1 / r .
$$

Hence, the mean for the gamma-Pareto distribution is

$$
\mu=\theta(1-c)^{-\alpha}, \quad c<1
$$

Note that when $\alpha=1$ in equation (4.2), $E\left(X^{r}\right)=\theta^{r}(1-r c)^{-1}$ which represents the noncentral moments for the Pareto distribution with parameters $1 / c$ and $\theta$.

Using the binomial expansion for $(X-\mu)^{r}$, the central moments $E(X-\mu)^{r}$ for any random variable $X$ can be written as

$$
E(X-\mu)^{r}=\sum_{k=0}^{r}\left(\begin{array}{l}
r \\
k
\end{array}\right)(-1)^{r-k} \mu^{r-k} E\left(X^{k}\right) .
$$

Using equations (4.2) and (4.3), the central moments for the gamma-Pareto random variable $X$ can be simplified to

$$
\begin{aligned}
& E(X-\mu)^{r}= \\
& \theta^{r} \sum_{k=0}^{r}\left(\begin{array}{l}
r \\
k
\end{array}\right)(-1)^{r-k}(1-c)^{(k-r) \alpha}(1-k c)^{-\alpha} .
\end{aligned}
$$

Note that equation (4.4) indicates that the central moments of the gamma-Pareto distribution is an increasing function of $\theta$. Using (4.4), the variance, the skewness and the kurtosis for the gamma-Pareto distribution are respectively expressed as

$$
\sigma^{2}=\theta^{2}\left[(1-2 c)^{-\alpha}-(1-c)^{-2 \alpha}\right], c<0.5
$$

$\gamma_{1}=\frac{(1-3 c)^{-\alpha}+2(1-c)^{-3 \alpha}-3(1-2 c)^{-\alpha}(1-c)^{-\alpha}}{\left((1-2 c)^{-\alpha}-(1-c)^{-2 \alpha}\right)^{3 / 2}}$ 


$$
\begin{aligned}
\gamma_{2}= & {\left[(1-2 c)^{-\alpha}-(1-c)^{-2 \alpha}\right]^{-2}\left\{(1-4 c)^{-\alpha}\right.} \\
& -3(1-c)^{-4 \alpha}+6(1-2 c)^{-\alpha}(1-c)^{-2 \alpha} \\
& \left.-4(1-3 c)^{-\alpha}(1-c)^{-\alpha}\right\}
\end{aligned}
$$

Equations (4.6) and (4.7) show that the skewness and the kurtosis are free of $\theta$. Theorem 4 shows that when $\alpha \geq 1$ (or $\alpha<1$ ), the non-central moments of gamma-Pareto distribution is bounded below (or above) by the non-central moments of the Pareto distribution.

Theorem 4

Let $X$ be a random variable that follows the gamma-Pareto distribution. If $\alpha \geq 1$, then $E\left(X^{s}\right) \geq \theta^{r} /(1-r c)$ and if $\alpha<1$, then $E\left(X^{s}\right)<\theta^{r} /(1-r c)$.
Theorem 4 Proof

$$
\text { Because } 0<r c<1 \text { or } 0<1-r c<1
$$

this implies that for $\alpha \geq 1,(1-r c)^{\alpha} \leq 1-r c$ and for $\alpha<1,(1-r c)^{\alpha}>1-r c$. Thus, if $\alpha \geq 1$, then $\theta^{r}(1-r c)^{-\alpha} \geq \theta^{r}(1-r c)^{-1}$ and if $\alpha<1$, then $\theta^{r}(1-r c)^{-\alpha}<\theta^{r}(1-r c)^{-1}$.

Table 2 provides the mode, mean, variance, skewness and kurtosis of the gammaPareto distribution for various values of $\alpha$ and $c$ when $\theta=1$. For fixed $\alpha$ and $\theta$, the mean, variance, skewness and kurtosis are increasing functions of $c$. For fixed $c$ and $\theta$, the mean, median and variance are increasing functions of $\alpha$. When $\alpha>1$, the following trends for the mode are observed: (1) it increases as $c$ increases with fixed $\alpha$ and $\theta$, and (2) it increases as $\alpha$ increases with fixed $c$ and $\theta$. Table 2 also shows that the skewness is always positive and for fixed $\alpha$ and it increases rapidly as $c$ increases.

Table 2: Mode, Mean, Variance, Skewness and Kurtosis

for Some Values of $\alpha$ and $c$ with $\theta=1$ (*: Undefined)

\begin{tabular}{|c|c|c|c|c|c|c|}
\hline$\alpha$ & $c$ & Mode & Mean & Variance & Skewness & Kurtosis \\
\hline \multirow{4}{*}{0.5} & 0.1 & 1 & 1.0541 & 0.0069 & 3.6850 & 27.6334 \\
\cline { 2 - 7 } & 0.2 & 1 & 1.1180 & 0.0410 & 5.5537 & 95.1825 \\
\cline { 2 - 7 } & 0.3 & 1 & 1.1952 & 0.1526 & 15.2326 & $*$ \\
\cline { 2 - 7 } & 0.4 & 1 & 1.2910 & 0.5694 & $*$ & $*$ \\
\hline \multirow{4}{*}{1} & 0.1 & 1 & 1.1111 & 0.0154 & 2.8111 & 17.8286 \\
\cline { 2 - 8 } & 0.2 & 1 & 1.2500 & 0.1042 & 4.6476 & 73.8000 \\
\cline { 2 - 8 } & 0.3 & 1 & 1.4286 & 0.4592 & 16.4438 & $*$ \\
\cline { 2 - 8 } & 0.4 & 1 & 1.6667 & 2.2222 & $*$ & $*$ \\
\hline \multirow{4}{*}{2} & 0.1 & 1.0952 & 1.2346 & 0.0383 & 2.2819 & 13.2512 \\
\cline { 2 - 8 } & 0.2 & 1.1814 & 1.5625 & 0.3364 & 4.4009 & 77.3004 \\
\cline { 2 - 8 } & 0.3 & 1.2596 & 2.0408 & 2.0851 & 26.1507 & $*$ \\
\cline { 2 - 8 } & 0.4 & 1.3307 & 2.7778 & 17.2840 & $*$ & $*$ \\
\hline \multirow{3}{*}{3} & 0.1 & 1.1994 & 1.3717 & 0.0714 & 2.1075 & 12.0304 \\
\cline { 2 - 7 } & 0.2 & 1.3956 & 1.9531 & 0.8149 & 4.6209 & 98.2327 \\
\cline { 2 - 7 } & 0.3 & 1.5865 & 2.9155 & 7.1251 & 47.9991 & $*$ \\
\cline { 2 - 7 } & 0.4 & 1.7708 & 4.6296 & 103.5670 & $*$ & $*$ \\
\hline
\end{tabular}




\section{GAMMA-PARETO DISTRIBUTION AND ITS APPLICATIONS}

The deviation from the mean and the median are used to measure the dispersion and spread in a population from the center. If the median is denoted by $M$, then the mean deviation from the mean , $D(\mu)$, and the mean deviation from the median, $D(M)$, can be written as

$$
\begin{aligned}
D(\mu) & =\int_{\theta}^{\mu}(\mu-x) g(x) d x+\int_{\mu}^{\infty}(x-\mu) g(x) d x \\
& =2 \int_{\theta}^{\mu}(\mu-x) g(x) d x \\
& =2 \mu G(\mu)-2 \int_{\theta}^{\mu} x g(x) d x .
\end{aligned}
$$

$$
\begin{aligned}
D(M) & =\int_{\theta}^{M}(M-x) g(x) d x+\int_{M}^{\infty}(x-M) g(x) d x \\
& =2 \int_{\theta}^{M}(M-x) g(x) d x+E(X)-M \\
& =2 M G(M)+\mu-M-2 \int_{\theta}^{M} x g(x) d x \\
& =\mu-2 \int_{\theta}^{M} x g(x) d x .
\end{aligned}
$$

Consider the integral

$$
\begin{aligned}
& \int_{\theta}^{m} x g(x) d x= \\
& \frac{1}{c^{\alpha} \Gamma(\alpha)} \int_{\theta}^{m}(\theta / x)^{1 / c}(\log (x / \theta))^{\alpha-1} d x .
\end{aligned}
$$

Using the substitution $u=\log (x / \theta)$, the equation (4.8) can be written as

$$
\int_{\theta}^{m} x g(x) d x=\frac{\mu}{\Gamma(\alpha)} \gamma\left[\alpha,\left(c^{-1}-1\right) \log (m / \theta)\right],
$$

and by using equations (2.3) and (4.9), the mean deviation from the mean is

$$
D(\mu)=\frac{2 \mu\left\{\begin{array}{l}
\left\{\gamma\left[\alpha, c^{-1} \log (\mu / \theta)\right]\right. \\
-\gamma\left[\alpha,\left(c^{-1}-1\right) \log (\mu / \theta)\right]
\end{array}\right\}}{\Gamma(\alpha)}, c<1,
$$

and the mean deviation from the median is

$D(M)=\mu\left\{1-\frac{2}{\Gamma(\alpha)} \gamma\left[\alpha,\left(\frac{1}{c}-1\right) \log \left(\frac{M}{\theta}\right)\right]\right\}, c<1$.

Parameter Estimation

When $\alpha<1$, the likelihood function for the gamma-Pareto distribution goes to infinity as $\theta$ approaches the sample minimum $x_{(1)}$; thus, when $\alpha<1$ and $\theta$ is estimated by $x_{(1)}$, no MLE for $\alpha$ and $c$ exists. A similar problem was studied by Smith (1985) who proposed an alternative approach for estimating the parameters as follows: If sample data $x_{1}, x_{2}, \ldots, x_{n}$ are observed, estimate the parameter $\theta$ by the sample minimum $x_{(1)}$ and then use the MLE method to estimate $\alpha$ and $c$ by excluding the sample minimum.

Applying Smith's method to obtain the MLE for the gamma-Pareto parameters, the loglikelihood function for the gamma-Pareto distribution is given by

$$
\begin{aligned}
& \log L(\alpha, c)=\sum_{x_{i} \neq x_{(1)}} \log g\left(x_{i} ; x_{(1)}, \alpha, c\right) \\
& =\sum_{x_{i} \neq x_{(1)}}\left\{-\alpha \log c-\log \Gamma(\alpha)-\log x_{(1)}-\left(1+c^{-1}\right)\right. \\
& \left.\quad \times \log \left(x_{i} / x_{(1)}\right)+(\alpha-1) \log \left(\log \left(x_{i} / x_{(1)}\right)\right)\right\} .
\end{aligned}
$$

The derivatives of (5.1) with respect to $\alpha$ and $c$ are given by

$$
\frac{\partial \log L}{\partial \alpha}=\sum_{x_{i} \neq x_{(1)}}\left\{-\log c-\psi(\alpha)-\log \left(\log \left(\frac{x_{i}}{x_{(1)}}\right)\right)\right\}
$$




$$
\frac{\partial \log L}{\partial c}=\sum_{x_{i} \neq x_{(1)}}\left\{\frac{-\alpha}{c}+\frac{1}{c^{2}} \log \left(\frac{x_{i}}{x_{(1)}}\right)\right\} .
$$

Setting (5.3) to zero and simplifying results in

$$
c=\frac{1}{\left(n-n^{\prime}\right) \alpha} \sum_{x_{i} \neq x_{(1)}} \log \left(x_{i} / x_{(1)}\right),
$$

where $n^{\prime}$ is the frequency of $x_{(1)}$. Equation (5.4) can be written as

$$
c=\frac{1}{\alpha} m_{1}^{*},
$$

where $m_{1}^{*}=\sum_{x_{i} \neq x_{(1)}} \log \left(x_{i} / x_{(1)}\right) /\left(n-n^{\prime}\right)$ is the sample mean for $\log \left(x_{i} / x_{(1)}\right)$ after excluding $x_{(1)}$.

Setting (5.2) to zero and using equation (5.5) results in

$$
\psi(\alpha)-\log (\alpha)+\log \left(m_{1}^{*}\right)-m_{2}^{*}=0,
$$

where $m_{2}{ }^{*}=\sum_{x_{i} \neq x_{(1)}} \log \left(\log \left(x_{i} / x_{(1)}\right)\right) /\left(n-n^{\prime}\right)$ is the sample mean for $\log \left(\log \left(x_{i} / x_{(1)}\right)\right)$ after excluding $x_{(1)}$. The MLE $\hat{\alpha}$ of $\alpha$ is the solution of equation (5.6) and the MLE $\hat{c}$ of $c$ can be determined by substituting the estimate $\hat{\alpha}$ in equation (5.5).

The initial values for the parameters $\alpha$ and $c$ can be obtained by assuming the random sample $Y_{i}=\log \left(X_{i} / x_{(1)}\right), i=1, \ldots, n$ are taken from the gamma distribution with parameters $\alpha$ and $c$. By equating the population mean and the population variance of gamma distribution (with parameters $\alpha$ and $c$ ) to the corresponding sample mean and sample variance of $y_{i}, i=1, \ldots, n$ and then solving for $\alpha$ and $c$, the initial values are $c_{0}=s_{y}^{2} / \bar{y}$ and $\alpha_{0}=\bar{y}^{2} / s_{y}^{2}$, where $s_{y}^{2}$ and $\bar{y}$ are the sample variance and the sample mean for $y_{1}, y_{2}, \ldots$, $y_{n}$.

\section{Lemma 3}

The Fisher information matrix for the gamma-Pareto distribution when $\theta$ is known is given by

$$
I=n\left[\begin{array}{ll}
\psi^{\prime}(\alpha) & 1 / c \\
1 / c & \alpha / c^{2}
\end{array}\right]
$$

Lemma 3 Proof

The Fisher information matrix is defined by $I=\left[I_{i j}\right] \quad$ with $I_{i j}=E\left\{\frac{-\partial^{2}}{\partial \tau_{i} \partial \tau_{j}} \log \left(L\left(x_{i}, \vec{\tau}\right)\right)\right\}$, where $\tau_{1}=\alpha$ and $\tau_{2}=c$. To find $I$, the second derivatives of $\log L(\alpha, c)=\sum_{i=1}^{n} \log g\left(x_{i} ; \theta, \alpha, c\right), \quad$ are needed. These can be obtained from the derivatives of (5.2) and (5.3), where $x_{(1)}$ is replaced by $\theta$ and the sums are taken form $i=1$ to $n$. The second derivatives of $\log L(\alpha, c)$ can be written as

$$
\begin{aligned}
& \partial^{2} \log L / \partial \alpha^{2}=-n \psi^{\prime}(\alpha), \\
& \partial^{2} \log L / \partial \alpha \partial c=-n / c
\end{aligned}
$$

and

$$
\partial^{2} \log L / \partial c^{2}=n \alpha c^{-2}-2 c^{-3} \sum_{i=1}^{n} \log \left(x_{i} / \theta\right) .
$$

From Lemma 1, it may be concluded that $E(\log (X / \theta))=\alpha c$. The results of (5.7) follow from taking the negative expected values of the second derivatives.

Theorem 5

The variance-covariance matrix for the gamma-Pareto distribution when $\theta$ is known is given by 


\section{GAMMA-PARETO DISTRIBUTION AND ITS APPLICATIONS}

$$
\Sigma=\frac{1}{n\left(\alpha \psi^{\prime}(\alpha)-1\right)}\left[\begin{array}{cc}
\alpha & -c \\
-c & c^{2} \psi^{\prime}(\alpha)
\end{array}\right]
$$

\section{Theorem 5 Proof}

The result follows by taking the inverse of the Fisher information matrix in (5.7). From (5.8), the variance of the ML estimates $\hat{\alpha}$ and $\hat{c}$ are respectively given by

$$
\begin{aligned}
\operatorname{var}(\hat{\alpha})= & \frac{\alpha}{n\left(\alpha \psi^{\prime}(\alpha)-1\right)} \\
& \text { and } \\
\operatorname{var}(\hat{c})= & \frac{c^{2} \psi^{\prime}(\alpha)}{n\left(\alpha \psi^{\prime}(\alpha)-1\right)} .
\end{aligned}
$$

From (5.9), the variance of $\hat{\alpha}$ does not depend on the parameter $c$. Also, as $c$ increases the variance of $\hat{c}$ increases. Using the approximation $\quad \psi^{\prime}(\alpha) \simeq \alpha^{-1}+\frac{1}{2} \alpha^{-2}+\frac{1}{6} \alpha^{-3}$ (Johnson, et al., 1994, page 357), equations (5.9) can be approximated as

$$
\begin{gathered}
\operatorname{var}(\hat{\alpha}) \simeq \frac{6 \alpha^{3}}{n(3 \alpha+1)} \\
\text { and } \\
\operatorname{var}(\hat{c}) \simeq \frac{c^{2}\left(6 \alpha^{2}+3 \alpha+1\right)}{n \alpha(3 \alpha+1)} .
\end{gathered}
$$

Based on the Central Limit Theorem, $(\hat{\alpha}-\alpha) / \operatorname{se}(\hat{\alpha}) \stackrel{d}{\longrightarrow} N(0,1) \quad$ and $(\hat{c}-c) / \operatorname{se}(\hat{c}) \stackrel{d}{\longrightarrow} N(0,1)$ where $\operatorname{se}(\hat{\alpha})$ and $\operatorname{se}(\hat{c})$ are the standard errors of $\hat{\alpha}$ and $\hat{c}$ respectively.

In the following, the uniformly minimum variance unbiased estimator (UMVUE) is derived for the parameter $c$ assuming that the parameters $\alpha$ and $\theta$ are known. The following theorem by Lehmann \& Scheffé (1950) is needed in order to find the UMVUE for the parameter $c$.
Theorem 6

Let $X_{1}, X_{2}, \ldots, X_{n}$ be a random sample from PDF $g(x, \beta), \beta \in \Omega$. Let $T$ be a sufficient statistic for $\beta$ and let the family $\{g(T, \beta), \beta \in \Omega\} \quad$ of probability density functions be complete. If there is a function of $T$ that is an unbiased estimator of $\beta$, then this function of $T$ is the UMVUE of $\beta$.

Lemma 4

If $\alpha$ and $\theta$ are known for the gammaPareto distribution, then $T=\sum_{i=1}^{n} \log \left(x_{i} / \theta\right)$ is a sufficient statistic for the parameter $c$.

Lemma 4 Proof

Let $X_{1}, X_{2}, \ldots, X_{n}$ be a random sample from the gamma-Pareto distribution, the joint density function is then given by

$$
\begin{aligned}
& g\left(x_{1}, x_{2}, \ldots, x_{n} \mid c\right)= \prod_{i=1}^{n} \frac{\left(\theta / x_{i}\right)^{1 / c}\left(\log \left(x_{i} / \theta\right)\right)^{\alpha-1}}{x_{i} c^{\alpha} \Gamma(\alpha)} \\
&= k(c, T) h\left(x_{1}, x_{2}, \ldots, x_{n}\right) \\
& \text { where } \quad k(c, T)=\exp \left\{-n \alpha \log c+\left(1+c^{-1}\right) T\right\}, \\
& h\left(x_{1}, x_{2}, \ldots, x_{n}\right)=\exp \{-\log (\theta \Gamma(\alpha)) \\
&\left.+(\alpha-1) \log \left(\log \left(x_{i} / \theta\right)\right)\right\}
\end{aligned}
$$

and $T=\sum_{i=1}^{n} \log \left(x_{i} / \theta\right)$. Thus, by using the factorization theorem, the statistic $T$ is sufficient for the parameter $c$ because the parameters $\alpha$ and $\theta$ are known.

Theorem 7

If $\alpha$ and $\theta$ are known for the gammaPareto distribution, then $m_{1}=(n \alpha)^{-1} \sum_{i=1}^{n} \log \left(x_{i} / \theta\right)$ is the UMVU estimator for the parameter $c$.

Theorem 7 Proof

By using Lemma 4, $T=\sum_{i=1}^{n} \log \left(x_{i} / \theta\right)$ is a sufficient statistic for c. It follows from Lemma 1 that 
$T=\sum_{i=1}^{n} \log \left(x_{i} / \theta\right)$ has a gamma distribution with parameters $n \alpha$ and $c$. Because gamma density belongs to the exponential family, this implies that $\{g(T, c), c>0\}$ is a complete family where $g(x)$ is the gamma-Pareto density. Also from Lemma $1, E\left(m_{1}\right)=c$, hence $m_{1}$ is an unbiased estimator for parameter $c$. By applying Theorem 6 , the statistic $m_{1}$ is the UMVUE of $c$. From equation (5.5), it is interesting to note that when $\theta\left(=x_{(1)}\right)$ and $\alpha$ are known, the MLE of $c$ is the UMVUE of parameter $c$.

\section{Applications}

The gamma-Pareto is applied to three data sets: The first data set (see Table 3) was analyzed by Akinsete, et al. (2008) and represents Floyd River flood rates for the years 1935-1973 in Iowa, USA. The second data set (see Table 5) is from Mahmoudi (2011) and it represents the fatigue life of 6061-T6 aluminum coupons cut parallel with the direction of rolling and oscillated at 18 cycles per second. The third data set (see Table 7) was analyzed by Eugene (2001) and represents the observed frequencies for Tribolium Confusum Strain \#3. The maximum likelihood estimates, the loglikelihood value and the AIC (Akaike Information Criterion) values for the fitted distributions are reported in Tables 4, 6 and 8 .

Akinsete (2008) fitted the data in Table 3 to the beta-Pareto distribution and compared the result with the Pareto and the generalized
Pareto distribution (Pickands, 1975). Results are shown in Table 4, along with the result obtained by fitting the gamma-Pareto distribution to the data. The results show that both beta-Pareto and gamma-Pareto distributions provide adequate fit to the data. Because the gamma-Pareto distribution has only three parameters, this is an advantage for using it over the four-parameter beta-Pareto distribution. In examining the distribution of this data, observe that the data has a reversed J-shape distribution; this suggests that the gamma-Pareto distribution performs well in modeling reversed J-shape distribution. Figure 4 displays the empirical and the fitted cumulative distribution functions and supports the results shown in Table 4.

Mahmoudi (2011) proposed a fiveparameter beta generalized Pareto distribution. He fitted the data (shown in Table 5) and compared the result with beta-Pareto, threeparameter generalized Pareto, Weibull and Pareto distributions. To conserve space, only the results of fitting beta generalized Pareto and beta-Pareto from Mahmoudi (2011) are reported in Table 6 along with the result of fitting the gamma-Pareto distribution to the data. The results in Table 6 indicate that the gamma-Pareto distribution provides the best fit among the distributions. The distribution of this data indicates that the data is approximately symmetric. This example suggests that the gamma-Pareto distribution does very well in fitting the distribution of data which is approximately symmetric. Figure 5 displays the empirical and the fitted cumulative distribution functions and supports the results shown in Table 6.

Table 3: Annual Flood Discharge Rates of the Floyd River Data

\begin{tabular}{|c|c|c|c|c|c|c|c|c|c|c|}
\hline Years & \multicolumn{10}{|c|}{ Flood Discharge (ft3/s) } \\
\hline $1935-1944$ & 1460 & 4050 & 3570 & 2060 & 1300 & 1390 & 1720 & 6280 & 1360 & 7440 \\
\hline $1945-1954$ & 5320 & 1400 & 3240 & 2710 & 4520 & 4840 & 8320 & 13900 & 71500 & 6250 \\
\hline $1955-1964$ & 2260 & 318 & 1330 & 970 & 1920 & 15100 & 2870 & 20600 & 3810 & 726 \\
\hline $1965-1973$ & 7500 & 7170 & 2000 & 829 & 17300 & 4740 & 13400 & 2940 & 5660 & \\
\hline
\end{tabular}


GAMMA-PARETO DISTRIBUTION AND ITS APPLICATIONS

Table 4: Parameter Estimates for the Floyd River Flood Data

\begin{tabular}{|c|c|c|c|}
\hline Distribution & $\begin{array}{c}\text { Parameter } \\
\text { Estimates }\end{array}$ & $\begin{array}{c}\text { Log } \\
\text { Likelihood }\end{array}$ & AIC \\
\hline Pareto & $\begin{array}{c}\hat{k}=0.4125 \\
\hat{\theta}=318\end{array}$ & -392.81 & 789.62 \\
\hline $\begin{array}{c}\text { Generalized } \\
\text { Pareto }\end{array}$ & $\begin{array}{c}\hat{k}=-0.3071 \\
\hat{\theta}=4520\end{array}$ & -379.55 & 763.09 \\
\hline Beta-Pareto & $\begin{array}{c}\hat{\alpha}=6.1550 \\
\hat{\beta}=24.2434\end{array}$ & -365.45 & 738.9 \\
$\hat{k}=0.0926$ & & \\
\hline $\begin{array}{c}\text { Gamma- } \\
\text { Pareto }\end{array}$ & $\begin{array}{c}\hat{\alpha}=318 \\
\hat{c}=0.4712 \\
\hat{\theta}=318\end{array}$ & -365.81 & 734.9 \\
\hline
\end{tabular}

Figure 4: CDF for Fitted Distributions for Floyd River Flood Data

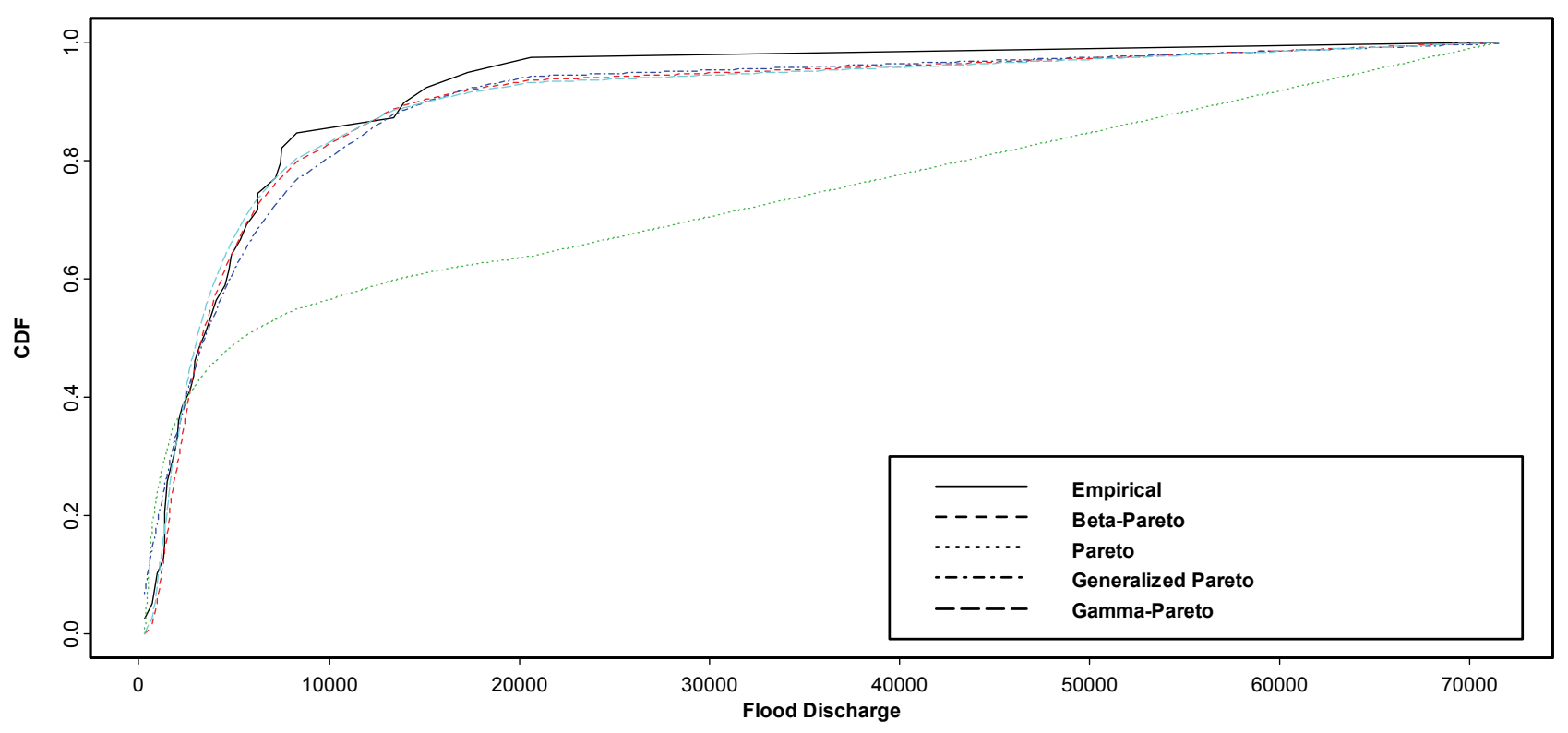


Table 5: Fatigue Life of 6061-T6 Aluminum Coupons Data

\begin{tabular}{|cccccccccc|}
\hline 70 & 90 & 96 & 97 & 99 & 100 & 103 & 104 & 104 & 105 \\
107 & 108 & 108 & 108 & 109 & 109 & 112 & 112 & 113 & 114 \\
114 & 114 & 116 & 119 & 120 & 120 & 120 & 121 & 121 & 123 \\
124 & 124 & 124 & 124 & 124 & 128 & 128 & 129 & 129 & 130 \\
130 & 130 & 131 & 131 & 131 & 131 & 131 & 132 & 132 & 132 \\
133 & 134 & 134 & 134 & 134 & 134 & 136 & 136 & 137 & 138 \\
138 & 138 & 139 & 139 & 141 & 141 & 142 & 142 & 142 & 142 \\
142 & 142 & 144 & 144 & 145 & 146 & 148 & 148 & 149 & 151 \\
151 & 152 & 155 & 156 & 157 & 157 & 157 & 157 & 158 & 159 \\
162 & 163 & 163 & 164 & 166 & 166 & 168 & 170 & 174 & 196 \\
212 & & & & & & & & & \\
\hline
\end{tabular}

Table 6: Parameter Estimates for the Fatigue Life of 6061-T6 Aluminum Coupons Data

\begin{tabular}{|c|c|c|c|}
\hline Distribution & $\begin{array}{c}\text { Parameter } \\
\text { Estimates }\end{array}$ & Log Likelihood & AIC \\
\hline Pareto & $\begin{array}{c}\hat{\alpha}=1.579 \\
\hat{\theta}=70\end{array}$ & -548 & 1100 \\
\hline Beta Pareto & $\begin{array}{c}\hat{\alpha}=485.47 \\
\hat{\beta}=162.06 \\
\hat{k}=0.3943 \\
\hat{\theta}=3.91\end{array}$ & -458.65 & 925.3 \\
\hline Beta- Generalized Pareto & $\begin{array}{c}\hat{\alpha}=12.112 \\
\hat{\beta}=1.702 \\
\hat{\mu}=40.564 \\
\hat{k}=0.273 \\
\hat{\theta}=54.837\end{array}$ & -457.85 & 925.7 \\
\hline Gamma-Pareto & $\begin{array}{c}\hat{\alpha}=15.0209 \\
\hat{c}=0.04258 \\
\hat{\theta}=70\end{array}$ & -448.53 & 900.6 \\
\hline
\end{tabular}




\section{GAMMA-PARETO DISTRIBUTION AND ITS APPLICATIONS}

Eugene (2001) proposed the beta-normal distribution and termed it the generalized normal distribution. Eugene (2001) fitted the data in Table 7 and compared the result with gamma distribution and Lagrange-gamma distribution proposed by Famoye and Govindarajulu (1998). These results are reported in Table 8 along with the result of fitting the data to the gamma-Pareto distribution. The results from the log-likelihood and AIC values indicate that the gamma-Pareto and the generalized normal distributions fit the data best. Figure 6 displays the empirical and the fitted cumulative distribution functions. Figure 6 shows that the generalized normal distribution does not fit the left tail very well, however, the gamma-Pareto distribution does provide a good fit. The distribution shows that the data has a long right tail. This example suggests that the gamma-Pareto distribution does very well in fitting the distributions of data with a long right tail characteristic.

\section{Conclusion}

This article defined the gamma- $X$ family and studied a special case of the gamma- $X$ family, the gamma-Pareto distribution. Various properties of the gamma-Pareto distribution were investigated, including moments, deviations from the mean and median, hazard function, unimodality, entropies and Fisher information matrix. Results of the uniformly minimum variance unbiased estimator was obtained for one of the shape parameters of the gamma-Pareto distribution. Three real data sets were fitted to the gamma-Pareto distribution and compared with other known distributions. Results show that the gamma-Pareto distribution provides a good fit to each data set and suggests that the gamma-Pareto distribution can be a good model to fit data with a reversed J-shape, approximately symmetric and long right tail characteristics.

Figure 5: CDF for Fitted Distributions for Fatigue Life of 6061-T6 Aluminum Data

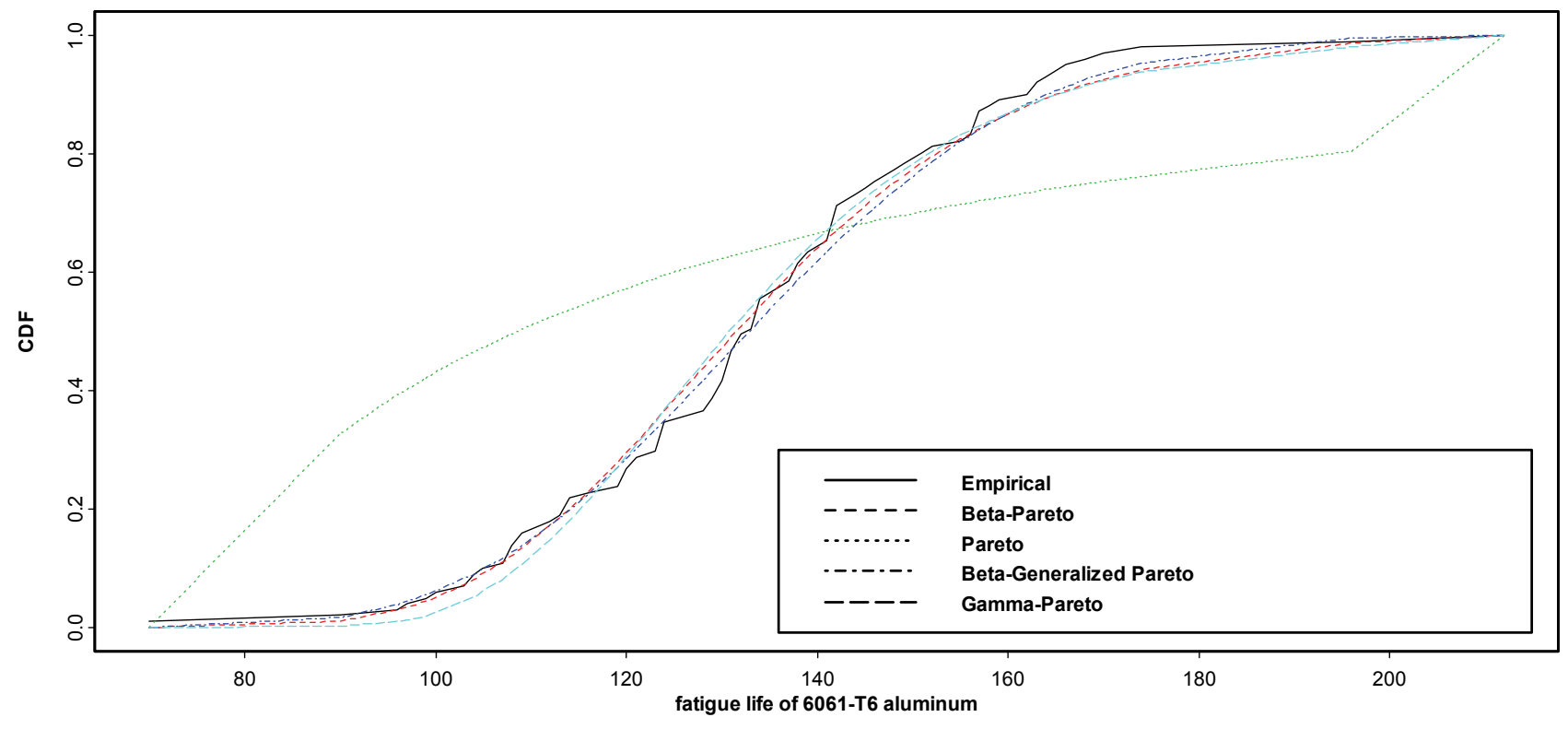


Table 7: Observed frequencies for Tribolium Confusum Strain \# 3

\begin{tabular}{|c|c|c|c|c|c|}
\hline $\mathrm{x}$-Values & Frequency & $\mathrm{x}$-Values & Frequency & $\mathrm{x}$-Values & Frequency \\
\hline 55 & 3 & 125 & 51 & 195 & 1 \\
\hline 65 & 20 & 135 & 20 & 205 & 2 \\
\hline 75 & 53 & 145 & 11 & 215 & 0 \\
\hline 85 & 78 & 155 & 6 & 225 & 1 \\
\hline 95 & 86 & 165 & 4 & 235 & 1 \\
\hline 105 & 86 & 175 & 7 & 245 & 1 \\
\hline 115 & 68 & 185 & 5 & & \\
\hline
\end{tabular}

Table 8: Parameter Estimates for the Tribolium Confusum Strain \# 3 Data

\begin{tabular}{|c|c|c|c|}
\hline Distribution & $\begin{array}{l}\text { Parameter } \\
\text { Estimates }\end{array}$ & $\begin{array}{c}\log \\
\text { Likelihood }\end{array}$ & AIC \\
\hline Gamma & $\begin{array}{c}\hat{\alpha}=15.15 \\
\hat{\beta}=6.92\end{array}$ & -2335.31 & 4674.62 \\
\hline $\begin{array}{l}\text { Lagrange- } \\
\text { gamma }\end{array}$ & $\begin{array}{c}\hat{r}=31 \\
\hat{\lambda}=0.36842 \\
\hat{\theta}=0.02913\end{array}$ & -2314.2 & 4640.41 \\
\hline $\begin{array}{l}\text { Generalized } \\
\text { Normal }\end{array}$ & $\begin{array}{c}\hat{\alpha}=28.68 \\
\hat{\beta}=0.20 \\
\hat{\mu}=30.65 \\
\hat{\sigma}=22.04\end{array}$ & -2290.85 & 4597.71 \\
\hline $\begin{array}{c}\text { Gamma- } \\
\text { Pareto }\end{array}$ & $\begin{array}{c}\hat{\alpha}=6.3513 \\
\hat{c}=0.09743 \\
\hat{\theta}=55\end{array}$ & -2297.7 & 4599.4 \\
\hline
\end{tabular}




\section{GAMMA-PARETO DISTRIBUTION AND ITS APPLICATIONS}

Figure 6: CDF for Fitted Distributions for Tribolium Confusum Strain \# 3 Data

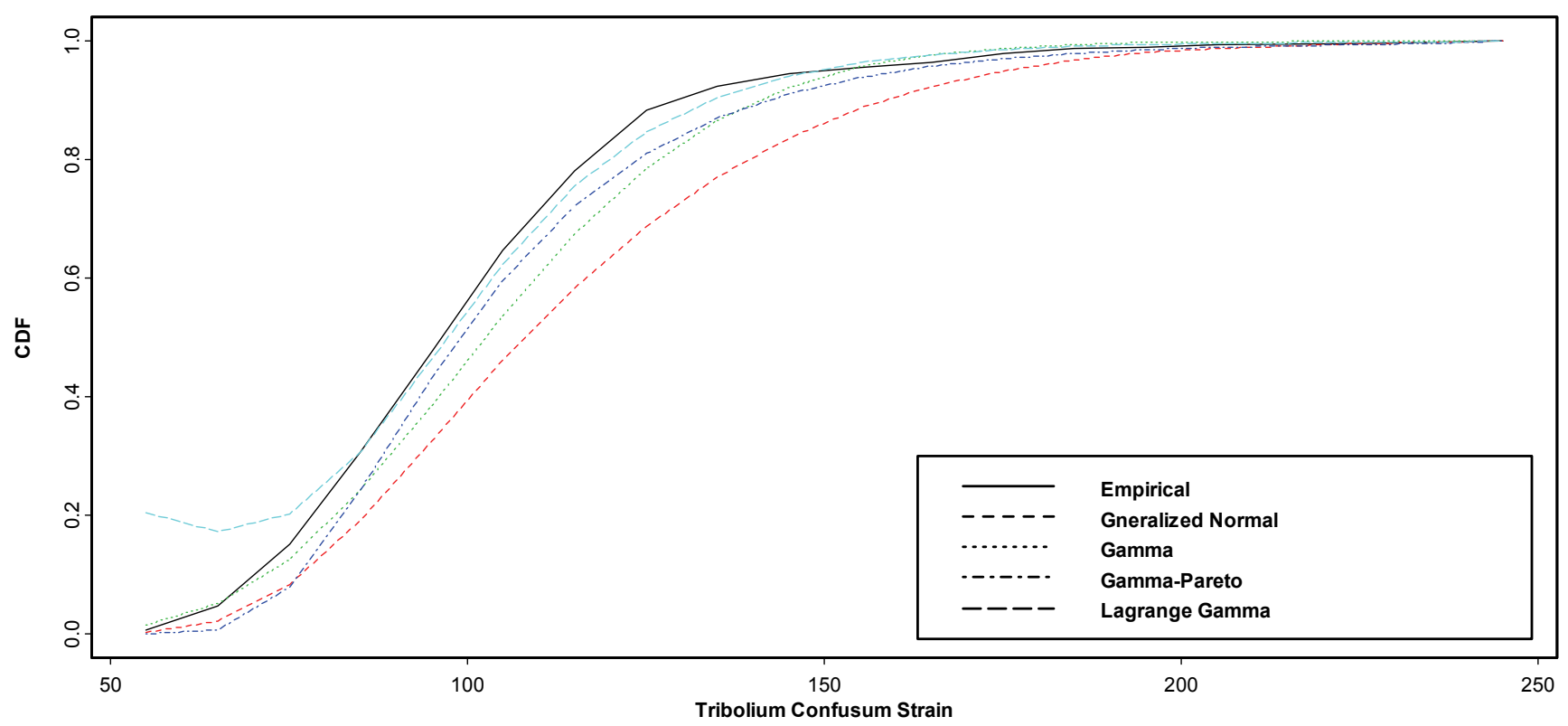

References

Ahsanullah, M., \& Houchens, R. L. (1989). A note on record values from a Pareto distribution. Pakistan Journal of Statistics, 5(1), 51-57.

Akinsete, A., Famoye, F., \& Lee, C. (2008). The beta-Pareto distribution, Statistics, 42, 547-563.

Alzaatreh, A., Lee, C., \& Famoye, F. (2012). A new method for generating families of continuous distributions. Sumbitted for publication.

Burroughs, S. M., \& Tebbens, S. F. (2001). Upper-truncated power law distributions. Fractals, 9(2), 209-222.

Eugene, N. (2001). A Class of Generalized Normal Distribution: Properties, Estimation and Applications (Doctoral Dissertation). Central Michigan University, Mount Pleasant, MI.

Famoye, F., \& Govindarajulu, Z. (1998). On the Lagrange gamma distribution. Computational Statistics and Data Analysis, 27, 421-431.

Johnson, N. L., Kotz, S., \& Balakrishnan, N. (1994) Continuous Univariate Distributions, Vol. 1, $2^{\text {nd }}$ Ed. Hoboken, NJ: John Wiley and Sons, Inc.
Lehmann, E. L., \& Scheffé, H. (1950). Completeness, similar regions, and unbiased estimation. Sankhyā, 10, 305-340.

Mahmoudi, E. (2011). The beta generalized Pareto distribution with application to lifetime data. Mathematics and Computers in Simulation, 81, 2414-2430.

Pickands, J. (1975) Statistical inference using extreme order statistics. Annals of Statistics, 3, 119-131.

Rényi, A. (1961). On measures of entropy and information. In: Proceedings of the Fourth Berkeley Symposium on Mathematical Statistics and Probability, I, University of California Press, Berkeley, 547-561.

Schroeder, B., Damouras, S., \& Gill, P. (2010). Understanding latent sector error and how to protect against them. ACM Transactions on Storage (TOS), 6(3), Article 8.

Shannon, C. E. (1948). A mathematical theory of communication. Bell System Technical Journal, 27, 379-432.

Smith, L. R. (1985). Maximum likelihood estimation in a class of nonregular cases. Biometrika, 72(1), 67-90. 


\title{
Comparison of Re-sampling Methods to Generalized Linear Models and Transformations in Factorial and Fractional Factorial Designs
}

\author{
Maher Qumsiyeh Gerald Shaughnessy \\ University of Dayton, \\ Dayton $\mathrm{OH}$
}

Experimental situations in which observations are not normally distributed frequently occur in practice. A common situation occurs when responses are discrete in nature, for example counts. One way to analyze such experimental data is to use a transformation for the responses; another is to use a link function based on a generalized linear model (GLM) approach. Re-sampling is employed as an alternative method to analyze non-normal, discrete data. Results are compared to those obtained by the previous two methods.

Key words: Factorial experiments, fractional factorial experiments, effect estimation, confidence intervals.

\begin{abstract}
Introduction
Transformation, generalized linear model (GLM) and bootstrap (re-sampling) are methods employed to examine data when systematic and error assumptions may not hold; this suggests a need for inference procedures which are not based on ordinary least squares. Montgomery and Myers (1997) used a GLM approach to analyze data from research conducted by Bisgaard and Fuller (1994) where the response variable was a count of the number of defects in car grille opening panels; they concluded that using GLMs will provide shorter confidence intervals for the mean predicted response compared to those obtained using transformations. This study shows that this is not necessarily true: it depends on the type of data at hand.
\end{abstract}

Benski (1994) compared nine different techniques to determine active factors. Qumsiyeh and Shaughnessy (2008) showed that

Maher Qumsiyeh is an Assistant Professor in the Department of Mathematics. Email him at: qumsiyeh@udayton.edu. Gerald Shaughnessy is a Professor Emeritus in the Department of Mathematics. Email him at: gshaughnessy1@udayton.edu. the bootstrap (re-sampling) could be used to determine the active factors (factors that have an effect on the response) without any requirements on the type of data provided. This study shows that this method of determining active factors agrees with other methods, such as the halfnormal plots, in addition this study shows how this method can be used to obtain confidence intervals for responses after determining active factors.

The bootstrap has been shown to provide better than normal estimates of distribution functions of studentized statistics (see Singh, 1981; Bickle \& Freedman, 1980; Babu \& Singh 1983; Babu and Singh 1984). Qumsiyeh (1994) demonstrated that bootstrap approximation for the distribution of the studentized least square estimate is asymptotically better, not only than the normal approximation, but also than the two-term Edgeworth expansion. Lahiri (1992) showed the superiority of the bootstrap for approximating the distribution of M-estimators. Bhattacharya and Qumsiyeh (1989) conducted an $\mathrm{L}^{\mathrm{p}}$ comparison between the bootstrap and Edgeworth expansions. Finally, Qumsiyeh and Shaughnessy (2010) showed that the bootstrap can be used to determine the active factors in two level designs with missing responses. 


\section{COMPARISON OF RE-SAMPLING METHODS}

\section{The Data}

The data consist of three examples taken from the literature, the first two are fractional factorial and the third is full factorial. The third example presents data that can be analyzed using ordinary least squares. Results obtained were compared with results obtained using ordinary least squares.

\section{Box, Hunter and Hunter}

Box, Hunter and Hunter (1978) presented an example where the response $Y_{1}$ is the glossiness of car paint measured as counts on a scale of 1 to 100; this measure depended on the setting of 8 factors and sixteen readings were taken, thus, it is part of a $2^{8-4}$ fractional factorial design. The first four factors are listed in standard order, other factors have the alias structure: $\mathrm{E}=\mathrm{ABC}, \mathrm{F}=\mathrm{ABD}, \mathrm{G}=\mathrm{ACD}, \mathrm{H}=\mathrm{BCD}$. Using normal plots, factors $\mathrm{A}$ and $\mathrm{B}$ appear to be the active factors. Using the transformation

$$
Y=\left(\sqrt{Y_{1}}+\sqrt{Y_{1}+1}\right) / 2
$$

the final model for the transformed responses is:

$$
\widehat{Y}=8.04+0.5173^{*} \mathrm{~A}+0.3916^{*} \mathrm{~B} .
$$

Bisgaard and Fuller

Bisgaard and Fuller (1994) presented an example of a $2^{9-5}$ fractional factorial. The response $\mathrm{Y}_{2}$ is the number of defects in the finish of sheet molded grille opening panels. The first four factors are listed in the standard order. Other factors have the alias structure: $E=B D$, $\mathrm{F}=\mathrm{BCD}, \mathrm{G}=\mathrm{AC}, \mathrm{H}=\mathrm{ACD}, \mathrm{J}=\mathrm{AB}$. Using the transformation

$$
Y=\left(\sqrt{Y_{2}}+\sqrt{Y_{2}+1}\right) / 2
$$

as well as normal plots, Bisgaard and Fuller determined that the final model for the transformed responses is:

$$
\widehat{Y}=2.513-0.996^{*} \mathrm{D}-1.21 * \mathrm{~F}-0.772 * \mathrm{BG},
$$

where $\mathrm{D}, \mathrm{F}=\mathrm{BCD}$ and $\mathrm{BG}=\mathrm{ABC}$ are the active factors.

Walpole and Myers

In this third example, the data are from a study to determine factors that influence the shrinkage of molded parts. The response variable $\mathrm{Y}_{3}$ is the deviation from nominal shrinkage in $\mathrm{cm} \times 10^{4}$ and it is part of a $2^{4}$ full factorial design. This example is from Walpole and Myers (1993) and differs from the other two examples, because ordinary least squares can be applied to this data without the need for transformation. Using the half-normal plot, it was determined that factors $\mathrm{A}, \mathrm{B}$ and the $\mathrm{AB}$ interaction are the active factors and the final model is:

$$
\widehat{Y}_{3}=80+5.28^{*} \mathrm{~A}+6.22 * \mathrm{~B}+5.70 * \mathrm{AB} .
$$

The experimental grid for the three examples is shown in Table 1 and the half-normal plots for the three examples are shown in Table 2. In Table 2, based on the normal plots, it is clear that factors $\mathrm{A}$ and $\mathrm{B}$ are the active factors for the first data set, factors $\mathrm{A}, \mathrm{B}$ and the $\mathrm{AB}$ interaction are the active factors for the third data set and for the second data set $\mathrm{D}$ and $\mathrm{F}$ and the two factor interaction $\mathrm{GH}$ are active.

Bootstrap Method to Determine Active Factors The bootstrap method can be used, without need for transformation, to determine the active factors (that is, factors that have an effect on the response) and provide models similar to equations (1), (2) and (3). The bootstrap can also be used to provide confidence intervals for the size of the effect. The procedure is as follows: Assume a data set has $\mathrm{N}$ total responses (16 in the examples provided). In order to test whether some factor, for example Z, is active ( $Z$ could be the interaction of other factors):

1) Sample $N / 2$ responses with replacement from the data at the $(+1)$ level of the factor Z. 


\section{QUMSIYEH \& SHAUGHNESSY}

Table 1: Experimental Grid for Examples

\begin{tabular}{|c|c|c|c|c|c|c|c|}
\cline { 2 - 8 } \multicolumn{1}{c|}{} & $\mathrm{A}$ & $\mathrm{B}$ & $\mathrm{C}$ & $\mathrm{D}$ & $\mathrm{Y}_{1}$ & $\mathrm{Y}_{2}$ & $\mathrm{Y}_{3}$ \\
\hline$(-)$ & -1 & -1 & -1 & -1 & 53 & 56 & 72.68 \\
\hline $\mathrm{A}$ & 1 & -1 & -1 & -1 & 60 & 17 & 71.74 \\
\hline $\mathrm{B}$ & -1 & 1 & -1 & -1 & 68 & 2 & 76.09 \\
\hline $\mathrm{AB}$ & 1 & 1 & -1 & -1 & 78 & 4 & 93.19 \\
\hline $\mathrm{C}$ & -1 & -1 & 1 & -1 & 48 & 3 & 71.25 \\
\hline $\mathrm{AC}$ & 1 & -1 & 1 & -1 & 67 & 4 & 70.59 \\
\hline $\mathrm{BC}$ & -1 & 1 & 1 & -1 & 55 & 50 & 70.92 \\
\hline $\mathrm{ABC}$ & 1 & 1 & 1 & -1 & 78 & 2 & 104.96 \\
\hline $\mathrm{D}$ & -1 & -1 & -1 & 1 & 49 & 1 & 73.52 \\
\hline $\mathrm{AD}$ & 1 & -1 & -1 & 1 & 68 & 0 & 75.97 \\
\hline $\mathrm{BD}$ & -1 & 1 & -1 & 1 & 61 & 3 & 74.28 \\
\hline $\mathrm{ABD}$ & 1 & 1 & -1 & 1 & 81 & 12 & 92.87 \\
\hline $\mathrm{CD}$ & -1 & -1 & 1 & 1 & 52 & 3 & 79.34 \\
\hline $\mathrm{ACD}$ & 1 & -1 & 1 & 1 & 70 & 4 & 75.12 \\
\hline $\mathrm{BCD}$ & -1 & 1 & 1 & 1 & 65 & 0 & 79.67 \\
\hline $\mathrm{ABCD}$ & 1 & 1 & 1 & 1 & 82 & 0 & 97.8 \\
\hline
\end{tabular}

2) Sample $N / 2$ responses with replacement at the $(-1)$ level of the factor $Z$.

3) Estimate the effect of that factor using the difference between the average at the $(+1)$ level and (-1) levels.

4) Repeat the sampling procedure a large number of times, for example, 500 .

5) Take the average of the differences of the averages at the +1 level and -1 levels across the 500 samples: this is the effect size estimate.

6) Determine the upper $(1-\alpha / 2)$ and lower $\alpha / 2$ percentile points of the re-sampled effect values.

7) Use these values to construct the $(1-\alpha)$ $\mathrm{x} 100 \%$ confidence interval for the effect size.
8) If the confidence interval does not contain zero then the factor is identified as an active factor.

The mean effect size and confidence intervals for the different effect sizes for the three different examples were determined using this procedure (see Table 3 ).

Table 3 shows the LCL (2.5 percentile lower confidence limit) and UCL (97.5 percentile upper confidence limit). The only confidence intervals that do not contain zero in the first example are those of $\mathrm{A}$ and $\mathrm{B}$, this matches the results of the normal plots and Box, Hunter and Hunter. In addition, the only confidence intervals that do not contain zero in the third example are those of $\mathrm{A}, \mathrm{B}$ and the $\mathrm{AB}$ interaction, this matches the results of the halfnormal plots and of Walpole and Myers. With respect to the second example, the only confidence intervals that do not contain zero are those of D and F. The two-factor interaction BG 
COMPARISON OF RE-SAMPLING METHODS

Table 2: Half-Normal Plots for the Three Examples

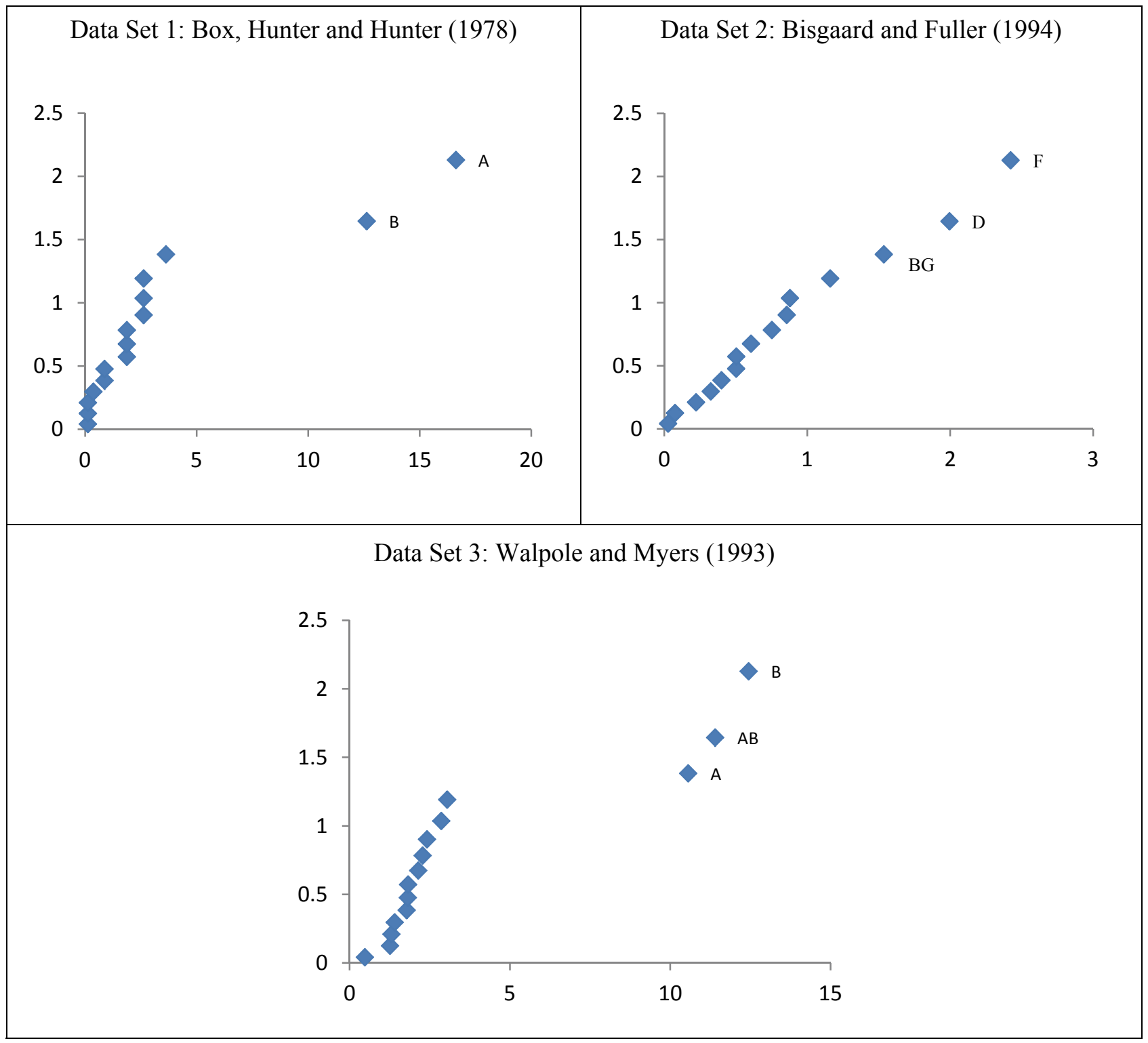

may also be considered active, because the upper confidence limit is very close to zero; this agrees with the results of Bisgaard and Fuller and the half-normal plots. The half-normal plots do not clearly show that $B G$ is active.

Bootstrap Method to Determine Confidence Intervals

Confidence intervals for the mean predicted responses can be obtained as follows: For each effect size determined in step (3) of the bootstrap method, find the predicted value of $Y_{1}$ for the first example, ( $\mathrm{Y}_{2}$ for the second example and $\mathrm{Y}_{3}$ for the third), using the two active factors found for the first example, A and B, and use as coefficients the effect size divided by two. (Note that there are 3 active factors for the second and the third examples). Because the data consists of counts for the first two examples, if the predicted value is negative, zero was substituted for its value. This was necessary for the Bisgaard and Fuller example, however it was not 
necessary for the first and third examples because none of the responses were zero and responses were large positive values. Resampling was repeated 1,000 times, and one thousand predicted values were produced at each setting of the active factors. These 1,000 values were used to construct $95 \%$ confidence intervals for the mean predicted responses. Results are summarized and shown Tables 4-8. Note that, for the first two examples, after finding the confidence intervals for the transformed mean and for the lower and upper confidence limit, the results are un-transformed using a software package that can solve for $x$, when $y$ is known in the equation: $y=(\sqrt{x}+\sqrt{x+1}) / 2$. For the third example, because no transformation was used, the confidence interval provided by the ordinary least squares method is reported.

Table 7 gives the mean predicted values and confidence intervals for the mean predicted values, these results were obtained using the SQL procedure in the statistical software SAS. Re-sampling was repeated 1,000 times. A slight difference occurred between re-sampling 500 and 1,000 times; the length of the confidence interval was slightly smaller when re-sampling 1,000 times. Very little difference was observed between re-sampling 1,000 times and resampling more than 1,000 times.

Table 8 shows the length of the confidence intervals using the different methods for the three different examples. For the Box, Hunter and Hunter example the Bootstrap gave shorter confidence intervals than those of the Generalized Linear Model (GLM). For the Bisgaard and Fuller example, the bootstrap results are not inferior to the untransformed or GLM procedures. For data with count responses where some of the responses are zeros using a transformation is questionable, therefore the bootstrap provides a good alternative. For data such as the third example taken from Walpole and Myers, ordinary least squares (OLS) can be applied and this method results in confidence intervals that are shorter than both the Bootstrap and GLM approaches.

Table 3: Mean Effect Size and Confidence Intervals for the Different Effect Sizes for the Three Different Examples

\begin{tabular}{|c|c|c|c|}
\hline \multirow{2}{*}{ Factors } & \multicolumn{3}{|c|}{ Box Hunter and Hunter } \\
\cline { 2 - 4 } & Mean & LCL & UCL \\
\hline A & 16.82 & 10.75 & 22.50 \\
\hline B & 13.12 & 6.00 & 20.75 \\
\hline C & 0.07 & -10.75 & 10.75 \\
\hline D & 2.59 & -6.75 & 12.50 \\
\hline AB & 1.19 & -10.63 & 13.38 \\
\hline AC & 3.03 & -6.00 & 13.38 \\
\hline AD & 1.69 & -9.00 & 12.25 \\
\hline BC & -2.27 & -11.63 & 7.75 \\
\hline BD & 0.18 & -9.88 & 10.75 \\
\hline CD & 2.75 & -7.00 & 13.00 \\
\hline ABC & -0.04 & -9.75 & 9.25 \\
\hline ABD & -0.60 & -11.50 & 10.38 \\
\hline ACD & -3.64 & -13.13 & 6.13 \\
\hline BCD & 2.11 & -10.00 & 12.63 \\
\hline ABCD & -0.32 & -10.75 & 10.00 \\
\hline
\end{tabular}

\begin{tabular}{|c|c|c|}
\hline \multicolumn{3}{|c|}{ Bisgaard and Fuller } \\
\hline Mean & LCL & UCL \\
\hline-9.16 & -24.63 & 4.38 \\
\hline-1.97 & -17.13 & 15.00 \\
\hline-4.04 & -20.50 & 11.75 \\
\hline-14.97 & -32.50 & -1.13 \\
\hline 0.06 & -16.88 & 16.00 \\
\hline-2.17 & -17.25 & 13.75 \\
\hline 11.58 & -2.38 & 27.50 \\
\hline 10.77 & -2.63 & 26.25 \\
\hline 3.87 & -13.88 & 19.75 \\
\hline 0.55 & -15.75 & 16.25 \\
\hline-11.84 & -28.38 & 0.68 \\
\hline 2.28 & -14.50 & 18.25 \\
\hline 0.88 & -14.88 & 17.00 \\
\hline-16.95 & -32.25 & -3.38 \\
\hline 10.24 & -5.13 & 29.00 \\
\hline
\end{tabular}

\begin{tabular}{|c|c|c|}
\hline \multicolumn{3}{|c|}{ Walpole and Myers } \\
\hline Mean & LCL & UCL \\
\hline 16.82 & 10.75 & 22.50 \\
\hline 13.12 & 6.00 & 20.75 \\
\hline 0.07 & -10.75 & 10.75 \\
\hline 2.59 & -6.75 & 12.50 \\
\hline 11.43 & 2.45 & 20.14 \\
\hline 1.30 & -8.33 & 11.90 \\
\hline-1.67 & -12.09 & 7.86 \\
\hline 2.01 & -8.28 & 12.02 \\
\hline-2.20 & -13.31 & 7.88 \\
\hline 1.45 & -9.30 & 11.11 \\
\hline 2.58 & -5.36 & 12.15 \\
\hline-1.94 & -11.70 & 7.38 \\
\hline-2.99 & -13.07 & 5.32 \\
\hline-0.29 & -10.19 & 9.22 \\
\hline-1.59 & -10.82 & 7.97 \\
\hline
\end{tabular}


Table 4: Results of the Box, Hunter and Hunter (1978) Example

\begin{tabular}{|c|c|c|c|c|c|c|}
\hline \multirow[b]{3}{*}{ Obs. } & \multicolumn{4}{|c|}{ Least Squares } & \multirow{2}{*}{\multicolumn{2}{|c|}{ GLM Poisson }} \\
\hline & \multicolumn{2}{|c|}{ Transformed } & \multicolumn{2}{|c|}{ Untransformed } & & \\
\hline & $\begin{array}{l}\text { Predicted } \\
\text { Value }\end{array}$ & $\begin{array}{c}95 \% \\
\text { Confidence } \\
\text { Interval }\end{array}$ & $\begin{array}{l}\text { Predicted } \\
\text { Value }\end{array}$ & $\begin{array}{c}95 \% \\
\text { Confidence } \\
\text { Interval }\end{array}$ & $\begin{array}{l}\text { Predicted } \\
\text { Value }\end{array}$ & $\begin{array}{c}95 \% \\
\text { Confidence } \\
\text { Interval } \\
\end{array}$ \\
\hline 1 & 7.14 & $(6.92,7.36)$ & 50.42 & $(47.32,53.63)$ & 51.26 & $(42.45,61.9)$ \\
\hline 2 & 8.17 & $(7.95,8.39)$ & 66.26 & $(62.7,69.92)$ & 11.74 & $(8.14,16.94)$ \\
\hline 3 & 7.92 & $(7.7,8.14)$ & 62.22 & $(58.76,65.76)$ & 1.12 & $(0.6,2.08)$ \\
\hline 4 & 8.95 & $(8.73,9.17)$ & 79.67 & $(75.76,83.68)$ & 4.88 & $(2.87,8.32)$ \\
\hline 5 & 7.14 & $(6.92,7.36)$ & 50.42 & $(47.32,53.63)$ & 1.12 & $(0.6,2.08)$ \\
\hline 6 & 8.17 & $(7.95,8.39)$ & 66.26 & $(62.7,69.92)$ & 4.88 & $(2.87,8.32)$ \\
\hline 7 & 7.92 & $(7.7,8.14)$ & 62.22 & $(58.76,65.76)$ & 51.26 & $(42.45,61.9)$ \\
\hline 8 & 8.95 & $(8.73,9.17)$ & 79.67 & $(75.76,83.68)$ & 11.74 & $(8.14,16.94)$ \\
\hline 9 & 7.14 & $(6.92,7.36)$ & 50.42 & $(47.32,53.63)$ & 0.81 & $(0.42,1.56)$ \\
\hline 10 & 8.17 & $(7.95,8.39)$ & 66.26 & $(62.7,69.92)$ & 0.19 & $(0.09,0.38)$ \\
\hline 11 & 7.92 & $(7.7,8.14)$ & 62.22 & $(58.76,65.76)$ & 1.96 & $(1.16,3.3)$ \\
\hline 12 & 8.95 & $(8.73,9.17)$ & 79.67 & $(75.76,83.68)$ & 8.54 & $(5.62,12.98)$ \\
\hline 13 & 7.14 & $(6.92,7.36)$ & 50.42 & $(47.32,53.63)$ & 1.96 & $(1.16,3.3)$ \\
\hline 14 & 8.17 & $(7.95,8.39)$ & 66.26 & $(62.7,69.92)$ & 8.54 & $(5.62,12.98)$ \\
\hline 15 & 7.92 & $(7.7,8.14)$ & 62.22 & $(58.76,65.76)$ & 0.81 & $(0.42,1.56)$ \\
\hline 16 & 8.95 & $(8.73,9.17)$ & 79.67 & $(75.76,83.68)$ & 0.19 & $(0.09,0.38)$ \\
\hline
\end{tabular}


QUMSIYEH \& SHAUGHNESSY

Table 5: Results of Montgomery and Myers (1997) for the Bisgaard and Fuller Example

\begin{tabular}{|c|c|c|c|c|c|c|}
\hline \multirow[b]{3}{*}{ Obs. } & \multicolumn{4}{|c|}{ Least Squares } & \multirow{2}{*}{\multicolumn{2}{|c|}{ GLM Poisson }} \\
\hline & \multicolumn{2}{|c|}{ Transformed } & \multicolumn{2}{|c|}{ Untransformed } & & \\
\hline & $\begin{array}{l}\text { Predicted } \\
\text { Value }\end{array}$ & $\begin{array}{c}95 \% \\
\text { Confidence } \\
\text { Interval }\end{array}$ & $\begin{array}{c}\text { Predicted } \\
\text { Value }\end{array}$ & $\begin{array}{c}95 \% \\
\text { Confidence } \\
\text { Interval }\end{array}$ & $\begin{array}{l}\text { Predicted } \\
\text { Value }\end{array}$ & $\begin{array}{c}95 \% \\
\text { Confidence } \\
\text { Interval }\end{array}$ \\
\hline 1 & 5.50 & $(4.13,6.84)$ & 29.75 & $(16.65,46.41)$ & 51.26 & $(42.45,61.9)$ \\
\hline 2 & 3.95 & $(2.6,5.3)$ & 15.11 & $(6.25,27.65)$ & 11.74 & $(8.14,16.94)$ \\
\hline 3 & 1.53 & $(0.17,2.88)$ & 1.86 & $(* *, 7.78)$ & 1.12 & $(0.6,2.08)$ \\
\hline 4 & 3.07 & $(1.71,4.42)$ & 8.9 & $(2.45,19.04)$ & 4.88 & $(2.87,8.32)$ \\
\hline 5 & 1.53 & $(0.17,2.88)$ & 1.86 & $(* *, 7.78)$ & 1.12 & $(0.6,2.08)$ \\
\hline 6 & 3.07 & $(1.71,4.42)$ & 8.9 & $(2.45,19.04)$ & 4.88 & $(2.87,8.32)$ \\
\hline 7 & 5.49 & $(4.13,6.84)$ & 29.61 & $(16.65,46.41)$ & 51.26 & $(42.45,61.9)$ \\
\hline 8 & 3.95 & $(2.6,5.3)$ & 15.11 & $(6.25,27.65)$ & 11.74 & $(8.14,16.94)$ \\
\hline 9 & 1.07 & $(-0.28,2.42)$ & 0.7 & $(*, 5.41)$ & 0.81 & $(0.42,1.56)$ \\
\hline 10 & -0.47 & $(-1.82,0.89)$ & $*$ & $(*, 0.36)$ & 0.19 & $(0.09,0.38)$ \\
\hline 11 & 1.96 & $(0.6,3.31)$ & 3.34 & $(0.04,10.49)$ & 1.96 & $(1.16,3.3)$ \\
\hline 12 & 3.49 & $(2.14,4.85)$ & 11.7 & $(4.13,23.1)$ & 8.54 & $(5.62,12.98)$ \\
\hline 13 & 1.96 & $(0.6,3.31)$ & 3.34 & $(0.04,10.49)$ & 1.96 & $(1.16,3.3)$ \\
\hline 14 & 3.49 & $(2.14,4.85)$ & 11.7 & $(4.13,23.1)$ & 8.54 & $(5.62,12.98)$ \\
\hline 15 & 1.07 & $(-0.28,2.42)$ & 0.7 & $(*, 5.41)$ & 0.81 & $(0.42,1.56)$ \\
\hline 16 & -0.47 & $(-1.82,0.89)$ & $*$ & $(*, 0.36)$ & 0.19 & $(0.09,0.38)$ \\
\hline
\end{tabular}

*values that can't be calculated using the transformation because of the negative predicted value;

**values that appear to be incorrectly calculated in Montgomery and Myers (1997) work. 
COMPARISON OF RE-SAMPLING METHODS

Table 6: Results of the Walpole and Myers (1993) Example

\begin{tabular}{|c|c|c|c|c|}
\hline \multirow[b]{2}{*}{ Obs. } & \multicolumn{2}{|c|}{ Least Squares } & \multicolumn{2}{|c|}{ GLM Poisson } \\
\hline & $\begin{array}{l}\text { Predicted } \\
\text { Value }\end{array}$ & $\begin{array}{l}\text { 95\% Confidence } \\
\text { Interval }\end{array}$ & $\begin{array}{l}\text { Predicted } \\
\text { Value }\end{array}$ & $\begin{array}{l}\text { 95\% Confidence } \\
\text { Interval }\end{array}$ \\
\hline 1 & 74.20 & $(69.82,78.57)$ & 74.2 & $(66.22,83.14)$ \\
\hline 2 & 73.36 & $(68.98,77.73)$ & 73.36 & $(65.42,82.25)$ \\
\hline 3 & 75.24 & $(70.87,79.61)$ & 75.24 & $(67.2,84.24)$ \\
\hline 4 & 97.21 & $(92.83,101.58)$ & 97.21 & $(88.01,107.36)$ \\
\hline 5 & 74.20 & $(69.82,78.57)$ & 74.2 & $(66.22,83.14)$ \\
\hline 6 & 73.36 & $(68.98,77.73)$ & 73.36 & $(65.42,82.25)$ \\
\hline 7 & 75.24 & $(70.87,79.61)$ & 75.24 & $(67.2,84.24)$ \\
\hline 8 & 97.21 & $(92.83,101.58)$ & 97.21 & $(88.01,107.36)$ \\
\hline 9 & 74.20 & $(69.82,78.57)$ & 74.2 & $(66.22,83.14)$ \\
\hline 10 & 73.36 & $(68.98,77.73)$ & 73.36 & $(65.42,82.25)$ \\
\hline 11 & 75.24 & $(70.87,79.61)$ & 75.24 & $(67.2,84.24)$ \\
\hline 12 & 97.21 & $(92.83,101.58)$ & 97.21 & $(88.01,107.36)$ \\
\hline 13 & 74.20 & $(69.82,78.57)$ & 74.2 & $(66.22,83.14)$ \\
\hline 14 & 73.36 & $(68.98,77.73)$ & 73.36 & $(65.42,82.25)$ \\
\hline 15 & 75.24 & $(70.87,79.61)$ & 75.24 & $(67.2,84.24)$ \\
\hline 16 & 97.21 & $(92.83,101.58)$ & 97.21 & $(88.01,107.36)$ \\
\hline
\end{tabular}


QUMSIYEH \& SHAUGHNESSY

Table 7: Bootstrap Mean and Confidence Interval for the Predicted Values for the Three Examples

\begin{tabular}{|c|c|c|c|c|c|c|}
\hline \multirow[b]{3}{*}{ Obs. } & \multicolumn{6}{|c|}{ Bootstrap (Re-sampling) } \\
\hline & \multicolumn{2}{|c|}{ Box, Hunter and Hunter } & \multicolumn{2}{|c|}{ Bisgaard and Fuller } & \multicolumn{2}{|c|}{ Walpole and Myers } \\
\hline & $\begin{array}{l}\text { Predicted } \\
\text { Value }\end{array}$ & $\begin{array}{l}95 \% \text { Confidence } \\
\text { Interval }\end{array}$ & $\begin{array}{l}\text { Predicted } \\
\text { Value }\end{array}$ & $\begin{array}{l}\text { 95\% Confidence } \\
\text { Interval }\end{array}$ & $\begin{array}{l}\text { Predicted } \\
\text { Value }\end{array}$ & $\begin{array}{c}95 \% \text { Confidence } \\
\text { Interval }\end{array}$ \\
\hline 1 & 50.87 & $(45.39,57.03)$ & 31.86 & $(19.22,45.56)$ & 73.95 & $(66.32,82.06)$ \\
\hline 2 & 65.88 & $(59.29,73.19)$ & 19.19 & $(5.06,33.72)$ & 73.65 & $(66.08,81.29)$ \\
\hline 3 & 61.88 & $(55.57,68.9)$ & 10.26 & $(0,26.78)$ & 75.18 & $(68.45,81.64)$ \\
\hline 4 & 80.12 & $(72.63,88.38)$ & 15.05 & $(3.44,27.34)$ & 97.22 & $(89.44,105.26)$ \\
\hline 5 & 50.87 & $(45.39,57.03)$ & 10.26 & $(0,26.78)$ & 73.95 & $(66.32,82.06)$ \\
\hline 6 & 65.88 & $(59.29,73.19)$ & 15.05 & $(3.44,27.34)$ & 73.65 & $(66.08,81.29)$ \\
\hline 7 & 61.88 & $(55.57,68.9)$ & 31.86 & $(19.22,45.56)$ & 75.18 & $(68.45,81.64)$ \\
\hline 8 & 80.12 & $(72.63,88.38)$ & 19.19 & $(5.06,33.72)$ & 97.22 & $(89.44,105.26)$ \\
\hline 9 & 50.87 & $(45.39,57.03)$ & 3.41 & $(0,15.06)$ & 73.95 & $(66.32,82.06)$ \\
\hline 10 & 65.88 & $(59.29,73.19)$ & 0.07 & $(0,0.91)$ & 73.65 & $(66.08,81.29)$ \\
\hline 11 & 61.88 & $(55.57,68.9)$ & 5.72 & $(0,16.69)$ & 75.18 & $(68.45,81.64)$ \\
\hline 12 & 80.12 & $(72.63,88.38)$ & 17.76 & $(5.44,30.38)$ & 97.22 & $(89.44,105.26)$ \\
\hline 13 & 50.87 & $(45.39,57.03)$ & 5.72 & $(0,16.69)$ & 73.95 & $(66.32,82.06)$ \\
\hline 14 & 65.88 & $(59.29,73.19)$ & 17.76 & $(5.44,30.38)$ & 73.65 & $(66.08,81.29)$ \\
\hline 15 & 61.88 & $(55.57,68.9)$ & 3.41 & $(0,15.06)$ & 75.18 & $(68.45,81.64)$ \\
\hline 16 & 80.12 & $(72.63,88.38)$ & 0.07 & $(0,0.91)$ & 97.22 & $(89.44,105.26)$ \\
\hline
\end{tabular}


COMPARISON OF RE-SAMPLING METHODS

Table 8: Length of Confidence Intervals for the Three Examples Using the Different Methods

\begin{tabular}{|c|c|c|c|c|c|c|c|c|}
\hline \multicolumn{9}{|c|}{ Confidence Interval Length } \\
\hline \multicolumn{2}{|c|}{ Box, Hunter and Hunter } & \multicolumn{3}{c|}{ Bisgaard and Fuller } & \multicolumn{3}{c|}{ Walpole and Myers } \\
\hline Untrans. & GLM & Bootstrap & Untrans. & GLM & Bootstrap & OLS & GLM & Bootstrap \\
\hline 6.31 & 11.64 & 9.44 & 29.76 & 19.45 & 26.34 & 8.75 & 16.92 & 15.75 \\
\hline 7.22 & 13.90 & 11.16 & 21.40 & 8.80 & 27.84 & 8.75 & 16.83 & 15.21 \\
\hline 7.00 & 13.34 & 11.16 & $* *$ & 1.47 & 26.78 & 8.75 & 17.04 & 13.20 \\
\hline 7.92 & 15.75 & 9.44 & 16.59 & 5.45 & 23.91 & 8.75 & 19.35 & 15.82 \\
\hline 6.31 & 11.64 & 9.44 & $* *$ & 1.47 & 26.78 & 8.75 & 16.92 & 15.75 \\
\hline 7.22 & 13.90 & 11.16 & 16.59 & 5.45 & 23.91 & 8.75 & 16.83 & 15.21 \\
\hline 7.00 & 13.34 & 11.16 & 29.76 & 19.45 & 26.34 & 8.75 & 17.04 & 13.20 \\
\hline 7.92 & 15.75 & 9.44 & 21.40 & 8.80 & 27.84 & 8.75 & 19.35 & 15.82 \\
\hline 6.31 & 11.64 & 9.44 & $*$ & 1.13 & 14.25 & 8.75 & 16.92 & 15.75 \\
\hline 7.22 & 13.90 & 11.16 & $*$ & 0.29 & 0.91 & 8.75 & 16.83 & 15.21 \\
\hline 7.00 & 13.34 & 11.16 & 10.45 & 2.14 & 16.69 & 8.75 & 17.04 & 13.20 \\
\hline 7.92 & 15.75 & 9.44 & 18.97 & 7.35 & 24.22 & 8.75 & 19.35 & 15.82 \\
\hline 6.31 & 11.64 & 9.44 & 10.45 & 2.14 & 16.69 & 8.75 & 16.92 & 15.75 \\
\hline 7.22 & 13.90 & 11.16 & 18.97 & 7.35 & 24.22 & 8.75 & 16.83 & 15.21 \\
\hline 7.00 & 13.34 & 11.16 & $*$ & 1.13 & 14.25 & 8.75 & 17.04 & 13.20 \\
\hline 7.92 & 15.75 & 9.44 & $*$ & 0.29 & 0.91 & 8.75 & 19.35 & 15.82 \\
\hline
\end{tabular}

*values that can't be calculated using the transformation because of the negative predicted value; **values that appear to be incorrectly calculated in Montgomery and Myers (1997) work.

\section{Conclusion}

The bootstrap method can be used to determine active factors and to construct confidence intervals for effect size and for a predicted mean response. The results are not inferior to those obtained using transformations or generalized linear models procedures and are actually better in some situations. The availability of computers and statistical software makes using re-sampling (bootstrap) easy and fast and provides good predictions.

\section{References}

Babu, G., \& Singh, K. (1983). Inference on means using the bootstrap. The Annals of Statistics, 11, 999-1003.
Babu, G. J., \& Singh, K.(1984). On one term Edgeworth correction by Efrons bootstrap. Sankhya, 46, Ser.A, 219-232.

Benski, C. (1994). Applicability of nine numerical techniques for detecting active factors in unreplicated experimental designs. ASA Proceedings of the Statistical Computing Section, 214-217. CISid: 154639. Alexandria, VA: American Statistical Association.

Bhattacharya, R. N., \& Qumsiyeh, M. (1989). Second order and Lp- comparison between the bootstrap and empirical Edgeworth expansion methodoligies. Annals of Statistics, 17, 160-169.

Bickel, P. J., \& Freedman, D. A. (1980). On Edgeworth expansions for the bootstrap. Unpublished. 


\section{QUMSIYEH \& SHAUGHNESSY}

Bisgaard, S., \& Fuller, H. (1994). Analysis of factorial experiments with defects or defectives as the response. Quality Engineering, 7(2), 429-443.

Box, G., Hunter, W., \& Hunter, J. (1978). Statistics for experiments. New York, NY: John Wiley.

Daniel, C. (1959). Using of half normal plots in interpreting factorial two-level experiments. Technometrics, 1, 311-341.

Efrom, B. (1979). Bootstrap methods: Another look at jackknife. The Annals of Statistics, 7, 1-26.

Lahiri, S. (1992). Bootstrapping Mestimators of a multiple linear regression parameter. The Annals of Statistics, 20(3), 15481570.

Lenth, R. (1989). Quick and easy analysis of unreplicated factorials. Technometrics, 31, 469-473.

Montgomry, D. and Meyers, R. (1997). A tutorial on generalized linear models. Journal of Quality Technology, 29(3), 274-291.
Qumsiyeh, M. (1994). Bootstrapping and empirical Edgeworth expansions in multiple linear regression models. Communications in Statistical Theory and Methods, 23(11), 32273239.

Qumsiyeh, M., \& Shaughnessy, G. (2008). Using the bootstrap to select active factors in unreplicated factorial experiment. JSM Proceedings, Statistical Computing Section. Alexandria, VA: American Statistical Association.

Qumsiyeh, M., \& Shaughnessy, G. (2010). Bootstrapping un-replicated two-level designs with missing responses. Journal of Statistics, Advances in Theory and Applications, 4, 91-106.

Singh, K. (1981). On the asymptotic accuracy of Efron's bootstrap. The Annals of Statistics, 9, 1187-1195.

Walpole, R. E., \& Myers, R. H. (1993). Probability and statistics for engineers and scientists, $5^{\text {th }}$ Ed. Englewood Cliffs, NJ: Prentice Hall. 


\title{
Estimation of Multinomial Proportions using Higher Order Moments of Scrambling Variables in Randomized Response Sampling
}

\author{
Cheng C. Chen Sarjinder Singh \\ Texas A\&M University, \\ Kingsville, TX
}

An extension to estimating multinomial proportions of potentially sensitive attributes in survey sampling is proposed using higher order moments of scrambling variables at the estimation stage to produce unbiased estimators. The variance and covariance expressions are derived and the relative efficiency of the proposed estimators based on scrambling variables is investigated.

Key words: Estimation of sensitive multinomial proportions, randomized response sampling, respondents protection.

\section{Introduction}

The problem of estimating the proportion of potentially sensitive attributes in survey sampling has been very well addressed in the literature following the pioneering work of Warner (1965), and the use of randomized response sampling in social, medical and environmental sciences has been well documented (Waltz, et al., 2004; Blank, 2008). Singh and Chen (2009) introduced the use of higher order moments of scrambling variables to improve single proportion estimates without affecting respondent cooperation in survey research.

The problem of estimating trinomial proportions has been very useful, especially during election periods in the United States of America. Voters in the US can be divided into three mutually exclusive groups: Democrat, Republican and Other. At this time, expressing preference for one of these three groups does not

Cheng Chen is an Associate Professor in the Department of Mathematics. Email him at: cheng.chen@tamuk.edu. Sarjinder Singh is an Associate Professor in the Department of Mathematics. Email him at: sarjinder@yahoo.com. pose a threat to an individual's privacy; however, the competition for the presidential position is becoming more difficult and there may be a time when voters will not feel safe disclosing their preferences to vote in the US. A new method is developed here that could be useful in such circumstances to organizations that conduct surveys about the prediction of future president for the US in the forthcoming elections. It is assumed that the partition of voters will remain trinomial because it may not be easy to establish a new competitive party as strong as those that are currently functioning; however, the proposed model can be extended to the case of a multinomial distribution if required.

This same argument can be extended to other applications if a population can be captured completely within three mutually exclusive groups. As noted by Singh, Kim and Grewal (2008), a sensitive question in one survey could be non-sensitive in another survey depending on the situation, particularly when there are three categories that are feasible when an answer is: exactly known, exactly unknown and not sure; hence leading to the problem of trinomial proportions estimation.

In a careful examination of the literature in randomized response sampling (Tracy \& Mangat, 1996), not much attention has been paid to estimate sensitive multinomial proportions. Abul-Ela, et al. (1967) extended Warner's (1965) design to the multichotomous case when 


\section{CHENG CHEN \& SARJINDER SINGH}

a population can be considered to be divided into $t$ disjoint classes $C_{j}$ with unknown proportions $\pi_{j} \quad\left(j=1,2, \ldots, t, \quad 0<\pi_{j}<1\right.$, $\left.\sum \pi_{j}=1\right)$. It is assumed that at least one of the classes carries a stigma and at least one carries no stigma. They suggested drawing $s \quad(=t-1)$ independent simple random with replacement samples sized $n_{i}\left(i=1,2, \ldots, s, \sum n_{i}=n\right)$, and then employing a randomized response device to each of the samples. Abul-Ela, et al. (1967) examined the extent of bias and the mean square error of estimators for $t=3$.

Bourke and Dalenious (1973, 1974) proposed a Latin square measurement design to extend Warner's model to the multinomial case; their design uses $t$ different possible responses and requires only one sample. A respondent is asked to select one of a $t$-type cards using a random device. Each of the $t$-mutually exclusive classes is described on each card, except that the order of the description is permuted from card to card and the permutation for $t$-cards forms a Latin square.

The respondent reads the cards selected and reports only the position of the card (i.e., $t=1,2, \ldots,(t-1)$ or $t)$ of the statement describing the class to which he/she belongs. The unrelated question design was also extended by Bourke (1974) to estimate the proportion of a population in each of $t$ mutually exclusive classes of which $(t-1)$ are sensitive. One sample is needed if the distribution of the unrelated character is known. The design uses a deck of cards, each of which contains a number of statements. The arrangement of the statements is a part of the design.

Hochberg (1975) outlined an alternative scheme for estimating the $t$ group proportions of which, at most, $(t-2)$ are stigmatizing. The realizations for any sampled individuals constitute a two-stage scheme. The second stage is conditional on the random individual's response in the first stage. Drane (1976) used a forced yes stochastic model to estimate the proportion of more than one sensitive character. The use of supplemented block, balanced incomplete block and spring balance weighing designs were introduced by Raghavarao and
Federer (1979); their models allow the surveyor to obtain answers to several sensitive questions. Mukhopadhyay (1980), Mukherjee (1981), Tamhane (1981), Bourke (1981, 1982, 1990), Silva (1983) and Christofides (2003) have also considered the estimation of multi-attribute parameters.

Guerriero and Sandri (2007) pointed out that the family of models proposed by Kuk (1990) is better than the Simmons' family (refer to Greenbeg et al. (1969) ) in terms of efficiency and privacy protection. From an empirical standpoint, van der Heijden, et al. (2000) showsed that Kuk's procedure performs slightly better than the forced-response procedure and markedly better than face-to-face direct questioning and computer assisted selfinterviewing.

They also noted that the recommendations and successful applications of Kuk's procedure have been reported in van den Hout and van der Heijden (2002), and these results should be even more marked for the model proposed by Christofides (2003). In addition, an adequate analysis of the efficiency and the respondents' protection is always necessary when proposing new randomized response models. Thus, following Guerriero and Sandri (2007), it is worthwhile to extend the Kuk (1990) and Franklin (1989) type models. Note that the the Mangat (1994), Mangat and Singh (1990), Gjestvang and Singh (2006) and Kuk (1990) models are special cases of the Franklin (1989) model. Additional work on randomized response sampling is available in Singh and Kim (2011), Diana and Perri (2009), Tan, et al. (2009) and Esponda and Guerrero (2009).

Proposed Randomized Response Technique

In the proposed randomized response device, if a person selected in the sample belongs to the first sensitive group $A_{1}$ then that person is requested to draw a random number $S_{1}$ from a density function $f_{1}(s)$ and report to the interviewer; if that person belongs to second sensitive group $A_{2}$ then that person is requested to draw a random number $S_{2}$ from a density function $f_{2}(s)$ and report to the interviewer; and if that person belongs to the third sensitive 


\section{MULTINOMIAL PROPORTION ESTIMATION USING HIGHER ORDER MOMENTS}

group $A_{3}$, then that person is requested to draw a random number $S_{3}$ from a density function $f_{3}(s)$ and report to the interviewer. The respondent is further requested not to disclose the mode of response.

Let $\Omega$ be the population under study; $\Omega=\bigcup_{k=1}^{3} A_{k}$ and the groups $A_{k}$ are mutually exclusive. The choice of the three densities $f_{1}(s), f_{2}(s)$ and $f_{3}(s)$ are comprised such that respondents should feel safe in reporting the random number drawn. In other words, to maintain the privacy of respondents from all the three groups, the mean values and the variances of the three densities should not deviate too greatly from each other. In particular, the densities $f_{1}(s), f_{2}(s)$ and $f_{3}(s)$ could be normal, beta, gamma or some other distribution.

Let $\pi_{1}, \pi_{2}$ and $\pi_{3}$ represent the true proportions of persons belonging to groups $A_{1}$, $A_{2}$ and $A_{3}$ respectively such that $\pi_{1}+\pi_{2}+\pi_{3}=1$. Assume that $E$ denotes the expected value over the proposed randomization response device, and let $\theta_{1}=E\left(S_{1}\right), \theta_{2}=E\left(S_{2}\right)$, $\theta_{3}=E\left(S_{3}\right)$,

and

$\gamma_{a b c}=E\left[\left(S_{1}-\theta_{1}\right)^{a}\left(S_{2}-\theta_{2}\right)^{b}\left(S_{3}-\theta_{3}\right)^{c}\right]$, where $a, b$ and $c$ are non-negative integers and are known moments of the three scrambling variables used in the proposed randomization device. Consider a simple random with replacement sample (SRSWR) of $n$ respondents. Interestingly, it can be shown that, based on only single sample information, three unbiased estimates of the three different parameters can be proposed. The distribution of the responses will be as follows:

$$
Z_{i}=\left\{\begin{array}{l}
S_{1} \text { with probability } \pi_{1} \\
S_{2} \text { with probability } \pi_{2} \\
S_{3} \text { with probability } \pi_{3}
\end{array}\right.
$$

If

$$
E\left(Z_{i}\right)=\pi_{1} \theta_{1}+\pi_{2} \theta_{2}+\left(1-\pi_{1}-\pi_{2}\right) \theta_{3}
$$

then, following Singh and Chen (2009),

$$
Z_{i}^{2}=\left\{\begin{array}{l}
S_{1}^{2} \text { with probability } \pi_{1} \\
S_{2}^{2} \text { with probability } \pi_{2} \\
S_{3}^{2} \text { with probability } \pi_{3}
\end{array}\right.
$$

where $E\left(S_{1}^{2}\right)=\gamma_{200}+\theta_{1}^{2}, \quad E\left(S_{2}^{2}\right)=\gamma_{020}+\theta_{2}^{2}$ and $E\left(S_{3}^{2}\right)=\gamma_{002}+\theta_{3}^{2}$.

If

$$
\begin{aligned}
E\left(Z_{i}^{2}\right)= & \pi_{1}\left(\gamma_{200}+\theta_{1}^{2}\right)+\pi_{2}\left(\gamma_{020}+\theta_{2}^{2}\right) \\
& +\left(1-\pi_{1}-\pi_{2}\right)\left(\gamma_{002}+\theta_{3}^{2}\right)
\end{aligned}
$$

and defining

$$
\begin{aligned}
\Delta= & \left(\theta_{1}-\theta_{3}\right)\left\{\left(\gamma_{020}+\theta_{2}^{2}\right)-\left(\gamma_{002}+\theta_{3}^{2}\right)\right\} \\
& -\left(\theta_{2}-\theta_{3}\right)\left\{\left(\gamma_{200}+\theta_{1}^{2}\right)-\left(\gamma_{002}+\theta_{3}^{2}\right)\right\},
\end{aligned}
$$

several theorems and lemmas may be put forth (see Appendix A).

Empirical Comparisons

It is possible to use the Warner (1965) model three times to estimate the three nonoverlapping parameters $\pi_{k}, k=1,2,3$. Each respondent selected in the sample could be provided with three randomization devices, for example, $R_{k}, k=1,2,3$. The randomization $R_{k}$ bears two types of statements, are you a member of group $A_{k}$ ?, and are you a member of group $A_{k}^{c}$ ? with probabilities $P_{k}$ and $\left(1-P_{k}\right)$, respectively. Based on a sample of $n$ respondents, if $n_{k}$ reports yes related to the $k^{\text {th }}$ group, then the unbiased estimator of $\pi_{k}$ is

$$
\begin{aligned}
& \hat{\pi}_{k(w)}=\frac{n_{k} / n-\left(1-P_{k}\right)}{2 P_{k}-1}, \\
& P_{k} \neq 0.5
\end{aligned}
$$

with variance 


\section{CHENG CHEN \& SARJINDER SINGH}

$$
V\left(\hat{\pi}_{k(w)}\right)=\frac{\pi_{k}\left(1-\pi_{k}\right)}{n}+\frac{P_{k}\left(1-P_{k}\right)}{n\left(2 P_{k}-1\right)^{2}} .
$$

The relative efficiency of the proposed estimator $\hat{\pi}_{k}$ (as defined in the Appendix) with respect to the corresponding estimator $\hat{\pi}_{k(w)}$ (Warner, 1965) is:

$$
\begin{aligned}
& R E(k)=\frac{V\left(\hat{\pi}_{k(w)}\right)}{V\left(\hat{\pi}_{k}\right)} \times 100 \%, \\
& k=1,2,3 .
\end{aligned}
$$

\section{Results}

Choosing $P_{k}=0.7, k=1,2,3$, based on Warner (1965) is a reasonable and practical choice for the model, considering the problem of estimation of $\pi_{k}$ with their respective estimators $\hat{\pi}_{k(w)}$ for $k=1,2,3$. A privacy protection criterion is suggested, that is:

$$
\lambda_{Z_{k, i}}=\frac{f\left(Z_{k} \mid k \in A_{i}\right)}{f\left(Z_{k} \mid k \notin A_{i}\right)}
$$

and refers to the privacy protection with respect to response $Z_{k}$ for a respondent $k$ being a member of $A_{i}$. For these measures $0 \leq \lambda_{Z_{k, i}}<\infty$ applies with $\lambda_{Z_{k, 1}}=1$ indicating data privacy protection for unit $k$ being a member of group $A_{i}$. This means that the value $Z_{k}$ contains absolutely no information on the variable of interest; the more the $\lambda$-measure differs from unity the more information on the variable under study is contained in the response, meaning the less the privacy protection. The maximum $\lambda_{Z_{k, i}}=\infty$ (or 0 ) describes a situation where membership or the non-membership of $A_{i}$ may be concluded based on the answer $Z_{k}$ directly. A respondent would answer untruthfully or not answer at all in such a case.

Bearing in mind the proposed privacy protection criterion in (3.4), choice of the known parameters of the scrambling variables was: $\theta_{1}=57, \quad \theta_{2}=62, \quad \theta_{3}=60, \quad \gamma_{200}=0.5$, $\gamma_{020}=3.5$, and $\gamma_{002}=4.5$ in the proposed model with three scrambling variables. Based on the three sigma empirical rule, most of the values of the scrambling variables $S_{1}, S_{2}$ and $S_{3}$ could be any real numbers in the ranges: (54.87, 59.12); (56.38, 67.61), and (53.63, 66.36) respectively, but those values are not $100 \%$ bounded to these intervals. Due to an overlap between the three intervals, it is difficult to guess the status of the respondents based on their reported responses. Using the four sigma rule the scrambling variables $S_{1}, S_{2}$ and $S_{3}$ could, respectively, be any real numbers in the ranges: $(54.17,59.82)$; $(54.51,69.48)$, and $(51.51,68.48)$, and the six sigma empirical rule can be considered in a similar manner.

To study the effect of known higher order moments, such as skewness and kurtosis, of the scrambling variables on the relative efficiencies $R E(k)$ of the proposed estimators we studied different values of $\gamma_{3}=\gamma_{300}=\gamma_{030}=\gamma_{003}$ as $-2,0,3,5,10$ and 20; and the values of the $\gamma_{4}=\gamma_{400}=\gamma_{040}=\gamma_{004}$ as 2 , 3,5 and 10 . The minimum relative efficiency of $103 \%$ was retained by assuming that a minimum $3 \%$ gain is enough if one methodology could gain over the other without affecting the respondents' cooperation (see Table 1).

Note that, while estimating rare attributes in two groups such as $\pi_{1}=0.1$, and $\pi_{2}=0.1$, then $\pi_{3}=0.8$ and based on 3 observations, the relative efficiencies $R E(1)$, $R E(2)$ and $R E(3)$ remain as $615.3 \%, 451.1 \%$, $713.4 \%$ for $\gamma_{300}=\gamma_{030}=\gamma_{003}=-2 \quad$ and $\gamma_{400}=\gamma_{040}=\gamma_{004}=10$. Keeping the same value of $\gamma_{400}=\gamma_{040}=\gamma_{004}=10, \quad$ for $\gamma_{300}=\gamma_{030}=\gamma_{003}=0$, the $\operatorname{RE}(1)$, and $R E(3)$ values become $919.0 \%, 306.8 \%$ and $562.5 \%$. Thus, changing the value of $\gamma_{300}=\gamma_{030}=\gamma_{003}$ from -2 to 0 , the $R E(1)$ increases from $615.3 \%$ to $919.0 \%$, but the value of $R E(2)$ decreases from $451.1 \%$ to $306.8 \%$, and the value of $R E(3)$ decreases from $713.4 \%$ to $562.5 \%$. As the values of $\gamma_{300}=\gamma_{030}=\gamma_{003}$ increase to 3 , there is huge increase in the value of $R E(1)$ to $3543.1 \%$, whereas a decrease in the values of $R E(2)$ and 
MULTINOMIAL PROPORTION ESTIMATION USING HIGHER ORDER MOMENTS

Table 1: Relative efficiencies, $R E(k), k=1,2,3$, of Proposed Estimators for Difference Choices of Known Higher Order Moments of the Three Scrambling Variables

\begin{tabular}{|c|c|c|c|c|c|c|c|c|c|c|c|}
\hline$\pi_{1}$ & $\pi_{2}$ & $\pi_{3}$ & $\gamma_{300}$ & $\gamma_{030}$ & $\gamma_{003}$ & $\gamma_{400}$ & $\gamma_{040}$ & $\gamma_{004}$ & $R E(1)$ & $R E(2)$ & $R E(3)$ \\
\hline \multirow{33}{*}{0.1} & \multirow{3}{*}{0.1} & \multirow{3}{*}{0.8} & -2 & -2 & -2 & 10 & 10 & 10 & 615.3 & 451.1 & 713.4 \\
\hline & & & 0 & 0 & 0 & 10 & 10 & 10 & 919.0 & 306.8 & 562.5 \\
\hline & & & 3 & 3 & 3 & 10 & 10 & 10 & 3543.1 & 207.3 & 426.9 \\
\hline & \multirow{12}{*}{0.2} & \multirow{12}{*}{0.7} & -2 & -2 & -2 & 2 & 2 & 2 & 977.6 & 358.7 & 1368.6 \\
\hline & & & -2 & -2 & -2 & 3 & 3 & 3 & 907.5 & 338.1 & 843.5 \\
\hline & & & -2 & -2 & -2 & 5 & 5 & 5 & 793.7 & 303.4 & 477.3 \\
\hline & & & -2 & -2 & -2 & 10 & 10 & 10 & 604.3 & 241.4 & 228.9 \\
\hline & & & 0 & 0 & 0 & 2 & 2 & 2 & 1633.8 & 246.8 & 548.7 \\
\hline & & & 0 & 0 & 0 & 3 & 3 & 3 & 1447.0 & 236.9 & 439.1 \\
\hline & & & 0 & 0 & 0 & 5 & 5 & 5 & 1177.7 & 219.3 & 313.8 \\
\hline & & & 0 & 0 & 0 & 10 & 10 & 10 & 803.8 & 185.0 & 183.1 \\
\hline & & & 3 & 3 & 3 & 3 & 3 & 3 & 13358.9 & 163.5 & 255.4 \\
\hline & & & 3 & 3 & 3 & 5 & 5 & 5 & 4294.3 & 154.9 & 207.3 \\
\hline & & & 3 & 3 & 3 & 10 & 10 & 10 & 1592.6 & 136.9 & 140.9 \\
\hline & & & 5 & 5 & 5 & 10 & 10 & 10 & 4606.9 & 116.7 & 122.1 \\
\hline & \multirow{11}{*}{0.3} & \multirow{11}{*}{0.6} & -2 & -2 & -2 & 2 & 2 & 2 & 950.1 & 220.8 & 282.2 \\
\hline & & & -2 & -2 & -2 & 3 & 3 & 3 & 883.8 & 213.1 & 250.7 \\
\hline & & & -2 & -2 & -2 & 5 & 5 & 5 & 775.5 & 199.2 & 204.9 \\
\hline & & & -2 & -2 & -2 & 10 & 10 & 10 & 593.6 & 171.2 & 140.6 \\
\hline & & & 0 & 0 & 0 & 2 & 2 & 2 & 1301.9 & 166.3 & 187.7 \\
\hline & & & 0 & 0 & 0 & 3 & 3 & 3 & 1180.5 & 161.9 & 173.2 \\
\hline & & & 0 & 0 & 0 & 5 & 5 & 5 & 994.9 & 153.7 & 150.0 \\
\hline & & & 0 & 0 & 0 & 10 & 10 & 10 & 714.2 & 136.5 & 112.4 \\
\hline & & & 3 & 3 & 3 & 2 & 2 & 2 & 2928.3 & 121.3 & 124.9 \\
\hline & & & 3 & 3 & 3 & 3 & 3 & 3 & 2378.1 & 119.0 & 118.3 \\
\hline & & & 3 & 3 & 3 & 5 & 5 & 5 & 1728.6 & 114.5 & 107.0 \\
\hline & \multirow{5}{*}{0.4} & \multirow{5}{*}{0.5} & -2 & -2 & -2 & 2 & 2 & 2 & 924.1 & 163.6 & 161.3 \\
\hline & & & -2 & -2 & -2 & 3 & 3 & 3 & 861.2 & 159.5 & 150.5 \\
\hline & & & -2 & -2 & -2 & 5 & 5 & 5 & 758.1 & 151.7 & 132.8 \\
\hline & & & 0 & 0 & 0 & 2 & 2 & 2 & 1082.1 & 127.8 & 115.2 \\
\hline & & & 0 & 0 & 0 & 3 & 3 & 3 & 996.9 & 125.2 & 109.6 \\
\hline & \multirow{2}{*}{0.5} & \multirow{2}{*}{0.4} & -2 & -2 & -2 & 2 & 2 & 2 & 899.5 & 131.5 & 113.5 \\
\hline & & & -2 & -2 & -2 & 3 & 3 & 3 & 839.8 & 128.8 & 108.0 \\
\hline
\end{tabular}




\section{CHENG CHEN \& SARJINDER SINGH}

Table 1 (continued): Relative efficiencies, $R E(k), k=1,2,3$, of Proposed Estimators for Difference Choices of Known Higher Order Moments of the Three Scrambling Variables

\begin{tabular}{|c|c|c|c|c|c|c|c|c|c|c|c|}
\hline$\pi_{1}$ & $\pi_{2}$ & $\pi_{3}$ & $\gamma_{300}$ & $\gamma_{030}$ & $\gamma_{003}$ & $\gamma_{400}$ & $\gamma_{040}$ & $\gamma_{004}$ & $R E(1)$ & $R E(2)$ & $R E(3)$ \\
\hline \multirow{37}{*}{0.2} & \multirow{10}{*}{0.1} & \multirow{10}{*}{0.7} & -2 & -2 & -2 & 2 & 2 & 2 & 529.7 & 657.2 & 1375.4 \\
\hline & & & -2 & -2 & -2 & 3 & 3 & 3 & 509.4 & 588.5 & 846.1 \\
\hline & & & -2 & -2 & -2 & 5 & 5 & 5 & 473.1 & 486.6 & 478.1 \\
\hline & & & -2 & -2 & -2 & 10 & 10 & 10 & 401.6 & 339.7 & 229.0 \\
\hline & & & 0 & 0 & 0 & 3 & 3 & 3 & 786.8 & 431.9 & 2202.0 \\
\hline & & & 0 & 0 & 0 & 5 & 5 & 5 & 703.5 & 374.4 & 733.2 \\
\hline & & & 0 & 0 & 0 & 10 & 10 & 10 & 556.3 & 280.9 & 274.9 \\
\hline & & & 3 & 3 & 3 & 5 & 5 & 5 & 2610.9 & 278.1 & 3673.9 \\
\hline & & & 3 & 3 & 3 & 10 & 10 & 10 & 1317.1 & 223.0 & 392.7 \\
\hline & & & 5 & 5 & 5 & 10 & 10 & 10 & 14931.8 & 196.1 & 549.8 \\
\hline & \multirow{14}{*}{0.2} & \multirow{14}{*}{0.6} & -2 & -2 & -2 & 2 & 2 & 2 & 521.9 & 287.3 & 282.5 \\
\hline & & & -2 & -2 & -2 & 3 & 3 & 3 & 502.1 & 274.0 & 250.9 \\
\hline & & & -2 & -2 & -2 & 5 & 5 & 5 & 466.9 & 250.7 & 205.0 \\
\hline & & & -2 & -2 & -2 & 10 & 10 & 10 & 397.1 & 206.8 & 140.7 \\
\hline & & & 0 & 0 & 0 & 2 & 2 & 2 & 743.9 & 230.5 & 282.5 \\
\hline & & & 0 & 0 & 0 & 3 & 3 & 3 & 704.4 & 221.8 & 250.9 \\
\hline & & & 0 & 0 & 0 & 5 & 5 & 5 & 636.9 & 206.3 & 205.0 \\
\hline & & & 0 & 0 & 0 & 10 & 10 & 10 & 513.8 & 175.7 & 140.7 \\
\hline & & & 3 & 3 & 3 & 2 & 2 & 2 & 2055.6 & 177.8 & 282.5 \\
\hline & & & 3 & 3 & 3 & 3 & 3 & 3 & 1780.2 & 172.6 & 250.9 \\
\hline & & & 3 & 3 & 3 & 5 & 5 & 5 & 1404.0 & 163.1 & 205.0 \\
\hline & & & 3 & 3 & 3 & 10 & 10 & 10 & 918.7 & 143.3 & 140.7 \\
\hline & & & 5 & 5 & 5 & 5 & 5 & 5 & 7125.6 & 143.0 & 205.0 \\
\hline & & & 5 & 5 & 5 & 10 & 10 & 10 & 1935.8 & 127.6 & 140.7 \\
\hline & \multirow{11}{*}{0.3} & \multirow{11}{*}{0.5} & -2 & -2 & -2 & 2 & 2 & 2 & 514.3 & 192.3 & 161.3 \\
\hline & & & -2 & -2 & -2 & 3 & 3 & 3 & 495.1 & 186.5 & 150.6 \\
\hline & & & -2 & -2 & -2 & 5 & 5 & 5 & 460.8 & 175.7 & 132.8 \\
\hline & & & 0 & 0 & 0 & 2 & 2 & 2 & 669.8 & 159.0 & 144.8 \\
\hline & & & 0 & 0 & 0 & 3 & 3 & 3 & 637.7 & 154.9 & 136.0 \\
\hline & & & 0 & 0 & 0 & 5 & 5 & 5 & 581.8 & 147.4 & 121.4 \\
\hline & & & 3 & 3 & 3 & 2 & 2 & 2 & 1226.0 & 126.1 & 125.5 \\
\hline & & & 3 & 3 & 3 & 3 & 3 & 3 & 1122.4 & 123.6 & 118.9 \\
\hline & & & 3 & 3 & 3 & 5 & 5 & 5 & 960.2 & 118.8 & 107.5 \\
\hline & & & 5 & 5 & 5 & 2 & 2 & 2 & 2745.7 & 110.9 & 115.2 \\
\hline & & & 5 & 5 & 5 & 3 & 3 & 3 & 2275.6 & 108.9 & 109.6 \\
\hline & \multirow{2}{*}{0.4} & \multirow{2}{*}{0.4} & -2 & -2 & -2 & 2 & 2 & 2 & 506.9 & 147.8 & 113.5 \\
\hline & & & -2 & -2 & -2 & 3 & 3 & 3 & 488.3 & 144.3 & 108.1 \\
\hline
\end{tabular}


MULTINOMIAL PROPORTION ESTIMATION USING HIGHER ORDER MOMENTS

Table 1 (continued): Relative efficiencies, $R E(k), k=1,2,3$, of Proposed Estimators for Difference Choices of Known Higher Order Moments of the Three Scrambling Variables

\begin{tabular}{|c|c|c|c|c|c|c|c|c|c|c|c|}
\hline$\pi_{1}$ & $\pi_{2}$ & $\pi_{3}$ & $\gamma_{300}$ & $\gamma_{030}$ & $\gamma_{003}$ & $\gamma_{400}$ & $\gamma_{040}$ & $\gamma_{004}$ & $R E(1)$ & $R E(2)$ & $R E(3)$ \\
\hline \multirow{36}{*}{0.3} & \multirow{11}{*}{0.1} & \multirow{11}{*}{0.6} & -2 & -2 & -2 & 2 & 2 & 2 & 383.8 & 444.7 & 282.8 \\
\hline & & & -2 & -2 & -2 & 3 & 3 & 3 & 373.4 & 412.2 & 251.1 \\
\hline & & & -2 & -2 & -2 & 5 & 5 & 5 & 354.1 & 359.5 & 205.2 \\
\hline & & & -2 & -2 & -2 & 10 & 10 & 10 & 313.7 & 272.4 & 140.8 \\
\hline & & & 0 & 0 & 0 & 2 & 2 & 2 & 567.7 & 410.2 & 570.8 \\
\hline & & & 0 & 0 & 0 & 3 & 3 & 3 & 545.2 & 382.3 & 454.9 \\
\hline & & & 0 & 0 & 0 & 5 & 5 & 5 & 505.1 & 336.5 & 323.6 \\
\hline & & & 0 & 0 & 0 & 10 & 10 & 10 & 426.7 & 259.1 & 188.0 \\
\hline & & & 3 & 3 & 3 & 5 & 5 & 5 & 1401.7 & 307.1 & 2416.8 \\
\hline & & & 3 & 3 & 3 & 10 & 10 & 10 & 928.3 & 241.3 & 378.2 \\
\hline & & & 5 & 5 & 5 & 10 & 10 & 10 & 4290.6 & 230.7 & 1163.0 \\
\hline & \multirow{15}{*}{0.2} & \multirow{15}{*}{0.5} & -2 & -2 & -2 & 2 & 2 & 2 & 379.8 & 239.7 & 161.4 \\
\hline & & & -2 & -2 & -2 & 3 & 3 & 3 & 369.6 & 230.3 & 150.7 \\
\hline & & & -2 & -2 & -2 & 5 & 5 & 5 & 350.7 & 213.6 & 132.9 \\
\hline & & & 0 & 0 & 0 & 2 & 2 & 2 & 524.9 & 216.3 & 194.9 \\
\hline & & & 0 & 0 & 0 & 3 & 3 & 3 & 505.6 & 208.6 & 179.4 \\
\hline & & & 0 & 0 & 0 & 5 & 5 & 5 & 470.9 & 194.9 & 154.8 \\
\hline & & & 0 & 0 & 0 & 10 & 10 & 10 & 402.0 & 167.3 & 115.3 \\
\hline & & & 3 & 3 & 3 & 2 & 2 & 2 & 1229.3 & 188.6 & 282.9 \\
\hline & & & 3 & 3 & 3 & 3 & 3 & 3 & 1128.4 & 182.8 & 251.4 \\
\hline & & & 3 & 3 & 3 & 5 & 5 & 5 & 969.2 & 172.1 & 205.6 \\
\hline & & & 3 & 3 & 3 & 10 & 10 & 10 & 716.5 & 150.2 & 141.2 \\
\hline & & & 5 & 5 & 5 & 2 & 2 & 2 & 11669.3 & 173.8 & 404.6 \\
\hline & & & 5 & 5 & 5 & 3 & 3 & 3 & 6310.2 & 168.9 & 343.1 \\
\hline & & & 5 & 5 & 5 & 5 & 5 & 5 & 3289.2 & 159.7 & 263.1 \\
\hline & & & 5 & 5 & 5 & 10 & 10 & 10 & 1497.2 & 140.7 & 166.2 \\
\hline & \multirow{10}{*}{0.3} & \multirow{10}{*}{0.4} & -2 & -2 & -2 & 2 & 2 & 2 & 375.9 & 170.4 & 113.6 \\
\hline & & & -2 & -2 & -2 & 3 & 3 & 3 & 365.9 & 165.8 & 108.1 \\
\hline & & & 0 & 0 & 0 & 2 & 2 & 2 & 488.1 & 152.3 & 118.4 \\
\hline & & & 0 & 0 & 0 & 3 & 3 & 3 & 471.3 & 148.6 & 112.5 \\
\hline & & & 3 & 3 & 3 & 2 & 2 & 2 & 883.5 & 131.3 & 126.4 \\
\hline & & & 3 & 3 & 3 & 3 & 3 & 3 & 830.1 & 128.5 & 119.7 \\
\hline & & & 3 & 3 & 3 & 5 & 5 & 5 & 740.6 & 123.4 & 108.1 \\
\hline & & & 5 & 5 & 5 & 2 & 2 & 2 & 1921.1 & 120.3 & 132.4 \\
\hline & & & 5 & 5 & 5 & 3 & 3 & 3 & 1685.4 & 118.0 & 125.0 \\
\hline & & & 5 & 5 & 5 & 5 & 5 & 5 & 1353.4 & 113.6 & 112.5 \\
\hline
\end{tabular}




\section{CHENG CHEN \& SARJINDER SINGH}

Table 1 (continued): Relative efficiencies, $R E(k), k=1,2,3$, of Proposed Estimators for Difference Choices of Known Higher Order Moments of the Three Scrambling Variables

\begin{tabular}{|c|c|c|c|c|c|c|c|c|c|c|c|}
\hline$\pi_{1}$ & $\pi_{2}$ & $\pi_{3}$ & $\gamma_{300}$ & $\gamma_{030}$ & $\gamma_{003}$ & $\gamma_{400}$ & $\gamma_{040}$ & $\gamma_{004}$ & $R E(1)$ & $R E(2)$ & $R E(3)$ \\
\hline \multirow{28}{*}{0.4} & \multirow{9}{*}{0.1} & \multirow{9}{*}{0.5} & -2 & -2 & -2 & 2 & 2 & 2 & 313.4 & 336.1 & 161.5 \\
\hline & & & -2 & -2 & -2 & 3 & 3 & 3 & 306.5 & 317.2 & 150.7 \\
\hline & & & -2 & -2 & -2 & 5 & 5 & 5 & 293.7 & 285.0 & 133.0 \\
\hline & & & 0 & 0 & 0 & 2 & 2 & 2 & 456.2 & 365.2 & 298.1 \\
\hline & & & 0 & 0 & 0 & 3 & 3 & 3 & 441.9 & 342.9 & 263.3 \\
\hline & & & 0 & 0 & 0 & 5 & 5 & 5 & 415.6 & 305.7 & 213.5 \\
\hline & & & 0 & 0 & 0 & 10 & 10 & 10 & 362.0 & 240.4 & 144.9 \\
\hline & & & 3 & 3 & 3 & 5 & 5 & 5 & 1102.4 & 342.9 & 2330.8 \\
\hline & & & 3 & 3 & 3 & 10 & 10 & 10 & 791.2 & 262.8 & 378.1 \\
\hline & \multirow{13}{*}{0.2} & \multirow{13}{*}{0.4} & -2 & -2 & -2 & 2 & 2 & 2 & 310.8 & 205.5 & 113.6 \\
\hline & & & -2 & -2 & -2 & 3 & 3 & 3 & 304.0 & 198.6 & 108.2 \\
\hline & & & 0 & 0 & 0 & 2 & 2 & 2 & 428.7 & 203.7 & 150.2 \\
\hline & & & 0 & 0 & 0 & 3 & 3 & 3 & 416.0 & 196.9 & 140.8 \\
\hline & & & 0 & 0 & 0 & 5 & 5 & 5 & 392.6 & 184.6 & 125.0 \\
\hline & & & 3 & 3 & 3 & 2 & 2 & 2 & 994.8 & 200.9 & 290.1 \\
\hline & & & 3 & 3 & 3 & 3 & 3 & 3 & 928.8 & 194.3 & 256.9 \\
\hline & & & 3 & 3 & 3 & 5 & 5 & 5 & 820.1 & 182.3 & 209.0 \\
\hline & & & 3 & 3 & 3 & 10 & 10 & 10 & 634.4 & 157.9 & 142.5 \\
\hline & & & 5 & 5 & 5 & 2 & 2 & 2 & 8315.3 & 199.1 & 765.8 \\
\hline & & & 5 & 5 & 5 & 3 & 3 & 3 & 5218.4 & 192.6 & 570.8 \\
\hline & & & 5 & 5 & 5 & 5 & 5 & 5 & 2990.7 & 180.8 & 378.2 \\
\hline & & & 5 & 5 & 5 & 10 & 10 & 10 & 1446.7 & 156.8 & 205.2 \\
\hline & \multirow{6}{*}{0.3} & \multirow{6}{*}{0.3} & 3 & 3 & 3 & 2 & 2 & 2 & 759.0 & 136.9 & 127.8 \\
\hline & & & 3 & 3 & 3 & 3 & 3 & 3 & 720.0 & 133.9 & 120.8 \\
\hline & & & 3 & 3 & 3 & 5 & 5 & 5 & 652.9 & 128.3 & 108.8 \\
\hline & & & 5 & 5 & 5 & 2 & 2 & 2 & 1829.2 & 131.4 & 157.1 \\
\hline & & & 5 & 5 & 5 & 3 & 3 & 3 & 1617.9 & 128.7 & 146.6 \\
\hline & & & 5 & 5 & 5 & 5 & 5 & 5 & 1314.4 & 123.5 & 129.3 \\
\hline
\end{tabular}


MULTINOMIAL PROPORTION ESTIMATION USING HIGHER ORDER MOMENTS

Table 1 (continued): Relative efficiencies, $R E(k), k=1,2,3$, of Proposed Estimators for Difference Choices of Known Higher Order Moments of the Three Scrambling Variables

\begin{tabular}{|c|c|c|c|c|c|c|c|c|c|c|c|}
\hline$\pi_{1}$ & $\pi_{2}$ & $\pi_{3}$ & $\gamma_{300}$ & $\gamma_{030}$ & $\gamma_{003}$ & $\gamma_{400}$ & $\gamma_{040}$ & $\gamma_{004}$ & $R E(1)$ & $R E(2)$ & $R E(3)$ \\
\hline \multirow{25}{*}{0.5} & \multirow{8}{*}{0.1} & \multirow{8}{*}{0.4} & -2 & -2 & -2 & 2 & 2 & 2 & 272.2 & 270.1 & 113.7 \\
\hline & & & -2 & -2 & -2 & 3 & 3 & 3 & 267.0 & 257.7 & 108.2 \\
\hline & & & 0 & 0 & 0 & 2 & 2 & 2 & 398.2 & 329.1 & 205.3 \\
\hline & & & 0 & 0 & 0 & 3 & 3 & 3 & 387.3 & 310.9 & 188.1 \\
\hline & & & 0 & 0 & 0 & 5 & 5 & 5 & 367.1 & 280.0 & 161.1 \\
\hline & & & 0 & 0 & 0 & 10 & 10 & 10 & 324.8 & 224.2 & 118.5 \\
\hline & & & 3 & 3 & 3 & 5 & 5 & 5 & 1020.9 & 388.1 & 3115.4 \\
\hline & & & 3 & 3 & 3 & 10 & 10 & 10 & 749.6 & 288.6 & 392.0 \\
\hline & \multirow{10}{*}{0.2} & \multirow{10}{*}{0.3} & 0 & 0 & 0 & 2 & 2 & 2 & 377.2 & 192.5 & 122.2 \\
\hline & & & 0 & 0 & 0 & 3 & 3 & 3 & 367.3 & 186.4 & 115.8 \\
\hline & & & 0 & 0 & 0 & 5 & 5 & 5 & 349.2 & 175.3 & 104.7 \\
\hline & & & 3 & 3 & 3 & 2 & 2 & 2 & 928.5 & 214.9 & 305.8 \\
\hline & & & 3 & 3 & 3 & 3 & 3 & 3 & 871.1 & 207.3 & 268.4 \\
\hline & & & 3 & 3 & 3 & 5 & 5 & 5 & 775.3 & 193.7 & 215.7 \\
\hline & & & 3 & 3 & 3 & 10 & 10 & 10 & 608.2 & 166.4 & 144.7 \\
\hline & & & 5 & 5 & 5 & 3 & 3 & 3 & 10163.3 & 224.1 & 2219.8 \\
\hline & & & 5 & 5 & 5 & 5 & 5 & 5 & 4162.8 & 208.3 & 735.2 \\
\hline & & & 5 & 5 & 5 & 10 & 10 & 10 & 1681.3 & 177.1 & 275.1 \\
\hline & \multirow{7}{*}{0.3} & \multirow{7}{*}{0.2} & 3 & 3 & 3 & 2 & 2 & 2 & 720.8 & 143.1 & 129.9 \\
\hline & & & 3 & 3 & 3 & 3 & 3 & 3 & 685.8 & 139.8 & 122.4 \\
\hline & & & 3 & 3 & 3 & 5 & 5 & 5 & 625.0 & 133.7 & 109.8 \\
\hline & & & 5 & 5 & 5 & 2 & 2 & 2 & 2216.4 & 144.9 & 197.4 \\
\hline & & & 5 & 5 & 5 & 3 & 3 & 3 & 1915.3 & 141.5 & 180.6 \\
\hline & & & 5 & 5 & 5 & 5 & 5 & 5 & 1506.2 & 135.3 & 154.4 \\
\hline & & & 5 & 5 & 5 & 10 & 10 & 10 & 981.8 & 121.8 & 113.3 \\
\hline
\end{tabular}




\section{CHENG CHEN \& SARJINDER SINGH}

Table 1 (continued): Relative efficiencies, $R E(k), k=1,2,3$, of Proposed Estimators for Difference Choices of Known Higher Order Moments of the Three Scrambling Variables

\begin{tabular}{|c|c|c|c|c|c|c|c|c|c|c|c|}
\hline$\pi_{1}$ & $\pi_{2}$ & $\pi_{3}$ & $\gamma_{300}$ & $\gamma_{030}$ & $\gamma_{003}$ & $\gamma_{400}$ & $\gamma_{040}$ & $\gamma_{004}$ & $R E(1)$ & $R E(2)$ & $R E(3)$ \\
\hline \multirow{17}{*}{0.6} & \multirow{5}{*}{0.1} & \multirow{5}{*}{0.3} & 0 & 0 & 0 & 2 & 2 & 2 & 365.7 & 299.5 & 157.2 \\
\hline & & & 0 & 0 & 0 & 3 & 3 & 3 & 356.4 & 284.4 & 146.7 \\
\hline & & & 0 & 0 & 0 & 5 & 5 & 5 & 339.2 & 258.3 & 129.5 \\
\hline & & & 3 & 3 & 3 & 5 & 5 & 5 & 1068.8 & 447.1 & 12053.1 \\
\hline & & & 3 & 3 & 3 & 10 & 10 & 10 & 773.7 & 320.0 & 424.2 \\
\hline & \multirow{5}{*}{0.2} & \multirow{5}{*}{0.2} & 3 & 3 & 3 & 2 & 2 & 2 & 967.3 & 230.9 & 334.1 \\
\hline & & & 3 & 3 & 3 & 3 & 3 & 3 & 904.8 & 222.2 & 288.7 \\
\hline & & & 3 & 3 & 3 & 5 & 5 & 5 & 801.3 & 206.7 & 227.1 \\
\hline & & & 3 & 3 & 3 & 10 & 10 & 10 & 623.1 & 175.9 & 148.0 \\
\hline & & & 5 & 5 & 5 & 10 & 10 & 10 & 2651.1 & 203.3 & 446.3 \\
\hline & \multirow{7}{*}{0.3} & \multirow{7}{*}{0.1} & 3 & 3 & 3 & 2 & 2 & 2 & 742.9 & 149.8 & 132.7 \\
\hline & & & 3 & 3 & 3 & 3 & 3 & 3 & 705.5 & 146.2 & 124.6 \\
\hline & & & 3 & 3 & 3 & 5 & 5 & 5 & 641.0 & 139.5 & 110.9 \\
\hline & & & 5 & 5 & 5 & 2 & 2 & 2 & 4297.9 & 161.4 & 279.0 \\
\hline & & & 5 & 5 & 5 & 3 & 3 & 3 & 3289.0 & 157.2 & 245.3 \\
\hline & & & 5 & 5 & 5 & 5 & 5 & 5 & 2238.2 & 149.5 & 197.4 \\
\hline & & & 5 & 5 & 5 & 10 & 10 & 10 & 1244.3 & 133.2 & 132.7 \\
\hline \multirow{9}{*}{0.7} & \multirow{4}{*}{0.1} & \multirow{4}{*}{0.2} & 0 & 0 & 0 & 2 & 2 & 2 & 348.7 & 274.8 & 126.9 \\
\hline & & & 0 & 0 & 0 & 3 & 3 & 3 & 340.1 & 262.0 & 119.7 \\
\hline & & & 0 & 0 & 0 & 5 & 5 & 5 & 324.1 & 239.7 & 107.6 \\
\hline & & & 3 & 3 & 3 & 10 & 10 & 10 & 880.7 & 359.0 & 488.1 \\
\hline & \multirow{5}{*}{0.2} & \multirow{5}{*}{0.1} & 3 & 3 & 3 & 2 & 2 & 2 & 1147.2 & 249.6 & 385.8 \\
\hline & & & 3 & 3 & 3 & 3 & 3 & 3 & 1058.8 & 239.4 & 324.1 \\
\hline & & & 3 & 3 & 3 & 5 & 5 & 5 & 917.4 & 221.5 & 245.5 \\
\hline & & & 3 & 3 & 3 & 10 & 10 & 10 & 687.8 & 186.5 & 152.9 \\
\hline & & & 5 & 5 & 5 & 10 & 10 & 10 & 36398.8 & 238.8 & 1620.7 \\
\hline \multirow{2}{*}{0.8} & \multirow{2}{*}{0.1} & \multirow{2}{*}{0.1} & 0 & 0 & 0 & 2 & 2 & 2 & 343.5 & 253.9 & 105.3 \\
\hline & & & 3 & 3 & 3 & 10 & 10 & 10 & 1177.1 & 408.8 & 624.8 \\
\hline
\end{tabular}




\section{MULTINOMIAL PROPORTION ESTIMATION USING HIGHER ORDER MOMENTS}

$R E(3)$ is $207.3 \%$ and $426.9 \%$. Thus, for this case the minimum values of the relative efficiencies $R E(1), R E(2)$ and $R E(3)$ are $615.3 \%, 207.3 \%$ and $426.9 \%$, while the maximum values are $3543.1 \%, 451.1 \%$ and $713.4 \%$ respectively.

Consider another situation: If one of the attributes is rare, $\pi_{1}=0.1$, and the second attribute is moderate, $\pi_{2}=0.3$, then $\pi_{3}=0.6$ and based on 11 observations, the average relative efficiencies $R E(1), R E(2)$ and $R E(3)$ remain as $1311.77 \%, 161.59 \%, 168.35 \%$ with standard deviations $742.70 \%, 37.46 \%$ and $58.19 \%$ respectively as the values different parameters of the scrambling variables changes. The medians of the relative efficiencies $R E(1)$, $R E(2)$ and $R E(3)$ remain $994.9 \%, 161.9 \%$ and $150.0 \%$. The minimum values of the relative efficiencies $R E(1), \quad R E(2)$ and $R E(3)$ are $593.6 \%, \quad 114.5 \%$ and $107.0 \%$ while the maximum values are $2928.3 \%, 220.8 \%$ and $282.2 \%$ respectively.

By contrast, when all three variables have moderate prevalence over the population as $\pi_{1}=0.3, \pi_{2}=0.3$ and $\pi_{3}=0.4$ then, based on 10 observations, the average relative efficiencies $R E(1), R E(2)$ and $R E(3)$ remain as $911.53 \%$, $137.22 \%, \quad 117.67 \%$ with standard deviations $558.53 \%, 20.52 \%$ and $8.19 \%$ respectively as the values different parameters of the scrambling variables changes. The medians of the relative efficiencies $R E(1), R E(2)$ and $R E(3)$ remain $785.35 \%, 129.90 \%$ and $166.00 \%$. The minimum values of the relative efficiencies $R E(1), R E(2)$ and $R E(3)$ are $365.90 \%, 113.60 \%$ and $108.1 \%$ while the maximum values are $1921.10 \%$, $170.40 \%$ and $132.40 \%$ respectively (see Table $1)$.

Note that in Table 1 the $R E(1), R E(2)$ and $R E(3)$ for $\pi_{1}=0.1, \pi_{2}=0.1$ and $\pi_{3}=0.8$ are not the same as for $\pi_{1}=0.8, \pi_{2}=0.1$ and $\pi_{3}=0.1$ because of different choices of mean and variances of the scrambling variables for the three categories. Further note that the choice of parameters considered herein, shows in majority when $\pi_{1}$ remains close to zero, for example $\pi_{1}=0.1$ and $\pi_{2}=0.1$ there are three situations where the proposed method remains efficient and as soon as $\pi_{1}$ becomes 0.8 the proposed method shows efficiency only in two situations.

Thus, the proposed randomization device should be considered for a situation when the first attribute is rare, the second attribute is moderate and the third attribute is widespread. It may be concluded that the proposed randomized response technique based on higher order moments of the scrambling variables can be used to estimate multinomial proportions. The choice of the scrambling variables for a particular study may require an expert to decide based on simulation studies or past experience. The FORTRAN codes used in the simulation study are provided in Appendix A.

Generalization to the Case of a Multinomial Distribution

Consider a population $\Omega$ consisting of $m$ mutually exclusive groups such that $\Omega=\bigcup_{k=1}^{m} A_{k}$. Let $\pi_{k}$ be the proportion of a sensitive attribute is the $k^{\text {th }}$ group. Then extending the proposed randomized response model from Section 2, that a respondent belonging to the $k^{\text {th }}$ group is requested to report a random number from the $k^{\text {th }}$ scrambling variable $S_{k}$ for $k=1,2, \ldots, m$, then $(m-1)$ unbiased estimates of the population proportion $\pi_{k}$ for $k=1,2, . .,(m-1)$ are given by

$$
\hat{\Pi}_{(m-1) \times 1}=\left(A^{-1}\right)_{(m-1) \times(m-1)} Z_{(m-1) \times 1}
$$

where

$$
\hat{\Pi}=\left[\begin{array}{l}
\hat{\pi}_{1} \\
\hat{\pi}_{2} \\
\\
\hat{\pi}_{m-1}
\end{array}\right],
$$

$$
Z=\left[\begin{array}{l}
\frac{1}{n} \sum_{i=1}^{n} Z_{i}-E\left(S_{m}\right) \\
\frac{1}{n} \sum_{i=1}^{n} Z_{i}^{2}-E\left(S_{m}^{2}\right) \\
\frac{1}{n} \sum_{i=1}^{n} Z_{i}^{m-1}-E\left(S_{m}^{m-1}\right)
\end{array}\right]
$$

and 


\section{CHENG CHEN \& SARJINDER SINGH}

$A=$
$\left[\begin{array}{l}E\left(S_{1}\right)-E\left(S_{m}\right), E\left(S_{2}\right)-E\left(S_{m}\right), \ldots \ldots E\left(S_{m-1}\right)-E\left(S_{m}\right) \\ E\left(S_{1}^{2}\right)-E\left(S_{m}^{2}\right), E\left(S_{2}^{2}\right)-E\left(S_{m}^{2}\right), \ldots \ldots E\left(S_{m-1}^{2}\right)-E\left(S_{m}^{2}\right) \\ E\left(S_{1}^{m-1}\right)-E\left(S_{m}^{m-1}\right), E\left(S_{2}^{m-1}\right)-E\left(S_{m}^{m-1}\right), \ldots E\left(S_{m-1}^{m-1}\right)-E\left(S_{m}^{m-1}\right)\end{array}\right]$

and, the unbiased estimate of the proportion $\pi_{m}$ is given by

$$
\hat{\pi}_{m}=1-\sum_{k=1}^{m-1} \hat{\pi}_{k} .
$$

The variance of $\hat{\Pi}$ is given by

and

$$
V(\hat{\Pi})=\left(A^{-1}\right) V(Z)\left(A^{-1}\right)^{t}
$$

$$
V\left(\hat{\pi}_{m}\right)=\sum_{k=1}^{m-1} V\left(\hat{\pi}_{k}\right)+2 \sum_{k<j=1}^{m-1} \operatorname{Cov}\left(\hat{\pi}_{k}, \hat{\pi}_{j}\right)
$$

where $V(Z)$ denotes the variance-covariance matrix of the scrambled responses which utilizes the higher order moments of the scrambling variables $Z_{i}^{l}, t=1,2,3, \ldots ., m$.

\section{Acknowledgements}

The authors are thankful to the Editor Professor Sawilowsky and to Dr. Julie M. Smith to bring the original manuscript in the present form. The authors are also thankful to Ms. Rebecca West at TAMUK for editing the original version of the manuscript.

Appendix A: Theorems and Proofs Theorem 2.1

An unbiased estimator of the population proportion $\pi_{1}$ is given by

$$
\begin{array}{r}
\left\{\left(\gamma_{020}+\theta_{2}^{2}\right)-\left(\gamma_{002}+\theta_{3}^{2}\right)\right\}\left\{\frac{1}{n} \sum_{i=1}^{n} Z_{i}-\theta_{3}\right\} \\
\hat{\pi}_{1}=\frac{-\left(\theta_{2}-\theta_{3}\right)\left\{\frac{1}{n} \sum_{i=1}^{n} Z_{i}^{2}-\left(\gamma_{002}+\theta_{3}^{2}\right)\right\}}{\Delta}
\end{array}
$$

Theorem 2.1 Proof

Solving (2.2) and (2.4) for $\pi_{1}$ and using the method of moments proves the theorem.

Theorem 2.2

An unbiased estimator of the population proportion $\pi_{2}$ is given by

$$
\begin{gathered}
\left(\theta_{1}-\theta_{3}\right)\left\{\frac{1}{n} \sum_{i=1}^{n} Z_{i}^{2}-\left(\gamma_{002}+\theta_{3}^{2}\right)\right\} \\
\hat{\pi}_{2}=\frac{-\left\{\left(\gamma_{200}+\theta_{1}^{2}\right)-\left(\gamma_{002}+\theta_{3}^{2}\right)\right\}\left(\frac{1}{n} \sum_{i=1}^{n} Z_{i}-\theta_{3}\right)}{\Delta}
\end{gathered}
$$

Theorem 2.2 Proof

Solving (2.2) and (2.4) for $\pi_{2}$ and using the method of moments proves the theorem.

Theorem 2.3

An unbiased estimator of the population proportion $\pi_{3}$ is given by

$$
\hat{\pi}_{3}=1-\hat{\pi}_{1}-\hat{\pi}_{2}
$$

Theorem 2.3 Proof

Theorem 2.3 is proven by taking expected values on both sides of (2.8).

Based on these theorems and proofs, the following lemmas are put forth. 


\section{MULTINOMIAL PROPORTION ESTIMATION USING HIGHER ORDER MOMENTS}

\section{Lemma 2.1}

The variance of $Z_{i}$ is given by

$$
\begin{aligned}
V\left(Z_{i}\right)= & A_{1} \pi_{1}+A_{2} \pi_{2}+A_{11} \pi_{1}\left(1-\pi_{1}\right) \\
& +A_{22} \pi_{2}\left(1-\pi_{2}\right)+A_{12} \pi_{1} \pi_{2}+A_{00}
\end{aligned}
$$

where

$$
\begin{gathered}
A_{1}=\left(\gamma_{200}-\gamma_{002}\right), \\
A_{2}=\left(\gamma_{020}-\gamma_{002}\right), \\
A_{00}=\gamma_{002}, \\
A_{11}=\left(\theta_{1}-\theta_{3}\right)^{2}, \\
A_{22}=\left(\theta_{2}-\theta_{3}\right)^{2}
\end{gathered}
$$

and

$$
A_{12}=-2\left(\theta_{1}-\theta_{3}\right)\left(\theta_{2}-\theta_{3}\right) .
$$

Lemma 2.2

The variance of $Z_{i}^{2}$ is given by

$$
\begin{aligned}
V\left(Z_{i}^{2}\right)= & B_{1} \pi_{1}+B_{2} \pi_{2}+B_{11} \pi_{1}\left(1-\pi_{1}\right) \\
& +B_{22} \pi_{2}\left(1-\pi_{2}\right)+B_{12} \pi_{1} \pi_{2}+B_{00}
\end{aligned}
$$

where

$$
\begin{aligned}
B_{1}= & \left(\gamma_{400}-\gamma_{004}\right)+4\left(\gamma_{300} \theta_{1}-\gamma_{003} \theta_{3}\right) \\
& +6\left(\gamma_{200} \theta_{1}^{2}-\gamma_{002} \theta_{3}^{2}\right)+\left(\theta_{1}^{4}-\theta_{3}^{4}\right) \\
& +\left(\gamma_{002}+\theta_{3}^{2}\right)^{2}-\left(\gamma_{200}+\theta_{1}^{2}\right)^{2} \\
B_{2}= & \left(\gamma_{040}-\gamma_{004}\right)+4\left(\gamma_{030} \theta_{2}-\gamma_{003} \theta_{3}\right) \\
& +6\left(\gamma_{020} \theta_{2}^{2}-\gamma_{002} \theta_{3}^{2}\right)+\left(\theta_{2}^{4}-\theta_{3}^{4}\right) \\
& +\left(\gamma_{002}+\theta_{3}^{2}\right)-\left(\gamma_{020}+\theta_{2}^{2}\right)^{2} \\
B_{11}= & {\left[\left(\gamma_{200}+\theta_{1}^{2}\right)-\left(\gamma_{002}+\theta_{3}^{2}\right)\right]^{2} } \\
B_{22}= & {\left[\left(\gamma_{020}+\theta_{2}^{2}\right)-\left(\gamma_{002}+\theta_{3}^{2}\right)\right]^{2} }
\end{aligned}
$$

$B_{12}=$$$
-2\left\{\left(\gamma_{200}+\theta_{1}^{2}\right)-\left(\gamma_{002}+\theta_{3}^{2}\right)\right\}\left\{\left(\gamma_{020}+\theta_{2}^{2}\right)-\left(\gamma_{002}+\theta_{3}^{2}\right)\right\}
$$

and

$$
B_{00}=\left(\gamma_{004}+4 \gamma_{003} \theta_{3}+4 \gamma_{002} \theta_{3}^{2}-\gamma_{002}^{2}\right) .
$$

Lemma 2.2 Proof

Based on the definition of variance,

$$
\begin{aligned}
V\left(Z_{i}^{2}\right)= & E\left(Z_{i}^{4}\right)-\left\{E\left(Z_{i}^{2}\right)\right\}^{2} \\
= & \left(\gamma_{400}+4 \gamma_{300} \theta_{1}+6 \gamma_{200} \theta_{1}^{2}+\theta_{1}^{4}\right) \pi_{1} \\
& +\left(\gamma_{040}+4 \gamma_{030} \theta_{2}+6 \gamma_{020} \theta_{2}^{2}+\theta_{2}^{4}\right) \pi_{2} \\
& +\left(1-\pi_{1}-\pi_{2}\right)\left(\gamma_{004}+4 \gamma_{003} \theta_{3}+6 \gamma_{002} \theta_{3}^{2}+\theta_{3}^{4}\right) \\
& -\left[\begin{array}{l}
\pi_{1}\left(\gamma_{200}+\theta_{1}^{2}\right)+\pi_{2}\left(\gamma_{020}+\gamma_{2}^{2}\right) \\
+\left(1-\pi_{1}-\pi_{2}\right)\left(\gamma_{002}+\theta_{3}^{2}\right)
\end{array}\right]^{2}
\end{aligned}
$$

which, on rearranging reduces to (2.10), and proves the lemma.

Lemma 2.3

The covariance between $Z_{i}$ and $Z_{i}^{2}$ is given by

$$
\begin{aligned}
\operatorname{Cov}\left(Z_{i}, Z_{i}^{2}\right)= & C_{1} \pi_{1}+C_{2} \pi_{2}+C_{11} \pi_{1}\left(1-\pi_{1}\right) \\
& +C_{22} \pi_{2}\left(1-\pi_{2}\right)+C_{12} \pi_{1} \pi_{2}+C_{00}
\end{aligned}
$$

where

$$
\begin{aligned}
C_{1}= & \left(\gamma_{300}-\gamma_{003}\right)+3\left(\theta_{1} \gamma_{200}-\theta_{3} \gamma_{002}\right) \\
& +\left(\theta_{1}^{3}-\theta_{3}^{2}\right)-\theta_{3}\left\{\left(\gamma_{200}+\theta_{1}^{3}\right)\right. \\
& \left.-\left(\gamma_{002}+\theta_{3}^{2}\right)\right\}-\left(\theta_{1}-\theta_{3}\right)\left(\gamma_{200}+\theta_{1}^{2}\right) \\
C_{2}= & \left(\gamma_{030}-\gamma_{003}\right)+3\left(\theta_{2} \gamma_{020}-\theta_{3} \gamma_{002}\right) \\
& +\left(\theta_{2}^{3}-\theta_{3}^{2}\right)-\theta_{3}\left\{\left(\gamma_{020}+\theta_{2}^{2}\right)\right. \\
& \left.-\left(\gamma_{002}+\theta_{3}^{2}\right)\right\}-\left(\theta_{2}-\theta_{3}\right)\left(\gamma_{020}+\theta_{2}^{2}\right) \\
C_{11}= & \left(\theta_{1}-\theta_{3}\right)\left\{\left(\gamma_{200}+\theta_{1}^{2}\right)-\left(\gamma_{002}+\theta_{3}^{2}\right)\right\},
\end{aligned}
$$




\section{CHENG CHEN \& SARJINDER SINGH}

$$
\begin{aligned}
C_{22}= & \left(\theta_{2}-\theta_{3}\right)\left\{\left(\gamma_{020}+\theta_{2}^{2}\right)-\left(\gamma_{002}+\theta_{3}^{2}\right)\right\} \\
C_{12}= & \left(\theta_{2}-\theta_{3}\right)\left\{\left(\gamma_{200}+\theta_{1}^{2}\right)-\left(\gamma_{002}+\theta_{3}^{2}\right)\right\} \\
& +\left(\theta_{1}-\theta_{3}\right)\left\{\left(\gamma_{020}+\theta_{2}^{2}\right)-\left(\gamma_{002}+\theta_{3}^{2}\right)\right\}
\end{aligned}
$$

and

$$
C_{00}=\left(\gamma_{003}+2 \theta_{3} \gamma_{002}\right) \text {. }
$$

Lemma 2.3 Proof

Based on the definition of covariance,

$\operatorname{Cov}\left(Z_{i}, Z_{i}^{2}\right)=E\left(Z_{i}^{3}\right)-E\left(Z_{i}\right) E\left(Z_{i}^{2}\right)$

$$
\begin{aligned}
= & \left(\gamma_{300}+3 \theta_{1} \gamma_{200}+\theta_{1}^{3}\right) \pi_{1} \\
& +\left(\gamma_{030}+3 \theta_{2} \gamma_{020}+\theta_{2}^{3}\right) \pi_{2} \\
& +\left(\gamma_{003}+3 \theta_{3} \gamma_{002}+\theta_{3}^{3}\right)\left(1-\pi_{1}-\pi_{2}\right) \\
& -\left[\begin{array}{l}
\left\{\pi_{1} \theta_{1}+\pi_{2} \theta_{2}+\left(1-\pi_{1}-\pi_{2}\right) \theta_{3}\right\} \\
\times\left\{\begin{array}{l}
\pi_{1}\left(\gamma_{200}+\theta_{1}^{2}\right)+\pi_{2}\left(\gamma_{020}+\theta_{2}^{2}\right) \\
+\left(1-\pi_{1}-\pi_{2}\right)\left(\gamma_{002}+\theta_{3}^{2}\right)
\end{array}\right\}
\end{array}\right.
\end{aligned}
$$

which, on rearranging, reduces to (2.11) and proves the lemma.

Consider the following theorems:

\section{Theorem 2.4}

The variance of the unbiased estimator $\hat{\pi}_{1}$ of the population proportion $\pi_{1}$ is given by

$$
\begin{aligned}
& V\left(\hat{\pi}_{1}\right)= \\
& \pi_{1}\{ \\
& \pi_{1}\left\{\begin{array}{l}
\left(\left(\gamma_{020}+\theta_{2}^{2}\right)-\left(\gamma_{002}+\theta_{3}^{2}\right)\right)^{2} A_{1}+B_{1}\left(\theta_{2}-\theta_{3}\right)^{2} \\
-2\left(\theta_{2}-\theta_{3}\right)\left(\left(\gamma_{020}+\theta_{2}^{2}\right)-\left(\gamma_{002}+\theta_{3}^{2}\right)\right) C_{1}
\end{array}\right\} \\
& +\pi_{2}\left\{\begin{array}{l}
\left(\left(\gamma_{020}+\theta_{2}^{2}\right)-\left(\gamma_{002}+\theta_{3}^{2}\right)\right)^{2} A_{2}+B_{2}\left(\theta_{2}-\theta_{3}\right)^{2} \\
-2\left(\theta_{2}-\theta_{3}\right)\left(\left(\gamma_{020}+\theta_{2}^{2}\right)-\left(\gamma_{002}+\theta_{3}^{2}\right)\right) C_{2}
\end{array}\right\} \\
& +\pi_{1}\left(1-\pi_{1}\right)\left\{\begin{array}{l}
\left(\left(\gamma_{020}+\theta_{2}^{2}\right)-\left(\gamma_{002}+\theta_{3}^{2}\right)\right) A_{11}+ \\
\left(\theta_{2}-\theta_{3}\right)^{2} B_{11}-2\left(\theta_{2}-\theta_{3}\right) \\
\left(\left(\gamma_{020}+\theta_{2}^{2}\right)-\left(\gamma_{002}+\theta_{3}^{2}\right)\right) C_{11}
\end{array}\right\} \\
& \frac{1}{n \Delta^{2}}\left\{+\pi_{2}\left(1-\pi_{2}\right)\left\{\begin{array}{l}
\left(\left(\gamma_{020}+\theta_{2}^{2}\right)-\left(\gamma_{002}+\theta_{3}^{2}\right)\right) A_{22} \\
+\left(\theta_{2}-\theta_{3}\right)^{2} B_{22}-2\left(\theta_{2}-\theta_{3}\right) \\
\left(\left(\gamma_{020}+\theta_{2}^{2}\right)-\left(\gamma_{002}+\theta_{3}^{2}\right)\right) C_{22}
\end{array}\right\}\right. \\
& +\pi_{1} \pi_{2}\left\{\begin{array}{l}
\left(\left(\gamma_{020}+\theta_{2}^{2}\right)-\left(\gamma_{002}+\theta_{3}^{2}\right)\right) A_{12}+ \\
\left(\theta_{2}-\theta_{3}\right)^{2} B_{12}-2\left(\theta_{2}-\theta_{3}\right) \\
\left(\left(\gamma_{020}+\theta_{2}^{2}\right)-\left(\gamma_{002}+\theta_{3}^{2}\right)\right) C_{12}
\end{array}\right\} \\
& +\left(\left(\gamma_{020}+\theta_{2}^{2}\right)-\left(\gamma_{002}+\theta_{3}^{2}\right)\right) A_{00}+ \\
& \left(\theta_{2}-\theta_{3}\right)^{2} B_{00}-2\left(\theta_{2}-\theta_{3}\right) \\
& {\left[\left(\left(\gamma_{020}+\theta_{2}^{2}\right)-\left(\gamma_{002}+\theta_{3}^{2}\right)\right) C_{00}\right.}
\end{aligned}
$$

Theorem 2.4 Proof

Based on the definition of variance,

$$
\begin{aligned}
& V\left(\hat{\pi}_{1}\right)= \\
& \frac{1}{n^{2} \Delta^{2}}\left[\begin{array}{l}
\left\{\left(\gamma_{020}+\theta_{2}^{2}\right)-\left(\gamma_{002}+\theta_{3}^{2}\right)\right\}^{2} \sum_{i=1}^{n} V\left(Z_{i}\right) \\
+\left(\theta_{2}-\theta_{3}\right)^{2} \sum_{i=1}^{n} V\left(Z_{i}^{2}\right)-2\left(\theta_{2}-\theta_{3}\right)\left\{\left(\gamma_{020}+\theta_{2}^{2}\right)\right. \\
\left.-\left(\gamma_{002}+\theta_{3}^{2}\right)\right\} \sum_{i=1}^{n} \operatorname{Cov}\left(Z_{i}, Z_{i}^{2}\right)
\end{array}\right]
\end{aligned}
$$

Using the lemmas, the following theorems are put forth. 


\section{MULTINOMIAL PROPORTION ESTIMATION USING HIGHER ORDER MOMENTS}

Theorem 2.5

The variance of the unbiased estimator $\hat{\pi}_{2}$ of the population proportion $\pi_{2}$ is given by

$$
\begin{aligned}
& V\left(\hat{\pi}_{2}\right)=
\end{aligned}
$$

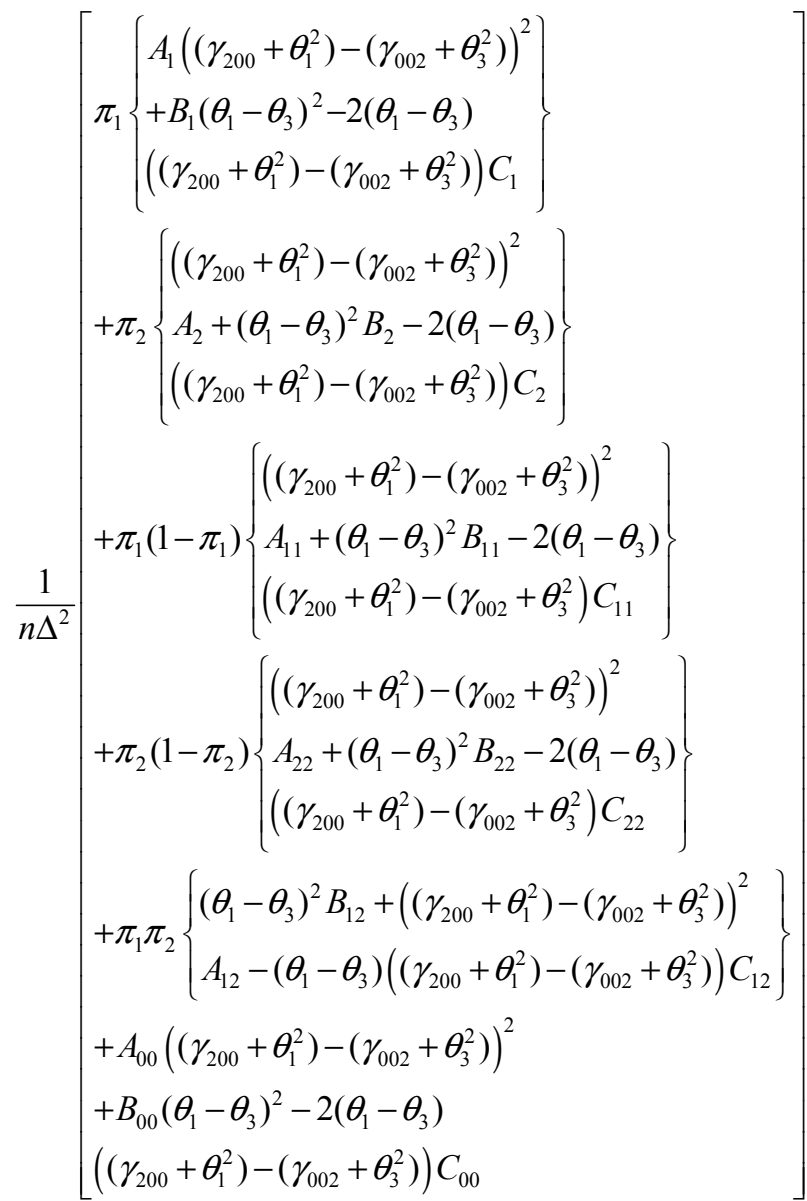

Theorem 2.5 Proof

Based on the definition of variance,

$$
V\left(\hat{\pi}_{2}\right)=
$$$$
\frac{1}{n^{2} \Delta^{2}}\left[\begin{array}{l}
\left(\theta_{1}-\theta_{3}\right)^{2} \sum_{i=1}^{n} V\left(Z_{i}^{2}\right)+ \\
\left(\left(\gamma_{200}+\theta_{1}^{2}\right)-\left(\gamma_{002}+\theta_{3}^{2}\right)\right)^{2} \sum_{i=1}^{n} V\left(Z_{i}\right) \\
-2\left(\theta_{1}-\theta_{3}\right)\left(\left(\gamma_{200}+\theta_{1}^{2}\right)-\left(\gamma_{002}+\theta_{3}^{2}\right)\right) \\
\sum_{i=1}^{n} \operatorname{Cov}\left(Z_{i}, Z_{i}^{2}\right)
\end{array}\right]
$$

Theorem 2.6

The covariance between the unbiased estimators $\hat{\pi}_{1}$ and $\hat{\pi}_{2}$ is given by

$\operatorname{Cov}\left(\hat{\pi}_{1}, \hat{\pi}_{2}\right)=$

$$
\frac{1}{n \Delta^{2}}\left[\begin{array}{c}
\pi_{1}\left\{\begin{array}{l}
C_{1} \Psi-B_{1}\left(\theta_{1}-\theta_{3}\right)\left(\theta_{2}-\theta_{3}\right) \\
-A_{1}\left(\left(\gamma_{020}+\theta_{2}^{2}\right)-\left(\gamma_{002}+\theta_{3}^{2}\right)\right) \\
\left(\left(\gamma_{200}+\theta_{1}^{2}\right)-\left(\gamma_{002}+\theta_{3}^{2}\right)\right)
\end{array}\right\} \\
+\pi_{2}\left\{\begin{array}{l}
C_{2} \Psi-B_{2}\left(\theta_{1}-\theta_{3}\right)\left(\theta_{2}-\theta_{3}\right) \\
-A_{2}\left(\left(\gamma_{020}+\theta_{2}^{2}\right)-\left(\gamma_{002}+\theta_{3}^{2}\right)\right) \\
\left(\left(\gamma_{200}+\theta_{1}^{2}\right)-\left(\gamma_{002}+\theta_{3}^{2}\right)\right)
\end{array}\right\} \\
\left\{\begin{array}{l}
C_{11} \Psi-B_{11}\left(\theta_{1}-\theta_{3}\right)\left(\theta_{2}-\theta_{3}\right) \\
-A_{11}\left(\left(\gamma_{020}+\theta_{2}^{2}\right)-\left(\gamma_{002}+\theta_{3}^{2}\right)\right) \\
\left(\left(\gamma_{200}+\theta_{1}^{2}\right)-\left(\gamma_{002}+\theta_{3}^{2}\right)\right)
\end{array}\right\} \\
+\pi_{2}\left(1-\pi_{2}\right)\left\{\begin{array}{l}
C_{22} \Psi-B_{22}\left(\theta_{1}-\theta_{3}\right)\left(\theta_{2}-\theta_{3}\right) \\
-A_{22}\left(\left(\gamma_{020}+\theta_{2}^{2}\right)-\left(\gamma_{002}+\theta_{3}^{2}\right)\right) \\
\left(\left(\gamma_{200}+\theta_{1}^{2}\right)-\left(\gamma_{002}+\theta_{3}^{2}\right)\right)
\end{array}\right\} \\
+C_{00} \Psi-B_{00}\left(\theta_{1}-\theta_{3}\right)\left(\theta_{2}-\theta_{3}\right) \\
+A_{00}\left\{\begin{array}{l}
\left(\gamma_{020}+\theta_{2}^{2}\right) \\
-\left(\gamma_{002}+\theta_{3}^{2}\right)
\end{array}\right\}\left\{\begin{array}{l}
\left(\gamma_{200}+\theta_{1}^{2}\right) \\
-\left(\gamma_{002}+\theta_{3}^{2}\right)
\end{array}\right\}
\end{array}\right\}
$$

where

$$
\begin{aligned}
\Psi= & \left(\theta_{1}-\theta_{3}\right)\left(\left(\gamma_{020}+\theta_{2}^{2}\right)-\left(\gamma_{002}+\theta_{3}^{2}\right)\right) \\
& +\left(\theta_{2}-\theta_{3}\right)\left(\left(\gamma_{200}+\theta_{1}^{2}\right)-\left(\gamma_{002}+\theta_{3}^{2}\right)\right)
\end{aligned}
$$

Theorem 2.6 Proof

Based on the definition of covariance, 


\section{CHENG CHEN \& SARJINDER SINGH}

$$
\begin{aligned}
& \operatorname{Cov}\left(\hat{\pi}_{1}, \hat{\pi}_{2}\right)= \\
& =\frac{\operatorname{Cov}\left[\begin{array}{l}
\left.\left(\gamma_{020}+\theta_{2}^{2}\right)-\left(\gamma_{002}+\theta_{3}^{2}\right)\right) \\
\sum_{i=1}^{n} Z_{i}-\left(\theta_{2}-\theta_{3}\right) \sum_{i=1}^{n} Z_{i}^{2},\left(\theta_{1}-\theta_{3}\right) \\
\sum_{i=1}^{n} Z_{i}^{2}-\left(\begin{array}{l}
\left(\gamma_{200}+\theta_{1}^{2}\right) \\
-\left(\gamma_{002}+\theta_{3}^{2}\right)
\end{array}\right) \sum_{i=1}^{n} Z_{i}
\end{array}\right]}{n^{2} \Delta^{2}} \\
& =\frac{1}{n^{2} \Delta^{2}}\left[\begin{array}{l}
\left(\theta_{1}-\theta_{3}\right)\left(\left(\gamma_{020}+\theta_{2}^{2}\right)-\left(\gamma_{002}+\theta_{3}^{2}\right)\right) \operatorname{Cov}\left(Z_{i}, Z_{i}^{2}\right) \\
-\left(\theta_{1}-\theta_{3}\right)\left(\theta_{2}-\theta_{3}\right) \sum_{i=1}^{n} \operatorname{Cov}\left(Z_{i}^{2}, Z_{i}^{2}\right) \\
-\left(\begin{array}{l}
\left(\gamma_{020}+\theta_{2}^{2}\right) \\
-\left(\gamma_{002}+\theta_{3}^{2}\right)
\end{array}\right)\left(\begin{array}{l}
\left(\gamma_{200}+\theta_{1}^{2}\right) \\
-\left(\gamma_{002}+\theta_{3}^{2}\right)
\end{array}\right) \\
\sum_{i=1}^{n} \operatorname{Cov}\left(Z_{i}, Z_{i}\right)+\left(\theta_{2}-\theta_{3}\right) \\
\left(\begin{array}{l}
\left(\gamma_{200}+\theta_{1}^{2}\right) \\
-\left(\gamma_{002}+\theta_{3}^{2}\right)
\end{array}\right) \sum_{i=1}^{n} \operatorname{Cov}\left(Z_{i}, Z_{i}^{2}\right)
\end{array}\right]
\end{aligned}
$$

These lemmas result in theorem 2.7.

Theorem 2.7

The variance of the unbiased estimator $\hat{\pi}_{3}$ of the parameter $\pi_{3}$ is given by

$$
V\left(\hat{\pi}_{3}\right)=V\left(\hat{\pi}_{1}\right)+V\left(\hat{\pi}_{2}\right)+2 \operatorname{Cov}\left(\hat{\pi}_{1}, \hat{\pi}_{2}\right)
$$

Theorem 2.7 Proof

Theorem 2.7 is proved based on the definition of variance.

\section{References}

Abul-Ela, A. L. A., Greenberg, B. G., \& Horvitz, D. G. (1967). A multi-proportion randomized response model. Journal of the American Statistical Association, 62, 990-1008.
Blank, S. G. (2008). Using the randomized response technique to investigate illegal fishing and contribute to abalone management in Northern California. A 90 point thesis submitted to Victoria University of Wellington, as partial fulfillment for the degree of Master of Environmental Studies. School of Geography, Environment and Earth Sciences, Victoria University of Wellington.

Bourke, P. D. (1974). Multi-proportions randomized response using the unrelated question technique. Report No. 74 of the Errors in Survey Research Project. Institute of Statistics, University of Stockholm (Mimeo).

Bourke, P. D. (1981). On the analysis of some multivariate randomized response designs for categorical data. Journal of Statistical Planning and Inference, 5, 165-170.

Bourke, P. D. (1982). RR multivariate designs for categorical data. Communications in Statistics - Theory and Methods, A, 11, 28892901.

Bourke, P. D. (1990). Estimating a distribution function for each category of a sensitive variable. Communications in Statistics - Theory and Methods, 19(9), 3233-3241.

Bourke, P. D., \& Dalenius, T. (1973). Multi-proportions randomized response using a single sample. Report No. 68 of the Errors in Survey Research Project. Institute of Statistics, University of Stockholm. (Mimeo).

Bourke, P. D., \& Dalenious, T. (1974). RR models with lying. Technical Report -71 . Institute of Statistics, University of Stockholm. (Mimeo).

Christofides, T. C. (2003). A generalized randomized response technique. Metrika, 57(2), 195-200.

Diana, G., \& Perri, P. F. (2009a). Estimating sensitive proportion through randomized response procedures based on auxiliary information. Statistical Papers, 50, 661-672.

Diana, G., \& Perri, P. F. (2009b). A class of estimators for quantitative sensitive data. Statistical Papers, doi: 10.1007/s00362009-0273-1. (Published online).

Drane, W. (1976). On the theory of randomized responses to two sentitive questions. Communications in Statistics - Theory and Methods, A, 5, 565-574. 


\section{MULTINOMIAL PROPORTION ESTIMATION USING HIGHER ORDER MOMENTS}

Esponda, F., \& Guerrero, V. M. (2009). Surveys with negative questions for sensitive items. Statistics and Probability Letters, 79, 2456-2461.

Franklin, L. A. (1989). A comparison of estimators for randomized response sampling with continuous distributions from a dichotomous population. Communications in Statistics - Theory and Methods, 18, 489-505.

Gjestvang, C. R., \& Singh, S. (2006). A new randomized response model. Journal of the Royal Statistical Society, Series B, 68, 523-530.

Greenberg, B.G., Abul-Ela, Abdel-Latif, A., Simmons, W.R. and Horvitz, D.G. (1969). The unrelated question RR model: theoretical framework. J. Amer. Statist. Assoc., 64, 520539.

Guerriero, M., \& Sandri, M. F. (2007). A note on the comparison of some randomized response procedures. Journal of Statistical Planning and Inference, 137, 2184-2190.

Hochberg, Y. (1975). Two-stage randomized response scheme for estimating a multinomial. Communications in Statistics Theory and Methods, 4, 1021-1032.

Kuk, A. Y. C. (1990). Asking sensitive questions indirectly. Biomerika, 77(2), 436-438.

Mangat, N. S. (1994). An improved randomized response strategy. Journal of the Royal Statistical Society, Series B, 56, 93-95.

Mangat, N. S., \& Singh, R. (1990). An alternative randomized response procedure. Biometrika, 77(2), 439-442.

Mukherjee, R. (1981). Inference on confidential characters from survey data. Calcutta Statistical Association Bulletin, 30, 7788.

Mukhopadhyay, P. (1980). On the estimation of some confidential characters from survey. Calcutta Statistical Association Bulletin, 29, 77-88.

Raghavarao, D., \& Federer, W. T. (1979). Block total response as an alternative to the randomized response method. Journal of the Royal Statistical Society, Series B, 41, 40-45.

Silva, L. C. (1983). On the generalized randomized response model with polychotomous variables. Review of Investment Operac., 4, III, 75-100.
Singh, S., \& Chen, C. C. (2009). Utilization of higher order moments of scrambling variables in randomized response sampling. Journal of Statistical Planning and Inference, 139, 3377-3380.

Singh, S., \& Kim, J-M. (2011). A pseudo-empirical log-likelihood estimator using scrambled responses. Statistics and Probability Letters, 81, 345-351.

Singh, S., Kim, J-M., \& Grewal, I. S. (2008). Imputing and Jackknifing scrambled responses. Metron, LXVI(2), 183-204.

Tamhane, A. C. (1981). Randomized response techniques for multiple attributes. Journal of the American Statistical Association, 76, 916-923.

Tan, M. T., Tian, G-L., \& Tang, M-L. (2009). Sample surveys with sensitive questions: A nonrandomized response approach. The American Statistician, 63(1), 9-16.

Tracy, D. S., \& Mangat, N. S. (1996). Some developments in randomized response sampling during the last decade: A follow up of review by Chaudhuri and Mukerjee. Journal of Applied Statistical Science, 4(2/3), 147-158.

van der Heijden, P. G. M., van Gils, G., Bouts, J., \& Hox, J. J. (2000). A comparison of randomized response, computer-assisted selfinterview, and face-to-face direct questioning. Eliciting sensitive information in the context of welfare and unemployment benefit. Sociological Methods \& Research, 28, 505-537.

van den Hout, A., \& van der Heijden, P. G. M. (2002). Randomized response, statistical disclosure control and misclassification: a review. International Statistical Review, 70, 269-288.

Waltz, C. F., Strickland, O. L., \& Lenz, E. R. (2004). Measurement in Nursing and Health Research, $3^{\text {rd }}$ Ed. New York, NY: Springer.

Warner, S. L. (1965). Randomized response: a survey technique for eliminating evasive answer bias. Journal of the American Statistical Association, 60, 63-69. 


\title{
Empirical Sampling from Permutation Space with Unique Patterns
}

\author{
Justice I. Odiase \\ University of Benin, \\ Benin, Nigeria
}

The exact distribution of a test statistic ultimately guarantees that the probability of a Type I error is exactly $\alpha$. Several methods for estimating the exact distribution of a test statistic have evolved over the years with inherent computational problems and varying degrees of accuracy. The unique pattern of permutations resulting from using experimental data to sample within the permutation space without the risk of repeating permutations is identified. The method presented circumvents the theoretical requirements of asymptotic procedures and the computational difficulties associated with an exhaustive enumeration of permutations. Results show that time and space complexities are drastically reduced without compromising accuracy even when enumeration is not exhaustive provided error tolerance is achieved. The exact distribution of the Siegel-Tukey test statistic is examined as an illustration.

Key words: Algorithm, exact test, permutation test, bootstrap, resampling.

Introduction

The first edition of Fisher (1935) contains descriptions of two tests of significance that depend on permutation: Fisher's exact test for analyzing categorical data, and the permutation test for the difference between means. Many studies have been designed to confirm the asymptotic equivalence of permutation and classical tests (Ludbrook, 1994). Fisher wrote that "the statistician does not carry out this very simple and very tedious process, but his conclusions have no justification beyond the fact that they agree with those which could have been arrived at by this elementary method" (1936, p 59). Ernst (2004) noted that with fast computers there is little reason for a statistician not to carry out this very tedious process.

The main problem with permutation tests is that their null distributions are generally very difficult to express in closed form and to calculate exactly. This is because they depend

Justice Odiase is a Senior Lecturer in the Department of Mathematics, University of Benin, Benin City, Nigeria. Email him at: justice.odiase@uniben.edu. on a specific data set; thus they vary as the data varies in the sample space, however, for several test statistics involving ranks, the null distributions only need to be computed once. For large sample sizes, direct calculations are practically impossible due to the very large cardinality of associated permutation sample spaces. For example, a data set consisting of four treatments with five observations per treatment, $n_{i}=5, i=1(1) 4$, demands as many as

$$
\frac{\left(\sum_{\mathrm{i}=1}^{4} \mathrm{n}_{\mathrm{i}}\right) !}{\prod_{i=1}^{4}\left(\mathrm{n}_{\mathrm{i}} !\right)}=\frac{20 !}{5 ! 5 ! 5 ! 5 !}=11,732,745,024
$$

configurations for an exhaustive enumeration of all permutations. Pesarin (2001) stated that, unless sample sizes are very large, the approximation of such distributions by means of asymptotic arguments is not always appropriate. No general agreement exists regarding how large a sample should be before applying asymptotic approximation (Fahoome, 2000). Pesarin (2001) observed that the algorithms for exact calculations are generally based on direct calculus of upper tail probabilities; a strategy which may become highly impractical, if not 


\section{EMPIRICAL SAMPLING FROM PERMUTATION SPACE WITH UNIQUE PATTERNS}

impossible, in multivariate problems because there is no general computing routine useful to identify the critical regions. This was also observed by Hall and Weierserman (1997). In the early years of research into exact statistical inference, Scheffé (1943) clearly identified the fact that the permutation approach is the only way of constructing the exact distribution of a test statistic.

To avoid the computational difficulty in exact permutation tests, the conditional Monte Carlo (CMC) method was adopted by Pesarin (2001). In CMC replicate resampling is conducted without-replacement on the given data set, which is considered as playing the role of a finite population, provided that sample sizes are finite. According to Opdyke (2003), all existing permutation procedures developed to date can perform conventional Monte Carlo sampling without replacement within a sample but none can avoid the possibility of drawing the same sample more than once. The consideration given by Odiase and Ogbonmwan (2007) is an exception but involves a complete enumeration of all the distinct permutations, which becomes impracticable when the sample size is large.

In this study, the unique pattern of each permutation resulting from experimental data is identified and exploited in sampling from the permutation space without the attendant risk of repeating permutations. The method presented circumvents the elaborate theoretical requirements of asymptotic procedures and the logical and computational difficulties associated with an exhaustive enumeration of permutations.

\section{Exhaustive Permutation Procedures}

The process of obtaining permutations begins by choosing the test statistic $T$ and the acceptable significance level $\alpha$. Let $\pi_{1}, \pi_{2}$, $\ldots, \pi_{\mathrm{N}}$ be a set of all distinct permutations of the observations or ranks of the observations in the experiment. Compute the test statistic $T_{i}$ for permutation $\pi_{i}$, that is, $T_{i}=T\left(\pi_{i}\right)$. Construct an empirical cumulative distribution for $T$ as:

$$
p_{0}=\mathrm{p}\left(T \leq T_{\mathrm{i}}\right)=\frac{1}{\mathrm{~N}} \sum_{\mathrm{i}=1}^{\mathrm{N}} \psi\left(\mathrm{t}_{0}-T_{\mathrm{i}}\right),
$$

$$
\psi(\cdot)= \begin{cases}1, & \text { if } \mathrm{t}_{0} \geq T_{\mathrm{i}} \\ 0, & \text { if } \mathrm{t}_{0}<T_{\mathrm{i}}\end{cases}
$$

Under the empirical distribution, if $p_{0} \leq \alpha$, reject the null hypothesis.

\section{Paired Permutation}

Given two paired samples $X=\left(x_{1}, x_{2}, \ldots\right.$, $\left.x_{n}\right)$ and $Y=\left(y_{1}, y_{2}, \ldots, y_{n}\right)$, suppose a sample of $n$ units from the population distribution $F_{X}$ is paired with a sample of $n$ units from the population distribution $F_{Y}$ and are simultaneously tested in an experiment with $T$ as the test statistic. For $k$ distinct values of the test statistic $T$, the probability distribution of the test statistic $T=\left(T_{1}, T_{2}, \ldots, T_{k}\right)$ under the null hypothesis $H_{0}: F_{X}=F_{Y}$ is given by

$$
\mathrm{P}\left(T_{j}=t_{0} \mid H_{0}\right)={ }_{\sum_{i=1}^{f}}^{f}\left(2^{-n}\right)=f_{j}\left(2^{-n}\right),
$$

where $f_{j}$ is the number of occurrences of $T_{j}$. For specified value of $n$ and the level of significance $\alpha$, the critical value $c$ corresponds to a level closest to $\alpha$. Ordering all the distinct occurrences of $T$ in ascending order of magnitude, and if $g$ is the position of the observed value of $T$, then the following significance level for the left tail of the distribution of the test statistic is

$$
\alpha=\mathrm{P}\left(T_{g} \leq c \mid H_{0}\right)=\sum_{j=1}^{g} \sum_{i=1}^{f}\left(2^{-n}\right)=\left(2^{-n}\right) \sum_{j=1}^{g} f_{j}
$$

and, for the right tail,

$$
\alpha=\mathrm{P}\left(T_{g} \geq c \mid H_{0}\right)=\left(2^{-n}\right) \sum_{j=g}^{k} f_{j} .
$$

For a two-tailed test, the left and right tails are summed. If the distribution of the test statistic is symmetric, then

$$
\sum_{j=1}^{g} f_{j}=\sum_{j=k-g+1}^{k} f_{j}
$$

where 


\section{JUSTICE I. ODIASE}

The distribution of the test statistic is obtained by tabulating the distinct values of the statistic against their probabilities of occurrence in the complete enumeration (see Odiase \& Ogbonmwan, 2007a for a detailed description of the implementation of the paired permutation algorithm). Given a balanced two-sample layout as

$$
\left(\begin{array}{cc}
x_{1} & y_{1} \\
x_{2} & y_{2} \\
\vdots & \vdots \\
x_{n} & y_{n}
\end{array}\right)
$$

where $x_{i} \in X$ and $y_{j} \in Y$. If $n=4$, then, for a two-sample problem, the number of permutations is $\sum_{i=0}^{n}\left(\begin{array}{l}n \\ i\end{array}\right)=2^{n}$.

The test statistic is computed for each permutation in the complete enumeration of the distinct permutations. The distribution of the test statistic is obtained by tabulating the distinct values of the statistic against their probabilities of occurrence in the complete enumeration, where all the permutations are equally likely.

Considering consecutive number of pairs for a given experiment, the growth rate of the permutations from $n-1$ pairs to $n$ pairs in a two-sample paired permutation experiment is $\frac{2^{n}}{2^{n-1}}=2$, meaning that it doubles each time a single pair of observations is added.

Independent Samples Experiment Given a multi-sample experiment with $X_{i}=\left(X_{i 1}, X_{i 2}, \ldots, X_{i n_{i}}\right)^{T}, i=1(1) p$ and $\boldsymbol{X}_{N}=$ $\left(X_{1}, X_{2}, \ldots, X_{p}\right)$. Under the null hypothesis, $X_{N}$ is composed of $N=\sum_{i=1}^{p} n_{i}$ independent and identically distributed random variables. An exhaustive permutation of the observations yields $\frac{\boldsymbol{N} !}{\boldsymbol{P}}$ permutations of the $N$ variates $\prod_{i=1}\left[\left(n_{i}\right) !\right]$

of $p$ subsets of size $n_{i}, i=1(1) p$, which are equally likely and each has the conditional probability $\left(\frac{N !}{\prod_{i=1}^{P}\left[\left(n_{i}\right) !\right]}\right)^{-1} \cdot \quad$ Odiase and

Ogbonmwan (2005a) showed that the number of permutations for a two-sample experiment is $\sum_{i=0}^{n}\left(\begin{array}{c}n_{1} \\ i\end{array}\right)\left(\begin{array}{c}n_{2} \\ i\end{array}\right), \mathrm{n}=\min \left(\mathrm{n}_{1}, \mathrm{n}_{2}\right)$. After obtaining the permutations of a two sample experiment, find the number of ways to permute any $n_{3}$ elements of the combined $\left(\mathrm{n}_{1}+\mathrm{n}_{2}+\mathrm{n}_{3}\right)$ variates of the three treatments. This yields:

$\left(\begin{array}{c}n_{1}+n_{2}+n_{3} \\ n_{3}\end{array}\right) \sum_{i=0}^{n}\left(\begin{array}{c}n_{1} \\ i\end{array}\right)\left(\begin{array}{c}n_{2} \\ i\end{array}\right)=\left(\begin{array}{c}\sum_{r=1}^{3} n_{r} \\ n_{3}\end{array}\right) \sum_{i=0}^{n}\left(\begin{array}{c}n_{1} \\ i\end{array}\right)\left(\begin{array}{c}n_{2} \\ i\end{array}\right)$.

By following the same procedure as for the case of three treatments, a complete enumeration of the distinct permutations for a four-treatment experiment yields:

$$
\begin{array}{r}
\left(\begin{array}{c}
\sum_{r=1}^{4} n_{r} \\
n_{4}
\end{array}\right)\left(\begin{array}{c}
\sum_{r=1}^{3} n_{r} \\
n_{3}
\end{array}\right) \sum_{i=0}^{n}\left(\begin{array}{c}
n_{1} \\
i
\end{array}\right)\left(\begin{array}{c}
n_{2} \\
i
\end{array}\right)= \\
\prod_{j=3}^{4}\left(\begin{array}{c}
\sum_{r=1}^{j} n_{r} \\
n_{j}
\end{array}\right) \sum_{i=0}^{n}\left(\begin{array}{c}
n_{1} \\
i
\end{array}\right)\left(\begin{array}{c}
n_{2} \\
i
\end{array}\right) .
\end{array}
$$

Continuing in this manner, for $\mathrm{p} \geq 3$ treatments, the distinct permutations are enumerated through the expression

$$
\prod_{j=3}^{p}\left(\begin{array}{c}
\sum_{r=1}^{j} n_{r} \\
n_{j}
\end{array}\right) \sum_{i=0}^{n}\left(\begin{array}{c}
n_{1} \\
i
\end{array}\right)\left(\begin{array}{c}
n_{2} \\
i
\end{array}\right)
$$

but

$$
\sum_{i=0}^{n}\left(\begin{array}{c}
n_{1} \\
i
\end{array}\right)\left(\begin{array}{c}
n_{2} \\
i
\end{array}\right)=\left(\begin{array}{c}
\sum_{r=1}^{2} n_{r} \\
n_{2}
\end{array}\right)
$$


Therefore, in a p-sample problem, the number of distinct permutations is $\prod_{j=1}^{p}\left(\begin{array}{c}\sum_{r=1}^{j} n_{r} \\ n_{j}\end{array}\right)$ (Odiase \& Ogbonmwan, 2005b). Observe that, for the balanced case, the number of distinct permutations is

$$
\prod_{\mathrm{j}=3}^{\mathrm{p}}\left(\begin{array}{c}
j n \\
n
\end{array}\right) \sum_{\mathrm{i}=0}^{\mathrm{n}}\left(\begin{array}{c}
\mathrm{n} \\
\mathrm{i}
\end{array}\right)^{2}=\prod_{\mathrm{j}=1}^{\mathrm{p}}\left(\begin{array}{c}
j n \\
n
\end{array}\right) .
$$

Again, considering consecutive number of treatments for a given experiment, the growth rate of the permutations from $p-1$ treatments to $p$ treatments is

$$
\prod_{\mathrm{j}=1}^{\mathrm{p}}\left(\begin{array}{c}
j n \\
n
\end{array}\right) / \prod_{\mathrm{j}=1}^{\mathrm{p}-1}\left(\begin{array}{c}
j n \\
n
\end{array}\right)=\left(\begin{array}{c}
\mathrm{np} \\
\mathrm{n}
\end{array}\right) \text {. }
$$

Repeated Measures (Block) Permutation

Repeated measures ANOVA tests the equality of means and is used when all members of a random sample are measured under varying conditions. In the repeated measures design, each trial represents the measurement of the same characteristic under a different condition. Given the layout of a multi-sample $\left(\begin{array}{lll}n & \mathrm{x} & k\end{array}\right)$ experiment as

$$
\left(\begin{array}{cccc}
x_{11} & x_{12} & \cdots & x_{1 k} \\
x_{21} & x_{22} & \cdots & x_{2 k} \\
\vdots & \vdots & \vdots & \vdots \\
x_{n 1} & x_{n 2} & \cdots & x_{n k}
\end{array}\right)
$$

where $\mathrm{x}_{\mathrm{ij}}$ is an observation in the $j^{\text {th }}$ treatment and the $i^{\text {th }}$ block and the total number of observations in the experiment is $n k$. Rank the observations for each row from 1 (smallest $x_{i j}$ on row $i$ ) to $k$ (largest $x_{i j}$ on row $i$ ). Let the layout of the ranks $\left(r_{i j}\right)$ of the observations $x_{i j}$ be

$$
L_{R}=\left(\begin{array}{cccc}
r_{11} & r_{12} & \cdots & r_{1 k} \\
r_{21} & r_{22} & \cdots & r_{2 k} \\
\vdots & \vdots & \vdots & \vdots \\
r_{n 1} & r_{n 2} & \cdots & r_{n k}
\end{array}\right)
$$

The data are arranged in $k$ columns (treatments) and $n$ rows (blocks), where each block contains $k$ repeated observations. Obviously, there are $k$ ! possible arrangements or permutations of each block and due to multiplication of choices, the entire layout of the $n \times k$ experiment requires $(k !)^{n}$ permutations of the observations to yield the exact distribution of a test statistic, with the permutations equally likely and each having the conditional probability $(k !)^{-n}$.

The first step in developing permutation algorithm is to formulate an initial configuration of the ranks of the variates of an experiment by taking the trivial configuration

$$
\begin{array}{cccccc} 
& \operatorname{Tr} .1 & \operatorname{Tr} .2 & \operatorname{Tr} .3 & \cdots & \operatorname{Tr} . k \\
\text { Block } 1 & 1 & 2 & 3 & \cdots & k \\
\text { Block2 } & 1 & 2 & 3 & \cdots & k \\
\text { Block } 3 & 1 & 2 & 3 & \cdots & k \\
\vdots & \vdots & \vdots & \vdots & \cdots & \vdots \\
\text { Blockn } & 1 & 2 & 3 & \cdots & k
\end{array}
$$

because any configuration of the ranks can engender all the distinct permutations. The test statistic is computed for each permutation in the complete enumeration of all the distinct permutations. The distribution of the test statistic is obtained by tabulating the distinct values of the test statistic against their probabilities of occurrence in the complete enumeration.

Considering two consecutive numbers of blocks for a given experiment, the growth rate of the permutations from $n-1$ blocks to $n$ blocks is $\frac{(k !)^{n}}{(k !)^{n-1}}=k !$ and the growth rate of the permutations from $k-1$ treatments to $k$ treatments is $\frac{(k !)^{n}}{[(k-1) !]^{n}}=k^{n}$ and clearly, $k$ ! grows faster than $k^{n}$ for a fixed $n$, and for a fixed 


\section{JUSTICE I. ODIASE}

$k, k$ ! is constant while $k^{n}$ explodes as $n$ increases. Therefore, the growth rate of the permutations is higher for a unit increase in blocks than a unit increase in treatments for a fixed number of treatments and the reverse is the case when it is the number of blocks that is fixed.

Sampling Permutations with Unique Patterns Given the layout of a two-sample experiment as

$$
\left(\begin{array}{cc}
x_{1} & y_{1} \\
x_{2} & y_{2} \\
\vdots & \vdots \\
x_{1 n_{1}} & y_{n_{2}}
\end{array}\right)
$$

where $x_{i}$ and $y_{i}$ are the $i^{\text {th }}$ observations of the independent random samples $\mathrm{X}$ and $\mathrm{Y}$ respectively, arrange the combined samples in ascending order of magnitude and rank all the $m=n_{1}+n_{2}$ observations from 1 (smallest) to $m$ (largest). Let the layout of the ranks $\left(r_{i j}\right)$ of the observations in a two-sample layout be

$$
L_{R}=\left(\begin{array}{cc}
r_{11} & r_{21} \\
r_{12} & r_{22} \\
\vdots & \vdots \\
r_{1 n_{1}} & r_{2 n_{2}}
\end{array}\right) \text {. }
$$

Under the null hypothesis, $L_{R}$ is composed of $m$ independent and identically distributed random variables and hence conditioned on the observed data set. An exhaustive permutation of the ranks yields $N=\frac{m !}{n_{1} ! n_{2} !}$ permutations of the $m$ ranks of the variates of two subsets of sizes $n_{1}$ and $n_{2}$, which are equally likely, each having the conditional probability $N^{-1}$.

The unique pattern of each permutation resulting from experimental data is identified by adopting the first sample, for example, in a twosample problem. This is carried out by concatenating the ranks or indices of observations in the experiment in a particular manner that makes the pattern unique for every distinct permutation. The unique patterns obtained are therefore exploited in sampling from the permutation space without the risk of repeating permutations already sampled. The benefit of this approach is that - even when enumeration is not exhaustive - the distribution of a test statistic can be obtained within a reasonable level of accuracy with reduced time and space complexities. This sampling approach therefore circumvents the elaborate theoretical requirements of asymptotic procedures and the logical and computational difficulties associated with an exhaustive enumeration of permutations.

\section{Methodology}

Let the initial configuration of the ranks of the variate in a two-sample experiment be $L_{R}$. The entire permutation space can be spanned by any of the permutations (configurations) of the observations or ranks of observations. In a twosample problem, only one of the samples is required to define each permutation because it is obvious that the remaining variates are in the second sample.

In a two-sample experiment

$$
\left(\begin{array}{cc}
x_{1} & y_{1} \\
x_{2} & y_{2} \\
\vdots & \vdots \\
x_{1 n_{1}} & y_{n_{2}}
\end{array}\right),
$$

where $x_{i}$ and $y_{i}$ are the $i^{\text {th }}$ observations of the independent random samples $\mathrm{X}$ and $\mathrm{Y}$ respectively, arrange the combined samples in ascending order of magnitude and rank all the $m=n_{1}+n_{2}$ observations from 1 (smallest) to $m$ (largest) to arrive at $L_{R}$. For the original permutation and subsequent permutations, sort $\mathrm{X}$ such that $x_{1} \leq x_{2} \leq \cdots \leq x_{n_{1}}$.

The variates are identified by their indices $(1,2, \ldots, m)$ or actual ranks, which are employed in obtaining the unique patterns. Attach 0 in front of the first nine indices or ranks $(01,02, \ldots, 09)$ to make each number two digits, leaving $10,11, \ldots, 99$ as they are and treat all the numbers as strings so that it will be possible to manipulate the numbers. Concatenate the indices or ranks of $\mathrm{X}$ and store as a single constant value. This now becomes the pattern of the 


\section{EMPIRICAL SAMPLING FROM PERMUTATION SPACE WITH UNIQUE PATTERNS}

given layout of the observations. (Concatenation is a standard operation in computer programming languages. It is the operation of joining two character strings end to end. In programming languages, string concatenation is a binary operation usually accomplished by putting a concatenation operator between two strings or operands.)

After a unique pattern is obtained, a resampling without replacement is carried out to obtain a random sample of $n_{l}$ variates from the original combined sample of $m$ variates. This is achieved by deleting points already selected at random. Again, sort the resampled $n_{l}$ variates and concatenate their indices or ranks to obtain a pattern. Compare this pattern with previously obtained patterns and store it only if it is unique, otherwise, resample without replacement again until a unique pattern is obtained. The chosen test statistic is computed for each unique permutation and the probability distribution of the test statistic is constructed. Finally, compute the cumulative probability distribution of the test statistic, $T$, under the null hypothesis and obtain the $p$-values such that the probability of making a Type I error is exactly $\alpha$.

As an illustration, consider an $n \times k$ experiment with $n=2$ treatments $(\mathrm{X}, \mathrm{Y})$ and $k=$ 5 variates in each treatment could have the trivial configuration or permutation of ranks represented as

$\begin{array}{cc}X & Y \\ 1 & 6 \\ 2 & 7 \\ 3 & 8 \\ 4 & 9 \\ 5 & 10\end{array}$

Using the methodology presented, the permutation is written as

$\begin{array}{ll}X & Y \\ 01 & 06 \\ 02 & 07 \\ 03 & 08 \\ 04 & 09 \\ 05 & 10\end{array}$

a total of $\frac{10 !}{5 ! \times 5 !}=252$ permutations are required for an exhaustive enumeration of all the distinct or unique permutations. The process starts thus: The entire permutation space can be spanned by the trivial permutation (configurations) of the observations or ranks of observations, any other permutation from the permutation space can also be adopted to span all the unique permutations. In a two-sample problem, only one of the samples $(\mathrm{X})$ is required to define each permutation because it is clear that the remaining variates are in the second sample.

The first permutation pattern is 0102030405. Assuming resampling from the permutation space yields $X=\{8,3,5,2,6\}$, then the second permutation pattern is obtained by first sorting to have $\{2,3,5,6,8\}$, leading to the pattern 0203050608. Resampling again, given $\{2,10,5,9,8\}$, the third permutation pattern is 0205080910. The resampling process continues until either all the patterns are enumerated for small samples or the error tolerance is achieved for large samples. See Table 1 for an exhaustive enumeration of the $\frac{10 !}{5 ! \times 5 !}=252$ permutation patterns for this illustration.

For very small samples, an exhaustive enumeration of all the unique permutations is achieved with the sampling method described. When sample size is large, enumeration of permutations does not need to be exhaustive. Instead, a subset of the permutation space (for example, 2,000) is obtained and the probability distribution of the test statistic is constructed. Take a second sample of same size and fuse it into the earlier distribution to obtain an updated probability distribution and compare with the earlier distribution. With a given level of error 


\section{JUSTICE I. ODIASE}

Table 1: Exhaustive Enumeration of Unique Permutation Patterns for $n=2, k=5$

\begin{tabular}{|c|c|c|c|c|c|}
\hline 0102030405 & 0304050710 & 0103050709 & 0405070809 & 0304070810 & 0105060708 \\
\hline 0203050608 & 0206070809 & 0105060910 & 0102030408 & 0203060708 & 0405060708 \\
\hline 0106070809 & 0102030910 & 0102040910 & 0205080910 & 0102060810 & 0102030506 \\
\hline 0104050809 & 0102050609 & 0102060709 & 0204050708 & 0304060708 & 0506080910 \\
\hline 0104080910 & 0102030709 & 0304050709 & 0102040608 & 0206070810 & 0305060710 \\
\hline 0104060708 & 0205060809 & 0104060809 & 0102030510 & 0204050610 & 0102070809 \\
\hline 0203040708 & 0104060710 & 0306070809 & 0307080910 & 0102040609 & 0102040710 \\
\hline 0507080910 & 0103040508 & 0103040810 & 0305080910 & 0103040507 & 0102040709 \\
\hline 0203060810 & 0306070810 & 0203040510 & 0205070810 & 0105080910 & 0106080910 \\
\hline 0102060710 & 0103050610 & 0204070910 & 0103050810 & 0203060809 & 0204050809 \\
\hline 0102030409 & 0102030407 & 0204060708 & 0506070809 & 0102050910 & 0205070910 \\
\hline 0304050910 & 0405060910 & 0102060809 & 0103060910 & 0104060709 & 0103040506 \\
\hline 0203040508 & 0205060910 & 0205060810 & 0103060709 & 0204060910 & 0203040610 \\
\hline 0304050708 & 0104070809 & 0103050710 & 0105060710 & 0102030509 & 0304050609 \\
\hline 0102030607 & 0406080910 & 0304050809 & 0103040708 & 0203050910 & 0204080910 \\
\hline 0203040810 & 0104070910 & 0102040509 & 0104050910 & 0203050609 & 0304070809 \\
\hline 0103060710 & 0103040608 & 0406070809 & 0103050609 & 0107080910 & 0104070810 \\
\hline 0106070810 & 0204050910 & 0305060708 & 0203040609 & 0102030609 & 0106070910 \\
\hline 0405060710 & 0304060910 & 0304050610 & 0103040609 & 0102050809 & 0102040506 \\
\hline 0203040506 & 0102030406 & 0104050607 & 0204070810 & 0103070810 & 0203040509 \\
\hline 0102040610 & 0304060809 & 0102040810 & 0103050708 & 0405060709 & 0203060910 \\
\hline 0203040607 & 0104050709 & 0204050709 & 0102080910 & 0206080910 & 0102030810 \\
\hline 0205060710 & 0103040809 & 0103060708 & 0203060710 & 0405060809 & 0204060710 \\
\hline 0104050609 & 0102060910 & 0104050710 & 0102070910 & 0103060810 & 0304080910 \\
\hline 0103060809 & 0203050607 & 0102040507 & 0102030708 & 0203040709 & 0204050810 \\
\hline 0105060709 & 0207080910 & 0304070910 & 0204050710 & 0203070809 & 0103040710 \\
\hline 0102040508 & 0305060809 & 0103050809 & 0102030410 & 0103040607 & 0205060709 \\
\hline 0105070810 & 0103040510 & 0405070910 & 0405060810 & 0607080910 & 0204060809 \\
\hline 0102030710 & 0102070810 & 0203080910 & 0204060810 & 0406070910 & 0405070810 \\
\hline 0203050809 & 0203060709 & 0204060709 & 0103080910 & 0105070809 & 0204050607 \\
\hline 0102030507 & 0103070809 & 0102050607 & 0103050910 & 0104050810 & 0105070910 \\
\hline 0203040608 & 0204050609 & 0104060810 & 0203040809 & 0103070910 & 0102040607 \\
\hline 0103040610 & 0103050607 & 0206070910 & 0102040809 & 0506070910 & 0304060709 \\
\hline 0305060810 & 0306080910 & 0203040710 & 0105060809 & 0102060708 & 0203050709 \\
\hline 0102050710 & 0203070910 & 0304060710 & 0104060910 & 0304050608 & 0102030608 \\
\hline 0203050610 & 0102030508 & 0102030809 & 0406070810 & 0103050608 & 0204070809 \\
\hline 0102030610 & 0203040507 & 0405080910 & 0102040708 & 0105060810 & 0103040509 \\
\hline 0305060910 & 0205070809 & 0103040709 & 0304050607 & 0203040910 & 0203050708 \\
\hline 0306070910 & 0305070910 & 0102040510 & 0304060810 & 0305070809 & 0203070810 \\
\hline 0204050608 & 0205060708 & 0103040910 & 0304050810 & 0407080910 & 0102050608 \\
\hline 0305060709 & 0104050608 & 0104050610 & 0203050810 & 0506070810 & 0102050610 \\
\hline 0305070810 & 0104050708 & 0203050710 & 0102050708 & 0102050709 & 0102050810 \\
\hline
\end{tabular}


tolerance, if the error tolerance is exceeded, another sample is taken and fused into the last update of the probability distribution to obtain another update and again compared with the previous update of the probability distribution. This process is continued until the error tolerance is achieved. Compare for every occurrence of the test statistic in the last two updates using the error tolerance as a guide and proceed to update the probability distribution if the error tolerance is not met. Compute the cumulative probability distribution of the Test Statistic, $T$, under the null hypothesis and obtain the p-values such that the probability of making a type I error is exactly $\alpha$.

\section{Unique Permutation Pattern Test Procedure}

Let $\pi_{1}, \pi_{2}, \ldots, \pi_{\mathrm{N}}$ be a set of all distinct permutations of the ranks of the data set in the experiment. The unique permutation pattern test procedure is as follows:

1. Read the original layout of observations.

2. Rank the combined observations of the experiment in (1) to obtain $L_{R}=\pi_{1}$ and compute the observed value of $T$ statistic to obtain $T_{1}=t_{0}$.

3. Store pattern of (1) or (2).

4. Obtain a distinct permutation $\pi_{i}$, of the ranks $\left(L_{R}\right)$ from (3) by sampling without replacement.

5. Obtain pattern of (4) and compare with previous patterns. If different, store pattern, if already exists, go to (4).

6. Compute the $T$ statistic $T_{\mathrm{i}}=T\left(\pi_{i}\right)$, for permutation $\pi_{i}$ in (5), where $i>1$; update probability distribution.

7. Perform (4) to (6) for $i=2,3, \ldots, k \leq N$. If sample size $(\mathrm{N})$ is large,

a) Assume a level of error tolerance $(0.00001)$ and take a subset of size $\mathrm{k}=$ 1,000 of the permutation space to obtain the probability distribution of the test statistic.

b) Take another subset of size $\mathrm{k}$ from the permutation space and fuse into the earlier probability distribution to obtain an updated distribution. Compare this distribution with the earlier distribution of the test statistic for every value of the test statistic.

c) If the error tolerance is exceeded for any value of the test statistic, go back to (b); continue this process until the error tolerance is achieved.

8. Construct the empirical cumulative probability distribution for the distinct values of $T$ and extract critical values.

$$
p_{0}=p\left(T \leq T_{i}\right)=\frac{1}{k} \sum_{i=1}^{k} \psi\left(T_{i}-t_{0}\right)
$$

where

$$
\psi\left(T_{i}-t_{0}\right)=\left\{\begin{array}{ll}
1, & \text { if } T_{i} \leq t_{0} \\
0, & \text { if } \mathrm{T}_{i}>t_{0}
\end{array} .\right.
$$

9. Under the empirical distribution, if $p_{0} \leq \alpha$, reject the null hypothesis.

In a two-sample problem, only one of the samples is important in the generation of permutation patterns because it is unique for each permutation, that is,

$$
\left(\begin{array}{l}
m \\
n_{1}
\end{array}\right)=\left(\begin{array}{l}
m \\
n_{2}
\end{array}\right)=\frac{m !}{n_{1} ! n_{2} !}, m=n_{1}+n_{2} .
$$

To provide exact critical values when ties occur, midranks are assigned as the ranks of tied observations, and the algorithm is implemented with $r_{i j}$ as input, composed of actual ranks containing ties. Tabulated exact critical values of a test statistic are usually provided for experiments with distinct observations, because it will be practically difficult to consider all possible occurrences of ties and create tables of exact critical values for each occurrence of ties 


\section{JUSTICE I. ODIASE}

for different sample sizes. This will result in several volumes of tables. In order to arrive at the critical values (see Table 2), the ranks of distinct observations (rij) were used as input in Algorithm for various sample sizes. See Appendix A for the unique permutation pattern algorithm. This algorithm identifies and compiles the unique permutation patterns of the layout of observations or rank of observations in a two-sample experiment. It is illustratively implemented to produce a table of critical values for the Siegel-Tukey test statistic

\section{Siegel-Tukey Test}

The Siegel-Tukey test is similar in procedure to the Wilcoxon Rank Sum test for difference in location. It is based on the logic that if two samples come from populations with the same median, the one with the greater variability will have more extreme scores. The hypotheses for a two-tailed test are:

$H_{0}$ : There is no difference in spread between the two populations

versus

$H_{1}$ : There is some difference in spread between the two populations.

The two samples are combined and ordered in ascending order of magnitude, keeping track of sample membership. For $m=n_{1}+n_{2}$, the ranking proceeds as follows: $\alpha(1)=1, \alpha(m)=2, \alpha(m-1)=3, \alpha(2)=4$, $\alpha(3)=5, \quad \alpha(m-2)=6, \quad \alpha(m-3)=7$, $\alpha(4)=8$, the ranking continues to alternate from lowest to highest, ranking two scores at each end. It tests for differences in scale between two groups.

Critical Values for the Siegel-Tukey Test Statistic

Figures 1a-1b illustrate that the normal distribution will poorly approximate the exact distribution of the Siegel-Tukey (S-T) test statistic for very small sample sizes. As group sample size increases, the shape of the distribution of the S-T test begins to look more like the normal distribution as shown in Figures $1 \mathrm{c}-1 \mathrm{~d}$. The critical values of the S-T test statistic shown in Table 2 were obtained from the enumeration of all distinct permutations of the ranks of the observations in an experiment $(m, n$ $<20$ ) combined with the idea of resampling while ensuring an error tolerance level $(m, n \geq$ 20). These critical values ensure that the probability of a Type I error in decisions arising from the use of the S-T test is exactly $\alpha$.

Results obtained from asymptotic procedures and resampling techniques are commonly adopted in several nonparametric tests as alternatives to tabulated exact critical values. Fahoome (2002) conducted a Monte Carlo study and recommended the asymptotic approximation of the S-T test when group sample sizes exceed 25 , based on conservative estimates of $0.045<$ Type I error rate $<0.055$ for $\alpha=0.05$; other authors recommended higher or lower sample sizes.

\section{Conclusion}

The critical values for a test statistic are determined by cutting off the most extreme $100 \alpha \%$ of the theoretical frequency distribution of the test statistic, where $\alpha$ is the level of significance (Siegel \& Castellan, 1988). Classical methods require that the theoretical distribution of the test statistic should agree with a mathematically definable frequency distribution, this often leads to a probability of Type I error greater than $\alpha$, particularly when sample sizes are small. The cost of such an error might be too high to risk. Therefore, the exact permutation paradigm methodology presented in this study is of value because it guarantees that the probability of a Type I error is exactly $\alpha$ with the attendant advantage of no distributional assumptions apart from the exchangeability of the observations. When sample sizes are large and it becomes practically difficult or impossible to construct the probability distribution, permutation sampling becomes very useful because it quickly converges to the actual distribution; Scheffe (1943) opined that this is the only sure way of constructing the exact distribution of a test statistic. 
Figure 1: Exact Distribution of Siegel-Tukey Test Statistic (S-T) for Different Sample Sizes with their Large Sample Approximations (Z)

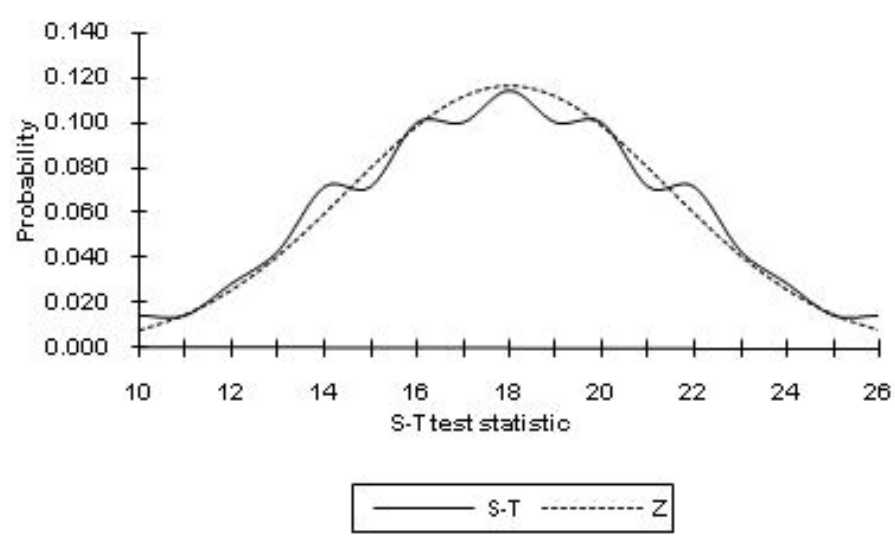

(a)

$$
n_{1}=4, n_{2}=4
$$

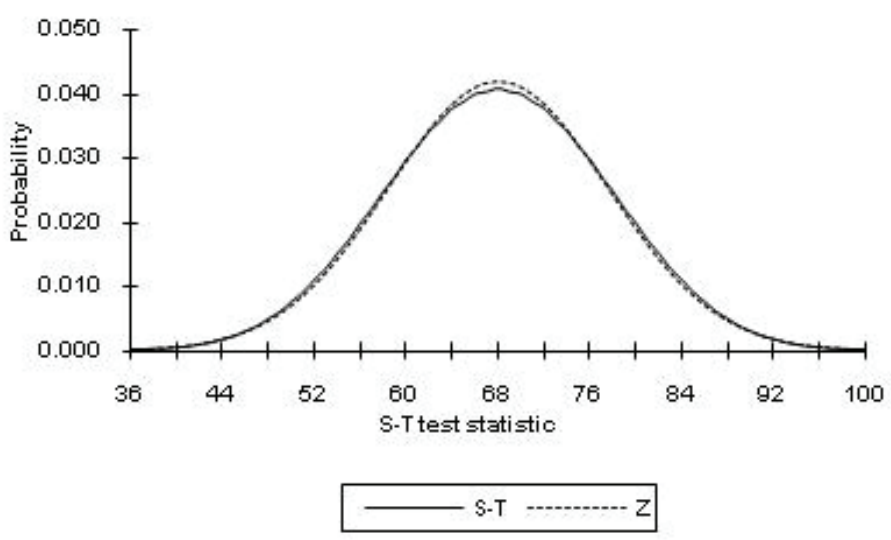

(c)

$n_{1}=8, n_{2}=8$

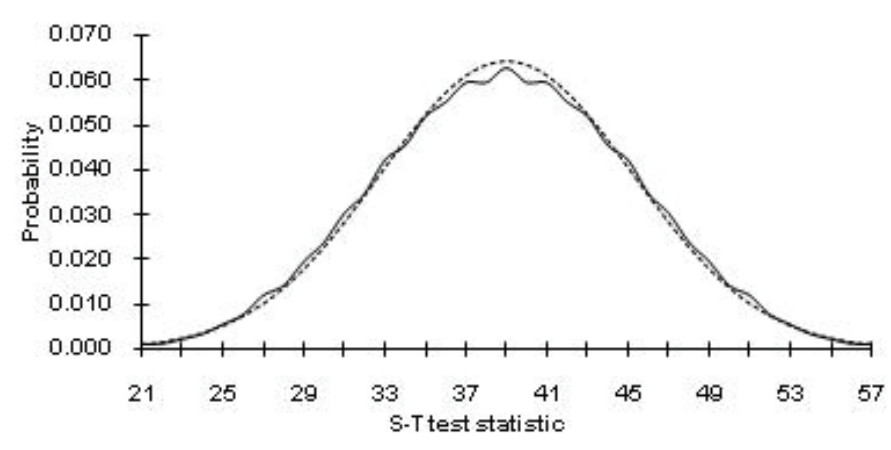

(b)

$n_{1}=6, n_{2}=6$

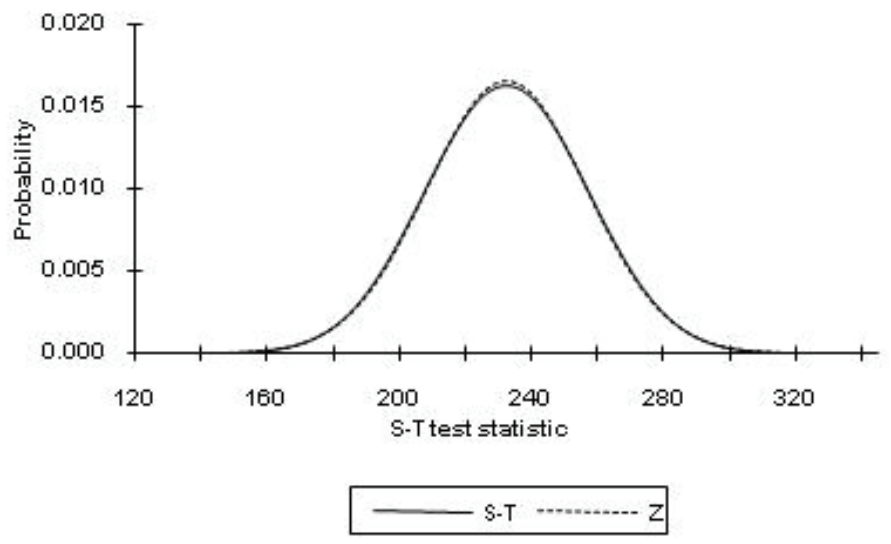

(d)

$n_{1}=15, n_{2}=15$ 


\section{JUSTICE I. ODIASE}

Figure 2: Distribution of Siegel-Tukey Test Statistic with Error Tolerance $=0.00001$ for Different Sample Sizes

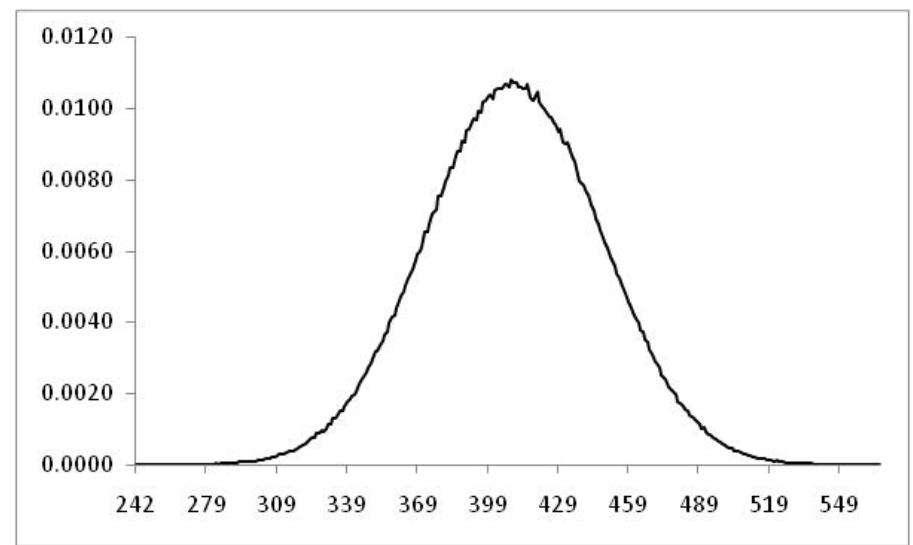

(a)

$n_{1}=20, n_{2}=20$

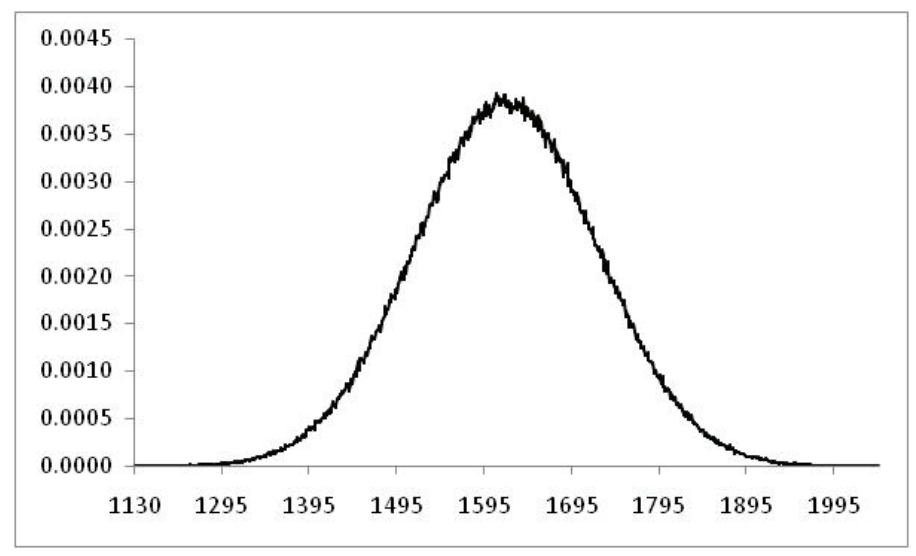

(c)

$n_{1}=40, n_{2}=40$

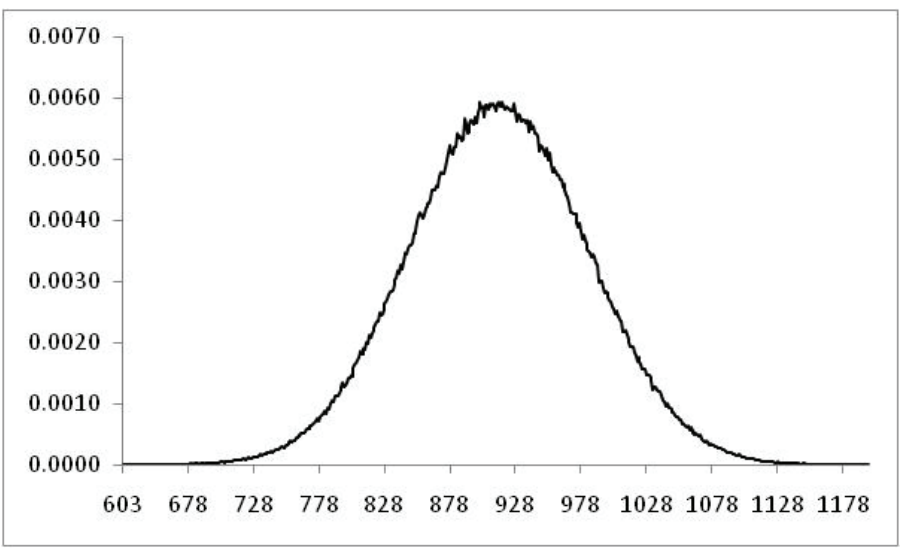

(b)

$$
n_{1}=30, n_{2}=30
$$

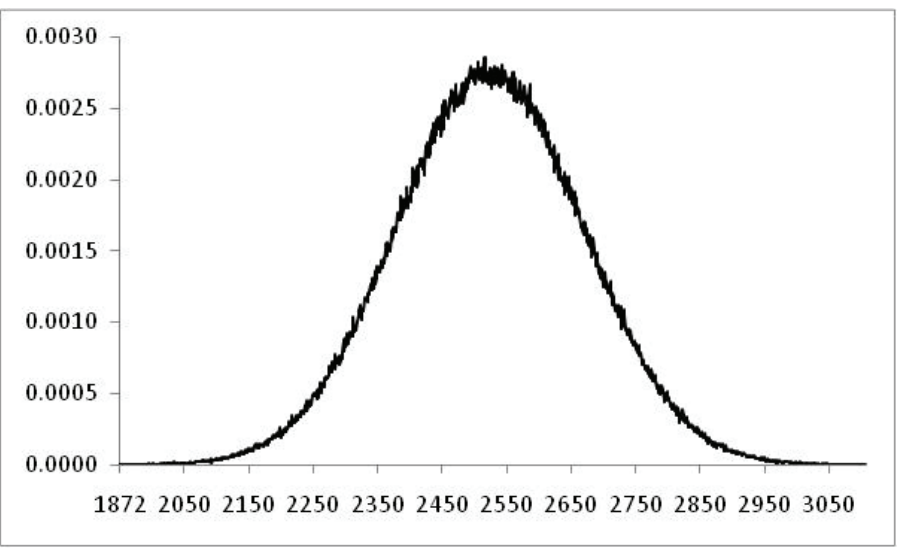

(d)

$n_{1}=50, n_{2}=50$ 
Figure 3: Distribution of Siegel-Tukey Test Statistic for Different Levels of Error Tolerance $\left(n_{l}=15, n_{2}=15\right)$

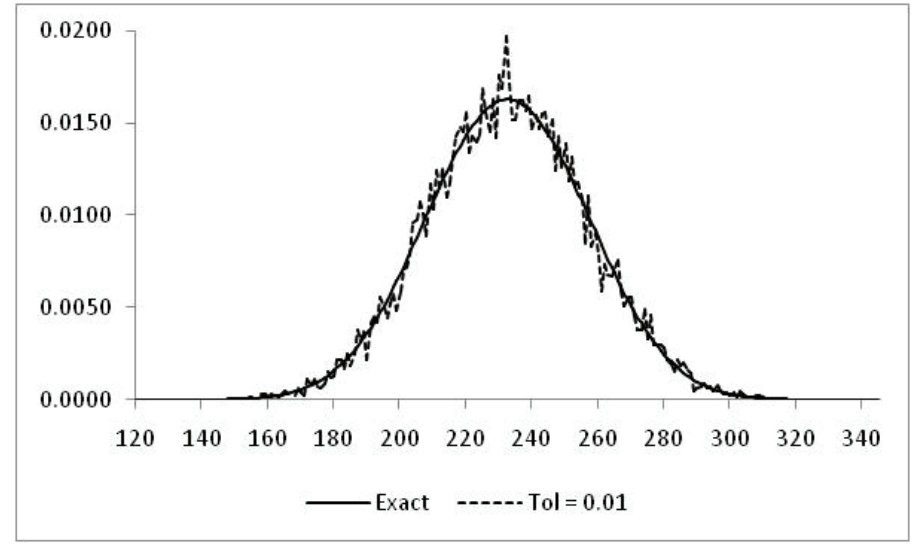

(a)

2 Iterations

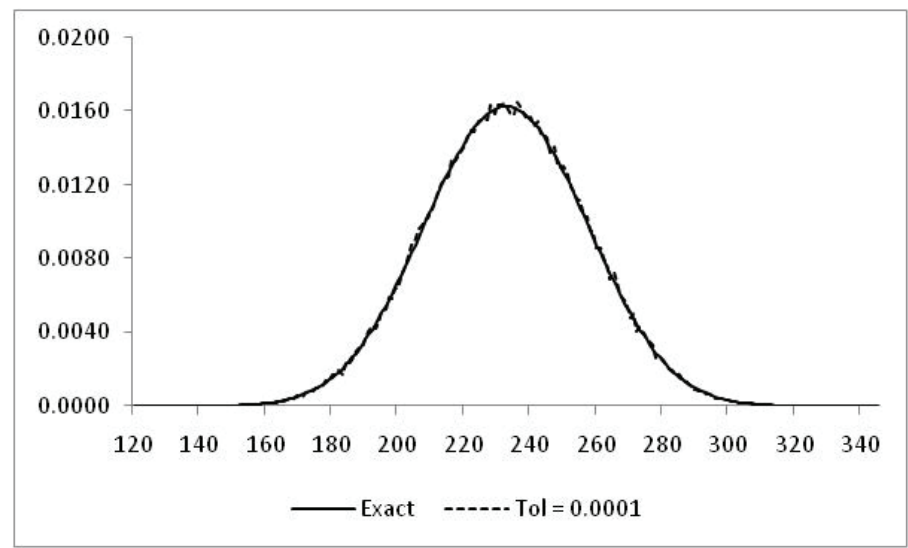

(c)

36 Iterations

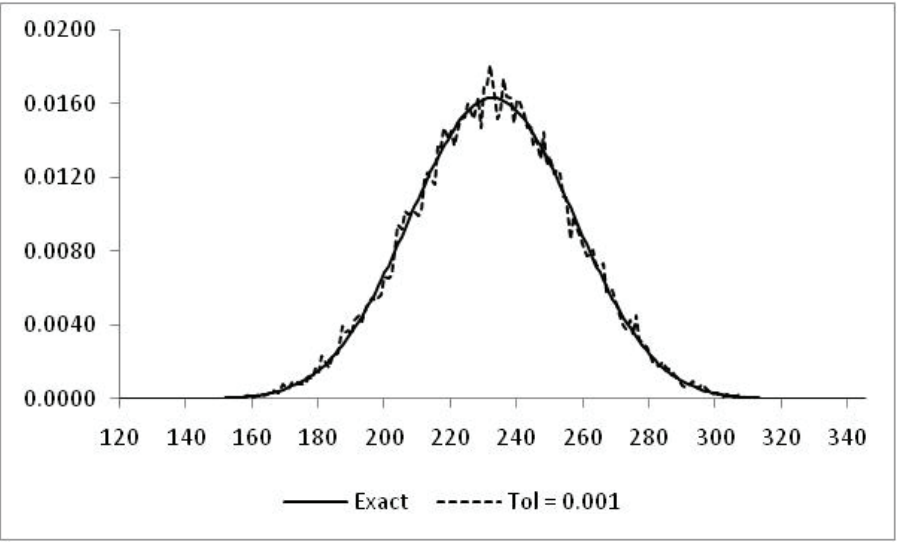

(b)

5 Iterations

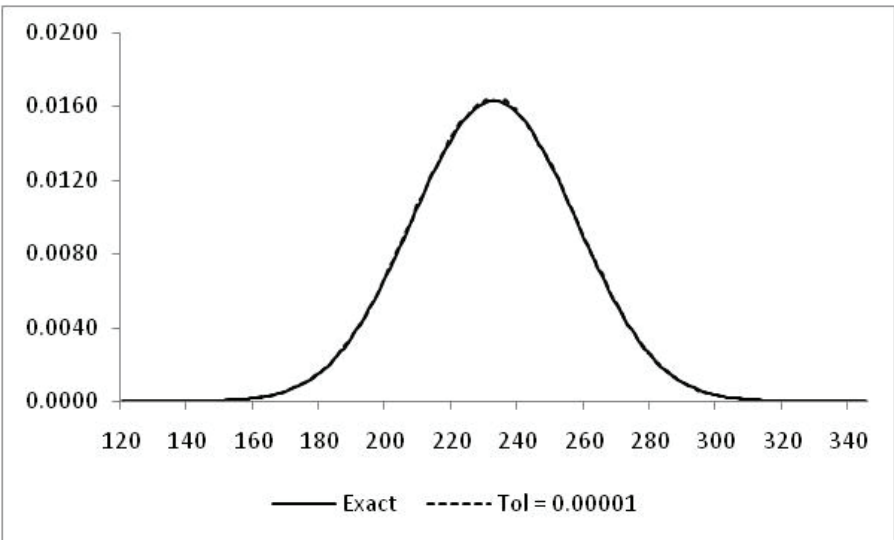

(d)

339 Iterations 


\section{JUSTICE I. ODIASE}

Figure 4: Distribution of Siegel-Tukey Test Statistic for Different Number Samples $\left(n_{1}=15, n_{2}=15\right)$

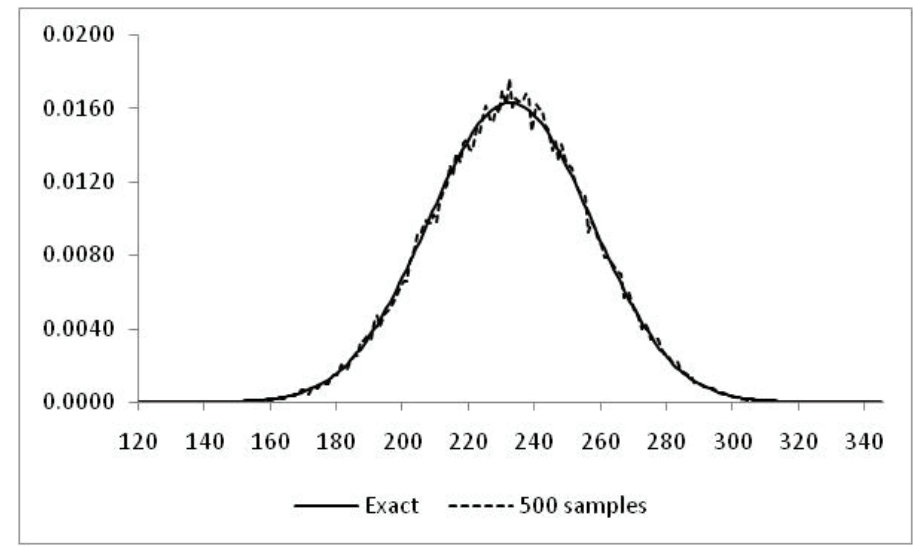

(a)

103 Iterations

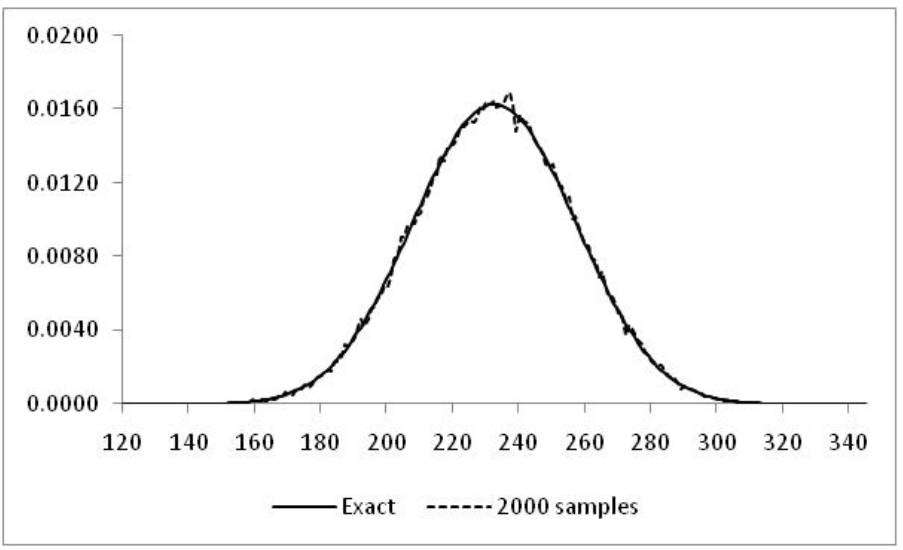

(c)

50 Iterations

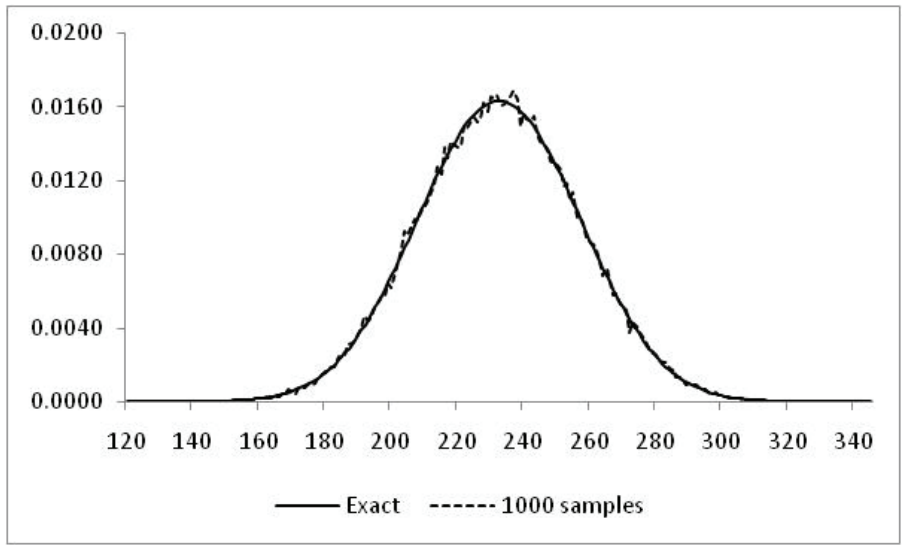

(b)

82 Iterations

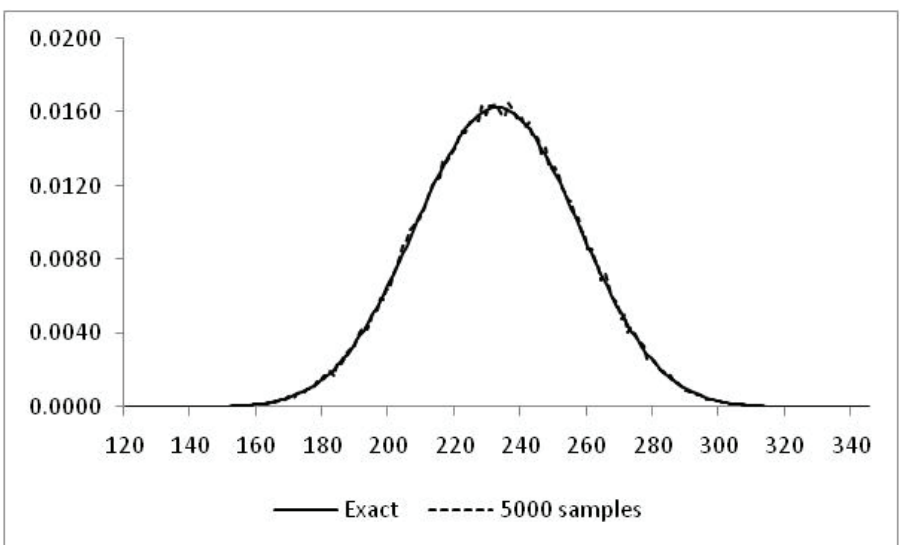

(d)

36 Iterations 
EMPIRICAL SAMPLING FROM PERMUTATION SPACE WITH UNIQUE PATTERNS

Table 2: Lower and Upper Critical Values for the Siegel-Tukey Test Statistic

\begin{tabular}{|c|c|c|c|c|c|c|c|c|}
\hline \multicolumn{2}{|c|}{ Sample Size } & \multirow{2}{*}{$\mathrm{ST}_{0.9000}$} & \multirow{2}{*}{$\mathrm{ST}_{0.9500}$} & \multirow{2}{*}{$\mathrm{ST}_{0.9750}$} & \multirow{2}{*}{$\mathrm{ST}_{0.9900}$} & \multirow{2}{*}{$\mathrm{ST}_{0.9950}$} & \multirow{2}{*}{$\mathrm{ST}_{0.9975}$} & \multirow{2}{*}{$\mathrm{ST}_{0.9990}$} \\
\hline $\mathrm{n}_{1}$ & $\mathrm{n}_{2}$ & & & & & & & \\
\hline 3 & 2 & $\begin{array}{c}6 \\
12\end{array}$ & & & & & & \\
\hline 3 & 3 & $\begin{array}{c}7 \\
14\end{array}$ & $\begin{array}{c}6 \\
15\end{array}$ & & & & & \\
\hline 4 & 2 & $\begin{array}{l}11 \\
17\end{array}$ & & & & & & \\
\hline 4 & 3 & $\begin{array}{l}12 \\
20\end{array}$ & $\begin{array}{l}11 \\
21\end{array}$ & & & & & \\
\hline 4 & 4 & $\begin{array}{l}13 \\
23\end{array}$ & $\begin{array}{l}12 \\
24\end{array}$ & $\begin{array}{l}11 \\
25\end{array}$ & & & & \\
\hline 5 & 2 & $\begin{array}{l}17 \\
23\end{array}$ & $\begin{array}{l}16 \\
24\end{array}$ & & & & & \\
\hline 5 & 3 & $\begin{array}{l}18 \\
27\end{array}$ & $\begin{array}{l}17 \\
28\end{array}$ & $\begin{array}{l}16 \\
29\end{array}$ & & & & \\
\hline 5 & 4 & $\begin{array}{l}20 \\
30\end{array}$ & $\begin{array}{l}18 \\
32 \\
\end{array}$ & $\begin{array}{l}17 \\
33 \\
\end{array}$ & $\begin{array}{l}16 \\
34 \\
\end{array}$ & & & \\
\hline 5 & 5 & $\begin{array}{l}21 \\
34\end{array}$ & $\begin{array}{l}20 \\
35\end{array}$ & $\begin{array}{l}18 \\
37\end{array}$ & $\begin{array}{l}17 \\
38\end{array}$ & $\begin{array}{l}16 \\
39\end{array}$ & $\begin{array}{l}16 \\
39\end{array}$ & \\
\hline 6 & 2 & $\begin{array}{l}23 \\
31\end{array}$ & $\begin{array}{l}22 \\
32\end{array}$ & & & & & \\
\hline 6 & 3 & $\begin{array}{l}25 \\
35 \\
\end{array}$ & $\begin{array}{r}24 \\
36 \\
\end{array}$ & $\begin{array}{l}23 \\
37 \\
\end{array}$ & & & & \\
\hline 6 & 4 & $\begin{array}{l}27 \\
39\end{array}$ & $\begin{array}{l}25 \\
41\end{array}$ & $\begin{array}{l}24 \\
42 \\
\end{array}$ & $\begin{array}{l}23 \\
43\end{array}$ & $\begin{array}{l}22 \\
44\end{array}$ & & \\
\hline 6 & 5 & $\begin{array}{l}29 \\
43\end{array}$ & $\begin{array}{l}27 \\
45\end{array}$ & $\begin{array}{l}25 \\
47\end{array}$ & $\begin{array}{l}24 \\
48\end{array}$ & $\begin{array}{l}23 \\
49\end{array}$ & $\begin{array}{l}22 \\
50\end{array}$ & \\
\hline 6 & 6 & $\begin{array}{l}31 \\
47 \\
\end{array}$ & $\begin{array}{l}29 \\
49 \\
\end{array}$ & $\begin{array}{l}27 \\
51 \\
\end{array}$ & $\begin{array}{l}25 \\
53 \\
\end{array}$ & $\begin{array}{l}24 \\
54 \\
\end{array}$ & $\begin{array}{r}23 \\
55 \\
\end{array}$ & \\
\hline 7 & 2 & $\begin{array}{l}30 \\
40 \\
\end{array}$ & $\begin{array}{l}29 \\
41 \\
\end{array}$ & & & & & \\
\hline 7 & 3 & $\begin{array}{l}33 \\
44 \\
\end{array}$ & $\begin{array}{l}31 \\
46\end{array}$ & $\begin{array}{l}30 \\
47\end{array}$ & $\begin{array}{l}29 \\
48\end{array}$ & & & \\
\hline 7 & 4 & $\begin{array}{l}35 \\
49\end{array}$ & $\begin{array}{l}33 \\
51\end{array}$ & $\begin{array}{l}32 \\
52\end{array}$ & $\begin{array}{l}30 \\
54\end{array}$ & $\begin{array}{l}29 \\
55\end{array}$ & & \\
\hline 7 & 5 & $\begin{array}{l}37 \\
54\end{array}$ & $\begin{array}{l}35 \\
56\end{array}$ & $\begin{array}{l}34 \\
57\end{array}$ & $\begin{array}{l}32 \\
59\end{array}$ & $\begin{array}{l}30 \\
61\end{array}$ & $\begin{array}{l}29 \\
62\end{array}$ & \\
\hline 7 & 6 & $\begin{array}{l}40 \\
58\end{array}$ & $\begin{array}{l}37 \\
61\end{array}$ & $\begin{array}{l}35 \\
63\end{array}$ & $\begin{array}{l}33 \\
65\end{array}$ & $\begin{array}{l}32 \\
66\end{array}$ & $\begin{array}{l}31 \\
67\end{array}$ & $\begin{array}{l}29 \\
69\end{array}$ \\
\hline 7 & 7 & $\begin{array}{l}42 \\
63 \\
\end{array}$ & $\begin{array}{l}40 \\
65 \\
\end{array}$ & $\begin{array}{l}37 \\
68 \\
\end{array}$ & $\begin{array}{l}35 \\
70\end{array}$ & $\begin{array}{l}33 \\
72 \\
\end{array}$ & $\begin{array}{l}32 \\
73\end{array}$ & $\begin{array}{l}30 \\
75 \\
\end{array}$ \\
\hline 8 & 2 & $\begin{array}{l}39 \\
49\end{array}$ & $\begin{array}{l}38 \\
50\end{array}$ & $\begin{array}{l}37 \\
51\end{array}$ & & & & \\
\hline 8 & 3 & $\begin{array}{l}42 \\
54 \\
\end{array}$ & $\begin{array}{l}40 \\
56\end{array}$ & $\begin{array}{l}39 \\
57\end{array}$ & $\begin{array}{l}37 \\
59\end{array}$ & & & \\
\hline 8 & 4 & $\begin{array}{l}44 \\
60\end{array}$ & $\begin{array}{l}42 \\
62\end{array}$ & $\begin{array}{l}41 \\
63\end{array}$ & $\begin{array}{l}39 \\
65\end{array}$ & $\begin{array}{l}38 \\
66\end{array}$ & $\begin{array}{l}37 \\
67\end{array}$ & \\
\hline 8 & 5 & $\begin{array}{l}47 \\
65\end{array}$ & $\begin{array}{l}45 \\
67\end{array}$ & $\begin{array}{l}43 \\
69\end{array}$ & $\begin{array}{l}41 \\
71\end{array}$ & $\begin{array}{l}39 \\
73\end{array}$ & $\begin{array}{l}38 \\
74\end{array}$ & $\begin{array}{l}37 \\
75\end{array}$ \\
\hline
\end{tabular}


JUSTICE I. ODIASE

Table 2 (continued): Exact Critical Values for Siegel-Tukey Test Statistic

\begin{tabular}{|c|c|c|c|c|c|c|c|c|}
\hline \multicolumn{2}{|c|}{ Sample Size } & \multirow{2}{*}{$\mathrm{ST}_{0.9000}$} & \multirow{2}{*}{$\mathrm{ST}_{0.9500}$} & \multirow{2}{*}{$\mathrm{ST}_{0.9750}$} & \multirow{2}{*}{$\mathrm{ST}_{0.9900}$} & \multirow{2}{*}{$\mathrm{ST}_{0.9950}$} & \multirow{2}{*}{$\mathrm{ST}_{0.9975}$} & \multirow{2}{*}{$\mathrm{ST}_{0.9990}$} \\
\hline & & & & & & & & \\
\hline 8 & 6 & $\begin{array}{l}50 \\
70\end{array}$ & $\begin{array}{l}47 \\
73\end{array}$ & $\begin{array}{l}45 \\
75\end{array}$ & $\begin{array}{l}43 \\
77\end{array}$ & $\begin{array}{l}41 \\
79\end{array}$ & $\begin{array}{l}40 \\
80\end{array}$ & $\begin{array}{l}38 \\
82\end{array}$ \\
\hline 8 & 7 & $\begin{array}{l}53 \\
75\end{array}$ & $\begin{array}{l}50 \\
78\end{array}$ & $\begin{array}{l}47 \\
81\end{array}$ & $\begin{array}{l}44 \\
84\end{array}$ & $\begin{array}{l}43 \\
85\end{array}$ & $\begin{array}{l}41 \\
87\end{array}$ & $\begin{array}{l}39 \\
89\end{array}$ \\
\hline 8 & 8 & $\begin{array}{l}56 \\
80\end{array}$ & $\begin{array}{l}52 \\
84\end{array}$ & $\begin{array}{l}50 \\
86\end{array}$ & $\begin{array}{l}46 \\
90\end{array}$ & $\begin{array}{l}44 \\
92\end{array}$ & $\begin{array}{l}43 \\
93\end{array}$ & $\begin{array}{l}41 \\
95\end{array}$ \\
\hline 9 & 2 & $\begin{array}{l}48 \\
60\end{array}$ & $\begin{array}{l}47 \\
61\end{array}$ & $\begin{array}{l}46 \\
62\end{array}$ & & & & \\
\hline 9 & 3 & $\begin{array}{l}51 \\
66\end{array}$ & $\begin{array}{l}49 \\
68\end{array}$ & $\begin{array}{l}48 \\
69\end{array}$ & $\begin{array}{l}47 \\
70\end{array}$ & $\begin{array}{l}46 \\
71\end{array}$ & & \\
\hline 9 & 4 & $\begin{array}{l}55 \\
71\end{array}$ & $\begin{array}{l}52 \\
74\end{array}$ & $\begin{array}{l}50 \\
76\end{array}$ & $\begin{array}{l}49 \\
77\end{array}$ & $\begin{array}{l}47 \\
79\end{array}$ & $\begin{array}{l}46 \\
80\end{array}$ & \\
\hline 9 & 5 & $\begin{array}{l}58 \\
77\end{array}$ & $\begin{array}{l}55 \\
80\end{array}$ & $\begin{array}{l}53 \\
82\end{array}$ & $\begin{array}{l}51 \\
84\end{array}$ & $\begin{array}{l}49 \\
86\end{array}$ & $\begin{array}{l}48 \\
87\end{array}$ & $\begin{array}{l}47 \\
88\end{array}$ \\
\hline 9 & 6 & $\begin{array}{l}61 \\
83\end{array}$ & $\begin{array}{l}58 \\
86\end{array}$ & $\begin{array}{l}56 \\
88\end{array}$ & $\begin{array}{l}53 \\
91\end{array}$ & $\begin{array}{l}51 \\
93\end{array}$ & $\begin{array}{l}50 \\
94\end{array}$ & $\begin{array}{l}48 \\
96\end{array}$ \\
\hline 9 & 7 & $\begin{array}{l}64 \\
89\end{array}$ & $\begin{array}{l}61 \\
92\end{array}$ & $\begin{array}{l}58 \\
95\end{array}$ & $\begin{array}{l}55 \\
98\end{array}$ & $\begin{array}{c}53 \\
100\end{array}$ & $\begin{array}{c}51 \\
102\end{array}$ & $\begin{array}{c}49 \\
104\end{array}$ \\
\hline 9 & 8 & $\begin{array}{l}68 \\
94\end{array}$ & $\begin{array}{l}64 \\
98\end{array}$ & $\begin{array}{c}61 \\
101\end{array}$ & $\begin{array}{c}57 \\
105\end{array}$ & $\begin{array}{c}55 \\
107\end{array}$ & $\begin{array}{c}53 \\
109\end{array}$ & $\begin{array}{c}51 \\
111\end{array}$ \\
\hline 9 & 9 & $\begin{array}{c}71 \\
100\end{array}$ & $\begin{array}{c}67 \\
104\end{array}$ & $\begin{array}{c}63 \\
108\end{array}$ & $\begin{array}{c}60 \\
111\end{array}$ & $\begin{array}{c}57 \\
114\end{array}$ & $\begin{array}{c}55 \\
116\end{array}$ & $\begin{array}{c}53 \\
118\end{array}$ \\
\hline 10 & 10 & $\begin{array}{c}88 \\
122\end{array}$ & $\begin{array}{c}83 \\
127\end{array}$ & $\begin{array}{c}79 \\
131\end{array}$ & $\begin{array}{c}75 \\
135\end{array}$ & $\begin{array}{c}72 \\
138\end{array}$ & $\begin{array}{c}69 \\
141\end{array}$ & $\begin{array}{c}66 \\
144\end{array}$ \\
\hline 11 & 11 & $\begin{array}{l}107 \\
146\end{array}$ & $\begin{array}{l}101 \\
152\end{array}$ & $\begin{array}{c}97 \\
156\end{array}$ & $\begin{array}{c}92 \\
161\end{array}$ & $\begin{array}{c}88 \\
165\end{array}$ & $\begin{array}{c}85 \\
168\end{array}$ & $\begin{array}{c}82 \\
171\end{array}$ \\
\hline 12 & 12 & $\begin{array}{l}128 \\
172\end{array}$ & $\begin{array}{l}121 \\
179\end{array}$ & $\begin{array}{l}116 \\
184\end{array}$ & $\begin{array}{l}110 \\
190\end{array}$ & $\begin{array}{l}106 \\
194\end{array}$ & $\begin{array}{l}103 \\
197\end{array}$ & $\begin{array}{c}99 \\
201\end{array}$ \\
\hline 13 & 13 & $\begin{array}{l}150 \\
201\end{array}$ & $\begin{array}{l}143 \\
208\end{array}$ & $\begin{array}{l}137 \\
214\end{array}$ & $\begin{array}{l}131 \\
220\end{array}$ & $\begin{array}{l}126 \\
225\end{array}$ & $\begin{array}{l}122 \\
229\end{array}$ & $\begin{array}{l}118 \\
233\end{array}$ \\
\hline 14 & 14 & $\begin{array}{l}175 \\
231\end{array}$ & $\begin{array}{l}167 \\
239\end{array}$ & $\begin{array}{l}161 \\
245\end{array}$ & $\begin{array}{l}153 \\
253\end{array}$ & $\begin{array}{l}148 \\
258\end{array}$ & $\begin{array}{l}144 \\
262\end{array}$ & $\begin{array}{l}138 \\
268\end{array}$ \\
\hline 15 & 15 & $\begin{array}{l}201 \\
264\end{array}$ & $\begin{array}{l}193 \\
272\end{array}$ & $\begin{array}{l}185 \\
280\end{array}$ & $\begin{array}{l}177 \\
288\end{array}$ & $\begin{array}{l}172 \\
293\end{array}$ & $\begin{array}{l}167 \\
298\end{array}$ & $\begin{array}{l}161 \\
304\end{array}$ \\
\hline 20 & 20 & $\begin{array}{l}362 \\
458\end{array}$ & $\begin{array}{l}349 \\
471\end{array}$ & $\begin{array}{l}338 \\
482\end{array}$ & $\begin{array}{l}325 \\
495\end{array}$ & $\begin{array}{l}316 \\
504\end{array}$ & $\begin{array}{l}308 \\
512\end{array}$ & $\begin{array}{l}298 \\
522\end{array}$ \\
\hline 30 & 30 & $\begin{array}{c}828 \\
1002\end{array}$ & $\begin{array}{c}804 \\
1027\end{array}$ & $\begin{array}{c}783 \\
1047\end{array}$ & $\begin{array}{c}759 \\
1071\end{array}$ & $\begin{array}{c}743 \\
1088\end{array}$ & $\begin{array}{c}728 \\
1102\end{array}$ & $\begin{array}{c}710 \\
1121\end{array}$ \\
\hline 40 & 40 & $\begin{array}{l}1487 \\
1754\end{array}$ & $\begin{array}{l}1449 \\
1791\end{array}$ & $\begin{array}{l}1417 \\
1824\end{array}$ & $\begin{array}{l}1380 \\
1861\end{array}$ & $\begin{array}{l}1354 \\
1886\end{array}$ & $\begin{array}{l}1331 \\
1910\end{array}$ & $\begin{array}{l}1303 \\
1937\end{array}$ \\
\hline 50 & 50 & $\begin{array}{l}2339 \\
2711\end{array}$ & $\begin{array}{l}2286 \\
2764\end{array}$ & $\begin{array}{l}2241 \\
2809\end{array}$ & $\begin{array}{l}2189 \\
2861\end{array}$ & $\begin{array}{l}2153 \\
2898\end{array}$ & $\begin{array}{l}2122 \\
2930\end{array}$ & $\begin{array}{l}2082 \\
2970\end{array}$ \\
\hline
\end{tabular}




\section{EMPIRICAL SAMPLING FROM PERMUTATION SPACE WITH UNIQUE PATTERNS}

\section{References}

Agresti, A. (1992). A survey of exact inference for contingency tables. Statistical Science, 7, 131-177.

Bayarri, M. J., \& Berger, J. O. (2004). The interplay of Bayesian and frequentist analysis. Statistical Science, 19, 58-80.

Darwin, C. (1878). The Effects of Cross and self-fertilization in the Vegetable Kingdom, $2^{\text {nd }} E d$. London: John Murray.

Devore, J. L. (1982). Probability and statistics for engineering and the sciences. California: Brooks/Cole Publishing Company.

Efron, B. (1982). The jackknife, the bootstrap, and other resampling plans. Philadelphia, PA: SIAM.

Ernst, M. D. (2004). Permutation methods: A basis for exact inference. Statistical Science, 19, 676-685.

Fahoome, G. (2002). Twenty nonparametric statistics and their large sample approximations. Journal of Modern Applied Statistical Methods, 1, 248-268.

Fisher, R. A. (1935). The design of experiments. Edinburgh: Oliver and Boyd.

Fisher, R. A. (1936). The coefficient of racial likeness and the future of Craniometry. Journal of the Royal Anthropological Institute, 66, 57-63.

Fisher, R. A. (1971). The design of experiments ( $\left.8^{\text {th }} E d.\right)$. New York: Hafner Publishing.

Friedman, M. (1937). The use of ranks to avoid the assumption of normality implicit in the analysis of variance. Journal of the American Statistical Association, 32(200), 675701.

Friedman, M. (1939). A correction: The use of ranks to avoid the assumption of normality implicit in the analysis of variance. Journal of the American Statistical Association, 34(205), 109.

Friedman, M. (1940). A comparison of alternative tests of significance for the problem of $\mathrm{m}$ rankings. The Annals of Mathematical Statistics, 11(1), 86-92.

Good, P. (2000). Permutation tests: a practical guide to resampling methods for testing hypotheses $\left(2^{\text {nd }} E d.\right)$. New York: Springer-Verlag.
Hall, P. and I. Weissman (1997). On the estimation of extreme tail probabilities. The Annals of Statistics, 25, 1311-1326.

Headrick, T. C. (2003). An algorithm for generating exact critical values for the Kruskal-Wallis one-way ANOVA. Journal of Modern Applied Statistical Methods, 2, 268-271.

Ludbrook, J., \& Dudley, H. (1998). Why permutation tests are superior to $\mathrm{t}$ and $\mathrm{F}$ tests in biomedical research. The American Statistician, 52, 127-132.

Odiase, J. I., \& Ogbonmwan, S. M. (2005a). An algorithm for generating unconditional exact permutation distribution for a two-sample experiment. Journal of Modern Applied Statistical Methods, 4, 319-332.

Odiase, J. I., \& Ogbonmwan, S. M. (2005b). Exact permutation critical values for the Kruskal-Wallis one-way ANOVA. Journal of Modern Applied Statistical Methods, 4(2), 609-620.

Odiase, J. I., \& Ogbonmwan, S. M. (2007a). Exact permutation of paired observations: The challenge of R. A. Fisher. Journal of Mathematics and Statistics, 3(3), 116-121.

Odiase, J. I., \& Ogbonmwan, S. M. (2007b). Exact permutation paradigm in multisample experiments. Advances and Applications in Statistics, 7(3), 417-434.

Opdyke, J. D. (2003). Fast permutation tests that maximize power under conventional Monte Carlo sampling for pairwise and multiple comparisons. Journal of Modern Applied Statistical Methods, 2, 27-49.

Scheffe, H. (1943) Statistical inference in the nonparametric case. The Annals of Mathematical Statistics, 14, 305-332.

Siegel, S., \& Castellan, N. J. (1989). Nonparametric statistics for the behavioural sciences ( $3^{\text {rd }} E d$.). New York: McGraw-Hill.

Spiegelhalter, D. J. (2004). Incorporating Bayesian ideas into health-care evaluation. Statistical Science, 19, 156-174.

Wald, A., \& Wolfowitz, J. (1944). Statistical tests based on permutation of the observations. The Annals of Mathematical Statistics, 15, 358-372. 
Appendix A: Unique Permutation Pattern Algorithm

This computer algorithm identifies and compiles the unique permutation patterns of the layout of observations or rank of observations in a two-sample experiment. It was implemented to produce a table of critical values for the Siegel-Tukey test statistic.

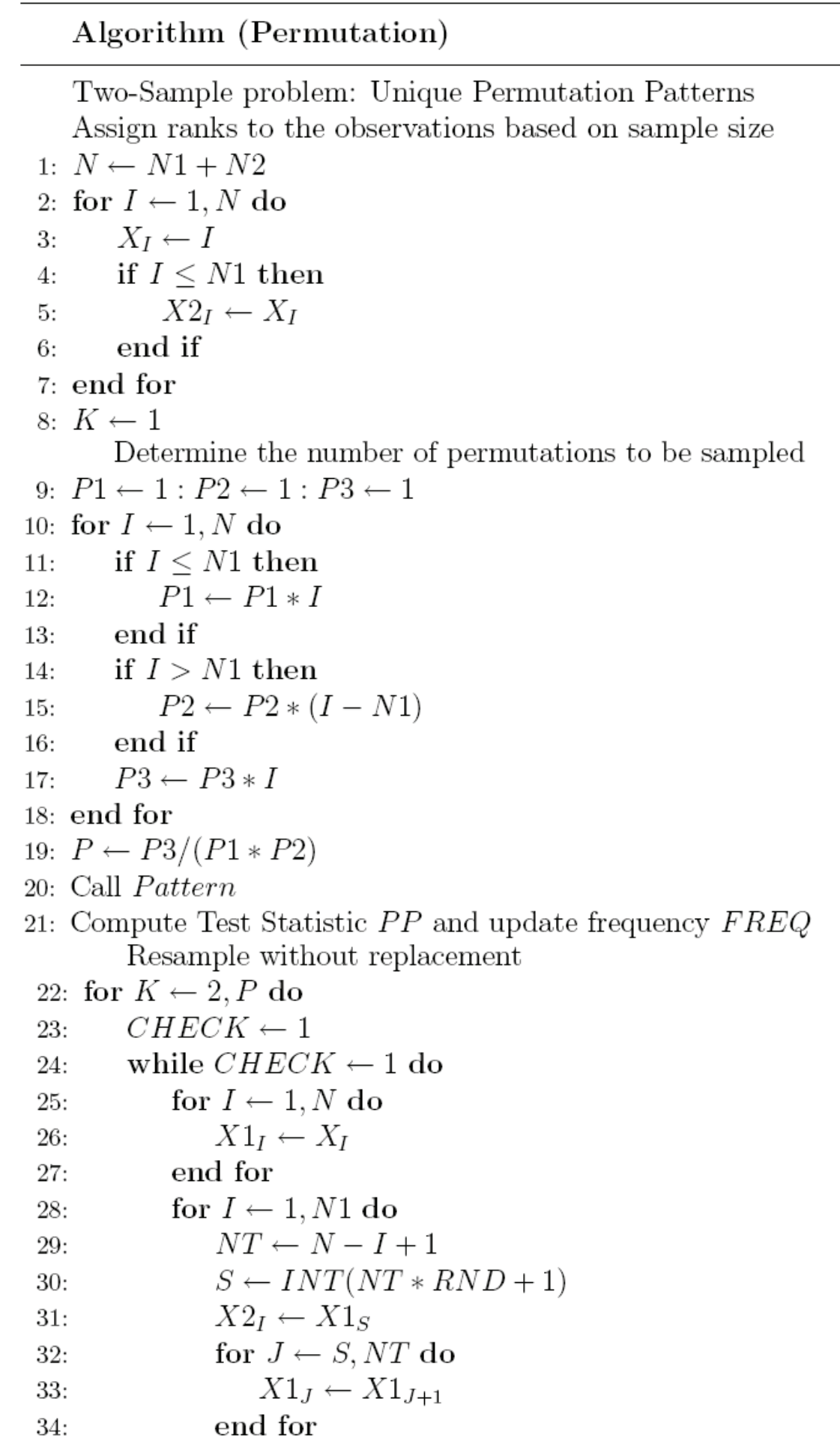


Appendix A (continued): Unique Permutation Pattern Algorithm

35: $\quad$ end for

36: Call Pattern

Ensure no repetition of permutations

37: $\quad C H E C K \leftarrow 0$

38: $\quad$ for $I 1 \leftarrow 1, K-1$ do

39: $\quad$ if $P A T_{K} \leftarrow P A T_{I 1}$ then

40: $\quad C H E C K \leftarrow 1$

41: $\quad$ end if

42: $\quad$ end for

43: $\quad$ if $C H E C K \leftarrow 1$ then

44: $\quad P A T_{K} \leftarrow " ”$

45: $\quad$ end if

46: $\quad$ end while

47: $\quad$ Compute Test Statistic $P P$ and update frequency $F R E Q$

48: end for

49: Sort $P P_{I}$ in ascending order for $I \leftarrow 1, C O U N T 1$

50: PRINT N1, N2

51: $P V \leftarrow 0$

52: for $I \leftarrow 1, C O U N T 1$ do

53: $\quad P D M \leftarrow F R E Q_{I} / P$

54: $\quad P V \leftarrow P V+P D M$

55: $\quad$ PRINT $P P_{I}, F R E Q_{I}, P D M, P V$

56: end for

\section{Algorithm (Pattern)}

1: Sort X2(I) for $I \leftarrow 1, N 1$ to have a unique pattern

2: for $J \leftarrow 1, N 1$ do

3: $\quad$ if $X 2_{J}<10$ then

4: $\quad P A T_{K} \leftarrow P A T_{K}+" 0 "+\operatorname{LTRIM}\left(\operatorname{RTRIM}\left(\operatorname{STR}\left(X 2_{J}\right)\right)\right)$

5: $\quad$ else $P A T_{K} \leftarrow P A T_{K}+\operatorname{LTRIM}\left(R T R I M\left(S T R\left(X 2_{J}\right)\right)\right)$

6: $\quad$ end if

7: end for 
Appendix A (continued): Unique Permutation Pattern Algorithm

\section{Algorithm (Permutation-Convergence)}

Unique Permutation Patterns: Iterate to convergence

Assign ranks to the observations based on sample size

1: $N \leftarrow N 1+N 2$

2: for $I \leftarrow 1, N$ do

3: $\quad X_{I} \leftarrow I$

4: $\quad$ if $I \leq N 1$ then

5: $\quad X 2_{I} \leftarrow X_{I}$

6: end if

7: end for

8: $B B \leftarrow 0$

9: $C K \leftarrow 1$

10: Ensure error tolerance is achieved

11: while $C K \leftarrow 1$ do

12: $\quad$ for $C 0 \leftarrow 1, C O U N T 1$ do

13: $\quad F R E Q_{C 0} \leftarrow 0$

14: end for

15: $\quad C O U N T 1 \leftarrow 0$

16: $\quad$ for $K \leftarrow 1, N N$ do

17: $\quad P A T_{K} \leftarrow " ”$

18: $\quad$ end for

19: $\quad B B \leftarrow B B+1$

Resample without replacement

20: $\quad$ for $K \leftarrow 1, N N$ do

21: $\quad C H E C K \leftarrow 1$

Ensure pattern is unique

22: while $C H E C K \leftarrow 1$ do

23:

for $I \leftarrow 1, N$ do

$X 1_{I} \leftarrow X_{I}$

end for

25:

26:

27:

for $I \leftarrow 1, N 1$ do

$N T \leftarrow N-I+1$

$S \leftarrow I N T(N T * R N D+1)$

$X 2_{I} \leftarrow X 1_{S}$

for $J \leftarrow S, N T$ do

$X 1_{J} \leftarrow X 1_{J+1}$

end for

end for

34:

Sort $X 2_{I}$ to have a unique pattern 
Appendix A (continued): Unique Permutation Pattern Algorithm

$35:$

36:

37:

38:

39:

40:

41:

42:

43:

44:

58:

59:

60:

61:

63:

64:

65:

66 :

67:

68:

69:

70 :

71 :

72 :

73:
Call Pattern

Ensure no repetition of permutations

$C H E C K \leftarrow 0$

for $I 1 \leftarrow 1, K-1$ do

if $P A T_{K} \leftarrow P A T_{I 1}$ then

$C H E C K \leftarrow 1$

end if

end for

if $C H E C K \leftarrow 1$ then

$P A T_{K} \leftarrow " "$

end if

end while

Compute Test Statistic $P P$ and update frequency FREQ

end for

Sort $P P_{I}$ in ascending order for $I \leftarrow 1, C O U N T 1$

Merge and Update pdf

if $B B>1$ then

$N P \leftarrow C O U N T 2+C O U N T 1$

for $I \leftarrow 1, N P$ do

if $I \leq C O U N T 2$ then

$U P D_{I} \leftarrow U P D 1_{I}$

$F R E Q_{I} \leftarrow F R E Q 1_{I}$

else

$U P D_{I} \leftarrow P P_{I-C O U N T 2}$

$F R E Q_{I} \leftarrow F R E Q 2_{I-C O U N T 2}$

end if

end for

for $J \leftarrow 1, C O U N T 2$ do

for $I \leftarrow C O U N T 2+1, N P$ do

if $U P D_{J} \leftarrow U P D_{I}$ then

$F R E Q_{J} \leftarrow F R E Q_{J}+F R E Q_{I}$

for $T \leftarrow I, N P-1$ do

$U P D_{T} \leftarrow U P D_{T+1}$

$F R E Q_{T} \leftarrow F R E Q_{T+1}$

end for

$N P \leftarrow N P-1$

end if

end for

end for

Sort the update $U P D$ in ascending order else 


\section{JUSTICE I. ODIASE}

Appendix A (continued): Unique Permutation Pattern Algorithm

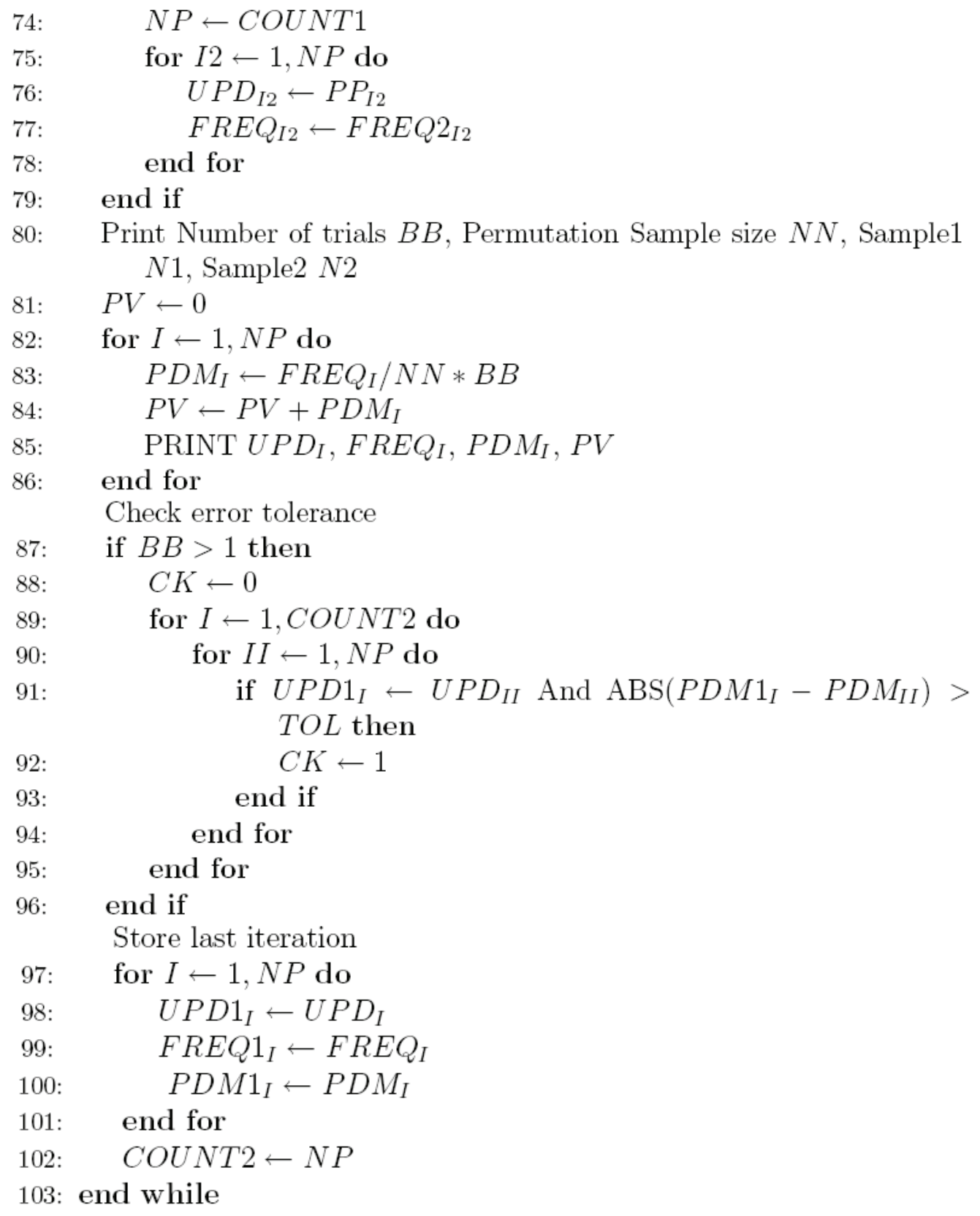




\title{
A Weighted Exponential Detection Function Model for Line Transect Data
}

\author{
Faisal Ababneh \\ Omar M. Eidous \\ Al-Hussian Bin Talal University, \\ Ma'an, Jordan \\ Yarmouk University, \\ Irbid, Jordan
}

A new parametric model is proposed for modeling the density function of perpendicular distances in line transects sampling. The model can be considered a weighted exponential model in the sense that it combines two exponential models with different weights. The proposed model is appealing because it is monotone decreasing with distance from transect line; in contrast to the classical exponential model, it satisfies the shoulder condition at the origin. Simulation results for a wide range of target densities show reasonable and good performances of the weighted exponential model in most considered cases compared to the classical exponential and the half-normal models.

Key words: Line transect sampling, exponential model, weighted exponential model, half-normal model.

Introduction

Transect methods, particularly line transect methods, are a practical and relatively inexpensive procedure for estimating the population density of certain objects in a given region; these methods have become a popular sampling scheme among ecologists. The estimation procedure can be achieved by walking distance $L$ following a deterministic transect line, counting the number of objects being investigated and recording the perpendicular distance, $X$, from the object sighted to the path of the observer (line transect center). When objects are observed from a line transect with a detection function $g(x)$, the distance $X$ to the observed object from a randomly placed transect will tend to have a probability density function (pdf) $f(x)$ of the same shape as $g(x)$, but scaled so that the area under $f(x)$ equals unity. Buckland, et al.

Omar M. Eidous is a Professor on the Faculty of Science in the Department of Statistics. Email him at: omarm@yu.edu.jo. Faisal Ababneh is Assistant Professor in the Department of Mathematics. Email him at: fmababneh@gmail.com.
(2001) and Burnham, et al. (1980) are the key references for this distance sampling procedure.

The logical assumption related to the detection function $g(x)$ indicates that $g(x)$ is monotonically decreasing and satisfies the shape criterion (i.e., $g^{\prime}(x)=0$ ). Accordingly, $f(x)$ is in turn monotonically decreasing with $f^{\prime}(0)=0$. Burnham and Anderson (1976) gave the fundamental relation for estimating the density of objects in a specific area, which is expressed as

$$
D=\frac{E(n) f(0)}{2 L},
$$

where $E(n)$ is the expected value of the number of detected objects. Given various assumptions (Burnham \& Anderson, 1976) show that the general estimate for $D$ is given by

$$
\hat{D}=\frac{n \hat{f}(0)}{2 L},
$$

where $\hat{f}(0)$ is an appropriate sample estimator of $f(0)$ based on $n$ observed perpendicular distances $x_{1}, x_{2}, \ldots, x_{n}$. Thus, the key aspects in line transects sampling are the modeling of $f(x)$ and the estimation of $f(0)$. 


\section{ABABNEH \& EIDOUS}

Let $f(x)$ be the unknown pdf of perpendicular distances $X_{1}, X_{2}, \ldots, X_{n}$, which are usually assumed to be a random sample (Buckland, et al., 1993). A parametric approach involves assuming that $f(x)$ is a member of a family of proper pdf of a known functional form, but depends on an unknown parameter $\theta$, where $\theta$ may take a vector value and should be estimated by using perpendicular distances. A variety of approaches to estimate $\theta$ will lead to $\hat{f}(0)=f(0, \hat{\theta})$.

A number of parametric models have been proposed for $f(x)$. The classical exponential model and the half normal model, each with one scale parameter, are the most prominent. Gates, et al. (1968) suggested the exponential model with detection function,

$$
g_{1}(x)=e^{-x / \alpha}, \quad x \geq 0
$$

and pdf,

$$
f_{1}(x)=e^{-x / \alpha} / \alpha, \quad x \geq 0 .
$$

The maximum likelihood (ML) method indicates that the ML estimator of $f(0)$ is $\hat{f}_{1, M L}(0)=1 / \bar{X}$, where $\bar{X}$ is the sample mean. The detection function $g_{1}(x)$ (or the pdf $f_{1}(x)$ ) do not satisfy the shoulder condition which minimizes the importance of utilizing this model in line transect sampling. In contrast to the exponential model, the half normal model suggested by Hemingway (1971) satisfies the shoulder condition assumption. The half normal detection function is given by

$$
g_{2}(x)=e^{-x^{2} / 2 \sigma^{2}}, \quad x \geq 0
$$

and the pdf is,

$$
f_{2}(x)=\frac{2}{\sigma \sqrt{2 \pi}} e^{-x^{2} / 2 \sigma^{2}}, x \geq 0
$$

The ML estimator of $f(0)$ is $\hat{f}_{2, M L}(0)=\left(\frac{2}{\pi T}\right)^{1 / 2}$ under the half normal model, where $T=\sum_{i=1}^{n} x_{i}^{2} / n$. Quinn and Gallucci (1980) derived the minimum variance unbiased estimator for $f(0)$ under Model (2), which is given by

$$
\hat{f}_{2, M V}(0)=\frac{1}{\beta(n)}\left(\frac{2}{\pi T}\right)^{1 / 2},
$$

where

$$
\beta(n)=\frac{\Gamma((n-1) / 2)}{\Gamma(n / 2)}\left(\frac{n}{2}\right)^{1 / 2} .
$$

Under Model (2), Zhang (2009) proposed the shrinkage estimator

$$
\hat{f}_{2, S H}(0)=\frac{n-2}{n} \beta(n)\left(\frac{2}{\pi T}\right)^{1 / 2} .
$$

The estimator $\hat{f}_{2, S H}(0)$ is biased for $f(0)$, but it achieves the smallest mean square error.

Comparing the estimators $\hat{f}_{2, M L}(0), \hat{f}_{2, M V}(0)$ and $\hat{f}_{2, S H}(0)$ it is observed that

$$
\begin{aligned}
\hat{f}_{2, S H}(0) & =\frac{n-2}{n} \beta^{2}(n) \hat{f}_{2, M V}(0) \\
& =\frac{n-2}{n} \beta(n) \hat{f}_{2, M L}(0)
\end{aligned}
$$

Because $\beta(n) \rightarrow 1$ (Magnus, et al., 1966) and $\frac{n-2}{n} \rightarrow 1$ as $n \rightarrow \infty$, the three estimators are asymptotically equivalent. A simulation study was performed using a finite sample for different target models; results indicate that the three estimators perform very similarly to each other, even for the target detection functions that are deviated from the half normal model.(For other parametric models with two parameters see: Burnham \& Anderson (1976); Pollock, (1978); 


\section{WEIGHTED EXPONENTIAL DETECTION MODEL FOR LINE TRANSECT DATA}

Burnham, et al. (1980); Buckland (1985); Eidous (2004).

A weighted exponential model with one parameter is proposed to fit line transects data. Two estimators under this model are derived to estimate $f(0)$ and hence the density of objects, $D$. The small-sample properties of the new estimators were studied and compared to both the classical exponential and the half normal estimators via simulation techniques.

The Model

Let $X_{1}, X_{2}, \ldots, X_{n}$ be $n$ perpendicular distances (assumed to be independent and identically distributed) following the detection function $g(x ; \theta, \gamma)$, where $\theta$ and $\gamma$ are two unknown parameters. The detection function is proposed to be,

$$
\begin{aligned}
& g(x ; \theta, \gamma)=2 e^{-\theta x}-e^{-\lambda x}, \\
& x \geq 0, \theta, \gamma>0
\end{aligned}
$$

According to this detection function, the probability of detecting an object given its perpendicular distance on the transect line is one (i.e., $g(0 ; \theta, \gamma)=1)$, which indicates that the probability of detecting on the line transect center is certain. However, the first derivative of $g(x ; \theta, \gamma)$ at $x=0$ is $-2 \theta+\gamma \quad$ (i.e. $\left.g^{\prime}(0 ; \theta, \gamma)=-2 \theta+\gamma\right)$, which indicates that $g(x ; \theta, \gamma)$ do not satisfy the shoulder condition unless $\gamma=2 \theta$. Therefore, the detection function for the perpendicular distances that satisfies the shoulder condition is proposed to be,

$$
g_{3}(x)=e^{-\theta x}\left(2-e^{-\theta x}\right),
$$

and the corresponding pdf is,

$$
f_{3}(x)=\frac{2}{3 \theta} e^{-\theta x}\left(2-e^{-\theta x}\right), \quad x \geq 0, \theta>0 .
$$

Because $g_{3}(0)=1$, the parameter $f_{3}(0)$ is given by

$$
f_{3}(0)=\frac{2}{3 \theta}
$$

model (3) can be expressed in terms of $f_{3}(0)$ as

$$
\begin{aligned}
& f_{3}(x)=f_{3}(0) e^{-3 f_{3}(0) x / 2}\left(2-e^{-3 f_{3}(0) x / 2}\right), \\
& x \geq 0
\end{aligned}
$$

It can be shown that the detection function $g_{3}(x)$ is monotonically decreasing in $x$. The first derivative of $g_{3}(x)$ is $g_{3}^{\prime}(x)=2 \theta\left(e^{-2 \theta x}-e^{-\theta x}\right)$, which equals zero at $x=0 ;$ thus, $g_{3}(x)$ is monotonically decreasing for $x \in(0, \infty)$ if $2 \theta\left(e^{-2 \theta x}-e^{-\theta x}\right)$ $<0 \Rightarrow e^{-2 \theta x}-e^{-\theta x}<0 \Rightarrow e^{-2 \theta x}<e^{-\theta x} \Rightarrow$ $-2 \theta x<-\theta x \Rightarrow 2 \theta x>\theta x$, which is true for all $x>0$ and $\theta>0$. Accordingly $f_{3}(x)$ is monotonically decreasing for $x \in(0, \infty)$.

Moments and Maximum Likelihood Estimators The parameter $f_{3}(0)$ in model (5) must be estimated. The expected value of $X$ based on this model is $7 /\left(9 f_{3}(0)\right)$, which gives $\hat{f}_{3, M O}(0)=7 /(9 \bar{X})$ as the moment estimator for $f_{3}(0)$.

Although the moment estimator for $f_{3}(0)$ is given in a closed form, the maximum likelihood estimator must be calculated using a numerical method. The likelihood function $L(f)$ based on model (5) is

$L(f)=\prod_{i=1}^{n} f_{3}\left(x_{i}\right)$

$=f_{3}(0)^{n} \exp \left(-3 f_{3}(0) \sum_{\mathrm{i}=1}^{\mathrm{n}} \frac{\mathrm{x}_{\mathrm{i}}}{2}\right) \prod_{\mathrm{i}=1}^{\mathrm{n}}\left(2-\exp \left(\frac{-3 f_{3}(0) \mathrm{x}_{\mathrm{i}}}{2}\right)\right)$.

To find the maximum likelihood estimator of $f_{3}(0)$, the following equation must be solved

$$
\frac{\partial \log L(f)}{\partial f}=0
$$

where 
$\frac{\partial \log L(f)}{\partial f}=$

$\frac{n}{f_{3}(0)}-\frac{3}{2} \sum_{i=1}^{n} x_{i}+\frac{3}{2} \sum_{i=1}^{n}\left(\frac{x_{i}}{2 \exp \left(3 f_{3}(0) x_{i} / 2\right)-1}\right)$.

The maximum likelihood estimator can be found by using numerical methods such as the Newton-Raphson, and a Mathematica Program for carrying out the estimation procedure can be written. Let $\hat{f}_{3, M L}(0)$ be the maximum likelihood estimator of $f_{3}(0)$, then as $n \rightarrow \infty$, $\hat{f}_{3, M L}(0)$ is asymptotically $N\left(f_{3}(0), \sigma_{f}^{2}\right)$, where $\sigma_{f}^{2}=\left\{-E\left(\frac{d^{2} \ln L(f)}{d f^{2}}\right)\right\}^{-1}$. Using the transformation $\quad u=2-\exp \left(-3 f_{3}(0) x / 2\right)$ results in,

$$
\begin{aligned}
& -E\left(\frac{d^{2} \ln L(f)}{d f^{2}}\right) \\
& =\frac{n}{f_{3}(0)^{2}}+\frac{9}{2} \sum_{i=1}^{n} E\left(\frac{X_{i}^{2} \exp \left(-3 f_{3}(0) X_{i} / 2\right)}{\left(2-\exp \left(-3 f_{3}(0) X_{i} / 2\right)\right)^{2}}\right) \\
& =\frac{n}{f_{3}(0)^{2}}+\frac{4 n}{3 f_{3}(0)^{2}} \int_{1}^{2} \frac{(2-u) \ln ^{2}(2-u)}{u} d u
\end{aligned}
$$

The Mathematica Program was used to compute the last integral, which gives,

$$
=\frac{0.80153 n}{f_{3}(0)^{2}} \text {. }
$$

Therefore,

$$
\sigma_{f}^{2}=\frac{f_{3}(0)^{2}}{0.80153 n}
$$

Replacing $f_{3}(0)$ by $\hat{f}_{3, M L}(0)$ leads to the estimate $\hat{\sigma}_{f}^{2}$ of $\sigma_{f}^{2}$ and the approximate largesample $(1-\alpha) 100 \%$ confidence interval (C.I.) for $f_{3}(0)$ is given by

$$
\hat{f}_{3, M L}(0) \pm Z_{\alpha / 2} \sqrt{\hat{\sigma}_{f}^{2}}
$$

For example, if $\alpha=0.05$, then $Z_{0.025}=1.96$ and thus, a $95 \%$ C.I. for $f_{3}(0)$ is $\hat{f}_{3, M L}(0) \pm$ $2.18925 \hat{f}_{3, M L}(0) / \sqrt{n}$.

\section{Methodology}

To assess the performances of the proposed estimators $\hat{f}_{3, M O}(0)$ and $\hat{f}_{3, M L}(0)$ of $f(0)$ under the weighted exponential family, a simulation study was performed. For comparison, the classical exponential estimator $\hat{f}_{1, M L}(0)$ and the half normal estimator $\hat{f}_{2, M L}(0)$ were also considered. Four target models were chosen for inclusion in the simulation based on the criterion that they are representative of many different shapes that might occur in the field. These four models are:

(1) Exponential Power (EP) Model (Pollock, 1978)

$$
\begin{aligned}
& f(x)=\frac{1}{\Gamma(1+1 / \beta)} e^{-x^{\beta}} \\
& x \geq 0, \quad \beta \geq 1
\end{aligned}
$$

(2) Hazard-Rate (HR) Model (Hayes and Buckland, 1983)

$$
\begin{aligned}
& f(x)=\frac{1}{\Gamma(1-1 / \beta)}\left(1-e^{-x^{-\beta}}\right) \\
& x \geq 0, \quad \beta>1
\end{aligned}
$$

(3) Beta (BE) Model (Eberhardt, 1968)

$$
\begin{aligned}
& f(x)=(1+\beta)(1-x)^{\beta} \\
& 0 \leq x<1, \beta \geq 0
\end{aligned}
$$

(4) General Polynomial (GP) Model (Zhang, 2009)

$$
\begin{aligned}
& f(x)=\frac{10 \Gamma(\beta)}{3 \sqrt{\pi} \Gamma(\beta-1 / 2)}\left(1+(x / 0.6)^{2}\right)^{-\beta} \\
& x \geq 0, \beta>1 / 2 .
\end{aligned}
$$




\section{WEIGHTED EXPONENTIAL DETECTION MODEL FOR LINE TRANSECT DATA}

Four models were selected from the following different models: EP, HR, BE and GP. For the EP model, parameter values $\beta=1.0,1.5,2.0,2.5$ and corresponding truncation points $w=5.0,3.0,2.5,2.0$. For the HR model with parameter values $\beta=1.5,2.0,2.5,3.0$ and corresponding truncation points $w=20,12,8,6$. The $\mathrm{BE}$ model parameter values $\beta=1.5,2.0,2.5,3.0$ and truncated point $w=1.0$ for all cases. For the GP model parameter values $\beta=0.6,0.9,1.9,3.5$ and the truncated point $w=3.0$ for all cases.

The 16 target models considered cover a wide range of perpendicular distance probability density functions that vary near zero from spike to flat. It should be noted that the EP model with $\beta=1$ and the $\mathrm{BE}$ model with different values of $\beta$ do not satisfy the shoulder condition assumption. These choices were made in order to assess the robustness of the considered estimators with respect to the violation of the shoulder condition assumption. Note also that the other considered models satisfy the shoulder condition assumption.

For each model 1,000 samples of perpendicular distances were randomly drawn for sample sizes $n=50,100,200$. Table (1) reports the simulated value of the relative bias $(R B)$ for each model and for each sample size,

$$
R B=\frac{E(\hat{f}(0))-f(0)}{f(0)},
$$

and the relative mean error $(R M E)$

$$
R M E=\frac{\sqrt{M S E(\hat{f}(0))}}{f(0)},
$$

for each considered estimator.

\section{Conclusion}

Based on the simulation, several conclusions can be drawn by inspecting the results with respect to $R B$ and $R M E$.
(1) The performance of the classical exponential estimator, $\hat{f}_{1, M L}(0)$ is effective when the target (underlying) model is exponential (EP with $\beta=1$ ). In this case, the $R B$ and $R M E$ associated with $\hat{f}_{1, M L}(0)$ were very small compared other considered estimators. However, the $R B$ and $R M E$ of $\hat{f}_{1, M L}(0)$ become very large when the underlying model deviates from the exponential. The $R M E$ values of $\hat{f}_{1, M L}(0)$ range between 0.080 (EP with $\beta=1$ and $n=200$ ) and 0.732 (EP with $\beta=2.5$ and $n=50$ ).

(2) The estimator $\hat{f}_{2, M L}(0)$ seems to be better than $\hat{f}_{1, M L}(0)$ for most considered cases. Regarding $R M E, \quad \hat{f}_{2, M L}(0)$ beats $\hat{f}_{1, M L}(0)$ for all considered cases except for (EP with $\beta=1$ and HR with $\beta=1.5$ and $\beta=2$ ). Despite that HR satisfies the shoulder condition, it decreases very sharply away $x=0$ when $\beta=1.5$ and $\beta=2$. This may explain the performances of $\hat{f}_{1, M L}(0)$ in these two cases. The performance of $\hat{f}_{2, M L}(0)$ is very good when the target model is half normal (EP with $\beta=2$ ) and when the shoulder condition of the target model is very large (EP with $\beta=2.5$ and HR with $\beta=3$ ). Except for the cases: EP with $\beta=1$, HR with $\beta=1.5$ and $\beta=2$, the performance of $\hat{f}_{2, M L}(0)$ is acceptable compared the other estimators. The RME values of $\hat{f}_{2, M L}(0)$ range from 0.052 (EP with $\beta=2$ and $n=200$ ) to 0.576 (EP with $\beta=1.5$ and $n=200$ ). 


\section{ABABNEH \& EIDOUS}

(3) The $R B$ and $R M E$ associated with the two proposed estimators $\hat{f}_{3, M O}(0)$ and $\hat{f}_{3, M L}(0)$ are very similar to each other in all considered cases. Therefore, $\hat{f}_{3, M O}(0)$ is recommended as opposed to $\hat{f}_{3, M L}(0)$ because the formal model takes a closed form, while $\hat{f}_{3, M L}(0)$ needs a numerical method to compute. Comparing the RME $\mathrm{s}$ of $\hat{f}_{3, M O}(0)$ and $\hat{f}_{2, M L}(0)$ it appears that $\hat{f}_{3, M O}(0)$ performs better than $\hat{f}_{2, M L}(0)$ in most cases. More specifically, if the shoulder condition of the target model seems to be large, then $\hat{f}_{2, M L}(0)$ beats $\hat{f}_{3, M O}(0)$ (e.g. EP with $\beta=2,2.5$ and HR with $\beta=3)$. Otherwise, $\hat{f}_{3, M O}(0)$ performs better than $\hat{f}_{2, M L}(0)$. The $R M E$ values of $\hat{f}_{3, M O}(0)$ range from 0.058 (GP with $\beta=0.6$ and $n=200$ ) to 0.357 (EP with $\beta=2.5$ and $n=50)$. Comparing the range of $R M E$ for different estimators indicates that $\hat{f}_{3, M O}(0)$ is more stable than the other estimators.

Therefore, based on results in this study, it may be concluded that the weighted exponential model fits the line transect data reasonably and it can be recommended as a promising parametric model to estimate the parameter $f(0)$ and the population density $D$.

\section{References}

Bain, L. J., \& Engelhardt, M. (1992).Introduction to probability and mathematical statistics, $2^{\text {nd }} E d$. Boston, MA: Duxbury Press.
Barabesi, L. (2001). Local parametric density estimation methods in line transect sampling. Metron, LIX, 21-37.

Buckland, S. T. (1985). Perpendicular distance models for line transect sampling. Biometrics, 41, 177-195.

Buckland, S. T. (1992). Fitting density functions using polynomials. Applied Statistics, 41, 63-76.

Buckland, S. T., Anderson, D. R., Burnham, K. P., Laake, J. L., Borchers, D. L., \& Thomas, L. (2001). Introduction to distance sampling. Oxford, England: Oxford University Press.

Burnham, K. P., \& Anderson, D. R. (1976). Mathematical models for nonparametric inferences from line transect data. Biometrics, 32, 325-336.

Burnham, K. P., Anderson, D. R., \& Laake, J. L. (1980). Estimation of density from line transect sampling of biological populations. Wildlife Monograph, No. 72, 1-202.

Chen, S. X. (1996). A kernel estimate for the density of a biological population by using line transect sampling. Applied Statistics, 45, 135-150.

Eidous, O. M. (2004). A parametric family for density estimation in line transect sampling. Abhath Al-Yarmouk, 13, 315-326.

Gates, C. E., Marshall, W. H., \& Olson, D. P. (1968).Line transect method of estimating grouse population densities. Biometrics, 24, 135145.

Hayes, R. J., \& Buckland, S. T. (1983). Radial distance models for line-transect method. Biometrics, 39, 29-42.

Magnus, W., Oberhettinger, F., \&Soni, R. P. (1966).Formulas and theorems for special functions of mathematical physics. New York, NY: Springer-Verlag.

Pollock, K. H. (1978). A family of density estimators for line transect sampling. Biometrics, 34, 475-478.

Zhang, S. (2009). On parametric estimation of population abundance for line transect sampling. Environmental and Ecological Statistics, 18, 79-92. 
WEIGHTED EXPONENTIAL DETECTION MODEL FOR LINE TRANSECT DATA

Table 1: RB and RME for Different Estimators when Data are Simulated from the Four Target Models

\begin{tabular}{|c|c|c|c|c|c|c|c|c|c|c|}
\hline \multirow[b]{3}{*}{$n$} & \multirow[b]{3}{*}{$\beta$} & \multirow[b]{3}{*}{$w$} & \multicolumn{8}{|c|}{ Estimator } \\
\hline & & & \multicolumn{2}{|c|}{$\hat{f}_{1, M L}(0)$} & \multicolumn{2}{|c|}{$\hat{f}_{2, M L}(0)$} & \multicolumn{2}{|c|}{$\hat{f}_{3, M O}(0)$} & \multicolumn{2}{|c|}{$\hat{f}_{3, M L}(0)$} \\
\hline & & & $\mathrm{RB}$ & RME & $\mathrm{RB}$ & RME & $\mathrm{RB}$ & RME & $\mathrm{RB}$ & RME \\
\hline \multicolumn{11}{|c|}{ Exponential Power (EP) Model } \\
\hline 50 & \multirow{3}{*}{1} & \multirow{3}{*}{5} & 0.055 & 0.154 & -0.384 & 0.393 & -0.179 & 0.211 & -0.187 & 0.219 \\
\hline 100 & & & 0.040 & 0.105 & -0.393 & 0.397 & -0.191 & 0.206 & -0.187 & 0.204 \\
\hline 200 & & & 0.041 & 0.080 & -0.395 & 0.397 & -0.190 & 0.198 & -0.186 & 0.199 \\
\hline 50 & \multirow{3}{*}{1.5} & \multirow{3}{*}{3} & 0.400 & 0.435 & -0.133 & 0.166 & 0.089 & 0.159 & 0.089 & 0.155 \\
\hline 100 & & & 0.391 & 0.408 & -0.141 & 0.156 & 0.082 & 0.122 & 0.081 & 0.118 \\
\hline 200 & & & 0.390 & 0.399 & -0.142 & 0.149 & 0.081 & 0.103 & 0.078 & 0.102 \\
\hline 50 & \multirow{3}{*}{2} & \multirow{3}{*}{2.5} & 0.593 & 0.617 & 0.017 & 0.104 & 0.239 & 0.274 & 0.248 & 0.281 \\
\hline 100 & & & 0.590 & 0.602 & 0.015 & 0.073 & 0.236 & 0.254 & 0.243 & 0.261 \\
\hline 200 & & & 0.580 & 0.586 & 0.008 & 0.052 & 0.229 & 0.238 & 0.233 & 0.242 \\
\hline 50 & \multirow{3}{*}{2.5} & \multirow{3}{*}{2} & 0.711 & 0.732 & 0.118 & 0.155 & 0.331 & 0.357 & 0.345 & 0.373 \\
\hline 100 & & & 0.697 & 0.708 & 0.109 & 0.132 & 0.320 & 0.334 & 0.330 & 0.343 \\
\hline 200 & & & 0.695 & 0.700 & 0.105 & 0.117 & 0.318 & 0.325 & 0.326 & 0.331 \\
\hline \multicolumn{11}{|c|}{ Hazard Rate (HR) Model } \\
\hline 50 & \multirow{3}{*}{1.5} & \multirow{3}{*}{20} & -0.120 & 0.199 & -0.565 & 0.571 & -0.316 & 0.339 & -0.311 & 0.337 \\
\hline 100 & & & -0.126 & 0.169 & -0.570 & 0.573 & -0.320 & 0.332 & -0.310 & 0.335 \\
\hline 200 & & & -0.131 & 0.152 & -0.575 & 0.576 & -0.324 & 0.329 & -0.323 & 0.331 \\
\hline 50 & \multirow{3}{*}{2} & \multirow{3}{*}{12} & 0.149 & 0.256 & -0.409 & 0.427 & -0.107 & 0.194 & -0.128 & 0.215 \\
\hline 100 & & & 0.126 & 0.187 & -0.427 & 0.434 & -0.124 & 0.164 & -0.126 & 0.175 \\
\hline 200 & & & 0.112 & 0.152 & -0.437 & 0.441 & -0.135 & 0.157 & -0.127 & 0.149 \\
\hline 50 & \multirow{3}{*}{2.5} & \multirow{3}{*}{8} & 0.387 & 0.443 & -0.226 & 0.270 & 0.079 & 0.186 & 0.067 & 0.161 \\
\hline 100 & & & 0.394 & 0.421 & -0.231 & 0.252 & 0.084 & 0.143 & 0.083 & 0.139 \\
\hline 200 & & & 0.374 & 0.386 & -0.250 & 0.258 & 0.068 & 0.102 & 0.069 & 0.109 \\
\hline 50 & \multirow{3}{*}{3} & \multirow{3}{*}{6} & 0.559 & 0.599 & -0.074 & 0.169 & 0.213 & 0.270 & 0.236 & 0.294 \\
\hline 100 & & & 0.559 & 0.579 & -0.084 & 0.135 & 0.213 & 0.242 & 0.226 & 0.255 \\
\hline 200 & & & 0.543 & 0.552 & -0.099 & 0.122 & 0.200 & 0.215 & 0.213 & 0.227 \\
\hline
\end{tabular}


ABABNEH \& EIDOUS

Table 1 (continued): RB and RME for Different Estimators when Data are Simulated from the Four Target Models

\begin{tabular}{|c|c|c|c|c|c|c|c|c|c|c|}
\hline \multirow[b]{3}{*}{$n$} & \multirow[b]{3}{*}{$\beta$} & \multirow[b]{3}{*}{$w$} & \multicolumn{8}{|c|}{ Estimator } \\
\hline & & & \multicolumn{2}{|c|}{$\hat{f}_{1, M L}(0)$} & \multicolumn{2}{|c|}{$\hat{f}_{2, M L}(0)$} & \multicolumn{2}{|c|}{$\hat{f}_{3, M O}(0)$} & \multicolumn{2}{|c|}{$\hat{f}_{3, M L}(0)$} \\
\hline & & & $\mathrm{RB}$ & RME & $\mathrm{RB}$ & RME & $\mathrm{RB}$ & RME & $\mathrm{RB}$ & RME \\
\hline \multicolumn{11}{|c|}{ Beta (BE) Model } \\
\hline 50 & \multirow{3}{*}{1.5} & \multirow{3}{*}{1} & 0.410 & 0.436 & -0.096 & 0.126 & 0.096 & 0.151 & 0.092 & 0.152 \\
\hline 100 & & & 0.406 & 0.419 & -0.099 & 0.115 & 0.093 & 0.124 & 0.103 & 0.134 \\
\hline 200 & & & 0.403 & 0.410 & -0.101 & 0.109 & 0.092 & 0.107 & 0.096 & 0.109 \\
\hline 50 & \multirow{3}{*}{2} & \multirow{3}{*}{1} & 0.350 & 0.382 & -0.148 & 0.171 & 0.050 & 0.130 & 0.067 & 0.134 \\
\hline 100 & & & 0.336 & 0.352 & -0.157 & 0.168 & 0.039 & 0.091 & 0.046 & 0.093 \\
\hline 200 & & & 0.332 & 0.340 & -0.158 & 0.163 & 0.036 & 0.066 & 0.041 & 0.072 \\
\hline 50 & \multirow{3}{*}{2.5} & \multirow{3}{*}{1} & 0.297 & 0.332 & -0.189 & 0.207 & 0.009 & 0.117 & 0.021 & 0.121 \\
\hline 100 & & & 0.298 & 0.315 & -0.189 & 0.198 & 0.010 & 0.080 & 0.010 & 0.075 \\
\hline 200 & & & 0.289 & 0.298 & -0.196 & 0.200 & 0.003 & 0.057 & -0.000 & 0.054 \\
\hline 50 & \multirow{3}{*}{3} & \multirow{3}{*}{1} & 0.263 & 0.301 & -0.216 & 0.231 & -0.018 & 0.115 & -0.011 & 0.116 \\
\hline 100 & & & 0.257 & 0.276 & -0.221 & 0.229 & -0.023 & 0.082 & -0.023 & 0.080 \\
\hline 200 & & & 0.256 & 0.267 & -0.223 & 0.227 & -0.023 & 0.061 & -0.025 & 0.060 \\
\hline \multicolumn{11}{|c|}{ General Polynomial (GP) Model } \\
\hline 50 & \multirow{3}{*}{0.6} & \multirow{3}{*}{3} & 0.254 & 0.290 & -0.220 & 0.243 & 0.030 & 0.117 & 0.033 & 0.123 \\
\hline 100 & & & 0.245 & 0.262 & -0.233 & 0.238 & 0.022 & 0.083 & 0.021 & 0.073 \\
\hline 200 & & & 0.246 & 0.259 & -0.217 & 0.229 & 0.018 & 0.058 & 0.014 & 0.061 \\
\hline 50 & \multirow{3}{*}{0.9} & \multirow{3}{*}{3} & 0.189 & 0.243 & -0.296 & 0.308 & -0.075 & 0.141 & -0.077 & 0.154 \\
\hline 100 & & & 0.181 & 0.211 & -0.302 & 0.308 & -0.081 & 0.117 & -0.095 & 0.128 \\
\hline 200 & & & 0.170 & 0.187 & -0.309 & 0.311 & -0.090 & 0.108 & -0.092 & 0.110 \\
\hline 50 & \multirow{3}{*}{1.9} & \multirow{3}{*}{3} & 0.280 & 0.332 & -0.262 & 0.289 & -0.004 & 0.138 & 0.001 & 0.141 \\
\hline 100 & & & 0.275 & 0.303 & -0.276 & 0.289 & -0.009 & 0.099 & -0.011 & 0.101 \\
\hline 200 & & & 0.267 & 0.283 & -0.284 & 0.291 & -0.015 & 0.074 & -0.019 & 0.075 \\
\hline 50 & \multirow{3}{*}{3.5} & \multirow{3}{*}{3} & 0.452 & 0.489 & -0.118 & 0.174 & 0.129 & 0.194 & 0.121 & 0.191 \\
\hline 100 & & & 0.435 & 0.454 & -0.135 & 0.163 & 0.116 & 0.154 & 0.119 & 0.155 \\
\hline 200 & & & 0.426 & 0.436 & -0.140 & 0.154 & 0.109 & 0.130 & 0.120 & 0.142 \\
\hline
\end{tabular}




\title{
Improved Estimator in the Presence of Multicollinearity
}

\author{
Ghadban Khalaf \\ King Khalid University, \\ Saudi Arabia
}

The performances of two biased estimators for the general linear regression model under conditions of collinearity are examined and a new proposed ridge parameter is introduced. Using Mean Square Error (MSE) and Monte Carlo simulation, the resulting estimator's performance is evaluated and compared with the Ordinary Least Square (OLS) estimator and the Hoerl and Kennard (1970a) estimator. Results of the simulation study indicate that, with respect to MSE criteria, in all cases investigated the proposed estimator outperforms both the OLS and the Hoerl and Kennard estimators.

Key words: Regression, multicollinearity, ridge regression, Monte Carlo simulation.

Introduction

Multiple regression fits a model to predict a dependent variable $(Y)$ from two or more independent variables $(X)$ :

$$
Y=\beta_{0}+\beta_{1} X_{1}+\beta_{2} X_{2}+\cdots+e
$$

If the model fits the data well, the overall $R^{2}$ value will be high and the corresponding $P$ value will be low. In addition to the overall $P$ value, multiple regression also reports an individual $P$ value for each independent variable; a low $P$ value indicates that a particular independent variable significantly improves the fit of the model.

If the overall $P$ value is very low, but all the individual $P$ values are high, this indicates that a model fits the data well, even though none of the $X$ variables has a statistically significant impact on predicting $Y$. This occurs when two or more variables are highly correlated. If both variables are removed from the model, the fit would be much worse; thus, the overall model fits the data but neither $X$ variable makes a significant contribution when it is added to the

Ghadban Khalaf is an Associate Professor on the Faculty of Science in the Department of Mathematics. Email him at: albadran50@yahoo.com. model last. When this occurs, the $X$ variables are collinear and the results show multicollinearity, meaning the variables are related.

If the goal is simply to predict $Y$ from a set of $X$ variables, then multicollinearity is not problematic. The predictions will be accurate and the overall $R^{2}$ quantifies how well the model predicts the $Y$ values. However, if the goal is to understand how the various $X$ variables impact $Y$, then multicollinearity poses a big problem. These problems are summarized as:

(1) The individual $P$ values can be misleading, that is, a $P$ value can be high, even though the variable is important.

(2) The confidence intervals on the regression coefficients will be very wide - and may include zero - which indicates that a researcher cannot be confident whether an increase in $X$ values is associated with an increase or decrease in $Y$ values. In addition, wide confidence intervals can change the coefficients and/or their signs.

For these reasons, multicollinearity must be examined and removed. Different methods exist that can be used to reduce or to eliminate the impact of multicollinearity, examples include: 


\section{GHADBAN KHALAF}

(1) Removing a variable: If one of the variables does not seem logically essential to the model, then removing it may be helpful.

(2) Combining the variables; for example, if height and weight are collinear independent variables, then it would be logical to remove height and weight from the model and instead use a variable such as surface area (calculated from height and weight).

(3) Increasing sample size: Another way to reduce the impact of collinearity is to increase sample size, this results in narrower confidence intervals, despite multicollinearity, with more data.

(4) Using a standard technique called ridge regression: Ridge regression was originally developed to overcome multicollinearity.

model:

Consider the standard linear regression

$$
\vec{Y}=X \vec{\beta}+\vec{e},
$$

formulated to result in a $X^{\prime} X$ in correlation form and where $X^{\prime} Y$ is the vector of correlation coefficients of the dependent variable with each explanatory variable. Also assume that $X$ is $n \times p$ of full rank $p<n, E(e)=0$ and $E\left(e e^{\prime}\right)=\sigma^{2} I_{n}$. The $p$ - vector of the OLS estimator $(\overrightarrow{\hat{\beta}})$, is then given by the solution of:

$$
X^{\prime} X \overrightarrow{\hat{\beta}}=X^{\prime} \vec{Y}
$$

so that,

$$
\overrightarrow{\hat{\beta}}=\left(X^{\prime} X\right)^{-1} X^{\prime} \vec{Y}
$$

Clearly, $\overrightarrow{\hat{\beta}}$ is an unbiased estimator of $\vec{\beta}$. There are many reasons why a data analyst is often not satisfied with OLS estimates. One of the reasons is prediction accuracy: OLS estimates often have low bias but large variance. Thus, prediction accuracy can occasionally be improved by shrinking some coefficients to zero.
In doing this, a little bias is sacrificed to reduce the variance of the predicted values and hence may improve overall prediction accuracy.

Many attempts have been made to improve the OLS estimator procedure. Hoerl and Kennard (1970a) suggested a new technique called ridge regression to improve OLS estimates. The ridge regression estimators $\overrightarrow{\hat{\beta}}^{*}$, for a fixed $k>0$, satisfy,

$$
\left(X^{\prime} X+k I_{p}\right) \overrightarrow{\hat{\beta}}^{*}=X^{\prime} \vec{Y},
$$

so that,

$$
\overrightarrow{\hat{\beta}}^{*}=\left(X^{\prime} X+k I_{p}\right)^{-1} X^{\prime} \vec{Y}
$$

as an alternative to the OLS estimator for use in the presence of multicollinearity, where $I$ denotes an identity matrix, and $k$ is a positive number known as ridge parameter, which must be estimated from the real data. The ridge regression MSE is given by:

$$
\begin{aligned}
\operatorname{MSE}\left(\overrightarrow{\hat{\beta}}^{*}\right) & =\sigma^{2} \sum_{i=1}^{p} \frac{\lambda_{i}}{\left(\lambda_{i}+k_{i}\right)^{2}}+\sum_{i=1}^{p} \frac{k_{i}^{2} \beta_{i}^{2}}{\left(\lambda_{i}+k_{i}\right)^{2}}, \\
& =\text { Variance }+(\text { Bias })^{2},
\end{aligned}
$$

where $\sigma^{2}$ represents the error variance of the model given by (1). When the reduction in variance exceeds the square of the bias, ridge estimates are preferred.

When using ridge estimates, the choice of $k$-values in (5) is crucially important and several methods have been proposed for this purpose (see Hoerl \& Kennard, 1970a; Saleh \& Kibria, 1993; Singh \& Tracy, 1999; Khalaf \& Shuker, 2005; Alkhamisi \& Shukur, 2008; Khalaf, 2011; Khalaf, 2011).

The Proposed Estimator

The general form of ridge regression suggested by Hoerl and Kennard (1970a) reduces $X^{\prime} X$ to a diagonal matrix by applying an orthogonal transformation $Q$, thus,

$$
Q\left(X^{\prime} X\right) Q^{\prime}=\Lambda
$$




\section{IMPROVED ESTIMATOR IN THE PRESENCE OF MULTICOLLINEARITY}

where $Q$ is a $p \times p$ orthogonal matrix, $\Lambda$ is a diagonal matrix whose diagonal elements $\lambda_{1}, \lambda_{2}, \cdots, \lambda_{p}$ are the eigenvalues of $X^{\prime} X$. If $X^{*}=X Q^{\prime}$ and $\alpha=Q \beta$, then model (1) may be rewritten as,

where

$$
\vec{Y}=X^{*} \alpha+\vec{e},
$$

$$
\left(X^{*}\right)^{\prime}\left(X^{*}\right)=\Lambda
$$

The general ridge estimation procedure is defined as,

$$
\hat{\alpha}^{*}=\left(\left(X^{*}\right)^{\prime}\left(X^{*}\right)+K\right)^{-1}\left(X^{*}\right)^{\prime} \vec{Y},
$$

where $K$ is a diagonal matrix with non-negative diagonal elements $k_{1}, k_{2}, \ldots, k_{p}, k_{i}>0$. It follows from Hoerl and Kennard (1970a) that the value of $k_{i}$ which minimizes the MSE of $\hat{\alpha}^{*}$ given by:

$$
\operatorname{MSE}\left(\overrightarrow{\hat{\alpha}}^{*}\right)=\sigma^{2} \sum_{i=}^{p} \frac{\lambda_{i}}{\left(\lambda_{i}+k_{i}\right)^{2}}+\sum_{i=1}^{p} \frac{k_{i}^{2} \alpha_{i}^{2}}{\left(\lambda_{i}+k_{i}\right)^{2}},
$$

is:

$$
k_{i}=\frac{\sigma^{2}}{\alpha_{i}^{2}},
$$

where $\sigma^{2}$ represents the error variance of model (1) and $\alpha_{i}$ is the $i^{\text {th }}$ element of $\vec{\alpha}$. Equation (8) gives a value of $k_{i}$ that is fully dependent on the unknown $\sigma^{2}$ and $\alpha_{i}$, and therefore must be estimated from observed data. Hoerl and Kennard (1970a) recommended replacing $\sigma^{2}$ and $\alpha_{i}$ by their corresponding unbiased estimators, that is:

$$
\hat{k}_{i}=\frac{\hat{\sigma}^{2}}{\hat{\alpha}_{i}^{2}},
$$

where $\hat{\sigma}^{2}=\frac{\sum_{i} e_{i}^{2}}{n-p}$ is the residual MSE, which is an unbiased estimator of $\sigma^{2}$, and $\hat{\alpha}_{i}$ is the element of $\overrightarrow{\hat{\alpha}}$ which is an unbiased estimator of $\vec{\alpha}$. Hoerl and Kennard found that the best method for achieving a better estimator $\overrightarrow{\hat{\alpha}}^{*}$ is to use $k_{i}=k$ for all $i$, and they suggested $k$ to be $\hat{k}_{H K}$ where:

$$
\hat{k}_{H K}=\frac{\hat{\sigma}^{2}}{\max \left(\hat{\alpha}_{i}^{2}\right)} .
$$

They showed that the estimator $\hat{k}_{H K}(\mathrm{HK})$ is sufficient to give ridge estimators with smaller MSEs than an OLS estimator. This article proposes a modification of the Hoerl and Kennared (1970a) estimator shown in (10) to obtain a new estimator, given by:

$$
\begin{aligned}
\hat{k}_{G K} & =\frac{\hat{\sigma}^{2}}{\max \left(\hat{\alpha}_{i}^{2}\right)}+\frac{1}{\frac{1}{2}\left(\lambda_{\max }+\lambda_{\min }\right)}, \\
& =\frac{\hat{\sigma}^{2}}{\max \left(\hat{\alpha}_{i}^{2}\right)}+\frac{2}{\left(\lambda_{\max }+\lambda_{\text {min }}\right)},
\end{aligned}
$$

where $\lambda_{\max }, \lambda_{\min }$, are the largest and smallest eigenvalues of the matrix $X^{\prime} X$, respectively. This estimator will be denoted by GK. Because $\frac{2}{\lambda_{\max }+\lambda_{\min }}>0$, then GK is greater than $\mathrm{HK}$.

Monte Carlo Simulation

Monte Carlo simulation was used to investigate the properties of the considered estimators. It is convenient to make the comparison among the OLS estimator, HK estimator given by (10) and the new proposed GK estimator given by (11). These choices were made for many reasons: First is that; interest herein lies in studying the properties of the proposed GK estimator as an alternative to the OLS estimator in the presence of multicollinearity. Second, GK is a modified version of $\mathrm{HK}$, so it is necessary to make a 


\section{GHADBAN KHALAF}

comparison between them. Finally, the HK estimator was the first ridge estimator proposed among all other estimators, therefore, most studies comparing ridge estimators consider the HK estimator.

A comparison was made based on MSE criterion. Following McDonald and Galarneau (1975), Wichern and Churchill (1978), Gibbons (1981) and Kibria (2003), the explanatory variables were generated using the device:

$$
\begin{gathered}
x_{i j}=\left(1-\rho^{2}\right)^{\frac{1}{2}} z_{i j}+\rho z_{i p}, \\
i=1,2, \ldots, n, \\
j=1,2, \ldots, p .
\end{gathered}
$$

where $z_{i j}$ are independent standard normal pseudo-random numbers, $\rho$ is specified so that the correlation between any two explanatory variables is given by $\rho^{2}$ and $p$ is the number of explanatory variables. Once more, the variables are standardized so that $X^{\prime} X$ and $X^{\prime} Y$ are in correlation forms. Four sets of correlations were considered corresponding to $\rho=0.7,0.8,0.9$ and 0.99 . Using the condition number, $C N=\frac{\lambda_{\text {max }}}{\lambda_{\text {min }}}$, it can be shown that these values of $\rho$ will include a wide range of low, moderate and high correlations between variables. The $n$ observations for the dependent variable $Y$ are determined by:

$$
\begin{gathered}
Y_{i}=\beta_{0}+\beta_{1} X_{i 1}+\beta_{2} X_{i 2}+\ldots+\beta_{p} X_{i p}+e_{i}, \\
i=1,2, \ldots, n
\end{gathered}
$$

where $e_{i}$ are independent normal $\left(0, \sigma^{2}\right)$ pseudo-numbers and $\beta_{0}$ is considered identically zero without loss of generality. Three different sample sizes, $n=20,30,50$ were used with 10,15 and 20 explanatory variables, respectively. These choices of $p$ were chosen to study the behavior of the estimators for small, moderate and large number of explanatory variables.

The parameter values were chosen so that $\sum_{j=1}^{p} \beta_{j}^{2}=1$, which is a common restriction in simulation studies (see Muniz \& Kibria, 2009). For given values of $p, n$ and $\rho$, the experiment was repeated 5,000 times by generating 5,000 samples. For each replicate $r(r=1,2, \ldots$, $5,000)$ the values of $k$ different proposed estimators and the corresponding ridge estimators were calculated using:

$$
\hat{\alpha}^{*}=(\Lambda+\hat{k} I)^{-1} X^{*} \vec{Y},
$$

where $\hat{k}=H K, G K$. The MSE for the estimators were calculated as follows:

$$
\operatorname{MSE}\left(\overrightarrow{\hat{\alpha}}^{*}\right)=\frac{1}{5000} \sum_{r=1}^{5000}\left(\vec{\alpha}_{r}^{*}-\vec{\alpha}\right)^{\prime}\left(\vec{\alpha}_{r}^{*}-\vec{\alpha}\right) .
$$

\section{Results}

The results of the simulations that compared the MSE to the other estimators are summarized in Tables 1-3. To compare the performances of the considered estimators, the MSE was calculated for each. The estimator that resulted in the minimum MSE was considered to be the best. The statistics package Minitab 14 was used for all calculations.

Tables 1-3 show that both HK and GK are better than the OLS estimator, and the GK estimator performs better than the HK estimator. This also reveals that for low correlation, $r=$ 0.7 , the performance of the GK estimator is slightly better than the HK estimator. Moreover, it was observed that, for given $n$ and $p$, the MSE for all estimators increased as the correlation among the explanatory variables increased. Conversely, as the sample size and the number of explanatory variables increase, the MSE of all estimators decreased. 
IMPROVED ESTIMATOR IN THE PRESENCE OF MULTICOLLINEARITY

Table 1: Estimated MSE and the values of CN with $p=10$ and $n=20$.

\begin{tabular}{|c|c|c|c|c|c|}
\cline { 2 - 6 } & $\rho$ & 0.99 & 0.9 & 0.8 & 0.7 \\
\cline { 2 - 6 } & $\mathrm{CN}$ & 980.58 & 90.17 & 44.24 & 18.93 \\
\hline \multirow{3}{*}{ Estimators } & OLS & 0.03877 & 0.02844 & 0.02147 & 0.01679 \\
\cline { 2 - 6 } & $\mathrm{HK}$ & 0.03453 & 0.02613 & 0.02015 & 0.01611 \\
\cline { 2 - 6 } & $\mathrm{GK}$ & 0.02820 & 0.02241 & 0.01826 & 0.01393 \\
\hline
\end{tabular}

Table 2: Estimated MSE and the values of CN with $p=15$ and $n=30$.

\begin{tabular}{|c|c|c|c|c|c|}
\cline { 2 - 6 } & $\rho$ & 0.99 & 0.9 & 0.8 & 0.7 \\
\cline { 2 - 6 } & $\mathrm{CN}$ & 2834.43 & 255.92 & 89.68 & 64.19 \\
\hline \multirow{3}{*}{ Estimators } & OLS & 0.01886 & 0.01299 & 0.00933 & 0.00738 \\
\cline { 2 - 6 } & $\mathrm{HK}$ & 0.01822 & 0.01275 & 0.00922 & 0.00733 \\
\cline { 2 - 6 } & $\mathrm{GK}$ & 0.01641 & 0.01112 & 0.00875 & 0.00701 \\
\hline
\end{tabular}

Table 3: Estimated MSE and the values of CN with $p=20$ and $n=50$.

\begin{tabular}{|c|c|c|c|c|c|}
\cline { 2 - 6 } & $\rho$ & 0.99 & 0.9 & 0.8 & 0.7 \\
\cline { 2 - 6 } & $\mathrm{CN}$ & 25266.30 & 2235.51 & 945.37 & 399.63 \\
\hline \multirow{3}{*}{ Estimators } & OLS & 0.01454 & 0.00922 & 0.00665 & 0.00505 \\
\cline { 2 - 6 } & $\mathrm{HK}$ & 0.01416 & 0.00910 & 0.00660 & 0.00503 \\
\cline { 2 - 6 } & $\mathrm{GK}$ & 0.00694 & 0.00436 & 0.00320 & 0.00488 \\
\hline
\end{tabular}

\section{Conclusion}

Several procedures for constructing ridge estimators have been proposed in the literature. These procedures aim at a rule for selecting the constant $k$ in equation (13). The best method for estimating $k$ remains an unsolved problem and no constant value of $k$ is certain to yield an estimator that is uniformly better in terms of MSE than the OLS estimators in all cases.

This study investigated the properties of a newly proposed method for estimating the ridge parameter $(k)$ in the presence of multicollinearity. The investigation used Monte Carlo experiments, where levels of correlation, numbers of explanatory variables and sample sizes were varied. Each combination was replicated 5,000 times. The evaluation of the new estimator was accomplished by comparing the MSE of this estimator with the OLS estimator and the Hoerl and Kennard (1970a) estimator. Results show that the proposed estimator uniformly dominates the other estimators. 


\section{GHADBAN KHALAF}

\section{References}

Alkhamisi, M., \& Shukur, G. (2008). Developing ridge parameters for SUR model. Communications in Statistics - Theory and Methods, 37, 544-564.

Gibbons, D. G. (1981). A simulation study of some ridge estimators. Journal of the American Statistical Association, 76(373), 131139.

Hoerl, A. E., \& Kennard, R.W. (1970a). Ridge regression: biased estimation for non orthogonal problems. Technometrics, 12, 55-67.

Khalaf, G. (2011). Suggested ridge regression estimators under multicollinearity. To appear in Journal of Natural and Applied Sciences, University of Aden, 15(2).

Khalaf, G. (2011). Suggested values of the ridge parameters. International Journal of Statistics and Analysis, 1(2), 109-118.

Khalaf, G., \& Shukur, G. (2005). Choosing ridge parameters for regression problems. Communications in Statistics Theory and Methods, 34, 1177-1182.
Kibria, B. M. G. (2003). Performance of some new ridge regression estimators. Communications in Statistics - Theory and Methods, 32, 419-435.

McDonald, G. C., \& Galarneau, D. I. (1975). A Monte Carlo evaluation of some ridge-type estimators. Journal of the American Statistical Association, 70, 407-416.

Muniz, G., \& Kibria, B. M. G. (2009). On some ridge regression estimators: An empirical comparison. Communications in Statistics -Simulation and Computation, 38, 621-630.

Saleh, A. K., \& Kibria, B. M. (1993). Performances of some new preliminary test ridge regression estimators and their properties. Communications in Statistics - Theory and Methods, 22, 2747-2764.

Singh, S., \& Tracy, D. S. (1999). Ridgeregression using scrambled responses. Metrika, 147-157.

Wichern, D., \& Churchill, G. (1978). A comparison of ridge estimators. Technometrics, 20, 301-311. 


\title{
New Approximate Bayesian Confidence Intervals for the Coefficient of Variation of a Gaussian Distribution
}

\author{
Vincent A. R. Camara \\ Research Center for Bayesian Applications, Inc., \\ Largo, FL
}

Confidence intervals are constructed for the coefficient of variation of a Gaussian distribution. Considering the square error and the Higgins-Tsokos loss functions, approximate Bayesian models are derived and compared to a published classical model. The models are shown to have great coverage accuracy. The classical model does not always yield the best confidence intervals; the proposed models often perform better.

Key words: Estimation, loss functions, confidence intervals, statistical analysis.

\section{Introduction}

A significant amount of research in Bayesian analysis and modeling has been published during the last thirty-five years. Bayesian analysis implies the exploitation of suitable prior information and the choice of a loss function in association with Bayes' Theorem. It is based on the notion that a parameter within a model is not merely an unknown quantity but rather behaves as a random variable that follows some distribution. In the area of life testing, it is realistic to assume that a life parameter is stochastically dynamic. This assertion is supported by the fact that the complexity of electronic and structural systems is likely to cause undetected component interactions resulting in an unpredictable fluctuation of the life parameter.

Drake (1966) gave an excellent account of the use of Bayesian statistics in reliability

Vincent A. R. Camara earned a Ph.D. in Mathematics/Statistics. His research interests include the theory and applications of Bayesian and empirical Bayes analyses with emphasis on the computational aspect of modeling. $\mathrm{He}$ is featured in Marquis Who's Who in America and in Marquis Who's Who in the World. E-mail him at: gvcamara@ij.net. problems. As he pointed out, "He (a Bayesian) realizes...that his selection of a prior (distribution) to express his present state of knowledge will necessarily be somewhat arbitrary. But he greatly appreciates this opportunity to make his entire assumptive structure clear to the world..." (Drake, 1966, p. 315-320).

This study considers a widely used and useful underlying model; that is, the normal underlying model characterized by

$$
\begin{gathered}
f(x)=\frac{1}{\sqrt{2 \pi} \sigma} e^{-\frac{1}{2}\left(\frac{x-\mu}{\sigma}\right)^{2}} ; \\
-\infty \prec x \prec \infty,-\infty \prec \mu \prec \infty, \sigma \succ 0 .
\end{gathered}
$$

For a given level of significance, the published classical model obtained by Miller (1991) considers the following confidence bounds for the coefficient of variation of a normal distribution.

$L_{M}=\frac{s}{X}-Z_{\alpha / 2}\left[m^{-1}\left(\frac{s}{\bar{X}}\right)^{2}\left(0.5+\left(\frac{s}{\bar{X}}\right)^{2}\right)\right]^{\frac{1}{2}}$ 
and

$$
U_{M}=\frac{s}{\bar{X}}+Z_{\alpha / 2}\left[m^{-1}\left(\frac{s}{\bar{X}}\right)^{2}\left(0.5+\left(\frac{s}{\bar{X}}\right)^{2}\right)\right]^{\frac{1}{2}}
$$

with $m=n-1$, where $n$ is the sample size. This classical approach uses the Z-table to construct confidence intervals for a normal population coefficient of variation. This article relies only on observations under study to construct new approximate Bayesian confidence intervals for the coefficient of variation of a normal population.

Methodology

For model (1), the following results will be used to derive the approximate Bayesian confidence intervals for the coefficient of variation of a normal distribution: approximate Bayesian confidence bounds for a normal population variance (Camara 2003) and approximate Bayesian confidence bounds for a normal population mean (Camara, 2009). Although no specific analytical procedure is available that allows the identification of the appropriate loss function to be used, the most common is the square error loss function. One reason for selecting this loss function is due to its analytical tractability in Bayesian analysis. As this study shows, selecting the square error loss does not always lead to the best approximate Bayesian confidence intervals. However, the obtained approximate Bayesian confidence intervals corresponding to the square error and the Higgins-Tsokos loss functions will be respectively compared to the classical model (2).

\section{Square Loss Error Function}

The popular square error loss function places a small weight on estimates near the true value and proportionately more weight on extreme deviations from the true value of the parameter. Its popularity is due to its analytical tractability in Bayesian modeling. The square error loss is defined as:

$$
L_{S E}(\stackrel{\Lambda}{\theta}, \theta)=(\stackrel{\wedge}{\theta}-\theta)^{2}
$$

Higgins-Tsokos Loss Function

The Higgins-Tsokos loss function places a heavy penalty on extreme over- or underestimation. That is, it places an exponential weight on extreme errors. The Higgins-Tsokos loss function is defined as:

$$
\begin{gathered}
L_{H T}(\hat{\theta}, \theta)=\frac{f_{1} e^{f_{2}(\hat{\theta}-\theta)}+f_{2} e^{-f_{1}(\hat{\theta}-\theta)}}{f_{1}+f_{2}}-1, \\
f_{1}, f_{2} \succ 0
\end{gathered}
$$

The Pareto prior was selected due to its mathematical tractability. The Pareto prior defined as follows:

$$
\begin{aligned}
& f_{1}(\theta)=\frac{a}{b}\left(\frac{b}{\theta}\right)^{a+1} ; \\
& \theta \geq b \succ 0, \quad a \succ 0 .
\end{aligned}
$$

where $\theta=1 / \sigma^{2}$.

The use of good approximations of the Pareto prior (5) along with the square error loss and the Higgins-Tsokos loss leads to the approximate Bayesian confidence bounds for a normal population variance and positive mean (Camara, 2003). For the square error loss function

$$
L_{o^{2}(S E)}=\frac{\sum_{i=1}^{n}\left(x_{i}-\bar{x}\right)^{2}}{n-2-2 \ln (\alpha / 2)}
$$

and

$$
U_{\sigma^{2}(S E)}=\frac{\sum_{i=1}^{n}\left(x_{i}-\bar{x}\right)^{2}}{n-2-2 \ln (1-\alpha / 2)}
$$


For the Higgins-Tsokos loss function

$$
L_{o^{2}((H T)}=
$$

$\frac{1}{\frac{n-1-2 \operatorname{Ln}(\alpha / 2)}{\sum_{i=1}^{n}\left(x_{i}-\bar{x}\right)^{2}}-\frac{1}{f_{1}+f_{2}} \operatorname{Ln}\left(\frac{\sum_{i=1}^{n}\left(x_{i}-\bar{x}\right)^{2}+f_{2}}{\sum_{i=1}^{n}\left(x_{i}-\bar{x}\right)^{2}-f_{1}}\right)}$

and

$$
\begin{aligned}
& U_{o^{2}(H T)}= \\
& \frac{1}{\frac{n-1-2 \operatorname{Ln}(1-\alpha / 2)}{\sum_{i=1}^{n}\left(x_{i}-\bar{x}\right)^{2}}-\frac{1}{f_{1}+f_{2}} \operatorname{Ln}\left(\frac{\sum_{i=1}^{n}\left(x_{i}-\bar{x}\right)^{2}+f_{2}}{\sum_{i=1}^{n}\left(x_{i}-\bar{x}\right)^{2}-f_{1}}\right)}
\end{aligned}
$$

The approximate Bayesian confidence interval for a positive normal population mean corresponding to the square error loss (Camara, 2009) is:

$$
L_{\mu(S E)}=\left(s+\bar{x}^{2}-\frac{\sum_{i=1}^{n}\left(x_{i}-\bar{x}\right)^{2}}{n-2-2 \ln (1-\alpha / 2)}\right)^{0.5}
$$

and

$$
U_{\mu(S E)}=\left(s^{2}+\bar{x}^{2}-\frac{\sum_{i=1}^{n}\left(x_{i}-\bar{x}\right)^{2}}{n-2-2 \ln (\alpha / 2)}\right)^{0.5}
$$

The approximate Bayesian confidence bounds for a positive normal population mean corresponding to the Higgins-Tsokos loss function (Camara, 2009) is:

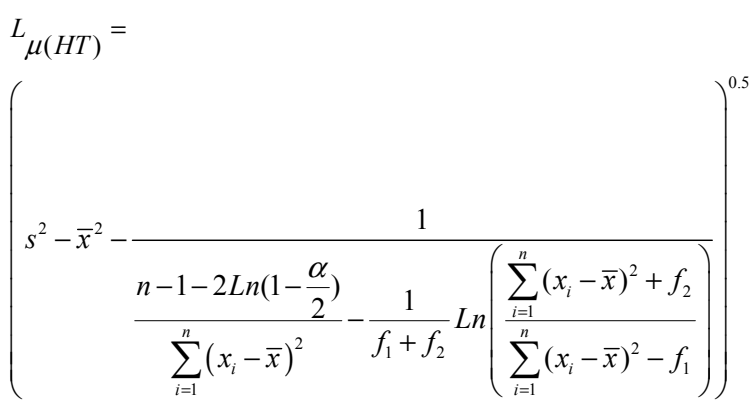

and

$U_{\mu(H T)}=$

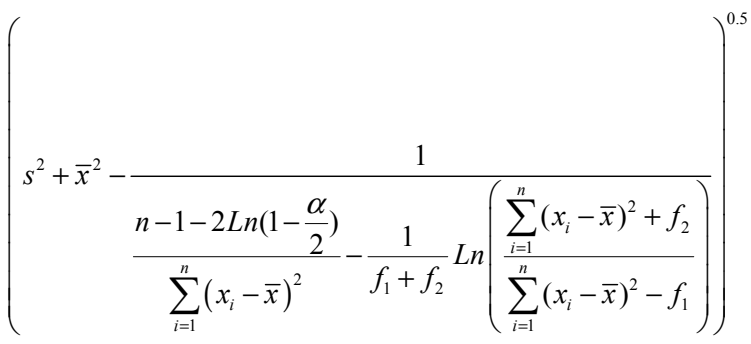

Using Equations (6), (7), (8) and (9), the approximate Bayesian confidence intervals are obtained for the coefficient of variation of a normal population corresponding to the square error loss function:

$$
\begin{aligned}
& L_{C v}(S E)= \\
& \left(\frac{n-1}{\left(1+\left(\frac{\bar{x}}{s}\right)^{2}\right)(n-2-2 \operatorname{Ln}(\alpha / 2))-n+1}\right)^{0.5}
\end{aligned}
$$

and

$$
U_{C v}(S E)=
$$

$$
\left(\frac{n-1}{\left(1+\left(\frac{\bar{x}}{s}\right)^{2}\right)(n-2-2 \operatorname{Ln}(1-\alpha / 2))-n+1}\right)^{0.5}
$$


The approximate Bayesian confidence interval for the coefficient of variation of a normal population corresponding to the HigginsStokes Loss functions are:

$$
\begin{aligned}
& L_{\text {Cir }}(H T)= \\
& \left.\left(s^{2}+\bar{x}^{2}\right)\left(\frac{n-1-2 \operatorname{Ln}\left(\frac{\alpha}{2}\right)}{\sum_{i=1}^{n}(x-x)^{-2}}-\frac{1}{f_{i}-f_{2}} \operatorname{Ln}\left(\frac{\sum_{i=1}^{n}\left(x_{i}-x\right)^{-2}+f_{2}}{\sum_{i=1}^{n}\left(x_{i}-x\right)^{-2}+f_{1}}\right)\right)-1\right)^{0.5}
\end{aligned}
$$

and

$$
\begin{aligned}
& U_{C r r}(H T)= \\
& \left(\left(s^{2}+\bar{x}^{2}\right)\left(\frac{n-1-2 \operatorname{Ln}\left(1-\frac{\alpha}{2}\right)}{\sum_{i=1}^{n}(x-x)^{-2}}-\frac{1}{f_{i}-f_{2}} L n\left(\frac{\sum_{i=1}^{n}\left(x_{i}-x\right)^{-2}+f_{2}}{\sum_{i=1}^{n}\left(x_{i}-x\right)^{-2}+f_{i}}\right)\right)-1\right)^{0.5}
\end{aligned}
$$

\section{Results}

To assess the proposed models, numerical results were obtained using SAS along with samples from normally distributed populations (Examples 1, 2, 3, .4, 7) as well as approximately normal populations (Examples 5, 6). Results were then compared to those obtained with the classical approach (2); for the Higgins-Tsokos loss function, $\mathrm{f} 1=1$ and $\mathrm{f} 2=1$ were considered. WC corresponds to the widths of the classical confidence intervals, and WSE and WHT denote the widths of the approximate Bayesian confidence intervals corresponding to the square error and the Higgins-Tsokos loss functions.

Example 1

Data: 24, 28, 22, 25, 24, 22, 29, 26, 25, 28, 19, 29 (Mann, 1998, p. 504).

Normal population distribution obtained with SAS:

Table 1: Classical (2) and Approximate Bayesian Confidence Intervals for the Population Coefficient of Variation Corresponding To Data Set 1

\begin{tabular}{|c|c|c|c|}
\hline C.L.\% & $\begin{array}{c}\text { Approximate } \\
\text { Bayesian Bounds } \\
\text { (SE) }\end{array}$ & $\begin{array}{c}\text { Approximate } \\
\text { Bayesian Bounds } \\
(\mathrm{HT})\end{array}$ & Classical Bounds I \\
\hline 80 & $0.1077-0.1291$ & $0.1132-0.1276$ & $0.0899-0.1587$ \\
\hline 90 & $0.1028-0.1298$ & $0.1077-0.1291$ & $0.0799-0.1687$ \\
\hline 95 & $0.0986-0.1301$ & $0.1028-0.1298$ & $0.0716-0.1770$ \\
\hline 99 & $0.0905-0.1303$ & $0.0937-0.1303$ & $0.0549-0.1937$ \\
\hline
\end{tabular}

\begin{tabular}{|c|c|c|c|c|}
\hline C.L.\% & $\begin{array}{c}\text { Lengths of } \\
\text { Approximate } \\
\text { Bayesian } \\
\text { Intervals (SE) }\end{array}$ & $\begin{array}{c}\text { Lengths of } \\
\text { Approximate } \\
\text { Bayesian } \\
\text { Intervals (HT) }\end{array}$ & $\underline{\underline{\mathrm{WC}}}$ & $\underline{\underline{\mathrm{WC}}}$ \\
\hline 80 & 0.0214 & 0.0144 & 3.215 & 4.778 \\
\hline 90 & 0.0270 & 0.0214 & 3.289 & 4.150 \\
\hline 95 & 0.0315 & 0.0270 & 3.346 & 3.904 \\
\hline 99 & 0.0398 & 0.0366 & 3.487 & 3.792 \\
\hline
\end{tabular}




\section{BAYESIAN CONFIDENCE INTERVALS FOR THE GAUSSIAN DISTRIBUTION}

$$
N(\mu=25.083, \sigma=3.1176)
$$

The corresponding coefficient of variation is:

$$
\bar{C}_{v}=\frac{\sigma}{\mu}=0.124
$$

Example 2

Data: $13,11,9,12,8,10,5,10,9,12$, 13 (Mann, 1998, p. 504).

Normal population distribution obtained with SAS:

$$
N(\mu=10.182, \sigma=2.4008)
$$

The corresponding coefficient of variation is:

$$
\bar{C}_{v}=\frac{\sigma}{\mu}=0.236
$$

Example 3

Data: 16, 14, 11, 19, 14, 17, 13, 16, 17, 18, 19, 12 (Mann, 1998, p. 504).

Normal population distribution obtained with SAS:

$$
N(\mu=15.5, \sigma=2.6799)
$$

The corresponding coefficient of variation is:

$$
\bar{C}_{v}=\frac{\sigma}{\mu}=0.173
$$

Example 4

Data: 27, 31, 25, 33, 21, 35, 30, 26, 25, 31, 33, 30, 28 (Mann, 1998, p. 504).

Normal population distribution obtained with SAS:

$$
N(\mu=28.846, \sigma=3.9549)
$$

The corresponding coefficient of variation is:

$$
\bar{C}_{v}=\frac{\sigma}{\mu}=0.137
$$

Example 5

Data: 52, 33, 42, 44, 41, 50, 44, 51, 45, 38,37,40,44, 50, 43 (McClave \& Sincich, 1997, p. 301).

Normal population distribution obtained with SAS:

$$
N(\mu=43.6, \sigma=5.4746)
$$

The corresponding coefficient of variation is:

$$
\bar{C}_{v}=\frac{\sigma}{\mu}=0.126
$$

\section{Example 6}

Data: 52, 43, 47, 56, 62, 53, 61, 50, 56, 52, 53, 60, 50, 48, 60, 55 (McClave \& Sincich, 1997, p. 301).

Normal population distribution obtained with SAS:

$$
N(\mu=53.625, \sigma=5.4145)
$$

The corresponding coefficient of variation is:

$$
\bar{C}_{v}=\frac{\sigma}{\mu}=0.101
$$

Example 7

The following observations were obtained from the collection of SAS data sets: $50,65,100,45,111,32,45,28,60,66,114$, $134,150,120,77,108,112,113,80,77,69,91$, $116,122,37,51,53,131,49,69,66,46,131$, $103,84,78$.

Normal population distribution obtained with SAS:

$$
N(\mu=82.861, \sigma=33.226)
$$

The corresponding coefficient of variation is:

$$
\bar{C}_{v}=\frac{\sigma}{\mu}=0.401
$$


Table 2 Classical (2) and Approximate Bayesian Confidence Intervals for the Population Coefficient of Variation Corresponding To Data Set 2

\begin{tabular}{|c|c|c|c|}
\hline C.L.\% & $\begin{array}{c}\text { Approximate } \\
\text { Bayesian Bounds } \\
(\mathrm{SE})\end{array}$ & $\begin{array}{c}\text { Approximate } \\
\text { Bayesian Bounds } \\
(\mathrm{HT})\end{array}$ & Classical Bounds I \\
\hline 80 & $0.2007-0.2463$ & $0.2122-0.2430$ & $0.1647-0.3069$ \\
\hline 90 & $0.1908-0.2478$ & $0.2007-0.2463$ & $0.1441-0.3275$ \\
\hline 95 & $0.1823-0.2486$ & $0.1908-0.2478$ & $0.1269-0.3447$ \\
\hline 99 & $0.1662-0.2492$ & $0.1724-0.2490$ & $0.0924-0.3792$ \\
\hline
\end{tabular}

\begin{tabular}{|c|c|c|c|c|}
\hline C.L.\% & $\begin{array}{c}\text { Lengths of } \\
\text { Approximate } \\
\text { Bayesian } \\
\text { Intervals (SE) }\end{array}$ & $\begin{array}{c}\text { Lengths of } \\
\text { Approximate } \\
\text { Bayesian } \\
\text { Intervals (HT) }\end{array}$ & $\frac{\mathrm{WC}}{\mathrm{WSE}}$ & $\frac{\underline{\mathrm{WC}}}{\mathrm{WHT}}$ \\
\hline 80 & 0.0456 & 0.0308 & 3.118 & 4.617 \\
\hline 90 & 0.0570 & 0.0456 & 3.218 & 4.022 \\
\hline 95 & 0.0663 & 0.0570 & 3.285 & 3.821 \\
\hline 99 & 0.0830 & 0.0766 & 3.455 & 3.744 \\
\hline
\end{tabular}

Table 3: Classical (2) and Approximate Bayesian Confidence Intervals for the Population Coefficient of Variation Corresponding To Data Set 3

\begin{tabular}{|c|c|c|c|}
\hline C.L.\% & $\begin{array}{c}\text { Approximate } \\
\text { Bayesian Bounds } \\
(\mathrm{SE})\end{array}$ & $\begin{array}{c}\text { Approximate } \\
\text { Bayesian Bounds } \\
(\mathrm{HT})\end{array}$ & Classical Bounds I \\
\hline 80 & $0.1495-0.1797$ & $0.1573-0.1776$ & $0.1243-0.2215$ \\
\hline 90 & $0.1427-0.1807$ & $0.1495-0.1797$ & $0.1103-0.2354$ \\
\hline 95 & $0.1368-0.1811$ & $0.14273-0.1807$ & $0.0985-0.2473$ \\
\hline 99 & $0.1255-0.1815$ & $0.1300-0.1842$ & $0.0750-0.2708$ \\
\hline
\end{tabular}

\begin{tabular}{|c|c|c|c|c|}
\hline C.L.\% & $\begin{array}{c}\text { Lengths of } \\
\text { Approximate } \\
\text { Bayesian } \\
\text { Intervals (SE) }\end{array}$ & $\begin{array}{c}\text { Lengths of } \\
\text { Approximate } \\
\text { Bayesian } \\
\text { Intervals (HT) }\end{array}$ & $\frac{\mathrm{WC}}{\mathrm{WSE}}$ & $\frac{\mathrm{WC}}{\mathrm{WHT}}$ \\
\hline 80 & 0.0302 & 0.0203 & 3.219 & 4.788 \\
\hline 90 & 0.0380 & 0.0302 & 3.292 & 4.142 \\
\hline 95 & 0.0443 & 0.0380 & 3.359 & 3.916 \\
\hline 99 & 0.0560 & 0.0542 & 3.496 & 3.613 \\
\hline
\end{tabular}


Table 4: Classical (2) and Approximate Bayesian Confidence Intervals for the Population Coefficient of Variation Corresponding To Data Set 4

\begin{tabular}{|c|c|c|c|}
\hline C.L.\% & $\begin{array}{c}\text { Approximate } \\
\text { Bayesian Bounds } \\
(\mathrm{SE})\end{array}$ & $\begin{array}{c}\text { Approximate } \\
\text { Bayesian Bounds } \\
(\mathrm{HT})\end{array}$ & Classical Bounds I \\
\hline 80 & $0.1200-0.1419$ & $0.1258-0.1404$ & $0.1006-0.1736$ \\
\hline 90 & $0.1150-0.1426$ & $0.1200-0.1419$ & $0.0901-0.18414$ \\
\hline 95 & $0.1104-0.1430$ & $0.1149-0.1426$ & $0.0812-0.1930$ \\
\hline 99 & $0.1018-0.1433$ & $0.1052-0.1432$ & $0.0636-0.2107$ \\
\hline
\end{tabular}

\begin{tabular}{|c|c|c|c|c|}
\hline C.L.\% & $\begin{array}{c}\text { Lengths of } \\
\text { Approximate } \\
\text { Bayesian } \\
\text { Intervals (SE) }\end{array}$ & $\begin{array}{c}\text { Lengths of } \\
\text { Approximate } \\
\text { Bayesian } \\
\text { Intervals (HT) }\end{array}$ & $\frac{\mathrm{WC}}{\mathrm{WSE}}$ & $\frac{\mathrm{WC}}{\mathrm{WHT}}$ \\
\hline 80 & 0.0219 & 0.0146 & 3.333 & 5.000 \\
\hline 90 & 0.0276 & 0.0219 & 3.406 & 4.292 \\
\hline 95 & 0.0326 & 0.0277 & 3.429 & 4.036 \\
\hline 99 & 0.0415 & 0.0380 & 3.545 & 3.871 \\
\hline
\end{tabular}

Table 5: Classical (2) and Approximate Bayesian Confidence Intervals for the Population Coefficient of Variation Corresponding To Data Set 5

\begin{tabular}{|c|c|c|c|}
\hline C.L.\% & $\begin{array}{c}\text { Approximate } \\
\text { Bayesian Bounds } \\
\text { (SE) }\end{array}$ & $\begin{array}{c}\text { Approximate } \\
\text { Bayesian Bounds } \\
(\mathrm{HT})\end{array}$ & Classical Bounds I \\
\hline 80 & $0.1118-0.1293$ & $0.1166-0.1282$ & $0.09472-0.1564$ \\
\hline 90 & $0.1076-0.1299$ & $0.1118-0.1293$ & $0.08580-0.1653$ \\
\hline 95 & $0.1039-0.1301$ & $0.1076-0.1299$ & $0.0783-0.1728$ \\
\hline 99 & $0.0964-0.1303$ & $0.0994-0.1303$ & $0.0634-0.1877$ \\
\hline
\end{tabular}

\begin{tabular}{|c|c|c|c|c|}
\hline C.L.\% & $\begin{array}{c}\text { Lengths of } \\
\text { Approximate } \\
\text { Bayesian } \\
\text { Intervals (SE) }\end{array}$ & $\begin{array}{c}\text { Lengths of } \\
\text { Approximate } \\
\text { Bayesian } \\
\text { Intervals (HT) }\end{array}$ & $\frac{\underline{\mathrm{WC}}}{\mathrm{WSE}}$ & $\frac{\underline{\mathrm{WC}}}{\mathrm{WHT}}$ \\
\hline 80 & 0.0175 & 0.0116 & 3.526 & 5.314 \\
\hline 90 & 0.0223 & 0.0175 & 3.565 & 4.543 \\
\hline 95 & 0.0262 & 0.0223 & 3.607 & 4.238 \\
\hline 99 & 0.0339 & 0.0309 & 3.667 & 4.023 \\
\hline
\end{tabular}


Table 6: Classical (2) and Approximate Bayesian Confidence Intervals for the Population Coefficient of Variation Corresponding To Data Set 6

\begin{tabular}{|c|c|c|c|}
\hline C.L.\% & $\begin{array}{c}\text { Approximate } \\
\text { Bayesian Bounds } \\
(\mathrm{SE})\end{array}$ & $\begin{array}{c}\text { Approximate } \\
\text { Bayesian Bounds } \\
(\mathrm{HT})\end{array}$ & Classical Bounds I \\
\hline 80 & $0.0906-0.1038$ & $0.0942-0.1029$ & $0.0771-0.1248$ \\
\hline 90 & $0.0874-0.1042$ & $0.0906-0.1038$ & $0.0702-0.1317$ \\
\hline 95 & $0.0845-0.1044$ & $0.0874-0.1042$ & $0.0645-0.1375$ \\
\hline 99 & $0.0787-0.1045$ & $0.0810-0.1045$ & $0.0529-0.1490$ \\
\hline
\end{tabular}

\begin{tabular}{|c|c|c|c|c|}
\hline C.L.\% & $\begin{array}{c}\text { Lengths of } \\
\text { Approximate } \\
\text { Bayesian } \\
\text { Intervals (SE) }\end{array}$ & $\begin{array}{c}\text { Lengths of } \\
\text { Approximate } \\
\text { Bayesian } \\
\text { Intervals (HT) }\end{array}$ & $\underline{\underline{\mathrm{WC}}}$ & $\underline{\underline{\mathrm{WC}}}$ \\
\hline 80 & 0.0132 & 0.0087 & 3.614 & 5.483 \\
\hline 90 & 0.0168 & 0.0132 & 3.661 & 4.659 \\
\hline 95 & 0.0199 & 0.0168 & 3.668 & 4.345 \\
\hline 99 & 0.0258 & 0.0235 & 3.725 & 4.089 \\
\hline
\end{tabular}

Table 7: Classical (2) and Approximate Bayesian Confidence Intervals for the Population Coefficient of Variation Corresponding To Data Set 7

\begin{tabular}{|c|c|c|c|}
\hline C.L.\% & $\begin{array}{c}\text { Approximate } \\
\text { Bayesian Bounds } \\
\text { (SE) }\end{array}$ & $\begin{array}{c}\text { Approximate } \\
\text { Bayesian Bounds } \\
(\mathrm{HT})\end{array}$ & Classical Bounds I \\
\hline 80 & $0.3790-0.4063$ & $0.3870-0.4047$ & $0.3305-0.4715$ \\
\hline 90 & $0.3714-0.4071$ & $0.3790-0.4063$ & $0.3101-0.4919$ \\
\hline 95 & $0.3643-0.4075$ & $0.3714-0.4071$ & $0.2930-0.5090$ \\
\hline 99 & $0.3492-0.4077$ & $0.3598-0.4077$ & $0.2588-0.5431$ \\
\hline
\end{tabular}

\begin{tabular}{|c|c|c|c|c|}
\hline C.L.\% & $\begin{array}{c}\text { Lengths of } \\
\text { Approximate } \\
\text { Bayesian } \\
\text { Intervals (SE) }\end{array}$ & $\begin{array}{c}\text { Lengths of } \\
\text { Approximate } \\
\text { Bayesian } \\
\text { Intervals (HT) }\end{array}$ & $\frac{\underline{\mathrm{WC}}}{\mathrm{WSE}}$ & $\frac{\mathrm{WC}}{\mathrm{WHT}}$ \\
\hline 80 & 0.0273 & 0.0177 & 5.165 & 7.966 \\
\hline 90 & 0.0357 & 0.0273 & 5.092 & 6.660 \\
\hline 95 & 0.0432 & 0.0357 & 5.000 & 6.050 \\
\hline 99 & 0.0585 & 0.0479 & 4.860 & 5.935 \\
\hline
\end{tabular}




\section{BAYESIAN CONFIDENCE INTERVALS FOR THE GAUSSIAN DISTRIBUTION}

Each of the seven randomly selected samples shows that the proposed approximate Bayesian confidence intervals contain the population coefficient of variation. The models are also strictly included in their counterparts obtained with classical approach (2). For each of the seven examples, the proposed approximate Bayesian approach has greater coverage accuracy than its classical counterpart (2).

\section{Conclusion}

New approximate Bayesian confidence intervals for the coefficient of variation of a normal population under two different loss functions were derived. The loss functions employed were the square error and the Higgins-Tsokos. Based on the above numerical results the following conclusions are put forth:

- The proposed approach and models rely only on the observations under study, contrary to the classical approach (2) that uses the standard normal distribution, The classical approach (2) used to constructing confidence intervals for the coefficient of variation of a normal population does not always perform better than the approximate Bayesian approach. In fact, in each of the examples provided, the obtained approximate Bayesian confidence intervals had greater coverage accuracy than those obtained with the classical approach (2). Each of the obtained approximate Bayesian confidence intervals contains the population coefficient of variation, and is strictly included in its classical counterpart obtained with the classical approach (2).

- With the proposed approach, approximate Bayesian confidence intervals for a normal population coefficient of variation may be obtained for any level of significance and any sample size.

- The approximate Bayesian approach under the square error loss function does not always yield the best approximate Bayesian results. In fact, in the examples provided, the Higgins-Tsokos loss function performs better.
- Bayesian analysis contributes to reinforcing well-known statistical theories such as the estimation theory.

\section{References}

Miller, S. G. (1991), Asymptotic test statistics for Coefficient of variation. Communications in Statistics - Theory and Methods, 20(10), 3351-3363.

Camara, V. A. R. (2003). Approximate Bayesian confidence intervals for the variance of a Gaussian distribution. Journal of Modern Applied Statistical Methods, 2(2), 350-358.

Higgins, J. J., \& Tsokos, C. P. (1976). Comparison of Bayes estimates of failure intensity for fitted priors of life data. In Proceedings of the Conference on the Theory an Applications of Reliability with Emphasis on Bayesian and Nonparametric Methods. New York, NY: Academic.

Mann, P. S. (1998). Introductory Statistics, $3^{\text {rd }}$ Edition. New York, NY: Wiley.

McClave, J. T., \& Sincich, T. A. (1997). First course in Statistics, $6^{\text {th }}$ Ed. New York, NY: Prentice Hall.

Camara, V. A. R. (2009). Approximate Bayesian confidence intervals for the mean of a Gaussian distribution versus Bayesian models. Journal of Modern Applied Statistical Methods, 8(2), 526-533.

Camara, V. A. R., \& Tsokos, C. P. (1998). Bayesian reliability modeling with applications. Michigan: UMI Publishing Company.

Drake, A. W. (1966). Bayesian statistics for the reliability eng. proc. Annual Symposium on Reliability, 315-320.

Camara, V. A. R. (2011). New approximate Bayesian confidence intervals for the coefficient of variation of a Gaussian distribution. In JSM Proceedings, Statistical Computing Section, 1121-1131. Alexandria, VA: American Statistical Association. 


\section{Parameter Estimation with Mixture Item Response Theory Models: A Monte Carlo Comparison of Maximum Likelihood and Bayesian Methods}

\author{
W. Holmes Finch \\ Ball State University, \\ Muncie, IN
}

\author{
Brian F. French \\ Washington State University, \\ Pullman, WA
}

The Mixture Item Response Theory (MixIRT) can be used to identify latent classes of examinees in data as well as to estimate item parameters such as difficulty and discrimination for each of the groups. Parameter estimation via maximum likelihood (MLE) and Bayesian estimation based on the Markov Chain Monte Carlo (MCMC) are compared for classification accuracy and parameter estimation bias for difficulty and discrimination. Standard error magnitude and coverage rates were compared across number of items, number of latent groups, group size ratio, total sample size and underlying item response model. Results show that MCMC provides more accurate group membership recovery across conditions and more accurate parameter estimates for smaller samples and fewer items. MLE produces narrower confidence intervals than MCMC and more accurate parameter estimates for larger samples and more items. Implications of these results for research and practice are discussed.

Key words: Mixture item response theory, differential item functioning, Bayesian estimation, Markov chain Monte Carlo estimation, maximum likelihood estimation.

\section{Introduction}

Mixture item response theory (MixIRT) has become an increasingly popular tool for investigating a variety of issues in educational and psychological assessment (Cohen \& Bolt, 2005; Bolt, Cohen \& Wollack, 2001). Use of the MixIRT model in a variety of contexts has been described in detail by a number of authors (Cohen \& Bolt, 2005; von Davier \& Yamamoto, 2004; von Davier \& Rost, 1995; Mislevy \& Verhelst, 1990; Rost, 1990; Yamamoto, 1987). For example, MixIRT has been recommended for identifying subsets of a population (latent

W. Holmes Finch is Professor of Psychology in the Department of Educational Psychology, and Educational Psychology Director of Research in the Office of Charter School. Email him at: whfinch@bsu.edu. Brian F. French is Associate Professor and Co-Director Learning and Performance Research Center Washington State University. Email him at: frenchb@wsu.edu. classes) which are characterized by different item response models for a particular measure or instrument ( $\mathrm{Li}$, et al., 2009). In this context, psychometricians have used MixIRT to detect and characterize differential item functioning (DIF) (Cohen \& Bolt, 2005; De Ayala, et al., 2002; Bolt, Cohen \& Wollack, 2002, 2001). This simulation study compares the parameter estimation accuracy for two methods of estimation used with MixIRT: Maximum Likelihood Estimation (MLE) and Baysian estimation using the Markov Chain Monte Carlo (MCMC) approach.

Prior research has demonstrated the utility of the MixIRT framework given its ability to identify differentially responding subgroups that exist organically in the data. This approach stands in contrast to the assumption that differential response patterns are inherently linked to easily identified grouping variables (e.g., gender) and that all (or most) members of such intact groups will demonstrate very similar responses to items; an assumption which underlies other statistical models used for similar purposes. For example, in the detection of DIF using standard methods such as logistic 


\section{MIXTURE ITEM RESPONSE THEORY MODEL PARAMETER ESTIMATION}

regression or the Mantel-Haenszel test, comparisons of item response patterns are made between known groups such as males and females. However, recent work in the area of DIF has demonstrated that the causes of DIF are often complex and not so clearly tied to easily identified groups (Cohen \& Bolt, 2005). In such cases, the utility of the MixIRT approach - and its sometimes superiority - has been demonstrated in gaining a deeper understanding into differential item response patterns such as those associated with DIF (Maij-de Meij, Kelderman \& van der Flier, 2010; Samuelson, 2008; Cohen, Cho \& Kim, 2005; Rost, 1990).

The MixIRT model, which combines the powerful statistical tools of latent class analysis (LCA) and item response theory (IRT), assumes that a population is composed of a finite number of latent examinee classes that can be differentiated based upon their item response patterns (Rost, 1997). In turn, these different response patterns will manifest themselves as differences in parameters of the item response model associated with each group. The 2parameter MixIRT (Mix2PL) model for dichotomous data takes the following form:

$$
P\left(U=1 \mid g, \theta_{i g}\right)=\frac{e^{\left(a_{j g}\left(\theta_{i g}-b_{j g}\right)\right)}}{1+e^{\left(a_{j g}\left(\theta_{i g}-b_{j g}\right)\right)}}
$$

Here latent class membership $(g=1,2, \ldots, G)$, within class difficulty for item $j\left(b_{j g}\right)$ within class discrimination for item $j\left(a_{j g}\right)$, and the within class level on the latent trait being measured for person $i\left(\theta_{i g}\right)$ are all model parameters to be estimated. In addition, each survey respondent is placed in a latent class, and the proportions of individuals in each class $\left(\pi_{g}\right)$, are also estimated, under the constraint that $\sum_{g=1}^{G} \pi_{g}=1$. Variants of this model including a pseudo-chance parameter (Mix3PL) and excluding both pseudo-chance and constraining discrimination to be equivalent across items (Mix1PL) are also available, as in the standard IRT context. The focus of this study is on dichotomous items for which chance responding is not applicable, such as behavior inventories; for this reason only the Mix1PL and Mix2PL models are examined.
The item parameter values carry the same meaning in the MixIRT context as in the more general IRT framework; thus, item difficulty provides information regarding the likelihood that an individual will endorse an item (or answer it correctly in the context of cognitive assessment), discrimination indicates how well the item differentiates between individuals with different levels of the construct being measured and pseudo-guessing is a measure of the likelihood that an examinee would respond to the item correctly due solely to chance (de Ayala, 2009).

When there are class differences in the item difficulty and discrimination parameter values, researchers conclude that members of the latent classes perform differently on the specific item (Cohen \& Bolt, 2005). For example, assume that the results of the analysis indicate the presence of two distinct latent classes in the population. In this case, if a specific item for latent class 1 has a higher value for $b_{j g}$ than class 2 , it is known that the item is more difficult for class 1 ; this in turn may provide insights into the types of individuals who tend to be in that class. Similarly, if latent class 2 has a higher $a_{j g}$ value on an item compared to class 1 , it can be concluded that the item is better able to differentiate among individuals with different levels of the latent trait for class 2 than for class 1. This approach to using MixIRT models has been particularly evident in the identification and characterization of DIF for achievement tests (Cohen \& Bolt, 2005), though it has also been used to identify different usage patterns of the not sure category in personality inventories (Maij-de Meij, Kelderman \& van der Flier, 2008) and to identify individuals engaging in impression management in organizational surveys (Eid \& Zickar, 2007).

Parameter Estimation

In the literature, model parameter estimation for MixIRT models has been examined using both MLE (Willse, 2011) and MCMC methods in the Bayesian context (von Davier \& Rost, 2007). Excellent discussions regarding the technical details of both approaches are present in the literature; the interested reader is referred to von Davier and Carstensen (2007) for a thorough treatment of a number of MixIRT models available. Although 


\section{FINCH \& FRENCH}

prior applied work has used both methods, there has been very little research empirically comparing the performance of the two estimation techniques to one another.

Based upon these prior applications, each approach has been shown to have specific advantages and disadvantages in practice. For example, MCMC has proven useful with complex MixIRT models because it does not require integration of the likelihood function (as does MLE) which can be extremely difficult when it is necessary to estimate many parameters (Junker, 1999).

Conversely, the MCMC approach is often very time consuming to implement (sometimes taking 10 days or more to fit a single model), and may encounter difficulties in converging to solutions for individual parameters ( $\mathrm{Li}$, et al., 2009). The issue of time is non-trivial when dealing with MixIRT models, as several different latent class solutions must typically be fit and then compared in order to determine which is optimal for the data at hand (Li, et al., 2009). MLE does not usually require such large amounts of time as MCMC and MLE has been used successfully in estimating MixIRT models (von Davier \& Rost, 2007); however, MLE can mistakenly converge on localized, rather than general maximum likelihood solutions, leading to suboptimal model parameter estimates. This problem can be overcome through the use of multiple random starting values, such as the 10 random starts used in this study (Rost, 1991). Of concern, though, is that using more starting values and increasing the maximum number of iterations in order to increase the probability of obtaining optimal fit, also increases the time necessary for the model to converge and provide parameter estimates.

Although relatively little work has been done explicitly comparing the performance of MLE and MCMC estimation techniques in the context of MixIRT models, Li, et al. (2009) conducted a simulation study in which they examined the performance of MCMC primarily in terms of identifying the optimal model selection criterion for dichotomous item response data. However, as a part of this study, MixIRT parameter estimation was also examined. Results of their research indicated that item parameter recovery was worse in the presence of more latent classes and better when there were more items and/or more examinees. Recovery of latent class membership was generally greater than $80 \%$, with the most accurate results for the Mix2PL model and the least accurate for the Mix3PL.

Cho and Cohen (2010) expanded on this work by investigating item parameter recovery for the multilevel Mix1PL model, in which information at both the student (level 1) and school (level 2) levels were taken into consideration. The estimation used in this simulation study was also MCMC and the model was restricted to the 1-Parameter Logistic form. The authors reported that recovery of both the item difficulty estimates and group membership was good for the MCMC methodology used in the study. A study by Willse (2011) examined the performance of a joint maximum likelihood estimator for the Rasch MixIRT model. He reported the results of a simulation study that showed good parameter recovery for group specific item difficulty values. No other simulation work examining the accuracy of parameter estimates in the MixIRT context was identified in the literature.

The goal of this simulation study is to compare the parameter recovery performance of the MLE and MCMC estimation procedures in the context of the MixIRT model for dichotomous item response data. Prior simulation work in this area has focused primarily on MCMC estimation and has not directly compared the ability of this approach and MLE in terms of parameter recovery accuracy, both for the items and for latent class membership. In addition, this work adds the additional simulation conditions of group size ratio, which has not been previously examined. Thus, this study adds to the literature by directly comparing these two popular methods of estimation across a range of conditions for dichotomous item response data. Prior applied work in this area has shown both methods to be potentially useful in many cases. However, given that both have distinct certain practical advantages in terms of their relative abilities to converge on the optimal solution and the time needed to use each; it would be helpful to understand whether one technique provides any 


\section{MIXTURE ITEM RESPONSE THEORY MODEL PARAMETER ESTIMATION}

methodological advantages over the other and, if so, under what conditions. If one approach does provide greater parameter estimation accuracy, researchers might be able to make decisions regarding which to use in light of this and the aforementioned practical concerns. Given that such a direct comparison has not been previously published, it is believed that this work will add valuable information to the literature on MixIRT models.

\section{Methodology}

The simulation study used to compare the parameter estimation accuracy for MLE and MCMC, involved the manipulation of several factors that have been shown pertinent in previous research. A total of 50 replications per combination of manipulated conditions were generated. The two estimation methods were fit using Mplus version 6.1 (Muthén \& Muthén, 2011). Several of the simulation conditions used in this study were based on those reported in $\mathrm{Li}$, et al. (2009). These were selected for use because they were used previously and have been shown to be related to the performance of the MCMC estimator.

Thus, given that something is known about how the MCMC approach performs under the various conditions, it was determined that they would be particularly informative for the comparison of this method and MLE. It should be noted that the simulating item parameter values were drawn from item responses to a behavioral checklist given to adolescents through the auspices of the 2009 administration of the Youth Risk Behavior Survey (Centers for Disease Control, 2009). A MixIRT study involving these items was published by Finch and Pierson (2011) in which they report results for four latent classes based on 16,000 adolescents' responses (yes or no) to items asking about participation in a variety of risky sexual and substance use behaviors. These data were fit with a Mix2PL model. The population item parameter values used in the generation of simulated data for the current study were drawn from this earlier work and are shown in Table 1.

Manipulated Conditions

A total of 2, 3, and 4 latent classes were simulated with sample sizes of 400, 1,000 and
2,000 examinees. Group sizes were either equal or unequal. In the unequal case for two groups, the ratio was $75 / 25$, for three groups the ratio was $50 / 25 / 25$ and for four groups the ratio was $40 / 20 / 20 / 20$. Two models were simulated, the Mix1PL and Mix2PL, and the appropriate model was fit for each replication. Specifically, when the Mix2PL model was used to generate the data, the Mix2PL model was fit to each simulated dataset. Finally, three conditions were simulated for the number of items, 5, 15 and 30. These were intended to simulate very short, moderate and somewhat longer instruments. The underlying latent trait was simulated to be unidimensional from the $N(0,1)$ distribution.

In order to differentiate the groups in the simulations, the item discrimination and item difficulty parameter values for the groups were made to differ (Table 1 shows the values for each group). For the 5 item condition, the first 5 item parameter sets were used, and for the 30 item condition, the 15 item set was used twice, in keeping with the methodology laid out in $\mathrm{Li}$, et al. (2009). The outcome variables of interest were the proportion of correctly placed individuals into the latent classes, the estimation bias for item difficulty and discrimination, mean standard error for parameters across replications and the coverage rates for the item parameters. In order to place all items on the same metric prior to estimating the outcome variables, methods outlined by Lloyd and Hoover (1980) were utilized.

\section{Model Convergence Issues}

Researchers using the MCMC approach to estimation must ensure that each time an analysis is run the results converge to the optimal solution. As a part of this, a burn-in period must be established, which means identifying a number of draws from the posterior distribution that will be ignored as the estimator seeks to converge to the solution for each parameter. After the burn-in has been established, samples are then drawn from subsequent values in the posterior in order to obtain the final parameter estimate. Based upon earlier work in this area, particularly that of $\mathrm{Li}$, et al. (2009) and Cho and Cohen (2010), as well as examination of auto-correlation plots from several of the simulated datasets, 10,000 
FINCH \& FRENCH

Table 1: Item Difficulty and Discrimination Parameters Used In the Monte Carlo Simulations

\begin{tabular}{|c|c|c|c|c|}
\hline \multirow[b]{2}{*}{ Item } & \multicolumn{4}{|c|}{ Difficulty } \\
\hline & Class 1 & Class 2 & Class 3 & Class 4 \\
\hline 1 & 2.817 & 1.188 & -2.522 & 1.824 \\
\hline 2 & -1.447 & 0.099 & 0.306 & 1.054 \\
\hline 3 & -2.507 & -0.835 & -2.002 & 2.819 \\
\hline 4 & 0.268 & -1.022 & -0.547 & 3.177 \\
\hline 5 & 1.743 & -0.001 & -2.569 & 2.535 \\
\hline 6 & -0.699 & -1.525 & 1.248 & 1.847 \\
\hline 7 & 0.022 & 0.206 & -0.262 & 2.025 \\
\hline 8 & 1.025 & 0.729 & -1.627 & 2.059 \\
\hline 9 & 1.201 & 0.747 & -1.766 & 2.037 \\
\hline 10 & 1.444 & 1.348 & -2.398 & 2.422 \\
\hline 11 & 1.299 & 0.867 & -2.085 & 2.207 \\
\hline 12 & 1.056 & 0.681 & -1.725 & 2.233 \\
\hline 13 & 0.713 & 0.626 & -1.048 & 1.948 \\
\hline 14 & 1.154 & 0.352 & -1.536 & 2.212 \\
\hline 15 & 0.546 & 0.001 & -0.851 & 1.868 \\
\hline \multirow[b]{2}{*}{ Item } & \multicolumn{4}{|c|}{ Discrimination } \\
\hline & Class 1 & Class 2 & Class 3 & Class 4 \\
\hline 1 & 0.096 & 1.735 & 0.083 & 0.689 \\
\hline 2 & 0.025 & 1.549 & 2.812 & 1.203 \\
\hline 3 & 0.236 & 1.146 & 0.057 & 0.351 \\
\hline 4 & 1.207 & 0.568 & 1.009 & 0.928 \\
\hline 5 & 0.845 & 0.279 & 0.547 & 0.483 \\
\hline 6 & 0.923 & 1.339 & 0.629 & 0.632 \\
\hline 7 & 0.918 & 2.105 & 0.836 & 1.062 \\
\hline 8 & 1.857 & 3.198 & 1.654 & 2.459 \\
\hline 9 & 1.075 & 2.106 & 0.722 & 0.978 \\
\hline 10 & 1.415 & 0.512 & 2.133 & 3.304 \\
\hline 11 & 2.477 & 0.163 & 1.765 & 0.853 \\
\hline 12 & 1.606 & 2.189 & 1.359 & 2.752 \\
\hline 13 & 0.432 & 1.918 & 0.529 & 0.547 \\
\hline 14 & 2.151 & 1.212 & 1.643 & 2.359 \\
\hline 15 & 1.029 & 2.130 & 1.150 & 1.009 \\
\hline
\end{tabular}




\section{MIXTURE ITEM RESPONSE THEORY MODEL PARAMETER ESTIMATION}

iterations were used as the burn-in, 10,000 post burn-in values were used to obtain parameter estimates with MCMC and thinning of the posterior draws was set at 50 .

Each method presented some difficulties in terms of convergence. The MLE approach had difficulty converging for the smallest sample size condition (400). Therefore additional simulations were run until the necessary 50 converged replicates were obtained for MLE. With respect to MCMC, difficulty was encountered in obtaining convergence for the 5 item condition for some of the replications. Thus, as with the MLE method, additional replications were run until the requisite 50 converged solutions were obtained. Although it was recognized that both conditions causing these problems (400 examinees and 5 items) might generally be viewed as problematic in practice, it is important to learn as much as possible about the relative performance of these two methods, including under relatively difficult circumstances such as these, given that such conditions are not uncommon in actual research practice, particularly for behavioral inventories and short mental health screening instruments.

\section{Label Switching}

An issue of some importance in any study involving latent class analysis is that of label switching, in which a given latent class might take one number (e.g., 1) in one case, and another number (e.g., 2) in another case. In reality, however, the group is constituted of the same individuals or type of individuals. In a simulation study involving MCMC estimation, label switching consists of two separate problems. First, within the context of Bayesian analysis, label switching can occur across repeated sampling from the posterior distribution within a single analysis. In order to detect this type of label switching, it is necessary to monitor the posterior densities of group membership. A multimodal distribution would be indicative of such label switching. During the simulation the densities were monitored and multimodal solutions did not present themselves, thus this type of label switching was eliminated as a concern.

The second type of label switching occurs across replications of a simulation study and is not limited to MCMC but can also occur for MLE. Essentially, it involves changing the arbitrary group label as described, but in this case from one replication to another. For this study, the methodology described in Cho, Cohen and Kim (2006) was used. Namely, the item parameter estimates from the individual sample replications were compared with those used to generate the data and the group labels from the sample replications were changed to match those to which they most closely conformed from the model generation groups.

\section{Results}

Classification Accuracy

In order to identify statistically significant effects among the manipulated factors described, a repeated measures analysis of variance (ANOVA) was used. The within replication variable was method, and the between replication variables were the manipulated factors including number of items, sample size, number of groups, group size ratio and the underlying model. The dependent variable was the mean classification accuracy across replications. In addition to statistical significance, effect sizes were also calculated for all main effects and interactions.

ANOVA results for the classification accuracy outcome variable indicate that the method of estimation interacted significantly with number of items $\left(F<0.001, \eta^{2}=0.363\right)$, number of subjects $\left(F=-0.025, \eta^{2}=0.07\right)$, group size ratio $\left(F=0.006, \eta^{2}=0.117\right)$ and number of groups $\left(F=0.003, \eta^{2}=0.108\right)$. In addition, method $\left(F<0.001, \eta^{2}=0.721\right)$ itself was statistically significant. Table 2 shows the classification accuracy rates for each of the manipulated variables by method. Across all other conditions, the MCMC approach yielded more accurate group classification than did MLE. This difference was most noticeable for fewer items, with the gap between the two estimation techniques narrowing as the number of items increased, in large part due to improvements in the accuracy of MLE. In addition, the MLE approach was more accurate at classifying individuals when the groups were of equal size, whereas the MCMC was largely impervious to the group size ratio. Across 


\section{FINCH \& FRENCH}

conditions MCMC yielded similar rates of correct classification, which were uniformly higher than 0.9, whereas MLE was much more likely to be influenced by the manipulated conditions and rarely had correct classification rates greater than 0.9 .

Item Discrimination Parameter Estimation

As with the classification accuracy results, ANOVA was used to identify significant study effects with regard to bias in the estimation of the item discrimination parameter.
The interaction terms of method by number of items $\left(F=0.025, \eta^{2}=0.07\right)$ and method by number of groups $\left(F<0.001, \eta^{2}=\right.$ 0.147 ) were significantly related to bias in the $a$ parameter estimate. Table 3 shows the mean bias results across replications for these two terms. Regardless of the number of items, the Bayesian method provided estimates of $a$ with bias under 0.15 in all cases. By contrast, MLE yielded very biased estimates in the case of 5 items, had comparable bias to the Bayesian for 15 items,

Table 2: Latent Class Classification Accuracy by Method, Number of Items, Number of Groups, Sample Size, Group Size Ratio and Underlying Model

\begin{tabular}{|c|c|c|}
\hline Items & MLE & MCMC \\
\hline 5 & 0.761 & 0.939 \\
\hline 15 & 0.889 & 0.972 \\
\hline 30 & 0.916 & 0.991 \\
\hline \multicolumn{3}{|c|}{ Sample Size } \\
\hline 400 & 0.852 & 0.971 \\
\hline 1,000 & 0.845 & 0.969 \\
\hline 2,000 & 0.827 & 0.947 \\
\hline \multicolumn{3}{|c|}{ Groups } \\
\hline 2 & 0.831 & 0.949 \\
\hline 3 & 0.880 & 0.970 \\
\hline 4 & 0.820 & 0.972 \\
\hline \multicolumn{3}{|c|}{ Group Ratio } \\
\hline Equal & 0.841 & 0.963 \\
\hline Unequal & 0.715 & 0.950 \\
\hline \multicolumn{3}{|c|}{ Model } \\
\hline $1 \mathrm{PL}$ & 0.844 & 0.951 \\
\hline $2 \mathrm{PL}$ & 0.822 & 0.969 \\
\hline
\end{tabular}


and had lower bias for 30 items. With respect to the number of groups, item discrimination bias for MLE increased concomitantly with increasing number of groups. In contrast, estimation accuracy for the Bayesian approach seemed largely unaffected by the number of groups in terms of the absolute size of bias, though for 4 groups the estimates were somewhat underestimated whereas for 2 and 3 groups they were somewhat overestimated.

In addition to parameter estimation bias, the coverage rates for the discrimination parameters were also estimated. These coverage rates represent the proportion of simulation replications for which the nominal 95\% confidence interval actually contained the true population value of $a$ : ideally they would be 0.95 . The results of the ANOVA indicated that the main effect of method $\left(F<0.001, \eta^{2}=\right.$
$0.762)$, as well as the interaction of method by number of items $\left(F<0.001, \eta^{2}=0.339\right)$ were statistically significant. Table 4 includes the coverage rates for each method by the number of items.

Across conditions, the coverage rates for the MCMC estimates were near 1.0 and were much higher than those of the MLE method. The latter estimation approach had higher coverage for tests with a larger number of items, though in no case were these rates comparable to those of the MCMC approach and they were generally lower than the nominal 0.95 level. The standard errors of these estimates also appear in Table 4, and show that those associated with MCMC were larger than those from MLE. These larger standard errors resulted in wider confidence intervals for the MCMC estimates, which contributed in part to the higher coverage rates for this approach.

Table 3: Item Discrimination Bias by Method, Number of Items and Number of Groups

\begin{tabular}{|c|c|c|}
\hline Items & MLE & MCMC \\
\hline 5 & 0.307 & -0.133 \\
\hline 15 & -0.084 & -0.082 \\
\hline 30 & 0.074 & 0.149 \\
\hline \multicolumn{2}{|c|}{ Groups } \\
\hline 2 & 0.097 & 0.118 \\
\hline 3 & 0.276 & 0.105 \\
\hline 4 & 0.302 & -0.080 \\
\hline
\end{tabular}

Table 4: Item Discrimination Coverage Rates (Mean Standard Error across Replications) By Method and Number of Items

\begin{tabular}{|c|c|c|}
\hline Items & MLE & MCMC \\
\hline 5 & $0.663(0.394)$ & $0.991(0.902)$ \\
\hline 15 & $0.788(0.378)$ & $1.000(0.886)$ \\
\hline 30 & $0.886(0.366)$ & $1.000(0.865)$ \\
\hline
\end{tabular}




\section{FINCH \& FRENCH}

Item Difficulty Parameter Estimation

The ANOVA results for the item difficulty parameter bias revealed that only the interaction of method by number of groups was statistically significant $\left(F=0.013, \eta^{2}=0.082\right)$. Table 5 includes the $b$ bias results for this interaction term. For both methods, underestimation bias of the $b$ parameter increased concomitantly with increases in the number of groups. The significant interaction appears to be a function of the fact that for 2 groups, the bias in the MCMC estimator was somewhat smaller than that of MLE; however, for 3 groups this pattern was reversed and for 4 groups the bias of the two methods was comparable.

The ANOVA for the $b$ parameter coverage rates showed that the main effect of method $\left(F<0.001, \eta^{2}=0.768\right)$ and the interaction of method by number of items $(F<$ $\left.0.001, \eta^{2}=0.263\right)$ were the two significant terms in this model. Table 6 includes the coverage rates for $b$ by method and number of items. Item difficulty coverage rates were uniformly 1.0 for the MCMC estimator, whereas for MLE these rates were below the nominal 0.95 level except for the 30 item condition. An examination of the average standard error for these estimates, also shown in Table 6, reveals that the MCMC estimator had a substantially larger standard error than did MLE, which in turn led to wider confidence intervals. Therefore, although the coverage rates for the MCMC approach were higher than those of MLE, the associated intervals were also wider, just as was the case for item discrimination.

\section{Conclusion}

It is hoped that the results of this study will prove useful to researchers and practitioners interested in using the MixIRT approach in order to gain a greater understanding of their data, whether in the context of characterizing DIF, or identifying specific item response profile groups, as was the case for the study upon which this work was built, or gaining further insights into the interplay of personality and item response profiles. In all of these cases, accurate estimation of item response and group membership parameters is crucial to obtaining useful results that can inform policy and practice. Prior applied research has focused on two different estimation methods, MCMC within the Bayesian framework, and MLE, and

Table 5: Item Difficulty Bias by Method and Number of Groups

\begin{tabular}{|c|c|c|}
\hline Groups & MLE & MCMC \\
\hline 2 & -0.029 & -0.019 \\
\hline 3 & -0.031 & -0.038 \\
\hline 4 & -0.057 & -0.055 \\
\hline
\end{tabular}

Table 6: Item Difficulty Parameter Coverage Rates (Mean Standard Error across Replications) by Method and Number of Items

\begin{tabular}{|c|c|c|}
\hline Items & MLE & MCMC \\
\hline 5 & $0.620(0.205)$ & $1.00(0.607)$ \\
\hline 15 & $0.814(0.195)$ & $1.00(0.598)$ \\
\hline 30 & $0.959(0.188)$ & $1.00(0.587)$ \\
\hline
\end{tabular}




\section{MIXTURE ITEM RESPONSE THEORY MODEL PARAMETER ESTIMATION}

has shown that both approaches appear to be useful for specific situations. In addition, a very brief simulation literature demonstrated some support for the MCMC technique in terms of parameter estimation, though no direct comparisons with MLE were made. At the same time, these earlier authors noted that the MCMC approach often requires a very lengthy time period in order to complete a single analysis $(\mathrm{Li}$, et al., 2009), a fact which has also been reported by other authors. Therefore, while previous work indicates that the MCMC estimation approach might hold promise in terms of parameter estimation, the logistics of using it in many real world situations might limit its practical value. Given that there has been little simulation work examining MixIRT in general, and no studies that could be found comparing the two major parameter estimation approaches with one another, this the current study should prove informative to practitioners considering the use of the MixIRT paradigm in research.

Study results herein indicate that for correctly identifying which group an individual belongs to, the MCMC approach would seem to be more effective. Across virtually all conditions simulated, it was more accurate than MLE in terms of correct group identification. Across all simulated conditions, MCMC correctly classified respondents in over $96 \%$ of cases, whereas MLE was correct only $84 \%$ of the time. Furthermore, there was very little variation in the rates of accuracy for MCMC across manipulated conditions, however, for MLE the accuracy rates varied greatly, particularly as a function of the number of items. Thus, for researchers whose primary goal is to gain insights into the types of respondents present in the population, it would seem that MCMC is the preferable estimation approach.

For researchers who are most interested in the accuracy and precision of class specific item difficulty and discrimination values, the results of the study are somewhat more ambiguous. It seems that with respect to item discrimination estimates, the MCMC approach might provide somewhat less biased estimates for shorter instruments. By contrast, item discrimination bias was lower for MLE when the instrument contained 30 items. With respect to item difficulty, the length of the instrument was not as salient as the number of latent classes, such that the presence of more groups was associated with greater item difficulty bias for both methods. It is possible that this relationship was due in part to the smaller number of individuals in the groups that was present when the number of groups increased.

In terms of estimate precision as measured by the average standard error value across replications and the coverage rates, MLE appears to have fared somewhat better than MCMC. It is true that the coverage rates for MCMC were uniformly higher than those of MLE, but this appears to have been due in the main to the larger standard errors associated with the Bayesian estimates. Thus, researchers using MCMC can be reasonably sure that the credible intervals for the estimates contain the population parameter value, but they also must be aware that these intervals will generally be wide. Such wide intervals may not be terribly informative to researchers interested in obtaining fairly precise estimates of the item difficulty and discrimination values.

Recommendations for Practice

Based on study results, some general recommendations for practice can be developed. First, when there are many items, the MLE approach might be optimal. With 30 items, MLE produced somewhat more accurate item parameter estimates than did MCMC and it had group classification accuracy rates above $90 \%$ (though this was lower than that of MCMC). In addition to the more accurate item parameter estimation in the presence of 30 items, MLE estimates were also more precise than those of MCMC, as witnessed in the narrower confidence intervals. However, when an instrument consists of very few items, MLE should probably be avoided, as it produced substantially more biased estimates than MCMC and will be less accurate in terms of classifying respondents. When researchers suspect that more than 3 groups are present, MCMC would also seem to be a better choice, particularly with regard to estimating item discrimination parameters. Such is not the case for item difficulty, which was compromised with equal severity for both estimation approaches for 4 groups. In short, situations in which many items are available to 


\section{FINCH \& FRENCH}

describe many examinees and few groups are ideal for the use of MLE, whereas cases in which the number of items is small and/or the number of groups is large may be better suited to MCMC. All of these recommendations must be considered in light of the fact that the MCMC estimation will probably take substantially more time than will MLE.

Finally, with respect to using MixIRT models with relatively small samples as previously discussed, with a sample size of 400 individuals, both estimation methods had difficulty reaching convergence for many of the replications in the study. This was particularly an issue for MLE, though the Bayesian approach was also less successful for an $N$ of 400 than for the larger sample sizes; thus, in practice researchers might find that they are unable to obtain useful estimates for this small sample size regardless of the method used. This problem was particularly acute for a larger number of groups in conjunction with the smaller sample size, because the number of individuals in each group became small. Therefore, one other recommendation for practice to come out of this study is that - for samples of 400 or fewer MixIRT may not be particularly viable, except perhaps for the simplest models.

Limitations and Areas for Future Research

As with any research, this study has some limitations which impact interpretations of the results that must be acknowledged. First, the Mix3PL model was not included in the study. This decision was made consciously, as the focus of the study was on instruments that are common in psychology, such as behavior inventories and personality assessments, for which chance responding is a negligible issue. In addition, the item parameter values used to generate the data were based on a behavior inventory. That this focus is believed to be appropriate for research in psychology, but it does limit the findings for those interested in cognitive assessments where chance responses to items are an issue. Future research should include a Mix3PL model. In addition, the current study examined a limited range of unequal group size conditions. Although this is the first study in this area to manipulate group sizes, it is recognized that more work in this area needs to be conducted and thus a wider range of unequal group size conditions should be simulated. In addition, it is believed that the settings of the MCMC and MLE techniques used in this study were in keeping with recommended practice, it would be helpful if a wider array of values for the burn-in period and post burn-in iterations for MCMC were used and if more conditions in terms of number of random starts and convergence criteria were investigated for MLE. Such research would provide more information regarding the optimal settings for use with these estimators.

\section{References}

Bolt, D. M., Cohen, A. S., \& Wollack, J. A. (2001). A mixture item response model for multiple-choice data. Journal of Educational and Behavioral Statistics, 26, 381-409.

Bolt, D. M., Cohen, A. S., \& Wollack, J. A. (2002). Item parameter estimation under conditions of test speededness: Application of a mixture Rasch model with ordinal constraints. Journal of Educational Measurement, 39, 331348.

Centers for Disease Control. (2009). Youth risk behavior survey: 2009 National YRBS data Users Manual. Atlanta, GA: Center for Disease Control.

Cho, S-J., \& Cohen, A. S. (2010). A multilevel mixture IRT model with an application to DIF. Journal of Educational and Behavioral Statistics, 35(3), 336-370.

Cho, S-J., Cohen, A. S., \& Kim, S-H. (2006). An investigation of priors on the probabilities of mixtures in the mixture Rasch model. Paper presented to the annual meeting of the Psychometric Society, Montreal, Canada.

Cohen, A. S., \& Bolt, D. M. (2005). A mixture model analysis of differential item functioning. Journal of Educational Measurement, 42(2), 133-148.

Cohen, A. S., Cho, S.J., \& Kim, S.H. (2005). A mixture testlet model for educational tests. Paper presented at the annual meeting of the American Educational Research Association, Montreal, Canada.

de, Ayala, R. J. (2009). The theory and practice of item response theory. New York, NY: The Guilford Press. 


\section{MIXTURE ITEM RESPONSE THEORY MODEL PARAMETER ESTIMATION}

de Ayala, R. J., Kim, S. H., Stapleton, L. M., \& Dayton, C. M. (2002). Differential item functioning: A mixture distribution conceptualization. International Journal of Testing, 2, 243-276.

Eid, M., \& Zickar, M. (2007). Detecting response styles and faking in personality and organizational assessment by mixed Rasch models. In Multivariate and Mixture Distribution Rasch Models, M. vonDavier \& C. Cartensen (Eds.), 255-270. New York: Springer.

Finch, W. H., \& Pierson, E. E. (2011). A mixture IRT analysis of risky youth behavior. Frontiers in Quantitative Psychology, 2, 1-10.

Junker, B. W. (1999). Some statistical models and computational methods that may be useful for cognitively-relevant assessment. Retrieved November 8, 2010, from http://citeseerx.ist.psu.edu/viewdoc/download?d $\mathrm{oi}=10.1 \cdot 1.142 .7053 \& \mathrm{rep}=$ rep1\&type $=\mathrm{pd}$.

Li, F., Cohen, A. S., Seok-Ho, K., \& Cho, S-J. (2009). Model selection methods for mixture dichotomous IRT models. Applied Psychological Measurement, 33(5), 353-373.

Maij-de Meij, A. M., Kelderman, H., \& van der Flier, H. (2010). Improvement in detection of differential item functioning using a mixture item response theory model. Multivariate Behavioral Research, 45(6), 975999.

Mislevy, R. J., \& Verhelst, N. D. (1990). Modeling item responses when different subjects employ different solution strategies. Psychometrika, 55(2), 195-215.

Muthén, L. K. \& Muthén, B. O. (2011). MPlus software, version 6.1. Los Angeles, CA: MPlus.

Rost, J. (1997). Logistic mixture models. In Handbook of modern item response theory, W. J. van der Linden \& R. K. Hambleton (Eds.), 449-463. New York, NY: Springer.
Rost, J. (1991). A logistic mixture distribution model for polychotomous item responses. British Journal of Mathematical and Statistical Psychology, 44, 75-92.

Rost, J. (1990). Rasch models in latent classes: An integration of two approaches to item analysis. Applied Psychological Measurement, 14, 271-282.

Samuleson, K. M. (2008). Examining differential item functioning from a latent class perspective. In Advances in latent variable mixture models, G. R. Hancock \& K. M. Samuelson (Eds.), 67-113. Charlotte, NC: Information Age Publishing.

von Davier, M. \& Rost, J. (1995). Polytomous mixed Rasch models. In Rasch models: Foundations, recent developments and applications, G. H. Fischer \& I. W. Molenaar (Eds.), 371-379. New York, NY: Sprinter.

von Davier, M. \& Carstensen, C.H. (2007). Multivariate and mixture distribution Rasch models. New York, NY: Springer.

von Davier, M., \& Rost, J. (2007). Mixture distribution item response models. In Handbook of statistics, 26: Psychometrics, C. R. Rao \& S. Sinharay (Eds.), 643-661. Amsterdam: Elsevier.

von Davier, M., \& Yamamoto, K. (2004). Partially observed mixture models of IRT models: An extension of the generalized partial credit model. Applied Psychological Measurement, 28, 389-406.

Willse, J. T. (2011). Mixture Rasch models with Joint Maximum Likelihood Estimation. Educational and Psychological Measurement, 17(1), 5-19.

Yamamoto, K. (1987). A model that combines IRT and latent class models. Unpublished doctoral dissertation, University of Illinois at Urbana-Champaign. 


\title{
Statistical Inferences for Lomax Distribution Based on Record Values (Bayesian and Classical)
}

\author{
Parviz Nasiri Saman Hosseini \\ University of Payame Noor, \\ Tehran, Iran
}

A maximum likelihood estimation (MLE) based on records is obtained and a proper prior distribution to attain a Bayes estimation (both informative and non-informative) based on records for quadratic loss and squared error loss functions is also calculated. The study considers the shortest confidence interval and Highest Posterior Distribution confidence interval based on records, and using Mean Square Error MSE criteria for point estimation and length criteria for interval estimation, their appropriateness to each other is examined.

Key words: Lomax distribution; record values, maximum likelihood estimation, method of moment, Bayesian estimation, shortest interval, highest posterior density (HPD) interval, quadratic loss function, squared error loss function, prior density, posterior density, simulation, MSE.

\section{Introduction}

Let $X_{1}, X_{2}, X_{3}$, be a sequence of independent and identically (iid) random variable with cumulative distribution (cdf) function $F(x)$ and probability density (pdf) $f(x)$ For $n \geq 1$ define

$$
\begin{aligned}
T(1) & =1, T(n+1) \\
& =\min \left\{j: X_{j} \geq X_{T_{(n)}}\right\} .
\end{aligned}
$$

The sequence $\left\{X_{T(n)}\right\}_{n=1}^{\infty}$ is known as an upper record value statistic and the sequence $\{T(n)\}_{n=1}^{\infty}$ is known as a record time sequence (Arnold, Balakrishnan \& Nagaraja, 1998). Chandler (1952) was one of the first to study record theory and he defined a mathematical model for record values. Record values arise naturally in many applications involving data

Parviz Nasiri is an Associate Professor in the Department of Statistics University of Payame Noor, 19395-4697 Tehran, Iran. Email him at: Pnasiri45@yahoo.com. Saman Hosseini is a Lecturer in Department of Statistics. Email him at: S.hosseini.stat@gmail.com. relating to weather, sports, economics and life testing studies. Many authors have studied records and their associated statistics as well as inference-based testing on records. Some of the best examples may be found in the works of Balakrishnan, Arnold, Nagaraja (1998), Ahsanullah (1998) and Nevzoroz (1946).

Sevgi, et al. (2005) examined the relationship between order statistics and records. Mohammad (2002) and Balakrishnan (1994) examined the recurrent relations between the moments for the generalized exponential and Lomax distributions. Ahsanullah (1974) studied record values received from Lomax distribution, and Ahsanullah and Holland (1994) discussed both scale and location estimation of the distribution of generalized extreme values based on records. Asgharzadeh (2009) discussed both MLE and Bayesian estimation based on record values and Chan (1998) presents interval estimation according to records for groups of scales and locations. Soliman and Abd Ellah (2006) compared Bayesian and Non-Bayesian estimation based on records.

The Lomax distribution plays an important role in reliability. Consider the oneparameter Lomax distribution with pdf 


$$
f(x ; \theta)=\frac{1}{\theta}(1+x)^{-\left(\frac{1}{\theta}+1\right)} \quad x \geq 0, \theta>0,
$$

and cdf

$$
F(x ; \theta)=1-(1+x)^{-\frac{1}{\theta}} \quad x \geq 0, \theta>0 .
$$

An application of the Lomax distribution in receiver operating characteristic (ROC) was presented by Campbell and Ratnaparkhi (1993). Distributional properties and recurrence relation moments of record values was studied by Balakrishnan (1994) and Ahsanullah (1991). Much work has been done with respect to estimating the parameters using both classical and Bayesian techniques, and parametric and nonparametric inference based on record values have also been studied extensively (for example, see Ahmadia, et al., 2009; Soliman \& AlAbound, 2008; Baklizi, 2008).

This study has several components: It considers Lomax parameter estimation based on record values. It estimates the parameter $\theta$ using maximum likelihood and method of moment (MME) based on record values. It uses an appropriate selection of density function for a prior distribution to derive a Bayesian estimation based on record values. For the latter, by applying an appropriate selection for the prior density, the society parameter is controlled; this means that the Mean Square Error MSE) of the Bayesian estimation is controlled by controlling the parameters of this distribution. Finally, it derives the shortest interval estimation and Highest Posterior Density (HPD) interval estimation based on record values. Examples are used to illustrate the various components.

Point Estimation of $\theta$ Parameter: The Method of Maximum Likelihood Estimation

If $X_{T(1)}, X_{T(2)}, \ldots, X_{T(n)}$ represents the first $n$ upper record values from the Lomax distribution in (1), then the joint distribution of $X_{T(1)}, X_{T(2)}, \ldots, X_{T(n)}$ is
$f\left(x_{T(1)}, x_{T(2)}, \ldots, x_{T(n)}\right)=f\left(x_{T(n)} ; \theta\right) \prod_{i=1}^{n-1} h\left(x_{T(i)} ; \theta\right)$,

where

$$
h\left(x_{T(i)} ; \theta\right)=\frac{f\left(x_{T(i)}\right)}{1-F\left(x_{T(i)}\right)} .
$$

Thus, for the Lomax distribution,

$$
f\left(x_{T(1)}, x_{T(2)}, \ldots, x_{T(n)}\right)=\theta^{-n} \frac{\left(1+x_{T(n)}\right)^{-\frac{1}{\theta}}}{\prod_{i=1}^{n}\left(1+x_{T(i)}\right)},
$$

and the log likelihood function is

$$
L=-n \ln \theta-\left(\frac{1}{\theta}\right) \ln \left(1+x_{T(n)}\right)-\sum_{i=1}^{n} \ln \left(1+x_{T(i)}\right) .
$$

The maximum likelihood estimation (MLE) based on records can be obtained from (4) as

$$
-\frac{n}{\theta}+\frac{\ln \left(1+x_{T(n)}\right)}{\theta^{2}}=0
$$

and

$$
\hat{\theta}=\frac{\ln \left(1+x_{T(n)}\right)}{n} .
$$

Using (2), the marginal pdf of $X_{T(n)}$ can be derived as

$$
\begin{aligned}
& f\left(x_{T(n)}\right)= \\
& \frac{1}{\theta^{n}(n-1) !}\left(1+x_{T(n)}\right)^{-\left(\frac{1}{\theta}+1\right)}\left(\ln \left(1+x_{T(n)}\right)\right)^{n-1},
\end{aligned}
$$

therefore,

$$
\left(\hat{\theta}_{M L E}\right)=\theta, \operatorname{Var}\left(\hat{\theta}_{m l e}\right)=\frac{\theta^{2}}{n} .
$$

conversely, if (3) is rewritten as 


$$
\begin{aligned}
& f\left(x_{T(1)}, x_{T(2)}, \ldots, x_{T(n)}\right)= \\
& \exp \left(-n \ln \theta-\frac{1}{\theta} \ln \left(1+x_{T(n)}\right)-\sum_{i=1}^{n} \ln \left(1+x_{T(i)}\right)\right),
\end{aligned}
$$

then $\ln \left(1+x_{n}\right)$ is a complete sufficient statistic for parameter $\theta$. Therefore $\hat{\theta}_{M L E}$ based on record is the equal to the Uniformly MinimumVariance Unbiased Estimator (UMVUE) for parameter $\theta$.

Point Estimation of $\theta$ Parameter: The Method of Moment Estimation

The MME, first introduced by Pearson (1894), was one of the first methods used to estimate the society parameter $\theta$ (for additional details and an example see Pearson, 1894). The Lomax parameter $\theta$ is estimated by the MME based on record values by using the density function (6), which results in

$$
E\left(X_{T(n)}\right)=\frac{1}{(1-\theta)^{n}}-1 .
$$

Next, solving the equation

$$
E\left(X_{T(n)}\right)=\bar{X}
$$

yields a MME based on record values, where $\bar{X}$ is average of the $n$ first records $\left(X_{T(1)}, X_{T(2)}, \ldots, X_{T(n)}\right)$. Thus,

$$
\hat{\theta}_{M M E}=1-\frac{1}{(1+\bar{X})^{\frac{1}{n}}} .
$$

\section{Bayesian Estimation of $\theta$ Parameter}

The Bayesian estimator of $\theta$ is obtained based on record values under the two following loss functions:

$$
L(\hat{\theta}, \theta)=\left(\frac{\hat{\theta}}{\theta}-1\right)^{2},
$$

$$
L(\hat{\theta}, \theta)=(\hat{\theta}-\theta)^{2},
$$

where $\hat{\theta}$ is an estimator of $\theta$. Assuming an inverse Weibull distribution $\operatorname{IWD}(\gamma, \beta, c=1)$, the prior for $\theta$ is conjugated as

$$
\pi(\theta)=\frac{1}{\Gamma(\gamma) \beta}\left(\frac{\beta}{\theta}\right)^{\gamma+1} \exp \left(-\frac{\beta}{\theta}\right),
$$

such that

$$
\begin{aligned}
& E(\theta)=\frac{\beta}{\gamma-1}, \\
& \operatorname{Var}(\theta)=\frac{\beta^{2}}{(\gamma-1)^{2}(\gamma-2)}
\end{aligned}
$$

where $\quad \gamma>0, \beta>0 . \quad$ Note that $\frac{1}{\theta} \sim \operatorname{gamma}(\gamma, \beta)$. This prior density has an advantage over other priors because it is easy to use and the parameter $(\gamma, \beta)$ can be chosen such that prior precision for the true value of $\theta$ is fulfilled because Bayesian estimations are functions of $(\gamma, \beta)$, therefore, the precision of the Bayesian estimations cannot be controlled by altering the prior distribution parameters. Combining likelihood function (3) with prior density (9), the posterior density of $\theta$ is obtained as

$$
\begin{aligned}
& \pi(\theta \mid x)= \\
& \left\{\begin{array}{l}
\frac{1}{\Gamma(n+\gamma)\left[\ln \left(1+x_{T(n)}\right)+\beta\right]}\left(\frac{\ln \left(1+x_{T(n)}\right)+\beta}{\theta}\right)^{n+\gamma 1} \\
\times \exp \left(-\frac{\ln \left(1+x_{T(n)}\right)+\beta}{\theta}\right)
\end{array}\right\}
\end{aligned}
$$

where $x=x_{T(1)}, \ldots, x_{T(n)}, \theta>0$. Note that

and 
$\left(\frac{1}{\theta} \mid x_{T(1)}, \ldots, x_{T(n)}\right) \sim \operatorname{gamma}\left(n+\gamma, \beta+\operatorname{Ln}\left(1+x_{T(n)}\right)\right)$.

Bayesian Estimator of $\theta$ Under Quadratic Loss Function

The posterior distribution of $\theta$ is $\operatorname{IWD}\left(n+\gamma, \beta+\ln \left(1+x_{n}\right), c=1\right)$, where IWD is Inverse Weibull Distribution (in other words $I W D=\frac{1}{W}$ where $\mathrm{W}$ is a Weibull variable) and the Bayes estimator of $\theta$ is based on record values under a quadratic loss function (7), for example $\hat{\theta}_{b, 1}$, as given by Berger (1985) is

$$
\begin{aligned}
\hat{\theta}_{b, 1}= & \frac{E\left(\omega(\theta) \mu(\theta) \mid X_{T(1)}, X_{T(2)}, \ldots, X_{T(n)}\right)}{E\left(\omega(\theta) \mid X_{T(1)}, X_{T(2)}, \ldots, X_{T(n)}\right)} \\
= & \frac{E\left(\frac{1}{\theta} \mid X_{T(1)}, X_{T(2)}, \ldots, X_{T(n)}\right)}{E\left(\frac{1}{\theta^{2}} \mid X_{T(1)}, X_{T(2)}, \ldots, X_{T(n)}\right)} \\
= & \frac{\beta+L n\left(1+x_{T(n)}\right)}{n+\gamma+1} .
\end{aligned}
$$

Bayesian Estimator of $\theta$ Under Squared Error Loss Function

Considering the posterior distribution of $\theta$ and loss function (8), the Bayes estimator based on record values, for example, $\hat{\theta}_{b, 2}$, is given as (Berger, 1985):

$$
\begin{aligned}
\hat{\theta}_{b, 2} & =E\left(\mu(\theta) \mid X_{T(1)}, X_{T(2)}, \ldots, X_{T(n)}\right) \\
& =E\left(\theta \mid X_{T(1)}, X_{T(2)}, \ldots, X_{T(n)}\right) \\
& =\frac{\operatorname{Ln}\left(1+x_{T(n)}\right)+\beta}{n+\gamma-1} .
\end{aligned}
$$

As a result, the Bayesian estimation is formed as a differentia combination of both prior distribution and sample distribution as:

$$
\hat{\theta}_{b, 2}=\frac{n}{n+\gamma-1} \frac{\ln \left(1+x_{T(n)}\right)}{n}+\frac{\gamma-1}{n+\gamma-1} \frac{\beta}{\gamma-1} .
$$

Interval Estimation of $\theta$ Based on Record Values: The Shortest Interval Estimation

To obtain the shortest $(1-\alpha) \%$ confidence interval estimation based on record values, a pivot quantity is chosen as a function of a minimal sufficient statistic for parameter $\theta\left(\hat{\theta}_{M L E}\right)$ such that

$$
Q=\frac{2 \ln \left(1+X_{T(n)}\right)}{\theta}
$$

From (6) it is clear that the distribution of $Q$ is $\chi_{2 n}$ for any constants $a$ and $b$, hence,

$$
\begin{aligned}
P(a<Q<b) & =\int_{a}^{b} f_{Q}(t) d t \\
& =1-\alpha .
\end{aligned}
$$

Algebraic manipulation results in the confidence interval

$$
\frac{2 \ln \left(1+x_{T(n)}\right)}{b}<\theta<\frac{2 \ln \left(1+x_{T(n)}\right)}{a},
$$

thus, the length of interval is obtained as

$$
L=2 \ln \left(1+x_{T(n)}\right)\left[\frac{1}{a}-\frac{1}{b}\right]
$$

To minimize (13) and satisfy (12), $a$ and $b$ are selected using the Lagrange multipliers method

$\psi(a, b, \lambda)=$

$$
2 \ln \left(1+x_{T(n)}\right)\left(\frac{1}{a}-\frac{1}{b}\right)+\lambda\left(\int_{a}^{b} f_{Q}(t) d t-(1-\alpha)\right) .
$$

After derivation by $\lambda, a$, and $b$, the following results: 


$$
\left\{\begin{array}{c}
\int_{a}^{b} f_{Q}(t) d t=1-\alpha \\
\frac{-2 \ln \left(1+x_{T(n)}\right)}{a^{2}}-\lambda f(a)=0 \Rightarrow\left\{\begin{array}{l}
\int_{a}^{b} f_{Q}(t) d t=1-\alpha \\
a^{2} f_{Q}(a)=b^{2} f_{Q}(b)
\end{array}\right. \\
\frac{2 \ln \left(1+x_{T(n)}\right)}{b^{2}}+\lambda f(b)=0
\end{array}\right.
$$

Accordingly, $a$ and $b$ must satisfy (14) to yield the shortest interval estimation for $\theta$ :

$$
\begin{aligned}
P(a<Q<b) & =\int_{a}^{b} f_{Q}(t) d t \\
& =1-\alpha, a^{2} f_{Q}(a) \\
& =b^{2} f_{Q}(b)
\end{aligned}
$$

Interval Estimation of $\theta$ Based on Record Values: Highest Posterior Density (HPD) $\theta$ Estimation

After obtaining the posterior distribution $\pi\left(\theta \mid X_{T(1)}, X_{T(2)}, \ldots, X_{T(n)}\right)$, the problem of the likelihood that the parameter $\theta$ lies within the interval $\left[c_{L}, c_{U}\right]$ arises. Bayesians call the interval based on the posterior distribution a credible interval; the interval $\left[c_{L}, c_{U}\right]$ is said to be a $(1-\alpha) \%$ credible interval for $\theta$ if

$$
\int_{c_{L}}^{c_{U}} \pi\left(\theta \mid X_{T(1)}, X_{T(2)}, \ldots, X_{T(n)}\right) d \theta=1-\alpha .
$$

The Highest Posterior Density (HPD) region is given by $\left\{A: \pi\left(\theta \mid X_{T(1)}, X_{T(2)}, \ldots, X_{T(n)}\right) \geq c\right\}$ where $\mathrm{c}$ is chosen so that

$$
\begin{gathered}
\int_{c_{L}}^{c_{U}} \pi\left(\theta \mid X_{T(1)}, X_{T(2)}, \ldots, X_{T(n)}\right) d \theta=1-\alpha \\
\pi\left(c_{L} \mid X_{T(1)}, X_{T(2)}, \ldots, X_{T(n)}\right)=\pi\left(c_{U} \mid X_{T(1)}, X_{T(2)}, \ldots, X_{T(n)}\right)
\end{gathered}
$$

The HPD interval estimation is optimal in the sense that it results in the shortest interval. Let $\lambda=\frac{1}{\theta}$, by this assumption the posterior distribution of $\lambda$ is Gamma $\left(n+\gamma, \ln \left(1+x_{n}\right)+\beta\right) . \quad$ After algebraic manipulation, an HPD estimation $(1-\alpha) \%$ for parameter $\theta$ based on records is given by

$$
\begin{aligned}
\frac{\Gamma^{*}\left(n+\gamma, A c_{L}, A c_{U}\right)}{\Gamma(n+\gamma)} & =1-\alpha,\left(\frac{c_{L}}{c_{U}}\right)^{n+\gamma-1} \\
& =\exp \left(c_{L} A-c_{U} A\right),
\end{aligned}
$$

where $A=\beta+\ln \left(1+x_{T(n)}\right)$ and $\Gamma^{*}$ is the generalized incomplete Gamma function. Therefore HPD interval estimation based on record values can be obtained as:

$$
\theta \in\left[\frac{1}{c_{U}}, \frac{1}{c_{L}}\right]
$$

MSE and Bias

Simulation and Examples

To illustrate the estimation techniques developed, consider the following simulated data from the Lomax distribution:

\begin{tabular}{|c|c|c|}
\hline 3.286379 & 2.652416 & 1.325698 \\
\hline 1.895476 & 16.420820 & 10.123657 \\
\hline 1.254875 & 14.852147 & 12.985314 \\
\hline 11.684235 & 15.365742 & 1085.950045 \\
\hline 50.254198 & 850.569874 & 32.154875 \\
\hline 950.548796 & 2423.065086 & 1.989562 \\
\hline 84.254187 & 1240.325487 & 7372.085167 \\
\hline 2.658474 & 352.325469 & 6524.123548 \\
\hline 15.987455 & 33.659874 & 5487.214587 \\
\hline 1.235478 & 3658.125489 & 9083.239327 \\
\hline 48.236584 & 6985.125489 & 6.325698 \\
\hline 448.125634 & 8754.215487 & 47739.689056 \\
\hline 125.258643 & 25.365987 & 12543.2158746 \\
\hline 25413.125487 & 256.326598 & 1254.365241 \\
\hline 1.36548 & 16845.362545 & 25.326874 \\
\hline 6985.125469 & 7.365214 & 121942.356923 \\
\hline
\end{tabular}


This data was obtained by using the transformation $x_{i}=\frac{1}{\left(1-u_{i}\right)^{\theta}}-1$, where $u_{i}$ is a uniformly distributed random variable. If only the upper record values have been observed, these are:

\begin{tabular}{|c|c|}
\hline 3.286379 & 7372.085167 \\
\hline 16.420820 & 9083.239327 \\
\hline 1085.950045 & 47739.689056 \\
\hline 2423.065086 & 121942.356923 \\
\hline
\end{tabular}

for a non-informative prior distribution with $\gamma=0, \beta=1$, and $\gamma=8, \beta=7.56$ for an informative prior distribution. Results from equations (5), (10) and (11) for the parameter $\theta$ computed for $n=4,5,6,7,8$ are presented in Table1.

Interval Estimation

Results from using equations (14) and (15) for the parameter, $\lambda=\frac{1}{\theta}$ computed for $n=4,5,6,7,8$ are presented in Tables 2 and 3 .

Table 1: Estimation, Bias and MSE

\begin{tabular}{|c|c|c|c|c|}
\hline $\begin{array}{c}\text { Number of } \\
\text { Records }(\mathrm{n}=)\end{array}$ & & Estimate & Bias & MSE \\
\hline 4 & & 1.948300 & 0 & 0.9489683 \\
5 & & 1.781118 & 0 & 0.6344765 \\
6 & $\hat{\theta}_{M L E}$ & 1.519049 & 0 & 0.3845852 \\
7 & & 1.539077 & 0 & 0.3383940 \\
8 & & 1.463914 & 0 & 0.2678805 \\
\hline 4 & & 1.758640 & -0.18966006 & 0.6433108 \\
5 & & 1.650932 & -0.13018638 & 0.4575572 \\
6 & $\hat{\theta}_{b, 1}$ & 1.444899 & -0.07414991 & 0.2880506 \\
7 & Non-Informative & 1.471692 & -0.06738463 & 0.2636236 \\
8 & & 1.412368 & -0.05154600 & 0.2143157 \\
\hline 4 & & 2.931067 & 0.9827668 & 2.6528857 \\
5 & & 2.476398 & 0.6952796 & 1.4747832 \\
6 & $\hat{\theta}_{b, 2}$ & 2.022859 & 0.5308099 & 0.8076270 \\
7 & Non-Informative & 1.962257 & 0.4231795 & 0.6396728 \\
8 & & 1.815902 & 0.3519877 & 0.4737801 \\
\hline 4 & & 1.181015 & -0.7672848 & 0.6785692 \\
5 & & 1.176114 & -0.6050046 & 0.4469587 \\
6 & $\hat{\theta}_{b, 1}$ & 1.111620 & -0.4074296 & 0.2275325 \\
7 & Informative & 1.145846 & -0.3932308 & 0.2194012 \\
8 & & 1.133607 & -0.3303074 & 0.1684260 \\
\hline 4 & & 1.395746 & -0.5525547 & 0.4308002 \\
5 & & 1.372133 & -0.4089857 & 0.2774215 \\
7 & & 1.282638 & -0.2364112 & 0.1378137 \\
\hline & & 1.309539 & -0.2295385 & 0.1372864 \\
& & 1.284754 & -0.1791599 & 0.1082954 \\
\hline
\end{tabular}


NASIRI \& HOSSEINI

Table2: Shortest $(1-\alpha) \%$ Confidence Interval Estimation Based On Record Values

\begin{tabular}{|c|c|c|c|c|}
\hline $\begin{array}{c}\text { Number of } \\
\text { Records } \\
(\mathrm{n}=)\end{array}$ & \multicolumn{2}{|c|}{ Interval } & Length & $\begin{array}{c}(1-\alpha) \% \\
\text { Confidence }\end{array}$ \\
\hline 4 & 0.7071710 & 4.549181 & 3.842010 & \\
5 & 0.7314714 & 3.743103 & 3.011632 & \\
6 & 0.6822589 & 2.965349 & 2.283090 & $90 \%$ \\
7 & 0.7401722 & 2.843073 & 2.102900 & \\
8 & 0.7432804 & 2.589680 & 1.846400 & \\
\hline 4 & 0.6255906 & 5.766975 & 5.141384 & \\
5 & 0.6532331 & 4.580130 & 3.930780 & \\
6 & 0.6139227 & 3.545798 & 2.931875 & $95 \%$ \\
7 & 0.6765692 & 3.340115 & 2.669904 & \\
8 & 0.6765692 & 3.001246 & 2.324677 & \\
\hline 4 & 0.5012662 & 9.505643 & 9.003677 & \\
5 & 0.5321775 & 7.002903 & 6.470725 & \\
6 & 0.5064018 & 5.142492 & 4.636090 & $99 \%$ \\
7 & 0.5586008 & 4.663650 & 4.104764 & \\
8 & 0.5689107 & 4.069324 & 3.500414 & \\
\hline
\end{tabular}

Table3: Highest Posterior Distribution (HPD) $(1-\alpha) \%$ Interval Estimation Based On Record Statistics

\begin{tabular}{|c|c|c|c|c|c|c|}
\hline $\begin{array}{c}\text { Number of } \\
\text { Records } \\
(\mathrm{n}=)\end{array}$ & \multicolumn{2}{|c|}{$\lambda \in\left(\lambda_{L}, \lambda_{U}\right)$} & \multicolumn{2}{c|}{$\theta \in\left[\frac{1}{\lambda_{U}}, \frac{1}{\lambda_{L}}\right]$} & Length & $\begin{array}{c}(1-\alpha) \% \\
\text { Confidence }\end{array}$ \\
\hline 4 & 0.414500 & 1.137700 & 0.878966 & 2.412545 & 1.533579 & \\
5 & 0.430000 & 1.140900 & 0.876501 & 2.325581 & 1.449080 & \\
6 & 0.472900 & 1.196200 & 0.835980 & 2.114612 & 1.278631 & $90 \%$ \\
7 & 0.467300 & 1.164000 & 0.859100 & 2.139900 & 1.280846 & \\
8 & 0.483600 & 1.173200 & 0.852369 & 2.067825 & 1.215455 & \\
\hline 4 & 0.3668000 & 1.237600 & 0.808015 & 2.726281 & 1.918266 & \\
5 & 383300 & 1.236500 & 0.808734 & 2.608923 & 1.800188 & \\
6 & 0.423500 & 1.293800 & 0.772917 & 2.361275 & 1.588358 & $95 \%$ \\
7 & 0.421100 & 1.254100 & 0.797384 & 2.374733 & 1.577384 & \\
8 & 0.437700 & 1.260800 & 0.793147 & 2.284670 & 1.491523 & \\
\hline 4 & 0.295500 & 1.416700 & 0.705865 & 3.384095 & 2.678229 & \\
5 & 0.312600 & 1.407700 & 0.710378 & 3.198976 & 2.488598 & \\
6 & 0.348500 & 1.468000 & 0.681198 & 2.869400 & 2.188242 & $99 \%$ \\
7 & 0.350100 & 1.415400 & 0.706514 & 2.856327 & 2.149813 & \\
8 & 0.366900 & 1.417600 & 0.705417 & 2.725538 & 2.020121 & \\
\hline
\end{tabular}


Figure 1: MSE's of the Estimators $\hat{\theta}_{M L E}, \hat{\theta}_{b, 1}$, and $\hat{\theta}_{b, 2}$ Informative and Non-Informative MSE's of the MLE and Bayesian estimator based on

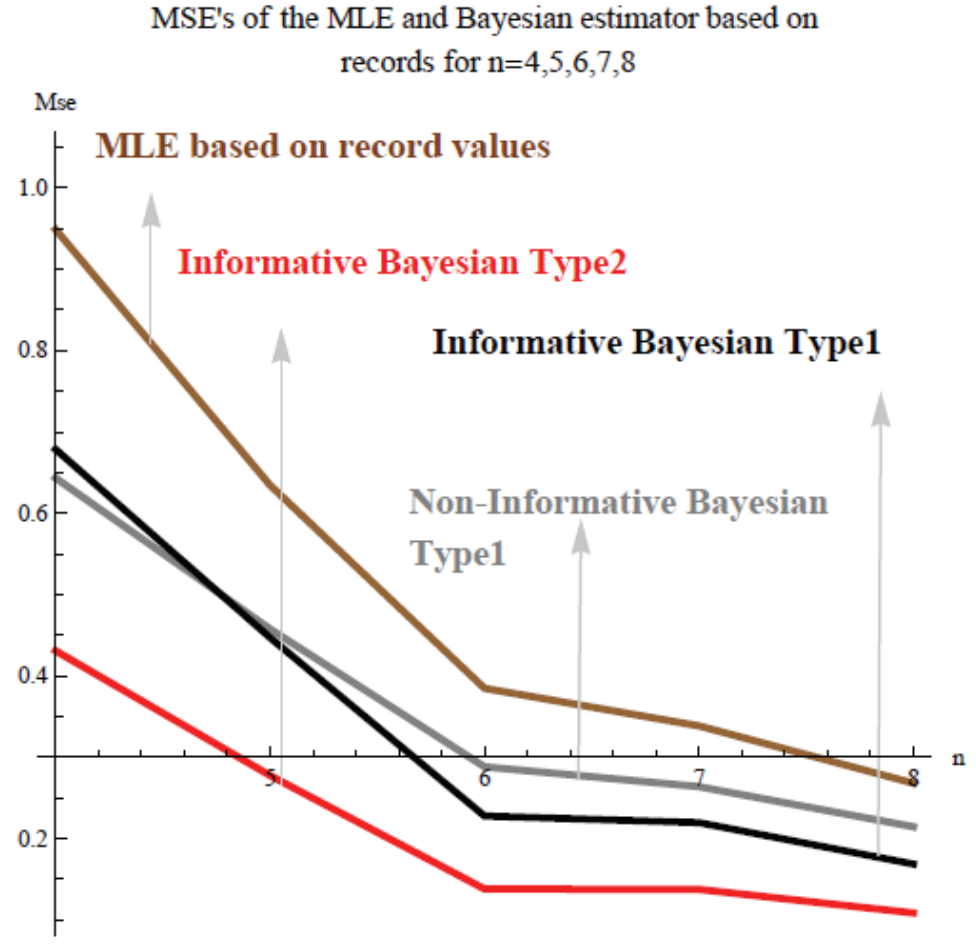

Figure 2: Lengths of the Shortest Interval and Highest Posterior Distribution (HPD) Estimations Based on Record Statistics for $90 \%$ Confidence for $n=4,5,6,7,8$ and confidence $=90$

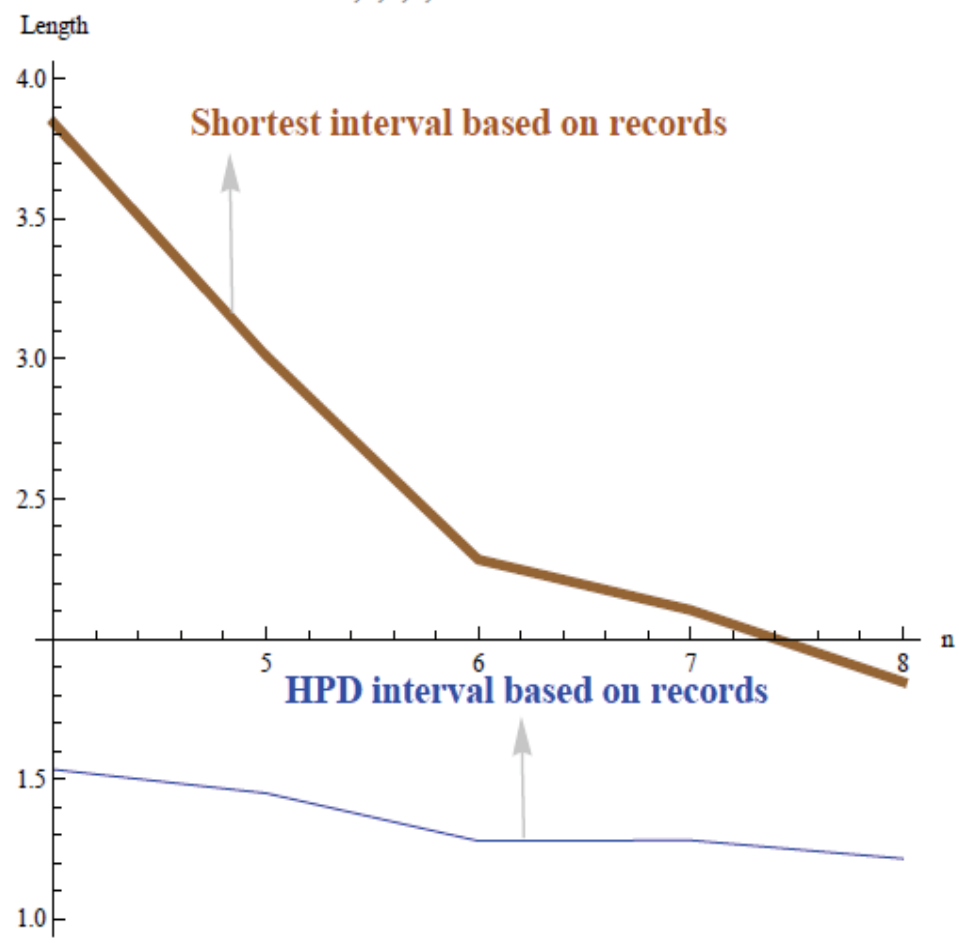


NASIRI \& HOSSEINI

Figure 3: Lengths of the Shortest Interval and Highest Posterior Distribution (HPD) Estimations Based on Record Statistics for 95\% Confidence

Lengths of the Shortest and HPD interval based on records

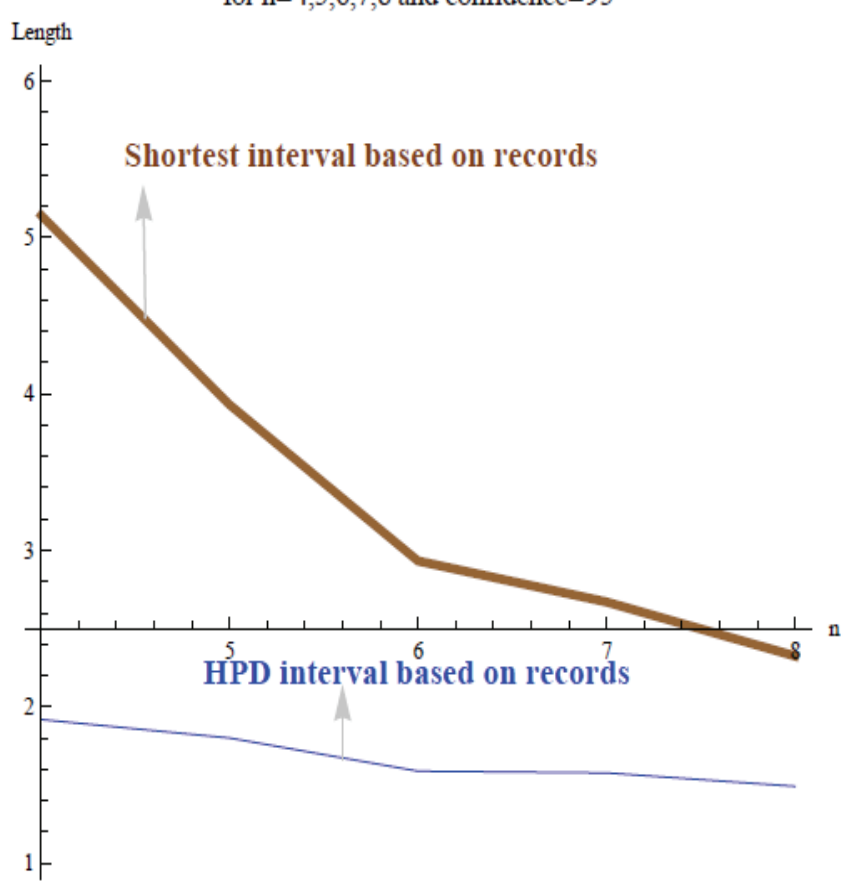

Figure 4: Lengths of the Shortest Interval and Highest Posterior Distribution (HPD) Estimations Based on Record Statistics for $99 \%$ Confidence

Lengths of the Shortest and HPD interval based on records

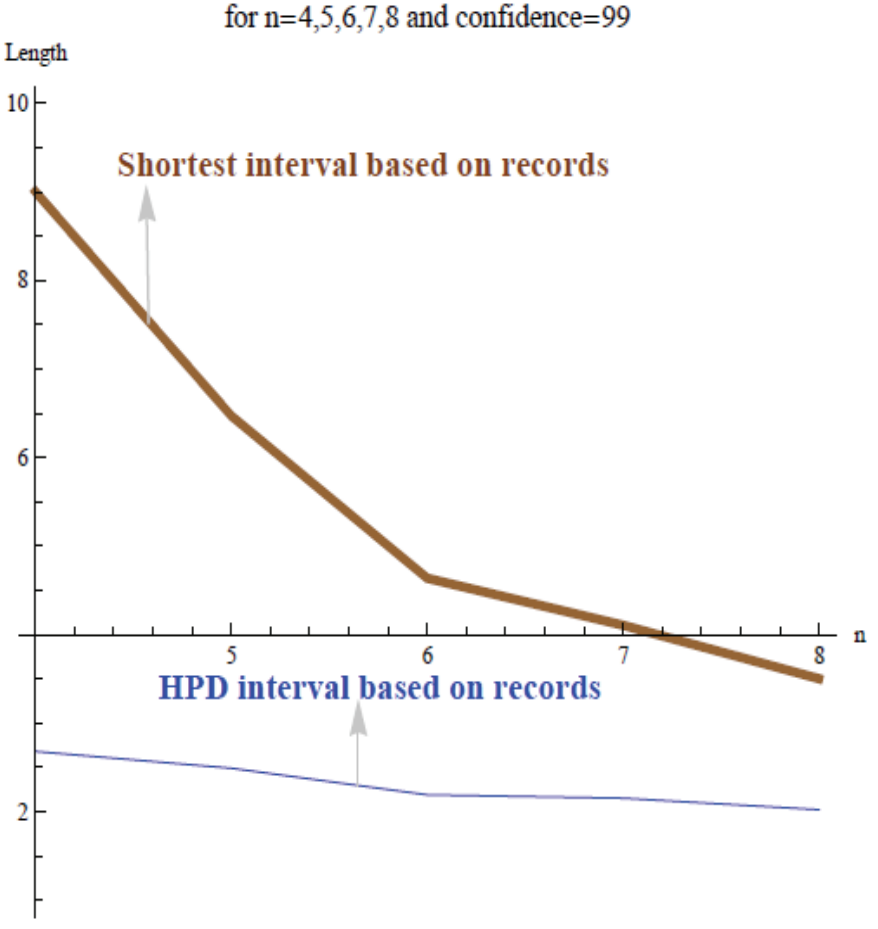


Figure 5: Lengths of the Shortest Interval and Highest Posterior Distribution (HPD) Estimations Based on

Record Statistics for $90 \%, 95 \%$ and $99 \%$ Confidence

Lengths of the Shortest and HPD interval based on records

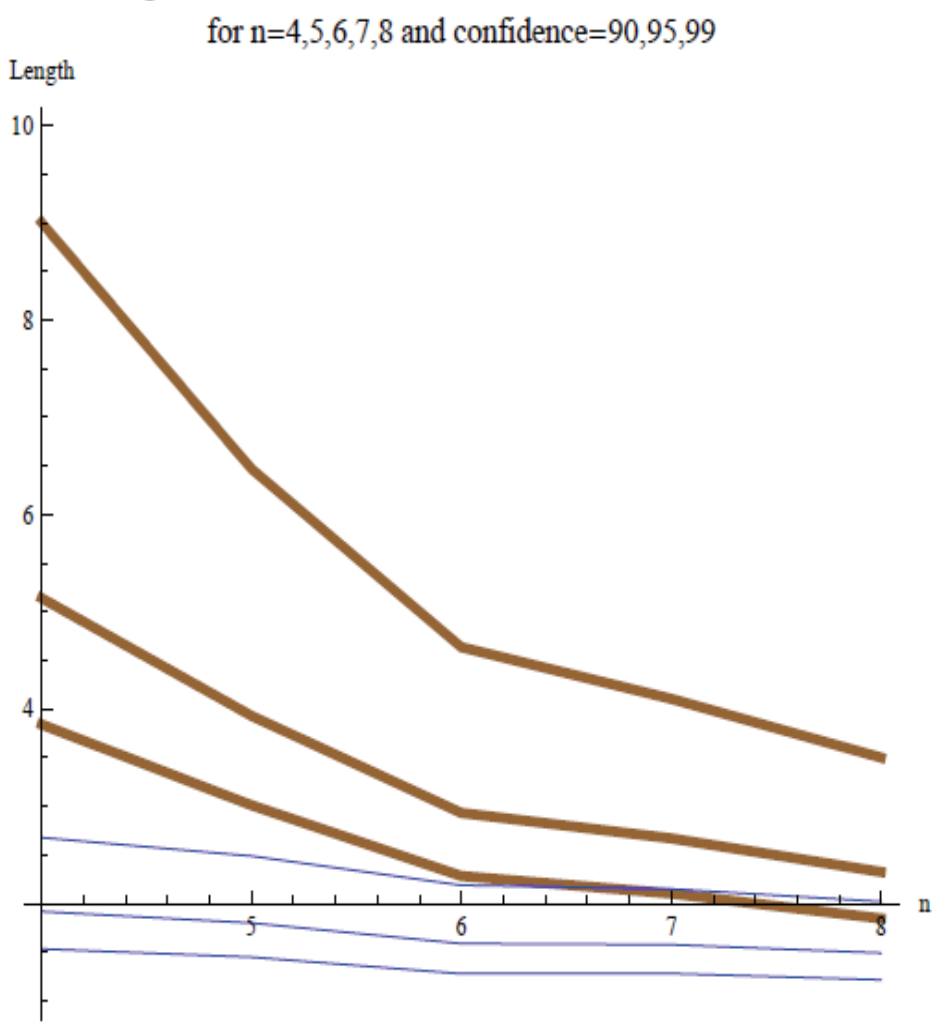

\section{Conclusion}

MLE and Bayesian estimations based on record values were obtained. For the Bayes estimations, in order to control the passive parameter of society, the prior distribution was assumed to be Gamma. In addition, Bayes estimations were obtained for two types of loss functions and, with a view of prior estimation, using an informative posterior density function, HPD estimations were obtained in a theoretic way (see Table 3). Conversely, the shortest confidence interval was obtained using a MLE based on records and equation (14) (Tate \& Klett, 1959); see Table 2 for results.

Theoretical results of the study are explained numerically by simulation in the following ways: Table 1 shows that an informative Bayesian estimation based on records under squared error loss function has the lowest MSE compared to the informative Bayesian estimation, which is based on records under a quadratic loss function with a noninformative Bayesian estimation under a squared error loss function.. This is also compared to a MLE based on records; comparisons are shown in Figure 1. Confidence intervals and their lengths for record numbers $4,5,6,7,8$ and confidence levels $90 \%, 95 \%$ and $99 \%$ were obtained. The longer the $n$, the shorter the interval distance (see Table 3). Comparing Tables 2 and 3, it the point at which HPD estimations have a shorter length than the confidence interval with optimal length is observed. This comparison is illustrated in Figures 2, 3 and 4 for various confidence levels; Figure 5 shows the comparison for all levels. 
Acknowledgments

The authors are thankful to the referees and editor for their valuable comments. This work was partially supported by the Tehran Payame Noor University (PNU) through a grant.

\section{References}

Ahmadia, J., Jozanib, M. J., Marchandc, E., \& Parsian, A. (2009). Bayes estimation based on $k$-record data from a general class of distributions under balanced type loss functions. Journal of Statistical Planning and Inference, 139, 1180-1189.

Ahmed A., Soliman, A. H., Abd Ellah, K. S. Sultan. (2006). Comparison of estimates using record statistics from Weibull model: Bayesian and non-Bayesian approaches. Computational Statistics \& Data Analysis, 51, 2065-2077.

Ahsanullah, M. (1991). Record values of the Lomax distribution. Statistica Neerlandica, 45, 21-29.

Ahsanullah, M. (1998). Introduction to record values. Needham Heights, MA: Ginn Press.

Ahsanullah, M., \& Holland, B. (1994). On the use of record values to estimate the location and scale parameters of the generalized extreme value distribution. The Indian Journal of Statistics, 56, 480-499.

Arnold, B. C., Balakrishnan, N., \& Nagaraja, H. N. (1998). Records. Canada: Wiley.

Asgharzadeh, A. (2009). On Bayesian estimation from exponential distribution based on records. Journal of the Korean Statistical Society, 38, 125-130.

Baklizi, A. (2008). Likelihood and Bayesian estimation of $\operatorname{Pr}(\mathrm{X}<\mathrm{Y})$ using lower record values from the generalized exponential distribution. Computational Statistics and Data Analysis, 52, 3468-3473.
Balakrishnan, M. (1994). Relations for single and product moments of record values from Lomax distribution. The Indian Journal of Statistics, 56, 140-146.

Berger, J. O. (1985). Statistical decision theory and Bayesian analysis. New York, Springer.

Campbell, G., \& Ratnaparkhi, M. V. (1993). An application of Lomax distribution in receiver operating characteristic (ROC) curve analysis. Communications in Statistics, 22, 1681-1687.

Chan, P. S. (1998). Interval estimation of location and scale parameters based on record values, Statistics \& Probability Letters, 37, 4958.

Mohammad, Z. R. (2002). Inferences for generalized exponential distribution Based on record statistics. Journal of Statistical Planning and Inference, 104, 339-350.

Nevzorov, V. B. (2001). Records: Mathematical theory. USA: American Mathematical Society.

Pearson, K. (1894). Contributions to the mathematical theory of evolution. Phil. Trans. Royal Soc.

Sevgi, Y. O., Mohammad, A., Fazil A. A., \& Funda, A. (2005).Switching record and order statistics via random contractions. Statistics \& Probability Letters, 73, 207-217.

Soliman, A. A., \& Al-Abound, F. M. (2008). Bayesian inference using record values from Rayleigh model with application. European Journal of Operational Research, 185, 659-672.

Tate, \& Klett. (1959). Optimal confidence intervals for the variance of a normal distribution. Journal of the American Statistical Association, 54(287), 674-682. 


\title{
Inverted Exponential Distribution Under a Bayesian Viewpoint
}

\author{
Gyan Prakash \\ S. N. Medical College, Agra, \\ U. P., India
}

The objective of this study was to examine the properties of Bayes estimators of the parameter, reliability function and hazard rate under the symmetric and asymmetric loss functions for the inverted exponential model. The Bayes predictive interval and the Bayes estimate of shift point are also determined. A simulation study was carried out to study the properties of the Bayes estimators.

Key words: Bayes estimators, LINEX loss function, squared error loss function, prediction limits.

\section{Introduction}

The exponential distribution is frequently used in lifetime data analysis, but its suitability is restricted to constant hazard (failure) rates. For situations where a failure rate is monotonically increasing or decreasing, the two-parameter Weibull and the Gamma distributions are popular for analyzing lifetime data. Both distributions have increasing and decreasing hazard rates depending on the shape parameter. However, one of the major disadvantages of the Gamma distribution is that its distribution and survival functions cannot be expressed in a closed form if the shape parameter is not an integer. Moreover, there are terms involving the incomplete Gamma function, thus, it is necessary to obtain distribution, survival or hazard functions by numerical integration. This makes the Gamma distribution less popular compared to the Weibull distribution, which has a closed form for the hazard and survival functions, but the Weibull distribution also has disadvantages. Bain \& Engelhardt (1991) demonstrated that the maximum likelihood estimators of the Weibull distribution might not behave properly for all parametric ranges.

Recently two new distributions have been introduced: the generalized Exponential (two - parameter) and the inverted Exponential (one - parameter) distributions. The generalized

Gyan Prakash is in the Department of Community Medicine. Email him at: ggyanji@yahoo.com. exponential distribution can be used effectively in situations where a skewed distribution is needed. Gupta \& Kundu (1999, 2002) and Raqab \& Ahsanullah (2001) investigated several properties of the two parameter generalized exponential distribution.

It is remarkable that most of the Bayesian inference procedures have been developed with the usual squared error loss function (SELF), which is symmetrical and associates equal importance to losses due to overestimation and underestimation of equal magnitude. However, such a restriction may be unrealistic in the most situations of practical importance. For example, in estimating reliability and hazard rate functions, an overestimation is usually much more serious than an underestimation. The use of a symmetrical loss function in Bayesian framework might be inappropriate (Parsian \& Kirmani, 2002).

A useful asymmetric loss function known as the LINEX loss function (LLF) was introduced by Varian (1975) and has been used in several studies. The LLF for any parameter $\theta$ is given by

$$
\mathrm{L}(\bar{\Delta})=\mathrm{e}^{\mathrm{a} \bar{\Delta}}-\mathrm{a} \bar{\Delta}-1 ; \bar{\Delta}=\hat{\theta}-\theta
$$

where $\mathrm{a}(\neq 0)$ is the shape parameter and $\hat{\theta}$ is any estimate of the parameter $\theta$.

The sign and magnitude of ' $a$ ' represents the direction and degree of 
asymmetry respectively. The positive (negative) value of ' $a$ ' is used when overestimation is more (less) serious than underestimation. The LLF (1.1) is approximately squared error and almost symmetric if $|\mathrm{a}|$ is near zero. Many authors have discussed estimation procedures under a LLF criterion, however a few recently presented studies using Bayesian and/or LLF criterions, for example see Xu \& Shi (2004), Ahmadi, et al. (2005), Son \& Oh (2006), Singh, et al. (2007) and Prakash (2011).

Present article examine the properties of Bayes estimators for the $\mathrm{r}^{\text {th }}$ power of the parameter $\theta$, reliability function, hazard rate and the shift point. Both the symmetric (SELF) and asymmetric (LLF) loss functions were considered and the behavior of the future observations is predicted in terms of the predictive interval.

The Model and the Prior Distributions

The model considered is the inverted Exponential distribution with a distribution function

$$
\mathrm{F}(\mathrm{x} ; \theta)=\mathrm{e}^{-1 / \mathrm{x} \theta} ; \mathrm{x}>0, \theta>0 .
$$

This distribution has no finite moments. The reliability function and hazard rate for a specific mission time $t(>0)$ are obtained as

$$
\psi(\mathrm{t})=1-\mathrm{e}^{-1 / t \theta}
$$

and

$$
\rho(\mathrm{t})=\frac{1}{\mathrm{t}^{2} \theta}\left(\mathrm{e}^{\mathrm{i} / \mathrm{t} \theta}-1\right)^{-1}
$$

If $\mathrm{x}_{1}, \mathrm{x}_{2}, \ldots, \mathrm{x}_{\mathrm{n}}$ are $\mathrm{n}$ independent random samples from model (2.1), then the likelihood function is obtained as

$$
\begin{aligned}
& \mathrm{L}\left(\mathrm{x}_{1}, \mathrm{x}_{2}, \ldots, \mathrm{x}_{\mathrm{n}} \mid \theta\right)=\frac{1}{\theta^{2}} \prod_{\mathrm{i}=1}^{\mathrm{n}} \mathrm{x}_{\mathrm{i}}^{-2} \exp \left(-\frac{\mathrm{T}_{\mathrm{n}}}{\theta}\right), \\
& \mathrm{T}_{\mathrm{n}}=\left\{\sum_{\mathrm{i}=1}^{\mathrm{n}} \mathrm{x}_{\mathrm{i}}^{-1}\right\} .
\end{aligned}
$$

The maximum likelihood estimate (MLE) of the parameter $\theta$ is $\hat{\theta}=\frac{1}{n} T_{n}$. Further, $\mathrm{x}_{\mathrm{i}}^{-1} ; \mathrm{i}=1,2, \quad \ldots, \mathrm{n}$ are iid Exponential with parameter $\theta$, and the distribution of $T_{n}$ is a Gamma distribution with a probability density function (pdf)

$$
f\left(T_{n}\right)=\frac{T_{n}^{n-1}}{\Gamma n} e^{-\frac{T_{n}}{\theta}} \theta^{-n} ; T_{n}>0 .
$$

It is assumed that, from a Bayesian viewpoint, there is clearly no way in which it can be stated that one prior is better than another (Arnold \& Press, 1983). More frequently the case is that attention to a given flexible family of priors is restricted and a prior is chosen from that family. Thus, in present case the conjugate prior of the parameter $\theta$ is considered as inverted Gamma distribution and is given as:

$$
\mathrm{g}_{1}(\theta)=\frac{\beta^{\alpha}}{\Gamma \alpha} \theta^{-(\alpha+1)} \mathrm{e}^{-\frac{\beta}{\theta}} ; \alpha>0, \beta>0
$$

Further, in a situation where a researcher has no or very little prior information about the parameter $\theta$, a family of priors defined as

$$
\mathrm{g}_{2}(\theta)=\theta^{-\delta} ; \delta>0
$$

If $\delta=0$ a diffuse prior is obtained, and if $\delta=1$ a non-informative prior is obtained. The posterior density of $\theta$ under the prior $\mathrm{g}_{1}(\theta)$ is given by

$$
Z_{1}(\theta)=\frac{\left(T_{n}+\beta\right)^{\alpha+n}}{\Gamma(\alpha+n)} e^{-\frac{\left(T_{n}+\beta\right)}{\theta}} \theta^{-(\alpha+n+1)}
$$

This is an inverted Gamma distribution with parameters $(\alpha+n)$ and $\left(T_{n}+\beta\right)$. Similarly, the posterior density of $\theta$ corresponding to $g_{2}(\theta)$ is 


$$
Z_{2}(\theta)=\frac{\left(T_{n}\right)^{\delta+n-1}}{\Gamma(\delta+n-1)} e^{-\frac{T_{n}}{\theta}} \theta^{-(\delta+n)} .
$$

Bayes Prediction Limits

Predicting the nature of the future behavior of an observation when sufficient information regarding the past and present behavior of an event or an observation are known or given is an important problem in lifetime models. Statistical prediction limits have many applications in quality control and reliability problems and the determination of these limits has been extensively investigated. It may be desirable to obtain confidence limits not only for any parameter of a distribution, but also for a future observation drawn from the same model. Such limits are called prediction limits.

If a $100 \varepsilon \%$ prediction limit for an additional observation is desired, for example $\mathrm{Y}$, given a random sample $\underline{\mathrm{X}}=\left(\mathrm{x}_{1}, \mathrm{x}_{2}, \ldots, \mathrm{x}_{\mathrm{n}}\right)$ from model (2.1), the problem is equivalent to determining the region $\mathrm{R}(\underline{\mathrm{X}})$ such that $\mathrm{R}(\underline{\mathrm{X}})$ covers the average proportion $\varepsilon$ of the distribution of $\mathrm{Y}$.

A wealth of literature is available regarding predictive inference for future failure distributions; examples of studies involving predictive inference for future observations include: Aitchison \& Dunsmore (1975), Bain (1978), Sinha (1990), Raqab (1997), Cramer \& Kamps (1998), Raqab \& Madi (2002), Ahmed et al. (2007) and Prakash \& Prasad (2010).

In the context of prediction, it may be stated that $(l, u)$ is a $100(1-\varepsilon) \%$ prediction interval for a future observation $\mathrm{Y}$ if

$$
\operatorname{Pr}(l \leq \mathrm{Y} \leq u)=1-\varepsilon
$$

where $l$ and $u$ are the lower and upper prediction limits for the random variable $\mathrm{Y}$, and $1-\varepsilon$ is termed the confidence prediction coefficient.

The predicative distribution of a future observation $\mathrm{Y}$ may be obtained from model (2.1) by simplifying

$$
\begin{gathered}
\mathrm{h}(\mathrm{y} \mid \underline{\mathrm{X}})=\int_{\theta} \mathrm{f}(\mathrm{y} ; \theta) \cdot \mathrm{Z}_{1}(\theta) \mathrm{d} \theta \\
\Rightarrow \mathrm{h}(\mathrm{y} \mid \underline{\mathrm{X}})=(\mathrm{n}+\alpha) \mathrm{y}^{-2} \frac{\left(\mathrm{T}_{\mathrm{n}}+\beta\right)^{\mathrm{n}+\alpha}}{\left(\mathrm{T}_{\mathrm{n}}+\beta+\mathrm{y}^{-1}\right)^{\mathrm{n}+\alpha+1}},
\end{gathered}
$$

and $100(1-\varepsilon) \%$ equal tail prediction interval is obtained by solving

$$
\int_{0}^{l} \mathrm{~h}(\mathrm{y} \mid \underline{\mathrm{X}}) \mathrm{dy}=\int_{u}^{\infty} \mathrm{h}(\mathrm{y} \mid \underline{\mathrm{X}}) \mathrm{dy}=\frac{\varepsilon}{2} .
$$

Hence, the Bayes prediction limits and length of the Bayes predictive interval are obtained as

$$
\begin{gathered}
l=\left[\left(\mathrm{T}_{\mathrm{n}}+\beta\right)\left\{\left(\frac{\varepsilon}{2}\right)^{-1 /(\alpha+\mathrm{n})}-1\right\}\right]^{-1}, \\
u=\left[\left(\mathrm{T}_{\mathrm{n}}+\beta\right)\left\{\left(1-\frac{\varepsilon}{2}\right)^{-1 /(\alpha+\mathrm{n})}-1\right\}\right]^{-1}
\end{gathered}
$$

and

$$
\mathrm{I}=u-l
$$

Bayes Estimators for Reliability Function and Hazard Rate

The Bayes estimates of $\psi(\mathrm{t})$ and $\rho(\mathrm{t})$ under the SELF corresponding to the posterior $\mathrm{Z}_{1}(\theta)$ are obtained as

$$
\psi_{1}=\mathrm{E}_{\mathrm{P}}(\psi(\mathrm{t}))=1-\left(1+\frac{1}{\mathrm{t}\left(\mathrm{T}_{\mathrm{n}}+\beta\right)}\right)^{-(\alpha+\mathrm{n})}
$$

and

$$
\begin{aligned}
& \rho_{1}=\mathrm{E}_{\mathrm{P}}(\rho(\mathrm{t}))=\frac{1}{\mathrm{t}^{2}} \mathrm{I}\left(0, \infty, \rho_{\mathrm{S} 1}\right) ; \\
& \rho_{\mathrm{S} 1}=\mathrm{z}\left(\mathrm{e}^{\mathrm{z} / \mathrm{t}}-1\right)^{-1},
\end{aligned}
$$


where

$$
I\left(z_{1}, z_{2}, f_{z}\right)=\frac{\left(T_{n}+\beta\right)^{\alpha+n}}{\Gamma(\alpha+n)} \int_{z_{1}}^{z_{2}} f_{z} \cdot e^{-\left(T_{n}+\beta\right) z} z^{\alpha+n-1} d z .
$$

Here $f_{z}$ is a function of $\mathrm{z}$ and suffix $\mathrm{P}$ indicates the expectation taken under posterior density.

Similarly, the Bayes estimators of the reliability function and hazard rate under the LLF-criterion corresponding to the posterior $\mathrm{Z}_{1}(\theta)$ are obtained by solving

$$
\begin{aligned}
\psi_{2} & =-\frac{1}{\mathrm{a}} \ln \mathrm{E}_{\mathrm{P}}\left(\mathrm{e}^{-\mathrm{a} \psi(\mathrm{t})}\right) \\
& =-\frac{1}{\mathrm{a}} \ln \left(\mathrm{e}^{-\mathrm{a}} \mathrm{I}\left(0, \infty, \psi_{\mathrm{L} 1}\right)\right), \\
\psi_{\mathrm{L} 1} & =\exp \left(\mathrm{ae}^{-\mathrm{z} / \mathrm{t}}\right)
\end{aligned}
$$

and

$$
\begin{aligned}
\rho_{2} & =-\frac{1}{\mathrm{a}} \ln \mathrm{E}_{\mathrm{P}}\left(\mathrm{e}^{-\mathrm{a} \rho(\mathrm{t})}\right) \\
& =-\frac{1}{\mathrm{a}} \ln \mathrm{I}\left(0, \infty, \rho_{\mathrm{L} 1}\right), \\
\rho_{\mathrm{L} 1} & =\exp \left(\frac{\mathrm{a}}{\mathrm{t}^{2}} \rho_{\mathrm{S} 1}\right) .
\end{aligned}
$$

The expressions of the risks for these estimators under the SELF and the LLF loss criterions are $\mathrm{R}_{(\mathrm{S})}\left(\psi_{\mathrm{i}}\right), \mathrm{R}_{(\mathrm{L})}\left(\psi_{\mathrm{i}}\right), \mathrm{R}_{(\mathrm{S})}\left(\rho_{\mathrm{i}}\right)$ and $\mathrm{R}_{(\mathrm{L})}\left(\rho_{\mathrm{i}}\right) ; \mathrm{i}=1,2$. Note that these do not exist in closed form. However, a numerical study has been carried out in later section.

\section{The Bayes Estimator for Shift Point}

In order to obtain information about their endurance, manufactured items such as mechanical or electronic components, are often put to life tests and life times are observed periodically. Physical systems manufacturing different items are often subject to random fluctuations and it may happen that, at some point, there is a change in the parameter. The objective of this study was to determine when and where this change starts occurring; this is called the shift point inference problem. Bayesian modeling may play an important role in the study of such shift point problems (Broemeling \& Tsurumi, 1987; Jani \& Pandya, 1999).

Consider first a sequence of independent random sample of size $\mathrm{n}$ such as $\mathrm{X}_{1}$, $\mathrm{x}_{2}, \ldots, \mathrm{x}_{\mathrm{m}}, \mathrm{x}_{\mathrm{m}+1}, \ldots, \mathrm{x}_{\mathrm{n}}$ from model (2.1) with a reliability function $\psi_{1}(\mathrm{t})$ at mission time $t(>0)$. If it is later found that there was a change in the system at some point in time $\mathrm{m}$, this will be reflected in the sequence after $x_{m}$ by a change in the reliability $\psi_{2}(\mathrm{t})$ at mission time $\mathrm{t}$.

Thus, from model (2.1), the pdf of the random samples $\mathrm{x}_{1}, \mathrm{x}_{2}, \ldots, \mathrm{x}_{\mathrm{m}}$ of size $\mathrm{m}$ is given by

$$
\begin{aligned}
& \mathrm{f}\left(\mathrm{x}_{\mathrm{i}} ; \theta_{1}\right)=\frac{1}{\theta_{1} \mathrm{x}_{\mathrm{i}}^{2}} \exp \left(-\frac{1}{\theta_{1} \mathrm{x}_{\mathrm{i}}}\right) ; \\
& \mathrm{i}=1,2, \ldots, \mathrm{m}, \theta_{1}>0 .
\end{aligned}
$$

Similarly, the remaining $\mathrm{x}_{\mathrm{m}+1}, \mathrm{x}_{\mathrm{m}+2}, \ldots, \mathrm{x}_{\mathrm{n}}$ components of size $(\mathrm{n}-\mathrm{m})$ follow model (2.1) with the pdf

$$
\begin{aligned}
& \mathrm{f}\left(\mathrm{x}_{\mathrm{i}} ; \theta_{2}\right)=\frac{1}{\theta_{2} \mathrm{x}_{\mathrm{i}}^{2}} \exp \left(-\frac{1}{\theta_{2} \mathrm{x}_{\mathrm{i}}}\right) \\
& \mathrm{i}=\mathrm{m}+1, \mathrm{~m}+2, \ldots, \mathrm{n}, \theta_{2}>0 .
\end{aligned}
$$

If prior information regarding the parameter is considered as the conjugate prior, then prior $g_{1}(\theta)$ is redefined as

$$
\begin{aligned}
& g_{3}\left(\theta_{\mathrm{i}}\right)=\frac{\beta^{\alpha}}{\Gamma \alpha} \theta_{\mathrm{i}}^{-(\alpha+1)} \mathrm{e}^{-\frac{\beta}{\theta_{\mathrm{i}}}} \\
& \alpha>0, \beta>0, \mathrm{i}=1,2 .
\end{aligned}
$$

Further, the prior distribution for shift point $\mathrm{mis}$ considered to be discrete uniform over the set $\{1,2, \ldots, n-1\}$. Hence, the joint posterior density for the parameters $\theta_{1}, \theta_{2}$ and $\mathrm{m}$ is 


$$
\begin{aligned}
& \mathrm{Z}_{3}\left(\theta_{1}, \theta_{2}, \mathrm{~m}\right)= \\
& \mathrm{k}^{-1} \exp \left(-\frac{\omega_{1}}{\theta_{1}}-\frac{\omega_{2}}{\theta_{2}}\right) \theta_{1}^{-\mathrm{m}-\alpha-1} \theta_{2}^{-\mathrm{n}+\mathrm{m}-\alpha-1}
\end{aligned}
$$

where

$$
\begin{gathered}
\mathrm{k}^{-1}=\sum_{\mathrm{m}=1}^{\mathrm{n}-1} \Delta, \\
\Delta=\left(\frac{\Gamma(\mathrm{m}+\alpha) \Gamma(\mathrm{n}-\mathrm{m}+\alpha)}{\omega_{1}^{\mathrm{m}+\alpha} \omega_{2}^{\mathrm{n}-\mathrm{m}+\alpha}}\right), \\
\omega_{1}=\beta+\sum_{\mathrm{i}=1}^{\mathrm{m}} \mathrm{x}_{\mathrm{i}}^{-1}
\end{gathered}
$$

and

$$
\omega_{2}=\beta+\sum_{i=m+1}^{n} x_{i}^{-1}
$$

This case may be verified without considering shift point situations with $\theta_{1}=\theta_{2}$.

The marginal posterior density for shift point $\mathrm{mis}$

$$
\mathrm{Z}_{4}(\mathrm{~m})=\mathrm{k}^{-1} \Delta
$$

Therefore, the Bayes estimator for shift point $\mathrm{m}$ under the SELF and LLF are obtained respectively as (suffixes $\mathrm{S}$ and Lindicates the loss criterion selected as the SELF and LLF respectively)

$$
\hat{\mathrm{m}}_{\mathrm{S}}=\mathrm{k}^{-1} \sum_{\mathrm{m}=1}^{\mathrm{n}-1}(\mathrm{~m} \Delta)
$$

and

$$
\hat{\mathrm{m}}_{\mathrm{L}}=-\frac{1}{\mathrm{a}} \ln \left\{\mathrm{k}^{-1} \sum_{\mathrm{m}=1}^{\mathrm{n}-1}\left(\mathrm{e}^{-\mathrm{am}} \Delta\right)\right\} .
$$

If no further information regarding $\theta_{\mathrm{i}} ; \mathrm{i}=1,2$ is available and they are assumed as a priori independent random variables, then the noninformative prior is considered from (2.7) with $(\delta=1)$ such that

$$
\mathrm{g}_{4}\left(\theta_{\mathrm{i}}\right)=\frac{1}{\theta_{\mathrm{i}}} ; \mathrm{i}=1,2,
$$

The Bayes estimators for shift point $\mathrm{m}$ under SELF and LLF are obtained from (5.6) by replacing $\beta=0=\alpha$ as:

$$
\hat{\mathrm{m}}_{\mathrm{S}}^{\prime}=\mathrm{k}_{1}^{-1} \sum_{\mathrm{m}=1}^{\mathrm{n}-1}\left(\mathrm{~m} \Delta^{\prime}\right)
$$

and

$$
\hat{\mathrm{m}}_{\mathrm{L}}^{\prime}=-\frac{1}{\mathrm{a}} \ln \left\{\mathrm{k}_{1}^{-1} \sum_{\mathrm{m}=1}^{\mathrm{n}-1}\left(\mathrm{e}^{-\mathrm{am}} \Delta^{\prime}\right)\right\} .
$$

where

$$
\mathrm{k}_{1}^{-1}=\sum_{\mathrm{m}=1}^{\mathrm{n}-1} \Delta^{\prime}
$$

$$
\Delta^{\prime}=\frac{\Gamma \mathrm{m} \Gamma(\mathrm{n}-\mathrm{m})}{\omega_{3}^{\mathrm{m}} \omega_{4}^{\mathrm{n}-\mathrm{m}}}
$$

$$
\omega_{3}=\sum_{i=1}^{m} x_{i}^{-1}
$$

and

$$
\omega_{4}=\sum_{i=m+1}^{n} x_{i}^{-1}
$$

The Bayes Estimator for Parameter $\theta$

The Bayes estimator for $\theta^{r}$ ( $r$ being any integer) obtained corresponding to the posterior $Z_{1}(\theta)$ under the SELF is

$$
\hat{\theta}_{\mathrm{S}}^{\mathrm{r}}=\frac{\Gamma(\mathrm{n}+\alpha-\mathrm{r})}{\Gamma(\mathrm{n}+\alpha)}\left(\mathrm{T}_{\mathrm{n}}+\beta\right)^{\mathrm{r}} .
$$

In particular, the Bayes estimators for the parameters $\theta(\mathrm{r}=1)$ and $\frac{1}{\theta}(\mathrm{r}=-1)$ are given respectively as

$$
\hat{\theta}_{\mathrm{S}}=\frac{\mathrm{T}_{\mathrm{n}}+\beta}{\mathrm{n}+\alpha-1}
$$

and 


$$
\hat{\theta}_{\mathrm{S}}^{-1}=\frac{\mathrm{n}+\alpha}{\mathrm{T}_{\mathrm{n}}+\beta}
$$

Similarly, the Bayes estimator for $\theta^{r}$ under the LLF is obtained with respect to the posterior $\mathrm{Z}_{1}(\theta)$ by solving

$$
\hat{\theta}_{\mathrm{L}}^{\mathrm{r}}=-\frac{1}{\mathrm{a}} \ln \mathrm{I}\left(0, \infty, \mathrm{e}^{-\mathrm{a} \theta^{\mathrm{r}}}\right)
$$

The Bayes estimator for parameter $\theta$ does not exist in a closed form. However, the Bayes estimator for $\frac{1}{\theta}$ is given as

$$
\hat{\theta}_{\mathrm{L}}^{-1}=\left(\frac{\mathrm{n}+\alpha}{\mathrm{a}}\right) \ln \left(1+\frac{\mathrm{a}}{\mathrm{T}_{\mathrm{n}}+\beta}\right) .
$$

Note that, all results discussed thus hold for the posterior distribution $Z_{2}(\theta)$ if $\alpha(=\delta-1)$ and $\beta(=0)$ are substituted.

Numerical Analysis

To assess and study the properties of the proposed Bayes estimators and prediction interval, the random samples are generated as follows:

1. For the given values of prior parameters $\alpha$ and $\beta$, generate $\theta$ using the prior density $\mathrm{g}_{1}(\theta)$. The values of $\alpha$ and $\beta$ are chosen to maintain the prior variance at 1.00 and the considered values are $(\beta, \alpha)=(02,03)$, $(10,06)$ and $(30,11)$.

2. Using $\theta$ obtained in (1), generate 10,000 random samples size $n=5,10,15$ from the considered model (2.1).

\section{Bayes Prediction Interval}

The Bayes prediction intervals were obtained with the level of significance $\varepsilon=99 \%, 95 \%, 90 \%$ and results are presented in Table 1. The intervals tend to be wider as the sample size $n$ increases when other parametric values are fixed. The opposite trend was observed when a combination of the prior parameters increases. It is also noted that when the confidence level decreases the intervals also decrease.

Bayes Estimators for Reliability Function

Results for $\mathrm{a}=0.50,1.00,1.50$ and $\mathrm{t}=2.50$ (hours) are presented in Table 2. As Table 2 shows, the risk of Bayes estimators $\psi_{1}$ and $\psi_{2}$ decrease as sample size $\mathrm{n}$ increases under both loss criteria, SELF and LLF. In addition, the risk of $\psi_{1}$ increases as 'a' increases under a LLF loss criterion. A similar trend was observed for $\psi_{2}$ when 'a' increases under both loss criteria. A decreasing trend in risk was observed when a set of prior parameters increased only for $\psi_{1}$ under both loss criterions with other fixed parametric values. The magnitude of the risk is nominal for both estimators under the LLF.

Bayes Estimators for Hazard Rate

The numerical findings are presented in Table 3 for a similar set of values of ' $a$ ' and $t$. The performances of Bayes estimators $\rho_{1}$ and $\rho_{2}$ are similar to the Bayes estimators of the reliability functions $\psi_{1}$ and $\psi_{2}$ when sample size $\mathrm{n}$ or ' $\mathrm{a}$ ' increase respectively. The magnitude of the risk is nominal for both estimators under the LLF loss criterion.

Bayes Estimators for Shift Point

For a similar set of values considered earlier with $\mathrm{a}=0.25,0.50,1.00,1.50$, samples were generated for $\mathrm{n}=10,15,20$ and results are presented in Tables 4 and 5. It was observed that, when sample size $\mathrm{n}$ increases, the magnitude of the Bayes estimator (under SELF) increases but the increment in magnitude is nominal (robust). Further, an opposite trend was observed when values of the set of prior parameters increase. Similar properties have been noted for the Bayes estimate of the shift point under LLF, and a decreasing trend in the 
magnitude of the estimate has also been observed when 'a' increases.

Bayes Estimators for the $r^{\text {th }}$ Power of the Parameter

The numerical findings presented in Tables 6 and 7 are for $\mathrm{a}=0.50,1.00,1.50$ and $\mathrm{r}= \pm 1, \pm 2$. Based on results show in the tables it may be concluded that the magnitude of the risk increases (decreases) when $a(n)$ increases when other parametric values are fixed. The increasing trend in the magnitude has also been observed when prior parameters increase (only for $r=-1,-2)$. Further, the magnitude of the risk is smaller for these estimators under the LLF.

\section{References}

Ahmad, A. A., Mohammad, Z. R., \& Mohamed, T. M. (2007). Bayesian prediction intervals for the future order statistics from the generalized Exponential distribution. JIRSS, $6(1), 17-30$.

Ahmadi, J., Doostparast, M., \& Parsian, A. (2005). Estimation and prediction in a twoparameter Exponential distribution Based on $\mathrm{k}-$ record values under LINEX loss function. Communications in Statistics - Theory and Methods, 34, 795-805.

Aitchison, J., \& Dunsmore, I. R. (1975).Statistical prediction analysis. Cambridge, MA: Cambridge University Press.

Table 1: Bayes Prediction Limits

\begin{tabular}{|c|c|c|c|c|c|c|c|}
\hline \multirow[b]{2}{*}{$\mathrm{n}$} & \multirow[b]{2}{*}{$\beta, \alpha$} & \multicolumn{2}{|c|}{$\varepsilon=99 \%$} & \multicolumn{2}{|c|}{$\varepsilon=95 \%$} & \multicolumn{2}{|c|}{$\varepsilon=90 \%$} \\
\hline & & $l$ & $u$ & $l$ & $u$ & $l$ & $u$ \\
\hline \multirow{3}{*}{05} & 02,03 & 1.2272 & 1.2648 & 1.2662 & 1.4722 & 1.2825 & 1.7349 \\
\hline & 10,06 & 0.8442 & 0.8697 & 0.9292 & 1.0784 & 0.8212 & 1.1070 \\
\hline & 30,11 & 0.5941 & 0.6118 & 0.5458 & 0.6326 & 0.5265 & 0.7077 \\
\hline \multirow{3}{*}{10} & 02,03 & 2.2409 & 2.3083 & 2.0216 & 2.3447 & 1.3851 & 1.8645 \\
\hline & 10,06 & 1.3329 & 1.3727 & 1.2600 & 1.4604 & 1.0571 & 1.4209 \\
\hline & 30,11 & 0.8032 & 0.8271 & 0.7453 & 0.8631 & 0.6920 & 0.9287 \\
\hline \multirow{3}{*}{15} & 02,03 & 2.0570 & 2.1284 & 2.7406 & 3.1751 & 1.7060 & 2.2914 \\
\hline & 10,06 & 1.4661 & 1.5097 & 1.4084 & 1.6311 & 1.3485 & 1.8098 \\
\hline & 30,11 & 0.8807 & 0.9068 & 0.9548 & 1.1052 & 0.7790 & 1.0445 \\
\hline
\end{tabular}


Table 2: Risks for Bayes Estimate of Reliability Function

\begin{tabular}{|c|c|c|c|c|c|c|c|c|}
\hline $\mathrm{n}$ & $\mathrm{a}$ & $\beta, \alpha$ & $\psi_{1}$ & $\mathrm{R}_{\mathrm{S}}\left(\psi_{1}\right)$ & $\mathrm{R}_{\mathrm{L}}\left(\psi_{1}\right)$ & $\psi_{2}$ & $\mathrm{R}_{\mathrm{S}}\left(\psi_{2}\right)$ & $\mathrm{R}_{\mathrm{L}}\left(\psi_{2}\right)$ \\
\hline \multirow{9}{*}{05} & \multirow{3}{*}{0.50} & 02,03 & 0.1125 & 14.7296 & 1.0523 & 0.0123 & 14.7012 & 1.0502 \\
\hline & & 10,06 & 0.0903 & 14.7612 & 1.0541 & 0.0780 & 14.8141 & 1.0607 \\
\hline & & 30,11 & 0.0584 & 14.8300 & 1.0578 & 0.0055 & 14.2740 & 1.0266 \\
\hline & \multirow{3}{*}{1.00} & 02,03 & 0.1059 & 14.7296 & 2.8066 & 0.2646 & 14.7132 & 2.8041 \\
\hline & & 10,06 & 0.0818 & 14.7612 & 2.8106 & 0.1418 & 14.8245 & 2.8180 \\
\hline & & 30,11 & 0.0559 & 14.8300 & 2.8191 & 0.0567 & 14.5478 & 2.7834 \\
\hline & \multirow{3}{*}{1.50} & 02,03 & 0.1190 & 14.7296 & 4.6586 & 0.2088 & 14.7194 & 4.6564 \\
\hline & & 10,06 & 0.0847 & 14.7612 & 4.6647 & 0.1302 & 14.8335 & 4.6724 \\
\hline & & 30,11 & 0.0561 & 14.8300 & 4.6777 & 0.0339 & 14.6418 & 4.6416 \\
\hline \multirow{9}{*}{10} & \multirow{3}{*}{0.50} & 02,03 & 0.1827 & 5.9753 & 0.4270 & 0.8166 & 6.0077 & 0.4283 \\
\hline & & 10,06 & 0.1179 & 5.9827 & 0.4274 & 1.3328 & 5.8511 & 0.4193 \\
\hline & & 30,11 & 0.0768 & 6.0081 & 0.4288 & 0.5438 & 5.1131 & 0.3785 \\
\hline & \multirow{3}{*}{1.00} & 02,03 & 0.1348 & 5.9753 & 1.1389 & 0.2641 & 6.0863 & 1.1399 \\
\hline & & 10,06 & 0.1057 & 5.9827 & 1.1398 & 0.6477 & 5.9076 & 1.1298 \\
\hline & & 30,11 & 0.0729 & 6.0081 & 1.1429 & 0.2365 & 5.5525 & 1.0852 \\
\hline & \multirow{3}{*}{1.50} & 02,03 & 0.1668 & 5.9753 & 1.8905 & 0.0643 & 6.1807 & 1.8912 \\
\hline & & 10,06 & 0.1119 & 5.9827 & 1.8919 & 0.5157 & 5.9292 & 1.8813 \\
\hline & & 30,11 & 0.0734 & 6.0081 & 1.8967 & 0.0950 & 5.7037 & 1.8382 \\
\hline \multirow{9}{*}{15} & \multirow{3}{*}{0.50} & 02,03 & 0.1932 & 0.4478 & 0.0321 & 1.5324 & 0.4472 & 0.0372 \\
\hline & & 10,06 & 0.1434 & 0.4489 & 0.0321 & 1.0564 & 0.5884 & 0.0393 \\
\hline & & 30,11 & 0.0856 & 0.4517 & 0.0323 & 0.8032 & 0.3951 & 0.0291 \\
\hline & \multirow{3}{*}{1.00} & 02,03 & 0.2639 & 0.4478 & 0.0856 & 0.3801 & 0.4658 & 0.0913 \\
\hline & & 10,06 & 0.1468 & 0.4489 & 0.0857 & 0.3046 & 0.6159 & 0.0938 \\
\hline & & 30,11 & 0.0824 & 0.4517 & 0.0861 & 0.2365 & 0.4217 & 0.0822 \\
\hline & \multirow{3}{*}{1.50} & 02,03 & 0.2005 & 0.4478 & 0.1421 & 0.7962 & 0.4796 & 0.1480 \\
\hline & & 10,06 & 0.1315 & 0.4489 & 0.1423 & 0.2168 & 0.6929 & 0.1505 \\
\hline & & 30,11 & 0.0870 & 0.4517 & 0.1429 & 0.0306 & 0.4310 & 0.1388 \\
\hline
\end{tabular}


INVERTED EXPONENTIAL DISTRIBUTION UNDER A BAYESIAN VIEWPOINT

Table 3: Risks for Bayes Estimate of Hazard Rate

\begin{tabular}{|c|c|c|c|c|c|c|c|c|}
\hline $\mathrm{n}$ & $\mathrm{a}$ & $\beta, \alpha$ & $\rho_{1}$ & $\mathrm{R}_{\mathrm{S}}\left(\rho_{1}\right)$ & $\mathrm{R}_{\mathrm{L}}\left(\rho_{1}\right)$ & $\rho_{2}$ & $\mathrm{R}_{\mathrm{S}}\left(\rho_{2}\right)$ & $\mathrm{R}_{\mathrm{L}}\left(\rho_{2}\right)$ \\
\hline \multirow{9}{*}{05} & \multirow{3}{*}{0.50} & 02,03 & 0.1181 & 14.1954 & 1.0230 & 0.3540 & 14.1651 & 1.0208 \\
\hline & & 10,06 & 0.1238 & 14.1827 & 1.0223 & 0.0195 & 14.2119 & 1.0294 \\
\hline & & 30,11 & 0.1398 & 14.2215 & 1.0244 & 0.1485 & 13.6402 & 0.9914 \\
\hline & \multirow{3}{*}{1.00} & 02,03 & 0.1411 & 14.1954 & 2.7397 & 0.1463 & 14.1760 & 2.7368 \\
\hline & & 10,06 & 0.1216 & 14.1827 & 2.7381 & 0.3392 & 14.2227 & 2.7464 \\
\hline & & 30,11 & 0.1291 & 14.2215 & 2.7430 & 0.1184 & 13.9077 & 2.7026 \\
\hline & \multirow{3}{*}{1.50} & 02,03 & 0.1157 & 14.1954 & 4.5564 & 0.0612 & 14.1813 & 4.5534 \\
\hline & & 10,06 & 0.1148 & 14.1827 & 4.5540 & 0.2712 & 14.2315 & 4.5631 \\
\hline & & 30,11 & 0.1317 & 14.2215 & 4.5615 & 0.1289 & 13.9996 & 4.5181 \\
\hline \multirow{9}{*}{10} & \multirow{3}{*}{0.50} & 02,03 & 0.1944 & 5.7601 & 0.4152 & 0.0918 & 5.7696 & 0.4169 \\
\hline & & 10,06 & 0.0770 & 5.7694 & 0.4157 & 1.2395 & 5.6419 & 0.4077 \\
\hline & & 30,11 & 0.1676 & 5.8155 & 0.4182 & 0.3497 & 4.8807 & 0.3650 \\
\hline & \multirow{3}{*}{1.00} & 02,03 & 0.1936 & 5.7601 & 1.1119 & 0.1672 & 5.7782 & 1.1138 \\
\hline & & 10,06 & 0.0883 & 5.7694 & 1.1130 & 0.6489 & 5.6963 & 1.1031 \\
\hline & & 30,11 & 0.1692 & 5.8155 & 1.1189 & 0.1737 & 5.3099 & 1.0537 \\
\hline & \multirow{3}{*}{1.50} & 02,03 & 0.1841 & 5.7601 & 1.8493 & 0.1399 & 5.7825 & 1.8514 \\
\hline & & 10,06 & 0.0745 & 5.7694 & 1.8510 & 0.4315 & 5.7171 & 1.8404 \\
\hline & & 30,11 & 0.1730 & 5.8155 & 1.8599 & 0.0694 & 5.4577 & 1.7899 \\
\hline \multirow{9}{*}{15} & \multirow{3}{*}{0.50} & 02,03 & 0.1314 & 0.4286 & 0.0310 & 1.1623 & 0.5327 & 0.0364 \\
\hline & & 10,06 & 0.1164 & 0.4258 & 0.0308 & 0.3103 & 0.4731 & 0.0386 \\
\hline & & 30,11 & 0.1303 & 0.4386 & 0.0316 & 0.5889 & 0.3813 & 0.0283 \\
\hline & \multirow{3}{*}{1.00} & 02,03 & 0.2328 & 0.4286 & 0.0832 & 0.8862 & 0.5482 & 0.0897 \\
\hline & & 10,06 & 0.1980 & 0.4258 & 0.0828 & 0.0166 & 0.5017 & 0.0921 \\
\hline & & 30,11 & 0.1870 & 0.4386 & 0.0844 & 0.0024 & 0.4074 & 0.0804 \\
\hline & \multirow{3}{*}{1.50} & 02,03 & 0.0677 & 0.4286 & 0.1384 & 0.5436 & 0.5566 & 0.1455 \\
\hline & & 10,06 & 0.1858 & 0.4258 & 0.1379 & 0.3606 & 0.5479 & 0.1479 \\
\hline & & 30,11 & 0.1560 & 0.4386 & 0.1403 & 0.0739 & 0.4165 & 0.1360 \\
\hline
\end{tabular}


Table 4: Bayes Estimate of Shift Point Under SELF

\begin{tabular}{|c|c|c|c|c|}
\hline \multirow{2}{*}{ Prior Density } & $(\beta, \alpha) \downarrow \mathrm{n} \rightarrow$ & 10 & 15 & 20 \\
\hline \multirow{2}{*}{ Conjugate } & 02,03 & 4.9919 & 5.0069 & 5.0169 \\
\cline { 2 - 5 } & 10,06 & 4.9493 & 4.9641 & 4.9740 \\
\cline { 2 - 5 } & 30,11 & 4.4590 & 4.4724 & 4.4813 \\
\hline Non-Informative & 00,00 & 4.8946 & 4.9112 & 4.9472 \\
\hline
\end{tabular}

Table 5: Bayes Estimate of Shift Point Under LLF

\begin{tabular}{|c|c|c|c|c|c|c|}
\hline Prior Density & $\mathrm{n}$ & $(\beta, \alpha) \downarrow \mathrm{a} \rightarrow$ & 0.25 & 0.50 & 1.00 & 1.50 \\
\hline \multirow{9}{*}{ Conjugate Prior } & \multirow{3}{*}{10} & 02,03 & 4.0051 & 3.2058 & 3.1096 & 3.0163 \\
\hline & & 10,06 & 3.7951 & 3.1965 & 3.1006 & 3.0076 \\
\hline & & 30,11 & 3.7196 & 3.1806 & 3.0852 & 2.9926 \\
\hline & \multirow{3}{*}{15} & 02,03 & 4.2321 & 3.6035 & 3.4954 & 3.3905 \\
\hline & & 10,06 & 3.8554 & 3.2342 & 3.1372 & 3.0431 \\
\hline & & 30,11 & 3.7907 & 3.2191 & 3.1225 & 3.0288 \\
\hline & \multirow{3}{*}{20} & 02,03 & 4.5051 & 3.7436 & 3.6313 & 3.5224 \\
\hline & & 10,06 & 3.8615 & 3.3673 & 3.2663 & 3.1683 \\
\hline & & 30,11 & 3.8356 & 3.3249 & 3.2252 & 3.1284 \\
\hline \multirow{3}{*}{ Non-Informative Prior } & 10 & 00,00 & 3.9477 & 3.2412 & 3.1440 & 3.0497 \\
\hline & 15 & 00,00 & 4.0255 & 4.0184 & 3.9657 & 3.8467 \\
\hline & 20 & 00,00 & 4.0926 & 4.0783 & 4.0424 & 3.9502 \\
\hline
\end{tabular}


INVERTED EXPONENTIAL DISTRIBUTION UNDER A BAYESIAN VIEWPOINT

Table 6: Risks for the Bayes Estimate of $\theta^{\mathrm{r}}$

\begin{tabular}{|c|c|c|c|c|c|c|c|c|c|c|}
\hline \multirow[b]{2}{*}{$\mathrm{n}$} & \multirow{2}{*}{$\mathrm{a}$} & \multirow{2}{*}{$\beta, \alpha$} & \multicolumn{4}{|c|}{$r=-2$} & \multicolumn{4}{|c|}{$r=2$} \\
\hline & & & $\mathrm{R}_{(\mathrm{S})}\left(\hat{\theta}_{\mathrm{S}}^{\mathrm{r}}\right)$ & $\mathrm{R}_{(\mathrm{L})}\left(\hat{\theta}_{\mathrm{S}}^{\mathrm{r}}\right)$ & $\mathrm{R}_{(\mathrm{S})}\left(\hat{\theta}_{\mathrm{L}}^{\mathrm{r}}\right)$ & $\mathrm{R}_{(\mathrm{L})}\left(\hat{\theta}_{\mathrm{L}}^{\mathrm{r}}\right)$ & $\mathrm{R}_{(\mathrm{S})}\left(\hat{\theta}_{\mathrm{S}}^{\mathrm{r}}\right)$ & $\mathrm{R}_{(\mathrm{L})}\left(\hat{\theta}_{\mathrm{S}}^{\mathrm{r}}\right)$ & $\mathrm{R}_{(\mathrm{S})}\left(\hat{\theta}_{\mathrm{L}}^{\mathrm{r}}\right)$ & $\mathrm{R}_{(\mathrm{L})}\left(\hat{\theta}_{\mathrm{L}}^{\mathrm{r}}\right)$ \\
\hline \multirow{9}{*}{05} & \multirow{3}{*}{0.50} & 02,03 & 13.288 & 0.9700 & 13.343 & 0.9726 & 10.285 & 4.5467 & 1.6360 & 1.9821 \\
\hline & & 10,06 & 13.719 & 0.9961 & 13.804 & 1.0061 & 11.384 & 7.0496 & 0.7671 & 1.5262 \\
\hline & & 30,11 & 14.246 & 1.0258 & 13.706 & 0.9949 & 14.270 & 9.1108 & 2.0452 & 2.9854 \\
\hline & \multirow{3}{*}{1.00} & 02,03 & 13.288 & 2.6178 & 13.440 & 2.6363 & 10.285 & 6.0364 & 2.3876 & 2.5377 \\
\hline & & 10,06 & 13.719 & 2.6776 & 13.816 & 2.6959 & 11.384 & 9.8003 & 1.6753 & 2.2181 \\
\hline & & 30,11 & 14.246 & 2.7460 & 13.981 & 2.7115 & 14.270 & 9.7628 & 5.7040 & 3.6989 \\
\hline & \multirow{3}{*}{1.50} & 02,03 & 13.288 & 4.4315 & 13.518 & 4.4154 & 10.285 & 8.4251 & 7.7043 & 2.8013 \\
\hline & & 10,06 & 13.719 & 4.4615 & 13.859 & 4.4899 & 11.384 & 11.112 & 6.9306 & 2.4745 \\
\hline & & 30,11 & 14.246 & 4.5661 & 14.082 & 4.5336 & 14.270 & 10.550 & 5.9482 & 4.2190 \\
\hline \multirow{9}{*}{10} & \multirow{3}{*}{0.50} & 02,03 & 5.4663 & 0.3986 & 5.4705 & 0.3995 & 3.9248 & 0.9999 & 0.1222 & 0.0180 \\
\hline & & 10,06 & 5.5413 & 0.4029 & 5.3734 & 0.3929 & 3.6741 & 0.9096 & 0.2512 & 0.0331 \\
\hline & & 30,11 & 5.7225 & 0.4131 & 4.8579 & 0.3637 & 9.1533 & 3.4073 & 0.6589 & 0.8112 \\
\hline & \multirow{3}{*}{1.00} & 02,03 & 5.4663 & 1.0738 & 5.4805 & 1.0754 & 3.9248 & 1.2036 & 0.1302 & 0.0354 \\
\hline & & 10,06 & 5.5413 & 1.0838 & 5.4371 & 1.0701 & 3.6741 & 8.8628 & 0.2709 & 0.0474 \\
\hline & & 30,11 & 5.7225 & 1.1071 & 5.2914 & 1.0512 & 9.1533 & 4.6310 & 0.6659 & 0.8903 \\
\hline & \multirow{3}{*}{1.50} & 02,03 & 5.4663 & 1.3599 & 5.4858 & 1.7949 & 3.9248 & 2.0292 & 0.1518 & 0.1044 \\
\hline & & 10,06 & 5.5413 & 1.8063 & 5.4661 & 1.7915 & 3.6741 & 9.0630 & 0.2838 & 0.0675 \\
\hline & & 30,11 & 5.7225 & 1.8419 & 5.4438 & 1.7871 & 9.1533 & 5.4232 & 0.7181 & 0.9334 \\
\hline \multirow{9}{*}{15} & \multirow{3}{*}{0.50} & 02,03 & 0.3864 & 0.0286 & 0.4302 & 0.0342 & 0.0087 & 0.0009 & 0.0368 & 0.0038 \\
\hline & & 10,06 & 0.3975 & 0.0292 & 0.4434 & 0.0364 & 0.0045 & 0.0005 & 0.0443 & 0.0045 \\
\hline & & 30,11 & 0.4208 & 0.0306 & 0.3550 & 0.0267 & 0.1127 & 0.0203 & 0.0034 & 0.0004 \\
\hline & \multirow{3}{*}{1.00} & 02,03 & 0.3864 & 0.0666 & 0.4416 & 0.0847 & 0.0087 & 0.0028 & 0.0462 & 0.0097 \\
\hline & & 10,06 & 0.3975 & 0.0791 & 0.4818 & 0.0873 & 0.0045 & 0.0017 & 0.0628 & 0.0104 \\
\hline & & 30,11 & 0.4208 & 0.0822 & 0.3807 & 0.0769 & 0.1127 & 0.1251 & 0.0045 & 0.0019 \\
\hline & \multirow{3}{*}{1.50} & 02,03 & 0.3864 & 0.1225 & 0.4565 & 0.1379 & 0.0087 & 0.0054 & 0.0525 & 0.0182 \\
\hline & & 10,06 & 0.3975 & 0.1322 & 0.5402 & 0.1406 & 0.0045 & 0.0034 & 0.0737 & 0.0178 \\
\hline & & 30,11 & 0.4208 & 0.1369 & 0.3900 & 0.1307 & 0.1127 & 0.4628 & 0.0070 & 0.0062 \\
\hline
\end{tabular}


Table 7: Risks for the Bayes Estimate of $\theta^{\mathrm{r}}$

\begin{tabular}{|c|c|c|c|c|c|c|c|c|c|c|}
\hline \multirow[b]{2}{*}{$\mathrm{n}$} & \multirow[b]{2}{*}{$\mathrm{a}$} & \multirow[b]{2}{*}{$\beta, \alpha$} & \multicolumn{4}{|c|}{$\mathrm{r}=-1$} & \multicolumn{4}{|c|}{$\mathrm{r}=1$} \\
\hline & & & $\mathrm{R}_{(\mathrm{S})}\left(\hat{\theta}_{\mathrm{S}}^{\mathrm{r}}\right)$ & $\mathrm{R}_{(\mathrm{L})}\left(\hat{\theta}_{\mathrm{S}}^{\mathrm{r}}\right)$ & $\mathrm{R}_{(\mathrm{S})}\left(\hat{\theta}_{\mathrm{L}}^{\mathrm{r}}\right)$ & $\mathrm{R}_{(\mathrm{L})}\left(\hat{\theta}_{\mathrm{L}}^{\mathrm{r}}\right)$ & $\mathrm{R}_{(\mathrm{S})}\left(\hat{\theta}_{\mathrm{S}}^{\mathrm{r}}\right)$ & $\mathrm{R}_{(\mathrm{L})}\left(\hat{\theta}_{\mathrm{S}}^{\mathrm{r}}\right)$ & $\mathrm{R}_{(\mathrm{S})}\left(\hat{\theta}_{\mathrm{L}}^{\mathrm{r}}\right)$ & $\mathrm{R}_{(\mathrm{L})}\left(\hat{\theta}_{\mathrm{L}}^{\mathrm{r}}\right)$ \\
\hline \multirow{9}{*}{05} & \multirow{3}{*}{0.50} & 02,03 & 12.002 & 0.8972 & 12.029 & 0.8983 & 2.0144 & 0.1875 & 2.3556 & 0.2156 \\
\hline & & 10,06 & 12.224 & 0.9110 & 12.332 & 0.9193 & 1.6934 & 0.1622 & 2.1786 & 0.2043 \\
\hline & & 30,11 & 12.723 & 0.9401 & 12.246 & 0.9116 & 0.7526 & 0.0784 & 0.7404 & 0.0763 \\
\hline & \multirow{3}{*}{1.00} & 02,03 & 12.002 & 2.4474 & 12.082 & 2.4583 & 2.0144 & 0.5928 & 2.7107 & 0.7636 \\
\hline & & 10,06 & 12.224 & 2.4806 & 12.333 & 2.4953 & 1.6934 & 0.5180 & 2.4282 & 0.7162 \\
\hline & & 30,11 & 12.723 & 2.5485 & 12.510 & 2.5191 & 0.7526 & 0.2664 & 1.0455 & 0.3536 \\
\hline & \multirow{3}{*}{1.50} & 02,03 & 12.002 & 4.1081 & 12.129 & 4.1356 & 2.0144 & 1.1044 & 3.0264 & 1.5302 \\
\hline & & 10,06 & 12.224 & 4.1595 & 12.337 & 4.1834 & 1.6934 & 0.9617 & 2.6799 & 1.4354 \\
\hline & & 30,11 & 12.723 & 4.2639 & 12.610 & 4.2399 & 0.7526 & 0.5180 & 1.3107 & 0.8213 \\
\hline \multirow{9}{*}{10} & \multirow{3}{*}{0.50} & 02,03 & 4.8368 & 0.3626 & 4.8418 & 0.3629 & 0.9737 & 0.0933 & 1.2338 & 0.1153 \\
\hline & & 10,06 & 4.8905 & 0.3657 & 4.7321 & 0.3559 & 0.9049 & 0.0880 & 1.1338 & 0.1057 \\
\hline & & 30,11 & 5.0740 & 0.3764 & 4.2866 & 0.3297 & 0.5090 & 0.0525 & 0.2913 & 0.0298 \\
\hline & \multirow{3}{*}{1.00} & 02,03 & 4.8368 & 0.9901 & 4.8420 & 0.9903 & 0.9737 & 0.2977 & 1.3538 & 0.3923 \\
\hline & & 10,06 & 4.8905 & 0.9976 & 4.7943 & 0.9843 & 0.9049 & 0.2833 & 1.2602 & 0.3689 \\
\hline & & 30,11 & 5.0740 & 1.0225 & 4.6961 & 0.9708 & 0.5090 & 0.1769 & 0.4723 & 0.1598 \\
\hline & \multirow{3}{*}{1.50} & 02,03 & 4.8368 & 1.6626 & 4.8461 & 1.6646 & 0.9737 & 0.5517 & 1.4573 & 0.7545 \\
\hline & & 10,06 & 4.8905 & 1.6742 & 4.8244 & 1.6604 & 0.9049 & 0.5279 & 1.3707 & 0.7208 \\
\hline & & 30,11 & 5.0740 & 1.7125 & 4.8414 & 1.6639 & 0.5090 & 0.3412 & 0.6191 & 0.3880 \\
\hline \multirow{9}{*}{15} & \multirow{3}{*}{0.50} & 02,03 & 0.3405 & 0.0259 & 0.3434 & 0.0312 & 0.1351 & 0.0121 & 0.1536 & 0.0164 \\
\hline & & 10,06 & 0.3488 & 0.0264 & 0.4113 & 0.0333 & 0.1200 & 0.0110 & 0.2155 & 0.0179 \\
\hline & & 30,11 & 0.3695 & 0.0276 & 0.3085 & 0.0239 & 0.0756 & 0.0073 & 0.0843 & 0.0080 \\
\hline & \multirow{3}{*}{1.00} & 02,03 & 0.3405 & 0.0713 & 0.3881 & 0.0778 & 0.1351 & 0.0369 & 0.1644 & 0.0427 \\
\hline & & 10,06 & 0.3488 & 0.0725 & 0.4367 & 0.0803 & 0.1200 & 0.0338 & 0.2174 & 0.0445 \\
\hline & & 30,11 & 0.3695 & 0.0754 & 0.3326 & 0.0702 & 0.0756 & 0.0236 & 0.1025 & 0.0298 \\
\hline & \multirow{3}{*}{1.50} & 02,03 & 0.3405 & 0.1203 & 0.3939 & 0.1273 & 0.1351 & 0.0659 & 0.1861 & 0.0728 \\
\hline & & 10,06 & 0.3488 & 0.1221 & 0.4865 & 0.1300 & 0.1200 & 0.0608 & 0.2617 & 0.0746 \\
\hline & & 30,11 & 0.3695 & 0.1265 & 0.3414 & 0.1205 & 0.0756 & 0.0438 & 0.1117 & 0.0578 \\
\hline
\end{tabular}




\section{INVERTED EXPONENTIAL DISTRIBUTION UNDER A BAYESIAN VIEWPOINT}

Arnold, B. C., \& Press, S. J. (1983).Bayesian inference for Pareto populations. Journal of Econometrics, 21, 287306.

Bain, L. J. (1978). Statistical analysis of reliability and life testing model. New York, NY: Marcel Dekker.

Bain, L. J., \& Engelhardt, M. (1991). Statistical analysis of reliability and life testing models: Theory and methods. New York, NY: Marcel Dekker.

Broemeling, L. D., \& Tsurumi, H. (1987). Econometrics and structural change, New York, NY: Marcel Dekker.

Cramer, E., \& Kamps, U. (1998). Sequential k-out-of-n systems with Weibull components. Economics and Quality Control, 13, 227-239.

Gupta, R. D., \& Kundu, D. (1999). Generalized exponential distribution. Australia and New Zealand Journal of Statistics, 41, 173188.

Gupta, R. D., \& Kundu, D. (2002). Generalized Exponential Distribution; Statistical Inferences, Journal of Statistical Theory and Applications, 1(1), 101-118.

Jani, P. N., \& Pandya, M. (1999). Bayes estimation of shift point in left truncated exponential sequence. Communication in Statistics-Theory and Methods, 28(11), 26232639.

Parsian, A., \& Kirmani, S. N. U. A. (2002). Estimation under LINEX loss function. In Handbook of applied econometrics and statistical inference, Aman Ullah, Alan T. K. Wan and Anoop Chaturvedi Eds., 53-76. New York, NY: Marcel Dekker.

Prakash, G. (2011). Some Bayes estimators for the repairable system. Model Assisted Statistics and Applications, 6(1), 57-62. DOI: 10.3233/MAS-2011-0173.
Prakash, G., \& Prasad, B. (2010). Bayes prediction intervals for the Rayleigh model. Model Assisted Statistics and Applications, 5(1), 43-50. DOI: 10.3233/MAS-2010-0128.

Raqab, M. Z. (1997). Modified maximum likelihood predictors of future order statistics from normal samples. Computational Statistics and Data Analysis, 25, 91-106.

Raqab, M. Z., \& Ahsanullah, M (2001). Estimation of location and scale parameter of generalized exponential distribution based on order statistics. Journal of Statistical Computation and Simulation, 69(2), 109-124.

Raqab, M. Z., \& Madi, M. T. (2002). Bayesian prediction of the total time on test using doubly censored Rayleigh Data. Journal of Statistical Computational and Simulation, 72, 781-789.

Singh, D. C., Prakash, G., \& Singh, P. (2007). Shrinkage testimators for the shape parameter of Pareto distribution using the LINEX loss function. Communication in Statistics - Theory and Methods, 36(4), 741-753.

Sinha, S. K. (1990). On the prediction limits for Rayleigh life distribution. Calcutta Statistical Association Bulletin, 39,105-109.

Son, Y. S., \& Oh, M. (2006). Bayesian estimation of the two-parameter Gamma distribution. Communications in StatisticsSimulation and Computation, 35, 285-293.

Varian, H. R. (1975). A Bayesian approach to real estate assessment. In Studies in Bayesian econometrics and statistics in honor of L.J. Savage, S. E. Feinberge and A. Zellner Eds., 195-208. Amsterdam: North Holland.

$\mathrm{Xu}$, Y., \& Shi, Y. (2004). Empirical Bayes test for truncation parameters using LINEX loss. Bulletin of the Institute of Mathematics Academia SINICA, 32(3), 207-220. 


\title{
Steady State Probabilities of a Three Preemptive Single Server Queue
}

\author{
Ameen Jameel Alawneh \\ Qatar University, \\ Qatar, Qatar
}

A three preemptive priority queuing system is considered where customers with three priorities joined a queue according to a Poisson process. A customer with higher priority needs to enter the service immediately upon arrival. The recursive formulas approach was extended to determine the steady state probabilities of such a priority queuing system.

Key words: Poisson process, recursive formulas based approach, steady state probabilities, three preemptive queues.

\begin{abstract}
Introduction
Queuing systems are used frequently and represent adequate models in many real life aspects. Examples of queuing systems include job processing, production lines, service centers (such as ATM machines), bus stations, phone calls and network service. Consider an $\mathrm{M} / \mathrm{M} / 1$ queuing system where customers arrive to a service facility with one server; the arrival process is Poisson with rate $\lambda$, and the service time distribution is exponential with rate $\mu$. If $\rho(=\lambda / \mu)<1$, then the system is in a steady state and steady state probabilities exist. Determining performance measures for $\mathrm{M} / \mathrm{M} / 1$ is simple and can be found in most elementary queuing system texts, such as Gross and Harries (1998).

Priority queues form a large class of queuing models where arrival customers are distinguished according to their importance. The customer with higher priority should be served before those of lower priorities. Examples include analysis of computer and communication systems, mobile phone
\end{abstract}

Ameen Alawneh is an Associate Professor of Statistics and Operations Research. $\mathrm{He}$ is on leave from JUST in Jordan and is currently working in the Department of Math, Statistics and Physics at Qatar University. His main research interest focuses on the stochastic process. Email him at: aalawneh@qu.edu.qa. networks, ambulances and surgeries. The performance analysis of multi-server queues with multiple classes of customers often experiences difficulty because the state space needed to capture the system behavior grows infinitely in multiple dimensions; Marks (1973) and Cidon and Sidi (1990) proposed different approaches to obtain the steady state probabilities of such systems.

\section{Two Levels Priority Queues}

Consider an $\mathrm{M} / \mathrm{M} / 1$ with two priority levels of customers: high and low. The high priority customers need to be served ahead of the low priority customers. The arrival process is Poisson with rates $\lambda_{1}$ and $\lambda_{2}$ for high and low priority customers, respectively. Also, the service time distribution is exponential with rates $\mu_{1}$ and $\mu_{2}$ for high and low priority customers, respectively. The service discipline within each priority level is first in, first out (FIFO).

Preemptive queues indicate that a customer with high priority must be served upon arriving unless there are already high priority customers in the queue or in service, that is., ahead of any low priority customers in the system. Thus, upon arrival of a high priority customer, if the customer in service is low priority then he/she is ejected from service and the high priority customer is serviced; the low priority customer returns to the service center and re-starts service from the beginning. Nonpreemptive queues are defined as those where a 
customer in service cannot be ejected from service upon the arrival of high priority customer.

Steady State Probabilities of Priority Queuing Systems

The steady state probabilities of queuing systems can be determined with ease when the queue is stable, however, it is not an easy task in the case of infinite queues, when the system has a very large number of states or when $\rho$ (the intensity factor) approaches 1 (Smith, 2002). Different approaches have been proposed in the literature to find steady state probabilities. Some are based on generating functions, difference equations or direct algebraic manipulations (Mark, 1973; Cidon \& Sidi, 1990). In their work, Smith (2002) and Osogami, et al. (2004) considered approaches based on manipulating queuing systems as Markov chains.

The limiting distribution of a Markov chain can be interpreted as a steady state probability. Another approach is termed indirect; this approach is based on identifying the stationary distribution of a Markov chain associated with the number of customers at a moment when a customer finishes service and leaves the system. The limiting distribution of the Markov chain is the steady state probability of the corresponding queuing system. Many authors have used this indirect approach in the literature (Osogami, et al.; 2004; Sheskin, 1985). Gail, et al. (1992) considered a Markov chain with two priorities and multiple servers: again, when the queue length is infinite, determining the stationary distribution of a Markov chain becomes challenging.

Heyman (1990) proposed an approach to ascertain approximate values of a stationary distribution of an infinite stochastic matrix in one dimension. Heyman's approach was extended to the case of two dimensional state space Markov chains and applied to a nonpreemptive queuing system (Alawneh, 1995). The truncated approach was used by Alawneh (2011) to determine approximate values of the steady state probabilities of $\mathrm{M} / \mathrm{M} / 2$ with infinite queues; results were compared with approaches put forth by Flatto and McKean (1977) and Flatto and Han (1977). This article presents an extension of a recursive formula based approach that may be used to find the steady state probabilities of three preemptive priority levels queuing system with one server.

Steady State Probabilities of Two Preemptive Priority Queue

Marks (1973) was the first to study nonpreemptive queuing systems. He developed a computational approach based on recursion formulas to determine the exact values of both preemptive and non-preemptive systems. Cidon \& Sidi (1990) developed a recursive formula based on a moment generating function to find the same probabilities. However, as model complexity increases, the required algebraic manipulations become more tedious. Pasternack and Drezener (1998) proposed a recursive formula based on difference equations to establish exact probabilities for priority queues; their technique requires less computation than Mark's (1973). Smith (2002) and Pasternack and Drezener (1998) proposed an alternate approach for use when a system is finite but the expected number of customers is large.

A recursive formula approach introduced in the literature to determine the exact values of the steady state probabilities of M/M/1 for customers with two priorities - high and low - was provided by Marks (1973). Consider an M/M/1 where arrival customers are classified into two types according to their priority of obtaining service: high and low. Four possible cases are possible for the state space: both $m=0$ and $n=0$, only $m=0$, only $n=0$; or $(n, m) \neq(0,0)$. Figure 1 illustrates the general case when both $\mathrm{m}$ and $\mathrm{n}$ are nonzero; that is, $(n$, $m) \neq(0,0)$. The other three cases may be obtained by using an appropriate substitution of $n$ and/or $m$.

Let $\mathrm{P}_{n m}$ be the probability of having $n$ high and $m$ low priority customers in the system at the moment when one customer finishes his service and leaves the system, where $n$ and $m$ are non-negative integers. The steady state probability is determined by solving equations (White \& Christie, 1958):

$$
\mu_{1} P(1,0)-\mu_{2} P(0,1)=\lambda P(0,0)
$$


Figure 1: Special Case of a State Diagram of M/M/1 with Two Priorities

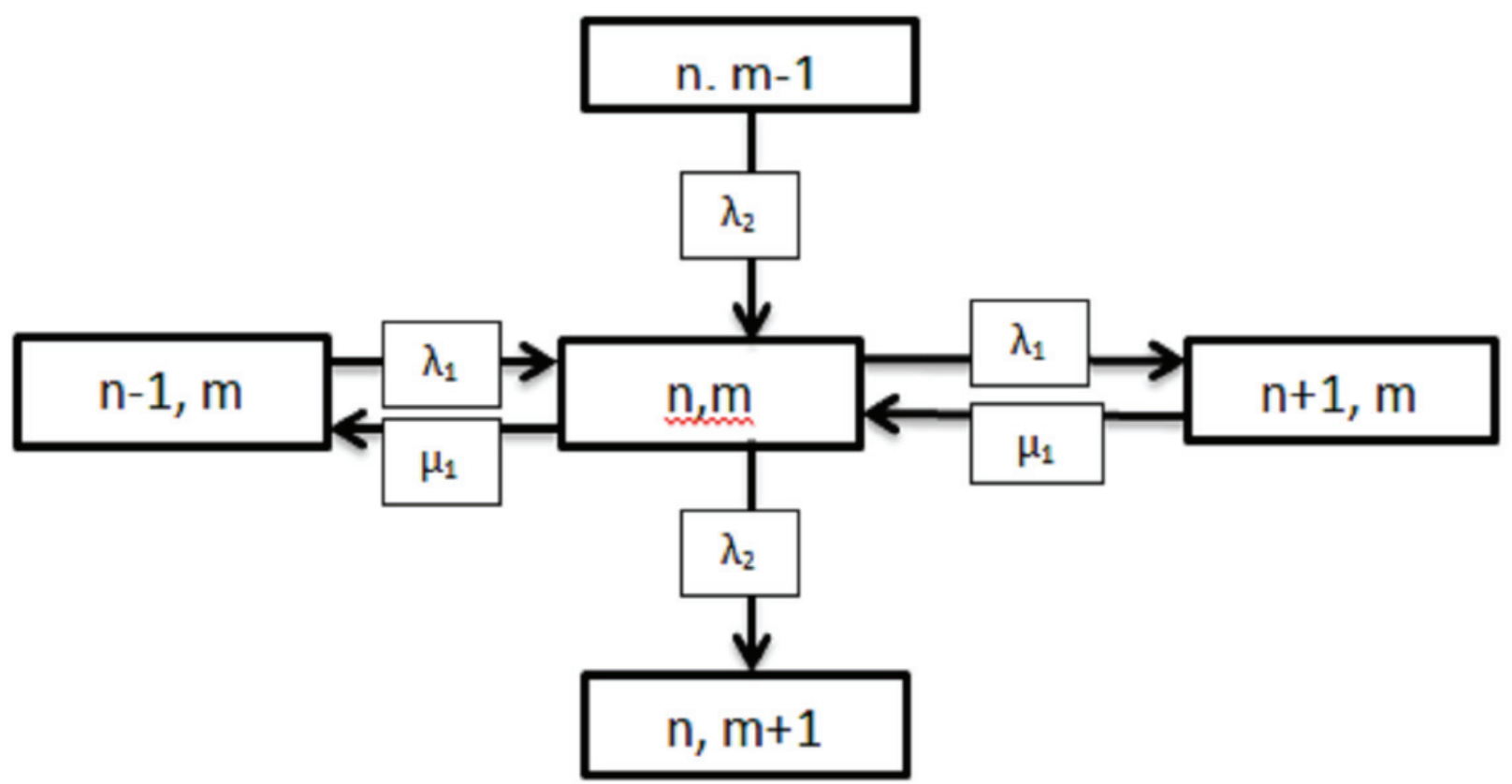

$\mu_{1} P(n+1,0)-\lambda_{1} P(n-1,0)=\left(\lambda+\mu_{1}\right) P(n, 0)$

$$
\left[\begin{array}{c}
\mu_{1} P(1, m)+\mu_{1} P(1, m) \\
+\mu_{2} P(1, m+1)+\lambda_{2} P(0, m-1)
\end{array}\right]=\left(\lambda+\mu_{2}\right) P(0, m)
$$

$$
\left[\begin{array}{c}
\mu_{1} P(n+1, m)+\lambda_{2} P(n, m-1) \mu \\
+\lambda_{2} P(n-1, m)
\end{array}\right]=\left(\lambda+\mu_{1}\right) P(n, m)
$$

for $n, m=0,1,2, \ldots$

The following ten-step algorithm was developed by Marks (1973) and may be used to solve equations (1) to (4) in order to determine the steady state probabilities of two preemptive queues:

1. Calculate $P_{00}=1-\rho_{1}-\rho_{2}$.

2. Set $\mathrm{B}_{00}=\mathrm{p}_{00}$.

3. Set $m=1$.
4. Calculate $\mathrm{B}_{11}$ as:

$$
B_{11}=\frac{1}{[\beta-\alpha]} \gamma \rho_{2} B_{00},
$$

where $0<\gamma<\infty$.

5. Calculate $\mathrm{B}_{01}$ as:

$$
B_{01}=\frac{[\beta-1]}{\gamma} B_{00}
$$

where

$$
\begin{aligned}
& \beta= \\
& \frac{1}{2}\left[\left(\rho_{1}+\gamma \rho_{2}+1\right)+\sqrt{\left(\rho_{1}+\gamma \rho_{2-}+1\right)^{2}-4 \rho_{1}}\right] .
\end{aligned}
$$

6. Increase $m$ by one.

7. Calculate $B_{i m}$ for $\mathrm{i}=\mathrm{m}, \mathrm{m}-1, \ldots, 1$, using the equation: 


$$
\begin{aligned}
& B_{i m}= \\
& {\left[\frac{1}{\beta-\alpha} i\right]\left\{\gamma \rho^{2} B_{i-1, m-1}+\sum_{k=i+1}^{k=m}\left(\begin{array}{c}
K \\
i-1
\end{array}\right)\left[\alpha+\beta(-1)^{k-i+1}\right] B_{k m l}\right\}}
\end{aligned}
$$

for $\mathrm{i}=m, m-1, \ldots, 1 ; m \geqq 1$.

8. Calculate $\mathrm{B}_{0 m}$ using:

$$
\begin{aligned}
& B_{0 m}= \\
& \left(\frac{\omega}{\gamma}\right) B_{0, m-1}-\rho_{2} B_{0, m-2}-\left(\frac{\alpha}{\gamma}\right) \sum_{k=0}^{m-1} B_{k, m-1}
\end{aligned}
$$

and

$$
\begin{aligned}
& \beta, \alpha= \\
& \frac{1}{2}\left[\left(\rho_{1}+\gamma \rho_{2}+1\right) \pm \sqrt{\left(\rho_{1}+\gamma \rho_{2}+1\right)^{2}-4 \rho_{1}}\right],
\end{aligned}
$$

where $0<\alpha<1, \beta>1, \omega=\rho_{1}+\rho_{2}+\gamma$.

9. Repeat steps 6 through 8 until all necessary coefficients are obtained.

10. Calculate the state probabilities $\mathrm{P}_{n m}$ using the formula:

$$
\begin{gathered}
P_{n m}=\alpha^{n} \sum_{k=0}^{k=m} B_{k m} \eta^{k}, \\
n \geq 0, m \geq 0 .
\end{gathered}
$$

Steady State Probabilities of Three Preemptive M/M/1 Queue

Next, the recursive formula approach is extended to the case where there are three preemptive priority levels. In this case, customers are classified according to their priorities into three types or classes. Type I, the highest priority, is followed by Type II, and lastly Type III, which is without priority. Assuming a preemptive priority, meaning customers with higher priority enter service upon arrival and ahead of any customer from a lower priority. In addition, a customer from the higher priority may eject any lower priority customer in service. The state space of the queuing system depends on the number of customers from each priority level in the system - both in the queue and in service. If $n, m$ and $l$ are the number of each priority level in the system, then all $n, m$ and $l$ are nonnegative integers. Eight possible cases for the state space may be considered based on $n, m$ and $l$. Figure 2 illustrates the most general case when $n, m$ and $l$ are all positive; the other seven cases may be obtained by appropriate substitutions of the values of $n, m$ and $l$.

If $\lambda_{i}$ for $i=1,2,3$ is the arrival rate from each priority level and $\mu_{i}$ is the service rate for each priority level, for $i=1,2,3$, then

$$
\rho_{i}=\frac{\lambda_{i}}{\mu_{i}} \text { for } i=1,2,3 \text {, }
$$

and $\lambda$ and $\mu$ are defined as: $\lambda=\lambda_{1}+\lambda_{2}+\lambda_{3}$ and $\mu=\mu_{1}+\mu_{2}+\mu_{3}$, respectively.

Steady state equations are derived from Figure 2 as follows:

$$
\mu_{1} P_{0,1,0}+\mu_{2} P_{0,1,0}+\mu_{3} P_{0,0,1}=\lambda P_{0,0,0}
$$

$$
\mu_{1} P_{n+1,0,0}+\lambda_{1} P_{n-1,0,0}=\left(\lambda+\mu_{1}\right) P_{n, 0,0}
$$

$$
\begin{gathered}
\mu_{1} P_{1, n, 0}+\mu_{2} P_{0, n+1,0}+\lambda_{2} P_{0, n-1,0}= \\
\left(\lambda+\mu_{3}\right) P_{0, n}
\end{gathered}
$$

$$
\begin{gathered}
\mu_{1} P_{1,0, l}+\mu_{2} P_{0,1, l}+\mu_{3} P_{0,0, l+1}+\lambda_{3} P_{0,0, l-1}= \\
\left(\lambda+\mu_{3}\right) P_{0,0, l} \\
\mu_{1} P_{n, m, 0}+\lambda_{1} P_{n-1, m, 0}+\lambda_{2} P_{n-1, m, 0}= \\
\left(\lambda+\mu_{1}\right) P_{n, m, 0} \\
\mu_{1} P_{n+1,0, l}+\lambda_{1} P_{n-1,0, l}+\lambda_{3} P_{n-1,0, l-1}= \\
\left(\lambda+\mu_{1}\right) P_{n, 0, l}
\end{gathered}
$$




\section{AMEEN JAMEEL ALAWNEH}

Figure 2: Special Case of the State Diagram of $M / M / 1$ with Three Priorities

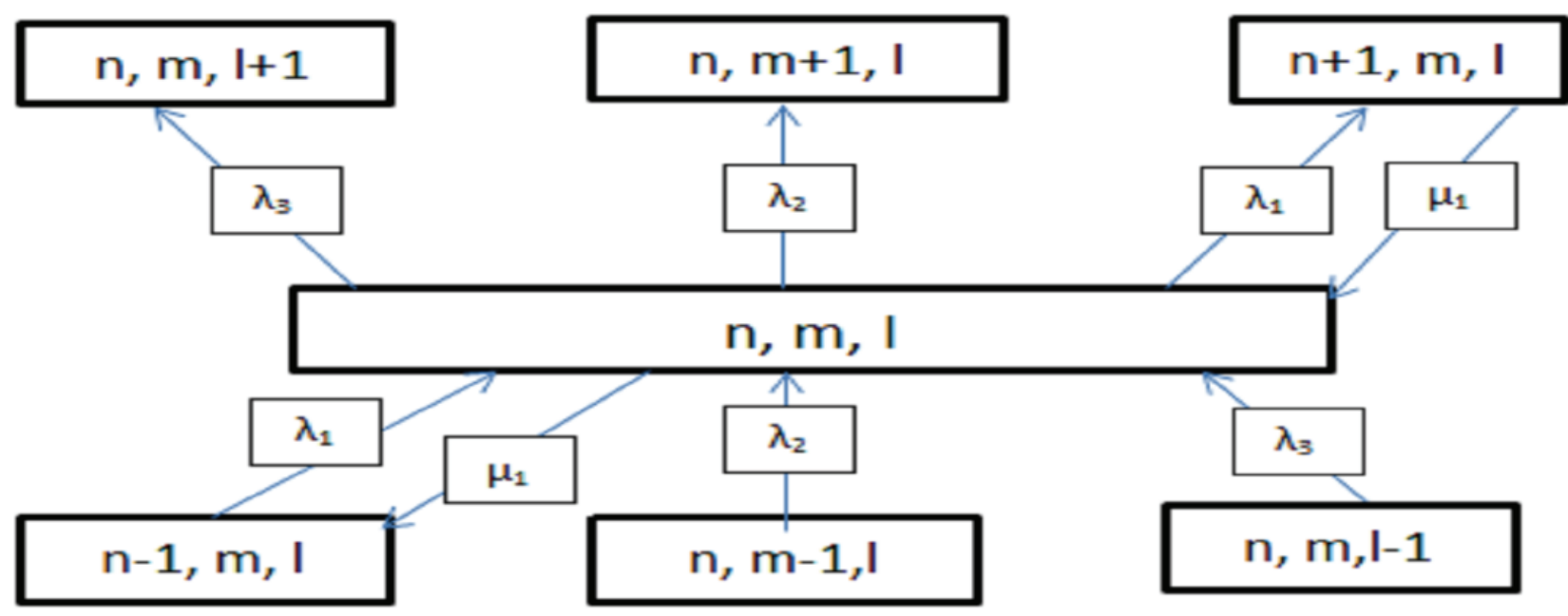

$$
\begin{gathered}
\mu_{1} P_{1, m, l}+\lambda_{2} P_{0, m-1, l}+\mu_{2} P_{0, m+1, l}+\lambda_{3} P_{0, m, l-1}= \\
\left(\lambda+\mu_{3}\right) P_{0, m, l}
\end{gathered}
$$

$$
\begin{gathered}
\mu_{1} P_{n+1, m, l}+\lambda_{1} P_{n-1, m, l}+\lambda_{2} P_{n, m-1, l}+\lambda_{3} P_{n, m, l-1}= \\
\left(\lambda+\mu_{1}\right) P_{n, m, l}
\end{gathered}
$$

and

$$
\sum_{l=0}^{\infty} \sum_{m=0}^{\infty} \sum_{n=0}^{\infty} P_{l, m, n}=1
$$

Defining $E P_{n, m, l}=P_{n+1, m, l}$ where $E$ is a difference operator and rewriting (2) using the difference operator $E$ results in:

$$
\begin{aligned}
\left(\mu_{1} E^{2}-\left(\lambda+\mu_{1}\right) E+\lambda_{1}\right) P_{l, 0,0} & =0 \\
& =\Psi(E) P_{l 00}
\end{aligned}
$$

where

$$
\Psi(E)=\left(\mu_{1} E^{2}-\left(\lambda+\mu_{1}\right) E+\lambda_{1}\right) .
$$

Substituting $m=1$ and rewriting (18) using difference operator $E$ results in:

$$
\begin{gathered}
\mu_{1} P_{n+1,1, l}+\lambda_{1} P_{n-1,1, l}+\lambda_{2} P_{n, 0, l}+\lambda_{3} P_{n, 0, l-1}= \\
\left(\lambda+\mu_{1}\right) P_{n, 0, l}
\end{gathered}
$$

and

$$
\Psi(E) P_{n, 1, m}=-\lambda_{3} P_{n, 0, l-1}-\lambda_{2} P_{n, 0, l}
$$

Using mathematical induction, for $n=0$,

$$
\Psi(E) P_{n, 1,0}=-\lambda_{2} P_{n, 0,0}
$$

and for $n=1$,

$$
\Psi(E) P_{n, 1,1}=-\lambda_{3} P_{n, 0,0}-\lambda_{2} P_{n, 0,1},
$$

$$
P_{n, 1,0}=B^{100} r^{n}+B_{101} n r^{n}
$$

and

$$
P_{n, 1,0}=B_{110} r^{n}+B_{111} n r^{n}+B_{112} n^{2} r n .
$$


Thus the general solution is

$$
P_{n, 1, l}=r^{n} \sum_{j=0}^{n+1} B_{i, m, j} n^{j}
$$

and, the general solution for the steady state probabilities is

$$
P_{n, m, l}=r^{n} \sum_{j=0}^{n+m} B_{i, m, j} n^{j}
$$

which shows that a recursive formula approach may be used to determine the steady state probabilities of a three preemptive queue.

Expected Number of Customers and the Average Waiting Time in $\mathrm{M} / \mathrm{M} / 1$

To compare an $\mathrm{M} / \mathrm{M} / 1$ with three priority levels but without priority according to the expected number of customers and average waiting time in the queue, let $\mathrm{L}_{i q}, i=1,2,3$ be the expected number of customers (average queue length) from the $i^{\text {th }}$ priority level in the queue, and let $\mathrm{W}_{i q}$ be the average waiting time for the $i^{\text {th }}$ priority level. The number of expected customers in the queue from the $i^{\text {th }}$ priority level is found using:

$$
\begin{aligned}
& L_{i q}=\frac{\rho_{i} \sum_{n=1}^{i} \rho_{n}}{\left(1-\sum_{n=1}^{i-1} \rho_{-} n\right)\left(1-\sum_{n=1}^{i} \rho_{n}\right)}, \\
& i=1,2,3 \text { and } \sum_{j=1}^{i} \rho_{j}<1 .
\end{aligned}
$$

If $\mathrm{L}_{q}$ is the expected number of customers in the queue then:

$$
L_{q}=\frac{1}{\lambda}\left(\lambda_{1} L_{1 q}+\lambda_{2} L_{2 q}+\lambda_{3} L_{3 q}\right)
$$

and the average waiting times in the queue is

$$
W q=\frac{1}{\lambda}\left(\begin{array}{lll}
\lambda_{1} & W_{1 q}+\lambda_{2} & W_{2 q}+\lambda_{3} W_{3 q}
\end{array}\right)
$$

where the average waiting time for the $i^{\text {th }}$ priority level is

$$
W_{i q}=\frac{L_{i q}}{\lambda}
$$

for $i=1,2,3$.

The expected number of customers and the average waiting times for an $\mathrm{M} / \mathrm{M} / 1$ without priorities are:

$$
\begin{gathered}
L_{q}=\frac{\rho^{2}}{1-\rho} \\
\text { and } \\
W_{q}=\frac{L_{q}}{\lambda}
\end{gathered}
$$

where the arrival rate for the non-priority is $\lambda=$ $\lambda_{1}+\lambda_{2}+\lambda_{3}$, and where $\lambda_{1}, \lambda_{2}$ and $\lambda_{3}$ are the same as in the priority queue. In addition, $\mu=\mu_{1}$ $+\mu_{2}+\mu_{3}$ and $\mu_{1}, \mu_{2}$ and $\mu_{3}$ are also the same as in the priority queue.

For the purpose of numerical comparisons between priority and non-priority customers, Table 1 shows different values for arrival and service rates for a priority model assuming $\lambda=\lambda_{1}+\lambda_{2}+\lambda_{3}$, and $\mu=\mu_{1}+\mu_{2}+\mu_{3}$ when customers are not prioritized.

\section{Conclusion}

Based on results shown in Table 1, the following conclusions are put forth:

1. The queue length in the non-priority systems always constitutes an upper bound for the queue length in the priority system.

2. The average waiting time for the nonpriority customers is an upper bound for the priority system.

3. Priority queues are more efficient when customers are classified according to their importance or needs for service.

4. As the intensity factor approaches one, the expected number of customers and the average waiting times increase for the highest priority customers.

This study shows that the recursive formal based approach may be used to find exact values of steady state probabilities for a three priority 
queuing system. Priority queues are more efficient than non-priority, particularly when customer arrivals are classified according to their importance or their service needs. In real life applications the number of the highest priority customers is limited; therefore, imposing such a condition on queue length will make using the recursive technique much easier.

Table 1: Expected Queue length and Waiting times for different values of $\mu$ and $\lambda$

\begin{tabular}{|c|c|c|c|c|c|c|c|c|}
\hline \multirow{2}{*}{$\mu$} & \multirow{2}{*}{$\lambda$} & \multirow{2}{*}{$\rho_{1}$} & \multirow{2}{*}{$\rho_{2}$} & \multirow{2}{*}{$\rho_{3}$} & \multicolumn{2}{|c|}{$\mathrm{L}_{\mathrm{q}}$} & \multicolumn{2}{|c|}{$\mathrm{W}_{\mathrm{q}}$} \\
\hline & & & & & With & Without & With & Without \\
\hline 6 & 3 & 0.167 & 0.167 & 0.167 & 0.128 & 0.500 & 0.064 & 0.167 \\
\hline 6 & 4 & 0.167 & 0.333 & 0.167 & 0.375 & 1.333 & 0.156 & 0.167 \\
\hline 6 & 4 & 0.167 & 0.167 & 0.333 & 0.533 & 1.333 & 0.267 & 0.167 \\
\hline 6 & 5 & 0.167 & 0.333 & 0.333 & 1.500 & 5.143 & 0.625 & 1.029 \\
\hline 6 & 4 & 0.167 & 0.333 & 0.167 & 0.375 & 1.333 & 0.156 & 0.167 \\
\hline 6 & 5 & 0.167 & 0.167 & 0.333 & 1.500 & 5.143 & 0.625 & 0.570 \\
\hline 7 & 5 & 0.167 & 0.333 & 0.333 & 0.918 & 0.714 & 0.394 & 0.357 \\
\hline 7 & 6 & 0.143 & 0.143 & 0.429 & 3.215 & 5.143 & 1.378 & 0.857 \\
\hline 7 & 6 & 0.143 & 0.143 & 0.571 & 1.615 & 5.143 & 0.519 & 0.857 \\
\hline 7 & 4 & 0.143 & 0.571 & 0.143 & 0.178 & 1.752 & 0.051 & 0.357 \\
\hline 7 & 5 & 0.286 & 0.143 & 0.143 & 0.426 & 1.786 & 0.106 & 0.357 \\
\hline 7 & 5 & 0.286 & 0.286 & 0.143 & 0.576 & 1.786 & 0.165 & 0.357 \\
\hline 7 & 4 & 0.286 & 0.143 & 0.286 & 0.178 & 1.752 & 0.051 & 0.355 \\
\hline 7 & 5 & 0.286 & 0.143 & 0.143 & 0.426 & 0.714 & 0.106 & 0.357 \\
\hline 7 & 6 & 0.286 & 0.286 & 0.286 & 0.576 & 5.143 & 0.165 & 0.857 \\
\hline 7 & 6 & 0.286 & 0.143 & 0.286 & 1.549 & 5.143 & 0.387 & 0.857 \\
\hline 7 & 6 & 0.286 & 0.286 & 0.429 & 2.313 & 5.143 & 0.661 & 0.857 \\
\hline 7 & 5 & 0.286 & 0.143 & 0.143 & 0.426 & 0.714 & 0.102 & 0.357 \\
\hline 7 & 6 & 0.429 & 0.143 & 0.143 & 1.077 & 5.143 & 0.231 & 0.857 \\
\hline 7 & 6 & 0.429 & 0.286 & 0.286 & 1.550 & 5.143 & 0.369 & 0.857 \\
\hline 7 & 6 & 0.571 & 0.143 & 0.143 & 1.147 & 5.143 & 0.246 & 0.857 \\
\hline
\end{tabular}




\section{References}

Alawneh, A. (1995). Approximating the stationary distribution of two-dimensional Markov chains. Unpublished Ph.D. Thesis, New Mexico State University, USA.

Alawneh, A. (2011). Numerical approach to compute the steady state probabilities of $\mathrm{M} / \mathrm{M} / 2$ with infinite queues. Accepted. International Journal of Applied Mathematics.

Ching, W., Zhang, S., Ng, M., \& Akustu, T. (2007). An approximation method for solving the steady state probability distribution of probabilistic Boolean networks. Bioinformatics, 23(12), 1611-1518.

Cidon, I., \& Sidi, M. (1990). Recursive computation of steady state probabilities in priority queues. Operations Research Letters, 9 , 249-256.

Flatto, L., \& McKean, H. P. (1977). Two queues in parallel. Communications on pure and applied mathematics, 30(2), 255-263.

Gail, H. R., Hantler, S. L., \& Taylor, B. A. (1992). On a preemptive Markovian queue with multiple servers and two priority classes. Mathematics of Operations Research, 17(2), 365-391.

Gross, D., \& Harris, C. (1998). Fundamentals of queuing systems, $3^{\text {rd }}$ Edition. New York, NY: John Wiley.

Heyman, D. P. (1991). Approximating the stationary distribution of an infinite stochastic matrix. Journal of Applied Probability, 28(1), 96-103.
Mark, P., \& Karlin, S. (2011). An Introduction to stochastic modeling, $4^{\text {th }}$ Edition. London: Academic Press.

Marks, B. I. (1973). State probabilities of $\mathrm{M} / \mathrm{M} / 1$ priority queues. Operations Research, 21, 974-987.

Miller, D. G. (1981). Computation of steady state probabilities for $\mathrm{M} / \mathrm{M} / 1$ priority queues. Operations Research, 29, 945-958.

Osogami, T., Wierman, A., HarcholBalter, M., \& Scheller-Wolf, A. (2004). A recursive analysis technique for multidimensionally infinite Markov chains. $A C M$ SIGMETRICS Performance Evaluation Review, 32(2), 3-5.

Pasternack, B. A., \& Drezener, Z. (1998). A note on calculating steady state results for $\mathrm{M} / \mathrm{M} / \mathrm{k}$ queuing systems when the ration of the arrival rate to the service rate is large. Journal of Applied Mathematics and Decision Science, 2(2), 201-203.

Sheskin, T. (1985). A Markov chain portioning algorithm for computing steady state probabilities. Operations Research, 33(1), 228235.

Smith, D. (2002). Calculation of M/M/ queues: Further approach. Journal of Applied Mathematics and Decision Science, 6(1), 43-50.

Wright, P. (1992). Two parallel processors with coupled inputs. Advances in Applied Probability, 24(4), 986-1007. 


\section{Weight: Does It Really Matter?}

\author{
Jennifer L. Brown \\ Columbus State University, \\ Columbus, GA
}

\author{
Gerald Halpin \\ Auburn University, \\ Auburn, AL
}

\author{
Glennelle Halpin \\ Auburn University, \\ Auburn, AL
}

Differential weighting promises to improve the validity of a measure. This study examines whether similar results would be found using weighted, unweighted and standardized $z$ scores from the All Stars Core survey. It was concluded that the weighted systems were developed to equate the questions within the scales and to ease the process for customers without access to data analysis programs; however, the standardized scores were the more appropriate method for equating the test items.

Key words: Differential weighting, standardized scores, z-scores.

Introduction

According to Russell and Hubley (2005), the multiplication model of weighting is typically used with subjective importance ratings. In studies, quality of life, self-esteem, job satisfaction and personality have been among the variables assessed (Streiner, Goldberg \& Miller, 1993; Trauer \& Mackinnon, 2001). With all methods of weighting, the most weight is given to the most desirable response, and the least weight is given to the least desirable response. In mathematical theory, differential weighting promises to substantially improve the validity of the measure; however, in practice, it does not prove to be worth the trouble, especially item weighting (Wang \& Stanley, 1970). Standardizing raw item data has been shown (Peterson, Kolen \& Hoover, 1989; Wang $\&$ Stanley) to have the closest representation to achieving equally effective weighting.

Jennifer Brown is an Assistant Professor of Educational Foundations in the Department of Teacher Education. Email her at: brown_jennifer2@columbusstate.edu. Gerald Halpin and Glennelle Halpin were Professors in the Department of Educational Foundations, Technology and Leadership.
This question as to whether weight matters, and more specifically, to determine if similar results would be found using weighted, unweighted and standardized $\mathrm{z}$ scores from the All Stars Core survey (Hansen, 2004) in reliability analyses and in repeated measures multivariate analyses of variance.

\section{Data Source}

$$
\text { Methodology }
$$

The data source for this study was from 393 sixth-grade students in a rural county in the southeastern United States. The sample included 190 (48.3\%) males and 203 (51.7\%) females who responded to the All Stars Core survey (Hansen, 2004) as a pre/post measure in an evaluation of a drug prevention intervention.

\section{Measures}

The All Stars Core survey consists of 62 multiple-choice items used to measure knowledge, attitudes, and behaviors regarding substance abuse. Items on the core measure were rationally combined to form scales, which measured alcohol, tobacco and other drug (ATOD) use factors and mediator factors. For this study, only six mediator factor scales were used:

1. Commitment: eight items assessing one's commitment to abstain from the use of alcohol, cigarettes, marijuana, and other drugs. 


\section{WEIGHT: DOES IT REALLY MATTER?}

2. School Bonding: seven items assessing one's perception of his or her teachers' attitudes towards him or her, acceptance, quality of school and teachers and sense of belonging at school.

3. Lifestyle Incongruence of Alcohol, Tobacco, and Other Drugs: six items assessing one's quality of life and ability to accomplish goals by avoiding alcohol, tobacco, and other drugs.

4. Normative Beliefs about Peer Drug Use: eight items assessing one's perception of his or her peers' use of and attitudes towards tobacco, alcohol, and other drugs.

5. Parent/Child Communication: three items assessing one's communication with parents about drugs and other important issues.

6. Parental Monitoring: three items assessing one's perception of parental knowledge regarding his or her friends.

The response scale for the mediator factors progressed from a rating of 1 , which represented the least desirable level of behaviors, attitudes, perceptions, and skills, to a rating of 4 , which represented the most desirable level. For the Normative Beliefs scale, four of the eight items had a response scale of 1 (None) to 5 (All). With different response scales, the response weights were on a scale ranging from 0 to 10 .

\section{Data Analysis}

For data analyses, three types of scores were created: unweighted, weighted and standardized. The unweighted items were left in raw form. The weighted items were weighted based on the curriculum developer's specifications. For the standardized items, the pre- and post- test responses were combined into a database and transformed into standardized $z$ scores; after standardization, the pre- and posttest items were separated into different variables. Reliabilities, repeated measures multivariate analysis of variance (MANOVA), and repeated measures analyses of variance (ANOVA) were conducted.

\begin{abstract}
Results
Reliabilities

The weighted item method rendered similar, but slightly higher, internal consistency reliabilities than the other two scaling methods. Table 1 presents the alpha reliability coefficients for the mediator factor scales using the unweighted, weighted, and standardized scores.
\end{abstract}

Analyses of Variance

Initially, scores on the mediator factor scales weighted according to Hansen's (2004) weighting method were entered as dependent variables into a MANOVA to determine the mean change or difference from pre- to posttest. The results of the multivariate test of group differences indicated that an optimally linear combination of the six mediator factor scales from pre- to post- test was statistically significant $\left(F(6,386)=474.17, p=0.00 ; \eta^{2}=\right.$ $0.88)$, and the eta square indicated an enormous effect size, which could be interpreted as the six mediator factor scales accounting for $88 \%$ of the variance of the linear composite; such results are very unusual in educational research. The follow-up analyses revealed a statistically significant difference for all six mediator factor scales (see Table 2) with inflated $F$ ratio and eta square results.

Subsequently, using the unweighted item scores, the results of the repeated measure multivariate test indicated that an optimal linear combination of the six mediator factor scales from pre- to posttest was also statistically significant $\left(F(6,386)=172.37, p=0.00 ; \eta^{2}=\right.$ $0.73)$; in addition, the eta square indicated a very strong effect size. The follow-up analyses revealed a statistically significant difference for all six mediator factor scales (see Table 3). The results were similar to the repeated measures MANOVA with follow-up univariate procedure that used the weighted item scores, but, with the unweighted item scores, the $F$ ratios and eta squares were slightly decreased. 
BROWN, HALPIN \& HALPIN

Table 1: Alpha Reliability Coefficients for Mediator Factor Scales

\begin{tabular}{|c|c|c|c|c|c|c|}
\hline \multirow{2}{*}{ Scale } & \multicolumn{2}{|c|}{ Unweighted } & \multicolumn{2}{c|}{ Weighted } & \multicolumn{2}{c|}{ Standardized } \\
\cline { 2 - 7 } & Pretest & Posttest & Pretest & Posttest & Pretest & Posttest \\
\hline Commitment & .69 & .70 & .75 & .76 & .69 & .74 \\
\hline School Bonding & .66 & .66 & .74 & .75 & .66 & .74 \\
\hline $\begin{array}{c}\text { Lifestyle Incongruence of Alcohol, } \\
\text { Tobacco, and Other Drugs }\end{array}$ & .44 & .44 & .46 & .48 & .44 & .47 \\
\hline $\begin{array}{c}\text { Normative Beliefs About Peer Drug } \\
\text { Use }\end{array}$ & .72 & .47 & .63 & .57 & .72 & .64 \\
\hline $\begin{array}{c}\text { Parent/Child Communication } \\
\text { Parental Monitoring }\end{array}$ & .63 & .63 & .64 & .66 & .64 & .65 \\
\hline
\end{tabular}

Table 2: Weighted Items: Means, Standard Deviations, and Univariates for the Mediator Factor Scales by Group as a Follow-up to the MANOVA Procedure

\begin{tabular}{|c|c|c|c|c|c|c|c|}
\hline \multirow{2}{*}{ Scale } & \multicolumn{2}{|c|}{ Pretest } & \multicolumn{2}{c|}{ Posttest } & \multirow{2}{*}{$F$} & \multirow{2}{*}{$\eta^{2}$} \\
\cline { 2 - 6 } Commitment & $\mathrm{M}$ & $\mathrm{SD}$ & $\mathrm{M}$ & $\mathrm{SD}$ & & \\
\hline School Bonding & 1.48 & 1.57 & 1.74 & 1.84 & 179.00 & .00 & .31 \\
\hline $\begin{array}{c}\text { Lifestyle Incongruence of Alcohol, } \\
\text { Tobacco, and Other Drugs }\end{array}$ & 1.09 & 1.30 & 1.25 & 1.46 & 156.19 & .00 & .29 \\
\hline $\begin{array}{c}\text { Normative Beliefs About Peer Drug } \\
\text { Use }\end{array}$ & 4.51 & 1.31 & 4.32 & 1.48 & 182.65 & .00 & .32 \\
\hline $\begin{array}{c}\text { Parent/Child Communication } \\
\text { Parental Monitoring }\end{array}$ & 1.54 & 1.97 & 1.53 & 2.00 & 1202.65 & .00 & .76 \\
\hline
\end{tabular}




\section{WEIGHT: DOES IT REALLY MATTER?}

Next, with the standardized item scores, the results of the repeated measure multivariate test indicated that an optimally linear combination of the six mediator factor scales from pre- to posttest was also statistically significant $\left(F(6,386)=2.95, p=0.01 ; \eta^{2}=\right.$ 0.04); however, the eta square indicated a weak effect size. The follow-up analyses revealed a statistically significant difference for only two of the six mediator factor scales: Lifestyle Incongruence and Parental Monitoring (see Table 4).

An examination of the statistical results for the weighted and unweighted item scores revealed the $F$ ratios, follow-up univariate $F$ ratios, and eta squares for each dependent variable entered into the MANOVA procedure were drastically out of the expected range for educational research. Conversely, the
MANOVA, follow-up univariate and eta square results with the standardized item scores yielded different findings compared to the other two methods. The $F$ ratios and eta squares were comprehensible, and the follow-up univariates yielded only two statistically significant changes from pretest to posttest. Further, a simple examination of the means and standard deviations calls the inflated $F$ ratios and eta squares into question.

After the repeated measures MANOVA indicated statistically significant differences, a series of separate repeated measures ANOVAs were conducted using the three types of scores to determine group mean differences from pretest to pretest. Tables 5, 6 and 7 display the separate univariate results for the mediator factor scales using unweighted, weighted and standardized items.

Table 3: Unweighted Items: Means, Standard Deviations, and Univariates for the Mediator Factor Scales by Group as a Follow-up to the MANOVA Procedure

\begin{tabular}{|c|c|c|c|c|c|c|c|}
\hline \multirow{2}{*}{ Scale } & \multicolumn{2}{|c|}{ Pretest } & \multicolumn{2}{c|}{ Posttest } & \multirow{2}{*}{$F$} & \multirow{2}{*}{$\eta^{2}$} \\
\cline { 2 - 7 } Commitment & $\mathrm{M}$ & $\mathrm{SD}$ & $\mathrm{M}$ & $\mathrm{SD}$ & & \\
\hline School Bonding & 1.45 & 0.47 & 1.53 & 0.56 & 177.65 & .00 & .31 \\
\hline $\begin{array}{c}\text { Lifestyle Incongruence of Alcohol, } \\
\text { Tobacco, and Other Drugs }\end{array}$ & 1.33 & 0.39 & 1.38 & 0.44 & 156.15 & .00 & .29 \\
\hline $\begin{array}{c}\text { Normative Beliefs About Peer Drug } \\
\text { Use }\end{array}$ & 1.89 & 0.55 & 2.03 & 0.53 & 186.75 & .00 & .32 \\
\hline $\begin{array}{c}\text { Parent/Child Communication } \\
\text { Parental Monitoring }\end{array}$ & 1.46 & 0.59 & 1.48 & 0.61 & 524.62 & .00 & .57 \\
\hline
\end{tabular}


Table 4: Standardized Items: Means, Standard Deviations, and Univariates for the Mediator Factor Scales by Group as a Follow-up to the MANOVA Procedure

\begin{tabular}{|c|c|c|c|c|c|c|c|}
\hline \multirow{2}{*}{ Scale } & \multicolumn{2}{|c|}{ Pretest } & \multicolumn{2}{c|}{ Posttest } & \multirow{2}{*}{$F$} & \multirow{2}{*}{$\eta^{2}$} \\
\cline { 2 - 6 } Commitment & $\mathrm{M}$ & $\mathrm{SD}$ & $\mathrm{M}$ & $\mathrm{SD}$ & & \\
\hline School Bonding & -0.06 & 0.51 & 0.03 & 0.62 & 0.31 & .58 & .00 \\
\hline $\begin{array}{c}\text { Lifestyle Incongruence of Alcohol, } \\
\text { Tobacco, and Other Drugs }\end{array}$ & -0.06 & 0.46 & 0.01 & 0.53 & 4.37 & .04 & .01 \\
\hline $\begin{array}{c}\text { Normative Beliefs About Peer Drug } \\
\text { Use }\end{array}$ & -0.08 & 0.55 & 0.06 & 0.54 & 2.50 & .11 & .01 \\
\hline $\begin{array}{c}\text { Parent/Child Communication } \\
\text { Parental Monitoring }\end{array}$ & -0.01 & 0.75 & 0.00 & 0.77 & 2.76 & .10 & .01 \\
\hline
\end{tabular}

Table 5: Unweighted Items: Means, Standard Deviations, and Univariates for the Mediator Factor Scales by Group Using Separate Repeated Measures ANOVAs

\begin{tabular}{|c|c|c|c|c|c|c|c|}
\hline \multirow{2}{*}{ Scale } & \multicolumn{2}{|c|}{ Pretest } & \multicolumn{2}{c|}{ Posttest } & \multirow{2}{*}{$F$} & $\eta^{2}$ \\
\cline { 2 - 7 } Commitment & $\mathrm{M}$ & $\mathrm{SD}$ & $\mathrm{M}$ & $\mathrm{SD}$ & & \\
\hline $\begin{array}{c}\text { School Bonding } \\
\text { Lifestyle Incongruence of Alcohol, } \\
\text { Tobacco, and Other Drugs }\end{array}$ & 1.45 & 0.47 & 1.53 & 0.56 & 11.07 & .00 & .03 \\
\hline $\begin{array}{c}\text { Normative Beliefs About Peer Drug } \\
\text { Use }\end{array}$ & 1.89 & 0.55 & 2.03 & 0.53 & 22.71 & .00 & .06 \\
\hline $\begin{array}{c}\text { Parent/Child Communication } \\
\text { Parental Monitoring }\end{array}$ & 1.46 & 0.59 & 1.48 & 0.61 & 0.16 & .69 & .00 \\
\hline
\end{tabular}


WEIGHT: DOES IT REALLY MATTER?

Table 6: Weighted Items: Means, Standard Deviations, and Univariates for the Mediator Factor Scales by Group Using Separate Repeated Measures ANOVAs

\begin{tabular}{|c|c|c|c|c|c|c|c|}
\hline \multirow{2}{*}{ Scale } & \multicolumn{2}{|c|}{ Pretest } & \multicolumn{2}{c|}{ Posttest } & \multirow{2}{*}{$F$} & \multirow{2}{*}{$\eta^{2}$} \\
\cline { 2 - 7 } Commitment & $\mathrm{M}$ & $\mathrm{SD}$ & $\mathrm{M}$ & $\mathrm{SD}$ & & \\
\hline $\begin{array}{c}\text { School Bonding } \\
\text { Lifestyle Incongruence of Alcohol, } \\
\text { Tobacco, and Other Drugs }\end{array}$ & 1.48 & 1.57 & 1.74 & 1.84 & 10.43 & .00 & .03 \\
\hline $\begin{array}{c}\text { Normative Beliefs About Peer Drug } \\
\text { Use }\end{array}$ & 4.51 & 1.31 & 4.32 & 1.48 & 6.65 & .01 & .02 \\
\hline $\begin{array}{c}\text { Parent/Child Communication } \\
\text { Parental Monitoring }\end{array}$ & 1.54 & 1.97 & 1.53 & 2.00 & 0.00 & .96 & .00 \\
\hline
\end{tabular}

Table 7: Standardized Items: Means, Standard Deviations, and Univariates for the Mediator Factor Scales by Group Using Separate Repeated Measures ANOVAs

\begin{tabular}{|c|c|c|c|c|c|c|c|}
\hline \multirow[b]{2}{*}{ Scale } & \multicolumn{2}{|c|}{ Pretest } & \multicolumn{2}{|c|}{ Posttest } & \multirow{2}{*}{$F$} & \multirow{2}{*}{$p$} & \multirow{2}{*}{$\eta^{2}$} \\
\hline & M & SD & M & $\mathrm{SD}$ & & & \\
\hline Commitment & -0.06 & 0.51 & 0.03 & 0.62 & 11.61 & .00 & .03 \\
\hline School Bonding & -0.08 & 0.53 & 0.09 & 0.64 & 32.37 & .00 & .08 \\
\hline $\begin{array}{l}\text { Lifestyle Incongruence of Alcohol, } \\
\text { Tobacco, and Other Drugs }\end{array}$ & -0.06 & 0.46 & 0.01 & 0.53 & 5.03 & .03 & .01 \\
\hline $\begin{array}{c}\text { Normative Beliefs About Peer Drug } \\
\text { Use }\end{array}$ & -0.08 & 0.55 & 0.06 & 0.54 & 23.52 & .00 & .06 \\
\hline Parent/Child Communication & -0.01 & 0.75 & 0.00 & 0.77 & 0.13 & .72 & .00 \\
\hline Parental Monitoring & -0.10 & 0.78 & 0.09 & 0.83 & 30.14 & .00 & .07 \\
\hline
\end{tabular}




\section{BROWN, HALPIN \& HALPIN}

Each ANOVA yielded similar results except for the Normative Beliefs scale. With unweighted items, the $F$ ratio for the Normative Beliefs scale was 22.71 with a $p$ value of 0.00 and eta square of 0.06 . The weighted items yielded an $F$ ratio of 6.65 with a $p$ value of 0.01 and eta square of 0.02 . With the standardized items, the $F$ ratio was 23.52 with a $p$-value of 0.00 and eta square of 0.06 . These analyses indicate that the results of the standardized items paralleled the results with the unweighted items; however, the ratio of mean square error was reduced with the weighted items.

\section{Conclusion}

The weighted systems were developed to equate the questions within the scales and to ease the process for customers without access to data analysis programs; however, the standardized scores were the more appropriate method of equating the test items. A word of caution should be given when analyzing weighted items using the MANOVA procedure with follow-up univariates regardless of the weighting method. The findings may yield results that are incomprehensible to the field of educational research despite internally consistent reliabilities.

In an effort to resolve this perplexing issue, Hansen (personal communication, November 2, 2007), the instrument developer, noted when the measure was constructed in the 1980s the items were standardized by combining the pre- and post- tests. The mean score was obtained for each scale. The average layman had difficulty interpreting a mean of 0 . In the 1990s, all scales were stretched to a response scale from 0 to 10 so a layman could consider a 10 as the most desirable score and a 0 as the least desirable score. The formula for stretching is:

Stretched Value $=$

$(O B S+@ A B S(\min )) \times\left(\frac{10}{((@ A B S(\min )+\max ))}\right)$

where OBS is observed value, min is minimum value, and max is maximum value. Using this formula, the developer found this method was always dependent on the actual distribution of scores within a study. More recently, a simpler method for weighting was implemented. For each item, the lowest score is always 0 and the highest score is always 10 . For a five-response item, scores are $0,2.5,5,7.5$ and 10 . In a fouritem response, scores are 0, 3.3, 6.7 and 10. When averaged across items within a scale, scores will always range from 0 to 10 . This simpler method allows the results within the database to be compared longitudinally.

When conducting research with weighted items, using both data analysis procedures, MANOVA with follow-up univariates and separate repeated measures ANOVAs is recommended. The MANOVA offers a more comprehensive analysis, but the $F$ ratios are determined based on estimated means and the procedure could yield inflated $F$ ratios and eta squares. By comparing the data analysis output, a researcher can decide which of the obtained analyses is most appropriate for their research question(s).

\section{References}

Hansen, W. B. (2004). All stars core (Version 2.4). Greensboro, NC: Tanglewood Research.

Peterson, N. S., Kolen, M. J., \& Hoover, H. D. (1989). Scaling, norming, and equating. In Educational measurement (3 ${ }^{\text {rd }}$ Ed.), R. L. Linn (Ed.), 221-262. New York, NY: MacMillan.

Russell, L. B., \& Hubley, A. M. (2005). Importance ratings and weighting: Old concerns and new perspectives. International Journal of Testing, 5, 105-130.

Streiner, D. L., Goldberg, J. O., \& Miller, H. R. (1993). MCMI-II item weights: Their lack of effectiveness. Journal of Personality Assessment, 60, 471-476.

Trauer, T., \& MacKinnon, A. (2001). Why are we weighting? The role of importance ratings in quality of life. Quality of Life Research, 10, 579-585.

Wang, M. W., \& Stanley, J. C. (1970). Differential weighting: A review of methods and empirical studies. Review of Educational Research, 40, 663-705. 


\section{Regression Models for Mixed Over-Dispersed Poisson and Continuous Clustered Data: Modeling BMI and Number of Cigarettes Smoked Per Day}

\author{
Folefac Atem \\ Brigham and Women's Hospital, \\ Boston, MA
}

\author{
Julius S. Ngwa \\ Boston University, \\ Boston, MA
}

\author{
Abidemi Adeniji \\ University of Pittsburgh, \\ Pittsburgh, PA
}

Clustered data, multiple observations collected on the same experimental unit, is common in epidemiological studies. Bivariate outcome data is often the result of interest in two correlated response variables. An efficient method is presented for dealing with bivariate outcomes when one outcome is continuous and the other is a count using a simple transformation to handle over-dispersed Poisson data. A multilevel analysis was performed on data from the National Health Interview Survey (NHIS) with body mass index (BMI) and the number of cigarettes smoked per day (NCS) as responses. Results show that these random effects models yield misleading results in cases where the data is not transformed.

Key words: Joint bivariate model, random effect model, multilevel, mixed model, GLIMMIX.

\section{Introduction}

Modeling bivariate outcome using joint multivariate random effect models (JMRE) is a popular approach in the medical field. There are a number of conditions where a disease under study is well understood when two outcomes are considered. For example, in clinical trials the clinician may be interested in the joint evolution of HIV RNA and CD4+t lymphocytes in a cohort of HIV-1 infected patients treated with active antiretroviral drugs. Bellamy (1995) studied the study of the risk factors associated with the progression of osteoarthritis (OA) of the knee. In this study two outcomes were collected, the Western Ontario and McMaster Universities (WOMAC) disability score and the number of missed work days for the past three months due to knee pain. The JMRE model allows the modeling of mixed effects and bivariate outcomes.

Folefac Atem is a Biometric Consultant. Email him at: folefac_atem@yahoo.com. Julius S. Ngwa is a Ph.D. candidate in the Biostatistics Department at the School of Public Health. Abidemi K. Adeniji has a Ph.D. in biostatistics and is a researcher at The Epidemiology Data Center (EDC), University of Pittsburgh.
There are several advantages of modeling bivariate outcomes (see Laird and Ware, 1982; Dempster, et al., 1984; Bagiella, 2000; Pantazis \& Touloumi, 2007; McCulloch, 2008; Atem, et al,. 2010). First, this model allows exploration of variations at different levels of a hierarchy and modeling of a correlation structure serially and across two outcomes. Second, the bivariate JMRE model achieves greater bias reduction in all model parameters compared to the two independent JMRE models. Third, there is greater flexibility in dealing with exploratory variables; the JMRE model can conveniently test hypotheses on either end point individually or simultaneously. JMRE can also handle missing data as the parameter estimates are obtained by techniques of maximum likelihood; the variance and means of the outcomes are estimated, thus the choice of the variance must be taken into consideration.

Rao (1973) suggested that irrespective of the chosen variance, the fixed effect estimates are unbiased; however, the estimates can achieve maximum efficiency only when the appropriate variance is specified. Lastly, multiple testing can be avoided by forming joint models without resorting to ad hoc methods such as the Bonferroni adjustment or using advanced methods such as those as presented by Dmitrienko, et al. (2009). Moreover, because the bivariate model is a regression model, 
classification and continuous predictors can be incorporated; the predictors can either be time variant or invariant.

This study develops a straightforward and efficient method to handle bivariate outcome data where one outcome is continuous and the other is count with over-dispersion; a test is proposed to identify possible overdispersion in the count outcome. For modeling, data from the National Health Interview Survey (NHIS) from 1997-2006 was used to establish the joint relationship between body mass index (BMI; continuous) and number of cigarettes smoked per day (NCS; count), adjusting for race, gender and age. The National Health Interview Survey (NHIS) was founded in 1957 as an annual nationwide survey of approximately 40,000 households in civilian, non-institutionalized populations. It is conducted by the National Center for Health Statistics (NCHS) and administered by the United States Census Bureau.

\section{Regression Techniques}

Regression techniques for hierarchical data have been referred to in the literature under different names, including random coefficient models (Rao, 1965) and hierarchical linear model (Bryk \& Raudenbush, 1987). Song, et al. (2008) refers to bivariate and multivariate analyses as structural level equations.

\section{Bivariate Model}

For the case of two continuous markers with correlated random effect superscripts $a$ and $b$ are used to distinguish the two markers. Subscripts $j$ and $i$ are used to denote the information of the $j^{\text {th }}$ measurement for the $i^{\text {th }}$ individual. Assuming that the marker trends can be explained by two linear mixed models with correlated random-effects, a bivariate model for the multilevel marker measurements can be presented as follows (Thiebaut, et al., 2002):

$$
\begin{aligned}
& y_{i j}^{a}=x_{i j}^{a} \beta^{a}+z_{i j}^{a} \lambda_{i}^{a}+e_{i j}^{a} \\
& \text { and } \\
& y_{i j}^{b}=x_{i j}^{b} \beta^{b}+z_{i j}^{b} \lambda_{i}^{b}+e_{i j}^{b}
\end{aligned}
$$

Vectors $\lambda_{i}^{a}\left(k^{a} \times 1\right)$ and $\lambda_{i}^{b}\left(k^{b} \times 1\right)$ contain random (subject-specific) regression coefficients for $k^{a}$ and $k^{b}$ predictor variables included in the corresponding design vectors $Z_{i j}^{a}(1 \times k)$ and $Z_{i j}^{b}(1 \times k)$ respectively. The joint distribution of $\lambda_{i}^{a}$ and $\lambda_{i}^{b}$ is assumed to be multivariate normal with zero means and variancecovariance matrix:

$$
\Sigma=\left(\begin{array}{cc}
\sum^{a} & \sum^{a b} \\
\sum^{a b} & \sum^{b}
\end{array}\right)
$$

Matrices $\sum^{a}\left(k^{a} \times k^{a}\right)$ and $\sum^{b}\left(k^{b} \times k^{b}\right)$ are variance and covariance of the random effects for both outcomes respectively, while $\sum^{a b}\left(k^{b} \times k^{a}\right)$ specifies the covariance structure of the random effects between outcomes.

$$
\text { Vectors } \beta^{a}\left(p^{a} \times 1\right) \text { and } \beta^{b}\left(p^{b} \times 1\right)
$$

contain fixed regression coefficients for both markers respectively; $\quad x_{i j}^{a}\left(1 \times p^{a}\right) \quad$ and $x_{i j}^{b}\left(1 \times p^{b}\right)$ are their corresponding design vectors containing the values of $p^{a}$ and $p^{b}$ exploratory variables. The coefficients $e_{i j}^{a}$ and $e_{i j}^{b}$ represent the level-1 residual for the two outcomes. The bivariate model can be presented in the matrix form:

$$
\left(\begin{array}{c}
Y_{i}^{a} \\
Y_{i}^{b}
\end{array}\right)=\left(\begin{array}{cc}
X_{i}^{a} & 0 \\
0 & X_{i}^{b}
\end{array}\right)\left(\begin{array}{l}
\beta^{a} \\
\beta^{b}
\end{array}\right)+\left(\begin{array}{cc}
Z_{i}^{a} & 0 \\
0 & Z_{i}^{b}
\end{array}\right)\left(\begin{array}{c}
\lambda_{i}^{a} \\
\lambda_{i}^{b}
\end{array}\right)
$$

Bivariate Model: Random Effects Approach The outcomes of interest, BMI and NCS are denoted by $Y_{1 i}(t)$ and $Y_{2 i}(t)$ respectively, for subject $i$ at time $t$. Assume that the outcomes can be described by two linear mixed models with correlated random effects. A joint model can be constructed from the basic random effect model proposed by Laird and Ware (1982): 


$$
\begin{aligned}
& Y_{1 i}(t)=\beta_{1}(t)+a_{1 i}+b_{1 i}(t)+\varepsilon_{1 i}(t) \\
& Y_{2 i}(t)=\beta_{2}(t)+a_{2 i}+b_{2 i}(t)+\varepsilon_{2 i}(t)
\end{aligned}
$$

where $\beta_{1}(t)$ and $\beta_{2}(t)$ refer to the average changes of the outcomes. The dependent variables (BMI and NCS) are linked together through the joint distribution of their random effects

$$
\left(\begin{array}{l}
a_{1 i} \\
a_{2 i} \\
b_{1 i} \\
b_{2 i}
\end{array}\right) \sim N(0, D)
$$

where $D$ is a matrix of the random effects with the following variance-covariance structure:

$$
\left(\begin{array}{ccc}
\sigma_{a 1}^{2} & \ldots & \sigma_{a 1 b 2} \\
\vdots & \ddots & \vdots \\
\sigma_{b 2 a 1} & \cdots & \sigma_{b 2}^{2}
\end{array}\right)
$$

The variance-covariance matrix of the model parameter is often derived by maximum likelihood or restricted maximum likelihood method.

The residual components are uncorrelated and independent of the random effects

$$
\left(\begin{array}{l}
\varepsilon_{1 i} \\
\varepsilon_{2 i}
\end{array}\right) \sim N\left(\left(\begin{array}{l}
0 \\
0
\end{array}\right),\left(\begin{array}{cc}
\sigma_{1}^{2} & 0 \\
0 & \sigma_{2}^{2}
\end{array}\right)\right)
$$

The variance-covariance structure implies that conditioning on the random effects, both responses are independent. Other cases can be obtained by making additional assumptions about the variance-covariance matrix $D$. Thiebaut, et al. (2002) outlined further procedures on a number of special cases of the $\mathrm{D}$ matrix. For these analyses, models were fitted using the SAS GLIMMIX procedure, while the random effects were introduced through the shared random effects procedures as described by De Gruttola, et al. (1994).

A common objective for joint modeling is to investigate how outcomes are correlated. For example, a researcher might be interested in how the correlation of BMI and NCS are associated with age, while controlling for race and gender. The correlation between outcome variables BMI and NCS is derived from the variance- covariance matrix of the random effect and is given by:

$$
r_{e}=\frac{\sigma_{b_{1} b_{2}}}{\sqrt{\sigma_{b_{1}}^{2}} \sqrt{\sigma_{b_{2}}^{2}}}
$$

The marginal correlation between BMI and NCS as a function of time $t$ is given by:

$r_{m}(t)=$

$\frac{\sigma_{a_{1} a_{2}}+t\left(\sigma_{a_{1} b_{2}}+\sigma_{a_{2} b_{1}}\right)+t^{2} \sigma_{b_{1} b_{2}}}{\sqrt{\sigma_{a_{1}}^{2}+2 t \sigma_{a_{1} b_{1}}+t^{2} \sigma_{b_{1}}^{2}+\sigma_{1}^{2}} \sqrt{\sigma_{2}^{2}+2 t \sigma_{a_{2} b_{2}}+t^{2} \sigma_{b_{2}}^{2}+\sigma_{a_{2}}^{2}}}$.

When $t=0$ the equation reduces to

$$
r_{m}(t)=\frac{\sigma_{a_{1} a_{2}}}{\sqrt{\sigma_{a_{1}}^{2}+\sigma_{1}^{2}} \sqrt{\sigma_{2}^{2}+\sigma_{a_{2}}^{2}}}
$$

This formula implies that the marginal correlation cannot be higher than the correlation between random intercepts. If the measurement errors of BMI and NCS are smaller, the marginal correlation at $t=0$ better approximates the correlation between the random intercepts. The covariance parameters of the random effects and the error components determine the shape of the marginal correlation function. Further, as $t$ increases, the marginal correlation converges to the correlation between the random slopes.

Simulation Study

$$
\text { Methodology }
$$

Yang, et al. (2007, 2009, 2010) discussed methods to simulate and test for an over-dispersed Poisson distribution. A simple and efficient way to simulate a mixture of 
bivariate continuous and over-dispersed Poisson distribution using the specified parameters is:

$$
\begin{aligned}
& \beta^{a}=(1,0.2,-0.2,1)^{\prime} a=1,5 \\
& \beta^{b}=(1,0.2,0.1,0.1)^{\prime}, \rho=0.5, \sigma^{2}=1 \\
& \lambda_{i}^{a}=\lambda_{i}^{b}=0.12, n=100, T=7
\end{aligned}
$$

Data were simulated using a negative binomial distribution for the case where $\alpha=5$. A negative binomial may result from a mixture of Poisson distributions with a Gamma distribution of the mean and a specified shape and scale parameters ( $\mathrm{R}$ - Documentation). The mean to variance ratio was about 1:5. See Table 1, Figure 1 for a table and plot of the mean and variance for a non-dispersed and see Table 2, Figure 2 for a dispersed Poisson distribution. Using equation 4, 5,000 samples were generated; each sample of size 100 represents a mixed Poisson and continuous longitudinal data with 7 time points. Both outcomes were generated as a linear function of 4 predictors $\left(x_{i}\right)$; the predictors were specified as both binary and continuous. The bias of the estimates using different transformations and correlations were approximately equal.

\section{Results}

Tables 3 - 5 summarize the results of the simulated dataset; Log likelihood (-2 Log L), $\mathrm{AIC}, \mathrm{AICC}$ and BIC together with the means estimates, standard errors and p-values of our estimates. The AIC, AICC and BIC as defined in Akaike (1973, 1985), Sakamota, et al. (1986) and Bozgogan (1987) are specified as:

$$
2(-\log g(x, \hat{\theta})+d), \quad A I C+\frac{2(d+1)(d+2)}{n-d-2}
$$

and $-2 \log g(x, \hat{\theta})+d\left(\log \left(n^{*}\right)\right)$ respectively.

These definitions of AIC, AICC and BIC imply that smaller AIC, AICC and BIC estimates provide a better fit for the model. Gurka, et al. (2011) showed that the model with independent correlation structure offers a parsimonious model, but may not always be the best model. However, this study shows that - in the case of mixed Poisson and continuous longitudinal data - the independent and the unstructured correlation tend to perform best and have the lowest AIC, AICC and BIC (see Table 3 ). In the case of a mixed over-dispersed Poisson and continuous clustered data a number of transformations for the outcome variables were examined. First, the case with no transformation on the outcome was considered; this model

Table 1: Mean and Variance for a Non-Dispersed Poisson Distribution

\begin{tabular}{|c|c|c|}
\hline \multicolumn{3}{|c|}{ Simulated Data Results (Non-Dispersed Poisson) } \\
\hline Replicate & Mean of Poisson & Variance of Poisson \\
\hline 1 & 4.46 & 4.95 \\
\hline 2 & 4.04 & 3.75 \\
\hline 3 & 3.98 & 3.08 \\
\hline 4 & 3.30 & 2.79 \\
\hline 5 & 4.54 & 3.56 \\
\hline 6 & 4.18 & 3.82 \\
\hline 7 & 4.32 & 3.81 \\
\hline
\end{tabular}


Figure 1: Mean and Variance Plots for a Non-Dispersed Poisson Distribution Results Obtained from one Replicate $(\mathrm{n}=100, \mathrm{q}=7)$

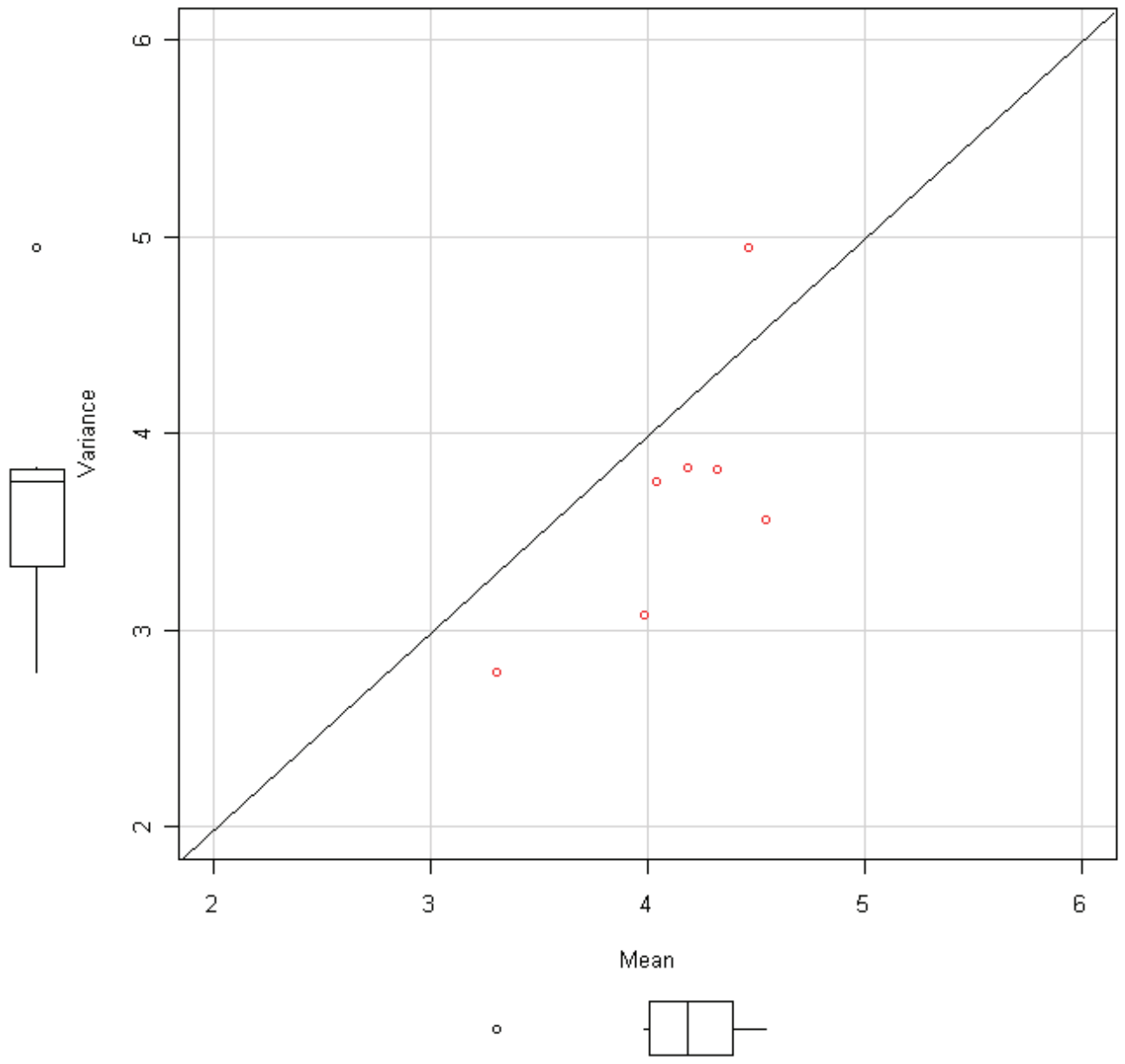

Table 2: Mean and Variance for a Dispersed Poisson Distribution

\begin{tabular}{|c|c|c|}
\hline \multicolumn{3}{|c|}{ Simulated Data Results (Dispersed Poisson) } \\
\hline Replicate & Mean of Poisson & Variance of Poisson \\
\hline 1 & 15.94 & 89.24 \\
\hline 2 & 16.56 & 90.62 \\
\hline 3 & 16.40 & 88.49 \\
\hline 4 & 16.12 & 83.94 \\
\hline 5 & 14.10 & 64.01 \\
\hline 6 & 16.72 & 102.21 \\
\hline 7 & 14.82 & 93.62 \\
\hline
\end{tabular}


Figure 2: Mean and Variance plots for a Dispersed Poisson Distribution Results Obtained from one Replicate $(n=100, q=7)$

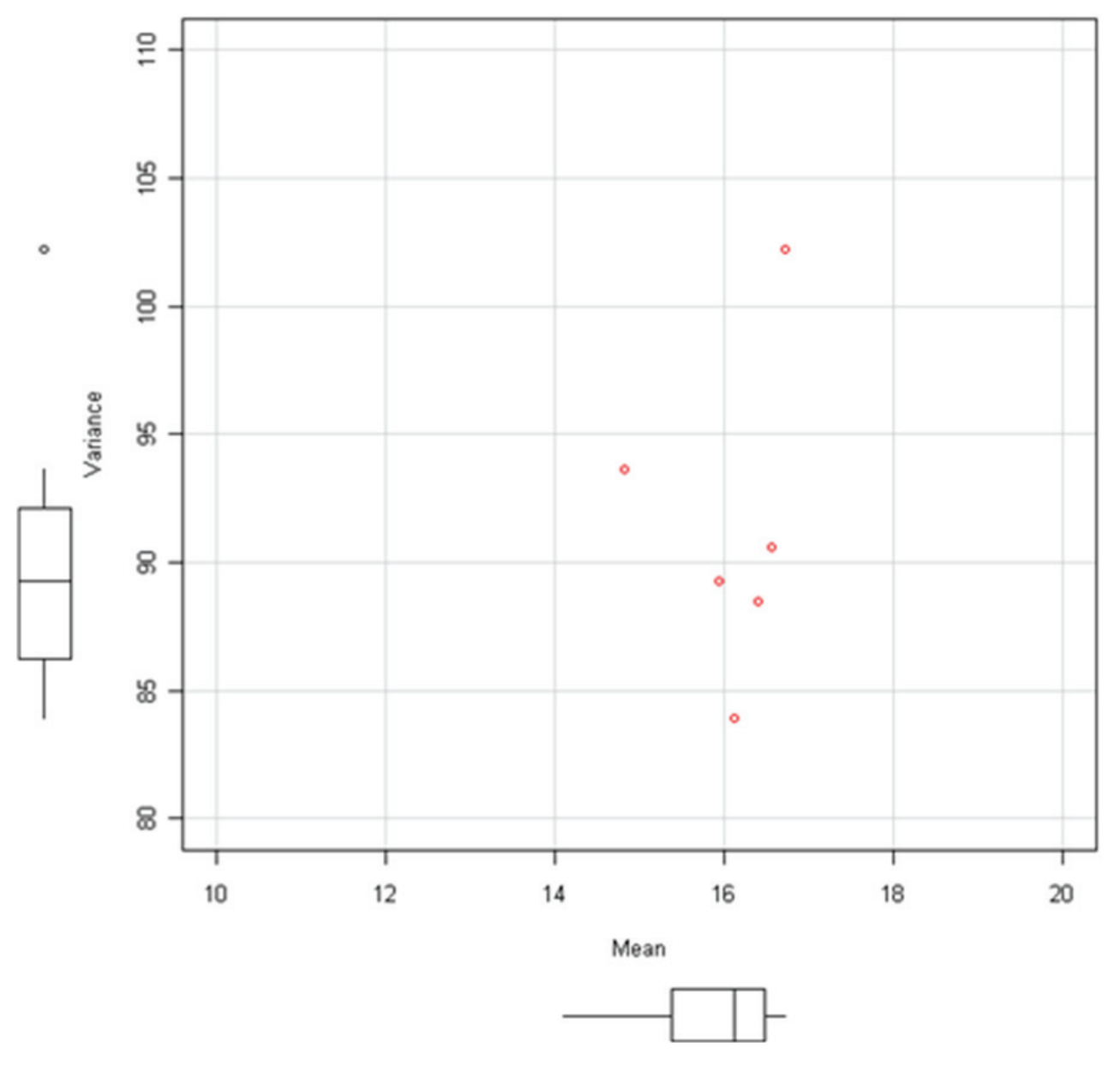

performed poorly with extremely high values for AIC, AICC and AICC (see Table 4). Next, a number of data transformations were performed and the best model was selected based on the AIC, AICC, BIC and the standard errors of the estimates. The transformation $\log (Y+1)$ performed best for both responses using the independent and the unstructured correlation structure for the data (see Table 5).

Using NHIS data, approximately 264,727 people were interviewed over the 10year period 1997-2006. The sample size for this analysis was reduced to 42,138 after cleaning. First, BMI values were analyzed with a general linear mixed model. The fixed effects in the model were gender, race and age. The random effects in this model were year (time) and residual (see Table 7). Next, NCS was analyzed with similar fixed effect and random effect variables. The univariate analysis assumed a Poisson error because NCS is count data (see Table 8); however, the resulting fitting algorithm did not converge. The Poisson error might not be a proper residual term for this analysis even though NCS is count data because the means are not equal to the variances (see Table 6). Overdispersion was verified using techniques proposed by Lindsey (1999). Assuming a normal error for NCS, the deviance is 323842.2 which is more than twice the degrees of freedom of 42139, hence the Poisson data is overdispersed. 
MODELS FOR OVER-DISPERSED POISSON AND CONTINUOUS CLUSTERED DATA

Table 3: Means, Standard Errors and p-values of Fixed Effects Parameter Estimates

\begin{tabular}{|c|c|c|c|c|c|}
\hline \multicolumn{6}{|c|}{$\begin{array}{l}\text { Simulated Data Results } \\
\text { (No Overdispersion) }\end{array}$} \\
\hline Variable & Independent & $\begin{array}{l}\text { Compound } \\
\text { Symmetry }\end{array}$ & $\begin{array}{l}\text { First Order } \\
\text { Autoregressive }\end{array}$ & Toeplitz & Unstructured \\
\hline$-2 \log L$ & 2500.50 & 2572.83 & 2572.83 & 2572.83 & 2485.63 \\
\hline AIC & 2507.60 & 2580.83 & 2580.83 & 2580.83 & 2507.63 \\
\hline AICC & 2507.65 & 2580.89 & 2580.89 & 2580.89 & 2508.02 \\
\hline $\mathrm{BIC}$ & 2514.39 & 2588.48 & 2588.48 & 2588.48 & 2528.66 \\
\hline$\alpha$ & -4.8887 & -4.7744 & -4.7744 & -4.7744 & -4.8593 \\
\hline$\beta 1$ & 27.1193 & 27.1193 & 27.1193 & 27.1193 & 27.1193 \\
\hline$\beta 2$ & 0.0034 & 0.0034 & 0.0034 & 0.0034 & 0.0034 \\
\hline$\beta 3$ & 0.6846 & 0.6846 & 0.6846 & 0.6846 & 0.6846 \\
\hline$\beta 4$ & -0.0063 & -0.0092 & -0.0092 & -0.0092 & -0.0070 \\
\hline \multicolumn{6}{|c|}{ Standard Error } \\
\hline$\alpha$ & 1.5987 & 1.0841 & 1.0841 & 1.0841 & 1.3286 \\
\hline$\beta 1$ & 0.3131 & 0.2424 & 0.2424 & 0.2424 & 0.3425 \\
\hline$\beta 2$ & 0.0274 & 0.0426 & 0.0426 & 0.0426 & 0.0283 \\
\hline$\beta 3$ & 0.0423 & 0.0547 & 0.0547 & 0.0547 & 0.0429 \\
\hline$\beta 4$ & 0.0394 & 0.0265 & 0.0265 & 0.0265 & 0.0324 \\
\hline \multicolumn{6}{|l|}{ p-value } \\
\hline$\alpha$ & 0.0150 & 0.0093 & 0.0093 & 0.0093 & 0.0053 \\
\hline$\beta 1$ & $<0.0001$ & $<0.0001$ & $<0.0001$ & $<0.0001$ & $<0.0001$ \\
\hline$\beta 2$ & 0.5084 & 0.6408 & 0.6408 & 0.6408 & 0.5179 \\
\hline$\beta 3$ & $<0.0001$ & $<0.0001$ & $<0.0001$ & $<0.0001$ & $<0.0001$ \\
\hline$\beta 4$ & 0.5675 & 0.4547 & 0.4547 & 0.4547 & 0.5820 \\
\hline
\end{tabular}


Table 4: Means, Standard Errors and p-values of Fixed Effects Parameter Estimates

\begin{tabular}{|c|c|c|c|c|c|}
\hline \multicolumn{6}{|c|}{$\begin{array}{c}\text { Simulated Data Results } \\
\text { (Overdispersed Poisson Without Transformation) }\end{array}$} \\
\hline Variable & Independent & $\begin{array}{l}\text { Compound } \\
\text { Symmetry }\end{array}$ & $\begin{array}{c}\text { First Order } \\
\text { Autoregressive }\end{array}$ & Toeplitz & Unstructured \\
\hline$-2 \log L$ & 3626.17 & 3668.83 & 3668.83 & 3668.83 & 3540.68 \\
\hline AIC & 3633.57 & 3676.83 & 3676.83 & 3676.83 & 3562.98 \\
\hline $\mathrm{AICC}$ & 3633.62 & 3676.89 & 3676.89 & 3676.89 & 3563.38 \\
\hline $\mathrm{BIC}$ & 3640.65 & 3684.48 & 3684.48 & 3684.48 & 3584.30 \\
\hline$\alpha$ & 4.8104 & 5.9763 & 5.9763 & 5.9763 & 6.0410 \\
\hline$\beta 1$ & 15.7333 & 15.7333 & 15.7333 & 15.7333 & 15.7333 \\
\hline$\beta 2$ & 0.0114 & 0.0114 & 0.0114 & 0.0114 & 0.0114 \\
\hline$\beta 3$ & 2.8471 & 2.8471 & 2.8471 & 2.8471 & 2.8471 \\
\hline$\beta 4$ & 0.0354 & 0.0061 & 0.0061 & 0.0061 & 0.0045 \\
\hline \multicolumn{6}{|c|}{ Standard Error } \\
\hline$\alpha$ & 3.3361 & 1.7362 & 1.7362 & 1.7362 & 2.1000 \\
\hline$\beta 1$ & 1.0875 & 1.0691 & 1.0691 & 1.0691 & 1.1838 \\
\hline$\beta 2$ & 0.0278 & 0.0436 & 0.0436 & 0.0436 & 0.0284 \\
\hline$\beta 3$ & 0.2171 & 0.2403 & 0.2403 & 0.2403 & 0.1950 \\
\hline$\beta 4$ & 0.0786 & 0.0342 & 0.0342 & 0.0342 & 0.0434 \\
\hline \multicolumn{6}{|l|}{ p-value } \\
\hline$\alpha$ & 0.2181 & 0.0308 & 0.0308 & 0.0308 & 0.0357 \\
\hline$\beta 1$ & $<0.0001$ & $<0.0001$ & $<0.0001$ & $<0.0001$ & $<0.0001$ \\
\hline$\beta 2$ & 0.4715 & 0.6047 & 0.6047 & 0.6047 & 0.4783 \\
\hline$\beta 3$ & $<0.0001$ & $<0.0001$ & $<0.0001$ & $<0.0001$ & $<0.0001$ \\
\hline$\beta 4$ & 0.4694 & 0.3689 & 0.3689 & 0.3689 & 0.4897 \\
\hline
\end{tabular}


Table 5: Means, Standard Errors and p-values of Fixed Effects Parameter Estimates

\begin{tabular}{|c|c|c|c|c|c|}
\hline \multicolumn{6}{|c|}{$\begin{array}{c}\text { Simulated Data Results } \\
\text { (Overdispersed Log Transformed Poisson Outcome) }\end{array}$} \\
\hline Variable & Independent & $\begin{array}{l}\text { Compound } \\
\text { Symmetry }\end{array}$ & $\begin{array}{c}\text { First Order } \\
\text { Autoregressive }\end{array}$ & Toeplitz & Unstructured \\
\hline$-2 \log \mathrm{L}$ & -373.94 & -336.83 & -336.83 & -336.83 & -466.84 \\
\hline $\mathrm{AIC}$ & -366.74 & -330.53 & -330.53 & -330.53 & -443.44 \\
\hline AICC & -366.69 & -330.50 & -330.50 & -330.50 & -443.00 \\
\hline $\mathrm{BIC}$ & -359.86 & -324.51 & -324.51 & -324.51 & -421.07 \\
\hline$\alpha$ & 1.9758 & 1.9803 & 1.9803 & 1.9803 & 1.9286 \\
\hline$\beta 1$ & 1.1255 & 1.1242 & 1.1242 & 1.1242 & 1.1809 \\
\hline$\beta 2$ & 0.0005 & 0.0005 & 0.0005 & 0.0005 & 0.0005 \\
\hline$\beta 3$ & 0.1852 & 0.1850 & 0.1850 & 0.1850 & 0.1953 \\
\hline$\beta 4$ & 0.0008 & 0.0007 & 0.0007 & 0.0007 & 0.0005 \\
\hline \multicolumn{6}{|c|}{ Standard Error } \\
\hline$\alpha$ & 0.1177 & 0.1027 & 0.1027 & 0.1027 & 0.1513 \\
\hline$\beta 1$ & 0.0827 & 0.0831 & 0.0831 & 0.0831 & 0.1288 \\
\hline$\beta 2$ & 0.0012 & 0.0016 & 0.0016 & 0.0016 & 0.0013 \\
\hline$\beta 3$ & 0.0181 & 0.0183 & 0.0183 & 0.0183 & 0.0193 \\
\hline$\beta 4$ & 0.0021 & 0.0015 & 0.0015 & 0.0015 & 0.0020 \\
\hline \multicolumn{6}{|l|}{ p-value } \\
\hline$\alpha$ & $<0.0001$ & $<0.0001$ & $<0.0001$ & $<0.0001$ & $<0.0001$ \\
\hline$\beta 1$ & $<0.0001$ & $<0.0001$ & $<0.0001$ & $<0.0001$ & $<0.0001$ \\
\hline$\beta 2$ & 0.6150 & 0.6922 & 0.6922 & 0.6922 & 0.6211 \\
\hline$\beta 3$ & $<0.0001$ & $<0.0001$ & $<0.0001$ & $<0.0001$ & $<0.0001$ \\
\hline$\beta 4$ & 0.6090 & 0.4955 & 0.4955 & 0.4955 & 0.6082 \\
\hline
\end{tabular}


BMI and NCS were analyzed using a joint bivariate model (see Table 8 ) with normal and Poisson residuals for the two outcome variables, respectively. The joint bivariate model appears to converge; however, the corresponding univariate analyses for the overdispersed Poisson error did not converge. This bivariate model is inappropriate because this analysis requires proper consideration of the correlation of the outcomes, which might not be true. In this case, the result of the joint bivariate analysis is similar to the univariate analysis of BMI as the outcome. This may be attributed to the correlation between the outcome variables BMI and cigarettes smoked not being accurately taken into consideration. In order to be sure that the model properly accounts for the association between both outcomes, it is important to ensure that the model assumptions of the independent univariate models are satisfied. For example, if a Poisson error for the univariate analysis of NCS as a dependent variable is assumed, the resulting model might be subject to over-dispersion (see Tables 2 \& 6). Yang, et al. (2009) discussed the method of testing and how to manage these types of situations. In order to deal with overdispersion, NCS is transformed using $\log (\mathrm{Y}+1)$, where $\mathrm{Y}$ is NCS. This model is very similar to the model in equation 1 with $y_{i j}^{a}=\log \left(y_{i j}^{a}+1\right)$.

After transforming to the count variable, NCS, the joint bivariate random effects model is fit. Fixed effects were gender, race, age, age interaction with age and the distribution (normal). The random effects were year and residual (see Table 9); this result is different from the previous joint bivariate model. Furthermore, the analysis of residual from the joint model with normal error for BMI and Poisson error for NCS shows that the model is not performing well. The Poisson model plot of residual is expected to have a funnel shape, but as Figure 3 shows, this is not the case. The model with normal error for BMI and normal error for log-transformed NCS tend to have improved residual plots, based on the distribution of the residuals. This model is also more efficient (see Figure 4) with $-2 \operatorname{logRe}=$ 579056.2, compared to the model with untransformed NCS (see Figure 3) with -2logRe $=663787.1$.

\section{Conclusion}

A number of methods have been discussed to handle correlated data with bivariate outcomes (Song, et al., 2008), Yang, et al., (2006, 2007, 2009), but little work has been done in cases with mixed over-dispersed Poisson and clustered continuous outcomes. Yang, et al., (2006, 2009) discussed estimation procedures for bivariate models with both complete and incomplete cases. Yang, et al., (2007, 2009) introduced various methods to test for over-dispersion using a univariate repeated measure data. Fieuws and Verbeke (2004) compared the univariate to the bivariate model with and without correlated random effects using the Hearing Data collected in the Baltimore Longitudinal Study of Aging (BLSA); they concluded that the bivariate correlated model performed best.

The purpose of this study was not to develop a new estimation technique, but rather to show that a simple transformation together with the correct correlation will stabilize the model. Modeling bivariate outcomes is essential when there is an association between primary outcomes or when the question of interest focuses on the joint behavior of multiple outcomes. The extent to which the dependent structure would be considered, however, depends on the question at hand. If interest is primarily on the population response means and the impact of covariates on these means, then a detailed consideration of the transformation and correlation mechanism may not be of significant importance. However, loss of efficiency could result if the assumed working correlation is far from the true correlation (Gardiner, 2009). On the other hand, if there is interest in both marginal and subject-specific inferences (for example, in estimating the growth trajectories of individuals (Potthoff \& Roy, 1964), a careful evaluation of the transformation mechanism and correlation structure is of tremendous importance.

The linear mixed model can be used for both marginal and subject specific inference. For example, on the subject-specific inference mean and the population mean, the significance test for this approach depends highly on the chosen covariance structure; therefore, careful consideration in choosing the right correlation structure is required. 
Table 6: Number Cigarettes per Day

\begin{tabular}{|c|c|c|c|}
\hline \multicolumn{5}{|c|}{ NHIS Data Results } \\
\hline Survey Year & N & Mean & Variance \\
\hline 1997 & 5578 & 16.27 & 146.87 \\
\hline 1998 & 4619 & 15.98 & 141.91 \\
\hline 1999 & 4316 & 15.39 & 144.02 \\
\hline 2000 & 4395 & 15.32 & 149.78 \\
\hline 2001 & 4616 & 14.96 & 134.03 \\
\hline 2002 & 4158 & 14.73 & 128.41 \\
\hline 2003 & 3900 & 14.33 & 131.44 \\
\hline 2004 & 3818 & 14.36 & 129.28 \\
\hline 2005 & 3840 & 13.85 & 120.16 \\
\hline 2006 & 2899 & 14.00 & 144.20 \\
\hline
\end{tabular}

Table 7: Modeling BMI with Normal Error

\begin{tabular}{|c|c|c|c|c|c|}
\hline \multicolumn{7}{|c|}{ NHIS Data Results } \\
\hline Effect & Estimate & Standard Error & DF & T-value & P-Value \\
\hline Intercept & 20.75 & 0.2043 & 9 & 101.56 & $<.0001$ \\
\hline Male vs. Female & 0.63 & 0.0366 & 84263 & 17.30 & $<.0001$ \\
\hline White vs. Others & -0.23 & 0.0825 & 84263 & -2.73 & 0.0064 \\
\hline Black vs. Others & 1.20 & 0.0944 & 84263 & 12.67 & 0.0013 \\
\hline Age & 0.25 & 0.0068 & 84263 & 37.13 & $<.0001$ \\
\hline Age*Age & $>-0.01$ & 0.0001 & 84263 & -37.22 & $<.0001$ \\
\hline
\end{tabular}

*The estimate of the Age interaction is -0.003 
Table 8: Bivariate Model with BMI and Number of Cigarette Smoked per Day

\begin{tabular}{|c|c|c|c|c|c|c|}
\hline \multicolumn{7}{|c|}{ NHIS Data Results } \\
\hline Effect & Distribution & Estimate & $\begin{array}{c}\text { Standard } \\
\text { Error }\end{array}$ & DF & T-value & p-Value \\
\hline Distribution & Normal & 24.62 & 0.0347 & 84262 & 709.21 & $<.0001$ \\
\hline Distribution & Poisson & 1.18 & 0.0229 & 84262 & 51.30 & $<.0001$ \\
\hline Male vs. Female & & 0.17 & 0.0025 & 84262 & 68.05 & $<.0001$ \\
\hline White vs. Others & & 0.39 & 0.0067 & 84262 & 58.72 & $<.0001$ \\
\hline Black vs. Others & & 0.08 & 0.0076 & 84262 & 10.56 & $<.0001$ \\
\hline Age & & 0.04 & 0.0005 & 84262 & 89.48 & $<.0001$ \\
\hline Age*Age & & $>-0.01$ & 0.0001 & 84262 & -71.98 & $<.0001$ \\
\hline
\end{tabular}

*The estimate of the Age interaction is -0.004

Table 9: Transformed Bivariate Model BMI and Number of Cigarette Smoked Per Day

\begin{tabular}{|c|c|c|c|c|c|c|}
\hline \multicolumn{7}{|c|}{ NHIS Data Results } \\
\hline Effect & Distribution & Estimate & $\begin{array}{c}\text { Standard } \\
\text { Error }\end{array}$ & DF & T-value & p-Value \\
\hline Distribution & Normal & 10.86 & 0.3838 & 84263 & 28.28 & $<.0001$ \\
\hline Male vs. Female & & 0.39 & 0.0857 & 84263 & 4.510 & $<.0001$ \\
\hline White vs. Others & & 0.10 & 0.1933 & 84263 & 0.540 & 0.5903 \\
\hline Black vs. Others & & 0.68 & 0.2213 & 84263 & 3.050 & 0.0023 \\
\hline Age & & 0.15 & 0.0159 & 84263 & 9.25 & $<.0001$ \\
\hline Age*Age $^{*}$ & & $>-0.01$ & 0.0002 & 84263 & -8.98 & $<.0001$ \\
\hline
\end{tabular}

*The estimate of the Age interaction is -0.0016 
Figure 3: Analysis of Residuals: Number of Cigarettes Smoked and BMI

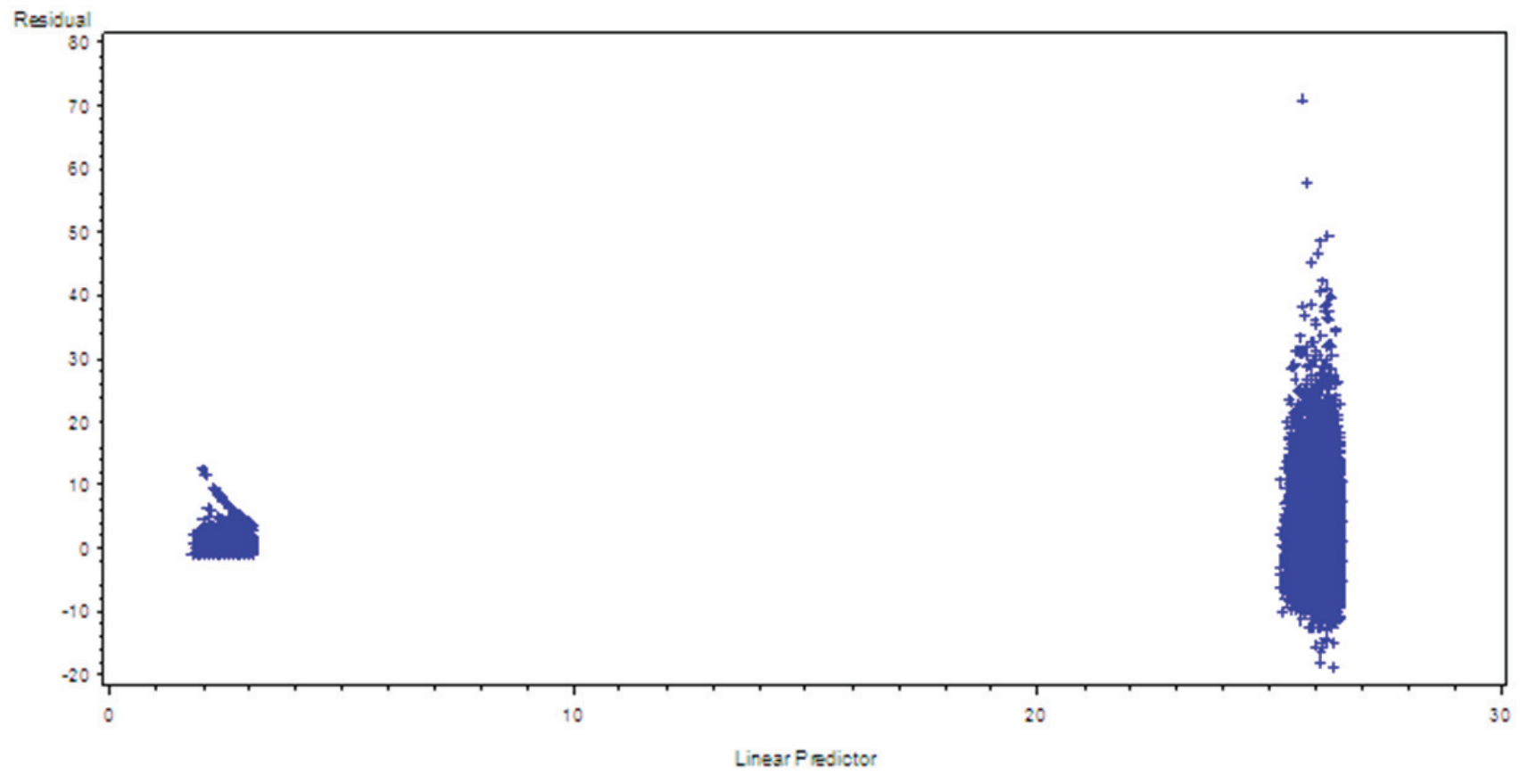

Figure 4: Analysis of Residuals; Transformed Number of Cigarettes Smoked and BMI

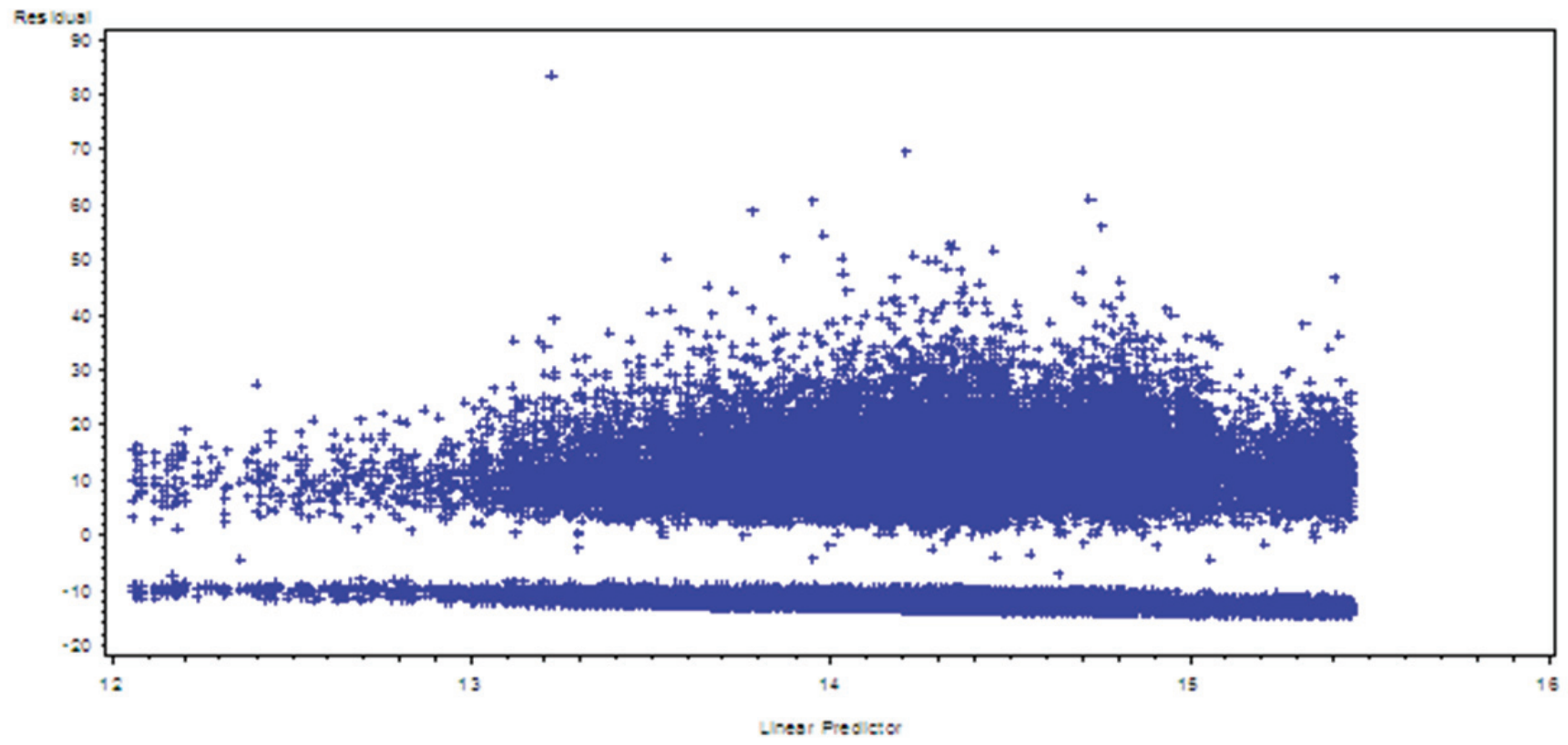




\section{ATEM, NGWA \& ADENIJI}

The fixed effects estimates with different covariance structures may yield the same values, even though the standard errors of these estimates can vary widely. One objective of data analysis using a linear mixed model is to define an adequate error covariance structure in order to obtain efficient estimates of regression parameters; however, to properly estimate the covariance structure, the normality assumption of the random effect must be met. After both conditions are met, multilevel models are most suitable for analysis of longitudinal data and data with hierarchical structure. (Bock, 1989; Bryk \& Raudenbush, 1996; Goldstein, 2003: Hoeksma \& Knol, 2001; Raudenbush, 1989; Snijders, 1996).

\section{References}

Akaike, H. (1973). Information theory and an extension of the maximum likelihood principle. In $2^{\text {nd }}$ International Symposium on Information Theory, B. N. Petrov and F. Csaki (Eds.), 267-281. Budapest: Akademia Kiado.

Akaike, H. (1974). A new look at the statistical model identification. IEEE Transactions on Automatic Control, 19(6), 716723.

Akaike, H. (1985). .Prediction and entropy. In A Celebration of Statistics, A. C. Atkinson and S. E. Fienberg (Eds.), 1-24. New York: Springer.

Atem, F. D., Sharma, R. K., \& Anderson, S. J. (2010). Fitting Bivariate Multilevel Models To Assess Long Term Changes in Body Mass Index (BMI) and Cigarette Smoking. Journal of Applied Statistics, 38(9), 1819-1831.

Bagiella, E., Sloan, R. P., \& Heitjan, D. F. (2000). Mixed-effects models in psychophysiology. Psychophysiology, 37, 13-20.

Bellamy, N. (1995). WOMAC osteoarthritis user's guide. Victoria Hospital Journal of Rheumatology Supplement, 43, 4951.

Bock, R. D. (1989). Multilevel analysis of educational data. San Diego, CA: Academic Press.

Bozdogan, H. (1987). Model selection and Akaike's Information Criterion (AIC): The general theory and its analytical extensions. Psychometrika, 52, 345-370.
Bryk, A. S., \& Raudenbush, S. W. (1987). Application of hierarchical linear models to assessing change. Psychological Bulletin, 101(1), 147-158.

Bryk, A., \& Raudenbush, S. W. (1992). Hierarchical Linear Models for Social and Behavioral Research: Applications and Data Analysis Methods. Newbury Park, CA: Sage.

Dempster, A. P., Selwyn, M. S., Patel, C. P., \& Roth, A. J. (1984). Statistical and computational aspects of mixed model analysis. Applied Statistics, 33, 203-214.

Dmitrienko, A., Tamhane, A. C., \& Bretz, F. (2009). Multiple problems in Pharmaceutical Statistics. New York, NY: Chapman and Hall/CRC Press.

Fieuws, S., \& Verbeke, G. (2004). Joint modeling of multivariate longitudinal profiles: pitfalls of the random-effect approach. Statistics in Medicine, 23, 3093-3104.

Gardiner, J. C., Lu, Z., \& Roman, L. A. (2009). Fixed effects, random effects and GEE: What are the differences? Statistics in Medicine, 28, 221-239.

Goldstein, H. (2003). Multilevel statistical models, $3^{\text {rd }}$ Ed. London: Oxford University Press.

Gomez, B. E., et al. (2005). Covariance Structure is Selected Using AIC and BIC. Communication in Statistics Simulation and Computation, 34, 377-392.

Gurka, M. J, Edwards, L. J., \& Muller, K. E. (2011). Avoiding bias in mixed model inference for fixed effects. Statistics in Medicine, 30(22), 2696-2707.

Hoeksma, J. B., \& Knol, D. L. (2001). Testing predictive developmental hypothesis. Multivariate Behavioral Research, 36, 227-248

Karlis, D., \& Meligkotsidou, L. (2005). Multivariate Poisson regression with covariance structure. Statistical Computations, 15, 255-265.

Laird, N. M., \&Ware, J. H. (1982). Random-effects models for longitudinal data. Biometric, 38, 963-974.

Lindsey, J. K. (1999). Models for Repeated Measurements, $2^{\text {nd }}$ Ed. Oxford: Oxford University Press.

McCulloch, C. (2008). Joint modeling of mixed outcome types using latent variables. Statistical methods in Medical Research, 17, 5373. 


\section{MODELS FOR OVER-DISPERSED POISSON AND CONTINUOUS CLUSTERED DATA}

Pantazis, N., \& Touloumi, G. (2007). Fitting bivariate models for longitudinal data with informative drop-outs using MLwiN. Multilevel Modeling Newsletter, 18, 10-18.

Pawitan, Y., \& Self, S. (1993). Modeling disease marker processes in AIDS. Journal of the American Statistical Association, 88, 719-726.

Rao, C. R. (1965). The theory of least squares when parameters are stochastic and its application to the analysis of growth curves. Biometrika, 52, 447-458.

Rao, C. R. (1973). Linear Statistical Inference and it Application, $2^{\text {nd }} E d$. New York, NY: Wiley.

Sakamoto, Y., Ishiguro, M., \& Kitagawa, G. (1886). Akaike information criterion statistics. Toyko, Japan: KTK Scientific Publisher.

Snijders, T. (1996). Analysis of longitudinal data using the hierarchical linear model. Quality and Quantity, 30, 405-426.

Song, X., Lee, S., \& Hser, Y. (2008). A two-level structural equation model approach for analyzing multivariate longitudinal responses. Statistics in Medicine, 27, 3017-3041.

Thiebaut, R., et al. (2002). Bivariate linear mixed models using SAS proc MIXED. Computer Methods Programs Biomed, 69, 249256.
Tsiatis, A. A, Degruttola, V., \& Wulfsohn, M. S. (1995). Modeling the relationship of survival to longitudinal data measured with error: Applications to survival and CD4 counts in patients with AIDS. Journal of the American Statistical Association, 90, 2737.

Yang, Y., \&Kang, J. (2010). Joint analysis of mixed Poisson and continuous longitudinal data with nonignorable missing values. Computational Statistics and Data Analysis, 54, 193-207.

Yang, Y., Kang, J., Mao, K., \& Zhang, J. (2007). Regression models for mixed Poisson and continuous longitudinal data. Statistics in Medicine, 26, 3782-3800.

Yang, Z., Hardin, J. W., \& Addy, C. L. (2009). A score test for overdispersion in Poisson regression based on the generalized Poisson-2 model. Journal of Statistical Planning and Inference, 139, 1514-1521.

Yang, Z., Hardin, J. W., Addy, C. L., \& Vuong, Q. H. (2007). Approaches for overdispersion in Poisson regression versus the generalized Poisson model. Biometrical Journal, 49(4), 565-584. 


\title{
A Poisson Regression Model for Female Radium Dial Workers
}

\author{
Tze-San Lee \\ Western Illinois University \\ Macomb, IL USA
}

A Poisson regression model with interaction terms was applied to study the dose response relationship for radium-induced skeletal cancers. The model showed that the expected frequency count of bone tumors depended not only on the logarithmic dose and the time since first exposure, but also on the interaction between the logarithmic dose and the time since first exposure, whereas the dose-response model for head tumors depended only on the logarithmic dose.

Key words: Bone sarcoma, confounding factor, head carcinoma, interaction, Poisson regression model, radium dial painters.

\section{Introduction}

The tragedy of female dial painters attributed to radiation poisoning was one of the first widely known incidents of occupational hazards. Because it was a well-paying job many young women were attracted to work in the dialpainting industry in the United States. Unaware of radium poisoning, a common practice adopted by dial painters was to tip their brushes with their lips in order to provide a fine point for painting. The luminous paint usually contained 10 microcurie $(\mu \mathrm{Ci})$ per gram; as a result, dial painters were exposed to the intake of radium into their bodies. Several years after leaving the plant, the former dial painters began developing a variety of mysterious medical problems; the most common symptoms experienced were teeth and jaw problems. For the story of this deadly glow tragedy see Mullner (1999).

A new dose-response model is proposed, specifically a Poisson regression model, for radium-induced skeletal cancers, bone sarcoma (osteogenic sarcoma or fibrosarcoma) and head carcinoma (carcinoma of paranasal sinuses or mastoid air cells), which occurred among the U.S. female radium-dial painters. The dose-response relationship for

Tze-San Lee is presently working at the Centers for Disease Control and Prevention, Mail Stop F-58, Chamblee, GA 34301, USA. Email: tj13@cdc.gov. radium-induced skeletal cancers is very important in the establishment of safety standards for the protection of the public health based on occupationally relevant exposure. Further, this study seeks to enhance understanding about the radiation effect of other $\alpha$-emitting radio-nuclides (e.g., plutonium) for which there are no human data available. (To learn more about the effect of radium poisoning, see Evans (1966, 1967, 1980, 1981), Evans, et al. (1969) and Loutit (1970).)

Evans (1943) established the radiation protection standard of $0.1 \mu \mathrm{Ci}$ of radium in the adult human. In 1967, data from separate studies were consolidated into a newly created Center for Human Radiology (CHR) at Argonne National Laboratory (ANL). Based on a followup of this consolidated data by the end of 1976, Rowland, et al. $(1978,1983)$ established a quasilog-linear model for the incidence rate of bone/head tumors as a function of the product between a quadratic function of exposure dose and an exponential function of exposure dose. They concluded that a model of dose-squaredexponential function provided the best fit for the bone sarcomas, and that an acceptable fit to the head carcinoma data was provided by the linear function of the dose. However, Rosenblatt, et al. (1971) showed that a plot of tumor incidence as a function of doses may potentially be erroneous and misleading. As a result, the theoretical support for Rowland, et al. chosen models might not be adequate. 


\section{A POISSON REGRESSION MODEL FOR FEMALE RADIUM DIAL WORKERS}

Based on this, a better dose-response model needs to be identified, and after reviewing literature on this topic, the author devised a different idea to model the dose-response curve. Because bone/head tumors are rare cancers, Poisson regression model was decided upon for use. The Poisson regression model has proven to be an effective statistical tool in the analysis of cancer death rates (Frome, 1983; Frome \& Checkoway, 1985; Frome, et al., 1990). In 2006 Lee showed that the tumor frequency was supposed to be not only a function of exposure dose levels, but also potential confounding factors including the age at first exposure, the duration of exposure and the time since first exposure. However, the Poisson regression model proposed in that study did not consider the interaction between the exposure variable (dose) and potential confounding variables. This study incorporates all interaction terms into the Poisson regression model.

\section{Study Population}

\section{Methodology}

The study population was a cohort of 4,337 females employed in the U.S. radium-dial industry which was maintained by the $\mathrm{CHR}$ at ANL. This is exactly the same cohort as that used by Rowland, et al. (1978), except that the cohort was enlarged due to extra effort to collect additional subjects after 1976. After the data was first consolidated in the CHR at ANL in 1967, all located subjects were followed for vital status by the staff of the CHR. Death certificates were obtained as soon as staff at the CHR had knowledge of the death and was coded $\left(8^{\text {th }}\right.$ International Classification of Diseases) by the national Center for Health Statistics.

An attempt was made to contact all living subjects annually by mail, and subjects would be contacted by telephone if they did not respond to the mail inquiry. Details of follow-up method, follow-up period, dose measurement and others were given in Argonne's internal report (Radiological and Environmental Research Annual Report, 1984). Excluding those with unknown birth dates or without the social security numbers, 3,688 cases were usable (see Table 1). The measured population contained most of the known radium-induced skeletal cancers. About 973 living cases were still unmeasured despite efforts to obtain their cooperation. Most of these women refused because they did not wish to be reminded of their association with the radium industry or for other reasons not related to their current state of health. There were no known skeletal cancers in this group.

\section{Exposure Data}

Measurements of radium body burden were conducted by whole body counting and radon breath tests as subjects proceeded through a medical examination by a nurse and physician from the medical group of CHR at Argonne. At the time of radium body burden measurement all subjects also received a complete clinical examination, electrocardiography, blood chemistries and urine tests. Due to the interest in bone changes due to radium, extensive sets of $\mathrm{x}$ rays emphasizing the skeleton were completed at each examination.

The complete measurement of radium in the body of a dial painter yielded two values, one for ${ }^{226} \mathrm{Ra}$ and one for ${ }^{228} \mathrm{Ra}$. Because the ratio of ${ }^{228} \mathrm{Ra}$ to ${ }^{226} \mathrm{Ra}$ could vary with each batch of paint being used it was not possible to compare radium cases on the basis of the quantity of radium within the body. What was needed was a method of defining a radium equivalent, so that all measured cases could be expressed in the same units. Two ways to calculate the radium equivalent dose are available.

It was found that an effectiveness ratio $\left({ }^{228} \mathrm{Ra}\right.$ to $\left.{ }^{226} \mathrm{Ra}\right)$ was 1.5 when average skeletal doses were used and 2.5 when initial systemic intake was used a measure of the risk. Because the initial systemic intake was used in Rowland, et al. (1978), the initial systemic intake is also used herein to define the risk of the induction of bone sarcoma in a given dial painter: it is the intake of radium until the end of follow-up in 1984. The average values for each class interval were calculated as the arithmetic mean of their respective individual subject's data in that interval (see Tables 2-3). 
Table 1: Female Radium Dial Workers with Known Status at the End of 1984

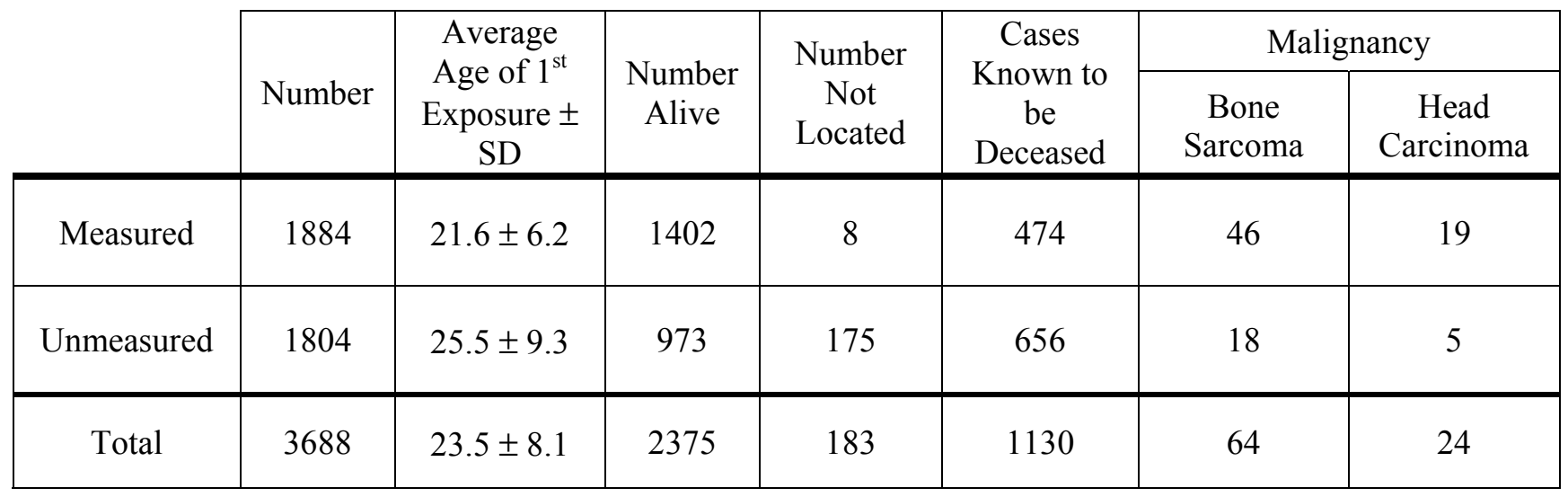

It is the sum of the activity of ${ }^{226} \mathrm{Ra}$, in $\mu \mathrm{Ci}$, that entered the body plus two and a half times the activity of ${ }^{228} \mathrm{Ra}$, in $\mu \mathrm{Ci}$, that entered the body. The head carcinoma was induced by radon $\left({ }^{222} \mathrm{Rn}\right)$ formed by decay of ${ }^{228} \mathrm{Ra}$ trapped within the air spaces in bone. Because the halflife of ${ }^{222} \mathrm{Rn}$ is only 55 seconds, it precludes its migration into these cavities; for this reason, ${ }^{228} \mathrm{Ra}$ was not considered and only ${ }^{226} \mathrm{Ra}$ activity was used for the systemic intake.

The panel data used for analysis of bone sarcomas and head carcinomas is summarized respectively in Tables 2 and 3. The interval sizes chosen were almost the same as that of Rowland, et al. (1978), except that the weighted average of systemic intake for the lowest dose $<$ 0.5 and the highest dose $>1,000$. The interval of the lowest dose $<0.5$ is broken into two intervals, $<0.25$ and $0.25-0.49$; in contrast to Rowland, et al. who ignored the measured dose of subjects in the lowest level. Similarly, the range of the highest dose $>1,000$ is broken into three intervals for bone sarcomas, designated as B1 $(1,000-2,499, \geq 2,500)$, and B2 (1,000-1,299, $1,300-1,599,1,600-1,899,1,900-2,199,2,200-$ $2,499, \geq 2,500$ ), and two intervals for head carcinomas, $\mathrm{H} 1(\geq 1,000)$ and $\mathrm{H} 2(1,000-1,499$, $\geq 1,500)$. However, little difference in the estimated model parameters with respect to different interval sizes for doses greater than $1,000 \mu \mathrm{Ci}$ were concerned; thus, only $\mathrm{B} 1$ and $\mathrm{H} 1$ were used for the purpose of estimating regression coefficients.
Person-years were calculated from the year of first employment to the time of diagnosis of a bone sarcoma, of death, or to the end of 1984. Person-years were summed across cases within exposure levels to estimate the rate denominator. Although the estimated latent period of bone sarcomas followed a lognormal distribution with a median of 22.0 years (or 27.5 years) for all 64 cases (or 46 measured cases), it was decided not to subtract any fixed amount of time from the total person-years to obtain the person-years at risk. The inclusion of those first few years of experience could help establish more precisely the baseline risk (Thomas, 1987). The weighted average systemic intake for each class is the sum of person-year micro-curies for that class divided by the number of person years in the class. Similarly, the time required between first exposure to radium and diagnosis of head carcinoma follows a lognormal distribution with a median 37.5 years for all 24-head carcinomas; no assumed $\log$ time was subtracted from the calculated person-years to estimate person-years at risk.

Three possible potential confounding variables were considered: the age at first exposure $(\mathrm{AFE})=$ age that a dial painter began to put the tip of the paint brush into her lips (years), the duration of exposure $(\mathrm{DOE})=$ period of time that took between the start of putting the tip of the paint brush into her lips and stop such a practice (days), and the time since first exposure $(\mathrm{TFE})=$ years since the first exposure 
A POISSON REGRESSION MODEL FOR FEMALE RADIUM DIAL WORKERS

Table 2: Case Distribution and Bone Sarcoma Experience as a Function of Dose Level and Potential Time-Related Confounding Factors

\begin{tabular}{|c|c|c|c|c|c|c|c|}
\hline \multicolumn{2}{|c|}{$\begin{array}{l}\text { B1: DOSE (Systemic intake/ } \\
\left.{ }^{226} \mathrm{Ra}+2.5 \times{ }^{228} \mathrm{Ra}\right)\end{array}$} & \multirow{2}{*}{$\begin{array}{c}\text { Number } \\
\text { of } \\
\text { Subjects }\end{array}$} & \multirow{2}{*}{$\begin{array}{c}\text { Person- } \\
\text { Years } \\
\text { (Years) }\end{array}$} & \multirow{2}{*}{$\begin{array}{c}\mathrm{N} \\
\text { (Bone } \\
\text { Sarcoma) }\end{array}$} & \multirow{2}{*}{$\begin{array}{c}\text { Average } \\
\text { Age at } 1^{\text {st }} \\
\text { Exposure } \\
\text { (AFE, } \\
\text { Years) }\end{array}$} & \multirow{2}{*}{$\begin{array}{c}\text { Average } \\
\text { Duration } \\
\text { of } \\
\text { Exposure } \\
\text { (DOE, } \\
\text { Days) }\end{array}$} & \multirow{2}{*}{$\begin{array}{l}\text { Average } \\
\text { Time } \\
\text { Since } 1^{\text {st }} \\
\text { Exposure } \\
\text { (TFE, } \\
\text { Years) }\end{array}$} \\
\hline $\begin{array}{c}\text { Range } \\
(\mu \mathrm{Ci})\end{array}$ & $\begin{array}{c}\text { Weighted } \\
\text { Average } \\
(\mu \mathrm{Ci})\end{array}$ & & & & & & \\
\hline$<0.25$ & 0.04 & 881 & 35054 & 0 & 21.2 & 159.1 & 39.8 \\
\hline $0.25-0.49$ & 0.36 & 190 & 8176 & 0 & 21.7 & 233.5 & 43.0 \\
\hline $0.5-0.99$ & 0.72 & 172 & 7784 & 0 & 21.7 & 233.2 & 45.3 \\
\hline $1.0-2.49$ & 1.52 & 193 & 9782 & 0 & 19.7 & 212.6 & 50.7 \\
\hline $2.5-4.9$ & 3.59 & 96 & 5100 & 0 & 19.0 & 195.6 & 53.1 \\
\hline $5-9.9$ & 6.99 & 78 & 4281 & 0 & 19.5 & 119.5 & 54.9 \\
\hline $10-24$ & 16.46 & 73 & 4144 & 0 & 19.5 & 156.0 & 56.8 \\
\hline $25-49$ & 26.12 & 52 & 2932 & 1 & 19.4 & 156.2 & 56.4 \\
\hline $50-99$ & 69.73 & 21 & 1188 & 0 & 18.3 & 301.1 & 56.6 \\
\hline $100-249$ & 160.5 & 28 & 1472 & 1 & 18.3 & 307.8 & 52.6 \\
\hline $250-499$ & 374.3 & 36 & 1639 & 12 & 19.3 & 251.6 & 45.5 \\
\hline $500-999$ & 683.1 & 21 & 835 & 10 & 19.5 & 268.1 & 39.8 \\
\hline $1,000-2,499$ & 1665.4 & 26 & 611 & 18 & 18.8 & 157.9 & 23.5 \\
\hline$\geq 2,500$ & 3576.6 & 17 & 310 & 4 & 18.5 & 185.8 & 18.2 \\
\hline
\end{tabular}


Table3: Case Distribution and Head Carcinoma Experience as a Function of Dose Level and Potential Time Related Confounding Factors

\begin{tabular}{|c|c|c|c|c|c|c|c|}
\hline \multicolumn{2}{|c|}{$\begin{array}{c}\text { H1: DOSE } \\
\left.\text { (Systemic Intake }{ }^{226} \mathrm{Ra}\right)\end{array}$} & \multirow{2}{*}{$\begin{array}{c}\text { Number } \\
\text { of } \\
\text { Subjects }\end{array}$} & \multirow{2}{*}{$\begin{array}{l}\text { Person- } \\
\text { Years } \\
\text { (Years) }\end{array}$} & \multirow{2}{*}{$\begin{array}{c}\mathrm{N}(\text { Head } \\
\text { Carcinoma) }\end{array}$} & \multirow{2}{*}{$\begin{array}{c}\text { Average } \\
\text { Age at } 1^{\text {st }} \\
\text { Exposure } \\
\text { (AFE, } \\
\text { Years) }\end{array}$} & \multirow{2}{*}{$\begin{array}{c}\text { Average } \\
\text { Duration } \\
\text { of } \\
\text { Exposure } \\
\text { (DOE, } \\
\text { Days) }\end{array}$} & \multirow{2}{*}{$\begin{array}{c}\text { Average } \\
\text { Time } \\
\text { Since } 1^{\text {st }} \\
\text { Exposure } \\
\text { (TFE, } \\
\text { Years) }\end{array}$} \\
\hline $\begin{array}{l}\text { Range } \\
(\mu \mathrm{Ci})\end{array}$ & $\begin{array}{c}\text { Weighted } \\
\text { Average } \\
(\mu \mathrm{Ci})\end{array}$ & & & & & & \\
\hline$<0.25$ & 0.04 & 884 & 36155 & 0 & 32.2 & 158.9 & 40.9 \\
\hline $0.25-0.49$ & 0.35 & 213 & 9801 & 0 & 21.2 & 210.5 & 46.0 \\
\hline $0.5-0.99$ & 0.71 & 198 & 9487 & 0 & 21.3 & 210.9 & 47.9 \\
\hline $1.0-2.49$ & 1.53 & 237 & 12489 & 0 & 20.1 & 191.0 & 52.7 \\
\hline $2.5-4.9$ & 3.50 & 85 & 4630 & 0 & 19.1 & 217.8 & 54.5 \\
\hline $5-9.9$ & 6.90 & 50 & 2826 & 0 & 19.1 & 155.0 & 56.5 \\
\hline $10-24.9$ & 16.0 & 59 & 3402 & 0 & 19.3 & 197.6 & 57.7 \\
\hline $25-49$ & 35.3 & 40 & 2148 & 1 & 18.6 & 217.4 & 53.7 \\
\hline $50-99$ & 68.6 & 23 & 1141 & 1 & 18.2 & 370.6 & 49.6 \\
\hline $100-249$ & 175. & 33 & 1303 & 6 & 17.9 & 143.4 & 39.5 \\
\hline $250-499$ & 364. & 33 & 1379 & 6 & 19.2 & 259.2 & 41.8 \\
\hline $500-999$ & 616. & 16 & 444 & 2 & 21.9 & 238.9 & 27.8 \\
\hline$\geq 1,000$ & 1566 & 13 & 289 & 3 & 17.8 & 179.2 & 22.2 \\
\hline
\end{tabular}




\section{A POISSON REGRESSION MODEL FOR FEMALE RADIUM DIAL WORKERS}

\section{Poisson Regression Model}

Because bone sarcomas and head carcinomas are rare cancers, the frequency count (Y) of bone sarcomas (or head carcinomas) was assumed to follow a Poisson process, that is, the probability of $\mathrm{N}$ bone (or head) tumors is given by

$$
P(Y=N)=e^{-\mu} \cdot \frac{\mu^{N}}{N !}, \mathrm{N}=0,1,2, \ldots,(\mu>0),
$$

where $\mu$ denotes the expected count number of bone (or head) tumor. The tumor rate is Y/PYR, where PYR denotes the total exposure person years. Further, a Poisson regression model with interaction terms was applied to model the expected frequency counts of bone (or head) tumor as a function of logarithmic dose, temporal confounding factors together with their interaction with the logarithmic dose as follows:

$$
\begin{aligned}
\ln \mu= & \alpha_{0}+\ln (P Y R)+\alpha_{1} L D O S E+\alpha_{2} A F E \\
& +\alpha_{3} D O E+\alpha_{4} T F E+\alpha_{5} L D O S E \cdot A F E \\
& +\alpha_{6} L D O S E \cdot D O E+\alpha_{7} L D O S E \cdot T F E
\end{aligned}
$$

where $\ln$, the natural algorithmic function, of the left-hand side of (2) denotes link function, $\ln (\mathrm{PYR})$ is the offset (McCullagh \& Nelder, 1989), and LDOSE is the natural logarithm of the weighted average systemic intake.

The reason the logarithm of the dose level (LDOSE) was used as opposed to the dose level was that the ratio between the highest to the lowest dose level was greater than 1,000 . The method of maximum likelihood estimation was employed in computing the unknown regression coefficients $\left(\alpha_{i}\right.$ 's) of equation 2 by setting distribution $=$ Poisson, link $=\log$, and offset $=\ln (\mathrm{PYR})$ in the Proc GENMOD provided by the SAS package (SAS/STAT User's Guide, 1999). Based upon the wellformulated hierarchical principle, a backward elimination procedure was employed to retain the significant terms in equation 2 (Kleinbaum, et al., 1982). A criterion of the best fit is that the ratio of the scaled deviance divided by the degrees of freedom (d.f.) associated with the fitted model equals to one (Fleiss, et al., 2003).

\section{Results}

A total of 64 and 24 subjects were diagnosed with bone sarcomas and head carcinomas respectively. The prevalence for bone sarcoma $(1.7 \%=64 / 3,688)$ is 2.7 times as large as that $(0.7 \%=24 / 3,688)$ for head carcinoma (see Table 1). The reason for this significant difference in the incidence rate was that the head carcinoma seems to appear much later. The time of appearance for bone sarcoma was approximately 5 years, whereas 19 years for head carcinoma if the time of appearance was plotted against the initial systemic intake (Rowland, 1994). The highest systemic intake was the age at first exposure (AFE); values ranged from 16.0 to 21.7 , which confirmed that the female radium dial workers were very young.

The average duration of exposure was shorter for low dose ranges than that for high dose ranges because the entire cohort in this study was comprised of two major sub-cohorts, pre-1930 and post-1930. A warning not put the tip of the paint brush into their mouth was issued by the government to workers in the dial painting industry in 1926; hence, workers in the post-1930 cohort received much less exposure. Similarly, the average time since first exposure (TFE) value for the class over $2,500 \mu \mathrm{Ci}$ was only 18.2 years which was far shorter than those in the range of less than $1,000 \mu \mathrm{Ci}$. This was because most of 17 measured in that class were already diseased. Incidentally, the highest systemic intake was $6,331 \mu \mathrm{Ci}$.

Table 4 shows the parameter estimate, standard error/p-value and scaled deviance/degrees of freedom associated with each of the risk factors used in (2) for the dataset B1. Using the p-value $<0.05$ as a criterion for variable inclusion, the final models for the bone tumor that represent the dataset B1 is given respectively by

$$
\begin{aligned}
\ln \mu= & 4.8-1.2 \cdot L D O S E-0.4 \cdot T F E \\
& +0.051 \cdot L D O S E \cdot T F E
\end{aligned}
$$


Table 4: Estimated Regression Coefficients (p-value) and Scaled Deviance (degrees of freedom) for Bone Sarcoma

\begin{tabular}{|c|c|c|c|c|c|}
\hline Data Set & $\begin{array}{c}\text { Intercept } \\
(\mathrm{p} \text {-value })\end{array}$ & $\begin{array}{c}\text { LDOSE } \\
(\mathrm{p} \text {-value })\end{array}$ & $\begin{array}{c}\text { TFE } \\
(\mathrm{p} \text {-value })\end{array}$ & $\begin{array}{c}\text { LDOSE*TFE } \\
\text { (p-value) }\end{array}$ & $\begin{array}{c}\text { Scaled Deviance } \\
\text { (d.f.) }\end{array}$ \\
\hline \multirow{3}{*}{ B1 } & 4.78 & -1.19 & -0.36 & 0.051 & 6.12 \\
& $(0.38)$ & $(0.09)$ & $(0.002)$ & $(0.001)$ & $(10)$ \\
\hline
\end{tabular}

for dataset $\mathrm{B} 1$, the interaction term LDOSE $\times$ TFE was significant. As a result, LDOSE and TFE (the lower order term) were retained in the model, even though the p-value for the term of LDOSE (p-value $=0.09$ ) in equation was not significant.

Table 5 shows the parameter estimates, standard errors, p-value, model deviances and degrees of freedom after fitting Poisson model of (2) to dataset H1. According to Table 5, the Poisson models for $\mathrm{H} 1$ is given respectively by

$$
\ln \mu=-11.29+0.978 \cdot \text { LDOSE . }
$$

\section{Discussion}

Time since first exposure (TFE) (see eq. 3), shown as a confounding factor, had an effect on the occurrence of bone sarcomas in addition to the logarithmic dose. Worse, a significant interaction existed between the logarithmic dose and the time since first exposure: This implies that, for different time since first exposure, the effect of the logarithmic dose on the expected frequency of bone tumors is different. In other words, time since first exposure is an effect modifier (Kleinbaum, et al., 1982). By contrast, time since first exposure is neither a confounder nor an effect modifier for the expected frequency of head carcinomas. An advantage of using the logarithmic dose level is reflected in that it is not necessary to be concerned if the term of dose-squared or the term of power higher than two is included or not. From a Taylor's series expansion, $\ln (\mathrm{DOSE})$ contains all powers of DOSE in the model.
The Environmental Protection Agency proposed the maximum level for radium in drinking water to be set at $5 \mathrm{pCi} /$ liter, where $\mathrm{pCi}$ denotes picocurie and one picocurie, onetrillionth of a curie (Train, 1975). Using the generally accepted values of 2.2 liters of water consumed per day and a gut absorption rate of $21 \%$, the systemic intake calculated by Rowland, et al. (1978) is $843 \mathrm{pCi}$ of ${ }^{226} \mathrm{Ra}$. Using the linear model for head carcinoma, the incidence rate after 1 -year intake calculated by Rowland, et al. is $1.3 \times 10^{-8}$. However, by using equation 4 , the incidence rate for head carcinomas is $1.2 \times 10^{-5}$. In a comparison with this estimate, the Rowland, et al. (1978) estimate seems too conservative. Rowland, et al. did not calculate the rate for bone sarcoma, however, using equation 3 , the rates for bone sarcomas were $3.4,0.09,0.002$, and $6.2 \times 10^{-5}$, respectively for $\mathrm{TFE}=10,20,30$, and 40 years.

Although the data for the frequency of bone/head tumors seemingly have excessive zeros over a wide range of logarithmic dose levels, the zero-inflated Poisson model (Lambert, 1992) was not able to be used for datasets $\mathrm{B} 1$ or $\mathrm{H} 1$ because it was not possible to model its frequency as a mixture of two models: one is a degenerated point mass function at zero count and the other is a Poisson model for count greater than one. When attempted using the SAS Proc GENMOD, the scaled deviance (36.7) was much larger than its degrees of freedom (11).

Baum (1973) claimed that the doseresponse curve of radiation induced tumors was often represented by a power function of dose with exponents less than one. By using the atomic bomb of surviving population in 
Table 5: Estimated Regression Coefficients (p-value) and Scaled Deviances (degrees of freedom) for Head Carcinoma

\begin{tabular}{|c|c|c|c|}
\hline Data Set & $\begin{array}{l}\text { Intercept } \\
\text { (p-value) }\end{array}$ & $\begin{array}{l}\text { LDOSE } \\
\text { (p-value) }\end{array}$ & $\begin{array}{c}\text { Scaled Deviance } \\
\text { (d.f.) }\end{array}$ \\
\hline $\mathrm{H} 1$ & $\begin{array}{c}-11.29 \\
(<0.0001)\end{array}$ & $\begin{array}{c}0.978 \\
(<0.0001)\end{array}$ & $\begin{array}{l}7.51 \\
(11)\end{array}$ \\
\hline
\end{tabular}

Hiroshima and Nagasaki, Japan, Baum found that for data on leukemia in Hiroshima and Nagasaki, the dose-response curve was adequately represented by power functions of dose with exponents between 0.65 and 1.0 . However, in view of results from this study, the expected frequency was a function of not only the logarithmic dose, but also potential timerelated confounding factors (time since first exposure) and the interaction between the logarithmic dose and time since first exposure. Hence, Baum's claim for the dose-response relationship is clearly invalid.

Recent studies have also addressed other aspects of radiation poisoning among U.S. radium dial workers. Carnes, et al. (1997) adopted Cox's (1972) hazard regression to build a dose response model. Although they incorporated AFE, a time-related confounding factor, into their model, the AFE was shown to be insignificant according the Poisson model used in this study. In addition, Carnes, et al. did not consider interaction in their study. An overview of studies of the U.S. radium dial workers was presented by Fry (1998). In addition, Rentztzi (2004) addressed the case of radium dial workers as human experimentation with radiation harmful effect.

\section{Conclusion}

Using the Poison regression model, data regarding radiation poisoning among female radium dial workers were re-analyzed. A doseresponse model was obtained respectively for bone sarcoma and head carcinoma. The model showed that the expected frequency of bone sarcomas was not only a function of the logarithmic dose and the time since first exposure, but also the interaction between the logarithmic dose and the time since first exposure, whereas the dose-response model for head carcinomas was a function of the logarithmic dose only. Among all dose-response models available in the literature, the Poisson regression model proposed in this article was deemed best because it is simple, precise and informative.

Acknowledgements

This work was initiated during a sabbatical leave from WIU for the period from August 20, 1990 to May 31, 1991 to visit the Argonne National Laboratory. The author wishes to thank and gratefully acknowledge the Biomedical Science Division of ANL for permission granted to work on the female radium dial painter data, T. J. Kotek for helping to familiarize me with database and Dr. Zhiyuan Liu for assisting me in calculating the AFE, DOE and TFE values listed in Tables 2-3.

\section{References}

Argonne National Laboratory. (1984). Radiological and Environmental Research Annual Report. ANL84-103, Part II, Appendix A, 98-185. Argonne, IL: Argonne National Laboratory.

Baum, J. W. (1973). Population heterogeneity hypothesis on radiation induced cancers, Health Physics, 25, 97-104.

Carnes, B. A., Groer, P. G., \& Kotek, T. J. (1997). Radium dial workers: issues concerning dose response and modeling. Radiation Research, 147, 707-714.

Comprehensive Epidemiologic Data Resource (CEDR). (1993). https://www.orau.gov/cedr.

Cox, D. R. (1972). Regression models and life tables (with discussion). Journal of the Royal Statistical Society, Series B, 34, 187-202. 


\section{TZE-SAN LEE}

Evans, R. D. (1943). Protection of radium dial workers and radiologists from injury by radium. Journal of Industrial Hygiene, 25, 253269.

Evans, R. D. (1966). The effect of skeletally deposited alpha-gray emitters in man. British Journal of Radiology, 39, 881-895.

Evans, R. D. (1967). The radium standard for boneseekers-evaluation of the data on radium patients and dial painters. Health Physics, 13, 267278.

Evans, R. D. (1980). Radium poisoning: a review of present knowledge. Health Physics, 38, 899-905.

Evans, R. D. (1981). Radium poisoning: a review of present knowledge. Health Physics, 41, 437-448.

Evans, R. D., Keane, A. T., Kolenkow, R. J., Neal, W. R., \& Shanahan, M. M. (1969). Radiogenic tumors in the radium and mesothorium cases studied at MIT. In Delayed effects of boneseeking radionuclides, C. W. Mays, Ed., 157-194. Salt Lake City, UT: University of Utah Press.

Fleiss, J. L., Levin, B., \& Paik, M. C. (2003). Statistical methods for rates and proportions, $3^{\text {rd }} E d$. New York, NY: Wiley.

Frome, E. L. (1983). The analysis of rates using Poisson regression models. Biometrics, 39, 665-674.

Frome, E. L., \& Checkoway, H. (1985). Use of Poisson regression models in estimating incidence rates and ratios. American Journal of Epidemiology, 121, 309-323.

Frome, E. L., Cragle, D. L., \& McLain, R. W. (1990). Poisson regression analysis of the mortality among a cohort of World War II nuclear industry workers. Radiation Research, 123, 138152.

Fry, S. A. (1998). Studies of U.S. radium dial workers: an epidemiological classic. Radiation Research, 150(supplement), S21-S29.

Gilbert, E. S., Fry, S. A., Wiggs, L. D., Volez, G. L., Cragle, G. L., \& Petersen, G. R. (1990). Methods for analyzing combined data from studies of workers exposed to low doses for radiation. American Journal of Epidemiology, 131, 917-927.

Kleinbaum, D. G., Kupper, L. L., \& Morgenstern, H. (1982). Epidemiologic research: Principles and quantitative methods. Belmonts, CA: Life Time Learning Publications.
Lambert, D. (1992). Zero-inflated Poisson regression with application to defects in manufacturing. Technometrics, 34, 1-14.

Lee, T-S. (2006). Dose-time-response models for female radium dial workers. Proceedings of the $3^{\text {rd }}$ Sino-International Symposium on Probability, Statistics, and Quantitative Management, 135-144. Taipei, Taiwan.

Loutit, J. F. (1970). Malignancy from radium. British Journal of Cancer, 24, 195-207.

McCullagh, P., \& Nelder, J. A. (1989). Generalized linear models, $2^{\text {nd }} E d$. London: Chapman \& Hall.

Mullner, R. (1999). Deadly glow: The radium dial worker tragedy. Washington, DC: American Public Health Association.

Rentetzi, M. (2004). The women radium dial painters as experimental subjects (1920-1990) or what counts as human experimentation. NTM Zeitschrift fur Geschichte der Wissenschaften, Technik und Medizin, 12, 233-248.

Rosenblatt, L. S., Hetherington, N. H., Goldman, M., \& Bustad, L. K. (1971). Evaluation of tumor incidence following exposure to internal emitters by application of the logistic doseresponse surface. Health Physics, 21, 869-875.

Rowland, R. E. (1994). Radium in humans: A review of U. S. studies. ANL/ER-3 UC408, Argonne, IL: Argonne National Laboratory.

Rowland, R. E., Stehney, A. F., \& Lucas, H. F. (1978). Dose-response relationships for female radium workers. Radiation Research, 76, 368-383.

Rowland, R. E., Stehney, A. F., \& Lucas, H. F. (1983). Dose-responses relationships for radium-induced bone sarcomas. Health Physics, 44 (supplement No. 1), 15-31.

SAS Institute, Inc. (1999). SAS/STAT user's guide, Version 9.2. Cary, NC: SAS Institute, Inc.

Thomas, D. C. (1987). Pitfalls in the analysis of exposure-time-response relationships. Journal of Chronic Disease, 40(Supplement No. 2), 71-78.

Train, R. E. (1975). Interim primary drinking water regulations. Federal Registry, 40, 324-334. 


\title{
Ordinal Regression Analysis: Using Generalized Ordinal Logistic Regression Models to Estimate Educational Data
}

\author{
Xing Liu \\ Hari Koirala \\ Eastern Connecticut State University, \\ Willimantic, CT
}

The proportional odds (PO) assumption for ordinal regression analysis is often violated because it is strongly affected by sample size and the number of covariate patterns. To address this issue, the partial proportional odds (PPO) model and the generalized ordinal logit model were developed. However, these models are not typically used in research. One likely reason for this is the restriction of current statistical software packages: SPSS cannot perform the generalized ordinal logit model analysis and SAS requires data restructuring. This article illustrates the use of generalized ordinal logistic regression models to predict mathematics proficiency levels using Stata and compares the results from fitting PO models and generalized ordinal logistic regression models.

Key words: Generalized ordinal logistic regression models, proportional odds models, partial proportional odds model, ordinal regression analysis, mathematics proficiency, stata, comparison.

\section{Introduction}

Ordinal data in education are substantive. Perhaps the most well-known model for estimating an ordinal outcome variable is the proportional odds (PO) model (Agresti, 1996, 2002, 2007; Anath \& Kleinbaum, 1997; Armstrong \& Sloan, 1989; Hardin \& Hilbe, 2007; Long, 1997; Long \& Freese, 2006; McCullagh, 1980; McCullagh \& Nelder, 1989; O'Connell, 2000, 2006; Powers \& Xie, 2000). Current general-purpose statistical software packages, such as SAS, SPSS and Stata, use this model as the default for ordinal regression analysis. The PO model is used to estimate the cumulative probability of being at or below a particular level of a response variable, or being beyond a particular level, which is the complementary direction. In this model, the effect of each predictor is assumed to be the

Xing Liu is an Associate Professor of Research and Assessment in the Education Department. Email him at: liux@easternct.edu. Hari Koirala is a Professor in the Education Department. Emailhim at:koiralah@easternct.edu. same across the categories of the ordinal dependent variable. This means that for each predictor, the effect on the odds of being at or below any category remains the same within the model. This restriction is referred to as the proportional odds, or the parallel lines, assumption.

The assumption of proportional odds is often violated, however, because it is strongly affected by sample size and the number of covariate patterns - for example, including continuous covariates or interactions as the predictors (Allison, 1999; Brant, 1990; O'Connell, 2006). It is misleading and invalid to interpret results if this assumption is not tenable. It has been suggested that the separate underlying binary logistic regression models are fitted and then are compared with the original PO model (Allison, 1999; Bender \& Grouven, 1998; Brant, 1990; Clogg \& Shihadeh, 1994; Long, 1997; O'Connell, 2000, 2006). Although this strategy would help researchers identify the reason why the overall PO assumption is violated, it is not clear how a well-fitting parsimonious model with a violated PO assumption is developed and interpreted.

To address this issue, the partial proportional odds (PPO) model (Peterson \& 


\section{XING LIU \& HARI KOIRALA}

Harrell, 1990) and the generalized ordinal logit model (Fu, 1998; Williams, 2006) were developed. The PPO model allows for interactions between a predictor variable that violates the PO assumption and different categories of the ordinal outcome variable. The analysis of a PPO model using SAS GENMOD procedure requires a restructured data set, which includes a new binary variable indicating whether an individual is at or beyond a particular level (O'Connell, 2006; Stokes, Davis \& Koch, 2000).

The generalized ordinal logit model developed by $\mathrm{Fu}$ (1998) and William (2006) relaxes the PO assumption by allowing the effect of each explanatory variable to vary across different cut points of the ordinal outcome variable without data restructuring. In addition, this model estimates parameters differently from the PPO model using SAS. Williams' gologit2 program (2006) for Stata is a more powerful extension of Fu's gologit (1998); it can estimate the generalized ordered logit model, the PPO model, the PO model and the logistic regression model within one program.

In educational research, the PO model is widely used. However, the use of the generalized ordinal logit model appears to be overlooked even in cases where the PO assumption is violated. One likely reason for this is the restriction of current statistical software packages: SPSS cannot perform the generalized ordinal logit model analysis and SAS requires data restructuring prior to data analysis, therefore, it is important to help educational researchers better understand this model and utilize it in practice.

The purpose of this study is to illustrate the use of generalized ordinal logistic regression models to predict mathematics proficiency levels using Stata and to compare the results of fitting PO and the generalized ordinal logistic regression models. This article is an extension of previous research focusing on the PO model (Liu, 2009), and the Continuation Ratio model with Stata (Liu, O'Connell, \& Koirala, 2011). Ordinal regression analyses are based on data from the 2002 Educational Longitudinal Study (ELS) in which the ordinal outcome of students' mathematics proficiency was forecast from a set of predictors, such as, using computers for fun, school work and to learn on their own.

Theoretical Framework

General Logistic Regression Model and the Proportional Odds Model

The binary logistic regression model estimates the odds of success or experiencing an event for the dichotomous response variable given a set of predictors. The logistic regression model can be defined as (Allison, 1999; Menard, 1995):

$$
\begin{aligned}
\ln \left(\mathrm{Y}^{\prime}\right) & =\operatorname{logit}[\pi(\underline{\mathrm{x}})] \\
& =\ln \left(\frac{\pi(\underline{\mathrm{x}})}{1-\pi(\underline{\mathrm{x}})}\right) \\
& =\alpha+\beta_{1} \mathrm{X}_{1}+\beta_{2} \mathrm{X}_{2}+\ldots+\beta_{\mathrm{p}} \mathrm{X}_{\mathrm{p}}
\end{aligned}
$$

In an ordinal logistic regression model, the outcome variable has more than two levels. It estimates the probability being at or below a specific outcome level given a collection of explanatory variables. The ordinal logistic regression model can be expressed in the logit form (Liu, 2009; Long, 1997; Long \& Freese, 2006) as follows:

$$
\begin{aligned}
\ln \left(\mathrm{Y}_{\mathrm{j}}^{\prime}\right) & =\operatorname{logit}[\pi(\mathrm{x})] \\
& =\ln \left(\frac{\pi(\mathrm{x})}{1-\pi(\mathrm{x})}\right) \\
& =\alpha_{\mathrm{j}}+\left(-\beta_{1} \mathrm{X}_{1}-\beta_{2} \mathrm{X}_{2}-\ldots-\beta_{\mathrm{p}} \mathrm{X}_{\mathrm{p}}\right)
\end{aligned}
$$

where $\pi_{\mathrm{j}}(\underline{\mathrm{x}})=\pi\left(\mathrm{Y} \leq \mathrm{j} \mid \mathrm{x}_{1}, \mathrm{x}_{2}, \ldots, \mathrm{x}_{\mathrm{p}}\right)$ is the probability of being at or below category $\mathrm{j}$, given a set of predictors, $\mathrm{j}=1,2, \ldots, \mathrm{J}-1, \alpha_{\mathrm{j}}$ are the cut points and $\beta_{1}, \beta_{2}, \ldots, \beta_{\mathrm{p}}$ are logit coefficients. When there are $\mathrm{j}$ categories, the PO model estimates J-1 cut points. This PO model assumes that the logit coefficient of any predictor is independent of categories, i.e., the coefficients for the underlying binary models are the same across all cutpoints. The equal logit slope or the proportional odds assumption can be assessed by the Brant test (Brant, 1990), which estimates logit coefficients for underlying binary logistic regressions, and provides the chi-square test 


\section{GENERALIZED ORDINAL LOGISTIC REGRESSION MODELS}

statistics for each predictor and the overall model in Stata.

To estimate the ln (odds) of being at or below the $\mathrm{j}^{\text {th }}$ category, the PO model can be rewritten as the following form:

$$
\begin{aligned}
\operatorname{logit} & {\left[\pi\left(Y \leq j \mid x 1, x 2, \ldots, x_{p}\right)\right] } \\
& =\ln \left(\frac{\pi\left(Y \leq j \mid x_{1}, x_{2}, \ldots, x_{p}\right)}{\pi\left(Y>j \mid x_{1}, x_{2}, \ldots, x_{p}\right)}\right) \\
& =\alpha_{j}+\left(-\beta_{1} X_{1}-\beta_{2} X_{2}-\ldots-\beta_{p} X_{p}\right) .
\end{aligned}
$$

Thus, this model predicts cumulative logits across $\mathrm{J}-1$ response categories. The cumulative logits can then be used to calculate the estimated cumulative odds and the cumulative probabilities being at or below the $\mathrm{j}^{\text {th }}$ category.

Different software packages may estimate parameters differently and the ordinal logistic regression model can be expressed in different forms (Liu, 2009). For example, Stata follows the above form with a negative sign before the logit coefficients. SAS, however, uses a different form when estimating the cumulative odds of being at or below a particular category using the ascending option.

The Generalized Ordinal Logistic Regression Model

The generalized ordinal logistic regression model extends the $\mathrm{PO}$ model by relaxing the $\mathrm{PO}$ assumption. In this model, if the assumption is violated by a certain predictor, then its effect can be estimated freely across different categories of the dependent variable. The model is expressed as:

$$
\begin{aligned}
\ln \left(Y_{j}{ }^{\prime}\right) & =\ln \left(\frac{\pi_{j}(\underline{x})}{1-\pi_{j}(\underline{x})}\right) \\
& =\alpha_{j}+\left(\beta_{1 j} X_{1}+\beta_{2 j} X_{2}+\ldots+\beta_{p j} X_{p}\right) .
\end{aligned}
$$

The above form can also be expressed as proposed by $\mathrm{Fu}$ (1998) and Williams (2006):

$$
\begin{aligned}
\operatorname{logit} & {\left[\pi\left(Y>j \mid x 1, x 2, \ldots, x_{p}\right)\right] } \\
= & \ln \left(\frac{\pi\left(Y>j \mid x_{1}, x_{2}, \ldots, x_{p}\right)}{\pi\left(Y \leq j \mid x_{1}, x_{2}, \ldots, x_{p}\right)}\right) \\
& =\alpha_{j}+\left(\beta_{1 j} X_{1}+\beta_{2 j} X_{2}+\ldots+\beta_{p j} X_{p}\right),
\end{aligned}
$$

where, in both equations, $\alpha_{j}$ are the intercepts or cutpoints, and $\beta_{1 \mathrm{j}}, \beta_{2 \mathrm{j}}, \ldots, \beta_{\mathrm{pj}}$ are logit coefficients. This model estimates the odds of being beyond a certain category relative to being at or below that category. A positive logit coefficient generally indicates that an individual is more likely to be in a higher category as opposed to a lower category of the outcome variable. To estimate the odds of being at or below a particular category, however, the signs before both the intercepts and logit coefficients in equation (5) must be reversed.

In this expression, all of the effects of the explanatory variables are allowed to vary across each of the cutpoints. If some of these effects are found to be stable, they can be constrained to be equal as in the PO model; thus, generalized ordinal logistic regression model refers to the case where at least one of the coefficients for a predictor varies across categories. Compared to SAS, neither data restructuring nor interactions between categories and predictor variables with non-proportional odds are required by Stata - this makes data analysis of the generalized ordinal logit model much easier.

Sample

\section{Methodology}

The data used in this study are from the Educational Longitudinal Study of 2002 (ELS: 2002). The ELS 2002 study, conducted by the National Center for Educational Statistics (NCES), was designed to provide longitudinal data regarding high school students' achievement, attitude and experiences, and their postsecondary school education and the labor market. In the 2002 base year of the study a cohort of more than 15,000 high school sophomores from a national sample of 752 public and private high schools participated in 


\section{XING LIU \& HARI KOIRALA}

the study by taking cognitive tests and responding to surveys.

The outcome variable was students' mathematics proficiency levels in high school, which was an ordinal variable with five levels (1 = students can do simple arithmetical operations on whole numbers; 2 = students can do simple operations with decimals, fractions, powers, and root; $3=$ students can do simple problem solving; $4=$ students can understand intermediate-level mathematical concepts and/or find multi-step solutions to word problems; and $5=$ students can solve complex multiple-step word problems and/or understand advanced mathematical material) (Ingels, Pratt, Roger, Siegel, \& Stutts, 2004, 2005). These five proficiency domains were hierarchically structured: mastery of higher proficiency level indicated mastery of all previous levels. Those students who failed to pass through level 1 were assigned to level 0 . Table 1 provides the categories and frequencies of all mathematics proficiency levels.
Data Analysis.

After the PO model was fitted with a single explanatory variable using the Stata ologit command the full-model was then fitted with all three explanatory variables. The brant test command, one of the Stata SPost (Long \& Freese, 2006) routines for the analysis of postestimations, was used to examine the PO assumption and identify predictors that violated the assumption. Stata gologit (Fu, 1998) and gologit2 (Williams, 2006) commands were then used to fit the generalized ordinal regression models and the results from both PO models and the generalized ordinal regression models were compared.

\section{Results}

Proportional Odds Model with a Single Explanatory Variable

A PO model with a single predictor, using computers for fun, was fitted first using the Stata ologit command with the logit function as default. Table 2 provides the results for the single-predictor PO model.

Table 1: Proficiency Categories and Frequencies (Proportions) for the Study Sample, ELS $2002(\mathrm{~N}=15,976)$

\begin{tabular}{|c|c|c|}
\hline $\begin{array}{l}\text { Proficiency } \\
\text { Category }\end{array}$ & Description & $\begin{array}{l}\text { Frequency and } \\
\text { Percent of Total Sample }\end{array}$ \\
\hline 0 & Did not pass level 1 & $\begin{array}{c}842 \\
(5.27 \%)\end{array}$ \\
\hline 1 & Can do simple arithmetical operations on whole numbers & $\begin{array}{c}3,882 \\
(24.30 \%)\end{array}$ \\
\hline 2 & $\begin{array}{l}\text { Can do simple operations with decimals, fractions, powers, } \\
\text { and root }\end{array}$ & $\begin{array}{c}3,422 \\
(21.42 \%)\end{array}$ \\
\hline 3 & Can do simple problem solving & $\begin{array}{c}4,521 \\
(28.30 \%)\end{array}$ \\
\hline 4 & $\begin{array}{l}\text { Can understand intermediate-level mathematical concepts } \\
\text { and/or find multi-step solutions to word problems }\end{array}$ & $\begin{array}{c}3,196 \\
(20.01 \%)\end{array}$ \\
\hline 5 & $\begin{array}{c}\text { Can solve complex multiple-step word problems and/or } \\
\text { understand advanced mathematical material }\end{array}$ & $\begin{array}{c}113 \\
(0.71 \%)\end{array}$ \\
\hline
\end{tabular}




\section{GENERALIZED ORDINAL LOGISTIC REGRESSION MODELS}

The log likelihood ratio Chi-Square test with 1 degree of freedom, LR $\chi_{(1)}^{2}=992.52, p<$ 0.001 , indicated that the logit regression coefficient of the predictor (using computer for fun) was statistically different from 0 , therefore, the model with one predictor provided a better fit than the null model with no independent variables. The Pseudo $R^{2}=0.023$, which is the likelihood ratio $R_{L}^{2}$, suggested that the relationship between the response variable, mathematics proficiency, and the predictor, using computer for fun, was small.

The estimated logit regression coefficient, $\beta=0.384, \mathrm{z}=31.28, \mathrm{p}<0.001$, indicated that the predictor variable, using computers for fun, had a significant effect on mathematics proficiency. Substituting the value of the coefficient into formula (3), logit $[\pi(Y \leq j$ $\left.\left.\mid \mathrm{X}_{1}\right)\right]=\alpha_{\mathrm{j}}+\left(-\beta_{1} \mathrm{X}_{1}\right)$, logit $[\pi(\mathrm{Y} \leq \mathrm{j} \mid$ fun $)]=$ $\alpha_{j}-0.384$ (fun). OR $=\mathrm{e}^{(-0.384)}=0.681$, indicated that the odds of being at or below a particular proficiency level relative to beyond that level decreased by a factor of 0.681 with one unit increase in the frequency of using computers for fun. In other words, a higher frequency of using computers for fun was related to the likelihood of being in a higher proficiency level. To estimate the probability of being beyond a category of mathematics proficiency, which is the complement of the probability of being at or below a category, it is only necessary to exponentiate 0.384 ; this results in $\mathrm{OR}=1.468$, indicating that the odds of being beyond a proficiency level was 1.468 time greater with one unit increase in the frequency of using computers for fun.

Full Proportional Odds Model with Three Predictor Variables

Next, the full PO model with all three predictor variables was fitted. Table 2 provides the results of the full model. The log likelihood ratio Chi-Square test, LR $\chi_{(3)}^{2}=1391.45, \mathrm{p}<$ 0.001 , indicated that the full model with three predictor provided a better fit than the null model with no independent variables. The likelihood ratio $R_{L}^{2}=0.032$ was larger than that of the single-variable model, but was still small. Compared with the single-variable model (3.020), the AIC statistic indicated that the fullmodel fitted the data slightly better (2.992).
The logit effects of all three predictors on mathematics proficiency were significant. Similar to the single variable PO model, the estimated logit regression coefficient for using computers for fun (fun), $\beta=0.314, \mathrm{z}=22.44, \mathrm{p}$ $<0.001$; the logit coefficient for using computers for school work (schoolwk), $\beta=$ $0.307, \mathrm{z}=19.815, \mathrm{p}<0.001$; and finally, for using computers to learn on their own (learn), $\beta$ $=-0.072, \mathrm{z}=-5.295, \mathrm{p}<0.001$. The predictors, using computers for fun and using computers for school work, were positively associated with the odds of being beyond a proficiency level. In terms of odds ratio (OR), the odds of being beyond a proficiency level were 1.369 times greater with one unit increase in the frequency of using computers for fun, and 1.360 times greater with one unit increase in the frequency of using computers for school work. For every one unit increase in using computers to learn on their own, however, the odds of being beyond a particular category decreased by a factor of $0.931(\mathrm{OR}=0.931)$.

The full model also estimated five cutpoints, which were used to differentiate adjacent categories of the mathematics proficiency. $\alpha_{1}=-1.022$, which was the cutpoint for the cumulative logit model for $Y>0 ; \alpha_{2}$ was the cutpoint for the cumulative logit model for $Y>1$; the final $\alpha_{5}$ was used as the cutpoint for the logit model when $Y>4$.

Brant Test of the Proportional Odds Assumption

The Brant test of the PO assumption was examined using the brant command of the Stata SPost (Long \& Freese, 2006) routines. Stata Brant test provided results of a series of separate binary logistic regression across different category comparisons, univariate Brant test results for each predictor and the omnibus test for the overall model. Table 3 shows five $(j-1)$ associated binary logistic regression models for the full PO model, where each split compares $\mathrm{Y}$ $>$ cat. $j$ to $Y \leq$ cat. $j$ because data were dichotomized according to probability comparisons. Examining the logit coefficient of all three variables across five logistic regression models, it is evident that the effect of using computers for fun was similar across these models. The effect of using computers for 
school work was similar across the first three models but it increased from model 3 to 5 . The logit coefficient in model 5 was almost double that observed in model 1. The effect of using computers to learn on their own was close among the first four logistic regression models, however, the direction of this effect changed in model 5. Visual examination provided only preliminary results of whether the parallel effects assumption was tenable.
To test the PO assumptions, the Brant test provided the results for the overall model and each predictor. Table 4 presents $\chi^{2}$ tests and $\mathrm{p}$ values for the full PO model and separate variables. The omnibus Brant test for the full model, $\chi_{12}^{2}=29.59, p=0.003$, indicates that the proportional odds assumption for the full model was violated. To identify which predictor variables violated the assumption, separate Brant tests were examined for each predictor variable.

Table 2: Results of the Single-Variable PO Model and the Full PO Model

\begin{tabular}{|c|c|c|c|c|}
\hline \multirow[b]{2}{*}{ Variable } & \multicolumn{2}{|c|}{ Single-Variable Model } & \multicolumn{2}{|c|}{ Full Model } \\
\hline & $\mathrm{b}(\mathrm{se}(\mathrm{b}))$ & OR & $\mathrm{b}(\mathrm{se}(\mathrm{b}))$ & OR \\
\hline$\alpha_{1}$ & -1.488 & & -1.022 & \\
\hline$\alpha_{2}$ & .627 & & 1.20 & \\
\hline$\alpha_{3}$ & 1.571 & & 2.082 & \\
\hline$\alpha_{4}$ & 2.900 & & 3.437 & \\
\hline$\alpha_{5}$ & 6.475 & & 7.033 & \\
\hline fun & $.384 * *$ & 1.468 & $.314 * *$ & 1.369 \\
\hline schoolwk & & & $.307 * *$ & 1.360 \\
\hline learn & & & $-.072 * *$ & .931 \\
\hline$L R R^{2}$ & \multicolumn{2}{|c|}{.023} & \multicolumn{2}{|c|}{.032} \\
\hline $\begin{array}{c}\text { Brant Test } \\
\text { (Omnibus Test) }\end{array}$ & \multicolumn{2}{|c|}{$\chi_{4}^{2}=5.14$} & \multicolumn{2}{|c|}{$\chi_{12}^{2}=29.59 * *$} \\
\hline Model Fit & \multicolumn{2}{|c|}{$\chi_{1}^{2}=992.52 * *$} & \multicolumn{2}{|c|}{$\chi_{3}^{2}=1391.45^{* *}$} \\
\hline
\end{tabular}

$* \mathrm{p}<0.05 ; * * \mathrm{p}<0.01$

Table 3: A Series ( $\mathrm{j}-1)$ of Associated Binary Logistic Regression Models for the Full PO Model, Each Split Compares $\mathrm{Y}>$ cat. j to $\mathrm{Y} \leq$ cat. $\mathrm{j}$

\begin{tabular}{|c|c|c|c|c|c|c|}
\cline { 2 - 7 } \multicolumn{1}{c|}{} & $\mathrm{Y}>0$ & $\mathrm{Y}>1$ & $\mathrm{Y}>2$ & $\mathrm{Y}>3$ & $\mathrm{Y}>4$ & $\begin{array}{c}\text { Brant Test } \\
\mathrm{p} \text { Value }\end{array}$ \\
\hline Variable & Logit (b) & Logit (b) & Logit (b) & Logit (b) & Logit (b) & \\
\hline Constant & 1.00 & -1.091 & -2.014 & -3.485 & -8.523 & \\
\hline fun & .357 & .327 & .316 & .285 & .316 & .328 \\
\hline schoolwk & .286 & .278 & .293 & .351 & .492 & $.02 *$ \\
\hline learn & -.094 & -.067 & -.081 & -.068 & .144 & .094 \\
\hline
\end{tabular}

$* \mathrm{p}<0.05 ; * * \mathrm{p}<0.01$ 


\section{GENERALIZED ORDINAL LOGISTIC REGRESSION MODELS}

Results revealed that the univariate Brant tests for the PO assumption were upheld for using computers for fun and using computers to learn on their own. Conversely, the Brant test was violated for using computers for school work.

Table 4: Brant Tests of the PO Assumption for Each Predictor and the Overall Model

\begin{tabular}{|c|c|c|}
\hline Variable & Test & $\mathrm{p}$ Value \\
\hline fun & $\chi^{2}{ }_{4}=4.62$ & .328 \\
\hline schoolwk & $\chi^{2}{ }_{4}=11.55$ & $.021^{*}$ \\
\hline learn & $\chi^{2}{ }_{4}=7.93$ & .094 \\
\hline $\begin{array}{c}\text { All } \\
\text { (Full-Model) }\end{array}$ & $\chi_{12}^{2}=29.59$ & $.003^{* *}$ \\
\hline
\end{tabular}

$* \mathrm{p}<0.05 ; * * \mathrm{p}<0.01$

Results of the Generalized Ordinal Logistic Regression Model Using Stata gologit

When the PO assumption is not satisfied, Stata gologit (Fu, 1998) relaxes the proportionality assumption by allowing the logits effects of predictor variables to vary across cutpoints, which dichotomize the underlying proficiency outcome. Similar to a series of underlying binary logistic regression models where the data were dichotomized across different categories, the effects of the predictor variables estimated by the generalized ordinal logistic regression model could vary freely.

Table 5 and Figure 1 show the results of the generalized ordinal model. The logit effects and corresponding odds ratios (OR) of all three variables were different across all five models comparing probabilities of being beyond category $j$ versus at or below that category. For example, the odds ratios for using computers for fun across five comparisons were 1.428, 1.385, $1.368,1.322$ and 1.314 , respectively. They were similar because the equal slopes assumption test was tenable for this predictor variable. After relaxing the $\mathrm{PO}$ assumption, Stata gologit estimated 12 more parameters in the generalized ordinal logistic regression model than the full PO model by Stata ologit.
Results of the Generalized Ordinal Logistic Regression Model Using gologit2

Stata gologit2 (Williams, 2006) is an extension of gologit (Fu, 1998), and can estimate various types of generalized models, including the partial proportional odds model (PPO), the PO model and the binary logistic regression model. Although Stata gologit2 allows the effects of all predictor variables to vary even when some violate the PO assumption, it can relax the $\mathrm{PO}$ assumption and put equal-slope constraints on those variables whose effects are constant across cutpoints: The model is then referred to as the PPO.

Table 6 and Figure 2 show the results of the generalized ordinal logistic regression model or the PPO model using gologit2. Because only one predictor variable, using computers for school work, violated the PO assumption, the other two variables, using computers for fun and using computers to learn on their own, were held constant in the PPO model. For example, the logit coefficients and odds ratios for using computers to learn on their own were the same across five comparisons $(\mathrm{OR}=0.913)$. The $\mathrm{OR}$ for using computer for school work were different across each cutpoint, they were: 1.331, $1.329,1.342,1.436$ and 1.906 , respectively. The PPO model estimated by Stata gologit 2 was more parsimonious than the generalized ordinal logistic regression model by gologit because the former model estimated five fewer parameters than the latter.

In terms of odds ratio, using computers for fun was positively associated with the odds of being above a particular mathematics proficiency level as opposed to being at or below that level $(\mathrm{OR}=1.372)$; however, using computers to learn on their own had a negative effect on the odds of being above a particular proficiency level $(\mathrm{OR}=0.93)$. Because the effect of using computers for school work was not invariant across five comparisons, separate interpretations were required. Using computers for school work was associated with the likelihood of being in a higher mathematics proficiency level. The effects became much stronger when mathematics proficiency level moved from low to high, further, the largest effect was identified among the final comparison (proficiency level 5 versus from 0 to 4 ). 


\section{Conclusion}

This article reviewed the proportional odds model and demonstrated the use of generalized ordinal regression models to estimate mathematics proficiency. The results of fitting the PO models and the generalized ordinal logistic regression models were interpreted. In addition, two different methods used to estimate the generalized ordinal models by Williams (2006) and $\mathrm{Fu}$ (1998) were illustrated and compared.

Compared to the PO model, the generalized ordinal logistic model provides a better solution when the proportional odds assumption is violated. The effects of the predictors which meet the $\mathrm{PO}$ assumption can be interpreted in the same way as that in the PO model. The effects of explanatory variables that violate the $\mathrm{PO}$ assumption must be interpreted separately at each comparison (i.e., being beyond a particular category versus at or below that category), and need more attention.

When the proportional odds assumption is untenable, the generalized ordinal logistic model proposed by Fu (1998) may be used for preliminary analysis. Williams' model (2006) is a more powerful extension of that of $\mathrm{Fu}$ (1998) in that it incorporated and expanded Fu's procedure. Numerous different types of generalized ordinal models can be estimated by Williams' gologit2 procedure, including the commonly used partial proportional odds model (PPO), which only relaxes the PO assumption when it is violated by one or a few predictors, but holds constant for those which have equal effects. Although not provided herein, methods of model diagnostics for ordinal logistic regression models were introduced by O’Connell and Liu (2011).

Table 5: Results of the Generalized Ordinal Logit Model Using Stata gologit

\begin{tabular}{|c|c|c|c|c|c|c|c|c|c|c|}
\hline \multirow[b]{2}{*}{ Variable } & \multicolumn{2}{|c|}{$\mathrm{Y}>0$ vs. $\mathrm{Y} \leq 0$} & \multicolumn{2}{|c|}{$Y>1$ vs. $Y \leq 1$} & \multicolumn{2}{|c|}{$Y>2$ vs. $Y \leq 2$} & \multicolumn{2}{|c|}{$Y>3$ vs. $Y \leq 3$} & \multicolumn{2}{|c|}{$Y>4$ vs. $Y \leq 4$} \\
\hline & $\begin{array}{c}\mathrm{b} \\
(\mathrm{se}(\mathrm{b}))\end{array}$ & OR & $\begin{array}{c}\mathrm{b} \\
(\mathrm{se}(\mathrm{b}))\end{array}$ & OR & $\begin{array}{c}\mathrm{b} \\
(\mathrm{se}(\mathrm{b}))\end{array}$ & OR & $\begin{array}{c}\mathrm{b} \\
(\mathrm{se}(\mathrm{b}))\end{array}$ & OR & $\begin{array}{c}\mathrm{b} \\
(\mathrm{se}(\mathrm{b}))\end{array}$ & OR \\
\hline fun & $\begin{array}{c}.356 \\
(.030)^{* *}\end{array}$ & 1.428 & $\begin{array}{c}.326 \\
(.016)^{* *}\end{array}$ & 1.385 & $\begin{array}{c}.313 \\
(.016)^{* *}\end{array}$ & 1.368 & $\begin{array}{c}.279 \\
(.022)^{* *}\end{array}$ & 1.322 & $\begin{array}{c}.273 \\
(.129)^{*}\end{array}$ & 1.314 \\
\hline schoolwk & $\begin{array}{c}.276 \\
(.037)^{* *}\end{array}$ & 1.318 & $\begin{array}{c}.277 \\
(.019)^{* *}\end{array}$ & 1.319 & $\begin{array}{c}.299 \\
(.018)^{* *}\end{array}$ & 1.348 & $\begin{array}{c}.370 \\
(.023)^{* *}\end{array}$ & 1.447 & $\begin{array}{c}.540 \\
(.116)^{* *}\end{array}$ & 1.716 \\
\hline \multirow[t]{2}{*}{ learn } & $\begin{array}{c}-.091 \\
(.035)^{* *}\end{array}$ & .913 & $\begin{array}{c}-.067 \\
(.017)^{* *}\end{array}$ & .935 & $\begin{array}{c}-.081 \\
(.016)^{* *}\end{array}$ & .922 & $\begin{array}{c}-.066 \\
(.019)^{* *}\end{array}$ & .936 & $\begin{array}{l}.180 \\
(.093)\end{array}$ & 1.197 \\
\hline & \multicolumn{2}{|c|}{$\alpha_{1}=1.022$} & \multicolumn{2}{|c|}{$\alpha_{2}=-1.083$} & \multicolumn{2}{|c|}{$\alpha_{3}=-2.024$} & \multicolumn{2}{|c|}{$\alpha_{4}=-3.520$} & \multicolumn{2}{|c|}{$\alpha_{5}=-8.642$} \\
\hline$L R R^{2}$ & \multicolumn{10}{|l|}{0.033} \\
\hline $\begin{array}{l}\text { Model } \\
\text { Fit }\end{array}$ & \multicolumn{10}{|c|}{$\chi_{15}^{2}=1429.62$} \\
\hline
\end{tabular}

$* \mathrm{p}<0.05 ; *{ }^{*} \mathrm{p}<0.01$ 


\section{GENERALIZED ORDINAL LOGISTIC REGRESSION MODELS}

Figure 1: Generalized Ordinal Logistic Model Using Stata gologit

gologit Profmath BYS45A_REC BYS45B_REC BYS45C_REC

Iteration 0: Log Likelihood $=-21943.368$

Iteration 1: Log Likelihood $=-21237.489$

Iteration 2: Log Likelihood $=-21228.561$

Iteration 3: Log Likelihood $=-21228.555$

Iteration 4: Log Likelihood $=-21228.555$

Generalized Ordered Logit Estimates

Number of obs $=14207$

Model $\operatorname{chi2}(15)=1429.62$

Log Likelihood $=-21228.5552037$

Prob > chi2 $=0.0000$

Pseudo R2 $=0.0326$

\begin{tabular}{|c|c|c|c|c|c|c|}
\hline Profmath | & Coef. & Std. Err. & $\mathrm{z}$ & $\mathrm{P}>|\mathrm{z}|$ & {$[95 \%$ Conf. } & Interval] \\
\hline \multicolumn{7}{|c|}{ 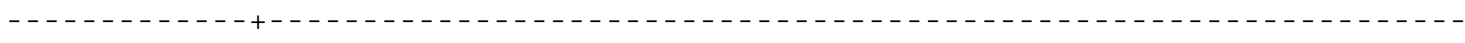 } \\
\hline \multicolumn{7}{|l|}{ mleq1 } \\
\hline BYS45A_REC | & .356211 & .0303759 & 11.73 & 0.000 & .2966753 & .4157468 \\
\hline BYS45B_REC | & .2763788 & .0372811 & 7.41 & 0.000 & .2033091 & .3494484 \\
\hline BYS45C_REC | & -.0914208 & .0350232 & -2.61 & 0.009 & -.1600651 & -.0227765 \\
\hline _cons | & 1.021761 & .1098978 & 9.30 & 0.000 & .8063653 & 1.237157 \\
\hline & -------- & $----\cdot--$ & ---- & ---- & -------- & --------- \\
\hline \multicolumn{7}{|l|}{ mleq2 } \\
\hline BYS45A_REC | & .3257729 & .01639 & 19.88 & 0.000 & .2936492 & .3578967 \\
\hline BYS45B_REC & .2768884 & .0189779 & 14.59 & 0.000 & .2396925 & .3140843 \\
\hline BYS45C_REC | & -.0673821 & .0171035 & -3.94 & 0.000 & -.1009042 & -.0338599 \\
\hline _cons | & -1.082717 & .0676152 & -16.01 & 0.000 & -1.21524 & -.9501931 \\
\hline-------------+ & ------- & -------- & ----- & $-\cdots--$ & --------- & - - - - - - - - \\
\hline \multicolumn{7}{|l|}{ mleq3 } \\
\hline BYS45A_REC & .3134542 & .0163592 & 19.16 & 0.000 & .2813907 & .3455177 \\
\hline BYS45B_REC & .2989051 & .0178569 & 16.74 & 0.000 & .2639063 & .333904 \\
\hline BYS45C_REC & -.081139 & .0155428 & -5.22 & 0.000 & -.1116022 & -.0506758 \\
\hline _cons | & -2.02405 & .0716248 & -28.26 & 0.000 & -2.164432 & -1.883668 \\
\hline----------- & --------- & --------- & $---\cdots$ & $-\cdots$ & ---------- & --------- \\
\hline \multicolumn{7}{|l|}{ mleq4 } \\
\hline BYS45A_REC & .2787754 & .0223384 & 12.48 & 0.000 & .234993 & .3225578 \\
\hline BYS45B_REC & .3697106 & .0227828 & 16.23 & 0.000 & .3250571 & .4143642 \\
\hline BYS45C_REC & -.0657926 & .018757 & -3.51 & 0.000 & -.1025556 & -.0290296 \\
\hline _cons | & -3.530265 & .1037457 & -34.03 & 0.000 & -3.733603 & -3.326927 \\
\hline----------+ & -------- & -------- & ----- & ----- & ---------- & ---------- \\
\hline \multicolumn{7}{|l|}{ mleq5 } \\
\hline BYS45A_REC & .27337 & .1291764 & 2.12 & 0.034 & .0201889 & .5265512 \\
\hline BYS45B_REC & .5401159 & .1155738 & 4.67 & 0.000 & .3135954 & .7666363 \\
\hline BYS45C_REC & .180175 & .0928936 & 1.94 & 0.052 & -.0018932 & .3622431 \\
\hline _cons | & -8.642421 & .6316348 & -13.68 & 0.000 & -9.880403 & -7.404439 \\
\hline
\end{tabular}


Compared to SAS, fitting generalized ordinal regression models using Stata is more straightforward because Stata does not require data restructuring and the results are easier to interpret (SAS needs an interaction term between the predictor with the violated effect and ordinal categories). The latest VGAM package (v. 0.8.3) for $\mathrm{R}$ (Yee, 2010, 2011) includes the functions of fitting the nonproportional odds and the partial proportional odds models, which is similar to Stata gologit and gologit2. Although different statistical packages may have advantages of fitting these models, the choice is left to researchers; this study does not imply an overall preference of one over another.

In educational research, the use of ordinal categorical data is common, thus, it is crucial for researchers to determine the most appropriate models to analyze ordinal categorical dependent variables. It is hoped that this demonstration clarifies some of the issues that researchers must consider in selecting appropriate statistical models for analyzing ordinal data when the PO assumption is violated.

\section{Notes}

Previous versions of this article were presented at the Modern Modeling Methods Conference in Storrs, CT (May, 2011), the $76^{\text {th }}$ Annual and the $17^{\text {th }}$ International Meeting of Psychometric Society (IMPS) in Hong Kong (July, 2011), the Northeastern Educational Research Association Annual Conference in Rocky Hill, CT (October, 2011), and the Annual Meeting of American Educational Research Association (AERA), Vancouver, British Columbia, Canada (April, 2012).

Table 6: Results of the Generalized Ordinal Logit Model/Partial Proportional Odds Model Using Stata gologit $2(\mathrm{Y}>$ cat. $\mathrm{j}$ vs. $\mathrm{Y} \leq$ cat. $\mathrm{j})$

\begin{tabular}{|c|c|c|c|c|c|c|c|c|c|c|}
\hline & \multicolumn{2}{|c|}{$\mathrm{Y}>0$ vs. $\mathrm{Y} \leq 0$} & \multicolumn{2}{|c|}{$\mathrm{Y}>1$ vs. $\mathrm{Y} \leq 1$} & \multicolumn{2}{|c|}{$\mathrm{Y}>2$ vs. $\mathrm{Y} \leq 2$} & \multicolumn{2}{|c|}{$\mathrm{Y}>3$ vs. $\mathrm{Y} \leq 3$} & \multicolumn{2}{|c|}{$\mathrm{Y}>4$ vs. $\mathrm{Y} \leq 4$} \\
\hline Variable & $\begin{array}{c}\mathrm{b} \\
(\mathrm{se}(\mathrm{b}))\end{array}$ & OR & $\begin{array}{c}\mathrm{b} \\
(\mathrm{se}(\mathrm{b}))\end{array}$ & OR & $\begin{array}{c}\mathrm{b} \\
(\mathrm{se}(\mathrm{b}))\end{array}$ & OR & $\begin{array}{c}\mathrm{b} \\
(\mathrm{se}(\mathrm{b}))\end{array}$ & OR & $\begin{array}{c}\mathrm{b} \\
(\mathrm{se}(\mathrm{b}))\end{array}$ & OR \\
\hline fun & $\begin{array}{c}.317 \\
(.014)^{* *}\end{array}$ & 1.373 & $\begin{array}{c}.317 \\
(.014)^{* *}\end{array}$ & 1.373 & $\begin{array}{c}.317 \\
(.014)^{* *}\end{array}$ & 1.373 & $\begin{array}{c}.317 \\
(.014)^{* *}\end{array}$ & 1.373 & $\begin{array}{c}.317 \\
(.014)^{* *}\end{array}$ & 1.373 \\
\hline schoolwk & $\begin{array}{c}.286 \\
(.032)^{* *}\end{array}$ & 1.331 & $\begin{array}{c}.284 \\
(.018)^{* *}\end{array}$ & 1.329 & $\begin{array}{c}.294 \\
(.017)^{* *}\end{array}$ & 1.342 & $\begin{array}{c}.362 \\
(.022)^{* *}\end{array}$ & 1.436 & $\begin{array}{c}.645 \\
(.116)^{* *}\end{array}$ & 1.906 \\
\hline learn & $\begin{array}{c}-.072 \\
(.014)^{* *}\end{array}$ & .931 & $\begin{array}{c}-.072 \\
(.014)^{* *}\end{array}$ & .931 & $\begin{array}{c}-.072 \\
(.014)^{* *}\end{array}$ & .931 & $\begin{array}{c}-.072 \\
(.014)^{* *}\end{array}$ & .931 & $\begin{array}{c}-.072 \\
(.014) * *\end{array}$ & .931 \\
\hline & \multicolumn{2}{|c|}{$\alpha_{1}=1.073$} & \multicolumn{2}{|c|}{$\alpha_{2}=-1.060$} & \multicolumn{2}{|c|}{$\alpha_{3}=-2.053$} & \multicolumn{2}{|c|}{$\alpha_{4}=-3.650$} & \multicolumn{2}{|c|}{$\alpha_{5}=-8.357$} \\
\hline$L R R^{2}$ & \multicolumn{10}{|l|}{.032} \\
\hline $\begin{array}{l}\text { Model } \\
\text { Fit }\end{array}$ & \multicolumn{10}{|c|}{$\chi_{7}^{2}=1414.05$} \\
\hline
\end{tabular}

$* \mathrm{p}<0.05 ; * * \mathrm{p}<0.01$ 


\section{GENERALIZED ORDINAL LOGISTIC REGRESSION MODELS}

Figure 2: Generalized Ordinal Logistic Model Using Stata gologit2

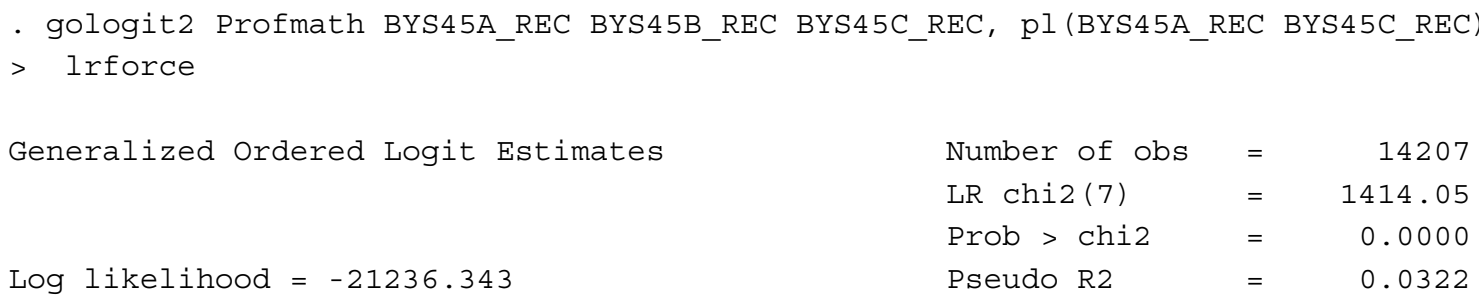

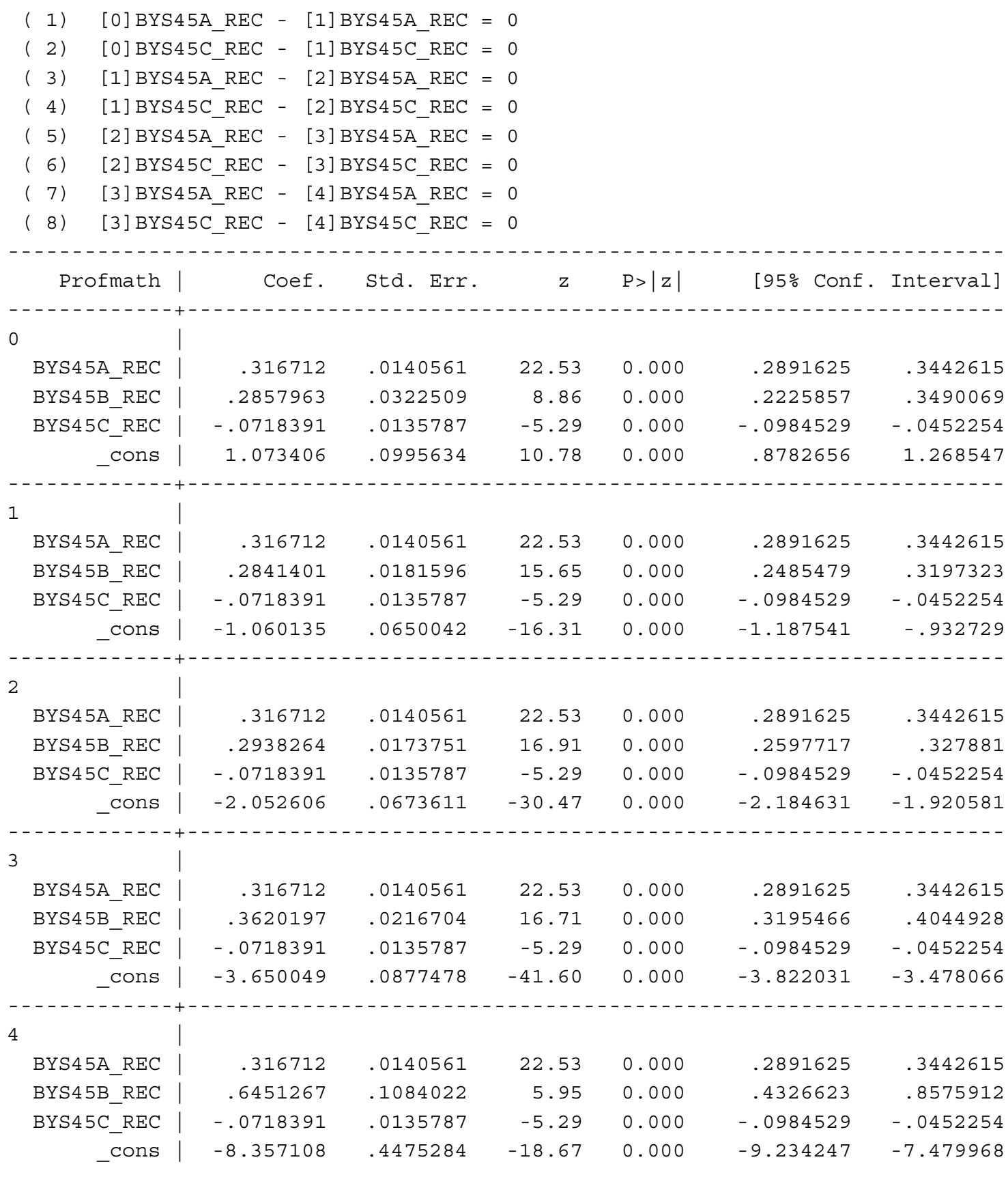




\section{XING LIU \& HARI KOIRALA}

\section{References}

Agresti, A. (1996). An introduction to categorical data analysis. New York, NY: John Wiley \& Sons.

Agresti, A. (2002). Categorical data analysis, $2^{\text {nd }} E d$. New York, NY: John Wiley \& Sons.

Agresti, A. (2007). An introduction to categorical data analysis, $2^{\text {nd }} E d$. New York, NY: John Wiley \& Sons.

Allison, P. D. (1999). Logistic regression using the SAS system: Theory and application. Cary, NC: SAS Institute, Inc.

Ananth, C. V., \& Kleinbaum, D. G. (1997). Regression models for ordinal responses: A review of methods and applications. International Journal of Epidemiology, 26, 1323-1333.

Armstrong, B. B., \& Sloan, M. (1989). Ordinal regression models for epidemiological data. American Journal of Epidemiology, 129(1), 191-204.

Bender, R., \& Benner, A. (2000). Calculating ordinal regression models in SAS and S-Plus. Biometrical Journal, 42(6), 677699.

Bender, R., \& Grouven, U. (1998). Using binary logistic regression models for ordinal data with non-proportional odds. Journal of Clinical Epidemiology, 51(10), 809-816.

Brant, R. (1990). Assessing proportionality in the proportional odds model for ordinal logistic regression. Biometrics, 46, 1171-1178.

Clogg, C. C., \& Shihadeh, E. S. (1994). Statistical models for ordinal variables. Thousand Oaks, CA: Sage.

Fienberg, S. E. (1980). The analysis of cross-classified categorical data. Cambridge, MA: The MIT Press.

$\mathrm{Fu}$, V. (1998). Estimating generalized ordered logit models. Stata Technical Bulletin, 44, 27-30.

Hardin, J. W., \& Hilbe, J. M. (2007). Generalized linear models and extensions, $2^{\text {nd }}$ $E d$. Texas: Stata Press.

Hosmer, D. W., \& Lemeshow, S. (2000). Applied logistic regression, $2^{\text {nd }} E d$. New York, NY: John Wiley \& Sons.
Ingels, S. J., Pratt, D. J., Roger, J., Siegel, P. H., \& Stutts, E. (2004). ELS: 2002 base year data file user's manual. Washington, DC: NCES (NCES 2004-405).

Ingels, S. J., Pratt, D. J., Roger, J., Siegel, P. H., \& Stutts, E. (2005). Education longitudinal study: 2002/04 public use base-year to first follow-up data files and electronic codebook system. Washington DC: NCES (NCES 2006-346).

Liu, X. (2009). Ordinal regression analysis: Fitting the proportional odds model using Stata, SAS and SPSS. Journal of Modern Applied Statistical Methods, 8(2), 632-645.

Liu, X., O'Connell, A. A., \& Koirala, H. (2011). Ordinal regression analysis: Predicting mathematics proficiency using the continuation ratio model. Journal of Modern Applied Statistical Methods, 10(2), 513-527.

Long, J. S. (1997). Regression models for categorical and limited dependent variables. Thousand Oaks, CA: Sage.

Long, J. S., \& Freese, J. (2006). Regression models for categorical dependent variables using Stata, $2^{\text {nd }} E d$. Texas: Stata Press.

McCullagh, P. (1980). Regression models for ordinal data (with discussion). Journal of the Royal Statistical Society, Series B, 42, 109-142.

McCullagh, P., \& Nelder, J. A. (1989). Generalized linear models, $2^{\text {nd }} E d$. London: Chapman and Hall.

Menard, S. (1995). Applied logistic regression analysis. Thousand Oaks, CA: Sage.

O'Connell, A. A., (2000). Methods for modeling ordinal outcome variables. Measurement and Evaluation in Counseling and Development, 33(3), 170-193.

O'Connell, A. A. (2006). Logistic regression models for ordinal response variables. Thousand Oaks, CA: SAGE.

O'Connell, A. A., \& Liu, X. (2011). Model diagnostics for proportional and partial proportional odds models. Journal of Modern Applied Statistical Methods, 10(1), 139-175.

Peterson, B., \& Harrell, F. E. (1990). Partial proportional odds models for ordinal response variables. Applied Statistics, 39(2), 205-217. 


\section{GENERALIZED ORDINAL LOGISTIC REGRESSION MODELS}

Powers, D. A., \& Xie, Y. (2000). Statistical models for categorical data analysis. San Diego, CA: Academic Press.

Stokes, M. E., Davis, C. S., \& Koch, G. G. (2000). Categorical data analysis using the SAS system. Cary, NC: SAS Institute Inc.

Williams, R. (2006). Generalized ordered logit/partial proportional odds models for ordinal dependent variables. The Stata Journal, 6(1), 58-82.
Yee, T. W. (2010). The VGAM package for categorical data analysis. Journal of Statistical Software, 32(10), 1-34.

Yee, T. W. (2011). The VGAM package for $R$. Retrieved from http://www.stat.auckland.ac.nz/ yee/VGAM. 


\section{The Length-Biased Lognormal Distribution and Its Application in the Analysis of Data from Oil Field Exploration Studies}

\author{
Makarand V. Ratnaparkhi \\ Wright State University, \\ Dayton, $\mathrm{OH}$
}

\author{
Uttara V. Naik-Nimbalkar \\ Pune University, \\ Pune, India
}

The length-biased version of the lognormal distribution and related estimation problems are considered and sized-biased data arising in the exploration of oil fields is analyzed. The properties of the estimators are studied using simulations and the use of sample mode as an estimate of the lognormal parameter is discussed.

Key words: Length-biased lognormal distribution, estimation, simulations, sample mode, bootstrap.

\section{Introduction}

The term length-biased data refers to sample data where the probability of recording an observation depends on the magnitude, for example $x$, of the observation. In particular, the larger the observation, the higher the probability of observing the related event and, hence, including the corresponding observation in the sample. Length-biased data occur in many research areas and in fields of application, such as, medical science, ecology and geological sciences. Further, the term size-biased data is used to describe the situation where the probability of inclusion of an observation depends on a certain function: $w(x)>0$ of $x$.

The length-biased version of the original probability density function (pdf.) that is of interest as a model is considered for modeling length-biased data. The lognormal distribution (LN) with parameters $(\mu, \sigma)$ is known to be a useful model in many applications. Therefore, it

Makarand V. Ratnaparkhi is a Professor of Statistics at Wright State University in Dayton, Ohio, USA. Email him at: makarand.ratnaparkhi@wright.edu. Uttara V. Naik-Nimbalkar is a Professor of Statistics at University of Pune, Pune, India. Email her at: uvnaik@stats.unipune.ac.in. is natural to expect the applications of lengthbiased lognormal distributions (LBLN) in some data analysis problems. For example, the lognormal distribution is commonly used in the analysis of data in geological studies, and Meisner and Demirmen (1981) observed that size-biased data occur in oil-field exploration studies. Yan (2004) considered the presence of length-biasedness in data on incubation periods arising in the SARS epidemic. Among many probability models that are considered for the analysis of these data, the length-biased lognormal distribution is one such model. Quin, et al. (2002) considered such a distribution for data on Breslow thickness in cancer research.

With respect to the properties of the length-biased lognormal distribution, in general, if $f(x ; \theta)$ is the original pdf of a non-negative random variable $X$ with $E(X)<\infty$, then its length-biased version is given by

$$
g\left(x ; \theta^{*}\right)=x f(x ; \theta) / E(X), x>0,
$$

where $\theta \varepsilon \Omega$ is a scalar or a vector of the parameters of the original distribution of $X$ and $\theta^{*} \varepsilon \Omega^{*}$ denotes a scalar or a vector of the parameters of the corresponding length-biased version. In some cases $\theta^{*}$ is the same as $\theta$. In practical situations, the interest is in estimating $\theta$, the parameter(s) of the original distribution using length-biased data. However, due to the nature of the available data (length-biased data) 


\section{THE LENGTH-BIASED LOGNORMAL DISTRIBUTION AND ITS APPLICATION}

the experimenter has no other choice but to use the length-biased version of the original distribution. Thus, there is a need to study the properties of the $\theta$ estimator with respect to $g(x$; $\left.\theta^{*}\right)$, but such an estimation problem is not straightforward for the lognormal distribution.

\section{Methodology}

The random variable $X$ is said to have a lognormal distribution with parameters $(\mu$, $\sigma)($ denoted by $\mathrm{LN}(\mu, \sigma))$, if its pdf is

$$
f(x ; \mu, \sigma)=\frac{1}{x \sigma \sqrt{2 \pi}} \exp \left[-\frac{1}{2 \sigma^{2}}(\log x-\mu)^{2}\right],
$$

where

$$
x>0,-\infty<\mu<\infty, \sigma>0 .
$$

Length-Biased Lognormal DistributionDefinition and Properties

Using the definition of length-biased distribution, the pdf of the length-biased lognormal distribution (denoted by LBLN $(\mu$, $\sigma)$ ) is given by

$$
\begin{aligned}
& g(x: \mu, \sigma)= \\
& \frac{1}{x \sigma \sqrt{2 \pi}} \exp \left[-\frac{1}{2 \sigma^{2}}\left(\log x-\left(\mu+\sigma^{2}\right)\right)^{2}\right]
\end{aligned}
$$

where

$$
x>0,-\infty<\mu<\infty, \sigma>\alpha .
$$

For convenience (2) will be expressed as

$$
g(x ; \mu, \sigma)=\frac{1}{x \sigma \sqrt{2 \pi}} \exp \left[-\frac{1}{2 \sigma^{2}}\left(\log x-\mu^{*}\right)^{2}\right]
$$

where

$$
x>0, \mu^{*}=\mu+\sigma^{2},-\infty<\mu<\infty \text { and } \sigma>0 .
$$

The properties of the pdfs (1) and (2) are presented in Table 1. The mode of LBLN shown in Table 1 depends only on $\mu$ and not on $\sigma^{2}$ as for LN. From Table 2, it is clear that the structure of the Fisher information for LBLN is not the same; hence the related results will not be the same when LBLN is used instead of LN in data analysis.

Parameter Spaces of LN $(\mu, \sigma)$ and $\operatorname{LBLN}(\mu$, $\sigma)$

In practical situations, for the analysis of length-biased data, the $\operatorname{LN}(\mu, \sigma)$ is replaced by LBLN $(\mu, \sigma)$. Further, examination of the pdfs for (1), (2) and (2a) shows that, although the listed pdfs seem to have the same form, there exists an in-built relationship between the parameters $(\mu, \sigma)$ of LBLN $(\mu, \sigma)$. Thus, studying the implications of this relationship is necessary for the interpretation and estimation of parameters. A brief discussion related to the parameter spaces of $(\mu, \sigma)$ for these two distributions is useful for identifying the underlying problems in data analysis.

Let $\Omega_{1}$ and $\Omega_{2}$ denote the respective parameters of $\operatorname{LN}(\mu, \sigma)$ and $\operatorname{LBLN}(\mu, \sigma)$. Then,

$$
\Omega_{1}=\{(\mu, \sigma) \mid-\infty<\mu<\infty, \sigma>0\}
$$

and

$$
\begin{aligned}
\Omega_{2} & =\left\{(\mu, \sigma) \mid-\infty<\mu^{*}\right. \\
& \left.=\mu+\sigma^{2}<\infty, \sigma>0\right\} .
\end{aligned}
$$

From (3) and (4) it is clear that if the $\operatorname{LBLN}(\mu$, $\sigma)$ is used as a model with $\mu^{*}=0$, then it will represent only those members of the original $\mathrm{LN}$ $(\mu, \sigma)$ model for which $\mu^{*}=\mu+\sigma^{2}=0$, i.e. $\mu=-\sigma^{2}$. A similar restriction will arise for other values, $\mu^{*}=\mathrm{c}$ for example, of $\mu^{*}$, where $\mathrm{c}$ is some constant. Thus, there is a built-in restriction on the choice of the LBLN distribution with respect to the selection of the appropriate model for representing the original LN.

Maximum Likelihood Estimation (MLE)

Let $\left(X_{1}, X_{2}, \ldots X_{n}\right)$ be a random sample from a $\operatorname{LBLN}(\mu, \sigma)$ distribution. The $\log$-likelihood function $l(\mu, \sigma)$ is then given as 


\section{RATNAPARKHI \& NAIK-NIMBALKAR}

Table 1: Properties of LN $(\mu, \sigma)$ and $\operatorname{LBLN}(\mu, \sigma)$

\begin{tabular}{|c|c|c|}
\hline Property & $\operatorname{LN}(\mu, \sigma)$ & $\operatorname{LBLN}(\mu, \sigma)$ \\
\hline Mean & $\exp \left(\mu+\sigma^{2} / 2\right)$ & $\exp \left(\mu^{*}+\sigma^{2} / 2\right)$ \\
\hline Median & $\exp (\mu)$ & $\exp \left(\mu^{*}\right)$ \\
\hline Mode & $\exp \left(\mu-\sigma^{2}\right)$ & $\exp \left(\mu^{*}-\sigma^{2}\right)=\exp (\mu)$ \\
\hline Variance & $\exp \left(2 \mu+\sigma^{2}\right)\left\{\exp \left(-\sigma^{2}\right)-1\right\}$ & $\exp \left(2 \mu^{*}+\sigma^{2}\right)\left\{\exp \left(-\sigma^{2}\right)-1\right\}$ \\
\hline
\end{tabular}

Table 2: The Fisher Information Matrix of $\operatorname{LN}(\mu, \sigma)$ and $\operatorname{LBLN}(\mu, \sigma)$

\begin{tabular}{|c|c|c|}
\hline \multirow{2}{*}{$\begin{array}{c}\text { Fisher } \\
\text { Information } \\
\text { Matrix }\end{array}$} & $\operatorname{LN}(\mu, \sigma)$ & $\operatorname{LBLN}(\mu, \sigma)$ \\
\cline { 2 - 3 } & $\mathrm{I}_{1}=\left[\begin{array}{cc}1 / \sigma^{2} & 0 \\
0 & 2 / \sigma^{2}\end{array}\right]$ & $\mathrm{I}_{2}=\left[\begin{array}{cc}1 / \sigma^{2} & 2 / \sigma \\
2 / \sigma & 4+2 / \sigma^{2}\end{array}\right]$ \\
\hline
\end{tabular}

$$
\begin{aligned}
l(\mu, \sigma)= & -n \sum \log x_{i}-n \log \sigma \\
& -\log \sqrt{2 \pi} \frac{1}{\sigma^{2}} \sum\left(\log x_{i}-\mu-\sigma^{2}\right)^{2} .
\end{aligned}
$$

The solutions to likelihood equations $\frac{\partial l}{\partial \mu}=0$ and $\frac{\partial l}{\partial \sigma}=0$ give the MLEs as:

$$
\hat{\mu}=\left(\sum \log x_{i} / n\right)-\hat{\sigma}^{2}
$$

and

$$
\hat{\sigma}^{2}=\frac{1}{n}\left[\sum\left(\log x_{i}\right)^{2}-\left(\sum \log x_{i}\right)^{2} / n\right] .
$$

To study the properties of $\left(\hat{\mu}, \hat{\sigma}^{2}\right)$ the transformation $Y=\log X$ is considered in (2). It is known that $Y \sim \operatorname{Normal}\left(\mu+\sigma^{2}, \sigma\right)$. This leads to the following estimates corresponding to (6):

$$
\hat{\mu}=\bar{Y}+c S^{2},
$$

where $c=-(n-1) / n, \quad \hat{\sigma}^{2}=\frac{(n-1)}{n} S^{2}$, and for the sample

$$
Y_{i}=\log X_{i}, i=1,2, \ldots, n, \bar{Y}=\sum \frac{Y_{i}}{n}
$$

and

$$
S^{2}=\sum \frac{\left(Y_{i}-\bar{Y}\right)^{2}}{(n-1)}
$$

From (7), it is clear that $\hat{\mu}$ is a biased estimator of $\mu$. Further, the distribution of $\hat{\mu}$ cannot be expressed in closed form. Thus, the distributional properties of $\hat{\mu}$, unlike in the case of the original LN distribution, are not readily 


\section{THE LENGTH-BIASED LOGNORMAL DISTRIBUTION AND ITS APPLICATION}

available for statistical inference. Therefore, simulations are considered in order to understand the properties of the above defined estimator of $\mu$. In particular, finding the confidence interval (C.I.) for $\mu$ is not straightforward. Hence, the bootstrap method for constructing the confidence interval for $\mu$ was considered to obtain the results.

\section{Results}

To illustrate the use of the methodology introduced above, data from oil field explorations (Meisner \& Demirmen, 1981) was analyzed.

Table 3: Sizes of Oil Fields Data $\left(X=\right.$ Field Size, Oil $\left(10^{6}\right.$ BBLS $\left.), n=58\right)$

\begin{tabular}{|c|c|c|c|c|}
\hline 28 & 26 & 775 & 114 & 31 \\
\hline 337 & 41 & 113 & 1328 & 21 \\
\hline 13 & 455 & 89 & 482 & 70 \\
\hline 215 & 62 & 58 & 6.9 & 154 \\
\hline 177 & 43 & 33 & 178 & 15 \\
\hline 22 & 11 & 8.1 & 35 & 25 \\
\hline 170 & 19 & 56 & 42 & 335 \\
\hline 21 & 50 & 181 & 93 & 75 \\
\hline 8.8 & 29 & 450 & 5.9 & 8.8 \\
\hline 49 & 100 & 10 & 8.8 & 17 \\
\hline 12 & 125 & 20 & 8.8 & 8.8 \\
\hline 6.9 & 25 & 100 & & \\
\hline
\end{tabular}

MLE of the Parameters $(\mu, \sigma)$ of LBLN

The MLE's of $\mu$ and $\sigma$ were obtained (see Table 4) using the formulas in (7). The amount of bias in the estimate of $\mu$ can also be estimated using (7).

Table 4: Estimates of $\mu$ and $\sigma$

\begin{tabular}{|c|c|c|}
\hline Parameter & Estimate & Standard Error \\
\hline$\mu$ & 2.0748 & 0.3729 \\
\hline$\sigma$ & 1.3317 & 0.1236 \\
\hline
\end{tabular}

Simulations for Studying the Properties of $\hat{\mu}$

As noted, the distribution of $\hat{\mu}$ is not available in closed form (see equation (7)); therefore, to understand the properties of $\hat{\mu}$ simulations were conducted. In particular, the amount of bias in the estimates of values of $\mu$ is of interest. For these simulations, different values of $n$ and $(\mu, \sigma)$ were used. The simulation results for $n=20$ and certain values of $(\mu, \sigma)$ are shown in Table 5. Results obtained were expected from (7), and show that the absolute value of the bias in the estimate $\hat{\mu}$ of $\mu$ increases as $\sigma$ increases. Results from the simulations for other values of $n$ were not different from those recorded above and therefore for brevity are not included.

Estimation of $\mu$ Based on the Mode of LBLN $(\mu, \sigma)$

The mode $\mathrm{M}$ of the LBLN $(\mu$, $\sigma)$ distribution from Table 1 is given by $\mathrm{M}=$ $\exp (\mu)$ which is free of $\sigma^{2}$ and, hence, leads to the formula $\mu=\ln (\mathrm{M})$. This expression can be employed to estimate $\mu$ using the sample mode. Note that such estimate of $\mu$, unlike the MLE of $\mu$, does not depend on the estimate of $\sigma^{2}$.

For data presented in Table 3, the estimate of $\mu$ using the sample mode is 2.1747 . This estimate is comparable with the MLE estimate of 2.0748 (see Table 4); however, because the sample mode is not known to be an efficient estimator of the location parameter it is not considered further.

\section{Bootstrap Estimation of $\mu$}

As noted previously, because the distribution of $\hat{\mu}$ is not available in closed form, the nonparametric bootstrap method was used to estimate $\mu$ and its related confidence interval; results are shown in Table 6 . Based on these results, the $95 \%$ and $90 \%$ C.I.s can be constructed.

The purpose of the above computations is for illustration, not for comparison of the results obtained herein with those obtained by Meisner and Demirmen (1981). However, the definition of the size-biased (also known as the weighted distribution) version of the $\operatorname{LN}(\mu, \sigma)$ 


\section{RATNAPARKHI \& NAIK-NIMBALKAR}

Table 5: Simulation Results for the Properties of $\hat{\mu}(\#$ of simulations $=5,000)$

\begin{tabular}{|c|c|c|c|c|c|c|c|c|c|c|c|}
\hline$\mu$ & $\sigma$ & $\begin{array}{c}\text { Mean } \\
\text { of } \hat{\mu}\end{array}$ & MSE & $\mu$ & $\sigma$ & $\begin{array}{c}\text { Mean } \\
\text { of } \hat{\mu}\end{array}$ & MSE & $\mu$ & $\sigma$ & $\begin{array}{c}\text { Mean } \\
\text { of } \hat{\mu}\end{array}$ & MSE \\
\hline-2 & 0.5 & -1.98 & 0.0186 & 0 & 0.5 & 0.0122 & 0.0186 & 1 & 0.5 & 1.0111 & 0.0189 \\
\hline-2 & 1.0 & -1.94 & 0.1416 & 0 & 1.0 & 0.0548 & 0.1486 & 1 & 1.0 & 1.0559 & 0.1417 \\
\hline-2 & 1.5 & -1.89 & 0.6199 & 0 & 1.5 & 0.1187 & 0.6086 & 1 & 1.5 & 1.1146 & 0.5893 \\
\hline-2 & 2.0 & -1.79 & 1.7518 & 0 & 2.0 & 0.2132 & 1.7597 & 1 & 2.0 & 1.2008 & 1.7003 \\
\hline
\end{tabular}

Table 6: Bootstrap Estimate of $\mu$ for the Oil Fields Data (Number of Replications: 3,000)

\begin{tabular}{|c|c|c|c|c|}
\hline Summary Statistics & Observed & Bias & Mean & SE \\
\hline Parameters & 2.075 & 0.03512 & 2.11 & 0.265 \\
\hline BCa Percentiles & $2.5 \%$ & $5 \%$ & $95 \%$ & $97.5 \%$ \\
\hline Parameters & 1.5028 & 1.5857 & 2.4702 & 2.5518 \\
\hline
\end{tabular}

given, and also considered by Meisner and Demirmen, may be useful to some readers.

Let $X \sim f(x ; \theta)$. If $w(x)>0$ is a function of $x$ such that $E[w(X)]<\infty$, then the weighted distribution of $X$ is defined by the pdf

$$
g(x ; \theta)=w(x) f(x ; \theta) / E[w(X)],
$$

where $w(x)$ is referred to as the weight function and $\theta$ is a scalar or a vector of parameters.

Meisner and Demirmen (1981) assumed that the original distribution of the size of the oil field, denoted by $X$, is $\operatorname{LN}(\mu, \sigma)$. Further, in the exploration of the oil field, the probability of discovering an oil field depends on the size of the oil field. Therefore, for modeling the collected sample data of the oil fields Meisner and Demirmen considered the weighted lognormal (WLN) with weight function $w(x)=x^{\beta}$. Using (8), the distribution of interest, the $\operatorname{WLN}(\mu, \sigma)$ with the pdf is given by

$$
\begin{aligned}
& g_{2}(x ; \mu, \sigma)= \\
& \frac{1}{x \sigma \sqrt{2 \pi}} \exp \left[-\frac{1}{2 \sigma^{2}}\left(\log x-\left(\mu+\beta \sigma^{2}\right)\right)^{2}\right],
\end{aligned}
$$

where

$$
x>0,-\infty<\mu<\infty, \sigma>0
$$

and $\beta$ may have a known or unknown value.

Meisner and Demirmen (1981) discussed the possible values of $\beta$. In particular, they noted that, because the sizes of oil fields change with the exploration period, the values of $\beta$ could be in a two-sided neighborhood of the value 1 . Therefore, this illustration, considering $\beta=1$, that is assuming the sizes of the oil fields have the $\operatorname{LBLN}(\mu, \sigma)$ distribution given by (2.2), is justified. Table 7 shows the estimates of $\mu$ for the other values of $\beta$; Meisner and Demirmen considered $\beta$ as a random variable and developed a Bayesian approach for the analysis of these data. To construct the estimates 


\section{THE LENGTH-BIASED LOGNORMAL DISTRIBUTION AND ITS APPLICATION}

in Table 7, the modified version of (7) for accommodating $\beta$ was used.

Table 7: Changes in the Estimate of $\mu$ for Values of $\beta$

\begin{tabular}{|c|c|c|c|}
\hline$\beta$ & 0.9 & 1.0 & 1.1 \\
\hline$\mu$ Estimate & 2.25 & 2.07 & 1.89 \\
\hline
\end{tabular}

If the estimate 2.1747 of $\mu$ is acceptable, then considering that the mode of $\operatorname{LBLN}(\mu, \sigma)$ is a function of $\mu$ alone, it can used for finding a value of $\beta$ (a sort of ad hoc estimate of $\beta$ ) by extending Table 5 to include more values of $\beta$ than may be necessary. In particular, using such a table it can be shown that if $\beta=0.94$ then $\hat{\mu}=$ 2.17 (approximately), which is close to the above estimate 2.1747 .

Note that, in view of the unstable behavior of the sample mode, such an estimate should be carefully considered. However, in this analysis, observations show that the assumption of $\beta=1$ (which is close to the value of $\beta=$ 0.94 ) used for modeling the data from Table 4 has some relevance. Further, it should be noted that other more robust methods exist for locating the sample mode (Bickel \& Fruthworth, 2006). The traditional method was used in this study for demonstration purposes.

\section{Conclusion}

The length-biased lognormal distribution was introduced along with an application in the analysis of data from oil field explorations. The maximum likelihood estimation of the parameters of the length-biased lognormal was discussed briefly. In particular, the properties of the estimator of $\mu$ are not tractable. Therefore, the related properties were studied using simulations. Results presented regarding the modal value of the length-biased lognormal show that the estimation of $\mu$ using the sample mode is straightforward, but the efficiency of such an estimator is doubtful. The concepts of weighted lognormal distribution as a generalization of the length-biased lognormal and related modeling problems were also briefly mentioned.

\section{References}

Bickel, D. R., \& Fruthwirth, R. (2006). On a fast, robust estimator of the mode: Comparison to other robust estimators with applications. Computational Statistics \& Data Analysis, 50, 3500-3530.

Meisner, J., \& Demirmen, F. (1981).The creaming method: a Bayesian procedure to Forecast future oil and gas discoveries in mature exploration provinces. Journal of the Royal Statistical Society, Series A, 144, 1-31.

Quin, I. J., Berwick, M., Ashbolt, R., \& Dwyer, T. (2002). Quantifying the change of melanoma incidence by Breslow thickness. Biometrics, 58, 665-670.

Yan, P. (2004). Estimation for the infection curves for the spread of severe acute respiratory syndrome (SARS) from a backcalculation approach. Report of the Modeling and Projection Section, Centre for Infectious Disease Prevention \& Control. Population and Public Health Branch Health Canada, 1-17. 


\title{
A Study on Underwriting Cycle of Property Insurance Industry of China
}

\author{
Lin Zhang Linjuan Tang \\ College of Hunan University, \\ Changsha Hunan Province, China
}

Methods in underwriting cycle research are compared. A second-order autoregressive model, which includes structural transition and Christiano-Fitzgerald (CF) Filter method, is used to analyze China's underwriting cycle with annual property insurance loss ratio data from 1982 to 2008. Results show that the underwriting cycle is 11-12 years and, from the phase of underwriting cycle, management suggestions about underwriting cycle phenomenon are provided.

Key words: Underwriting cycle, property insurance, second-order autoregressive model, CF filter, structural transition.

\begin{abstract}
Introduction
An underwriting cycle refers to a period phenomenon of underwriting profit that fluctuates over time. A comprehensive underwriting cycle moves through four stages: mature hard market, immature soft market, mature soft market and immature hard market. As a specific phenomenon of the property insurance industry, the underwriting cycle has a significant impact. The phenomenon of underwriting cycles penetrates into all aspects of business of insurance companies and influences product pricing, claims payments, marketing strategy decisions and reserve calculations. Ernst \& Young (2009) lists the future top ten risks of the Chinese insurance industry in their Annual Report of Insurance Industry Operating Risk in China Market. They state that regulatory intervention, underwriting cycle management and climate change-among others - will
\end{abstract}

Lin Zhang is a Professor in the department of Finance and Statistics and is the Director of the Insurance Institute of China. She is a Fellow member of the Actuaries Association of China. Her research interests include non-life actuarial science. Email her at: lindazhang0203@126.com. Linjuan Tang is a student in the department of Finance. Email her at: tangljyouyou@163.com. become major risk factors for the Chinese insurance industry in the future, and that the underwriting cycle is the most important among the various risk factors. For this reason, research into property insurance underwriting cycles is particularly important. Although some professionals have studied underwriting cycles of property insurance, there is not a generally accepted empirical result regarding underwriting cycles.

Underwriting cycles were proposed by a broker company in the United States. Venezian (1985) proofed the existence of underwriting cycles for the property insurance industry, and Cummins and Outreville (1987) confirmed their existence in insurance markets outside of the U.S.A. Chen (1997) found the underwriting cycle existed in Asian emerging markets (including Japan, Singapore and Malaysia); he also notes that, even if there no underwriting cycle of whole non-life insurance industry exists in an individual country, there is an underwriting cycle phenomenon for some business lines, such as property insurance in Korea and motor insurance in Chinese Taipei.

In China, there have been a few studies on the underwriting cycle. Cai (2005) introduced the principle of an underwriting cycle in the world, his research focuses on insurance supply and demand. Wang and Shi (2006) used a second order autoregressive model with simple loss ratio data and concluded that there was no underwriting cycle in the Chinese property 


\section{UNDERWRITING CYCLE OF PROPERTY INSURANCE INDUSTRY OF CHINA}

insurance industry. However, they recognized there was an underwriting cycle in the motor insurance business line that is about 6 years. A similar study was conducted by Zhang and Zhu (2007). Li and Li (2010) used a filter method and simple loss ratio data and reported that the underwriting cycle for non-life insurance industries of China was 4-5 years. Ji and Zheng (2009) used a second order autoregressive model and spectral analysis with gross ratio; they identified both long underwriting cycles of 12.516.7 years, and medium underwriting cycles of 5.6 years.

Wang and Shi (2006) and Ji and Zheng (2009) used same method, but came to opposite conclusions. This raised the question if the difference is due to the difference between a simple loss ratio and a gross ratio. If so, the cycle phenomenon on simple loss ratio and gross ratio will be consistent after excluding abnormal data. Differences between findings of $\mathrm{Li}$ and $\mathrm{Li}$ (2010) and Wang and Shi (2006) also raises the question if it was abnormal data in the underwriting cycle, invisible in second order autoregressive model, that led to their conclusions.

A second order autoregressive model with a structural transition is used here to analyze whether simple loss ratio time-series data exhibits a structural transition. It determines the reason for structural transition and introduces dummy variables at points in the structural transition to analyze the underwriting cycle of the property insurance industry in China. Taking into account that the lack of an underwriting cycle is caused by abnormal data in a simple loss ratio time-series, a ChristianoFitzgerald Filter was used to separate the trend and periodic components of simple loss ratio time-series one by one, and then to identify the existence of underwriting cycle of property insurance in China by use of a second order autoregressive model.

\section{Measure Methods on Underwriting Cycle}

Four methods are typically used to measure an underwriting cycle:

(1) Direct Observation. Direct observation uses some simple chart to express loss ratio data and operating profit data, the direction of chart or peak can be observed and used to judge the existence of an underwriting cycle and to estimate the length of the cycle. This method is most intuitive, but is also the most rough; thus, very few of applications rely on it because it is not possible to obtain an exact length of an underwriting cycle.

(2) Autoregressive Model. This method is applied widely and results in an exact length of an underwriting cycle by using a second order autoregressive model. Some researchers have identified the existence of structural transitions that caused the underwriting cycle to disappear and have shown that the structural transition, or presence of a transition, affects the test of an underwriting cycle and its length measurement (see Venezian, 1985; Cummins and Outreville, 1987; Chen, 1997; Leng, 2006). However, if dummy variables are introduced at a transition point, the length of the underwriting cycle can be obtained. This method can calculate the length of underwriting cycle and find the transition and its causes so that the phenomenon of the underwriting cycle can be understood. The downsides of this method are that data for analysis cannot be too short a time span and it cannot explain either the start or end points.

(3) Spectral Analysis. Spectral analyses were first used in physics, but have recently been employed to study economic cycles. These analyses can estimate economic cycles with shorter time series. Ji and Zheng (2009) used this approach to study underwriting cycles. This method has no requirement for data time-series and all data involved in the equation without losing the sample point. Because all data is placed into equations and there are clear calculation and process criteria, this approach can avoid subjectivity in analysis. However, this method results in different lengths of underwriting cycles for different data time-series even if the data comes from the same business line: Sometimes the difference is huge and the model cannot explain the difference. 


\section{ZHANG \& TANG}

(4) Christiano-Fitzgerald (CF) Filter Analysis. Filter analysis starts from time series volatility. It removes white noise from a time series and then obtains the regularity of its implied. This approach mainly used to study economic cycles and was used by $\mathrm{Li}$ and $\mathrm{Li}$ (2010) to study underwriting cycles. The method provides different results under different hypotheses for different filters. Generally, the CF filter is used to study economic cycles. CF filters can isolate trend and periodic components in time series data. Studying a periodic component can result in identifying an underwriting cycle with high credibility, but the chart cycle from this method has no higher credibility.

Each method has its own advantages and disadvantages. As an objective phenomenon, an underwriting cycle will have almost same length regardless of what method is used. This article uses a CF filter and second order autoregressive model with a structural transition to study the underwriting cycle of the property insurance industry in China.

\section{Methodology}

The Existence of an Underwriting Cycle: Indicators Selected and Data Resource

The best indicator is gross ratio or underwriting profit margin. Venezian (1985) used underwriting profit margin as the variable to test the existence of underwriting cycles in the property insurance industry in the United States. Gross loss ratio, or underwriting profit margin, arose in many references as an indicator of testing. Because there is no consistency of expense ratio in China, this study used a simple loss ratio, which is equal to ratio of losses and premiums. The gross loss ratio is equal to the simple loss ratio plus gross expenses ratio; because the expenses ratio is typically stable, a simple loss ratio can be substituted for the gross loss ratio. Data in this study is 1982-2008 property insurance collected from the China Insurance Yearbook and China Statistical Yearbook.
The Existence of an Underwriting Cycle: Stationary Test

It is first necessary to judge whether a time series is stationary both in the second order autoregressive model and the CF filter. A common test used for time series data is the Augmented Dickey-Fuller (ADF) test statistic; ADF analyzes the stationary of data and tests whether the data has unit root. Results of this test are shown in Table 1.

Table 1 shows a t-statistic equal to -1.110974 ; this result indicates that the null hypothesis cannot be rejected, thus, the timeseries data has unit root, which is not smooth. From this, it may be determined that outliers are present in the simple loss rate time series; for this reason, a dummy variable was introduced into the second order autoregressive model and analyzed by $\mathrm{CF}$ filter after treatment of abnormal values to conduct empirical analysis about the existence of underwriting cycle.

Empirical Analysis: Second Order Autoregressive Method

Next, the stability of loss rate timeseries is tested. The loss rate time-series is stability when autocorrelation and partial autocorrelation tests are used. Structural transition exists in loss rate time-series (Leng, 2002), but the exact point of transition is unknown, so the Chow Square Test is used to judge that point. Table 2 shows that the F-value is 5.023 , which is higher than the $1 \%$ level, and the LLR $=14.598$ in 1991. These are the largest in all results and they indicated the presence of an abnormal phenomenon in 1991; this is the structural transition.

Compared with the situation of the property insurance industry development in China in 1991, it is postulated that there may be two reasons for the s structural transition in that year. First, with increased competition in domestic market the loss rate increased. The insurance industry carried out an open policy in all of China in 1991, so more companies entered the insurance industry and competition increased. Insurance companies competed for market share and lower underwriting levels led to a surge of loss rate so that the loss rate after 1991 is significantly higher than before. Second, the American insurance market is a very 


\section{UNDERWRITING CYCLE OF PROPERTY INSURANCE INDUSTRY OF CHINA}

important market in the world; it has huge premiums, especially liability premiums. Chinese insurance companies possibly did not understand the coverage of American liabilities or the legislation associated with those liabilities, so Chinese companies may have assumed a large insurance liability blindly in world market in 1980s. In addition, Chinese insurance companies assumed a lot of claims of liability insurance in 1991 and ignored the characteristics of reinsurance claims lags at that same time.

Due to this, a dummy variable is introduced in a second order autoregressive model as:

$$
\begin{aligned}
C R(t)= & c+a C R(t-1)+b C R(t-2)+d D \\
& +e D C R(t-1)+f D C R(t-2)+u
\end{aligned}
$$

Using Eviews 6.0 (statistical software) for analysis results in the regression shown in Table 3.

After introducing the dummy variable (Table 3), $a>0$ and $b<0$ can be found; $a$ and $b$ are then used to calculate the underwriting cycle of the property insurance industry, which is 11.938 .

$$
\begin{aligned}
T & =\frac{2 \pi}{\cos ^{-1}(a / 2 \sqrt{-b})} \\
& =\frac{2 \pi}{\cos ^{-1}(0.862382 / 2 \sqrt{-(-0.340534)})} \\
& =11.938
\end{aligned}
$$

Empirical Analysis: CF Filter

Economic variables include trend, periodic and random components. If the trend and random components can be separated from the periodic component, the periodic component can be used to measure the cycle of an economic variable. Normally, the random component is ignored because it has little effect and the CF filter is used to separate the trend and periodic components and to analyze the periodic component (Table 4).

Use of a second order autoregressive

\begin{tabular}{|c|c|c|}
\hline & t-Statistic: & Prob.: \\
\hline Augrmented Dickey-Fuller test statistic & -1.110974 & D. 6950 \\
\hline $\begin{array}{cc}\text { Test critical walues: } & 1 \% \text { lewel } \\
5 \% \text { lewel } & 10 \% \text { level }\end{array}$ & $\begin{array}{l}-3.724070 \\
-2.986225 \\
-2.632604\end{array}$ & \\
\hline
\end{tabular}
model to test the periodic component results in

\begin{tabular}{|c|c|c|c|}
\hline Variable & $\mathrm{c}$ & $\mathrm{a}$ & $\mathrm{b}$ \\
\hline Coefficient & -0.00127 & 1.73261 & -0.96809 \\
\hline $\begin{array}{c}\text { Standard } \\
\text { Deviation }\end{array}$ & $6.80 \mathrm{E}-05$ & 0.008428 & 0.008202 \\
\hline t-Value & -1.86578 & 205.5874 & -118.076 \\
\hline $\mathrm{R}^{2}$ & \multicolumn{3}{|c|}{0.999643} \\
\hline
\end{tabular}

$$
\begin{aligned}
T & =\frac{2 \pi}{\cos ^{-1}(a / 2 \sqrt{-b})} \\
& =\frac{2 \pi}{\cos ^{-1}(1.732610 / 2 \sqrt{-(-0.968092)})} \\
& =11.720
\end{aligned}
$$

Table 1: ADF Test Output

*Mackinnon (1996) one-sided p-values. 


\section{ZHANG \& TANG}

Table 2: Chow Square Test - Structural Transition

\begin{tabular}{|c|c|c|c|c|}
\hline Year & F-value & Probability & LLR & Probability \\
\hline 1987 & 1.286 & 0.308 & 4.621 & 0.202 \\
\hline 1988 & 1.633 & 0.215 & 5.735 & 0.125 \\
\hline 1989 & $2.446^{*}$ & 0.095 & $8.164^{* *}$ & 0.043 \\
\hline 1990 & $2.901^{*}$ & 0.062 & $9.427^{* *}$ & 0.024 \\
\hline 1991 & $5.023^{* * *}$ & 0.010 & $14.598^{* * *}$ & 0.002 \\
\hline 1992 & $2.524^{*}$ & 0.088 & $8.386^{* *}$ & 0.039 \\
\hline 1993 & $2.981^{*}$ & 0.057 & $9.643^{* *}$ & 0.022 \\
\hline 1994 & $3.329^{* *}$ & 0.042 & $10.56^{* *}$ & 0.014 \\
\hline 1995 & 1.599 & 0.223 & 5.627 & 0.131 \\
\hline 1996 & 1.954 & 0.155 & $6.723^{*}$ & 0.081 \\
\hline 1997 & 0.986 & 0.420 & 3.619 & 0.306 \\
\hline 1998 & 0.352 & 0.788 & 1.351 & 0.717 \\
\hline 1999 & 0.541 & 0.660 & 2.049 & 0.562 \\
\hline 2000 & 0.508 & 0.681 & 1.930 & 0.587 \\
\hline 2001 & 0.424 & 0.738 & 1.618 & 0.655 \\
\hline 2002 & 0.729 & 0.547 & 2.723 & 0.436 \\
\hline 2003 & 1.177 & 0.345 & 4.262 & 0.235 \\
\hline 2004 & 1.370 & 0.282 & 4.895 & 0.180 \\
\hline 2005 & 1.361 & 0.285 & 4.867 & 0.182 \\
\hline 2006 & 1.282 & 0.309 & 4.609 & 0.203 \\
\hline
\end{tabular}

*Means 10\% level, **Means 5\% level, ***Means 1\% level. Structure transition is in 1991.

Table 3: Regression Analysis Table - Introduced Dummy Variable

\begin{tabular}{|c|c|c|c|c|}
\hline Variable & Coefficient & $\begin{array}{c}\text { Standard } \\
\text { Deviation }\end{array}$ & t-Value & $\mathrm{R}^{2}$ \\
\hline $\mathrm{c}$ & 0.155 & 0.165 & 0.935 & \multirow{6}{*}{0.669} \\
\hline $\mathrm{a}$ & 0.862 & 0.487 & 1.771 & \\
\hline $\mathrm{b}$ & -0.249 & 0.497 & -0.500 & \\
\hline $\mathrm{d}$ & 0.278 & 0.209 & 1.330 & \\
\hline $\mathrm{e}$ & -0.968 & 0.534 & -1.814 & \\
\hline $\mathrm{f}$ & 0.540 & 0.534 & 1.012 & \\
\hline
\end{tabular}




\section{UNDERWRITING CYCLE OF PROPERTY INSURANCE INDUSTRY OF CHINA}

\section{Conclusion}

The existence of a structural transition in 1991 in the Chinese property insurance industry is verified by using simple loss rate data. A second order autoregressive model and CF filter were also used to calculate the underwriting cycle of the $\mathrm{P} \& \mathrm{C}$ insurance industry in China; the cycles are approximately 12 years in length. At the end of the paper, we give two suggestions to underwriting cycle management. Because the phenomenon of underwriting cycles is a risk factor, it is necessary to strengthen the management of underwriting cycles to lower their influence of volatility on the insurance business. Two strategies are suggested.

Recommendations for Underwriting Cycle Management

(1) Procyclical Policy: This policy's target is to maintain the market shares of insurance companies. At the very stage, the underwriting strategy will be maintained, regardless of changes in prices. The internal management of insurance companies may lead to increased cycle phenomenon and also to increased procyclical leverage effects. Insurance companies need to analyze all kinds of risks in operating procedures and allow a limit fluctuation.

(2) Countercycle Policy: This policy's target is to maintain the profitability of insurance companies. Insurance companies need to reduce their business line in soft market and only keep some profitable businesses so that they will have sufficient capital and surplus to expand in hard market.

\section{References}

Boor, J. (2000). The impact of the insurance economic cycle on insurance pricing. CAS study note. http://casualtyactuaries.com/library/studynotes/b oor5.2.pdf.

Venezian, E. (1985). Ratemaking methods and profit cycles in property and liability insurance. Journal of Risk and Insurance, 52, 477-500.

Cummins, \& Outreville. (1987). An international analysis of underwriting cycles in property-liability insurance, Journal of Risk and Insurance, 54, 246-262.

Ernst \& Young. (2009). Annual report of insurance industry operating risk in China market. http://www.ey.com/Publication/ vwLUAssets/Business_Risk_Insurance_Report_ 2009_Chinese/\$FILE/Business\%20Risk_Insuran ce_Report_2009_Chinese.pdf

Ji, Y., \& Zheng, H. (2009). Existence analysis of underwriting cycle of $\mathrm{P} \& \mathrm{C}$ insurance industry of China. Journal of University of Aeronautics and Astronautics, 22(4), 1-3.

Leng. C-C. (2006). Stationarity and stability of underwriting profits in propertyliability insurance: Part II. Journal of Risk Finance, 1, 49-63

Li, X., \& Li, J. (2010). Study on underwriting cycle of $\mathrm{P} \& \mathrm{C}$ insurance industry of China. Insurance Studies, 2, 44-52.

Qiujie, C. (2005). The general characteristics and reasons of interaction between insurance supply and demand. Journal of Jiangxi Finance University, 39, 33-37.

Wang, B., \& Shi, A. (2006). Study on underwriting cycle of $\mathrm{P} \& \mathrm{C}$ insurance industry and test in China market. Shanghai Finance, 7, $37-40$

Zhang, L., \& Zhu, Y. (2007). Existence analysis of auto insurance underwriting cycle of China. China Economical Review, 69, 66-70. 
Table 4 Trend and Periodic Component Filter Table

\begin{tabular}{|c|c|c|c|c|c|}
\hline Year & $\begin{array}{c}\text { Trend } \\
\text { Component }\end{array}$ & $\begin{array}{c}\text { Periodic } \\
\text { Component }\end{array}$ & Year & $\begin{array}{c}\text { Trend } \\
\text { Component }\end{array}$ & $\begin{array}{c}\text { Periodic } \\
\text { Component }\end{array}$ \\
\hline 1982 & 0.43178 & 0.023206 & 1996 & 0.55212 & 0.025064 \\
\hline 1983 & 0.46395 & 0.026539 & 1997 & 0.53744 & 0.019601 \\
\hline 1984 & 0.4877 & 0.023344 & 1998 & 0.56252 & 0.009925 \\
\hline 1985 & 0.4252 & 0.014245 & 1999 & 0.53197 & -0.00145 \\
\hline 1986 & 0.41764 & 0.001426 & 2000 & 0.51837 & -0.01179 \\
\hline 1987 & 0.39252 & -0.01192 & 2001 & 0.50494 & -0.01881 \\
\hline 1988 & 0.40884 & -0.02244 & 2002 & 0.53785 & -0.02118 \\
\hline 1989 & 0.43324 & -0.02749 & 2003 & 0.56653 & -0.01878 \\
\hline 1990 & 0.41533 & -0.02584 & 2004 & 0.52721 & -0.01254 \\
\hline 1991 & 0.51941 & -0.01797 & 2005 & 0.54965 & -0.0042 \\
\hline 1992 & 0.46525 & -0.00592 & 2006 & 0.52357 & 0.004285 \\
\hline 1993 & 0.48249 & 0.007262 & 2007 & 0.49964 & 0.011113 \\
\hline 1994 & 0.55175 & 0.018337 & 2008 & 0.6019 & 0.015071 \\
\hline 1995 & 0.41103 & 0.024713 & & & \\
\hline
\end{tabular}




\title{
The Weighted Hellinger Distance for Kernel Distribution Estimator of Function of Observations
}

\author{
Abdel-Razzaq Mugdadi \\ Jordan University of Science and Technology, \\ Irbid, Jordan
}

The asymptotic mean weighted Hellinger distance (AMWHD) is derived for the kernel distribution estimator of a function of observations. In addition, the AMWHD is compared with the asymptotic mean integrated square error (AMISE) of the estimator. A completely data based method is proposed to select the bandwidth in the estimator using the mean weighted Hellinger distance (MWHD).

Key words: Kernel estimation, distribution function estimation, bandwidth, Hellinger distance, mean square error, function of random variables.

\section{Introduction}

Given a random sample $X_{1}, X_{2}, \ldots, X_{n}$ from a distribution $F(x)$ with unknown density function $f(x)$, the kernel density estimator (Rosenblatt, 1956) of $f(x)$ is given by

$$
\hat{f}(x)=\frac{1}{n b} \sum_{i=1}^{n} k\left(\frac{x-X_{i}}{b}\right),
$$

where $b$ is the smoothing bandwidth and $k$ is a symmetric function satisfying $\int k(x) d x=1$. The kernel distribution function estimator (Nadaraya, 1964) of $F(x)$ is given by

Abdel-Razzaq Mugdadi is an Associate Professor of Statistics in the Department of Mathematics and Statistics. He worked as an Assistant and Associate Professor with tenure at Southern Illinois University in Carbondale, IL from 2000 to 2010 . He has published more than 15 research articles in publications including the Journal of Statistical Planning and Inference, Journal of Nonparametric Statistics, Computational Statistics and Data Analysis, IEEE transactions on Reliability and others. Email him at: aamugdadi@just.edu.jo.

$$
\hat{F}(x)=\frac{1}{n} \sum_{i=1}^{n}\left(\frac{x-X_{i}}{b}\right),
$$

where $K$ is the distribution function of the kernel $k, K(x)=\int_{-\infty}^{x} k(u)(d u)$, and $b$ is the bandwidth.

Consider the function $g\left(X_{1}, X_{2}, \ldots, X_{m}\right)$ that depends on $m \geq 1$ observations. Assume that $g$ is a real value and is symmetric in its $m$ arguments. Frees (1994) proposed an estimate for the density function $h(t)$ of random variable $g\left(X_{1}, X_{2}, \ldots, X_{m}\right)$ which is given by

$$
\hat{h}(t)=\frac{1}{b\left(\begin{array}{l}
n \\
m
\end{array}\right)} \sum_{(n, m)} w\left(\frac{t-g\left(X_{i_{1}}, \ldots, X_{i_{m}}\right.}{b}\right),
$$

where $b$ is the bandwidth, the sum extends over all $1 \leq i_{1}<i_{2}<\ldots<i_{m} \leq n$, and $w($.$) is a$ kernel function. If $m=1$ and $g(x)=x$, then the estimator $\hat{h}(t)$ reduces to the estimator $\hat{f}(x)$. 
The estimator $\hat{h}(t)$ has many applications in real life. For example, in spatial statistics $g$ can be the inter point distance between pairs of objects and in insurance $g$ can be the sum of $m$ claims (Frees, 1994; Ahmad \& Fan, 2001; Mugdadi \& Ahmad, 2004).

Nadaraya (1964) and Mugdadi and Ghebregiorgis (2005) proposed a kernel distribution estimator of the distribution function of function of observations $H(t)$ as:

$$
\hat{H}(t)=\frac{1}{\left(\begin{array}{l}
n \\
m
\end{array}\right)} \sum_{(n, m)} W\left(\frac{t-g\left(X_{i_{1}}, \ldots, X_{i_{m}}\right.}{b}\right),
$$

where $W(x)=\int_{-\infty}^{x} w(t) d t, b$ is the bandwidth and the sum extends over all $1 \leq i_{1}<i_{2}<\ldots<i_{m} \leq n$. Theoretical and simulation analyses show that choice of kernel is not crucial for distribution function estimation in the case of independent and identically (i.i.d) random variables; the most important choice is that of bandwidth. A typical way to select the bandwidth is to minimize one error measure, and the most commonly used is the mean integrated square error (MISE) and its asymptotic (AMISE), where

$$
\operatorname{MISE}(\hat{H}(t))=E \int[\hat{H}(t)-H(t)]^{2} d t .
$$

Another criterion is the mean Hellinger distance (MHD), where

$$
\operatorname{MHD}(\hat{H}(t))=E \int\left[\hat{H}^{\frac{1}{2}}(t)-H^{\frac{1}{2}}(t)\right]^{2} d t .
$$

Kanzawaa (1993) discussed the relationship between the asymptotic mean Hellinger distance (AMHD) and the AMISE for $\hat{f}(x)$, Ahmad and Mugdadi (2006) examined the relationship between asymptotic mean weighted Hellinger distance (AMWHD) and the AMISE for both $\hat{f}(x)$ and $\hat{F}(x)$, and Mugdadi (2004) studied the AMWHD for $\hat{h}(t)$. This investigation examines the relationship between the AMWHD and the AMISE for $\hat{H}(t)$ and proposes a data method to select the bandwidth for $\hat{H}(t)$ based on the AMWHD $(\hat{H}(t))$.

The Asymptotic Mean Weighted Hellinger Distance

One error criterion used to evaluate the estimator is the mean weighted Hellinger distance (MWHD) and its asymptotic (AMWHD), where $\operatorname{MWHD}(\hat{H}(t))$ is defined by:

$$
M W H D(\hat{H}(t))=E \int\left[\hat{H}^{\frac{1}{2}}(t)-H^{\frac{1}{2}}(t)\right]^{2} H(t) d t .
$$

It can be argued that

$$
\operatorname{MWHD}(\hat{H}(t))=\frac{1}{4} \operatorname{MISE}(\hat{H}(t)),
$$

assuming that $\hat{H}(t) \approx H(t)$ results in:

$$
\begin{aligned}
\operatorname{MISE}(\hat{H}(t)) & =\int E[\hat{H}(t)-H(t)]^{2} d t \\
& =\int E\left[\hat{H}^{\frac{1}{2}}(t)-H^{\frac{1}{2}}(t)\right]^{2}\left[\hat{H}^{\frac{1}{2}}(t)+H^{\frac{1}{2}}(t)\right]^{2} d t \\
& \approx 4 E \int\left[\hat{H}^{\frac{1}{2}}(t)-H^{\frac{1}{2}}(t)\right]^{2} H(t) d t \\
& =4 M W H D(\hat{H}(t))
\end{aligned}
$$

Next, the $A M W H D(\hat{H}(t))$ is derived and compared with the AMISE $(\hat{H}(t))$. Mugdadi and Ghebregiorgis (2005) derived $\operatorname{AMISE}(\hat{H}(t))$ as:

$$
\begin{aligned}
& \operatorname{AMISE}(\hat{H}(t))= \\
& \qquad \frac{T(H)}{\left(\begin{array}{l}
n \\
m
\end{array}\right)}-\frac{b}{\left(\begin{array}{l}
n \\
m
\end{array}\right)} \rho(w)+\frac{b^{4}}{4} \mu_{2}(w)^{2} R\left(H^{\prime \prime}\right),
\end{aligned}
$$

where 


\section{WEIGHTED HELLINGER DISTANCE FOR KERNEL DISTRIBUTION ESTIMATOR}

$$
\begin{gathered}
\rho(w)=2 \int u W(u) w(u) d u \\
T(H)=\int H(t)[1-H(t)] d t
\end{gathered}
$$

and

$$
R(h)=\int h^{2}(t) d t
$$

Theorem

If the fourth derivative of $H(t)$ exists, then

$$
\begin{aligned}
& \operatorname{AMISE}(\hat{H}(t))= \\
& \frac{T(H)}{4\left(\begin{array}{l}
n \\
m
\end{array}\right)}-\frac{b}{4\left(\begin{array}{l}
n \\
m
\end{array}\right)} \rho(w)+\frac{b^{4}}{16} \mu_{2}(w)^{2} R\left(H^{\prime \prime}\right) .
\end{aligned}
$$

Proof

$$
\begin{aligned}
& M W H D(\hat{H}(t))= \\
& \int E \hat{H}(t) H(t) d t-2 \int E \hat{H}^{\frac{1}{2}}(t) H^{\frac{3}{2}}(t) d t+\int H^{2}(t) d t .
\end{aligned}
$$

Using integration by parts and expanding $H(t-b u)$ in a $2^{\text {nd }}$ order Taylor's series about $t$ results in:

$$
\begin{aligned}
E(\hat{H}(t)) & =E\left(\frac{1}{\left(\begin{array}{l}
n \\
m
\end{array}\right)} \sum_{(n, m)} W\left(\frac{t-g\left(X_{1}, X_{2}, \ldots, X_{m}\right)}{b}\right)\right) \\
& =\int w(u)\left(\begin{array}{l}
H(t)-b u H^{\prime}(t)+\frac{b^{2} u^{2} H^{\prime \prime}(t)}{2} \\
-\frac{b^{3} u^{3} H^{(3)}(t)}{6}+\frac{b^{4} u^{4} H^{(4)}(t)}{24} \\
+o\left(b^{4}\right)
\end{array}\right) d u \\
& \approx H(t)+\frac{b^{2} H^{\prime \prime}(t)}{2} \mu_{2}(w)+\frac{b^{4}}{24} \mu_{4}(w) H^{(4)}(t)
\end{aligned}
$$

therefore, $\int E[\hat{H}(t) H(t)] d t \approx$

$$
\left[\begin{array}{l}
\int H^{2}(t) d t+\frac{b^{2} \mu_{2}(w)}{2} \int H^{\prime \prime}(t) H(t) d t \\
+\frac{b^{4} \mu_{4}(w)}{24} \int H^{(4)}(t) H(t) d t
\end{array}\right]
$$

If $Z$ is a random variable with a standard normal distribution, then

$$
\hat{H}(t) \approx E(\hat{H}(t))+Z \sqrt{\operatorname{Var}(\hat{H}(t))} .
$$

Mugdadi and Ghebregiorgis (2005) derived $\operatorname{Var}(\hat{H}(t))$, this is given by:

$\operatorname{Var}(\hat{H}(t))=$

$$
\left[\begin{array}{l}
O\left(\frac{1}{n}\right)+O\left(\frac{b^{2}}{n}\right)+\left(\begin{array}{c}
n \\
m
\end{array}\right)^{-1} H(t)(1-H(t)) \\
+\left(\begin{array}{c}
n \\
m
\end{array}\right)^{-1} o(b)-\left(\begin{array}{l}
n \\
m
\end{array}\right)^{-1} b H^{\prime}(t) \rho(w)
\end{array}\right] .
$$

Thus,

$\hat{H}(t)$

$$
\begin{aligned}
& \approx H(t)+\frac{b^{2} H^{\prime \prime}(t)}{2} \mu_{2}(w)+\frac{b^{4} H^{(4)}(t)}{24} \mu_{4}(w) \\
& \left.+Z\left[\begin{array}{c}
O\left(\frac{1}{n}\right)+O\left(\frac{b^{2}}{n}\right)+\left(\begin{array}{l}
n \\
m
\end{array}\right)^{-1} H(t)(1-H(t)) \\
+\left(\begin{array}{l}
n \\
m
\end{array}\right)^{-1} o(b)-\left(\begin{array}{l}
n \\
m
\end{array}\right)^{-1} b H^{\prime}(t) \rho(w)
\end{array}\right]\right] \\
& 1+\frac{b^{2} H^{\prime \prime}(t) \mu_{2}(w)}{2 H(t)}+\frac{b^{4} \mu_{4}(w) H^{(4)}(t)}{24 H(t)} \\
& \approx H(t) \\
& \left.+Z\left(\frac{1-H(t)}{\left(\begin{array}{l}
n \\
m
\end{array}\right) H(t)}-\frac{b H^{\prime}(t) \rho(w)}{\left(\begin{array}{l}
n \\
m
\end{array}\right) H^{2}(t)}+O\left(\frac{1}{n}\right)+O\left(b^{2}\right)\right)^{\frac{1}{2}}\right]
\end{aligned}
$$


therefore,

$$
\begin{aligned}
& \hat{H}^{\frac{1}{2}}(t) \approx \\
& {\left[\begin{array}{c}
1+\frac{b^{2} H^{\prime \prime}(t) \mu_{2}(w)}{4 H(t)}+\frac{b^{4} \mu_{4}(w) H^{(4)}(t)}{48 H(t)} \\
+\frac{Z}{2}\left[\begin{array}{l}
\frac{1-H(t)}{\left(\begin{array}{l}
n \\
m
\end{array}\right) H(t)}-\frac{b H^{\prime}(t) \rho(w)}{\left(\begin{array}{l}
n \\
m
\end{array}\right) H^{2}(t)} \\
+O\left(\frac{1}{n}\right)+O\left(b^{2}\right)
\end{array}\right]^{\frac{1}{2}} \\
-\frac{b^{4}}{32}\left(\frac{H^{\prime \prime}(t)}{H(t)}\right)^{2}\left(\mu_{2}(w)\right)^{2}
\end{array}\right]}
\end{aligned}
$$

and

$$
\begin{aligned}
E\left(\hat{H}^{\frac{1}{2}}(t)\right) & \approx \\
H^{\frac{1}{2}}(t) & {\left[\begin{array}{l}
1+\frac{b^{2} H^{\prime \prime}(t) \mu_{2}(w)}{4 H(t)}+\frac{b^{4} \mu_{4}(w) H^{(4)}(t)}{48 H(t)} \\
-\frac{b^{4}}{32}\left(\frac{H^{\prime \prime}(t)}{H(t)}\right)^{2}\left(\mu_{2}(w)\right)^{2} \\
-\frac{1}{8}\left(\frac{1-H(t)}{\left(\begin{array}{l}
n \\
m
\end{array}\right) H(t)}-\frac{b H^{\prime}(t) \rho(w)}{\left(\begin{array}{l}
n \\
m
\end{array}\right) H^{2}(t)}\right) \\
+O\left(\frac{1}{n}\right)+O\left(b^{2}\right)
\end{array}\right] }
\end{aligned}
$$

thus

$$
\int\left[E \hat{H}^{\frac{1}{2}}(t) H^{\frac{3}{2}}(t)\right] d t \approx
$$

$$
\left[\begin{array}{l}
\int H^{2}(t) d t+\frac{b^{2}}{4} \mu_{2}(w) \int H^{\prime \prime}(t) H(t) d t \\
+\frac{b^{4}}{48} \mu_{4}(w) \int H^{(4)}(t) H(t) d t \\
-\frac{b^{4}}{32} \mu_{2}^{2}(w) \int\left(H^{\prime \prime}(t)\right)^{2} d t \\
-\frac{1}{8\left(\begin{array}{l}
n \\
m
\end{array}\right)} \int H(t)(1-H(t)) d t \\
+\frac{b\left(\begin{array}{c}
n \\
m
\end{array}\right)}{8(w)+O\left(\frac{1}{n}\right)+O\left(b^{2}\right)}
\end{array}\right]
$$

therefore,

$$
\begin{aligned}
& A M W H D(\hat{H}(t)) \approx \\
& {\left[\begin{array}{l}
\frac{b^{4}}{16} \mu_{2}^{2}(w) R\left(H^{\prime \prime}\right) \\
+\frac{1}{4\left(\begin{array}{c}
n \\
m
\end{array}\right)} \int(H(t) \\
\times\left[1-H(t) d t-\frac{b}{4\left(\begin{array}{c}
n \\
m
\end{array}\right)} \rho(w)\right.
\end{array}\right]}
\end{aligned}
$$

Under these conditions, the following corollaries can be proven.

Corollary 1

$$
A M W H D(\hat{H}(t)) \approx \frac{A M I S E(\hat{H}(t)}{4} .
$$

Similar to Powell and Stocker (1996), the optimal bandwidth to minimize the $A M W H D(\hat{H}(t))$ is shown in corollary 2 . 


\section{WEIGHTED HELLINGER DISTANCE FOR KERNEL DISTRIBUTION ESTIMATOR}

Corollary 2

$$
b_{o p t}=\left[\frac{\rho(w)}{\mu_{2}^{2}(w) R\left(h^{\prime}\right)}\right]^{\frac{1}{3}}\left(\begin{array}{l}
n \\
m
\end{array}\right)^{\frac{-1}{3}}
$$

Bandwidth Selection

The choice of bandwidth is very important in the Kernal density estimator as well as in the Kernal distribution estimator. One of the simplest methods to select bandwidth is based on equation (2.8). Assume that the data is from a normal distribution with mean equal to zero and variance $\sigma_{1}^{2}$. If $s^{2}$ is the variance of the data $g\left(X_{i 1}, \ldots, X_{i m}\right)$ for all $1 \leq i_{1}<\ldots<i_{m} \leq n$, then $R\left(h^{\prime}\right)=\frac{1}{4 \pi s^{3}}$, thus

$$
b_{o p t}=\left[\frac{\rho(w) 4 \pi}{\pi_{2}^{2}(w)}\right]^{\frac{1}{3}}\left(\begin{array}{c}
n \\
m
\end{array}\right)^{\frac{-1}{3}} S
$$

This shows that $b_{\text {opt }}$ depends only on the standard deviation and on the Kernel function.

A completely different data based method is proposed to select the bandwidth for a Kernal distribution estimator of the function of observations. The method is based on minimizing the $\operatorname{MWHD}(\hat{H}(t))$. The $\operatorname{MWHD}(\hat{H}(t)$ is defined as:

$$
\begin{aligned}
\operatorname{MWHD}(\hat{H}(t)) & =E \int\left[\hat{H}^{\frac{1}{2}}(t)-H^{\frac{1}{2}}(t)^{2} H(t) d t\right. \\
& =\left[\begin{array}{l}
\left.E \int \hat{H}(t) H(t) d t+E \int H^{2}(t) d t\right] \\
-2 E \int \hat{H}^{\frac{1}{2}} H^{\frac{3}{2}}(t) d t
\end{array}\right] \\
& =\left[\begin{array}{l}
E \int \hat{H}(t) H(t) d t+E \int H^{2}(t) d t \\
-2 \int\left(E \hat{H}^{\frac{1}{2}}(t)\right) H^{\frac{3}{2}}(t) d t
\end{array}\right] .
\end{aligned}
$$

Minimizing $\operatorname{MWHD}(\hat{H}(t))$ is therefore equivalent to minimizing $\operatorname{MWHD}(\hat{H}(t))$, where,

$$
\begin{aligned}
& M W H D 1(\hat{H}(t))= \\
& \quad \int(E \hat{H}(t)) H(t) d t-2 \int\left(E \hat{H}^{\frac{1}{2}}(t)\right) H(t)^{\frac{2}{3}} d t
\end{aligned}
$$

Thus, $M W H D 1(\hat{H}(t))$ can be estimated as follows. Let $m_{(1)}$ be a fixed choice of $m$ variables and let $I(A)$ be the indicator function. Also, define

$$
H_{n, m_{(1)}}(t)=\frac{1}{\left(\begin{array}{l}
n \\
m
\end{array}\right)-1} \sum_{(n, m), m \neq m_{(1)}} I\left(t-g\left(X_{i_{1}}, \ldots, X_{i_{m}}\right)\right)
$$

and

$$
\hat{H}_{-m_{(1)}}(t)=\frac{1}{\left(\begin{array}{l}
n \\
m
\end{array}\right)-1} \sum_{(n, m), m \neq m_{(1)}} W\left(\frac{t-g\left(X_{i_{1}}, \ldots, X_{i_{m}}\right)}{b}\right)
$$

which is the distribution estimated based on a sample with $m_{(1)}$ deleted. Thus, $\operatorname{MWHD} 1(\hat{H}(t))$ is estimated by

$$
\begin{aligned}
& \text { MWHD.EST }= \\
& \frac{1}{\left(\begin{array}{l}
n \\
m
\end{array}\right)} \sum_{1 \leq i_{1}<\ldots<i_{m}<n}\left[\begin{array}{l}
\hat{H}_{-m_{(1)}}\left(g\left(X_{i_{1}}, \ldots, X_{i_{m_{(1)}}}\right)\right. \\
\times H_{n, m_{(1)}}\left(g\left(X_{i_{1}}, \ldots, X_{i_{m_{(1)}}}\right)\right.
\end{array}\right]
\end{aligned}
$$

As noted, there are many applications for $\hat{H}(t)$. One example, introduced by Free (1993), regards an insurance claims problem. Table 1 shows total hospital charges (in dollars) in one Wisconsin (USA) hospital for females aged 3049 in the year 1989.

Consider the case $m=2$ and the function $\quad g\left(X_{1}, X_{2}\right)=X_{1}+X_{2}$. By minimizing MWHD.EST, the bandwidth is determined to be 0.437 . Figure 1 shows the kernel distribution function for $g\left(X_{1}, X_{2}\right)$ using data in Table 1. It is clear that the kernel 
estimate is smooth and $\hat{H}(t)$ is 0 when $t \leq 0$ because the sum of the charges should be positive; also, $\lim _{t \rightarrow \infty} \hat{H}(t)=1$.

Table 1: Total 1989 Hospital Charges (USD) for Females Aged 30-49

\begin{tabular}{|l|l|l|}
\hline 2337 & 1765 & 1802 \\
\hline 2179 & 2467 & 2011 \\
\hline 2348 & 3609 & 2270 \\
\hline 4765 & 2141 & 3425 \\
\hline 3041 & 1850 & 3558 \\
\hline 2088 & 3191 & 2315 \\
\hline 2872 & 3020 & 1642 \\
\hline 1924 & 2473 & 5878 \\
\hline 2294 & 1898 & 2101 \\
\hline 2182 & 7787 & 2242 \\
\hline 2138 & 6169 & 5746 \\
\hline
\end{tabular}

Figure 1: Kernel Distribution Function for $g\left(X_{1}, X_{2}\right)$

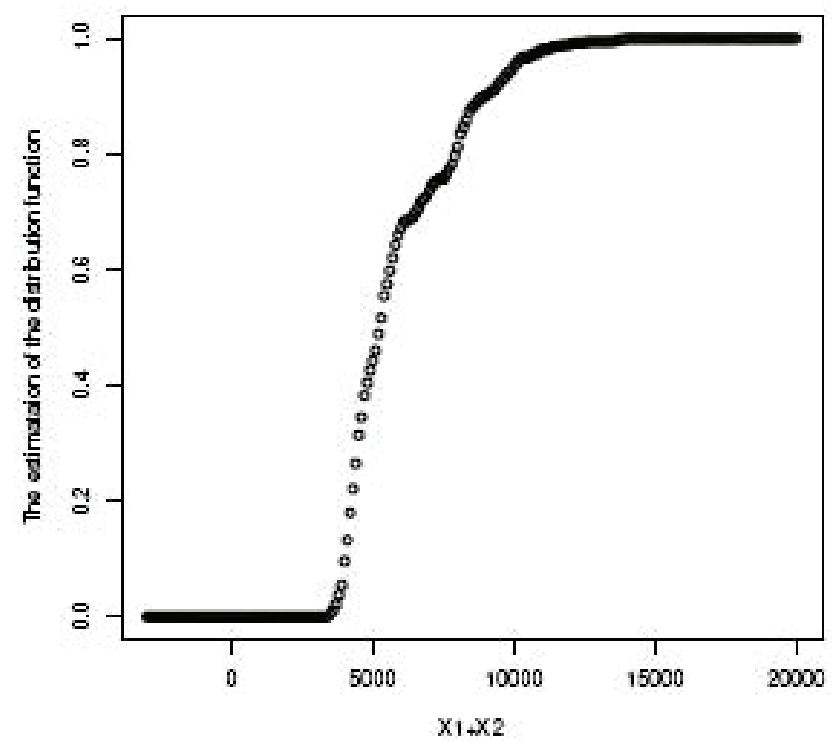

References

Ahmad, I. A., \& Fan, Y. (2001). Optimal bandwidth for kernel density estimator of functions of observations. Statistics and Probability Letters, 51(3), 245-251.

Ahmad, I. A., \& Mugdadi, A. R. (2006). A weighted Hellinger distance as an error criterion for bandwidth selection for kernel estimation. Journal of Nonparametric Statistics, 18, 215-226.

Bolanc, C., Guilln, M., \& Nielsen, J. (2010). Transformation kernel estimation of insurance claim cost distribution. In Mathematical and Statistical Methods for Actuarial Sciences and Finance, 43-51. Milan, Italy: Springer Italia.

Frees, E. (1994). Estimating densities of functions of observations. Journal of the American Statistical Association, 89, 517-525

Mugdadi, A. R., \& Ahmad, I. A. (2004). A bandwidth selection for Kernal density estimation of functions of random variables. Computational Statistics and Data Analysis, 47, 49-62.

Mugdadi, A. R., \& Ghebregiorgis, G. (2005). The Kernal distribution estimator of functions of random variables. Journal of Nonparametric Statistics, 17(7), 807-818.

Mugdadi, A. R. (2004). Bandwidth selection for the function of observations using Hellinger distance. Journal of Applied Statistical Science, 13(3), 231-240.

Nadraya, E. A. (1964). Some new estimates for distribution function. Theory and Probability Applications, 9, 497-500.

Powel, J. L., \& Stoker, T. M. (1996). Optimal bandwidth choice for density-weighted averages. Journal of Econometrics, 75, 291-316.

Rosenblatt, M. (1956). Remarks on some nonparametric estimates of a density function. Annals of Mathematical Statistics, 27, 832-837.

Sarda, P. (1993). Smoothing parameter selection for smooth distribution functions. Journal of Statistical Planning and Inference, $35,65-75$. 


\title{
Brief Reports \\ Four Period Crossover Designs
}

\author{
James F. Reed III \\ Christiana Care Hospital System, \\ Newark, Delaware
}

In higher-order four period crossover designs with two treatments, sixteen possible treatment sequences can result: AAAA, AAAB, AABA, AABB, ABAA, ABAB, ABBA, ABBB and their duals. Higher-order crossover designs are useful for several reasons: they allow estimation of a treatment effect even in the presence of a carry-over effect, they provide estimates of intra-subject variability and they draw inference on the carry-over effect. The real question related to a two-treatment four-period crossover design is the real world application of these designs. This article considers four designs: Design I: ABBA and its dual; Design II: ABBA, AABB and their duals, Design III: ABBA, ABAA and their duals, Design IV: ABBA, $\mathrm{ABAB}$ and their duals. A traditional model that specifies a first-order carryover effect is assumed and methods for estimating treatment and first-order carryover effects in the set of four period trials are outlined.

Key words: Four period crossover design, carryover effect, bioequivalence.

\section{Introduction}

The crossover design, which compares two treatments over two periods, has held a dominant position in the application of crossover designs to the extent that - in the majority of articles and texts - it is referred to as the crossover design. Crossover designs are popular for comparing several non-curative treatments for their efficacy. For convenience, a crossover design with $t$ treatments, $p$ periods and $s$ sequences is denoted as a $\mathrm{C}(\mathrm{t}, \mathrm{p}, \mathrm{s})$.

Critiques of the $\mathrm{C}(2,2,2)$ with sequences $\mathrm{AB}$ and $\mathrm{BA}$ are well known; the most serious of these is that the carryover effect is confounded with sequence by period effects leading to erroneous analyses. To overcome the disadvantages of the $\mathrm{C}(2,2,2)$, a higher-order crossover design may be used. Two strategies can be employed to overcome the problems

James F. Reed III, Ph.D., is the Director, Clinical Business Intelligence Biostatistician, Adventist Health. Email him at: ReedJF@ah.org. inherent in the $\mathrm{C}(2,2,2)$ design. The first strategy is to extend the number of sequences, such as Balaam's C $(2,2,4)$ design (Balaam, 1968); this design has four sequences, $\mathrm{AA}|\mathrm{AB}| \mathrm{BA} \mid \mathrm{BB}$. In addition, unbiased estimates of treatment and carryover effect can be formulated using a strategy outlined by Matthews (Matthews, 1994; Laska, Meisner \& Kushner, 1983; Reed, 2010). The second strategy is to extend the classic design by adding a third period and/or a fourth period and repeating one of the two treatments.

Higher-order four period crossover designs with two treatments can result in sixteen possible treatment sequences: AAAA, AAAB, AABA, AABB, ABAA, ABAB, ABBA, ABBB and their duals. This article considers four designs: Design I: ABBA and its dual; Design II: ABBA, AABB and their duals, Design III: ABBA, ABAA and their duals, Design IV: $\mathrm{ABBA}, \mathrm{ABAB}$ and their duals. This article presents methods for estimating treatment and first-order carryover effects in the set of four period trials, assuming a traditional model that specifies a first-order carryover effect. 
Crossover Design Model with Continuous Data

Assume that the primary goal is to compare two treatments $\mathrm{A}$ and $\mathrm{B}$ used in a study by estimating the treatment contrast $\tau_{\mathrm{A}}-\tau_{\mathrm{B}}$ and period effects $\pi_{1}$ and $\pi_{2}$; first order carryover effects $\lambda_{A}, \lambda_{B}$ and $\mu$ are regarded as nuisance parameters. Also assume that the response variable is continuous and that there is one response from each subject in each period. Finally, it is assumed that each treatment has a simple first-order carryover effect that does not interact with the direct effect of the treatment in the subsequent period. This model then assumes the following for the response of patient $y_{\mathrm{ij}}$.

If $y_{i j k}$ denotes the observed response of subject $j(j=1, \ldots, n)$ in period $i(i=1, \ldots, p)$, then

$$
\mathrm{y}_{\mathrm{ijk}}=\mu+\pi_{\mathrm{i}}+\tau_{\mathrm{d}(\mathrm{i}, \mathrm{j})}+\lambda_{\mathrm{d}(\mathrm{i}-1, \mathrm{j})}+\beta_{\mathrm{j}}+\varepsilon_{\mathrm{ij}}
$$

where $\pi_{\mathrm{i}}$ is the effect of period I, $\tau_{\mathrm{d}(\mathrm{i}, \mathrm{j})}$ is the direct effect of treatment $\mathrm{D}, \lambda_{\mathrm{d}(\mathrm{i}-1, \mathrm{j})}$ is the simple first-order carryover effect of treatment $\mathrm{D}$, and $\mathrm{d}(\mathrm{i}, \mathrm{j})$ is the treatment allocated to patient $\mathrm{j}$ in period i. $\lambda_{\mathrm{d}(0, \mathrm{j})}=0$ for all $\mathrm{j}$. It is assumed that all effects are fixed effects. $\beta_{\mathrm{j}}$ is the effect of patient $\mathrm{j}$ and $\varepsilon_{\mathrm{ij}}$ is the error term. The random subject effect, $\beta_{\mathrm{j}}$, and the experimental error, $\varepsilon_{\mathrm{ij}}$, are assumed to be mutually independently distributed as $\mathrm{N}\left(0, \sigma_{\beta}^{2}\right)$ and $\mathrm{N}\left(0, \sigma_{\varepsilon}^{2}\right)$.

\section{Design I: ABBA and Its Dual}

An optimal two-sequence three period crossover design is the $\mathrm{ABB} / \mathrm{BAA}$ design (Laska \& Meisner, 1985; Hedayat \& Stufken, 2003). This design is sometimes referred to as the twosequence dual design because it allows an estimate of both treatment and carryover effect. The first design considered extends this by adding a fourth period and considering the sequence ABBA and its dual (see Table 1).

In sequence $\mathrm{ABBA}$, the contrast, $\mathrm{c}_{1}=\mathrm{y}_{11}$ $+2 \mathrm{y}_{12}+\mathrm{y}_{13}+\mathrm{y}_{14}$ has expectation $5 \mu+\left(\pi_{1}+2 \pi_{2}\right.$ $\left.+\pi_{3}+\pi_{4}\right)+\left(2 \tau_{\mathrm{A}}+3 \tau_{\mathrm{B}}\right)+\left(2 \lambda_{\mathrm{A}}+2 \lambda_{\mathrm{B}}\right) . \mathrm{In}$ sequence $\mathrm{BAAB}$, the contrast $\mathrm{c}_{2}=\mathrm{y}_{21}+2 \mathrm{y}_{22}+$ $\mathrm{y}_{32}-\mathrm{y}_{42}$ has expectation $5 \mu+\left(\pi_{1}+2 \pi_{2}+\pi_{3}+\right.$ $\left.\pi_{4}\right)+\left(2 \tau_{\mathrm{B}}+3 \tau_{\mathrm{A}}\right)+\left(2 \lambda_{\mathrm{A}}+2 \lambda_{\mathrm{B}}\right)$. The difference between contrast $\mathrm{c}_{1}$ and $\mathrm{c}_{2}$ forms an unbiased estimator of $\tau_{\mathrm{A}}-\tau_{\mathrm{B}}$. For the construction of an unbiased estimator of any carryover effect, $\lambda_{\mathrm{A}}$ -
$\lambda_{\mathrm{B}}$, consider $\mathrm{c}_{3}=\mathrm{y}_{11}-\mathrm{y}_{12}+\mathrm{y}_{13}-\mathrm{y}_{14}$ and $\mathrm{c}_{4}=\mathrm{y}_{21}$ $-y_{22}+y_{32}-y_{42}$. The difference between $c_{3}$ and $\mathrm{c}_{4}$ forms an unbiased estimate of $\lambda_{\mathrm{A}}-\lambda_{\mathrm{B}}$. Note that if the last period is omitted, this design reduces to an optimal two-sequence dual design. If the last two periods are eliminated, the design becomes the standard $\mathrm{AB} / \mathrm{BA}$ crossover design.

Design II: ABBA, AABB and Their Duals

This four-sequence, four-period design is a combination of two Balaam designs. The first two periods are the same as Balaam's design, while periods 3 and 4 are copies of Balaam's design reversed (see Table 2).

In sequence $\mathrm{ABBA}$, the contrast $\mathrm{c}_{1}=\mathrm{y}_{11}$ $+\mathrm{y}_{12}-\mathrm{y}_{13}+\mathrm{y}_{14}$ has expectation $2 \mu+\left(\pi_{1}+\pi_{2}-\right.$ $\left.\pi_{3}+\pi_{4}\right)+\left(2 \tau_{\mathrm{A}}+\lambda_{\mathrm{A}}\right)$. In AABB, the contrast $\mathrm{c}_{2}=$ $\mathrm{y}_{21}+\mathrm{y}_{22}-\mathrm{y}_{23}-\mathrm{y}_{24}$ has expectation $2 \mu+\left(\pi_{1}+2\right.$ $\left.\pi_{2}-\pi_{3}+\pi_{4}\right)+\left(2 \tau_{\mathrm{A}}+\lambda_{\mathrm{B}}\right)$. In sequence $\mathrm{BAAB}$, the contrast $c_{3}=y_{31}+y_{32}-y_{33}-y_{34}$ has expectation $2 \mu+\left(\pi_{1}+2 \pi_{2}-\pi_{3}+\pi_{4}\right)+\left(2 \tau_{\mathrm{B}}+\right.$ $\left.\lambda_{\mathrm{B}}\right)$, and in BBAA the contrast $\mathrm{c}_{4}=\mathrm{y}_{41}+\mathrm{y}_{22}-$ $\mathrm{y}_{43}-\mathrm{y}_{44}$ has expectation $2 \mu+\left(\pi_{1}+2 \pi_{2}-\pi_{3}+\right.$ $\left.\pi_{4}\right)+\left(2 \tau_{\mathrm{B}}+\lambda_{\mathrm{A}}\right)$. A combination of $\left(\mathrm{c}_{1}-\mathrm{c}_{4}\right)+\left(\mathrm{c}_{2}\right.$ $-c_{3}$ ) forms an unbiased estimate of treatment effect $\left(\tau_{\mathrm{A}}-\tau_{\mathrm{B}}\right)$.

In sequence $\mathrm{ABBA}$, the expected value of $\mathrm{E}\left[\mathrm{c}_{5}\right]=\mathrm{E}\left[\mathrm{y}_{11}+2 \mathrm{y}_{12}+\mathrm{y}_{13}+\mathrm{y}_{14}\right]=5 \mu+\left(\pi_{1}+\right.$ $\left.2 \pi_{2}+\pi_{3}+\pi_{4}\right)+\left(2 \tau_{\mathrm{A}}+3 \tau_{\mathrm{B}}\right)+\left(2 \lambda_{\mathrm{A}}+2 \lambda_{\mathrm{B}}\right)$; in $\mathrm{AABB}$, the expected value of $\mathrm{E}\left[\mathrm{c}_{6}\right]=\mathrm{E}\left[\mathrm{y}_{21}+\right.$ $\left.2 \mathrm{y}_{22}+\mathrm{y}_{23}-\mathrm{y}_{24}\right]=5 \mu+\left(\pi_{1}+2 \pi_{2}+\pi_{3}+\pi_{4}\right)+$ $\left(3 \tau_{\mathrm{A}}+2 \tau_{\mathrm{B}}\right)+\left(3 \lambda_{\mathrm{A}}+\lambda_{\mathrm{B}}\right)$; in BAAB, the expected value of $\mathrm{E}\left[\mathrm{c}_{7}\right]=\mathrm{E}\left[\mathrm{y}_{31}+2 \mathrm{y}_{32}+\mathrm{y}_{33}-\mathrm{y}_{34}\right]=5 \mu+$ $\left(\pi_{1}+2 \pi_{2}+\pi_{3}+\pi_{4}\right)+\left(2 \tau_{\mathrm{B}}+3 \tau_{\mathrm{A}}\right)+\left(2 \lambda_{\mathrm{A}}+2 \lambda_{\mathrm{B}}\right) ;$ and in BBAA, the expected value of $\mathrm{E}\left[\mathrm{c}_{8}\right]=$ $\mathrm{E}\left[\mathrm{y}_{41}+2 \mathrm{y}_{22}+\mathrm{y}_{43}-\mathrm{y}_{44}\right]=5 \mu+\left(\pi_{1}+2 \pi_{2}+\pi_{3}+\right.$ $\left.\pi_{4}\right)+\left(3 \tau_{\mathrm{B}}+2 \tau_{\mathrm{A}}\right)+\left(\lambda_{\mathrm{A}}+3 \lambda_{\mathrm{B}}\right)$. A combination of $\left(c_{1}-c_{3}\right)+\left(c_{2}-c_{4}\right)$ forms an unbiased estimate of treatment effect $\left(\lambda_{A}-\lambda_{B}\right)$.

Design III: ABBA, ABAA and Their Duals

In sequences $\mathrm{ABBA}, \mathrm{ABAA}, \mathrm{BAAB}$, and $\mathrm{BABB}$ the expected values of $\mathrm{E}\left[\mathrm{c}_{1}\right], \mathrm{E}\left[\mathrm{c}_{2}\right]$, $\mathrm{E}\left[\mathrm{c}_{3}\right]$ and $\mathrm{E}\left[\mathrm{c}_{4}\right]$ are: $\mathrm{E}\left[\mathrm{y}_{11}+\mathrm{y}_{12}+\mathrm{y}_{13}-\mathrm{y}_{14}\right]=2 \mu$ $+\left(\pi_{1}+\pi_{2}+\pi_{3}-\pi_{4}\right)+\left(2 \tau_{\mathrm{B}}+\lambda_{\mathrm{A}}\right), \mathrm{E}\left[\mathrm{y}_{21}+\mathrm{y}_{22}+\right.$ $\left.\mathrm{y}_{23}-\mathrm{y}_{24}\right]=2 \mu+\left(\pi_{1}+\pi_{2}+\pi_{3}-\pi_{4}\right)+\left(\tau_{\mathrm{A}}+\tau_{\mathrm{B}}-\right.$ $\left.\lambda_{\mathrm{A}}+\lambda_{\mathrm{B}}\right), \mathrm{E}\left[\mathrm{y}_{31}+\mathrm{y}_{32}+\mathrm{y}_{33}-\mathrm{y}_{34}\right]=2 \mu+\left(\pi_{1}+\pi_{2}\right.$ $\left.+\pi_{3}-\pi_{4}\right)+\left(2 \tau_{\mathrm{A}}+\lambda_{\mathrm{B}}\right)$ and $\mathrm{E}\left[\mathrm{y}_{41}+\mathrm{y}_{22}+\mathrm{y}_{43}-\right.$ $\left.\mathrm{y}_{44}\right]=2 \mu+\left(\pi_{1}+\pi_{2}+\pi_{3}-\pi_{4}\right)+\left(\tau_{\mathrm{A}}+\tau_{\mathrm{B}}+\lambda_{\mathrm{A}}-\right.$ 


\section{FOUR PERIOD CROSSOVER DESIGNS}

$\lambda_{B}$ ), respectively (see Table 3 ). The linear combination of $\left(\mathrm{c}_{1}-\mathrm{c}_{4}\right)-\left(\mathrm{c}_{2}-\mathrm{c}_{3}\right)$ forms an unbiased estimate of treatment effect $\left(\lambda_{A}-\lambda_{B}\right)$, while the linear combination of $2\left(c_{1}-c_{3}\right)+\left(c_{2}-\right.$ $\mathrm{c}_{4}$ ) forms an unbiased estimate of treatment effect $\left(\tau_{\mathrm{A}}-\tau_{\mathrm{B}}\right)$.

Design V: ABBA, ABAB and Their Duals

In sequence $\mathrm{ABBA}$, the expected value of $\mathrm{E}\left[\mathrm{c}_{1}\right]=\mathrm{E}\left[\mathrm{y}_{11}-\mathrm{y}_{12}+\mathrm{y}_{13}+\mathrm{y}_{14}\right]=2 \mu+\left(\pi_{1}-\pi_{2}\right.$ $\left.+\pi_{3}+\pi_{4}\right)+\left(2 \tau_{\mathrm{A}}+\lambda_{\mathrm{B}}-\lambda_{\mathrm{A}}\right)$; in ABAA, the expected value of $E\left[c_{2}\right]=E\left[y_{21}-y_{22}+y_{23}+y_{24}\right]$ $=2 \mu+\left(\pi_{1}-\pi_{2}+\pi_{3}+\pi_{4}\right)+\left(2 \tau_{\mathrm{A}}+\lambda_{\mathrm{B}}\right)$. For sequence $\mathrm{BAAB}$, the expected value of $\mathrm{E}\left[\mathrm{c}_{3}\right]=$ $\mathrm{E}\left[\mathrm{y}_{31}-\mathrm{y}_{32}+\mathrm{y}_{33}+\mathrm{y}_{34}\right]=2 \mu+\left(\pi_{1}-\pi_{2}+\pi_{3}+\pi_{4}\right)$ $+\left(2 \tau_{\mathrm{B}}+\lambda_{\mathrm{A}}-\lambda_{\mathrm{B}}\right)$, and in BABB, the expected value of $\mathrm{E}\left[\mathrm{c}_{4}\right]=\mathrm{E}\left[\mathrm{y}_{41}-\mathrm{y}_{22}+\mathrm{y}_{43}+\mathrm{y}_{44}\right]=2 \mu+$ $\left(\pi_{1}-\pi_{2}+\pi_{3}+\pi_{4}\right)+\left(2 \tau_{\mathrm{B}}+\lambda_{\mathrm{A}}\right)$ (see Table 4). The linear combination of $\left(\mathrm{c}_{1}-\mathrm{c}_{2}\right)-\left(\mathrm{c}_{3}-\mathrm{c}_{4}\right)$ forms an unbiased estimate of treatment effect $\left(\lambda_{A}-\lambda_{B}\right)$, while the linear combination of $2\left(c_{2}-\right.$ $\left.c_{4}\right)+\left(c_{1}-c_{3}\right)$ forms an unbiased estimate of treatment effect $\left(\tau_{\mathrm{A}}-\tau_{\mathrm{B}}\right)$.

\section{Discussion}

This article considered four two-treatment in four-period crossover designs. For a simple one period carryover effect model, the four designs presented are ideal because the design efficiencies are optimal. The designs with the highest efficiency $(100 \%)$ are the AABB, ABBA and their duals (Table 2). The other designs considered weigh in at 91\% (Hedayat \& Stufken, 2003). All designs are robust, simple and easily implemented.

Higher-order crossover designs are useful because they allow a treatment effect to be estimated even in the presence of a carry-over effect, they provide estimates of intra-subject variability and they draw inference on the carryover effect (Chow \& Lu, 1992). Are these fourperiod crossover designs useful? Perhaps. The true question relates to the application of these designs.

\section{Acknowledgements}

The Journal of Modern Applied Statistical Methods has been kind to me over the years. I wish to thank the editor, associated editors and production staff for their helpful and constructive comments. Manuscript reviewers perform an invaluable service to The Journal of Modern Applied Statistical Methods. Their thoughtful and careful appraisal of manuscripts and their suggestions for improvement are aimed at making a manuscript better and suitable for the readership of this journal. For those insights, please accept my sincere thanks.

Table 1: Expected Values for Design I

\begin{tabular}{|c|c|c|c|c|}
\hline Sequence & $\begin{array}{c}\text { Period 1 } \\
(\mathrm{k}=1)\end{array}$ & $\begin{array}{c}\text { Period 2 } \\
(\mathrm{k}=2)\end{array}$ & $\begin{array}{c}\text { Period 3 } \\
(\mathrm{k}=3)\end{array}$ & $\begin{array}{c}\text { Period 4 } \\
(\mathrm{k}=4)\end{array}$ \\
\hline $\begin{array}{c}\mathrm{ABBA} \\
(\mathrm{i}=1)\end{array}$ & $\mathrm{y}_{11}=\mu+\pi_{1}+\tau_{\mathrm{A}}$ & $\mathrm{y}_{21}=\mu+\pi_{2}+\tau_{\mathrm{B}}+\lambda_{\mathrm{A}}$ & $\mathrm{y}_{31}=\mu+\pi_{3}+\tau_{\mathrm{B}}+\lambda_{\mathrm{B}}$ & $\mathrm{y}_{14}=\mu+\pi_{4}+\tau_{\mathrm{A}}+\lambda_{\mathrm{B}}$ \\
\hline $\begin{array}{c}\mathrm{BAAB} \\
(\mathrm{i}=2)\end{array}$ & $\mathrm{y}_{21}=\mu+\pi_{1}+\tau_{\mathrm{B}}$ & $\mathrm{y}_{22}=\mu+\pi_{2}+\tau_{\mathrm{A}}+\lambda_{\mathrm{B}}$ & $\mathrm{Y}_{23}=\mu+\pi_{3}+\tau_{\mathrm{A}}+\lambda_{\mathrm{A}}$ & $\mathrm{Y}_{24}=\mu+\pi_{4}+\tau_{\mathrm{B}}+\lambda_{\mathrm{A}}$ \\
\hline
\end{tabular}

Expected values for sequences:

- $\quad \mathrm{ABBA}, \mathrm{E}\left[\mathrm{c}_{1}\right]=\mathrm{E}\left[\mathrm{y}_{11}+2 \mathrm{y}_{12}+\mathrm{y}_{13}+\mathrm{y}_{14}\right]=5 \mu+\left(\pi_{1}+2 \pi_{2}+\pi_{3}+\pi_{4}\right)+\left(2 \tau_{\mathrm{A}}+3 \tau_{\mathrm{B}}\right)+\left(2 \lambda_{\mathrm{A}}+2 \lambda_{\mathrm{B}}\right)$

- $\mathrm{BAAB}, \mathrm{E}\left[\mathrm{c}_{2}\right]=\mathrm{E}\left[\mathrm{y}_{21}+2 \mathrm{y}_{22}+\mathrm{y}_{32}-\mathrm{y}_{42}\right]=5 \mu+\left(\pi_{1}+2 \pi_{2}+\pi_{3}+\pi_{4}\right)+\left(2 \tau_{\mathrm{B}}+3 \tau_{\mathrm{A}}\right)+\left(2 \lambda_{\mathrm{A}}+2 \lambda_{\mathrm{B}}\right)$

- ABBA, $\mathrm{E}\left[\mathrm{c}_{3}\right]=\mathrm{E}\left(\mathrm{y}_{11}-\mathrm{y}_{12}+\mathrm{y}_{13}-\mathrm{y}_{14}\right]=\left(\pi_{1}-\pi_{2}+\pi_{3}-\pi_{4}\right)-\lambda_{\mathrm{A}}$

- $\mathrm{BAAB}, \mathrm{E}\left[\mathrm{c}_{4}\right]=\mathrm{E}\left(\mathrm{y}_{21}-\mathrm{y}_{22}+\mathrm{y}_{32}-\mathrm{y}_{42}\right]=\left(\pi_{1}-\pi_{2}+\pi_{3}-\pi_{4}\right)-\lambda_{\mathrm{B}}$ 
JAMES F. REED III

Table 2: Expected Values for Design II: ABBA, AABB and Their Duals

\begin{tabular}{|c|c|c|c|c|}
\hline Sequence & $\begin{array}{c}\text { Period 1 } \\
(\mathrm{k}=1)\end{array}$ & $\begin{array}{c}\text { Period } 2 \\
(\mathrm{k}=2)\end{array}$ & $\begin{array}{c}\text { Period 3 } \\
(\mathrm{k}=3)\end{array}$ & $\begin{array}{c}\text { Period } 4 \\
(\mathrm{k}=4)\end{array}$ \\
\hline $\begin{array}{c}\mathrm{ABBA} \\
(\mathrm{i}=1)\end{array}$ & $\mu+\pi_{1}+\tau_{\mathrm{A}}$ & $\mu+\pi_{2}+\tau_{\mathrm{B}}+\lambda_{\mathrm{A}}$ & $\mu+\pi_{3}+\tau_{\mathrm{B}}+\lambda_{\mathrm{B}}$ & $\mu+\pi_{4}+\tau_{\mathrm{A}}+\lambda_{\mathrm{B}}$ \\
\hline $\begin{array}{c}\mathrm{AABB} \\
(\mathrm{i}=2)\end{array}$ & $\mu+\pi_{1}+\tau_{\mathrm{A}}$ & $\mu+\pi_{2}+\tau_{\mathrm{A}}+\lambda_{\mathrm{A}}$ & $\mu+\pi_{3}+\tau_{\mathrm{B}}+\lambda_{\mathrm{A}}$ & $\mu+\pi_{4}+\tau_{\mathrm{B}}+\lambda_{\mathrm{B}}$ \\
\hline $\begin{array}{c}\mathrm{BAAB} \\
(\mathrm{i}=3)\end{array}$ & $\mu+\pi_{1}+\tau_{\mathrm{B}}$ & $\mu+\pi_{2}+\tau_{\mathrm{A}}+\lambda_{\mathrm{B}}$ & $\mu+\pi_{3}+\tau_{\mathrm{A}}+\lambda_{\mathrm{A}}$ & $\mu+\pi_{4}+\tau_{\mathrm{B}}+\lambda_{\mathrm{A}}$ \\
\hline $\begin{array}{c}\mathrm{BBAA} \\
(\mathrm{i}=4)\end{array}$ & $\mu+\pi_{1}+\tau_{\mathrm{B}}$ & $\mu+\pi_{2}+\tau_{\mathrm{B}}+\lambda_{\mathrm{B}}$ & $\mu+\pi_{3}+\tau_{\mathrm{A}}+\lambda_{\mathrm{B}}$ & $\mu+\pi_{4}+\tau_{\mathrm{A}}+\lambda_{\mathrm{A}}$ \\
\hline
\end{tabular}

Expected values for sequences:

- $\mathrm{ABBA}, \mathrm{E}\left[\mathrm{c}_{1}\right]=\mathrm{E}\left[\mathrm{y}_{11}+\mathrm{y}_{12}-\mathrm{y}_{13}+\mathrm{y}_{14}\right]=2 \mu+\left(\pi_{1}+\pi_{2}-\pi_{3}+\pi_{4}\right)+\left(2 \tau_{\mathrm{A}}+\lambda_{\mathrm{A}}\right)$

- $\mathrm{AABB}, \mathrm{E}\left[\mathrm{c}_{2}\right]=\mathrm{E}\left[\mathrm{y}_{21}+\mathrm{y}_{22}-\mathrm{y}_{23}+\mathrm{y}_{24}\right]=2 \mu+\left(\pi_{1}+\pi_{2}-\pi_{3}+\pi_{4}\right)+\left(2 \tau_{\mathrm{A}}+\lambda_{\mathrm{B}}\right)$

- $\mathrm{BAAB}, \mathrm{E}\left[\mathrm{c}_{3}\right]=\mathrm{E}\left[\mathrm{y}_{31}+\mathrm{y}_{32}-\mathrm{y}_{33}+\mathrm{y}_{34}\right]=2 \mu+\left(\pi_{1}+\pi_{2}-\pi_{3}+\pi_{4}\right)+\left(2 \tau_{\mathrm{B}}+\lambda_{\mathrm{B}}\right)$

- $\mathrm{BBAA}, \mathrm{E}\left[\mathrm{c}_{4}\right]=\mathrm{E}\left[\mathrm{y}_{41}+\mathrm{y}_{42}-\mathrm{y}_{43}+\mathrm{y}_{44}\right]=2 \mu+\left(\pi_{1}+\pi_{2}-\pi_{3}+\pi_{4}\right)+\left(2 \tau_{\mathrm{B}}+\lambda_{\mathrm{A}}\right)$

- $\mathrm{ABBA}, \mathrm{E}\left[\mathrm{c}_{5}\right]=\mathrm{E}\left[\mathrm{y}_{11}+2 \mathrm{y}_{12}+\mathrm{y}_{13}+\mathrm{y}_{14}\right]=5 \mu+\left(\pi_{1}+2 \pi_{2}+\pi_{3}+\pi_{4}\right)+\left(2 \tau_{\mathrm{A}}+3 \tau_{\mathrm{B}}\right)+\left(2 \lambda_{\mathrm{A}}+2 \lambda_{\mathrm{B}}\right)$

- $\mathrm{AABB}, \mathrm{E}\left[\mathrm{c}_{6}\right]=\mathrm{E}\left[\mathrm{y}_{21}+2 \mathrm{y}_{22}+\mathrm{y}_{23}-\mathrm{y}_{24}\right]=5 \mu+\left(\pi_{1}+2 \pi_{2}+\pi_{3}+\pi_{4}\right)+\left(3 \tau_{\mathrm{A}}+2 \tau_{\mathrm{B}}\right)+\left(3 \lambda_{\mathrm{A}}+\lambda_{\mathrm{B}}\right)$

- $\mathrm{BAAB}, \mathrm{E}\left[\mathrm{c}_{7}\right]=\mathrm{E}\left[\mathrm{y}_{31}+2 \mathrm{y}_{32}+\mathrm{y}_{33}-\mathrm{y}_{34}\right]=5 \mu+\left(\pi_{1}+2 \pi_{2}+\pi_{3}+\pi_{4}\right)+\left(2 \tau_{\mathrm{B}}+3 \tau_{\mathrm{A}}\right)+\left(2 \lambda_{\mathrm{A}}+2 \lambda_{\mathrm{B}}\right)$

- $\quad \mathrm{BBAA}, \mathrm{E}\left[\mathrm{c}_{8}\right]=\mathrm{E}\left[\mathrm{y}_{41}+2 \mathrm{y}_{42}+\mathrm{y}_{43}-\mathrm{y}_{44}\right]=5 \mu+\left(\pi_{1}+2 \pi_{2}+\pi_{3}+\pi_{4}\right)+\left(3 \tau_{\mathrm{B}}+2 \tau_{\mathrm{A}}\right)+\left(\lambda_{\mathrm{A}}+3 \lambda_{\mathrm{B}}\right)$

Table 3: Expected Values for Design III: ABBA, ABAA and Their Duals

\begin{tabular}{|c|c|c|c|c|}
\hline Sequence & $\begin{array}{c}\text { Period 1 } \\
(\mathrm{k}=1)\end{array}$ & $\begin{array}{c}\text { Period 2 } \\
(\mathrm{k}=2)\end{array}$ & $\begin{array}{c}\text { Period 3 } \\
(\mathrm{k}=3)\end{array}$ & $\begin{array}{c}\text { Period 4 } \\
(\mathrm{k}=4)\end{array}$ \\
\hline $\begin{array}{c}\mathrm{ABBA} \\
(\mathrm{i}=1)\end{array}$ & $\mu+\pi_{1}+\tau_{\mathrm{A}}$ & $\mu+\pi_{2}+\tau_{\mathrm{B}}+\lambda_{\mathrm{A}}$ & $\mu+\pi_{3}+\tau_{\mathrm{B}}+\lambda_{\mathrm{B}}$ & $\mu+\pi_{4}+\tau_{\mathrm{A}}+\lambda_{\mathrm{B}}$ \\
\hline $\begin{array}{c}\mathrm{ABAA} \\
(\mathrm{i}=2)\end{array}$ & $\mu+\pi_{1}+\tau_{\mathrm{A}}$ & $\mu+\pi_{2}+\tau_{\mathrm{B}}+\lambda_{\mathrm{A}}$ & $\mu+\pi_{3}+\tau_{\mathrm{A}}+\lambda_{\mathrm{B}}$ & $\mu+\pi_{4}+\tau_{\mathrm{A}}+\lambda_{\mathrm{A}}$ \\
\hline $\begin{array}{c}\mathrm{BAAB} \\
(\mathrm{i}=3)\end{array}$ & $\mu+\pi_{1}+\tau_{\mathrm{B}}$ & $\mu+\pi_{2}+\tau_{\mathrm{A}}+\lambda_{\mathrm{B}}$ & $\mu+\pi_{3}+\tau_{\mathrm{A}}+\lambda_{\mathrm{A}}$ & $\mu+\pi_{4}+\tau_{\mathrm{B}}+\lambda_{\mathrm{A}}$ \\
\hline $\begin{array}{c}\mathrm{BABB} \\
(\mathrm{i}=4)\end{array}$ & $\mu+\pi_{1}+\tau_{\mathrm{B}}$ & $\mu+\pi_{2}+\tau_{\mathrm{A}}+\lambda_{\mathrm{B}}$ & $\mu+\pi_{3}+\tau_{\mathrm{B}}+\lambda_{\mathrm{A}}$ & $\mu+\pi_{4}+\tau_{\mathrm{B}}+\lambda_{\mathrm{B}}$ \\
\hline
\end{tabular}

Expected values for sequences:

- $\mathrm{ABBA}, \mathrm{E}\left[\mathrm{c}_{1}\right]=\mathrm{E}\left[\mathrm{y}_{11}+\mathrm{y}_{12}+\mathrm{y}_{13}-\mathrm{y}_{14}\right]=2 \mu+\left(\pi_{1}+\pi_{2}+\pi_{3}-\pi_{4}\right)+\left(2 \tau_{\mathrm{B}}+\lambda_{\mathrm{A}}\right)$

- $\mathrm{ABAA}, \mathrm{E}\left[\mathrm{c}_{2}\right]=\mathrm{E}\left[\mathrm{y}_{21}+\mathrm{y}_{22}+\mathrm{y}_{23}-\mathrm{y}_{24}\right]=2 \mu+\left(\pi_{1}+\pi_{2}+\pi_{3}-\pi_{4}\right)+\left(\tau_{\mathrm{A}}+\tau_{\mathrm{B}}-\lambda_{\mathrm{A}}+\lambda_{\mathrm{B}}\right)$

- $\mathrm{BAAB}, \mathrm{E}\left[\mathrm{c}_{3}\right]=\mathrm{E}\left[\mathrm{y}_{31}+\mathrm{y}_{32}+\mathrm{y}_{33}-\mathrm{y}_{34}\right]=2 \mu+\left(\pi_{1}+\pi_{2}+\pi_{3}-\pi_{4}\right)+\left(2 \tau_{\mathrm{A}}+\lambda_{\mathrm{B}}\right)$

- $\mathrm{BABB}, \mathrm{E}\left[\mathrm{c}_{4}\right]=\mathrm{E}\left[\mathrm{y}_{41}+\mathrm{y}_{42}+\mathrm{y}_{43}-\mathrm{y}_{44}\right]=2 \mu+\left(\pi_{1}+\pi_{2}+\pi_{3}-\pi_{4}\right)+\left(\tau_{\mathrm{A}}+\tau_{\mathrm{B}}+\lambda_{\mathrm{A}}-\lambda_{\mathrm{B}}\right)$ 


\section{FOUR PERIOD CROSSOVER DESIGNS}

Table 4: Expected Values for Design IV: ABBA, ABAB and Their Duals

\begin{tabular}{|c|c|c|c|c|}
\hline Sequence & $\begin{array}{c}\text { Period 1 } \\
(\mathrm{k}=1)\end{array}$ & $\begin{array}{c}\text { Period 2 } \\
(\mathrm{k}=2)\end{array}$ & $\begin{array}{c}\text { Period 3 } \\
(\mathrm{k}=3)\end{array}$ & $\begin{array}{c}\text { Period 4 } \\
(\mathrm{k}=4)\end{array}$ \\
\hline $\begin{array}{c}\mathrm{ABBA} \\
(\mathrm{i}=1)\end{array}$ & $\mu+\pi_{1}+\tau_{\mathrm{A}}$ & $\mu+\pi_{2}+\tau_{\mathrm{B}}+\lambda_{\mathrm{A}}$ & $\mu+\pi_{3}+\tau_{\mathrm{B}}+\lambda_{\mathrm{B}}$ & $\mu+\pi_{4}+\tau_{\mathrm{A}}+\lambda_{\mathrm{B}}$ \\
\hline $\begin{array}{c}\mathrm{ABAB} \\
(\mathrm{i}=2)\end{array}$ & $\mu+\pi_{1}+\tau_{\mathrm{A}}$ & $\mu+\pi_{2}+\tau_{\mathrm{B}}+\lambda_{\mathrm{A}}$ & $\mu+\pi_{3}+\tau_{\mathrm{A}}+\lambda_{\mathrm{B}}$ & $\mu+\pi_{4}+\tau_{\mathrm{B}}+\lambda_{\mathrm{A}}$ \\
\hline $\begin{array}{c}\mathrm{BAAB} \\
(\mathrm{i}=3)\end{array}$ & $\mu+\pi_{1}+\tau_{\mathrm{B}}$ & $\mu+\pi_{2}+\tau_{\mathrm{A}}+\lambda_{\mathrm{B}}$ & $\mu+\pi_{3}+\tau_{\mathrm{A}}+\lambda_{\mathrm{A}}$ & $\mu+\pi_{4}+\tau_{\mathrm{B}}+\lambda_{\mathrm{A}}$ \\
\hline $\begin{array}{c}\mathrm{BABA} \\
(\mathrm{i}=4)\end{array}$ & $\mu+\pi_{1}+\tau_{\mathrm{B}}$ & $\mu+\pi_{2}+\tau_{\mathrm{A}}+\lambda_{\mathrm{B}}$ & $\mu+\pi_{3}+\tau_{\mathrm{B}}+\lambda_{\mathrm{A}}$ & $\mu+\pi_{4}+\tau_{\mathrm{A}}+\lambda_{\mathrm{B}}$ \\
\hline
\end{tabular}

Expected values for sequences:

- $\mathrm{ABBA}, \mathrm{E}\left[\mathrm{c}_{1}\right]=\mathrm{E}\left[\mathrm{y}_{11}-\mathrm{y}_{12}+\mathrm{y}_{13}+\mathrm{y}_{14}\right]=2 \mu+\left(\pi_{1}-\pi_{2}+\pi_{3}+\pi_{4}\right)+\left(2 \tau_{\mathrm{A}}+\lambda_{\mathrm{B}}-\lambda_{\mathrm{A}}\right)$

- $\mathrm{ABAA}, \mathrm{E}\left[\mathrm{c}_{2}\right]=\mathrm{E}\left[\mathrm{y}_{21}-\mathrm{y}_{22}+\mathrm{y}_{23}+\mathrm{y}_{24}\right]=2 \mu+\left(\pi_{1}-\pi_{2}+\pi_{3}+\pi_{4}\right)+\left(2 \tau_{\mathrm{A}}+\lambda_{\mathrm{B}}\right)$

- $\mathrm{BAAB}, \mathrm{E}\left[\mathrm{c}_{3}\right]=\mathrm{E}\left[\mathrm{y}_{31}-\mathrm{y}_{32}+\mathrm{y}_{33}+\mathrm{y}_{34}\right]=2 \mu+\left(\pi_{1}-\pi_{2}+\pi_{3}+\pi_{4}\right)+\left(2 \tau_{\mathrm{B}}+\lambda_{\mathrm{A}}-\lambda_{\mathrm{B}}\right)$

- $\mathrm{BABB}, \mathrm{E}\left[\mathrm{c}_{4}\right]=\mathrm{E}\left[\mathrm{y}_{41}-\mathrm{y}_{42}+\mathrm{y}_{43}+\mathrm{y}_{44}\right]=2 \mu+\left(\pi_{1}-\pi_{2}+\pi_{3}+\pi_{4}\right)+\left(2 \tau_{\mathrm{B}}+\lambda_{\mathrm{A}}\right)$

\section{References}

Balaam, L. N. (1968). A two-period design with $\mathrm{t}^{2}$ experimental units. Biometrics, 24, 61-73.

Chow, S. C., \& Liu, J. P. (1992). On assessment of bioequivalence under a higherorder crossover design. Journal of Biopharmaceutical Statistics, 2, 239-256.

Hedayat, A. S., \& Stufken, J. (2003). Optimal and efficient crossover designs under different assumptions about the carryover effects. Journal of Biopharmaceutical Statistics, 13, 519-528.

Laska, E., Meisner, M., \& Kushner, H. B. (1983). Optimal crossover designs in the presence of carryover effects. Biometrics, 39, 1087-1091.
Matthews, J. N. S. (1994). Multi-period crossover trials. Statistical Methods in Medical Research, 3, 383-405.

Reed III, J. F. (2011). Extension of Grizzle's Classic Crossover Design. Journal of Modern Applied Statistical Methods, 10(1), 322328.

Reed III, J. F. (2011). Higher Order C (t, p, s) Crossover Designs. Journal of Modern Applied Statistical Methods, 10(2), 686-691. 


\section{Ratio Type Estimator of Ratio of Two Population Means in Stratified Random Sampling}

\author{
Rajesh Tailor \\ Vikram University, \\ Ujjain, M.P., India
}

\author{
Sunil Chouhan \\ ShriVaishnav Institute of Management, \\ Indore, M.P., India
}

A ratio estimator is proposed for the ratio of two population means using auxiliary information in stratified random sampling. Bias and mean squared error expressions are obtained under large sample approximation, and the proposed estimator is compared both theoretically and empirically with the conventional estimator of ratio for two population means in stratified random sampling.

Key words: Ratio of two population means, auxiliary information, stratified random sampling, bias, mean squared error.

\section{Introduction}

The problem of estimating population means is considered in a wide range of applications in many areas of human activities. A ratio of two population means is also applicable in many situations, such as per hectare production of crops, per capita income and per kilometer population density. Many researchers have studied the estimation of the ratio of two population means in simple random sampling (Singh, 1965; Rao\&Pareira, 1968; Shah \& Shah, 1978: Ray \& Singh, 1985; Upadhyaya\& Singh, 1985; Upadhyaya, et al., 1985; Singh \& Rani, 2005, 2006; Sindhu, et al., 2009). Other sampling designs have not attracted much attention; in many situations, it has been observed that stratified random sampling provides efficient estimators compared to those of simple random sampling. Thus, this article estimates the ratio of two population means under stratified random sampling.

Rajesh Tailor is a Reader in the School of Studies in Statistics. His research interests are in the field of sampling techniques. Email him at: tailorraj@gmail.com. Sunil Chouhan is an Assistant Professor at the Vaishnav Institute of Management, Indore, M. P. India. Email him at: chouhansd@gmail.com.
Consider a finite population $U=U_{1}, U_{2}, \ldots, U_{N}$ of size $\mathrm{N}$. This population $U$ is divided into $L$ strata each of size $N_{h}$ and sample of size $n_{h}$ is drawn from each stratum such that $n=\sum_{h=1}^{L} n_{h}(h=1,2, \ldots \ldots L)$. If $y_{0}$ and $y_{1}$ are the study variates, $x$ is an auxiliary variate, and $y_{0 h i}, y_{1 h i}$ and $x_{h i}$ $\left(h=1,2, \ldots . L ; i=1,2, \ldots ., N_{h}\right) \quad$ are the observations taken from the $i^{\text {th }}$ unit of the $h^{\text {th }}$ stratum on study variates $y_{0}, y_{1}$ and auxiliary variate $x$ respectively, then the following are defined:

$h^{\text {th }}$ stratum mean for study variate $y_{0}$ :

$$
\bar{Y}_{0 h}=\frac{1}{N_{h}} \sum_{i=1}^{N_{h}} y_{0 h i}
$$

$h^{\text {th }}$ stratum mean for study variate $y_{1}$ :

$$
\bar{Y}_{1 h}=\frac{1}{N_{h}} \sum_{i=1}^{N_{h}} y_{1 h i}
$$


$h^{\text {th }}$ stratum mean for auxiliary variate $x$ :

$$
\bar{X}_{h}=\frac{1}{N_{h}} \sum_{i=1}^{N_{h}} y_{1 h i}
$$

Population mean of study variate $y_{0}$ :

$$
\bar{Y}_{0}=\frac{1}{N} \sum_{h=1}^{L} \sum_{i=1}^{N_{h}} y_{0 h i}=\frac{1}{N} \sum_{h=1}^{L} N_{h} \bar{Y}_{0 h}=\sum_{h=1}^{L} W_{h} \bar{Y}_{0 h}
$$

Population mean of study variate $y_{1}$ :

$$
\bar{Y}_{1}=\frac{1}{N} \sum_{h=1}^{L} \sum_{i=1}^{N_{h}} y_{1 h i}=\frac{1}{N} \sum_{h=1}^{L} N_{h} \bar{Y}_{1 h}=\sum_{h=1}^{L} W_{h} \bar{Y}_{1 h}
$$

Population mean of auxiliary variate $x$ :

$$
\bar{X}=\frac{1}{N} \sum_{h=1}^{L} \sum_{i=1}^{N_{h}} x_{h i}=\frac{1}{N} \sum_{h=1}^{L} W_{h} \bar{X}_{h}
$$

Sample mean of study variate $y_{0}$ for $h^{\text {th }}$ stratum:

$$
\bar{y}_{0 h}=\frac{1}{n_{h}} \sum_{i=1}^{n_{h}} \bar{y}_{0 h i}
$$

Sample mean of study variate $y_{1}$ for $h^{\text {th }}$ stratum:

$$
\bar{y}_{1 h}=\frac{1}{n_{h}} \sum_{i=1}^{n_{h}} \bar{y}_{1 h i}
$$

Sample mean of auxiliary variate $x$ for $h^{\text {th }}$ stratum:

$$
\bar{x}_{h}=\frac{1}{n_{h}} \sum_{i=1}^{n_{h}} \bar{x}_{h i}
$$

Stratum weight of $h^{\text {th }}$ stratum:

$$
W_{h}=\frac{N_{h}}{N}
$$

Ratio of two population means:

$$
R=\frac{\bar{Y}_{0}}{\bar{Y}_{1}}
$$

The usual unbiased estimators of population means $\bar{Y}_{0}, \bar{Y}_{1}$ and $\bar{X}$ are

$$
\bar{y}_{0 s t}=\sum_{h=1}^{L} W_{h} \bar{y}_{0 h}
$$

$$
\begin{gathered}
\bar{y}_{1 s t}=\sum_{h=1}^{L} W_{h} \bar{y}_{1 h} \\
\bar{x}_{s t}=\sum_{h=1}^{L} W_{h} \bar{x}_{h}
\end{gathered}
$$

and the estimator of the ratio of two population means $R$ in stratified random sampling is:

$$
\widehat{R}_{s t}=\left(\frac{\bar{y}_{0 s t}}{\bar{y}_{1 s t}}\right) \text {. }
$$

Suggested Ratio Estimator

When the population mean $\bar{X}$ of auxiliary variate $x$ is known, Singh (1965) suggested an estimator for $R$ in simple random sampling as

$$
T_{1}=\left(\frac{\bar{y}_{0}}{\bar{y}_{1}}\right)\left(\frac{\bar{X}}{\bar{x}}\right)=R\left(\frac{\bar{X}}{\bar{x}}\right) .
$$

In stratified random sampling $T_{1}$ is defined as

$$
T_{1 s t}=\left(\frac{\bar{y}_{0 s t}}{\bar{y}_{1 s t}}\right)\left(\frac{\bar{X}}{\bar{x}_{s t}}\right)
$$

In order to derive the bias and mean squared error expressions of estimators $\widehat{R}_{s t}$ and $T_{1 s t}$ it is assumed that $\bar{y}_{0 h}=\bar{Y}_{0 h}\left(1+e_{0 h}\right)$, $\bar{y}_{1 h}=\bar{Y}_{1 h}\left(1+e_{1 h}\right)$, and $\bar{x}_{h}=\bar{X}_{h}\left(1+e_{2 h}\right)$, such that, $E\left(e_{0 h}\right)=E\left(e_{1 h}\right)=E\left(e_{2 h}\right)=0$. 


\section{RAJESH TAILOR \& SUNIL CHOUHAN}

To the first degree of approximation the bias and mean squared error are

$$
B\left(\hat{R}_{s t}\right)=R \sum_{h=1}^{L} W_{h}^{2} \gamma_{h}\left(\frac{S_{1 h}^{2}}{\bar{Y}_{1}^{2}}-\frac{S_{01 h}}{\bar{Y}_{0} \bar{Y}_{1}}\right)
$$

$$
\begin{aligned}
& B\left(T_{1 s t}\right)= \\
& R \sum_{h=1}^{L} W_{h}^{2} \gamma_{h}\left(\frac{S_{1 h}^{2}}{\bar{Y}_{1}^{2}}+\frac{S_{x h}^{2}}{\bar{X}^{2}}-\frac{S_{01 h}}{\bar{Y}_{0} \bar{Y}_{1}}-\frac{S_{0 x h}}{\bar{Y}_{0} \bar{X}}+\frac{S_{1 x h}}{\bar{Y} \bar{X}}\right)
\end{aligned}
$$

$$
\operatorname{MSE}\left(\hat{R}_{s t}\right)=R^{2} \sum_{h=1}^{L} W_{h}^{2} \gamma_{h}\left(\frac{S_{0 h}^{2}}{\bar{Y}_{0}^{2}}+\frac{S_{1 h}^{2}}{\bar{Y}_{1}^{2}}-2 \frac{S_{01 h}}{\bar{Y}_{0} \bar{Y}_{1}}\right)
$$

$$
\operatorname{MSE}\left(T_{1 s t}\right)=R^{2} \sum_{h=1}^{L} W_{h}^{2} \gamma_{h}\left\{\begin{array}{l}
\frac{S_{0 h}^{2}}{\bar{Y}_{0}^{2}}+\frac{S_{1 h}^{2}}{\bar{Y}_{1}^{2}}+\frac{S_{x h}^{2}}{\bar{X}^{2}} \\
-2\left(\frac{S_{01 h}}{\bar{Y}_{0} \bar{Y}_{1}}+\frac{S_{0 x h}}{\bar{Y}_{0} \bar{X}}-\frac{S_{1 x h}}{\bar{Y}_{1} \bar{X}}\right)
\end{array}\right\}
$$

where

$$
\begin{gathered}
S_{0 h}^{2}=\frac{1}{N_{h}-1} \sum_{i=1}^{N_{h}}\left(y_{o h i}-\bar{y}_{o h}\right)^{2}, \\
S_{1 h}^{2}=\frac{1}{N_{h}-1} \sum_{i=1}^{N_{h}}\left(y_{1 h i}-\bar{y}_{1 h}\right)^{2}, \\
S_{x h}^{2}=\frac{1}{N_{h}-1} \sum_{i=1}^{N_{h}}\left(x_{h i}-\bar{x}_{h}\right)^{2}, \\
S_{01 h}=\frac{1}{N_{h}-1} \sum_{i=1}^{N_{h}}\left(y_{0 h i}-\bar{y}_{0 h}\right)\left(y_{1 h i}-\bar{y}_{1 h}\right), \\
S_{0 x h}=\frac{1}{N_{h}-1} \sum_{i=1}^{N_{h}}\left(y_{0 h i}-\bar{y}_{0 h}\right)\left(x_{h i}-\bar{x}_{h}\right)
\end{gathered}
$$

$$
S_{1 x h}=\frac{1}{N_{h}-1} \sum_{i=1}^{N_{h}}\left(y_{1 h i}-\bar{y}_{1 h}\right)\left(x_{h i}-\bar{x}_{h}\right) .
$$

Efficiency Comparison of Estimators

A comparison of equations (2.3) and (2.4) shows that the suggested estimator $T_{1 s t}$ will be more efficient than conventional estimator $\hat{R}_{s t}$ if

$$
\operatorname{MSE}\left(T_{1 s t}\right)-\operatorname{MSE}\left(\hat{R}_{s t}\right)<0
$$

that is, if

$$
\sum_{h=1}^{L} W_{h}^{2} \gamma_{h}\left\{\frac{S_{x h}^{2}}{\bar{X}^{2}}-2\left(\frac{S_{0 x h}}{\bar{Y}_{0} \bar{X}}-\frac{S_{1 x h}}{\bar{Y}_{1} \bar{X}}\right)\right\}<0
$$

If condition (3.1) is satisfied, the suggested estimator would be more efficient than the conventional estimator.

Empirical Study

Two natural datasets were used to compare the proposed estimator numerically (See Tables 1 and 2).

\section{Conclusion}

The theoretical comparison provides the condition under which the suggested estimator is more efficient than the conventional estimator $\hat{R}_{s t}$. Table 3 shows that there is a considerable gain in efficiency by using the proposed ratio estimator $T_{1 S T}$ in comparison to the conventional estimator $\widehat{R}_{s t}$. Thus, if information regarding population mean $\bar{X}$ is available, the suggested estimator $T_{1 S T}$ is recommended for use in practice.

and 
Table 1: Population 1 ( $y_{0}$ : Number of Workers, $y_{1}$ : Fixed Capital, $x$ : Output)

\begin{tabular}{|c|c|c|c|c|}
\hline & $n_{1}=2$ & $n_{2}=2$ & $N_{1}=5$ & $N_{2}=5$ \\
\cline { 2 - 5 } & $\bar{Y}_{01}=51.80$ & $\bar{Y}_{02}=60.60$ & $\bar{Y}_{11}=214.4$ & $\bar{Y}_{12}=333.8$ \\
\cline { 2 - 5 } $\mathrm{N}=10$ & $\bar{X}_{1}=1925.8$ & $\bar{X}_{2}=315.6$ & $S_{y_{01}}=0.75$ & $S_{y_{02}}=4.84$ \\
\cline { 2 - 5 } $\mathrm{n}=4$ & $S_{y_{11}}=74.87$ & $S_{y_{12}}=66.35$ & $S_{x 1}=615.92$ & $S_{x_{2}}=340.38$ \\
\cline { 2 - 5 } & $S_{01_{1}}=38.08$ & $S_{01_{2}}=287.92$ & $S_{0 x 1}=411.16$ & $S_{0 x 2}=1536.24$ \\
\cline { 2 - 5 } & $S_{1 \times 1}=39360.68$ & $S_{1 x_{2}}=22356.52$ & & \\
\hline
\end{tabular}

Source: Murthy, 1967

Table 2: Population 2 ( $y_{0}$ : Area (in 000 Hectare), $y_{1}$ : Production ( in 000 MT), $x$ : Productivity (MT/Hectare))

\begin{tabular}{|c|c|c|c|c|}
\hline & $n_{1}=4$ & $n_{2}=4$ & $N_{1}=10$ & $N_{2}=10$ \\
\cline { 2 - 5 } & $\bar{Y}_{01}=6.2$ & $\bar{Y}_{02}=80.67$ & $\bar{Y}_{11}=3.53$ & $\bar{Y}_{12}=111.61$ \\
\cline { 2 - 5 } $\mathrm{N}=20$ & $\bar{X}_{1}=0.5$ & $\bar{X}_{2}=1.41$ & $S_{y_{01}}=1.2$ & $S_{y_{02}}=10.82$ \\
\cline { 2 - 5 } $\mathrm{n}=8$ & $S_{y_{11}}=74.87$ & $S_{y_{12}}=66.35$ & $S_{x 1}=615.92$ & $S_{x_{2}}=340.38$ \\
\cline { 2 - 5 } & $S_{01_{1}}=1.75$ & $S_{01_{2}}=-92.02$ & $S_{0 x 1}=-0.02$ & $S_{0 x 2}=-7.04$ \\
\cline { 2 - 5 } & $S_{1 x 1}=1.60$ & $S_{1 x_{2}}=144.87$ & & \\
\hline
\end{tabular}

Source: National Horticulture Board of India, Official Web Site, http://nhb.gov.in/statistics/areaproduction-statistics.html

Table 3: Percent Relative Efficiencies of $\widehat{R}_{s t}$ and $T_{1 s t}$ with respect to $\widehat{R}_{s t}$

\begin{tabular}{|c|c|c|}
\hline Estimator & $\hat{R}_{s t}$ & $T_{1 s t}$ \\
\hline Population 1 & 100 & 9274.573 \\
\hline Population 2 & 100 & 400747.3 \\
\hline
\end{tabular}




\section{RAJESH TAILOR \& SUNIL CHOUHAN}

\section{References}

Murthy, \& Singh, M. P. (1965).On the estimation of ratio and product of the population parameters. Sankhya, B, 27, 321-328.

Official

National Horticulture Board India.

http://nhb.gov.in/statistics/area-productionstatistics.html.

Rao, J. N. K., \& Pereira, N. P. (1968).On double ratio estimators. Sankhya, A, 30, 83-90.

Ray, S. K., \& Singh, R. K. (1985).Some estimators for the ratio and product of population parameters. Journal of the Indian Society of Agricultural Statistics, 37(1), 1-10.

Shah, S. M., \& Shah, D. N. (1978). Ratio-cum-product estimator for estimating ratio (product) of two population parameters. Sankhya, C, 40(2), 156-166.
Sindhu, S. S., Tailor, R., \& Singh, S. (2009). On the estimation of population proportion. Applied Mathematical Science, 3(35), 1739-1744.

Singh, G. N., \& Rani, R. (2005, 2006). Some linear transformations on auxiliary variable for estimating the ratio of two population means in sample surveys. Model Assisted. Statistics and Applications, 1(1), IOS Press, 1-5.

Upadhyaya, L. N., \& Singh, H. P. (1985). A class of estimators using auxiliary information for estimating ratio of two finite population means. Gujarat Statistical Review, $12(2), 7-16$.

Upadhyaya, L. N., Singh, H. P., \&Vos, J. W. E. (1985). On the estimation of population means and ratios using supplementary information. Statistica. Neerlandica, 39(3), 309318. 


\section{JMASM Algorithms and Code JMASM32: SAS Template for Single-Subject Experimental Designs}

\author{
Hyewon Chung \\ Chungnam National University, \\ Deajeon, Korea
}

\author{
Jiseon Kim \\ University of Washington, \\ Seattle, WA
}

\author{
Ryoungsun Park \\ University of Texas, \\ Austin, TX
}

Meta-analysis has been used to synthesize research findings and to evaluate the effectiveness of treatments or the accuracy of diagnostic tools. Although meta-analytic techniques were developed to synthesize the results of several studies, controversy exists as to how to quantify the results from singlesubject experimental designs (SSEDs). The most commonly used metrics are reviewed, including nonregression and regression based methods. The application of the SAS template is demonstrated through simulated data sets. The SAS templates can be modified to accommodate a more complex data structure.

Key words: Single-subject experimental designs (SSED), SAS template.

\section{Introduction}

Meta-analysis is a "statistical analysis of a large collection of results from individual studies for the purpose of integrating the findings" (Glass, 1976, p. 3). Meta-analyses have been adapted in a variety of settings, such as, special education (Swanson \& Sachse-Lee, 2000), counseling (Hagen \& Nordahl, 2008) and behavioral intervention research (Filter \& Horner, 2009).

Hyewon Chung is an Assistant Professor in the Department of Education at Chungnam National University, Daejeon, Korea. Her research interests are multilevel models, psychometric models and single case design. Email her at: hyewonchung7@gmail.com. Jiseon Kim is a Post-Doctoral researcher in the Department of Rehabilitation Medicine at University of Washington, Seattle, WA, USA. Her research interests are item response theory, computerized adaptive testing, multistage testing and automated test assembly. Email her at: jiseonk@u.washington. edu. Ryoungsun Park is a Ph.D. student in the Department of Educational Psychology at University of Texas, Austin, TX, USA. His research interests are automated test assembly, linear programming, item response theory and computerized adaptive testing. Email him at: rosepark222@gmail.com.
The majority of literature on metaanalyses focuses on group comparison experimental studies; single-subject experimental designs (SSEDs) studies are frequently excluded. Although these metaanalytic techniques effectively summarize and evaluate the results of studies with relatively large sample sizes, on-going controversy exists as to synthesizing results from SSEDs (Faith, Allison \& Gorman, 1996). SSED research has grown in popularity in educational and psychological research designed to assess a treatment's effect and different approaches for analyzing single-subject data have been considered over the past decades.

SSEDs have been traditionally evaluated by visual analysis. Bulté and Onghena (2011) suggested that an investigation of treatment effects starts with a visual exploration of the single-case data as opposed to subjective interpretation based on visual analysis; however, evidence-based practice (EBP) emphasizes the importance of more objective outcome measures, especially magnitude of effect indices or effect sizes (Horner, et al., 2005). Methodologists have developed a number of quantitative descriptors as options to describe a treatment's effect in SSEDs. Two main types of statistical summary approaches have been proposed: non-regression based and regression based approaches (Wendt, 2009). Although several metrics have been developed and used 


\section{CHUNG, KIM \& PARK}

for SSEDs, a question remains regarding what effect size metrics are most appropriate to measure effect size for SSEDs. Thus, this study was designed to develop a SAS procedure to test several metrics to compare their performance.

Regression Based Approaches: Four-Parameter Model

Huitema and McKean (2000) modified the piece-wise regression model by Center, Skiba and Casey (1985-1986) and introduced further regression coefficients that describe change in intercepts as well as in slope from the baseline (A) to treatment (B) phase:

$$
Y_{t}=\beta_{0}+\beta_{1} T_{t}+\beta_{2} D_{t}+\beta_{3}\left[T_{t}-\left(n_{1}+1\right)\right] D_{t}+e_{t}
$$

$Y_{t}$ represents outcome score at time $t, T_{t}$ is the time/session point, $D$ indicates phase either A or $\mathrm{B}, n_{l}$ is the number of time points in baseline, $\beta_{0}$ represents baseline intercept (i.e., $Y$ at time $=0$ ), $\beta_{1}$ expresses baseline linear trend, $\beta_{2}$ indicates difference in intercept predicted from treatment phase data and that predicted for time $=n_{1}+1$ from baseline phase data, and $\beta_{3}$ represents difference in slope between baseline and treatment, $e_{t} \sim N\left(0, \sigma^{2}\right)$. Thus, $\beta_{2}$ and $\beta_{3}$ provide estimates of a treatment's effect on intercept and on slope, respectively (Wendt, 2009).

The four parameter model interprets the results using $\Delta \mathrm{R}^{2}$ approaches, which can be converted to Cohen's $d$, and uses all data in both baseline and treatment phases (Wendt, 2009). The regression based approaches assume normality, equal variance and serial independence. Because these are not usually met by SSED data, careful interpretation is required depending on whether the data met these assumptions.

Regression Based Approaches: Multilevel Modeling

Researchers have proposed a multilevel modeling approach as an option for combining SSED data (Ferron, et al., 2009; Ferron, Farmer \& Owens, 2010; Shadish \& Rindskopf, 2007; Van den Noortgarte \& Onghena, 2003a, 2003b). Repeated measures are nested within subjects; lowest level measures within subject effect change from baseline to intervention. The multilevel modeling approaches can explain why some subjects show more change than others; the variability among individuals in the size of treatment effects can be explained by including study and/or characteristics such as an individual's gender or study intervention type in the model. The multilevel modeling approach can resolve many of the limitations of traditional ordinary least square regression approaches (e.g., independent error assumption).

Non-Regression Based Approaches: Percentage of Non-Overlapping Data (PND)

Percentage of Non-Overlapping Data (PND) computes non-overlap between baseline and treatment phases (Scruggs, Mastropieri \& Casto, 1987). PND identifies the highest data point in the baseline and calculates the percentage of data points during treatment exceeding this level. If a study includes several experiments, PND scores are aggregated by taking the median rather than mean because scores are usually not distributed normally (Wendt, 2009). The typical interpretation of PND is: The higher the percentage, the more effective the treatment. Readers can find specific criteria for interpreting PND scores in Scruggs, Mastropieri, Cook and Escobar (1986). Although PND is easy to interpret, it ignores all baseline data except one data point and PND cannot detect slope changes (Wendt, 2009).

Non-Regression Based Approaches: Percentage of All Non-Overlapping Data (PAND)

Percentage of All Non-Overlapping Data (PAND) computes the total number of data points that do not overlap between baseline and treatment phases (Parker, Hagan-Burke \& Vannest, 2007). PAND determines overlapping data points: the minimum number that would have to be transferred across phases for complete data separation. It calculates $\%$ overlap by dividing the number of overlapping points by the total number of points and then subtracts this percent from 100 to obtain PAND (Wendt, 2009). Unlike PND, PAND uses all data points across both phases. It may be translated to $P h i$ and $P h i^{2}$ to determine an effect size such as Cohen's $d$ (Wendt, 2009). 
Non-Regression Based Approaches: Percentage of Data Points Exceeding the Median (PEM)

Percentage of Data Points Exceeding the Median (PEM) computes the percentage of data points exceeding the median of baseline phase (Ma, 2006). It first locates the median point in baseline data and then draws a horizontal middle line passing through the median of the baseline into the treatment phase. PEM calculates the percentage of treatment phase data points above the middle line if behavior increase is anticipated, and below the middle line if behavior decrease is anticipated (Wendt, 2009). It is assumed that - if treatment is ineffective data points will continually fluctuate around the middle line. The interpretation of PEM is: Values higher than 0.9 reflect highly effective treatment; values between 0.7 and 0.9 indicate moderately effective treatment; values less than 0.7 reflect questionable or ineffective treatment. In the presence of floor or ceiling data points, PEM reflects effect size while PND fails to reflect effect size. However, PEM is insensitive to magnitude of data points above the median, thus, it does not consider trend and variability in data points of treatment phase. It might reflect only partial improvement if an orthogonal slope exists in a baseline treatment pair after the first treatment phase (Wendt, 2009).

Non-Regression Based Approaches: Pairwise Data Overlap (PDO)

Pairwise Data Overlap (PDO) computes the overlap of all possible paired data comparisons between baseline and treatment phases (Parker \& Vannest, in press). PDO first compares the baseline data points with all treatment data points, and then it determines the number of overlapping and non-overlapping points. PDO calculates the total number of nonoverlapping points divided by the total number of comparison (Wendt, 2009). Compared to other indices, PDO takes slightly longer to calculate and requires that individual data point results be written down and added. The calculation is time consuming for long and crossed data series (Wendt, 2009).
Non-Regression Based Approaches: Percentage Reduction Data (PRD)

Percentage Reduction Data (PRD) computes the reduction of targeted behavior due to treatment (O'Brien \& Repp, 1990). PRD is also termed Mean Baseline Reduction (MBR, Campbell, 2003, 2004). PRD determines the mean of the last three data points from the baseline $(\mu \mathrm{A})$ and of the last three data points from the treatment $(\mu \mathrm{B})$. It computes the amount of change between the baseline and treatment:

$$
[(\mu \mathrm{A}-\mu \mathrm{B}) \div \mu \mathrm{A}] \times 100
$$

Non-Regression Based Approaches: Percentage of Zero Data (PZD)

Percentage of Zero Data (PZD) computes the degree to which treatment completely suppresses targeted behavior (Scotti, et al., 1991). PZD identifies the first data point to reach zero in the treatment phase. It computes the percentage of data points that remain at zero from the first zero point onwards (Wendt, 2009). Interpretation of PZD scores are: PZD lower than $18 \%$ reflects ineffectiveness; PZD between $18 \%$ - 54\% reflects questionable effectiveness; PZD between $55 \%$ - $80 \%$ reflects fair effectiveness; PZD higher than $80 \%$ reflects high effectiveness.

\section{SAS Template for SSEDs \\ Methodology \\ The majority of literature on single case} data noted that research on SSED effect size metrics requires the use of multiple metrics and the metrics are compared against each other. The comparisons must compare the metrics within the family of non-overlap as well as across regression versus non-regression based approaches. Although researchers are aware of the methodological issues regarding several SSED tools discussed, difficulties are frequently encountered in running and comparing the metrics due to the complexity of each metric involved. This study develops a SAS procedure consisting of several SAS macros to test a number of metrics for SSEDs. Four sets of single case data were generated using SAS macros written by Fan, Felsovalyi, Sivo and Keenan (2002). Table 1 shows one of the four 


\section{CHUNG, KIM \& PARK}

simulated $\mathrm{AB}$ datasets (condition 1). The total number of observations $\left(T_{t}\right)$ is 18 , Phase $\left(D_{t}\right)$ is coded with a value of 0 for baseline (A) and 1 for treatment $(\mathrm{B})$. In this dataset, the number of observations $\left(n_{1}\right)$ for $\mathrm{A}$ phase is 8 and the number of observations $\left(n_{2}\right)$ for B phase is 10 .

Table 1: Simulated AB Dataset

\begin{tabular}{|c|c|c|c|}
\hline $\begin{array}{c}\text { Outcome } \\
(\mathrm{Y})\end{array}$ & $T_{t}$ & $D_{t}$ & $\left.T_{t}-\left(n_{1}+1\right)\right] D_{t}$ \\
\hline 1.7 & 1 & 0 & 0 \\
\hline 1.9 & 2 & 0 & 0 \\
\hline 1.6 & 3 & 0 & 0 \\
\hline 1.6 & 4 & 0 & 0 \\
\hline 2.4 & 5 & 0 & 0 \\
\hline 3.9 & 6 & 0 & 0 \\
\hline 4.9 & 7 & 0 & 0 \\
\hline 4.4 & 8 & 0 & 0 \\
\hline 6.5 & 9 & 1 & 0 \\
\hline 8.1 & 10 & 1 & 1 \\
\hline 9.2 & 11 & 1 & 2 \\
\hline 10.1 & 12 & 1 & 3 \\
\hline 10.4 & 13 & 1 & 4 \\
\hline 10.1 & 14 & 1 & 5 \\
\hline 11.1 & 15 & 1 & 6 \\
\hline 12.6 & 16 & 1 & 7 \\
\hline 13.8 & 17 & 1 & 8 \\
\hline 15.4 & 18 & 1 & 10 \\
\hline & & & \\
\hline
\end{tabular}

The SAS procedure in this article provides standardized parameter estimates for Huitema and McKean's model (2000): standardized slope estimates for phase A and standardized intercept and slope estimates for phase B. It also calculates the following nonregression metrics described above: PND, PAND, PEM, PZD and PRD (see Appendix).

\section{Conclusion}

It is not surprising that single-case designs are becoming increasingly more popular in diverse fields given the emphasis on establishing an evidence base for practice. This is due to the difficulties encountered in conducting large group experimental studies. Recently, a single case design standard was released by What Works Clearinghouse (Kratochwill, Hitchcock, Horner, Levin, Odom, Rindskopf \& Shadish, 2010). Methodologists have developed several SSED metrics to assess and compare the efficiency of the design standards. Several methodological concerns have been expressed; controversy exists as to how to quantify the results of SSEDs. In addition, a few studies resulted in user friendly software capable of performing SSED metrics including both nonregression and regression based methods. The use of a SAS template developed in this study will assist researchers to calculate and compare most the commonly used SSED metrics and to synthesize the results from single-case datasets more efficiently.

\section{References}

Bulté, I., \& Onghena, P. (2011). R functions to facilitate the visual analysis of single-case data. Paper presented at the Annual Meeting of the American Educational Research Association, April, New Orleans, LA.

Campbell, J. M. (2003). Efficacy of behavioral interventions for reducing problem behavior in persons with autism: A quantitative synthesis of single-subject research. Research in Developmental Disabilities, 24(2), 120-138.

Campbell, J. M. (2004). Statistical comparison of four effect sizes for single-subject designs. Behavior Modification, 28(2), 234-246.

Center, B., Skiba, R., \& Casey, A. (1985-1986). A methodology for the quantitative synthesis of intra-subject design research. The Journal of Special Education, 19(4), 387-400.

Faith, M. S., Allison, D. B., \& Gorman, D. B. (1996). Meta-analysis of single-case research. In Design and analysis of single-case research, R. D. Franklin, D. B. Allison, \& B. S. Gorman (Eds.), 245-277. Mahwah, NJ: Lawrence Erlbaum. 
Fan, X., Felsovalyi, A., Sivo, S. A., \& Keenan, S. C. (2002). SAS for Monte Carlo Studies: A Guide for Quantitative Researchers. Cary, NC: SAS Institute Inc.

Ferron, J. M., Bell-Ellison, B. A., Rendina-Gobioff, G., Hibbard, S. T., \& Hess, M. R. (2009). Making treatment effect inferences from multiple baseline data: The utility of alternative multilevel modeling approaches. Behavior Research Methods, 41(2), 372-384.

Ferron, J. M., Farmer, J. L. \& Owens, C. M. (2010). Estimating individual treatment effects from multiple-baseline data: a Monte Carlo study of multilevel-modeling approaches. Behavior Research Methods, 42(4), 930-943.

Filter, K. J. \& Horner, R. H. (2009). Function-based academic interventions for problem behavior. Education and Treatment of Children, 32(1), 1-19.

Glass, G. V. (1976). Primary, secondary, and meta-analysis of research. Educational Researcher, 5(10), 3-8.

Hagen, R., \& Nordahl, H. M. (2008). Behavioral experiments in the treatment of paranoid schizophrenia: A single case study. Cognitive Behavioral Practice, 15(3), 296-305.

Horner, R. H., Carr, E. G., Halle, J., McGee, G., Odom, S., \& Wolery, M. (2005). The use of single-subject research to identify evidence-based practice in special education. Exceptional Children, 71(2), 165-179.

Huitema, B., \& McKean, J., ( (2000). Design specification issues in time-series intervention models. Educational and Psychological Measurement, 60(1), 38-58.

Kratochwill, T. R., et al. (2010). Singlecase designs technical documentation. Retrieved from What Works Clearinghouse website: http://ies.ed.gov/ncee/wwc/pdf/wwc_scd.pdf.

Ma, H. (2006). An alternative method for quantitative synthesis of single-subject researchers: Percentage of data points exceeding the median. Behavior Modification, 30(5), 598617.

O’Brien, S., \& Repp, A. C. (1990). Reinforcement based reductive procedures: A review of 20 years of their use with persons with severe or profound retardation. The Journal of the Association for Persons with Severe Handicaps, 15(3), 148-159.
Parker, R. I., Hagan-Burke, S., \& Vannest, K. (2007). Percentage of all nonoverlapping data (PAND): An alternative to PND. The Journal of Special Education, 40(4), 194-204.

Parker, R. I., \& Vannest, K. J. (in press). Pairwise data overlap for single case research. School Psychology Review.

Scotti, J. R., Evans, I. M., Meyer, L. H., \& Walker, P. (1991). A meta-analysis of intervention research with problem behavior: Treatment validity and standards of practice. American Journal on Mental Retardation, 96(3), 233-256.

Scruggs, T. E., Mastropieri, M. A., \& Casto, G. (1987). The quantitative synthesis of single subject research methodology: Methodology and validation. Remedial and Special Education, 8(2), 24-33.

Scruggs, T. E., Mastropieri, M. A., Cook, S. B., \& Escobar, C. (1986). Early intervention for children with conduct disorders: A quantitative synthesis of single-subject research. Behavioral Disorders, 11, 260-271.

Shadish, W. R., \& Rindskopf, D. M. (2007). Methods for evidence-based practice: Quantitative synthesis of single-subject designs. New Directions for Evaluation, 113, 95-109.

Swanson, H. L., Sachse-Lee, C. (2000). A meta-analysis of single-subject design intervention research for students with LD. Journal of Learning Disabilities, 33(2), 114-36.

Van den Noortgarte, W., \& Onghena, P. (2003a). Combining single-case experimental data using hierarchical linear models. School Psychology Quarterly, 18(3), 325-346.

Van den Noortgarte, W., \& Onghena, P. (2003b). Hierarchical linear models for the quantitative integration of effect sizes in singlecase research. Behavior Research Methods, Instruments and Computers, 35(1), 1-10.

Wendt, O. (2009). Calculating effect sizes for single-subject experimental designs: An overview and comparison. Paper presented at the $7^{\text {th }}$ Annual International Campbell Collaboration Colloquium, May 2011. Oslo, Norway. 


\section{CHUNG, KIM \& PARK}

Appendix: SAS Procedure

The SAS procedure provides standardized regression parameter estimates as well as PND, PAND, PEM, PZD and PRD.

OPTIONS LINESIZE=100 NOSOURCE NOSOURCE2 NONOTES;

LIBNAME AUTOREG 'C: $\backslash$ Users $\backslash$ Desktop \Single Case';

\%MACRO AUTOREG_N (ITER, N1,N2,N, RES,AR, ERR, BETA1, BETA2, BETA3, CONDIT) ;

/* Generate Dataset */;

DATA TEMP1;

ARRAY SERIEA $\{\& N\}$ SERIEA1-SERIEA\&N;

$\operatorname{SERIEA}(1)=\operatorname{RANNOR}(-1)$;

DO J $=2$ TO $\& N$;

$\operatorname{SERIEA}(J)=\operatorname{RANNOR}(-11) * \operatorname{SQRT}(\& R E S)+\operatorname{SERIEA}(J-$

1) * $\operatorname{SQRT}(\& A R)$;

END;

KEEP SERIEA1-SERIEA\&N;

OUTPUT;

PROC TRANSPOSE DATA=TEMP1 OUT=TEMP1;

DATA TEMP1;

SET TEMP1;

$\mathrm{ID}=\mathrm{N}_{-} ;$

SERIE $\overline{\mathrm{S} A}=\mathrm{COL} 1$;

OUTPUT;

DROP COLI NAME_;

DATA TEMP2;

TIME $=1$;

DO ID $=1$ TO $\& N$;

TIME = ID;

IF ID $<=\& N 1$ THEN $D=0$;

ELSE D $=1 ;$

TIMED $=($ TIME $-(\& N 1+1)) * D ;$

$\mathrm{Y}=\mathrm{TIME} * \operatorname{SQRT}(\& B E T A 1)+\mathrm{D} * \operatorname{SQRT}(\& B E T A 2)+$

TIMED*SQRT (\&BETA3);

OUTPUT ;

END;

DATA INPUT1;

MERGE TEMP1 TEMP2;

BY ID; 


\section{SAS TEMPLATE SSED}

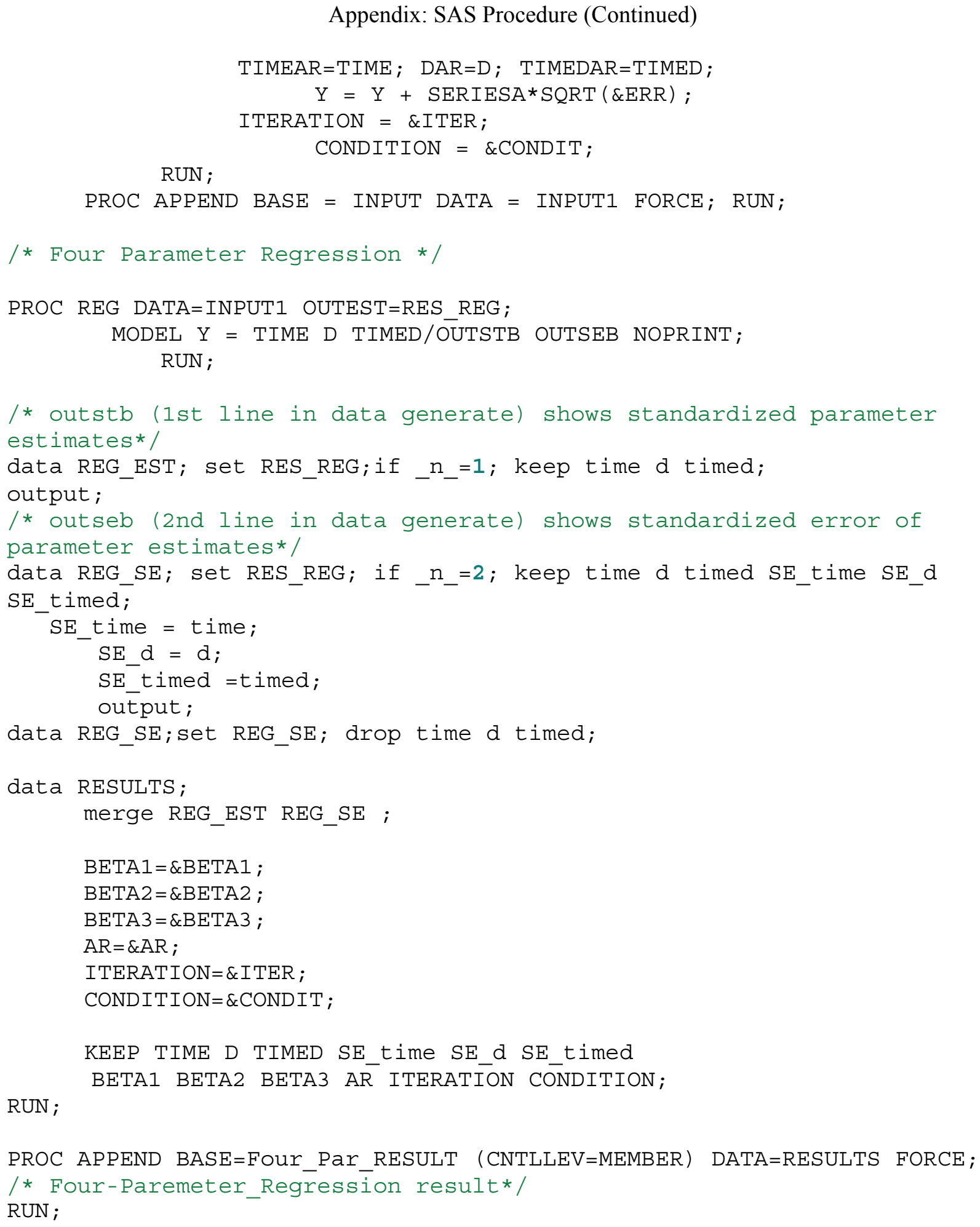




\section{CHUNG, KIM \& PARK}

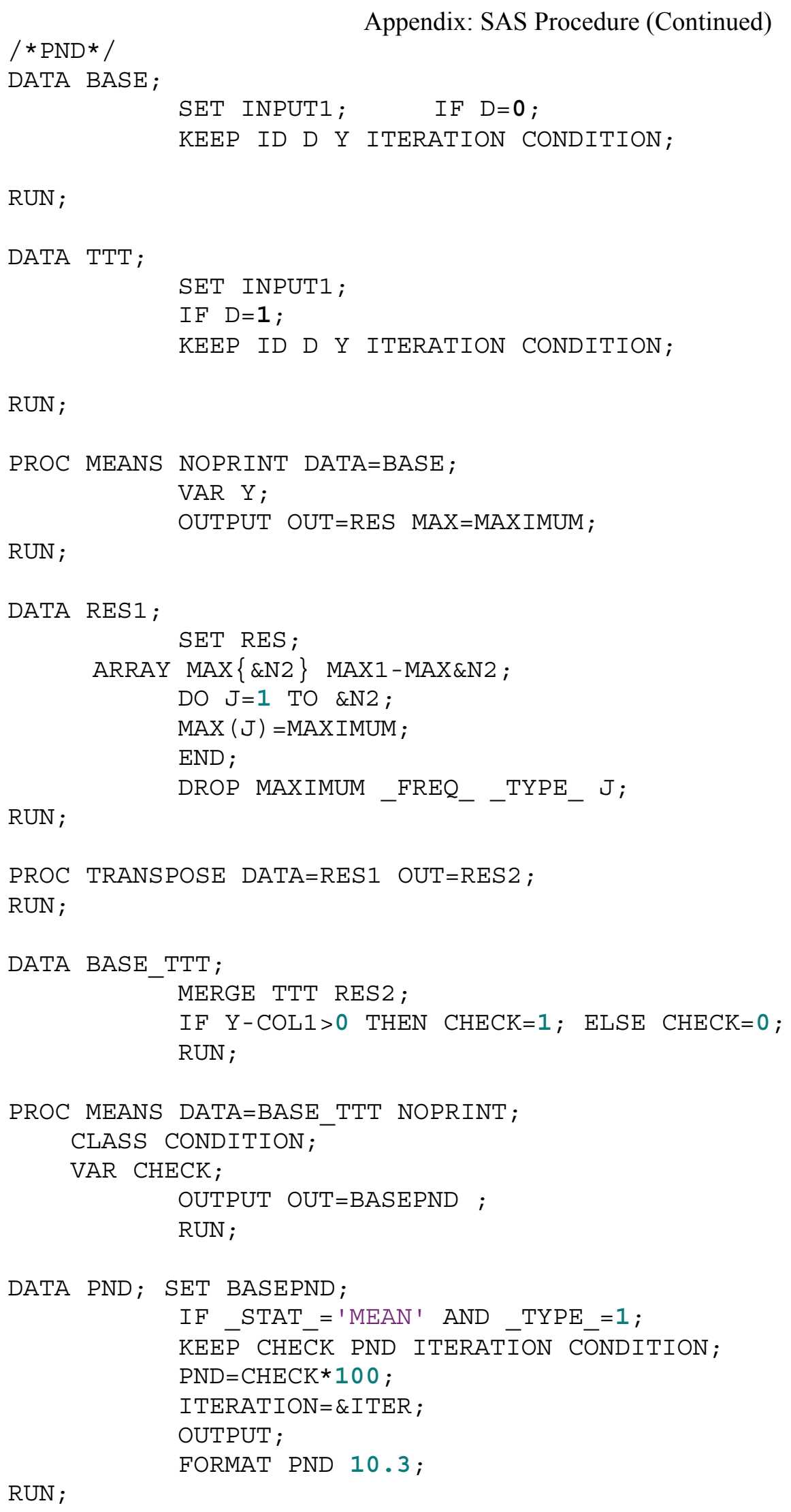

RUN ;

DATA TTT;

RUN ;

PROC MEANS NOPRINT DATA=BASE;

VAR Y;

OUTPUT OUT=RES MAX=MAXIMUM;

RUN ;

DATA RES1;

RUN ; 


\section{SAS TEMPLATE SSED}

\section{Appendix: SAS Procedure (Continued)}

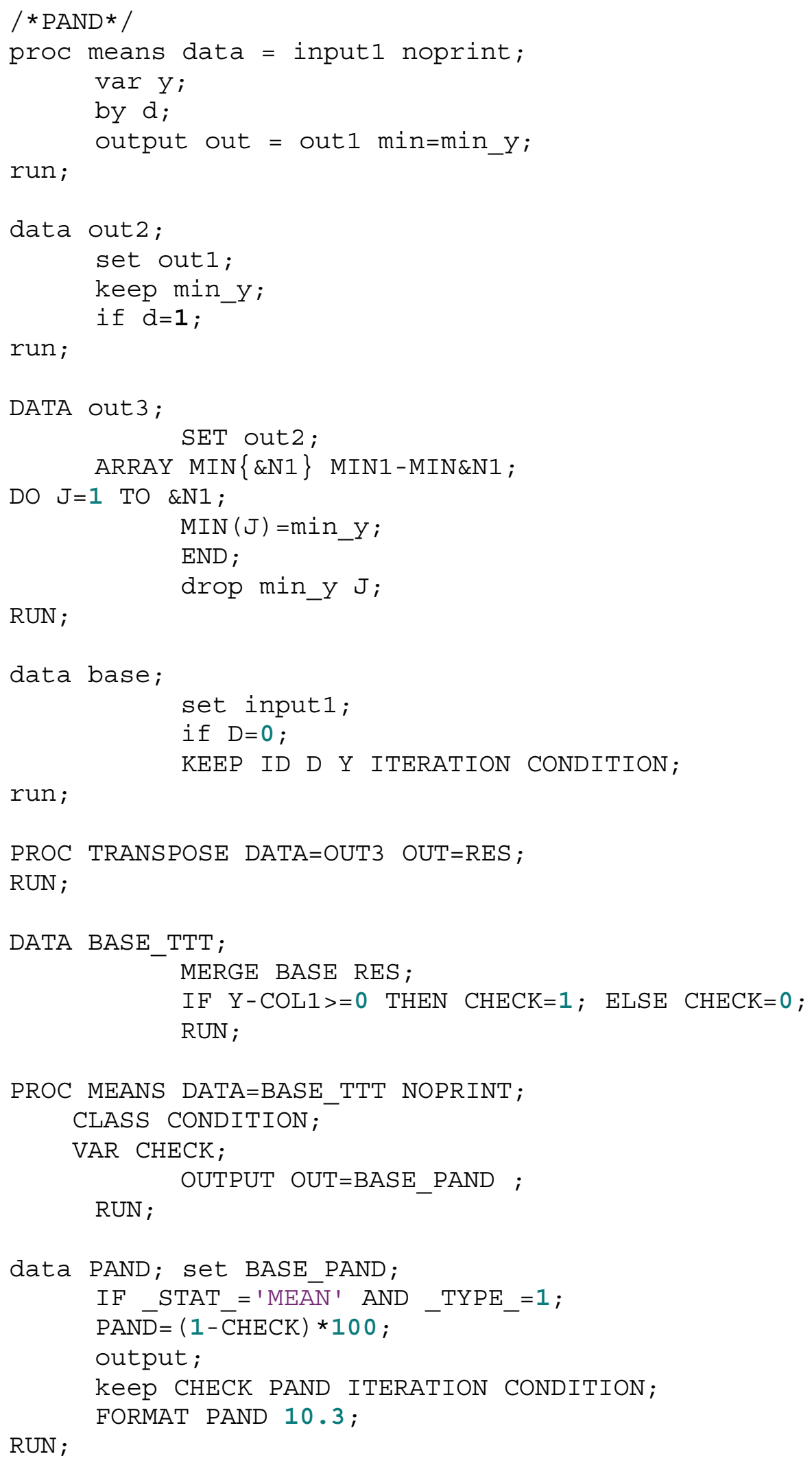




\section{CHUNG, KIM \& PARK}

\section{Appendix: SAS Procedure (Continued)}

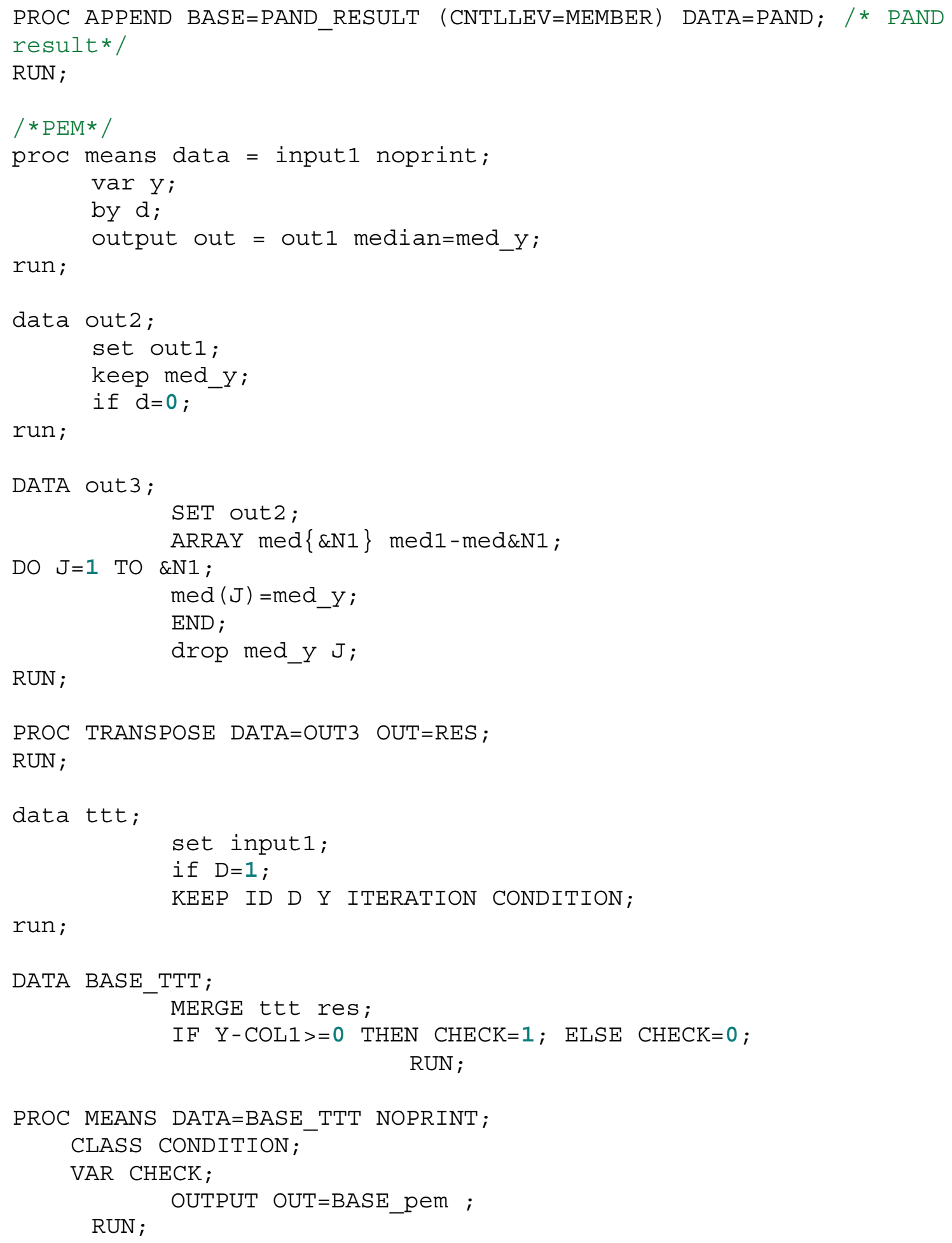




\section{SAS TEMPLATE SSED}

Appendix: SAS Procedure (Continued)

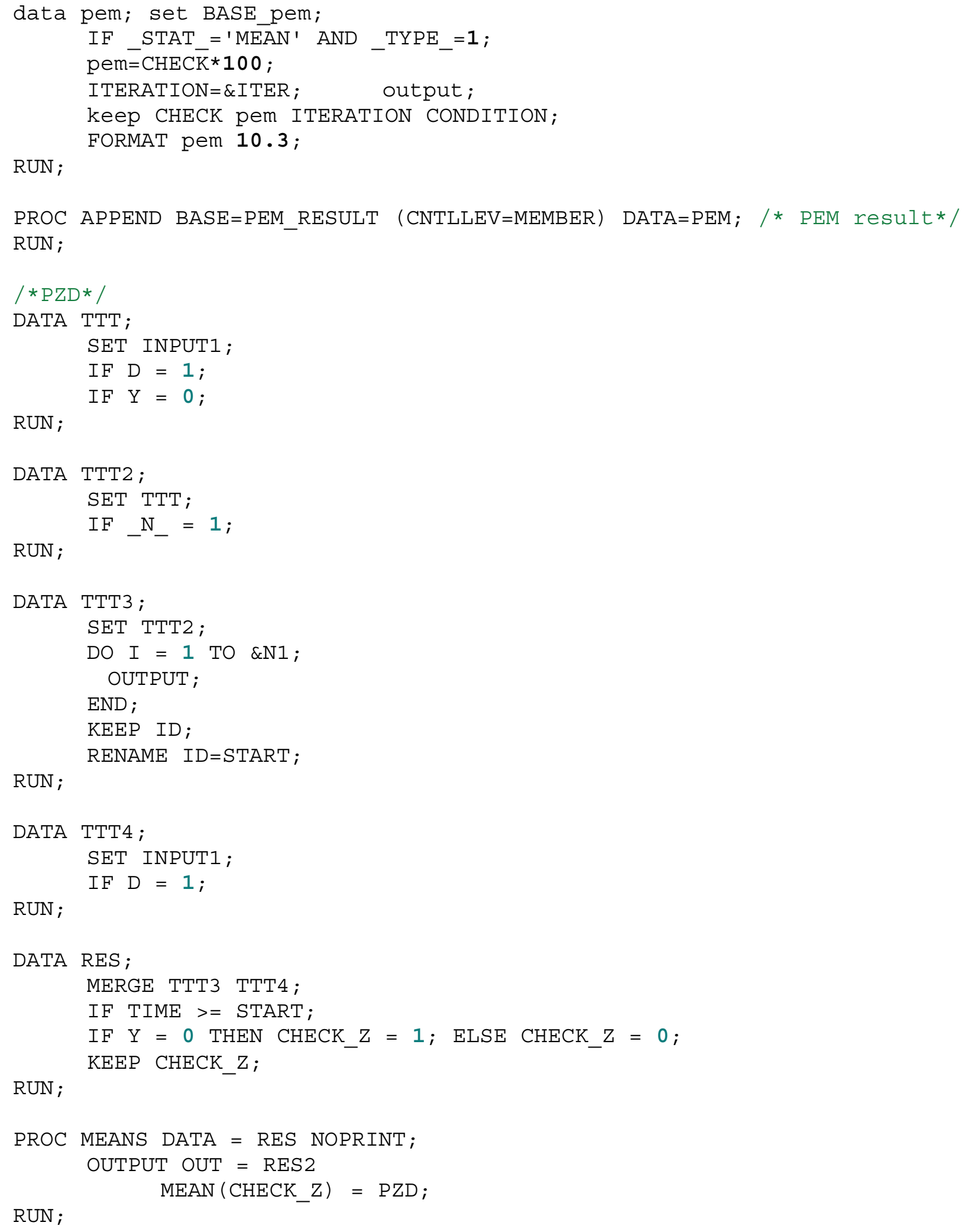




\section{CHUNG, KIM \& PARK}

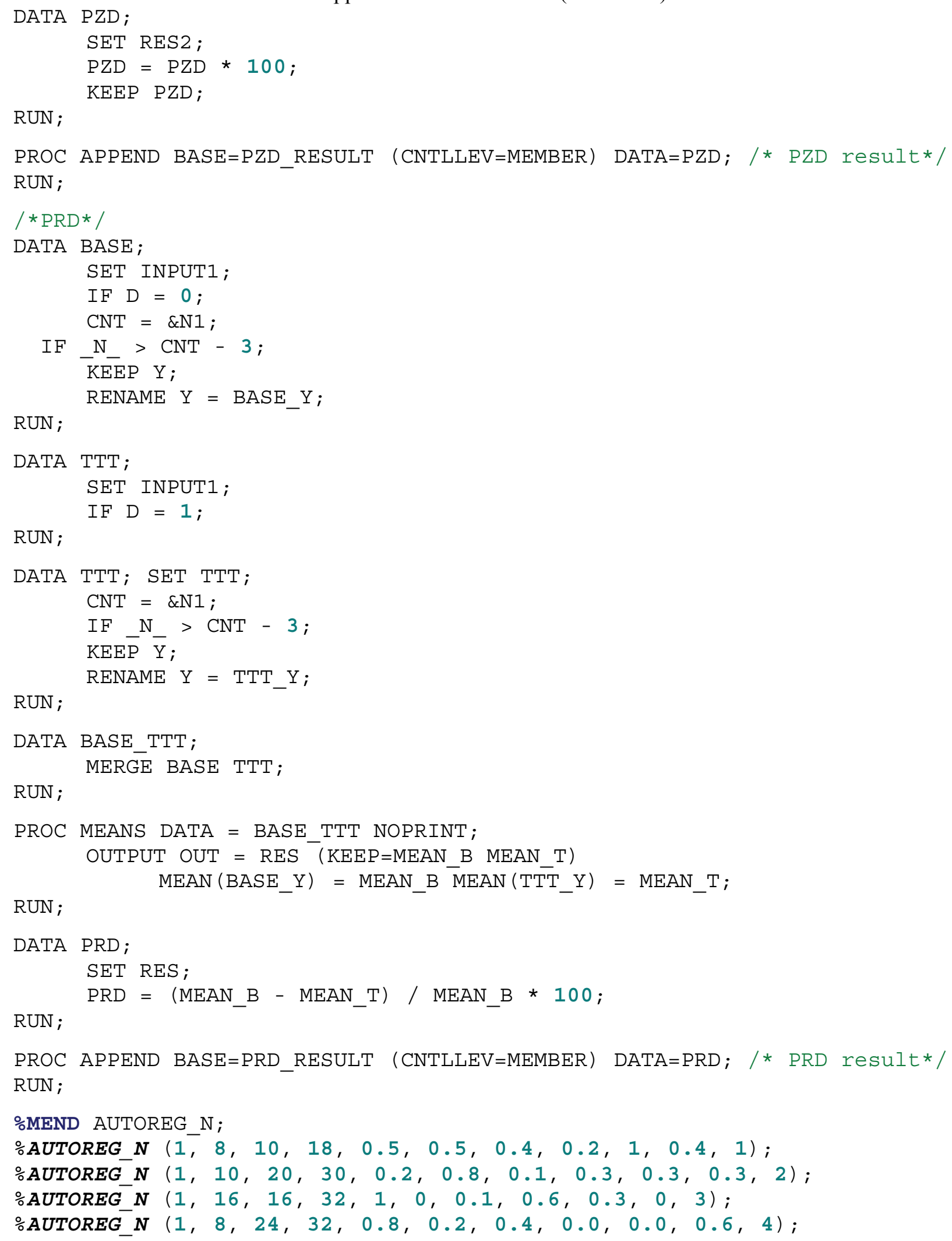




\section{Instructions For Authors}

Follow these guidelines when submitting a manuscript:

1. JMASM uses a modified American Psychological Association style guideline.

2. Submissions are accepted via e-mail only. Send them to the Editorial Assistant at ea@jmasm.com. Provide name, affiliation, address, e-mail address, and 30 word biographical statements for all authors in the body of the email message.

3. There should be no material identifying authorship except on the title page. A statement should be included in the body of the e-mail that, where applicable, indicating proper human subjects protocols were followed, including informed consent. A statement should be included in the body of the e-mail indicating the manuscript is not under consideration at another journal.

4. Provide the manuscript as an external e-mail attachment in MS Word for the PC format only. (Wordperfect and .rtf formats may be acceptable - please inquire.) Please note that Tex (in its various versions), Exp, and Adobe .pdf formats are designed to produce the final presentation of text. They are not amenable to the editing process, and are NOT acceptable for manuscript submission.

5. The text maximum is 20 pages double spaced, not including tables, figures, graphs, and references. Use 11 point Times New Roman font.

6. Create tables without boxes or vertical lines. Place tables, figures, and graphs "in-line", not at the end of the manuscript. Figures may be in .jpg, tif, .png, and other formats readable by Adobe Photoshop.

7. The manuscript should contain an Abstract with a 50 word maximum, following by a list of key words or phrases. Major headings are Introduction, Methodology, Results, Conclusion, and References. Center headings. Subheadings are left justified; capitalize only the first letter of each word. Sub-subheadings are left-justified, indent optional. Do not number headings or subheadings.

8. Number all formulas, tables, figures, and graphs, but do not use italics, bold, or underline.

9. Do not use underlining in the manuscript. Do not use bold, except for (a) matrices, or (b) emphasis within a table, figure, or graph. Do not number references. Do not use footnotes or endnotes.

10. In the References section, do not put quotation marks around titles of articles or books. Capitalize only the first letter of books. Italicize journal or book titles, and volume numbers. Use " $\&$ " instead of "and" in multiple author listings.

11. Suggestions for style: Instead of "I drew a sample of 40 " write "A sample of 40 was selected". Use "although" instead of "while", unless the meaning is "at the same time". Use "because" instead of "since", unless the meaning is "after". Instead of "Smith (1990) notes" write "Smith (1990) noted". Do not strike spacebar twice after a period.

\section{Print Subscriptions}

Print subscriptions including postage for professionals are US \$95 per year; for graduate students are US $\$ 47.50$ per year; and for libraries, universities and corporations are US \$195 per year. Subscribers outside of the US and Canada pay a US \$10 surcharge for additional postage. Online access is currently free at http://www.jmasm.com/. Mail subscription requests with remittances to JMASM, P. O. Box 48023, Oak Park, MI, 48237. Email journal correspondence, other than manuscript submissions, to ea@jmasm.com.

\section{Notice To Advertisers}

Send requests for advertising information to ea@jmasm.com. 